DIFFUSION OF HELIUM ISOTOPES IN SILICATE GLASSES AND MINERALS: IMPLICATIONS FOR PETROGENESIS AND GEOCHRONOLOGY

$$
\begin{aligned}
& \text { by } \\
& \text { THOMAS W. TRULL } \\
& \text { B.S., University of Michigan, } 1981 \\
& \text { submitted in partial fulfillment } \\
& \text { of the requirements for the degree of } \\
& \text { DOCTOR OF PHILOSOPHY } \\
& \text { at the } \\
& \text { MASSACHUSETTS INSTITUTE OF TECHNOLOGY } \\
& \text { and the }
\end{aligned}
$$

WOODS HOLE OCEANOGRAPHIC INSTITUTION

$$
\text { April, } 1989
$$

(C) Thomas W. Trull

The author hereby grants to MIT and WHOI permission to reproduce and distribute copies of this thesis document in whole or in part.

Signature of Author:

Joint Program in 0ceanography, Massachusetts Institute of Technology/Woods Hole Oceanographic Institution, and the Department of Earth, Atmospheric, and Planetary Sciences, Massachusetts Institute of Technology.

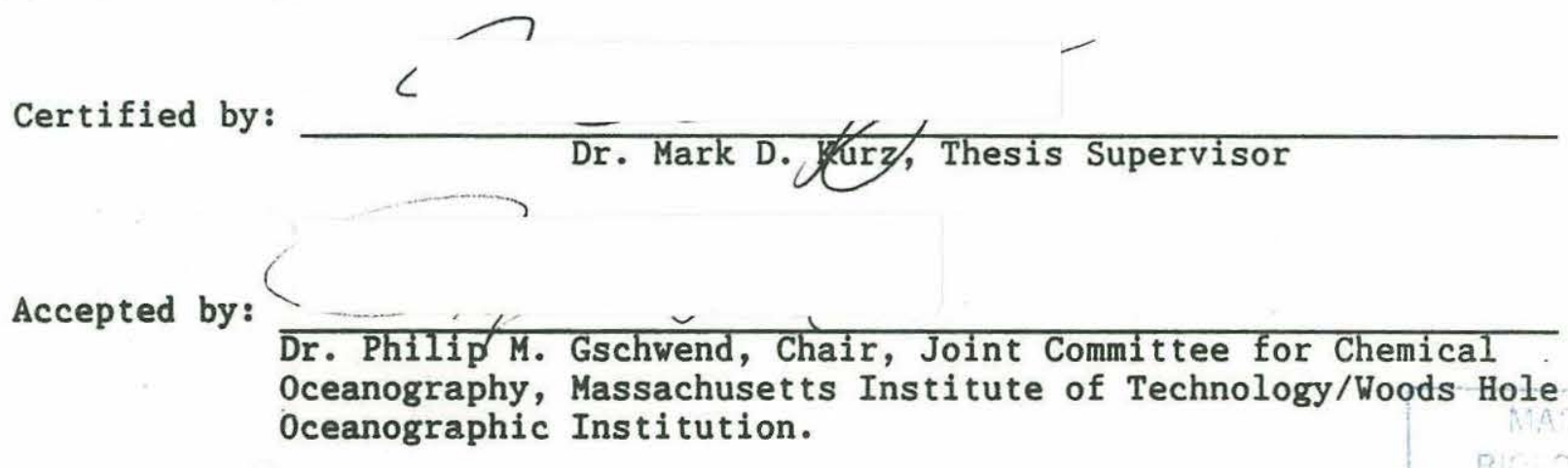
Oceanographic Institution.
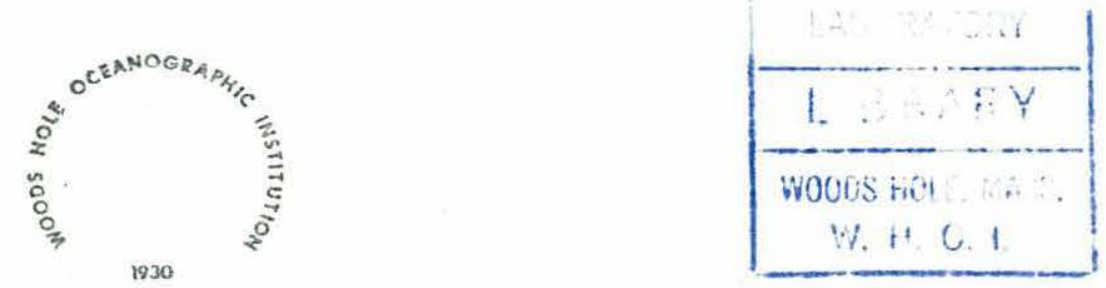



\section{DIFFUSION OF HELIUM ISOTOPES IN SILICATE GLASSES AND MINERALS: IMPLICATIONS FOR PETROGENESIS AND GEOCHRONOLOGY}

by

THOMAS W. TRULL

\section{ABSTRACT}

Helium mobility in geologic materials is a fundamental constraint on the petrogenetic origins of helium isotopic variability and on the application of radiogenic and cosmogenic helium geochronology.

${ }^{3} \mathrm{He}$ and ${ }^{4} \mathrm{He}$ volume diffusivities determined at $25-600^{\circ} \mathrm{C}$ in basaltic glasses by incremental-heating and powder storage experiments (using a diffusion model incorporating grain size and shape information to obtain high precision) are three to four orders of magnitude greater than for common cations. Diffusion in tholeiitic glass can be described by an Arrhenius relation with activation energy $=16.85 \pm .13 \mathrm{Kcal} / \mathrm{mole}$ and $\log \mathrm{D}$ 。 $=-2.37 \pm .06$, although low temperature data are better described by a dis $-^{\circ}$ tribution of activation energies model. The best estimate for $D$ at $0^{\circ} \mathrm{C}$ in tholeitic glass is $5 \pm 2 \times 10^{-16} \mathrm{~cm}^{2} / \mathrm{s}$, an order of magnitude higher than the results of Kurz and Jenkins (1981) but lower than suggested by Jambon, Weber and Begemann (1985). Measurements in an alkali basalt show that helium diffusion is composition dependent $\left(E_{2}=14.4 \pm .5 \mathrm{Kcal} / \mathrm{mole} ; \log \mathrm{D}_{0}=\right.$ $3.24 \pm .2$ ), and roughly five times faster than in tholeites at seafloor temperatures. The corresponding timescales for $50 \%$ helium loss or exchange with seawater ( $1 \mathrm{~cm}$ spheres) are about one million years for mid-oceanridge-basalts, and about 100,000 years in seamount alkali basalts. Radiogenic ${ }^{4} \mathrm{He}$ diffusion has a higher activation energy $\left(27 \pm 2 \mathrm{Kcal} / \mathrm{mole} ; \log \mathrm{D}_{0}=\right.$ $+2.4 \pm 1.0$ ) than inherited (magmatic) helium, suggesting very low mobility (D $=3 \times 10^{-19} \mathrm{~cm}^{2} / \mathrm{s}$ at $0^{\circ} \mathrm{C}$; factor of 5 uncertainty) and that $\mathrm{U}+\mathrm{Th} /{ }^{4} \mathrm{He}$ geochronology of fresh seafloor basalt glasses is unlikely to be hampered by helium loss.

Measured isotopic diffusivity ratios, $D^{3} \mathrm{He} / D^{4} \mathrm{He}$, are not composition dependent, average $1.08 \pm .02$, and vary slightly with temperature, consistent with an activation energy difference of $60 \pm 20 \mathrm{cal} / \mathrm{mole}$. This result differs from the inverse-square-root of mass prediction of 1.15 , and may be explained by quantization of helium vibrational energies. These results suggest preferential loss of ${ }^{3} \mathrm{He}$ will be minimal at low temperature $\left(D^{3} \mathrm{He} / D^{4} \mathrm{He}=1.02 \pm .03\right.$ at $\left.0^{\circ} \mathrm{C}\right)$. Therefore, alteration of magmatic ${ }^{3} \mathrm{He} /{ }^{4} \mathrm{He}$ ratios in basaltic glasses on the seafloor will occur only by helium exchange with seawater, and be important only for samples with low helium contents $\left(<10^{-8} \mathrm{ccSTP} / \mathrm{g}\right)$, such as those found in island arc environments. Extrapolating the glass results to magmatic temperatures yields diffusivities similar to melt values, and suggests $D^{3} \mathrm{He} / \mathrm{D}^{4} \mathrm{He}$ approaches 1.15 at these and higher temperatures.

Helium diffusivities in olivine and pyroxene at magmatic and mantle temperatures $\left(900-1400^{\circ} \mathrm{C}\right)$ are higher than for cations, $(E=100 \pm 5 \mathrm{Kcal} /$ $\mathrm{mole}, \log \mathrm{D}_{2}=+5.1 \pm .7 ;$ and $70 \pm 10 \mathrm{Kcal} / \mathrm{mole}, \log \mathrm{D}=+2.1 \pm 1.2$, respectively), but are still too low to transport or homogenize helium in the mantle 
or even in magma chambers. However, diffusion equilibrates melts and mantle minerals within decades, and interaction with wall-rocks may be enhanced for helium in comparison to other isotopic tracers because of its greater mobility. Rapid exchange of helium within xenoliths and with their host magmas set limits on origin depths and transport times for xenoliths which exhibit helium isotopic disequilibrium between minerals, or between the magma and the xenolith. Phenocrysts equilibrate helium too rapidly to exhibit zoned isotopic compositions, and are likely to retain magmatic helium quantitatively in rapidly cooled volcanic extrusives. The 100 -fold higher He diffusivity in pyroxene than olivine at $1000^{\circ} \mathrm{C}$ allows diffusive loss effects to be evaluated in more slowly cooled rocks, when cogenetic minerals can be measured.

Diffusivities of cosmic-ray produced ${ }^{3} \mathrm{He}$ in surface exposed rocks are several orders of magnitude higher than for inherited helium. However, activation energies for olivine and quartz, $25 \pm 4 \mathrm{Kcal} / \mathrm{mole}(\log \mathrm{D}=-$ $3.7 \pm .8)$ and $25.2 \pm .9 \mathrm{Kcal} / \mathrm{mole}(\log \mathrm{D}=+.2 \pm .4)$ respectively, still suggest low diffusivities at surface temperatures of approximately $10^{-22}$ and $10^{-20}$ $\mathrm{cm}^{2} / \mathrm{s}$. Equations for simultaneous helium production and diffusive loss allow model ages for surface exposure to be corrected for helium loss, and demonstrate that cosmogenic ${ }^{3} \mathrm{He}$ geochronology will not be limited by helium loss for timescales of approximately 1 million years in quartz and 10 million years or more in olivine. The measurements also suggest that radiogenic ${ }^{4} \mathrm{He}$ produced by $U$ and Th decay may be a useful dating method in quartz.

Application of the diffusion measurements demonstrates that part of the wide range of ${ }^{3} \mathrm{He} /{ }^{4} \mathrm{He}$ ratios (.01 to $9 \mathrm{R}$ ) of a suite of dredged basalts and andesites from the Woodlark Basin, (western Pacific) reflects post-eruptive helium addition, from seawater in glasses with low He contents and from $U$ and $T h$ decay in mafic mineral separates. In unaltered samples, ${ }^{3} \mathrm{He} /{ }^{4} \mathrm{He}$ ratios for tholeiites from the Woodlark Spreading Center are 8-9 R, similar to mid-ocean-ridges, but distinctly different than the ratio of $6.9 \pm .2 R$ observed in Kavachi submarine volcano basaltic andesites. Helium isotopic systematics in cogenetic pyroxenes and olivines from these samples demonstrate that this is a magmatic signature, and not the result of preferential ${ }^{3} \mathrm{He}$ loss by diffusion. Coupled $\mathrm{Sr}$ and $\mathrm{He}$ isotopic systematics in these and other samples from the region suggest the sub-arc mantle has been enriched in radiogenic helium supplied by subducted Pacific lithosphere.

Thesis Supervisor: Dr. Mark D. Kurz, Associate Scientist. 
This thesis is dedicated to Christine Coughanowr, without whom it might never have been completed. I thank her for love, late night assistance, financial support and listening to my complaints.

My thesis advisor, Mark Kurz, and his advisor, Bill Jenkins, made my time in the helium lab exciting. I thank them for insights, encouragement and patience. I thank Stan Hart, the other member of my thesis committee, for initiating my interest in hard rock geochemistry with an excellent course and for his continued interest in my work. I thank Bruno Giletti for stimulating comments and sharing his expertise with the details of diffusion measurements. Thanks to Michael Perfit, coauthor of chapter 6 , for his cheerful collaboration and unending encouragement. Thanks to Dempsey Lott for his instrumental ministrations and continual cheer, to Dave Graham for sharing his library and ideas, and to Billy Spitzer and Mirielle Polve for their interest and advice.

I thank the people who donated samples: Charlie Langmuir, Ray Binns, Michael Perfit, Bob Kay. Thanks to the Chemistry Department, and the WHOI Education office for providing financial support and a nice place to work. Parts of this research was funded by NSF grants OCE85-16082, EAR86-10611, and OCE87-16970.

Finally I thank my friends for all our great times together and especially my family for always being there. 


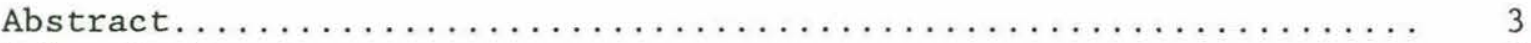

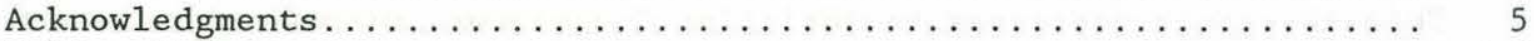

Table of Contents..............................

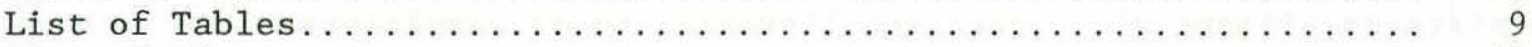

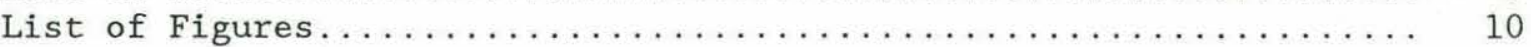

CHAPTER 1. INTRODUCTION

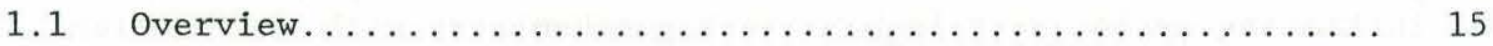

1.2 Terrestrial Helium Isotopic Systematics and Diversity...... 16

1.3 Origins of Helium Isotopic Variability in Oceanic

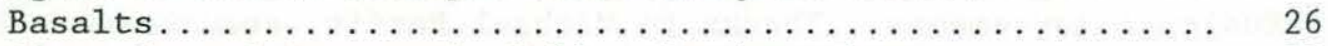

1.4 Radiogenic and Cosmogenic Helium Geochronology........... 28

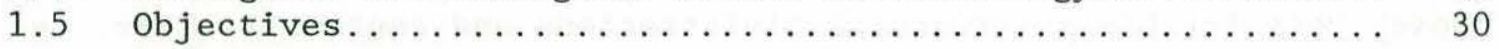

References............................. 32

CHAPTER 2. EXPERIMENTAL METHODS

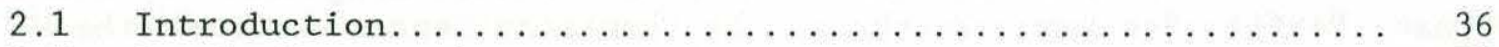

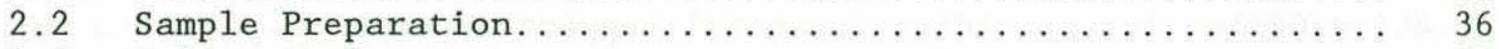

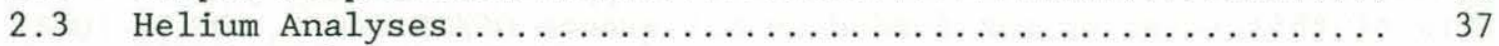

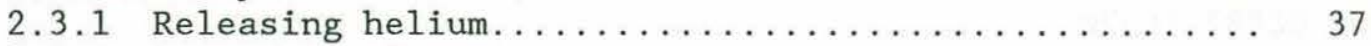

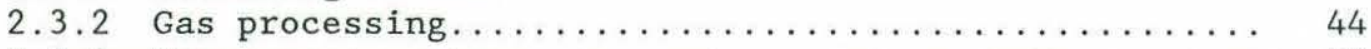

2.3.3 Mass spectrometry...................... 47

2.4 The Determination of Diffusion Coefficients from Emanation

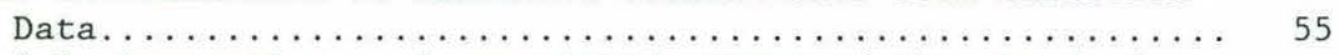

2.4.1 Assumptions and associated errors of the standard spherical model..................... 55

2.4.2 A refined model incorporating shape and size

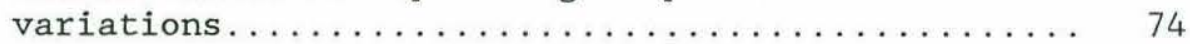

References................................. 74

CHAPTER 3. THE DIFFUSIVITY OF ${ }^{3} \mathrm{HE}$ and ${ }^{4} \mathrm{HE}$ IN BASALTIC GLASSES

3.1 Introduction. . . . .

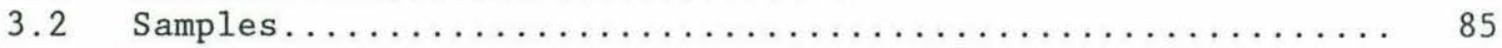

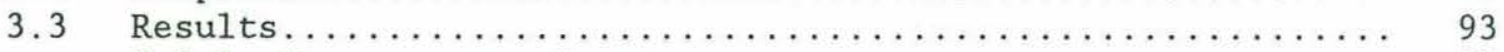

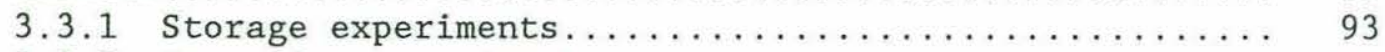

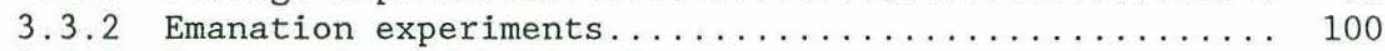

3.3.3 Systematics of helium loss................. 101

3.3.4 Calculation of diffusion coefficients from a standard model........................... 112

3.3.5 A refined model for diffusion coefficient

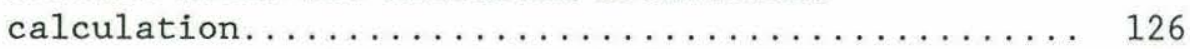

3.3.6 Temperature dependence of the helium diffusivity.... 132

3.3.7 Compositional effects on helium mobility......... 150

3.3.8 Diffusion of radiogenic helium............... 153

3.3.9 Isotopic fractionation during diffusion.......... 154

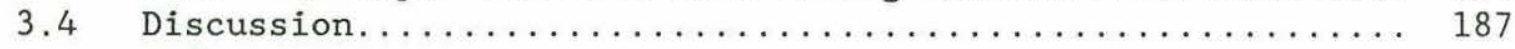

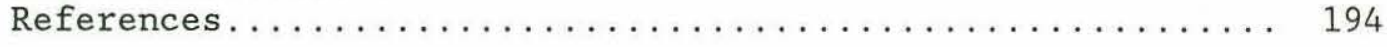


CHAPTER 4. DIFFUSION OF HELIUM IN OLIVINE AND PYROXENE AT MAGMATIC AND MANTLE TEMPERATURES

4.1 Introduction. . . . . . . . . . 201

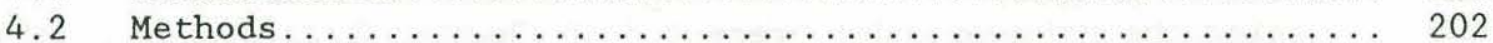

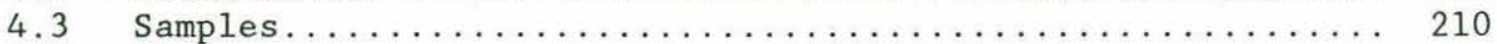

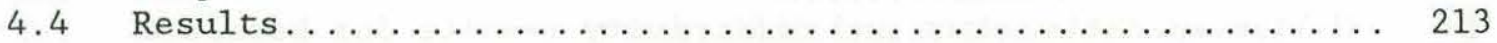

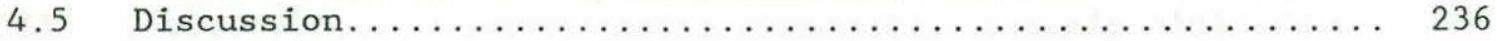

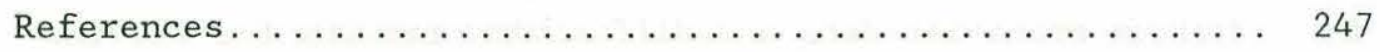

CHAPTER 5. DIFFUSION OF COSMOGENIC ${ }^{3} \mathrm{HE}$ IN OLIVINE AND QUARTZ:

IMPLICATIONS FOR SURFACE EXPOSURE DATING

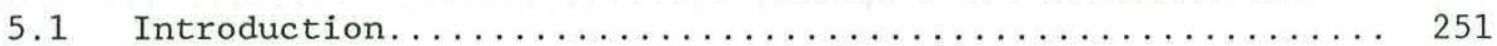

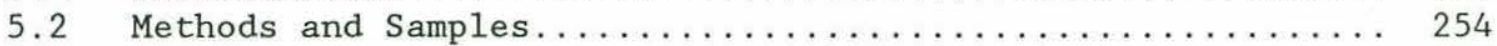

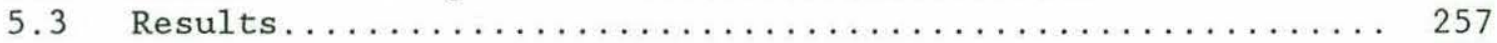

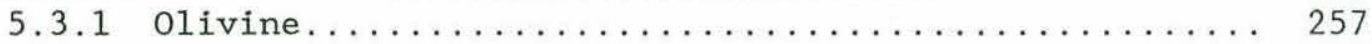

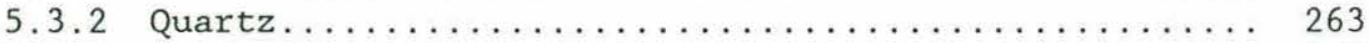

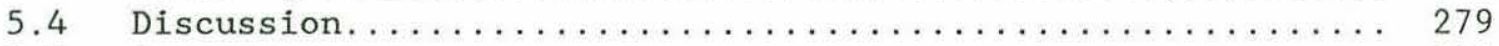

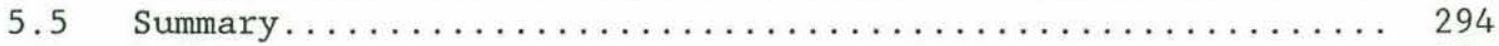

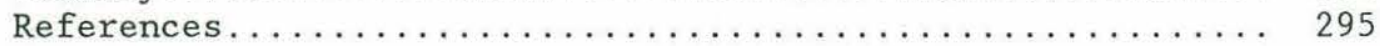

CHAPTER 6. HELIUM AND STRONTIUM ISOTOPIC CONSTRAINTS ON THE ORIGIN OF ISLAND ARC MAGMAS IN THE WOODLARK BASIN - SOLOMON ISLANDS REGION

6.1 Introduction. . . . . . . . . . . . . . . . . . . . . . 298 6.1.1 Petrogenisis in the Woodlark Basin - Solomon Islands

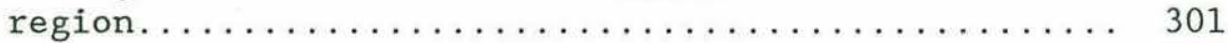

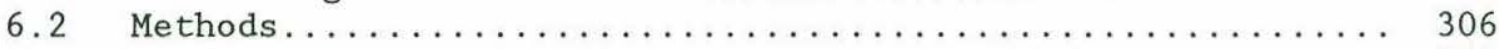

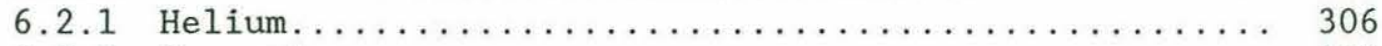

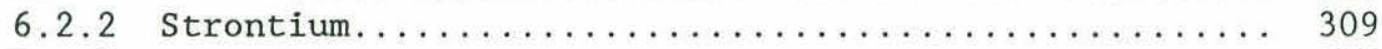

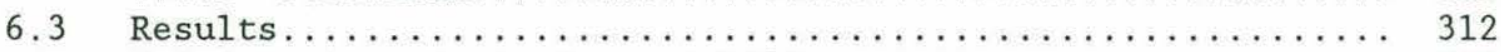

6.3.1 Helium isotopic variability............... 312

a. Alteration of magmatic ${ }^{3} \mathrm{He} /{ }^{4} \mathrm{He}$ ratios after eruption................... 315

b. Summary of the origins of He isotopic

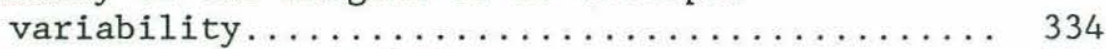

6.3.2 Strontium isotopic variability............. 336

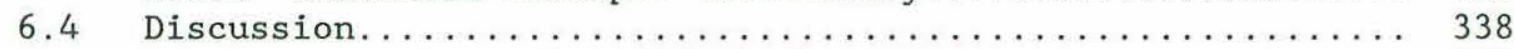

6.4.1 Magma degassing and chamber residence times....... 338

6.4.2 Sources of radiogenic $\mathrm{Sr}$ and $\mathrm{He}$ to WB-SI magmas...... 342

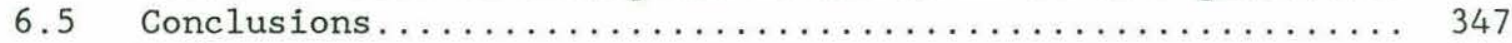

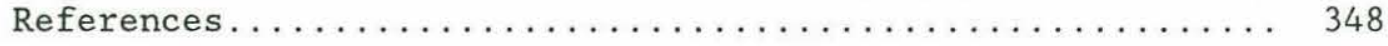


APPENDICES:

A: $\quad$ FORTRAN77 programs for calculating diffusion coefficients using the refined model presented in Chapter 2 . . . . . . 354

B: Helium emanation data and diffusivity results for basaltic basaltic glasses ......................... 373

C: Helium emanation data and diffusivity results for olivine and pyroxene at high temperature............... 391

D: Finite difference determination of simultaneous production and diffusion for a sphere................... 396 
3.1 Henry's law coefficients for helium solubility in basaltic

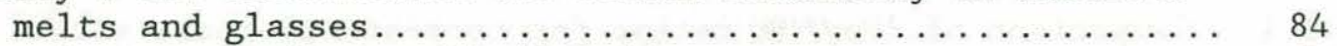

3.2a Sample descriptions and bulk helium contents........... 87

$3.2 \mathrm{~b}$ Chemical compositions of basaltic glasses............. 88

3.2c Molar compositions of major network formers/modifiers...... 88

3.3 Helium contents of powders stored at room temperature...... 97

3.4 Basaltic glass step-heating helium diffusivity results..... 102

3.5 Helium diffusivity estimates for basaltic glass........... 149

3.6 The isotopic diffusivity ratio of helium in tholeiitic tholeiitic glass......................... 171

\section{CHAPTER 4}

4.1 Sample compositions and bulk helium contents........... 212

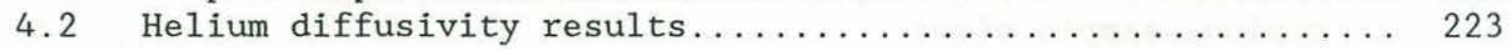

\section{CHAPTER 5}

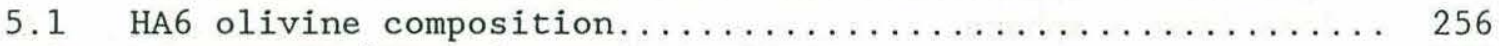

5.2 Cosmogenic ${ }^{3} \mathrm{He}$ release from olivine.................. 258

5.3 Cosmogenic ${ }^{3} \mathrm{He}$ and radiogenic ${ }^{4} \mathrm{He}$ from quartz............. 264

5.4 Total helium contents of BW84-105 quartz grains.......... 272

5.5 Diffusion parameters for cosmogenic ${ }^{3} \mathrm{He}$ and radiogenic ${ }^{4} \mathrm{He}$

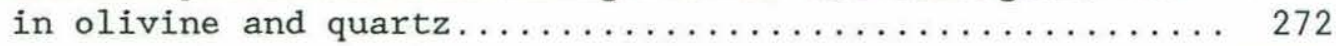

\section{CHAPTER 6}

6.1 Helium isotope data for Woodlark Basin basalts........... 310

6.2 Sr isotope and trace element data for Woodlark Basin

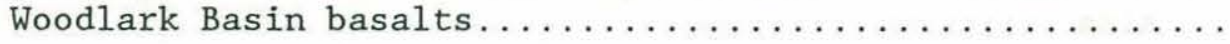




\section{CHAPTER 1}

1.1 Comparison of ${ }^{3} \mathrm{He} /{ }^{4} \mathrm{He}$ ratios for terrestrial rocks with likely primordial sources and Earth's atmosphere.........

1.2 Radioactive decay series for the production of ${ }^{4} \mathrm{He}$ from

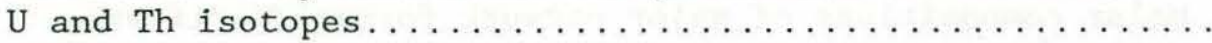

1.3 Fractional change in ${ }^{3} \mathrm{He} /{ }^{4} \mathrm{He}$ ratios as a function of

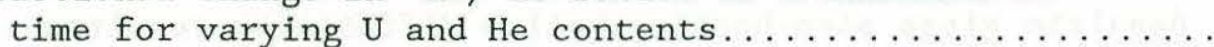

\section{CHAPTER 2}

2.1 The "Four-shooter" device for loading furnace samples......

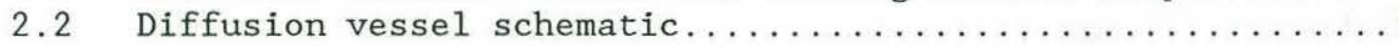

2.3 Gas processing line for Mass spectrometer $2 \ldots \ldots \ldots \ldots \ldots \ldots$

2.4 Relationship between sample size and measured ratio for typical sample sizes....................... 51

2.5 "Linearity" relationship for very large samples.......... 53

2.6 Effect of prior diffusive loss on apparent diffusivities... 61

2.7 Comparison of approximate and exact solutions for diffusive

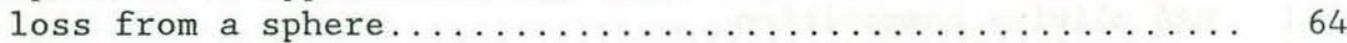

2.8 Effect of shape variations on apparent diffusivities...... 67

2.9 Effect of grain size distributions on apparent diffusivities......................... 70

2.10 Isotopic effects of grain size distribution............ 72

\section{CHAPTER 3}

3.1 Approximate fraction of helium in a glass sample that may

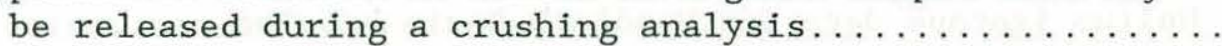

3.2 Helium content of basaltic glass as a function of grain

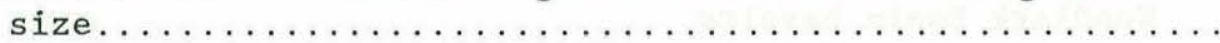

3.3 Comparison of helium contents of stored glass powders with expected loss curves........................ 98

3.4 Fractional release versus time plots for each of the

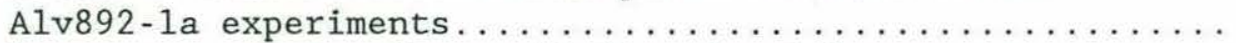

3.5 Release systematics for small fractional losses in

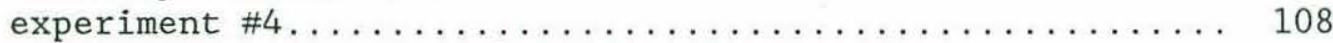

3.6 Release systematics for tholeiite glass powders at room temperature............................. 110

3.7 Spherical-model helium diffusivities calculated for: Alv892-1a tholeiite glass chip experiments............ 114 Alv892-1a tholeiite glass powder experiments........... 116 Charco 98-11T tholeiite and Aftermath D9 alkali basalt.... 118 1854B alkali basalt experiments................ 120

3.8 Effect of prior diffusive loss on apparent diffusivities... 123

3.9 Successive corrections for prior helium loss in expt. \#5... 127

3.10 Effect of unrecognized prior loss on apparent diffusion coefficients and activation energies............. 130

3.11 Arrhenius plots for the Alv892-1a tholeiite glass chip experiments.............................. 134

3.12 Arrhenius plots for Alv892-1a powder experiments \#5 and \#6.. 136 
3.13 Refined model Arrhenius plots for experiments with tholeitic Charco 98-11, alkali basalt Aftermath D9 and ${ }^{* 4} \mathrm{He}$ diffusion in alkali basalt 1854B............. 138

3.14 Comparison of Arrhenius slopes for all experiments......... 140

3.15 Temperature dependence of helium diffusion in tholeiitic glass..................................... 145

3.16 Isotopic compositions of sequential releases in the Alv892-1a glass chip experiments.................. 155

$3.17{ }^{3} \mathrm{He} /{ }^{4} \mathrm{He}$ ratios versus extent of release for the Alv892-1a

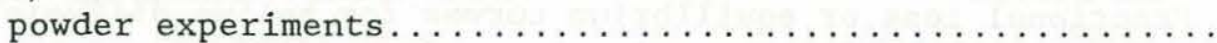

$3.18{ }^{3} \mathrm{He} /{ }^{4} \mathrm{He}$ ratios for tholeiitic sample Charco $98-11 \mathrm{~T}$ and

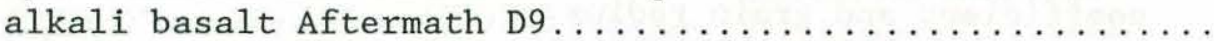

3.19 Isotopic diffusivity ratios for the Alv892-1a chip experiments as a function of release................

Isotopic diffusivity ratios for the Alv892-1a powder
experiments as a function of release $\ldots \ldots \ldots \ldots \ldots \ldots \ldots \ldots$

Isotopic diffusivity ratios for the Alv892-1a powder
experiments as a function of release $\ldots \ldots \ldots \ldots \ldots \ldots \ldots \ldots$ and for the alkali basalt Aftermath D9.............. 166

3.22 Examination of the temperature dependence of the isotopic diffusivity ratio, $\mathrm{D}^{3} \mathrm{He} / \mathrm{D}^{4} \mathrm{He}$ for tholeiitic basalt glass..

3.23 Theoretical relation for the isotopic effect on correlation in vacancy diffusion calculated for helium isotopes...... 175

3.24 Comparison of effective atomic radii for helium.......... 178

3.25 Comparison of diffusivities for helium and common ions in basaltic glass and obsidian................. 180

3.26 Timescales for helium loss and exchange in seafloor basalts.............................. 188

3.27 Effect of seawater exchange on helium isotopic

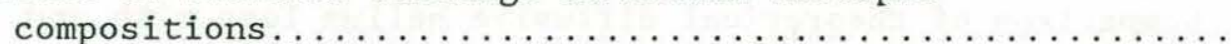

4.1 Apparent pyrometric temperature as a function of furnace

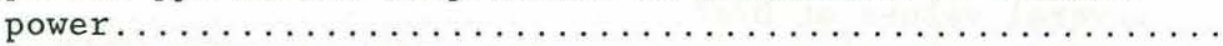

4.2 Comparison of pyrometric temperature calibration with fusion data for pure metals ................... 207

4.3 Fractional release of He versus time and versus the square

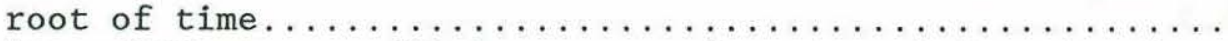

4.4 Helium diffusion coefficients as a function of fractional release, $F$, for each experiment................ 216

4.5 Arrhenius diagrams for helium diffusion in olivine and

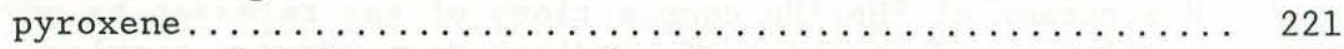

4.6 Comparison of diffusivity results for pyroxene and olivine.. 224

4.7 Helium isotopic data for emanation experiments.......... 228

4.8 Reduction in initial ${ }^{3} \mathrm{He} /{ }^{4} \mathrm{He}$ ratio as a function of fractional helium loss for several values of the isotopic

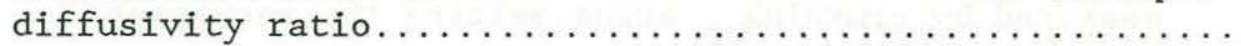


4.9 Comparison of the helium diffusivity in diopside and olivine with argon and common cation diffusion coefficients in

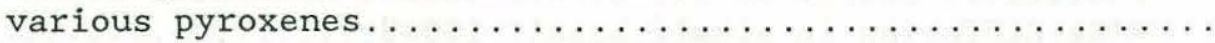

4.10 Diagram comparing the helium diffusivity and activation energy in olivine to a compensation trend for cations in

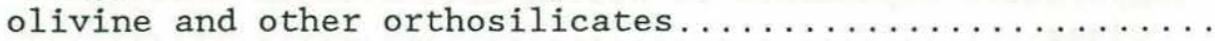

4.11 Characteristic diffusive length scales for helium diffusion in olivine and diopside at magmatic and mantle

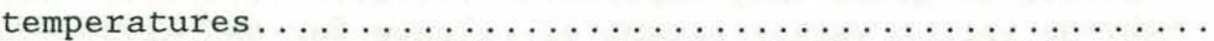

4.12 Fractional loss or equilibrium curves for helium diffusion from a sphere for several values of the diffusion

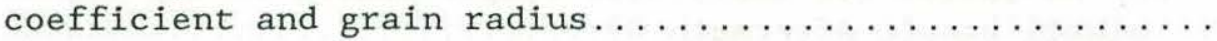

\section{CHAPTER 5}

5.1 Arrhenius relation for the release of cosmogenic ${ }^{3} \mathrm{He}$ from

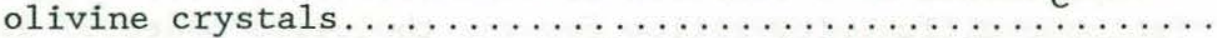

5.2 Comparison of cosmogenic and inherited helium release from

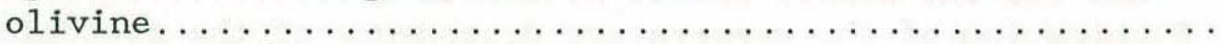

5.3 Comparison of ${ }^{3} \mathrm{He}$ and ${ }^{4} \mathrm{He}$ release from quartz grains during

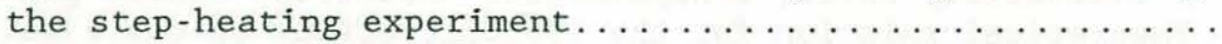

5.4 Isotopic compositions of sequential releases from BW84-105

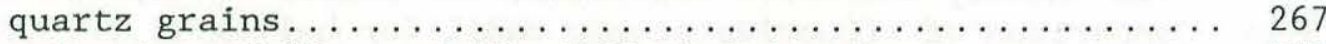

5.5 Examination of ${ }^{3} \mathrm{He}$ and ${ }^{4} \mathrm{He}$ diffusivities in quartz........

5.6 Arrhenius relations for cosmogenic ${ }^{3} \mathrm{He}$ and radiogenic ${ }^{4} \mathrm{He}$

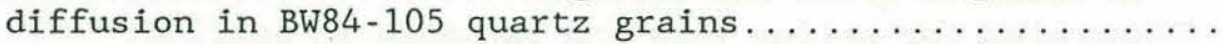

5.7 Comparison of Arrhenius relations for ${ }^{3} \mathrm{He}$ and ${ }^{4} \mathrm{He}$ in

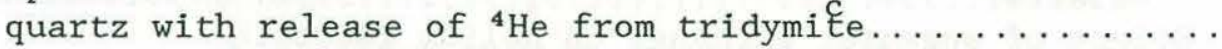

5.8 Comparison of theoretical diffusive helium loss with and

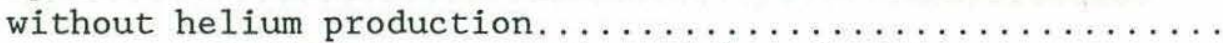

5.9 Comparison of diffusive profiles with and without

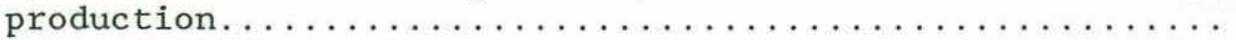

5.10 Effect of diffusive loss on cosmogenic helium model ages for

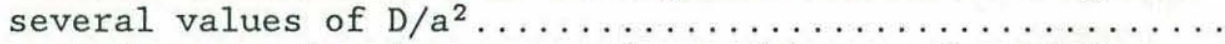

5.11 Expanded scale plot for correcting model ages for diffusive loss, drawn for an age range of .1 to 1 million years.....

\section{CHAPTER 6}

6.1 Tectonic map for the Woodlark Basin and Solomon Islands

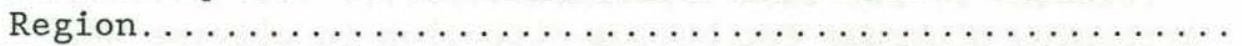

6.2 Histograms of ${ }^{3} \mathrm{He} /{ }^{4} \mathrm{He}$ compositions of gas released by powder melting and crushing of Woodlark Basin dredge samples....

6.3 Comparison of the quantity of helium released by crushing versus melting powdered mineral seperates............

6.4 Internal isotopic disequilibrium of the ${ }^{3} \mathrm{He} /{ }^{4} \mathrm{He}$ ratios measured by crushing, versus melting the resultant

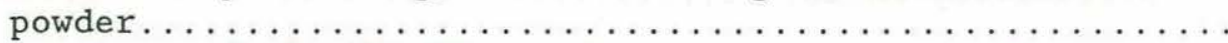

6.5 Comparison of the ${ }^{3} \mathrm{He} /{ }^{4} \mathrm{He}$ ratio measured by crushing versus the model quantity for samples that exhibit significant

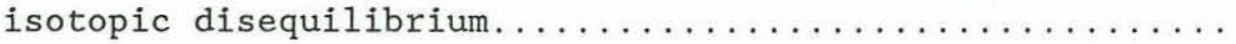


CHAPTER 6 (contd.)

6.6 The amount of He added by radiodecay for different uranium contents as a function of time................. 326

6.7 Comparison of observed helium concentrations with expected values for equilibrium with seawater.............. 329

6.8 Histogram of the amounts and isotopic compositions of helium released by crushing, melting and step-heating spherulitic

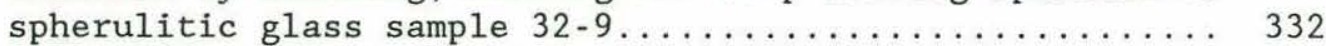

$6.9{ }^{3} \mathrm{He} /{ }^{4} \mathrm{He},{ }^{87} \mathrm{Sr} /{ }^{86} \mathrm{Sr}$ and $\mathrm{Sr} / \mathrm{Zr}$ versus distance from thench the trench............................. 339

$6.10{ }^{3} \mathrm{He} /{ }^{4} \mathrm{He}$ versus ${ }^{87} \mathrm{Sr} /{ }^{86} \mathrm{Sr}$ for Woodlark Basin samples....... 344 
Chapter 1. Introduction 


\subsection{OVERVIEW}

Helium isotopes provide a unique combination of properties for the study of igneous petrogenesis. Helium is one of the few volcanic volatiles for which there are both a primordial stable isotope, ${ }^{3} \mathrm{He}$, and a radiogenic stable isotope, ${ }^{4} \mathrm{He}$. This combination allows the timescales of mantle and magma degassing processes to be investigated, and the presence of juvenile, (i.e dating from the time of Earth's formation) volatiles to be identified. The generation of radiogenic helium is intimately tied to the heat budget of the Earth and to $\mathrm{Pb}$ isotopic systematics because the same nuclear decay chains that generate ${ }^{4} \mathrm{He}$ produce a large proportion of the heat presently being lost from the deep Earth, and terminate in stable lead isotopes. This means that the loss of heat, gaseous elements, and less volatile species during the chemical differentiation of the earth can be investigated by comparing helium isotopic systematics to other elements and to present-day heat flow estimates. In addition, because both isotopes are lost to space, the low concentration of helium in the atmosphere makes determining the isotopic composition of helium in rocks relatively free from contamination.

Helium isotopic studies have had important impacts on understanding the formation of Earth's atmosphere, the internal structure and dynamics of the mantle, and basaltic volcanism. However, an of ten poorly constrained aspect of these studies has been the mobility of helium in geologic materials. This is an important issue in the interpretation of isotopic variability as resulting from closed system evolution or bulk mixing of different source materials. The rate at which helium, as a light neutral atom, can permeate rocks or the silicate mantle is generally assumed to be fast, but has not been thoroughly investigated. In addition, the possible diffusive separation of ${ }^{3} \mathrm{He}$ from ${ }^{4} \mathrm{He}$ because of their large mass difference and different origins has not been previously quantified. Diffusive mobility of helium in individual crystals and basaltic glasses is also important to geochronology efforts using radiogenic ${ }^{4} \mathrm{He}$ and cosmogenic ${ }^{3} \mathrm{He}$. 
For these reasons, this thesis focuses on determining the diffusivities of ${ }^{3} \mathrm{He}$ and ${ }^{4} \mathrm{He}$ in silicate minerals and glasses. The results are used to interpret helium isotopic variability in rocks from mid-oceanic-ridges, island arcs, seamounts, and hotspot volcanos. In addition, the impact of helium mobility on radiogenic helium $\left({ }^{* 4} \mathrm{He}\right)$ dating of basaltic glasses and cosmogenic helium $\left({ }^{3} \mathrm{He}_{c}\right)$ surface exposure dating of lava flows and other rocks is considered and quantified.

\subsection{TERRESTRIAL HELIUM ISOTOPIC SYSTEMATICS AND DIVERSITY}

Two stable isotopes of helium exist in the universe, ${ }^{3} \mathrm{He}$, and ${ }^{4} \mathrm{He}$ which is by far the most abundant. For example, stars, meteorites, and our solar wind, have ratios of a few times $10^{-4}$ (e.g. Mamyrin and Tolstikhin, 1984). On Earth, the isotopic composition of helium varies from these high values to several orders of magnitude further depletion of ${ }^{3} \mathrm{He}$ (figure 1.1). This variability is related to the different origins of the two isotopes. Almost all of the ${ }^{3}$ He presently in the earth's interior remains from planetary formation. However, ${ }^{4} \mathrm{He}$ is continuously produced by decay of ${ }^{235} \mathrm{U},{ }^{238} \mathrm{U}$, and ${ }^{232} \mathrm{Th}$ and their radioactive daughters. These decays (listed in figure 1.2 ) also produce very small amounts of ${ }^{3}$ He through secondary nuclear reactions (predominantly with ${ }^{6} \mathrm{Li}$ ), yielding a ${ }^{3} \mathrm{He} /{ }^{4} \mathrm{He}$ radiogenic production ratio of $10^{-8}$ to $10^{-7}$ for common silicate rocks (e.g. Morrison and Pine, 1955; Mamyrin and Tolstikhin, 1984).

Over the age of the Earth, addition of radiogenic ${ }^{4} \mathrm{He}$ has reduced the ${ }^{3} \mathrm{He} /{ }^{4} \mathrm{He}$ ratio of silicate rocks relative to likely initial compositions (figure 1.2). For example, most continental rocks, degassed and enriched in $U$ and Th during their formation, contain virtually no primordial ${ }^{3} \mathrm{He}$ and exhibit ${ }^{3} \mathrm{He} /{ }^{4} \mathrm{He}$ ratios corresponding to the radiogenic production ratio (figure 1.2 ). 0ther rock types, such as young oceanic volcanics, still retain significant ${ }^{3} \mathrm{He}$, suggesting their sources have not been completely degassed, and reflecting their relatively low $U$ and Th contents. The exact helium isotopic composition of a sample reflects its initial composition, time-integrated $U$ and $T h$ 
Figure 1.1 Comparison of ${ }^{3} \mathrm{He} /{ }^{4} \mathrm{He}$ ratios for terrestrial rocks with likely primordial sources and Earth's atmosphere. The lowered ratios reflect ${ }^{3} \mathrm{He}$ loss, and ${ }^{4} \mathrm{He}$ gain by radiodecay of $\mathrm{U}$ and $\mathrm{Th}$. Continental rocks have isotopic compositions similar to the radiogenic production ${ }^{3} \mathrm{He} /{ }^{4} \mathrm{He}$ ratio, and have retained far less ${ }^{3} \mathrm{He}$ than samples from the oceanic mantle. The large range in ${ }^{3} \mathrm{He} /{ }^{4} \mathrm{He}$ ratios allows different components involved in petrogenesis and mantle mixing to be distinguished (data fields are approximate, for a compilation see Mamyrin and Tolstikhin, 1984; figure adapted from M.D. Kurz, unpublished). 
Figure 1.1

$$
\text { Terrestrial Helium }
$$

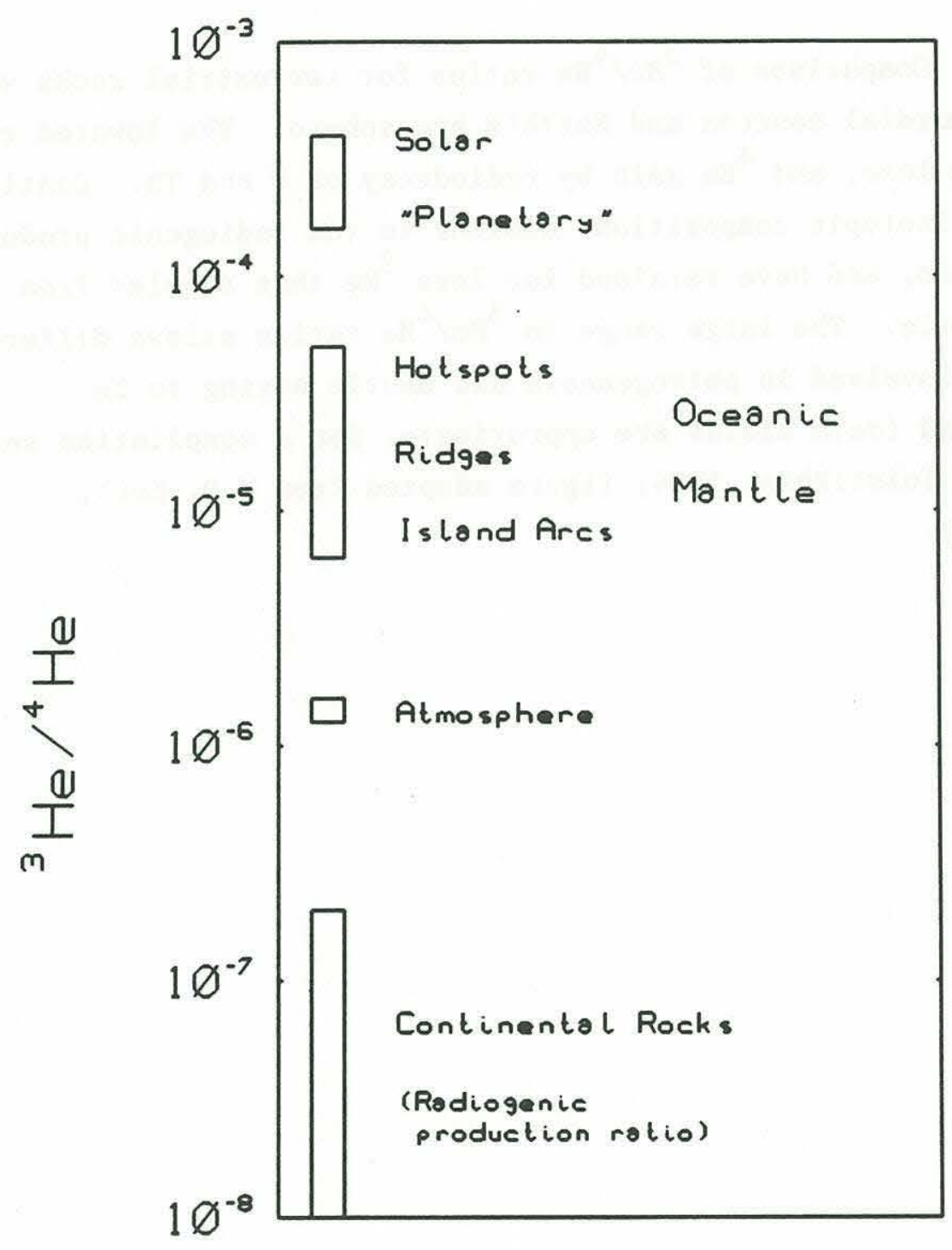


Figure 1.2 Radioactive decay series for the production of ${ }^{4} \mathrm{He}$ from $\mathrm{U}$ and Th isotopes. Decay half-lives and alpha-particle energies (MeV) are also shown. 


\begin{tabular}{|c|c|c|c|c|c|c|c|c|c|c|c|c|c|c|c|c|c|}
\hline \multirow{2}{*}{\begin{tabular}{|l} 
Element \\
Nepteorthem
\end{tabular}} & \multicolumn{7}{|c|}{ U-230 Serles } & \multicolumn{5}{|c|}{ Th-232 Series } & \multicolumn{5}{|c|}{ U-235 Sertes } \\
\hline & & & & & & & & & & & & & & & & & \\
\hline Uranivom & 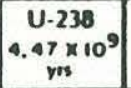 & & {$\left[\begin{array}{c}\mathrm{U} .234 \\
2.45 \times 10^{5} \\
\mathrm{rm}^{\mathrm{m}}\end{array}\right]$} & & & & & & & & & & $\left|\begin{array}{c}\mathrm{U}-2255 \\
.04 \times 10^{\circ} \\
\mathrm{m}\end{array}\right|$ & & & & \\
\hline Protactiontum & 4.20 & $\begin{array}{c}\mathrm{Pa}-234^{4} \\
1.10 \\
0 \mathrm{men}\end{array}$ & ${ }^{4} i^{78}$ & & & & & & & & & & 4.40 & $\mid \begin{array}{c}\mathrm{Pa}-231 \\
3.28 \times 10^{4} \\
\mathrm{~m}^{4}\end{array}$ & & & \\
\hline Thoothem & $\begin{array}{c}\text { Th-234 } \\
24.1 \\
\text { ders }\end{array}$ & & $\begin{array}{c}\text { Th-230 } \\
7.54 \times 10^{4} \\
n^{3}\end{array}$ & & & & & $\begin{array}{c}\text { Th-232 } \\
1.40 \times 10^{10} \\
\mathrm{~mm}\end{array}$ & & $\begin{array}{l}\text { Th-228 } \\
1.91 \\
\text { im }\end{array}$ & & & $\begin{array}{c}\text { Th-231 } \\
25.9 \\
h \mathrm{hr}\end{array}$ & $\begin{array}{c}5.01 \\
1\end{array}$ & $\begin{array}{c}\text { Th.227 } \\
18.7 \\
\text { dery } \\
\end{array}$ & & \\
\hline Actholvean & & & $\begin{array}{c}4.69 \\
1\end{array}$ & & & & & 4.01 & $\begin{array}{c}\mathrm{Ac}-22 \mathrm{C}^{\circ} \\
6.13^{\circ} \\
\mathrm{hrs}\end{array}$ & $\begin{array}{c}5.42 \\
1\end{array}$ & & & & $\mid \begin{array}{c}A c-227 \\
21.0 \\
y r s\end{array}$ & 6.04 & & \\
\hline Recllown & & & \begin{tabular}{|c|}
$\mathrm{Ra}-226$ \\
$1.60 \times 10^{3}$ \\
$m^{3}$
\end{tabular} & & & & & $\begin{array}{c}\mathrm{Ra}-228 \\
9.76 \\
\mathrm{ym}\end{array}$ & & \begin{tabular}{|c|c|c|c|c|}
$R a-224$ \\
3.66 \\
dems
\end{tabular} & & & & & \begin{tabular}{|c|}
$\mathrm{Ra}-223$ \\
11.4 \\
$\operatorname{don}$
\end{tabular} & & \\
\hline Francieonn & & & $\begin{array}{c}4.79 \\
1\end{array}$ & & & & & & & 5.69 & & & & & 5.72 & & \\
\hline Radon & & & $\begin{array}{c}R_{n-222} \\
\text { s.ev } \\
\text { dern }\end{array}$ & & & & & & & $\begin{array}{c}\mathrm{Rn} \cdot 220 \\
59.6 \\
\text { sec }\end{array}$ & & & & & \begin{tabular}{|c|} 
Rn-219 \\
3.9.96 \\
sec
\end{tabular} & & \\
\hline Astative & & & 5.49 & & & & & & & 6.29 & & & & & $\begin{array}{c}6.82 \\
1\end{array}$ & & \\
\hline Polonkem & & & $\begin{array}{c}\text { Po-218 } \\
311 \\
\text { min }\end{array}$ & & 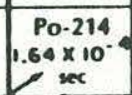 & & $\begin{array}{l}\text { Po-210 } \\
130 \\
\text { ders }\end{array}$ & & & $\begin{array}{c}\text { Po-216 } \\
0.15 \\
\text { sec }\end{array}$ & $64 \%$ & $\begin{array}{l}\text { Po-212 } \\
3.0 \times 10^{-7} \\
0 \times 10 c\end{array}$ & & & $\left|\begin{array}{|c|}\text { Po-215 } \\
\text { 1. } \\
\max \times 0^{-3}\end{array}\right|$ & & \\
\hline Eleomeneth & & & 6.00 & 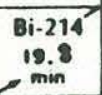 & 7.69 & $\begin{array}{l}8 \mathrm{Bi}-210^{-} \\
5.01 \\
\text { dors }\end{array}$ & 5.30 & & & $\begin{array}{c}6.78 \\
1\end{array}$ & $\begin{array}{l}\text { Bi-212 } \\
60.6 \\
\min \end{array}$ & 11.65 & & & $\begin{array}{c}7.39 \\
1\end{array}$ & $\begin{array}{r}8 i-211 \\
2.14 \\
-\min ^{2}\end{array}$ & \\
\hline lead & & & \begin{tabular}{|c|}
$\mathrm{Pb}-214$ \\
$26 .{ }^{\circ}$ \\
$\min$
\end{tabular} & & $\begin{array}{c}\mathrm{Pb}-210^{\prime} \\
22.3 \\
\mathrm{rm}\end{array}$ & & $\begin{array}{c}\mathrm{Pb}-206 \\
\text { stable lead } \\
\text { (brotope) }\end{array}$ & & & \begin{tabular}{|c|} 
Pb-212 \\
10.6 \\
his
\end{tabular} & 8.78 & \begin{tabular}{|c|c|} 
Pb-208 \\
"alble lead \\
(notope)
\end{tabular} & & & $\begin{array}{c}\mathrm{Pb}-211 \\
36.1 \\
\min \end{array}$ & 6.62 & 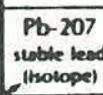 \\
\hline Thalllum & & & & & & & & & & & $\begin{array}{c}1 \mathrm{~T}-200^{-} \\
3.05 \\
\min \end{array}$ & & & & & $\begin{array}{l}T 1.207 \\
4.77 \\
\operatorname{man}\end{array}$ & \\
\hline
\end{tabular}


contents, and the degree to which it has exchanged helium with other reservoirs.

For a closed system, the helium isotopic composition at some time, $\mathrm{t}$, is related to its initial composition by:

$$
\left({ }^{3} \mathrm{He} /{ }^{4} \mathrm{He}\right)_{\mathrm{t}}=\left({ }^{3} \mathrm{He}_{\mathrm{o}}+{ }^{* 3} \mathrm{He}\right) /\left({ }^{4} \mathrm{He}_{\mathrm{o}}+{ }^{* 4} \mathrm{He}\right)
$$

where o indicates initial helium contents, and * denotes helium produced by radioactive decay over the time, $t$. Provided the geologic system is in secular equilibrium, the production of ${ }^{* 4} \mathrm{He}$ is given by:

$$
{ }^{* 4} \mathrm{He}=8\left[{ }^{238} \mathrm{U}\left(1-\mathrm{e}^{-\lambda t}\right)\right]+7\left[{ }^{235} \mathrm{U}\left(1-\mathrm{e}^{-\lambda t}\right)\right]+6\left[{ }^{232} \operatorname{Th}\left(1-\mathrm{e}^{-\lambda t}\right)\right]
$$

in which the parent nuclide concentrations are those at the start of production. In terms of final $U$ and Th contents, as are usually measured, this equation can be rewritten:

$$
{ }^{* 4} \mathrm{He}=8\left[{ }^{238} \mathrm{U}\left(\mathrm{e}^{\lambda \mathrm{t}}-1\right)\right]+7\left[{ }^{235} \mathrm{U}\left(\mathrm{e}^{\lambda t}-1\right)\right]+6\left[{ }^{232} \mathrm{Th}\left(\mathrm{e}^{\lambda t}-1\right)\right]
$$

The decay constants are $1.55125 \times 10^{-10}, 9.8485 \times 10^{-10}$, and $4.9475 \times 10^{-11}$ decays per year for ${ }^{238} \mathrm{U},{ }^{235} \mathrm{U}$ and ${ }^{232}$ Th respectively (Steiger and Jager, 1977). For short times and the present day ${ }^{238} \mathrm{U} /{ }^{235} \mathrm{U}$ ratio of 137.8 , is sometimes linearized (e.g. Graham et al, 1987):

$$
{ }^{* 4} \mathrm{He}=2.80 \times 10^{-8}\{[\mathrm{U}](4.35+\mathrm{Th} / \mathrm{U}\} \lambda \mathrm{t}
$$

where $\mathrm{Th} / \mathrm{U}$ is the atom ratio, [U] is concentration is $\mathrm{ppm}$, and ${ }^{* 4} \mathrm{He}$ is in $\mathrm{cm}^{3} \mathrm{STP} / \mathrm{g}$ (Graham et al, 1987). The radiogenic production of ${ }^{3} \mathrm{He}$ is:

$$
{ }^{* 3} \mathrm{He}=\mathrm{R}_{\mathrm{p}}{ }^{* 4} \mathrm{He}
$$

where $R_{p}$ designates the radiogenic production ratio, ${ }^{* 3} \mathrm{He} /{ }^{* 4} \mathrm{He}$. As mentioned above, $R_{p}$ is very small and varies from $10^{-8}$ to $10^{-7}$ depending primarily on lithium content and on the matrix composition. 
Equations 1.1 and 1.5 can be combined to obtain an expression for fractional change in the closed system's ${ }^{3} \mathrm{He} /{ }^{4} \mathrm{He}$ ratio with time, designating ${ }^{3} \mathrm{He} /{ }^{4} \mathrm{He}$ ratios by $\mathrm{R}$, leads to: :

$$
\begin{aligned}
F & =R_{t} / R_{0}=\left({ }^{3} \mathrm{He} /{ }^{4} \mathrm{He}\right)_{t} /\left({ }^{3} \mathrm{He} /{ }^{4} \mathrm{He}\right)_{0} \\
& =\left[1+Q^{0}\left(\mathrm{R}_{\mathrm{p}} / \mathrm{R}_{\mathrm{o}}\right)\right] /\left[1+0^{0}\right]
\end{aligned}
$$

where $Q^{0}$ is written for ${ }^{*}{ }^{4} \mathrm{He} /{ }^{4} \mathrm{He}$ or for convenience; $Q^{0}$ is the quotient of radiogenic to initial helium. This equation shows that radiogenic production of ${ }^{*} \mathrm{He}$ will have a negligible effect (less than . $1 \%$ ) on $\mathrm{F}$ for systems such as oceanic rocks, where $R_{0}$ is much (1000 times) larger than $R_{p}$. In these cases, taking $R_{p}$ equal to zero gives the approximation:

$$
\mathrm{F}=1 /\left(1+Q^{0}\right)={ }^{4} \mathrm{He}_{\mathrm{o}} /\left({ }^{4} \mathrm{He}_{\mathrm{O}}+{ }^{* 4} \mathrm{He}\right)
$$

These expressions can be used to predict the timescales over which different geologic materials will change their ${ }^{3} \mathrm{He} /{ }^{4} \mathrm{He}$ ratios by radiodecay.

F can also be written in terms of measured (rather than initial) helium contents and used to calculate initial ${ }^{3} \mathrm{He} /{ }^{4} \mathrm{He}$ ratios, when age estimates can be made. Defining $Q^{t}$ as the ratio of radiogenic to total helium in the sample:

$$
Q^{t}={ }^{* 4} \mathrm{He} /{ }^{4} \mathrm{He} \mathrm{t}_{\mathrm{t}}
$$

allows $\mathrm{F}$ to be written:

$$
F=\left\{1+\left[Q^{t} /\left(1-Q^{t}\right)\right]\left[1-\left(R_{p} / R_{t}\right)\right]\right\}^{-1}
$$

This form of $\mathrm{F}$ makes clear that ${ }^{*}$ He production is not important when 
the measured ratio, $R_{t}$, is large in comparison to the production ratio, $\mathrm{R}_{\mathrm{p}}$, giving the approximation:

$$
\mathrm{F}=1-\mathrm{Q}^{\mathrm{t}}=\left({ }^{4} \mathrm{He}_{\mathrm{t}}-{ }^{* 4} \mathrm{He}\right) /{ }^{4} \mathrm{He}_{\mathrm{t}}
$$

Given measured $U$ and $T h$ contents and an age, ${ }^{* 4}$ He can be obtained from equation 1.2 , and used along with the measured helium content to calculate $\mathrm{F}$ from equation 1.9 . The initial ratio is then obtained by multiplying the measured ${ }^{3} \mathrm{He} /{ }^{4} \mathrm{He}$ ratio by $\mathrm{F}$.

$F$ values for different $U$ and He contents ratios are plotted in figure 1.3. This figure reveals that the timescales to change the ${ }^{3} \mathrm{He} /{ }^{4} \mathrm{He}$ ratio of different rocks and source regions varies dramatically, from 1000 years to $10^{8}$ years, primarily because of large variations in He contents. This phenomenon offers the advantage that many different processes in petrogenesis can be investigated through helium isotopic systematics, but the concomitant disadvantage that the ${ }^{3} \mathrm{He} /{ }^{4} \mathrm{He}$ ratios of even relatively young rocks may have changed since eruption. In addition, it is clear that departures from closed system behavior, such as loss of helium by degassing from the mantle or a magma, or by diffusion from an erupted rock have a large influence on the subsequent evolution of helium isotopic compositions.

Earth's atmosphere is an open reservoir for He. Its helium content is quite low (5.24 ppm, e.g. Mamyrin and Tolstikhin, 1984) in comparison to other noble gases because helium is lost to space. Control of the helium isotopic composition of the atmosphere is quite complex and reflects a balance between radiogenic additions from continental crust, primordial additions from active volcanic regions, and several upper atmosphere processes, including accretion of helium from solar wind, helium production by cosmic ray spallation, and both thermal and photoionization losses to space (Johnson and Axford, 1969; Lupton, 1973; Mamyrin and Tolstikhin, 1984). These processes maintain the atmospheric ${ }^{3} \mathrm{He} /{ }^{4} \mathrm{He}$ ratio above that for radiogenic production (figure 1.1 ). Both the low helium concentration of air and its distinct isotopic composition are important to helium isotope geochemistry of silicate rocks, because contamination problems are minimized and readily 
Figure 1.3 Fractional change in ${ }^{3} \mathrm{He} /{ }^{4} \mathrm{He}$ ratios as a function of time for varying $U$ and He contents. Curves are calculated for $T h / U=3$, ${ }^{3} \mathrm{He} /{ }^{4} \mathrm{He}$ radiogenic production ratio of $10^{-8}$, and initial ${ }^{3} \mathrm{He} /{ }^{4} \mathrm{He}$ of 1.38 $\times 10^{-6}$, but are accurate to better than $1 \%$ for all initial ${ }^{3} \mathrm{He} /{ }^{4} \mathrm{He}$ ratios greater than $1 \mathrm{Ra}$ (see text). The individual curves correspond to initial $\mathrm{U}$ and He contents as follows:

U ppm He ccSTP/g Example

\begin{tabular}{llll}
\hline 1 & 0.3 & $10^{-7}$ & Back-arc basin basaltic glass \\
2 & 1.0 & $10^{-7}$ & \\
3 & $0.3,3.0$ & $10^{-8}$ & Island arc basaltic glass (Chap. 6) \\
4 & 1.0 & $10^{-8}$ & Island arc andesitic glass (Chap.6) \\
5 & $0.3,3.0$ & $10^{-9}$ & \\
6 & 1.0 & $10^{-9}$ & Dacite glass \\
7 & 3.0 & $10^{-9}$ & \\
\hline
\end{tabular}

Curves for mid-ocean-ridge basalt glasses and mantle source regions are not shown, but with He contents on the order of $10^{-5}$ to $10^{-6} \mathrm{cc} / \mathrm{g}$ and low $U$ contents of 100 to $10 \mathrm{ppb}$, the timescales are $10^{8}$ to $10^{10}$ years. (figure adapted from Graham, 1987) 
Figure 1.3

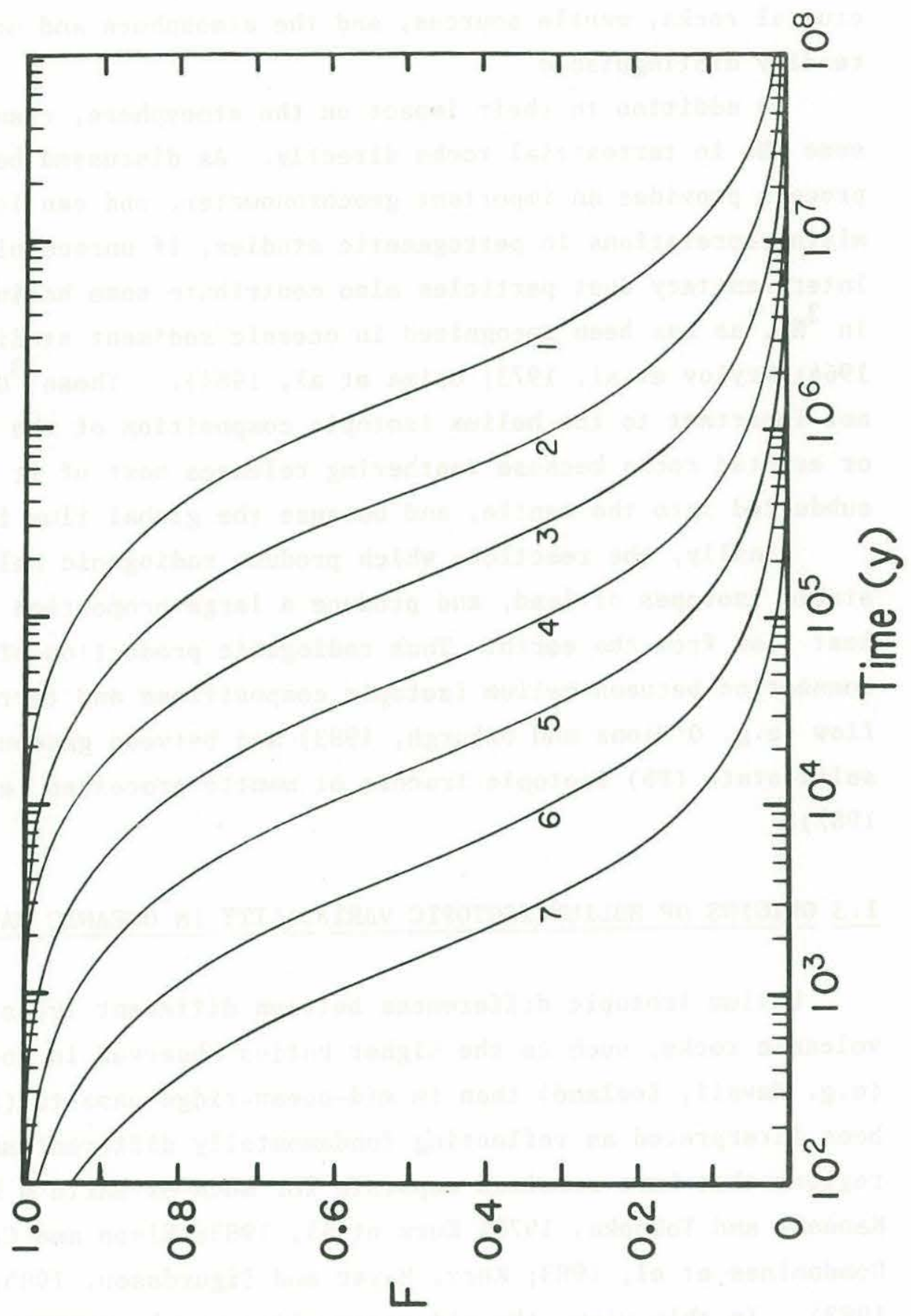


identified. This is true more generally, in that large differences in helium isotopic compositions allow the petrogenetic involvement of crustal rocks, mantle sources, and the atmosphere and oceans to be readily distinguished.

In addition to their impact on the atmosphere, cosmic rays produce some ${ }^{3} \mathrm{He}$ in terrestrial rocks directly. As discussed below, this process provides an important geochronometer, and can lead to misinterpretations in petrogenetic studies, if unrecognized. Interplanetary dust particles also contribute some helium which is high in ${ }^{3} \mathrm{He}$, as has been recognized in oceanic sediment studies (Merrihue, 1964; Krylov et al, 1973; 0zima et al, 1984). These ${ }^{3}$ He sources are not important to the helium isotopic composition of the earth's interior or erupted rocks because weathering releases most of it before it is subducted into the mantle, and because the global flux is small.

Finally, the reactions which produce radiogenic helium terminate in stable isotopes of lead, and produce a large proportion of the present heat flow from the earth. Thus radiogenic production of ${ }^{4} \mathrm{He}$ provides a connection between helium isotopic compositions and terrestrial heat flow (e.g. 0'Nions and 0xburgh, 1983) and between gaseous (He) and solid-state $(\mathrm{Pb})$ isotopic tracers of mantle processes (e.g. Graham, 1987).

\subsection{ORIGINS OF HELIUM ISOTOPIC VARIABILITY IN OCEANIC BASALTS.}

Helium isotopic differences between different types of oceanic volcanic rocks, such as the higher ratios observed in hotspot volcanos (e.g. Hawaii, Iceland) than in mid-ocean-ridge basalts (figure 1.1) have been interpreted as reflecting fundamentally different mantle source regions that have remained separate for much of Earth's history (e.g. Kaneoka and Takaoka, 1978; Kurz et al, 1983; Rison and Craig, 1983; Condomines et al, 1983; Kurz, Meyer and Sigurdsson, 1985; Allegre et al, 1983). In this view, the mid-ocean-ridge-basalt (MORB) source has lost primordial helium over time and decay has lowered its ${ }^{3} \mathrm{He} /{ }^{4} \mathrm{He}$ ratio to a greater degree than in the source region of hotspot volcanos. This interpretation assumes that parts of the mantle remain isolated from 
each other for long times and that helium does not migrate within the mantle at faster rates than bulk convection. The few measurements available for mafic minerals (Gramlich and Naughton, 1972; Hart, 1984) suggest helium diffusion rates are too slow to homogenize helium isotopic compositions on mantle length scales (i.e. km), even over the age of the earth. This result is confirmed by further measurements in chapter 4 on olivine and pyroxene crystals. Thus both the relative homogeneity of the MORB helium isotopic compositions $\left(7-9 R_{a}\right)$ and the difference between hotspots and mid-ocean ridge basalts must represent characteristics of the mixing process (e.g. Kurz and Jenkins, 1981) or possibly the relative nature of $U$, Th and He extraction during melt generation (Lupton, 1983).

In addition to these large differences, there are helium isotopic variations within hotspot volcanos and along oceanic ridges which are well outside measurement error. For example, Kurz et al (1987) have shown that the ${ }^{3} \mathrm{He} /{ }^{4} \mathrm{He}$ ratio of lavas erupted from Hawaiian volcanos vary with time, generally decreasing from the initial hotspot values of 20 to $30 \mathrm{R}_{\mathrm{a}}$ to values near $8 \mathrm{R}_{\mathrm{a}}$ as the volcanos age. This has been interpreted as a change of magma source from hotspot plume to residual heated lithosphere as the Pacific tectonic plate moves away from the plume source (op. cit.). However, other interpretations are possible, including sequential tapping of a magma chamber which is steadily degassing helium and accumulating radiogenic ${ }^{4} \mathrm{He}$, or preferential degassing of ${ }^{3} \mathrm{He}$ by diffusion (e.g. Condomines et al, 1983; Zindler and Hart, 1986).

Similarly, while Mid-oceanic-ridge-basalts have generally uniform helium isotopic compositions $\left(6-9 \mathrm{R}_{\mathrm{a}}\right)$ there are significant local and regional variations. For example, ${ }^{3} \mathrm{He} /{ }^{4} \mathrm{He}$ ratios along the midAtlantic-ridge display smooth variations that may represent mixing of mantle components with different $U$ contents and degassing histories (Kurz et al, 1982; Poreda et al, 1980; Lupton, 1983). In addition, the helium isotopic composition of spreading ridge basalts in the southwestern Pacific may be relatively enriched in ${ }^{3} \mathrm{He}$ in comparison to other regions (Craig and Poreda, 1986; data in chapter 6). These differences may offer important information about mantle heterogeneity 
or melt segregation and transport processes, particularly with respect to separation of a gas phase. If these variations reflect source differences, understanding the mobility of helium in the mantle in the presence of melt is essential to determining mantle structure. However, it is possible that part or all of this variability derives from isotopically fractionating gas loss from the magmas or the basalts after eruption (Zindler and Hart, 1986; Craig and Lupton, 1976).

Another aspect of interpreting helium isotopic studies of rocks is accounting for processes that may affect the samples measured, which are usually small basaltic glass fragments, or separated mineral grains. Helium loss by diffusion during magma transport, lava cooling, or from the subsamples can affect helium concentration determinations and isotopic compostitions if the loss process is isotopically fractionating. Thus, helium diffusion rates in glasses are important in assessing contamination of samples on the seafloor, especially when the samples contain very little helium so that a small amount of helium from seawater can drastically alter their isotopic compositions. Because low helium contents are common in samples from island arc regions and seamounts and subaerial volcanics, extending helium isotopic tracer studies to these regions requires a better understanding of helium diffusion rates.

\subsection{RADIOGENIC AND COSMOGENIC HELIUM GEOCHRONOLOGY}

The production of radiogenic helium, ${ }^{\star 4} \mathrm{He}$, by uranium and thorium series nuclear decay provides a method for dating rocks. However, ages obtained by this method have often been unreasonably young given geologic settings (e.g. Hurley, 1954; Fanale, 1964; Leventhal, 1975) This problem generally reflects radiogenic helium loss by diffusion, of ten exacerbated because $U$ and Th tend to be located on grain boundaries or in fine-grained accessory minerals. Recently radiogenic helium geochronology has been applied to alkali basalt glasses where heterogeneous uranium and thorium distribution is not a problem, but in which corrections for inherited magmatic helium must be made (Graham et al, 1987). The ${ }^{3} \mathrm{He} /{ }^{4} \mathrm{He}$ ratio measured in gas-filled vesicles is assumed 
to represent magmatic helium and combined with the ${ }^{3}$ He content of helium dissolved within the glass to correct the total ${ }^{4} \mathrm{He}$ of the glass for inherited helium. Therefore, both the loss of radiogenic helium and the exchange of helium between vesicles and glass can lead to incorrect age estimates.

Graham et al (1987) considered this problem using a diffusivity measured for tholeitic basalt (Kurz and Jenkins, 1981). However, higher diffusivities have been measured in similar rocks (Jambon, Weber, and Begemann, 1985) and the mobility of radiogenic helium may differ from inherited helium because of the nature of its generation. Thus, the accuracy of this technique remains uncertain. A similar problem is the determination of magmatic ${ }^{3} \mathrm{He} /{ }^{4} \mathrm{He}$ ratios from phenocryst fluid inclusion studies in older rocks which contain significant radiogenic helium. Determining the diffusive mobility of ${ }^{* 4} \mathrm{He}$ in these samples allows the contamination of the fluid inclusions to be constrained. In addition to the dominantly primordial origin of ${ }^{3} \mathrm{He}$ in the silicate earth, small amounts of ${ }^{3} \mathrm{He}\left({ }^{3} \mathrm{He}_{c}\right)$ are produced in surface rocks by interactions with energetic cosmic rays (Kurz et al, 1985; Kurz, 1986a,b; Craig and Poreda, 1986). This process is significant to the study of silicate rocks in two ways. The presence of ${ }^{3} \mathrm{He}_{c}$ can interfere with the determination of magmatic ${ }^{3} \mathrm{He} /{ }^{4} \mathrm{He}$ ratios, although this problem can be avoided simply by sampling rocks at depth (approximately a meter; Kurz, 1986b). More importantly, ${ }^{3} \mathrm{He}_{\mathrm{c}}$ production provides a means of obtaining surface exposure ages and erosion rates (Kurz, 1986b; Craig and Poreda, 1986).

Several nuclear reactions contribute to the ${ }^{3} \mathrm{He}_{\mathrm{c}}$ production. Most important is spallation of major element target atoms by high energy neutrons, which are produced as secondary particles in the atmosphere when the primary cosmic rays (predominantly protons, but containing some neutrons and heavier nuclei) collide with atmospheric atoms. Another production reaction is the interaction of thermalized secondary neutrons (from cosmic ray interactions) with ${ }^{6} \mathrm{Li}$ to yield equal amounts of ${ }^{3} \mathrm{He}$ and ${ }^{4} \mathrm{He}$. For rocks with lithium contents of a few parts per million this mechanism is only about $1 \%$ as effective as spallation. A third mechanism produces much less ${ }^{3} \mathrm{He}$ and only becomes relatively important 
at depths below where the secondary neutrons have been largely absorbed (on the order of a meter). This is the interaction of cosmic muons with major element nuclei to produce neutrons which then interact with lithium by the reaction already described. More detailed discussion of these production processes, the energies involved, and attenuation depths are presented in chapter 5, and have been recently reviewed by Lal $(1987,1988)$.

Using cosmogenic helium contents as an indicator of surface exposure duration requires determining its production rate, that inherited and cosmogenic helium can be distinguished and that no cosmogenic helium has been lost since formation. Accounting for inherited helium appears to be possible in basaltic phenocrysts by separating helium contained in fluid inclusions from that in the crystal matrix (Kurz, 1986a,b). However, the diffusivity of cosmogenic helium has not yet been determined for any minerals, and may differ significantly from diffusivity of inherited helium because of the particle energies involved in its generation. For this reason, the effect of ${ }^{3} \mathrm{He}_{\mathrm{c}}$ loss on surface exposure and erosion studies is unknown.

\subsection{OBJECTIVES}

The objectives of this research are 1) to quantify the diffusive mobility of helium isotopes in silicate glasses and minerals important to petrogenesis and geochronology studies, and 2) to apply the results in interpreting helium isotopic variability in oceanic volcanic rocks and evaluating model ages based on radiogenic and cosmogenic helium contents. Chapter 3 addresses the diffusivity of inherited ${ }^{3} \mathrm{He}$, inherited ${ }^{4} \mathrm{He}$ and radiogenic ${ }^{4} \mathrm{He}$ in basaltic glasses, with implications for low temperature loss of helium, exchange of helium with seawater, and alteration of inherited helium isotopic compositions via preferential ${ }^{3} \mathrm{He}$ loss. It also discusses the mobility of helium in basaltic melts as a guide to quantifying volcanic degassing processes, including the separation of helium from other elements, and the relative loss rates of helium and heat from magma bodies. Chapter 4 examines helium mobility in mafic minerals at high temperature to quantify the 
mobility of helium in the mantle and the extent to which phenocrysts and xenoliths retain magmatic helium. Chapter 5 discusses the mobility of cosmogenic ${ }^{3} \mathrm{He}_{\mathrm{c}}$ in olivine and quartz crystals to constrain the effects of diffusive loss on surface exposure dating. Chapter 6 is a study of the helium (and strontium) isotopic compositions of volcanic rocks from the Woodlark Basin/Solomon Islands lithospheric subduction region in the western Pacific. The large helium isotopic variability observed is considered in light of the diffusion measurements for contamination affects occuring on the seafloor in addition to source variations. 


\section{REFERENCES}

ALLEGRE C.J., STAUDACHER T., SARDA P. and KURZ M. (1983) Constraints on evolution of earth's mantle from rare gas systematics. Nature 303, $762-766$.

CONDOMINES M., GRONVOLD K., HOOKER P.J., MUEHLENBACHS K., O'NIONS R.K., OSKARSSON N. and OXBURGH E.R. (1983) Helium, oxygen, strontium and neodymium isotopic relationships in Icelandic volcanics. Earth Plan. Sci. Let. 66, 125-136.

CRAIG H. and LUPTON J.E. (1976) Promordial neon, helium and hydrogen in oceanic basalts. Earth Plan. Sci. Let. 31, 369-385.

CRAIG H. and POREDA R. (1986) Papatua Expedition Legs V and VI: Studies of methane and helium in hydrothermal vent plumes, spreading-axis basalts, and volcanic island lavas and gases in southwest Pacific marginal basins. Scripps Institute of 0ceanography Reference 87-14. FANALE F.P. (1964) Helium in magnetite. Columbia University Ph.D., (Geology), $135 \mathrm{p}$.

GRAHAM D.V., JENKINS W.J., KURZ M.D. and BATIZA R. (1987) Helium isotope disequilibrium and geochronology of glassy submarine basalts. Nature 326(6111), 384-386.

GRAMLICH J.W. and NAUGHTON J.J. (1972) Nature of source material for ultramafic minerals from Salt Lake Crater, Hawaii, from measurment of helium and argon diffusion. J. Geophys. Res. 77-17, 3032-3042.

HART S.R. (1984) He diffusion in olivine. Earth Plan. Sci. Let. 70, 297-302.HESS P.C. (1980) Polymerization model for silicate melts. In Physics of Magmatic Processes (ed. R.B. HARGRAVES) pp.3-44. Princeton Univ. Press, Princeton, N.J.

HURLEY P.M. (1954) The helium age method and the distribution and migration of helium in rocks. In Nuclear Geology (ed. H. FAUL) pp. 301-329. J. Wiley and Sons, New York.

JAMBON A., WEBER H.W. AND BEGEMANN F. (1985) Helium and Argon from an Atlantic MORB glass: concentration, distribution and isotopic composition. Earth Plan. Sci. Let. 73 255-267. 
JOHNSON H.E. and AXFORD W.I. (1969) Production and loss of ${ }^{3}$ He in the earth's atmosphere. J. Geophy. Res. 74, 2433.

KANEOKA I. and TAKAOKA N. (1978) Excess ${ }^{129} \mathrm{Xe}$ and high ${ }^{3} \mathrm{He} /{ }^{4} \mathrm{He}$ ratios in olivine phenocrysts of Kapuho lava and xenolithic dunites from Hawaii. Earth Plan. Sci. Let. 39, 382-386.

KRYLOV A.Y., MAMYRIN B.A., SILIN, Y.I. and KHABARIN L.V. (1973) Helium isotopes in ocean sediments. Geochem. Int. 10, 202.

KURZ M.D. and JENKINS W.J. (1981) The distribution of helium in oceanic basalt glasses. Earth Plan. Sci. Let. 53, 41-54.

KURZ M.D., JENKINS W.J., SCHILLING J.G. and HART S.R. (1982) Helium isotopic variations beneath the central North Atlantic 0cean. Earth Plan. Sci. Let. 58, 1-14.

KURZ M.D., JENKINS W.J., HART S.R. and CLAGUE D. (1983) Helium isotopic variations in the volcanic rocks from Loihi Seamount and the island of Hawaii. Earth Plan. Sci. Let. 66,

KURZ M.D., MEYER P.S. and SIGGURDSON H. (1985) Helium isotopic systematics within the neovolcanic zones of Iceland. Earth Plan. Sci. Let. 74, 291-305.

KURZ M.D. (1986a) Cosmogenic helium in a terrestrial igneous rock. Nature 320, 435-439.

KURZ M.D. (1986b) In situ production of terrestrial cosmogenic helium and some applications to geochronology. Geochim. Cosmo. Acta 50, 2855-2862.

KURZ M.D., GARCIA M.0., FREY F.A. and 0'BRIAN P.A. (1987) Temporal helium isotopic variations within Hawaiian volcanoes: basalts from Mauna Loa and Haleakala. Geochim Cosmo. Acta 51, 2905-2914.

LAL D. (1987) Production of ${ }^{3}$ He in terrestrial rocks. Chemical Geology (Isotope Geoscience Section) 66, 89-98.

LAL D. (1988) In situ-produced cosmogenic isotopes in terrestrial rocks. Ann. Rev. Earth Planet. Sci. 16, 355-388.

LEVENTHAL J.S. (1975) An evaluation of the U-Th-He method for dating young basalts. J. Geophys. Res. 80, 1911-1914. 
LUPTON J.E. (1983) Terrestrial inert gases: isotope tracer studies and clues to primordial components in the mantle. Ann. Rev. Earth Planet. Sci. 11, 371-414.

LUPTON J.E. (1973) Direct accretion of ${ }^{3}$ He and ${ }^{3} \mathrm{H}$ from cosmic rays. J. Geophys. Res. 78, 8330-8337.

MAMYRIN B.A. and TOLSTIKHIN I.N. (1984) Helium Isotopes in Nature. Elsevier, Amsterdam.

MERRIHUE C. (1964) C. Ann. N.Y. Acad. Sci. 119, 351-367.

MORRISON P. and PINE J. (1955) Radiogenic origin of the helium isotopes in rock. Ann. N.Y. Acad. Sci. 62, 71-92.

0'NIONS R.K. and OXBURGH E.R. (1983) Heat and helium in the earth. Nature 306, 429-431.

OZIMA M., TAKAYANAGI M., ZASHU S. and AMARI S. (1984) High ${ }^{3} \mathrm{He} /{ }^{4} \mathrm{He}$ ratio in ocean sediments. Nature $311,448-450$.

POREDA R., CRAIG H. and SCHILLING J.G. (1980) ${ }^{3} \mathrm{He} /{ }^{4} \mathrm{He}$ variations along the Reykjanes Ridge. E0S, Trans. Am. Geophys. Union 61, 1158.

RISON W. and CRAIG H. (1983) Helium isotopes and mantle volatiles in Loihi Seamount and Hawaiian Island basalts and xenoliths. Earth Plan. Sci. Let. 66, 407-426.

STEIGER R.H. and JAGER E. (1977) Subcommission on geochronology: convention on the use of decay constants in geo- and cosmochronology. Earth Plan. Sci. Let. 36, 359-362.

ZINDLER A. and HART S. (1986) Helium: problematic primordial signals. Earth Plan. Sci. Let. 79, 1-8. 
Chapter 2. Experimental Methods 


\subsection{INTRODUCTION}

This chapter describes the selection and preparation of rock samples, techniques for releasing helium from rocks, and details of helium isotope mass spectrometry. It also reviews the assumptions and deficiencies of standard equations for determining diffusivities from emanation data, and presents a refined calculation scheme incorporating grain shape and size variations developed to improve the accuracy of diffusivity estimates for basaltic glasses (chapter 3).

\subsection{SAMPLE PREPARATION}

The main sample types used were submarine basaltic glasses, phenocryst separates from both submarine and subaerial basaltic and andesitic rocks, and mineral separates from ultramafic xenoliths. In all cases the whole rock samples were examined in hand specimen and by preparing a thin section prior to dissection. Interior portions of the whole rocks were chosen for study to avoid weathered or thermally altered margins; when necessary altered portions were removed by abrasive sawing or with hammer and chisel. The fresh rock interior pieces were broken with a hydraulic sample splitter to produce centimeter size pieces which were then crushed with a ceramic jaw crusher to approximately .5 to $3 \mathrm{~mm}$ fragments. The rock chips were then sieved and directly hand-picked for mafic minerals when abundant .5 to $2 \mathrm{~mm}$ crystals were present. For samples with less abundant, smaller phenocrysts a preliminary separation of minerals from fine-grained rock matrix fragments was carried out with a Frantz Isodynamic Separator, prior to hand-picking.

In some cases the first mineral separates were further crushed using a hardened steel mortar and pestle to obtain grain fragments without adhering matrix or to produce smaller grain sizes for diffusion studies. Glass samples and xenolith minerals were crushed directly with the mortar and pestle, without preliminary steps. Glass and mineral separates were sieved in standard stainless sieves (Newark Wire Cloth Co., N.J.) and cleaned by ultrasonic agitation in water, acetone and 
methanol and dried in air at room temperature. Final selection of sample grains was done by individual examination in reflected and transmitted light in air and in ethanol at 20 to $80 \mathrm{x}$ magnification. Polished grain mounts were prepared from splits of the samples analysed to examine fluid inclusion or vesicle abundances and extent of internal fracturing. These mounts were also used for chemical analysis with the JEOL electron microprobe under the direction of Dr. Steve Recca at MIT. Multiple spot analyses were always performed, and appropriate calibration standards used. Other details of sample selection and preparation are given in individual chapters as appropriate.

\subsection{HELIUM ANALYSES}

\section{$\underline{2.3 .1}$ Releasing helium}

Helium was released from the rock samples using three different extraction methods, all of which were performed under high vacuum while attached to the processing line for the noble gas mass spectrometer used for rocks (MS2). Helium contained in gas or fluid inclusions was released by crushing glass and mineral separates in 3/4" diameter stainless cylinders connected to the processing lines with VCR fittings and copper gaskets. Crushing was accomplished with a magnetic stainless-steel cylindrical slug that was lifted and driven down onto the sample by three stacked electromagnetic coils. This was done in an automated mode by computer control. Fifty cycles generally were enough to reduce millimeter sized phenocrysts to powders of less than 100 microns, although a few larger grains (250 um or so) often remained, and some very fine powder was produced. Crushing efficiency dropped as sample size was increased, particularly if more than 200-300 $\mathrm{mg}$ of material was used. The ram is quite forceful and diamonds have been successfully fractured.

Cleaning of the crusher canisters and rams was extensive to avoid inter-sample contamination and to reduce pump out times. Cleaning steps included polishing of the slugs, high-speed wire brushing of the canisters and a sequence of washings and sonic agitations in water, $8 \mathrm{~N}$ 
nitric acid, acetone and methanol of both canisters and slugs. In addition, small disk inserts of non-magnetic stainless steel were used to protect the bottom of the sample container, and were replaced for each analysis to minimize memory effects. The crushers have been previously described by Kurz et al (1987). The only important improvement was the replacement of four $\operatorname{VCR}^{T M}$ vacuum fittings with welds and one Mini-Conflat ${ }^{\mathrm{TM}}$ connections in the crusher manifold, which reduced the leak background.

Helium release was also achieved by sample heating and/or fusion in a resistively-heated furnace. The furnace consists of a tantalum crucible sealed to the gas processing line with a gold wire o-ring and isolated from the secondary vaccum jacket with a second gold o-ring. The vacuum jacket contains a basket-shaped tungsten resistance element along with a series of concentric tungsten and molybdenum heat shields isolated from each other with high temperature ceramic spacers. The water cooled copper feedthroughs are electrically insulated with teflon and the vacuum seal is achieved with viton o-rings. The secondary vacuum is maintained at less than $2 \times 10^{-7}$ torr with a dedicated $200 / \mathrm{s}$ oil diffusion pump. The power supply is a 220v line controlled by a large variable transformer which supplies current to a secondary transformer. Temperature control relies on a constant power input and has been calibrated using pyrometry, and the melting of metal standards. The furnace can achieve temperatures up to $2000{ }^{\circ} \mathrm{C}$, can reach basaltic fusion temperatures $\left(1200{ }^{\circ} \mathrm{C}\right)$ in about 5 minutes, and will remain at constant temperature within 5-8 degrees for days on end without regulation. The furnace has been described before by Kurz et al (1987) and is similar to the design presented by Staudacher et al (1978).

Samples were loaded into the furnace with either a rotary "sixshooter" or a magnetic-piston "four-shooter". Both are thermallyisolated sample holders which sit above the crucible, from which multiple samples can be delivered to the hot zone without breaking vacuum. In this way, the furnace can be baked out, and a blank determination performed prior to sample analysis. The six-shooter has been described before (Kurz et al, 1987). The four-shooter was built for the diffusion experiments and has the advantage of a viewing port 
Figure 2.1. The "Four-shooter" device for loading furnace samples. Construction is welded stainless steel with copper gasket knife-edge seals. Samples can be loaded either as loose grains or wrapped in aluminum or tantalum foil. Loose grains are first loaded into a small cup made from a permanent magnet (ALNICO), which is then lowered into the central tube and magnetically connected to the permanent magnet push ram. Foil wrapped samples are simply pushed into the sample arms with forceps. Loading samples requires venting the furnace (there is no valve between the sample holder and the crucible), and unbolting the flange between the window section and the sample section. Cleaning the sapphire window (Varian), shutter and sample loader is done by swabbing with a solution of $45 \%$ conc. Nitric acid, $5 \%$ conc. HF and $50 \%$ water, followed by copious water rinsing, and a final methanol rinse. Care must be taken not to expose the knife-edges and especially the bronze window seal to the acid. Sample loading is done by repelling the permanent magnet push rods towards the axial tubes using an external cylindrical magnet. For the loose grains, the cup is first repelled into the center and then rotated by rolling the external magnet around the loading arm. Similarly, the stainless steel shutter is actuating with an external magnet, and protects the viewing window from becoming coated by fine particles from the crucible, although gases can still reach the port and coat it. 
Figure 2.1

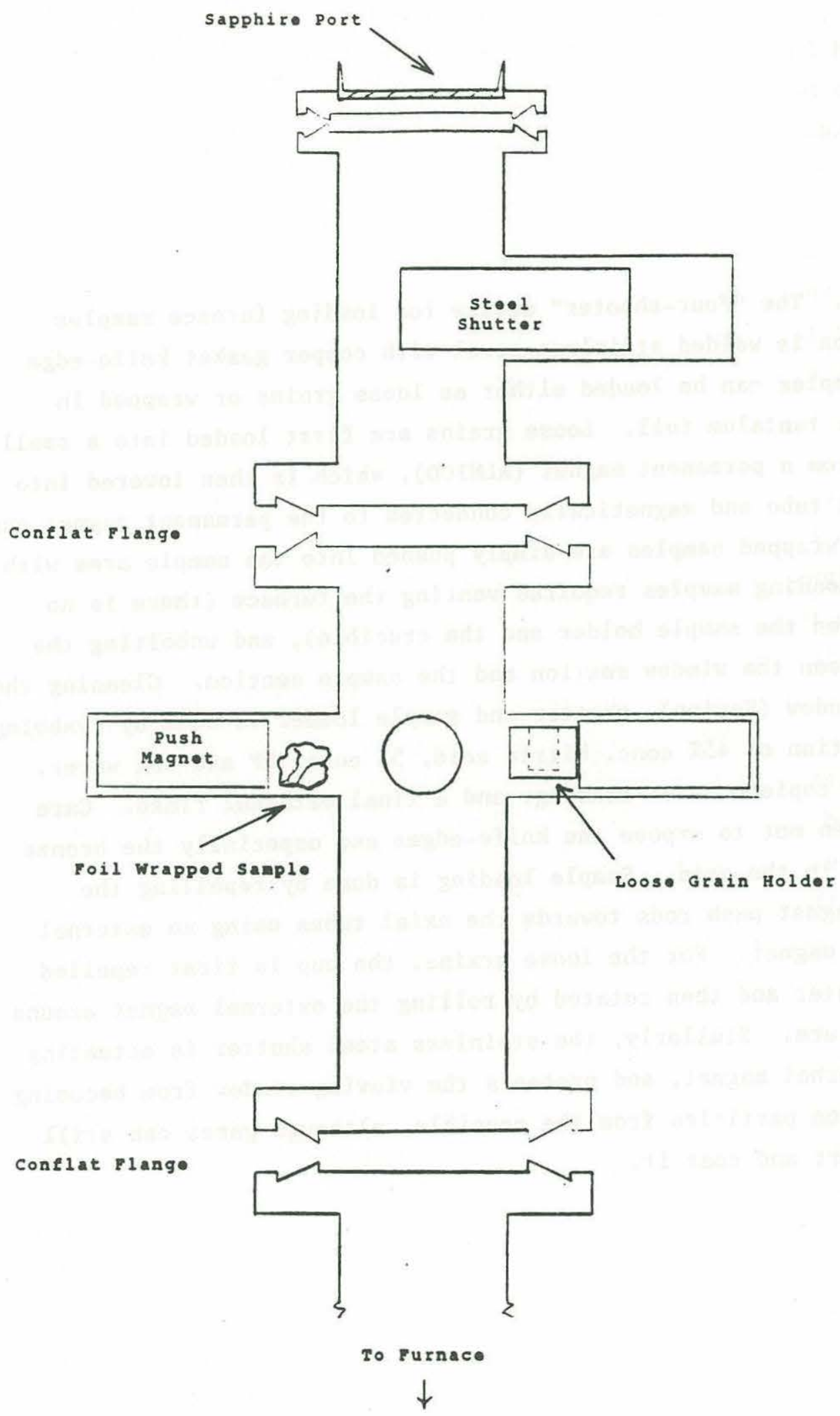


(figure 2.1). In general, the furnace was operated with a tantalum thin-walled inner crucible. This insert was replaced between samples during step-heating experiments to avoid chemical reactions that might release gases, and protected the outer crucible from direct contact with molten silicates during fusion studies. However, the outer tantalum crucibles are subject to hardening and fracture by recrystallization, and this required their replacement after roughly 100 heat cycles. Aspects of the furnace that have not been described before include the addition of the four-shooter, temperature calibration using standard metals, new copper feedthroughs, and a nitrogen venting line which allows the primary vacuum side of the furnace to be vented without bringing the processing line up to atmospheric pressure.

Measurements to determine the helium diffusivity of silicate glasses and crystals at low temperature used a specially constructed vessel designed for incremental heating of small samples in the temperature range or $20-600^{\circ} \mathrm{C}$. This diffusion vessel consists of a copper sample holder inserted in a .065" wall stainless steel canister, with an exterior thermocouple and resistance heater (figure 2.2). Good thermal contact is provided by the copper insert which surrounds the sample grains. The surfaces between the insert and the vessel bottom are polished to improve thermal contact between the sample and the thermocouple. In order to examine thermal equilibrium between the sample and the external thermocouple, a test was performed in which a second thermocouple was installed in the copper sample holder, and the temperature between the two was compared. In these tests the internal temperature was slightly lower, the differences ranged from $0{ }^{\circ} \mathrm{C}$ at 25 ${ }^{\circ} \mathrm{C}$ to $14{ }^{\circ} \mathrm{C}$ at $600{ }^{\circ} \mathrm{C}$ and the subsequent experiments were corrected for this offset. This temperature differential was reproducible and does not represent an important uncertainty in the experimental temperatures. The .015" Cr-Al bare wire thermocouples were insulated with high temperature ceramic tubing and glass wool, to avoid strong temperature gradients along the leads, and the junction was well within the heater's hot zone (see figure 2.2). The thermocouples were calibrated to $1{ }^{\circ} \mathrm{C}$ at the endpoints $0{ }^{\circ} \mathrm{C}$ and $600{ }^{\circ} \mathrm{C}$ using a distilled water/ice bath and a specially constructed DC millivolt supply, providing a factory-specified 
Figure 2.2. Diffusion Vessel schematic showing sample chips enclosed in a copper sample holder with copper lid, which is attached to the stainless vessel with a central screw. Outside the vacuum, a $\mathrm{Cr}-\mathrm{Al} .15$ " bare-wire thermocouple insulated with high-temperature ceramic tubes is attached to the vessel floor. The vessel wall thickness is .065". Both the vessel floor and the copper sample holder are highly polished to promote good thermal contact. Surrounding the sample hoder and the glass wool insulated thermocouple well is a Nichrome wire heater (20 ohm) constructed in the laboratory by winding the wire around an asbestos-wrapped stainless steel sleeve and insulating with high temperature ceramic (Ceramacast Inc.). The heater and thermocouple leads are run to a microprocessor-based controller (0mega 4002) and a variable power supply (0-140V, Variac.). 
Figure 2.2

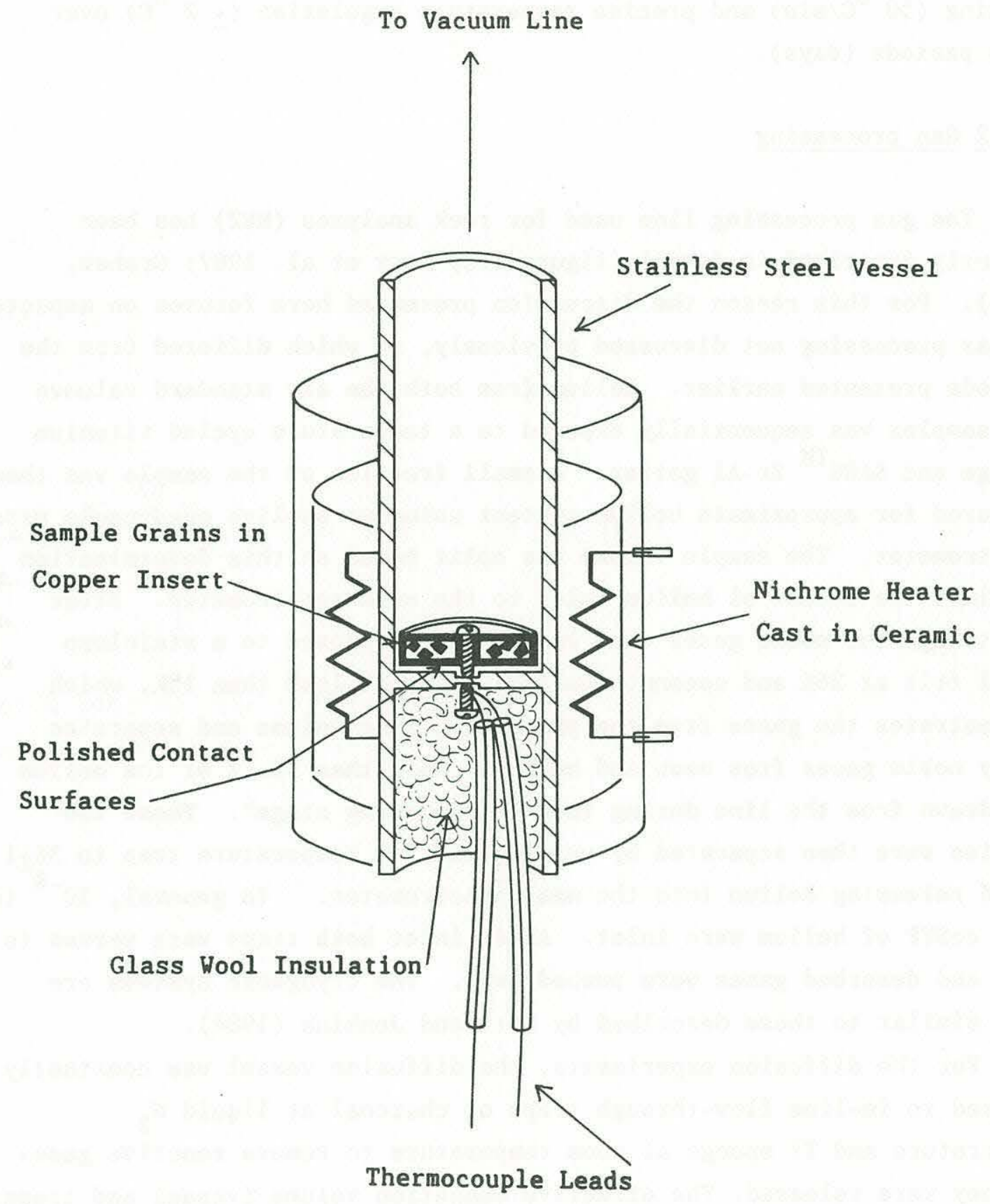


accuracy of better than $3{ }^{\circ} \mathrm{C}$ over this temperature range. The combination of a variable power supply (0-140v AC) and a timeproportional controller (OMEGA model 4002) allowed rapid temperature ramping $\left(50{ }^{\circ} \mathrm{C} / \mathrm{min}\right)$ and precise temperature regulation $\left( \pm 2{ }^{\circ} \mathrm{C}\right)$ over long periods (days).

\section{$\underline{2.3 .2}$ Gas processing}

The gas processing line used for rock analyses (MS2) has been recently described in detail (figure 2.3; Kurz et al. 1987; Graham, 1987). For this reason the discussion presented here focuses on aspects of gas processing not discussed previously, or which differed from the methods presented earlier. Helium from both the air standard volumes and samples was sequentially exposed to a temperature cycled titanium sponge and SAES ${ }^{\mathrm{TM}} \mathrm{Zr}-\mathrm{Al}$ getter. A small fraction of the sample was then measured for approximate helium content using an on-line quadrupole mass spectrometer. The sample volume was split based on this determination to limit the amount of helium inlet to the mass spectrometer. After splitting, the noble gases were sequentially exposed to a stainless steel frit at $26 \mathrm{~K}$ and coconut-charcoal chips at less than $15 \mathrm{~K}$, which concentrates the gases from the processing line volume and separates heavy noble gases from neon and helium. More than $99.7 \%$ of the helium was drawn from the line during this "cryopumping stage". These two species were then separated by warming the low temperature trap to $36 \pm 1$ $\mathrm{K}$ and releasing helium into the mass spectrometer. In general, $10^{-9^{-}}$to $10^{-8}$ ccSTP of helium were inlet. After inlet both traps were warmed to $65 \mathrm{~K}$ and desorbed gases were pumped away. The cryogenic systems are very similar to those described by Lott and Jenkins (1984).

For the diffusion experiments, the diffusion vessel was constantly exposed to in-line flow-through traps of charcoal at liquid $\mathrm{N}_{2}$ temperature and $\mathrm{Ti}$ sponge at room temperature to remove reactive gases as they were released. The effective emanation volume (vessel and traps) was about $130 \mathrm{cc}$, which guaranteed that helium release from the glass or crystal grains occured into an effectively zero concentration surrounding space. Another difference from standard processing for the 
Figure 2.3. Gas Processing Line for Mass Spectrometer 2, designed by M.D. Kurz and D.E. Lott. LTCT=low temperature cryogenic trap (charcoal), HTCT=high temperature cryogenic trap (steel frit), $\mathrm{RGA}=$ residual gas analyzer (quadrupole mass spectrometer), $\mathrm{TP}=$ turbomolecular pump, IP=ion pump, $\mathrm{RP}=$ rotary pump (figure from Graham, 1987). 


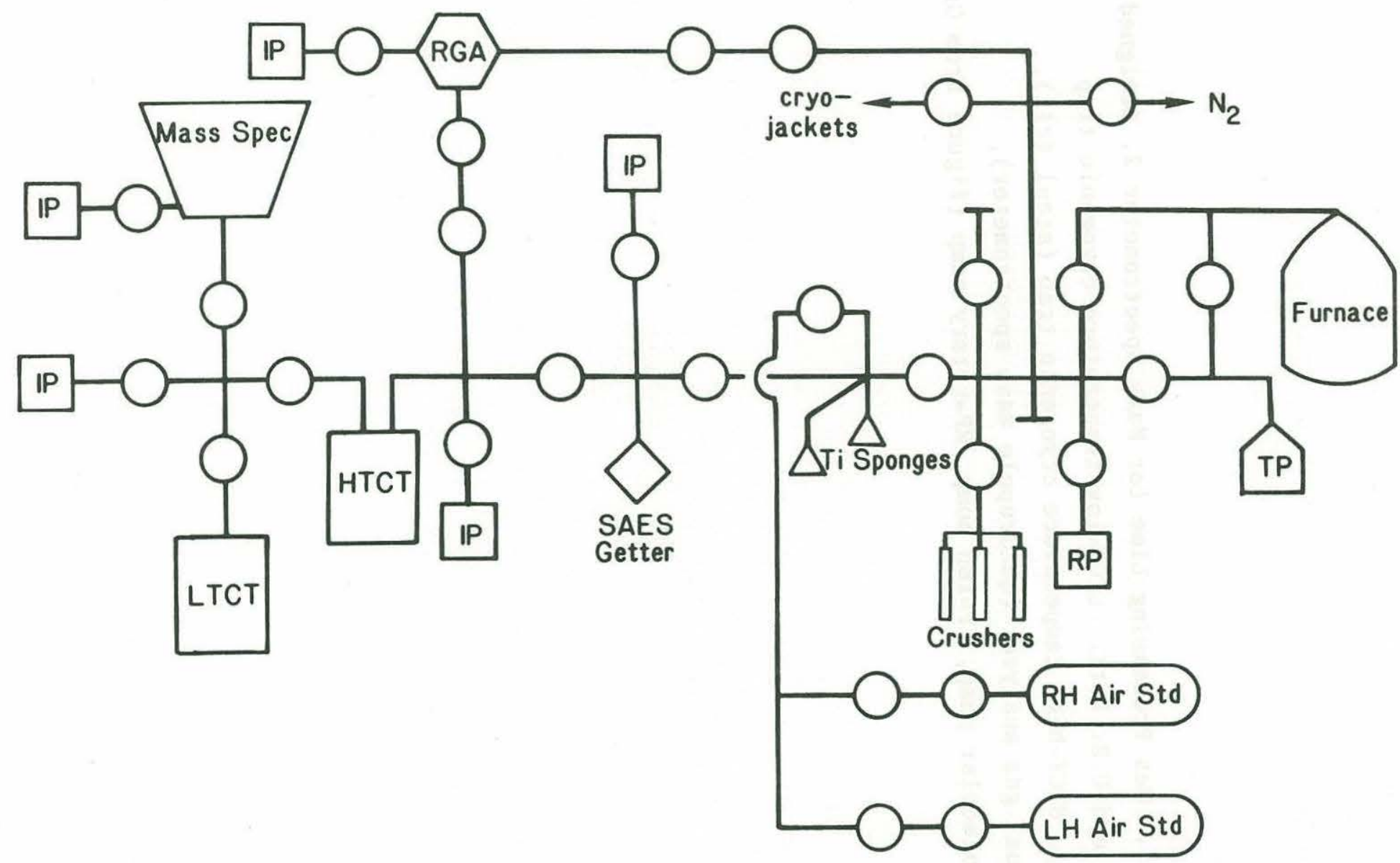


diffusion measurements was that the samples could not be split without losing some of the continuously emanating gas, so relatively large amounts of helium were occasionally inlet to the mass spectrometer.

The entire gas processing operation as well as the mass spectrometry has been automated using solenoid actuated pneumatic valves (thanks to Dr. W.J. Jenkins, Mr. D.E. Lott III and Dr. M.D. Kurz). This is an important aspect of the analysis because automation allows highly reproducible run conditions, including expansion, cryopumping, and inlet times; as well as pumping times between samples. Gas processing and analysis follows an approximately 30 minute cycle, with two samples being processed at any time, one in the mass spectrometer, the other in the processing line. Computer control is via a PDP11/23, recently upgraded from the previous $11 / 03 \mathrm{CPU}$ to allow the use of multitasking software (TSX-Plus version 6.31; S\&H computer systems, Inc.).

Gas processing and mass spectrometry are controlled by different software jobs (Fortran 77) which run at different priorities, providing uninterrupted servicing of analysis routines (program RATI05), while allowing the computer to be used for real time data analysis. The gas processing program (SMPLR5) was specifically tailored for the diffusion experiments. Data is recorded on a hard disk and on paper, and is automatically backed up to floppy disks every 24 hours. The dedicated PDP11 computer is networked to a central computer for the laboratory (a PDP11 173). This connection provides access to graphics routines, plotters, laser printers, other computers and communication lines. Modem access to the mass spectrometer at all hours greatly relieved the machine-side duties required during the long duration diffusion experiments. Data transfer to a SUN $3 / 260$ computer made the computationally intensive diffusion coefficient determinations possible.

\section{$\underline{2.3 .3}$ Mass spectrometry}

The mass spectrometer dedicated to rock analyses (MS2) has a 10 inch radius, $90^{\circ}$ sector electromagnet and an all metal branched flighttube. The source is a modified Nier design with a half-circle filament, and a source electromagnet outside the vacuum, to extend the electrons' 
flight paths through the ionizing chamber. A faraday cup is used to measure ${ }^{4} \mathrm{He}$ with a resolution of $1: 230$ and a electron-multiplier (JLI model MM-2) operated in analog mode with its entrance slit set to a resolution of 625 to separate ${ }^{3} \mathrm{He}$ from the much larger $\mathrm{HD}-\mathrm{H}_{3}$ peak. The instrument has been described before (Kurz, 1982; Graham, 1987) and is of the general design outlined by Jenkins (1974) and Clarke et al (1976). It was constructed in the Woods Hole laboratory. Measurement of the ion beams is done under computer control. Peak-switching is done by adjusting the accelerating voltage rather than the magnetic field strength. After sample inlet (duration 30 seconds), the machine is centered on the HD peak, and then offset to ${ }^{3}$ He. The ${ }^{3}$ He peak height (56 second integration) and baseline (14 second integration) are measured in 15 voltage switching cycles. During each on-peak cycle ${ }^{4} \mathrm{He}$ is also measured, but the ${ }^{4} \mathrm{He}$ baseline is measured only once (25 second integration) prior to inlet of the sample, because secondary electrons from the large ${ }^{4} \mathrm{He}$ ion beam lead to incorrect baseline estimates. Baseline corrections are done for each cycle, using the mean of adjacent baseline measurements for ${ }^{3} \mathrm{He}$ and the initial baseline determination for ${ }^{4} \mathrm{He}$.

Because both depletion and "grow-in" of background occur during the static analysis, the sequential peak determinations (and their ratio at each cycle) are fit to a linear trend and extrapolated to the time of sample inlet to obtain the peak heights prior to depletion. The ratios determined at each cycle display a more linear time dependence than the individual peaks, so the extrapolated ratio is a more reliable determination of the isotopic ratio of a sample than the ratio of individually extrapolated ${ }^{3} \mathrm{He}$ and ${ }^{4} \mathrm{He}$ peaks. The extrapolated ratio is then combined with the extrapolated ${ }^{4}$ He contents to obtain the best determination of the ${ }^{3} \mathrm{He}$ contents. For a wide range of ${ }^{3} \mathrm{He} /{ }^{4} \mathrm{He}$ ratios, this ${ }^{3}$ He estimate is found to be within the uncertainty (associated with linear regression) of the directly extrapolated ${ }^{3}$ He peak value, but with smaller uncertainties (by 20 to $50 \%$ ).

The mass spectrometer is capable of detecting small amounts of helium and in practice is limited by the ability to reduce the atmospheric background, or processing blank, rather than absolute 
sensitivity. Detection limits (estimated as twice the uncertainty in the determination of line blanks) are $5 \times 10^{-12} \operatorname{ccSTP}{ }^{4} \mathrm{He}$ and about $3 \mathrm{x}$ $10^{-16}$ ccSTP ${ }^{3} \mathrm{He}$. Under normal run conditions, the processing blanks range as follows, with uncertainties at any time of $10 \%$ or so: $2-3 \mathrm{x}$ $10^{-11}$ ccSTP He for the "dry" section of the line (that is the mass spectrometer, inlet line and cryotraps), and $3-5 \times 10^{-11}$ ccSTP He including the "wet" line (getters, quadrupole inlet line, crushers and/or furnace). It is not possible to precisely determine the isotopic composition of individual processing blanks, but their daily and longer term average has an atmospheric ${ }^{3} \mathrm{He} /{ }^{4} \mathrm{He}$ ratio. This isotopic composition and the increased blank associated with increased line sections is consistent with the blank being caused by very small leaks of atmospheric gases into the line at $\mathrm{VCR}^{\mathrm{TM}}$ and Mini-Conflat ${ }^{\mathrm{TM}}$ connections. In this regard, replacing VCR fittings with Mini-Conflats in the crusher manifold section reduced the processing blank from 5-6 $\mathrm{x}$ $10^{-11}$ to $3-4 \times 10^{-11}$ ccSTP He. There are additional backgrounds associated with long term closures of both the diffusion vessel (owing to several VCR connections) and the furnace when the Varian 5/8" diameter sapphire viewing port is used, of $8 \pm 1 \times 10^{-16} \mathrm{cc} / \mathrm{s}$ and $1.5 \pm 1 \mathrm{x}$ $10^{-16} \mathrm{cc} / \mathrm{s}$ respectively, both with atmospheric isotopic composition. Calibration of both sample size and isotopic composition is by comparison to air aliquots. An aliquot of approximately $1.6 \times 10^{-8}$ ccSTP He with atmospheric isotopic composition is automatically analyzed repeatedly throughout the day (usually 15-25 times) and is alternated with blank, sample, and other size air aliquot determinations. The size and ratio of this daily air standard may vary by a few percent during a 24 hour period (primarily due to changes in filament characteristics), so temporal trends are examined and used to calibrate sample determinations based on when they were run.

The dependence of apparent isotopic composition on sample size is examined on a roughly monthly basis from accumulated determinations on air standards of different sizes, or whenever instrumental modifications are made, or failures occur. In contrast to the strong dependence of

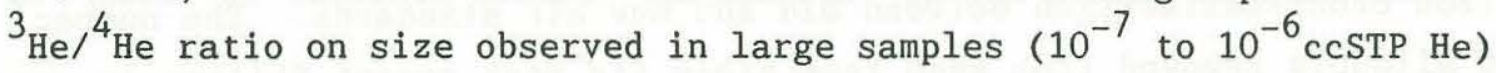
by Kurz (1982), no size effect is detectable in the much smaller sample 
range now used (generally less than a few times $10^{-8}$ ccSTP; figure 2.4). However, on the order of $3 \%$ decrease in the ${ }^{3} \mathrm{He} /{ }^{4} \mathrm{He}$ ratio with increasing size was found for samples larger than $10^{-7} \mathrm{cc}$ STP (figure 2.5).

A few diffusion experiment releases were this large (and could not be readily split) and have been corrected for this "linearity" effect. Possible "memory" effects for these large samples were explored by examining the size and isotopic composition of adjacent air standards and processing blanks, and were found to be unimportant. The uncertainties reported for the ${ }^{4} \mathrm{He}$ contents and ${ }^{3} \mathrm{He} /{ }^{4} \mathrm{He}$ ratios were derived from the statistical errors of measuring the ion beams and extrapolating to time of inlet combined with uncertainties propagated through the standard calibration, blank subtraction and linearity correction equations. Typical one sigma errors for a sample of $10^{-8}$ cCSTP He with a ${ }^{3} \mathrm{He} /{ }^{4} \mathrm{He}$ ratio of $1.2 \times 10^{-5}$ (8.4 Ra, typical of MORB) are $.1 \%$ on the size and $1 \%$ on the ratio.

Primary air standards are taken in a stainless-steel canister (about $40 \mathrm{ml}$ ) with copper stem-tipped stainless steel bellows valve. Its volume has been calibrated by weighing before and after filling with water and more recently by measuring the $\mathrm{N}_{2}$ content of the canister with a quadropole mass spectrometer. These determinations are in good agreement and provide a calibration volume precision of $.05 \%$. The air standard volume is corrected to standard temperature and pressure (including humidity) using values measured at the collection site, which is located well away from laboratory influences (Chemotaxis beach, Quissett Campus).

The air standard is then exposed to a charcoal trap at liquid $\mathrm{N}_{2}$ temperature to remove argon, $\mathrm{H}_{2} \mathrm{O}, \mathrm{CO}_{2}, \mathrm{O}_{2}$, etc. and expanded into a $10 \mathrm{~L}$ reservoir. Standard aliquots are taken from the reservoir during automated running for use in size and isotopic ratio calibration. The standard aliquot volumes were calibrated by comparison with a mercury calibrated glass volume using ${ }^{4}$ He peak height measurements. Two of these large reservoirs with separate aliquotting valves are used to allow cross-calibration between old and new air standards. The number of aliquots removed from each tank since its most recent filling is used 
Figure 2.4. Relationship between sample size and measured ratio for typical sample sizes (data from July, 1987). Samples used are splits of the air standard, obtained by isolating different sections of the processing line prior to cryotrapping helium. Errors in measuring small samples increase because of ion-counting statistics. There is no evidence for any dependence of the observed ${ }^{3} \mathrm{He} /{ }^{4} \mathrm{He}$ ratio on size in this range. This data is typical of normal running conditions. 
Figure 2.4

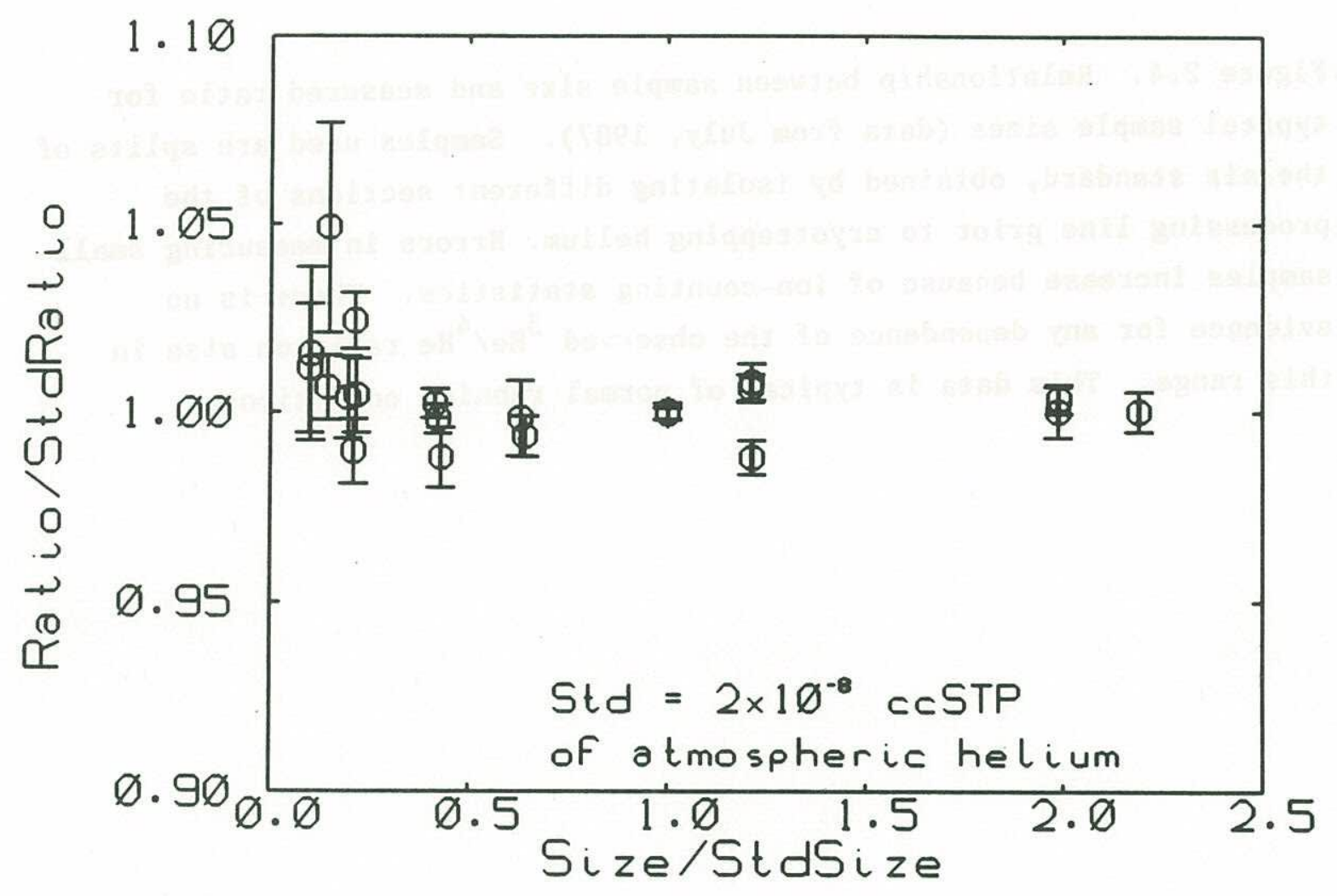


Figure 2.5. "Linearity" relationship for very large samples (data from August, 1987). In contrast to smaller samples, the apparent ${ }^{3} \mathrm{He} /{ }^{4} \mathrm{He}$ ratio varies with size for samples greater than about $8 \times 10^{-9}$ ccSTP, decreasing by $3 \%$ or so over the range of $2 \times 10^{-8}$ to $2 \times 10^{-7} \operatorname{ccSTP}$ (the large size data points were determined twice each, and the mean is shown). 
Figure 2.5

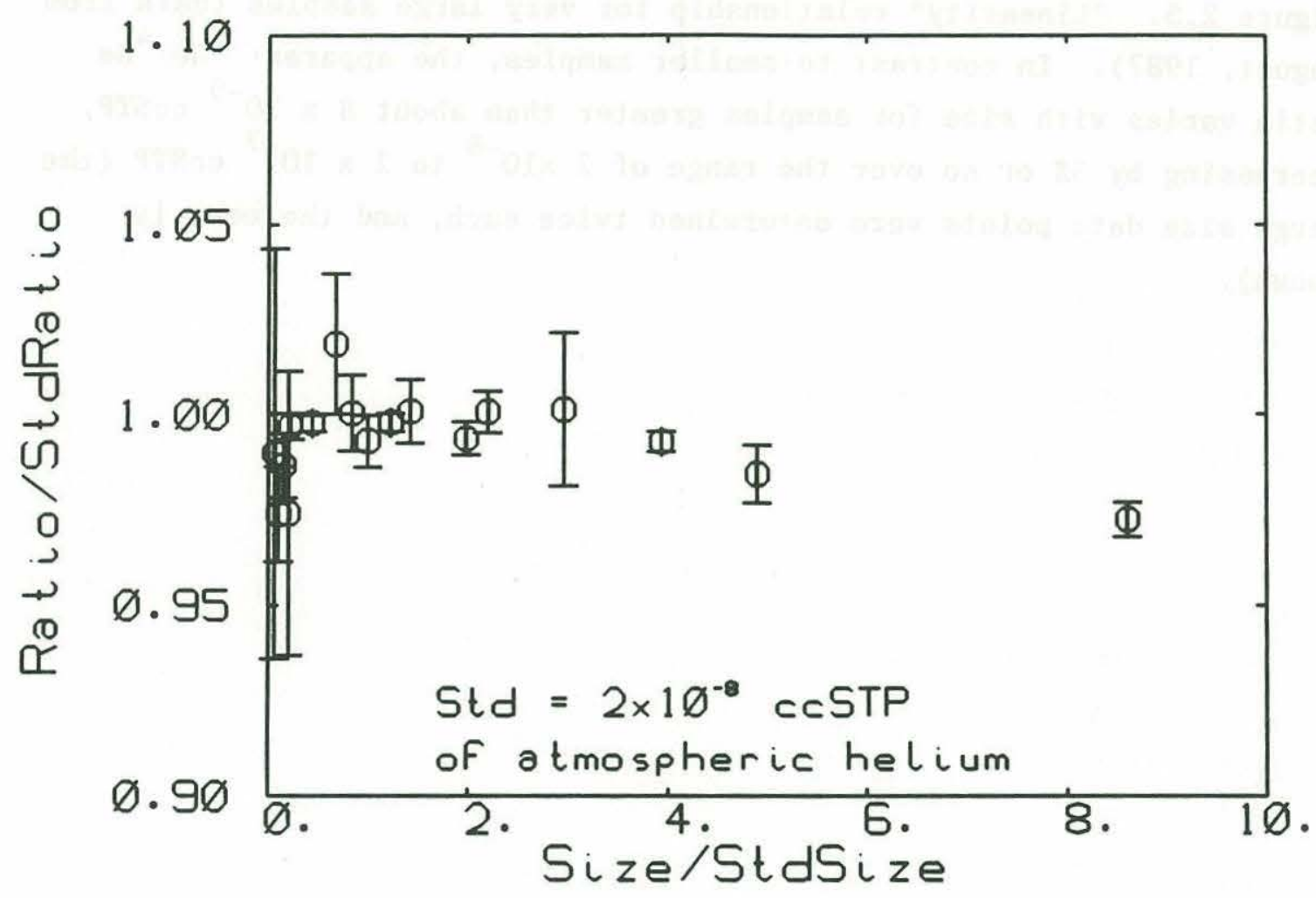


in correcting each standard's size for depletion. Overall, the precisions involved in calibrating the standard volume, aliquotting volumes, reservoir volumes, and expansion line volumes suggest an uncertainty of less than $<.5 \%$ in the absolute size of the air standards. This uncertainty is much smaller than errors associated with sample analyses

\subsection{THE DETERMINATION OF DIFFUSION COEFFICIENTS FROM EMANATION DATA}

\subsubsection{Assumptions and associated errors of the standard spherical model}

Emanation experiments measure the rate of release of a gas from a condensed phase, in this case a collection of solid particles. The release may occur by one or more mechanisms and may be controlled by processes that occur within the solid, at its surface, or in the surrounding medium. In order to use emanation data to characterize transport of the gas within the solid, it is neccesary to establish the physical nature of the release. This generally requires knowledge of the physical properties of the solid, diffusing gas, surrounding medium, and experimental setup, but to some degree the systematics of the measured release (for instance its time dependence) can distinguish different release processes. In these experiments, we desired to determine the rate of volume diffusion of helium isotopes within silicate glasses and minerals. In order to do this it was necessary to establish that helium release was governed by volume diffusion within the solid and not by processes such as diffusion along cracks, leaks from vesicles, surface desorption, or passage through a surface layer on the particles. This was done by comparing the time and temperature dependence of release from different grain sizes and from samples with and without vesicles over large extents of fractional release, as discussed in Chapter 3.

Volume diffusion, or diffusion in a locally isotropic medium, provides the fundamental hypothesis that the rate of transfer of a 
diffusing substance through a section is proportional to the concentration gradient normal to the section. This is Fick's first law:

$$
\mathrm{F}=-\mathrm{DdC} / \mathrm{dx}
$$

where $\mathrm{x}$ is measured normal to the section (e.g. Crank, 1975). This equation is the basis of the determination of diffusion coefficients in steady-state experiments. Conservation of the diffusing substance leads to the diffusion equation:

$$
\mathrm{dC} / \mathrm{dt}=-\operatorname{div}[D \operatorname{grad}(\mathrm{C})]
$$

which expresses the temporal change in concentration in terms of the spatial variations in both $C$ and $D$ that drive diffusive fluxes and allows the determination of diffusion coefficients in time dependent situations. Both equations 2.1 and 2.2 can be derived from the vantage of statistical mechanics by envisioning a random walk with characteristic step length and frequency (Einstein, 1905).

If $\mathrm{D}$ is independent of concentration and does not vary with location in the material of interest then equation 2.2 reduces to:

$$
\mathrm{dC} / \mathrm{dt}=-\mathrm{D} \operatorname{div}^{2}[\mathrm{C}]
$$

When $D$ varies with time, as in a step-heating experiment, solutions to this equation yield diffusivities defined by the mean value theorem, that is D becomes

$$
D=1 /\left(t-t_{0}\right) \int_{t_{0}}^{t} D(t) d t
$$

For diffusion of a trace component such as helium in an isotropic material like basaltic glass the assumption that $D$ varies only with time and temperature is extremely reasonable. In addition, since fastdiffusing helium is a neutral species and interacts only minimally with other atoms its diffusivity will not be coupled to other species by 
electroneutrality or mutual diffusion conditions so that a tracer diffusion coefficient may be directly obtained.

Evaluating equation 2.3 requires a description of the spatial nature of the system. Spherical geometry provides a useful limiting case because this is the shape with the lowest surface to volume ratio, the property which controls the rate of emanation for low extents of releases. For a sphere, considering only radial diffusion, equation 2.3 becomes:

$$
\mathrm{dC} / \mathrm{dt}=\mathrm{D}\left\{\mathrm{d}^{2} \mathrm{C} / \mathrm{dr} \mathrm{r}^{2}+2 / \mathrm{r}(\mathrm{dC} / \mathrm{dr})\right\}
$$

where $\mathrm{D}$ is defined by equation 2.4. The initial condition is given by the concentration profile, $\mathrm{C}(\mathrm{r})$ at time zero, and loss from the grain can be described by either evaporation or constant surface concentration boundary conditions.

For evaporation: the flux from the surface is proportional to the concentration difference at the boundary:

$$
-D \cdot d C / d r=h\left(C_{S}-C_{0}\right)
$$

where $\mathrm{C}_{\mathrm{S}}$ is the concentration just inside the sphere, $\mathrm{C}_{0}$ is the concentration in the surrounding medium, and $\mathrm{h}$ is a proportionality constant, essentially determined by the impedence to evaporation into the medium. As $\mathrm{h}$ increases, $\mathrm{C}_{\mathrm{S}}$ and $\mathrm{C}_{\mathrm{O}}$ tend to approach each other and for the limit of $\mathrm{h}=$ infinity the evaporation condition is equivalent to the constant surface concentration boundary condition:

$$
\mathrm{C}_{\mathrm{S}}=\mathrm{C}_{\mathrm{O}}
$$

In the helium emanation experiments, the greatest impedence to helium loss is likely to occur within the solid rather than at the surface (i.e. h is very large because there is little resistance to release of a helium atom from the surface to the surrounding medium) so that $2.6 \mathrm{~b}$ is a reasonable boundary condition. In addition, the diffusion vessel was designed so that the helium concentration in the space 
surrounding the grains was always negligible so that $\mathrm{C}_{0}$ can be taken as zero (the vessel volume was about $130 \mathrm{cc}$, and less than $10^{-6} \mathrm{cc}$ He were present in any sample volume of about $1 \mathrm{cc}$ ). Together with the initial distribution $C_{i}(r)$ this leads to the solution, for a sphere of radius a :

$$
C(t)=\sum_{1}^{\infty} A_{n} \exp \left(-n^{2} \pi^{2} / a^{2} D t\right)
$$

with:

$$
A_{n}=6 / a^{3} \int a\left(\int_{0}^{a} r C_{i}(r) \sin (n \pi r / a) d r\right) r / a \sin (n \pi r / a) d r
$$

Provided the initial helium distribution is known, this solution gives the fractional loss as a function of time, which is the relation needed to obtain diffusivities from emanation data. For $C_{i}(r)$ constant, equations 2.7 and 2.8 yield:

$$
F=1-6 / \pi^{2} \sum_{1}^{\infty} 1 / n^{2} \exp \left(-n^{2} \pi^{2} / a^{2} D t\right)
$$

where $\mathrm{F}$ is the fraction released. This equation reveals that loss is governed by the ratio $\mathrm{D} / \mathrm{a}^{2}$ for a sphere, and is a frequent starting point for analyses of emanation data. However, helium distributions in natural minerals and glasses can differ from homogeneity for two fundamental reasons, the grains may have formed with heterogeneous distributions, or the helium content of the grains may have evolved away from an initial constant concentration profile.

Vesicles and fluid inclusions contain large fractions of the total helium in some of the basaltic glasses studied in chapter 3 , and in the olivine and pyroxene mineral grains studied in chapter 4 . Thus, a homogeneous initial distribution is clearly an approximation, but will be a reasonable one provided the vesicles and inclusions are small in comparison to grain sizes and the rate-limiting step for loss is passage through the glass or mineral volume. Emanation rates for glasses with approximately $1 \%$ and $50 \%$ vesicle helium contents were very similar 
(chapter 3 ), suggesting the approximation is reasonable for these samples, and that dissolution at the gas-solid interface is not a ratelimiting process.

In the mineral studies, 50 to $90 \%$ of the helium was contained in the fluid inclusions, which tended to be aligned in planar arrays. It is possible this geometry enhances loss, so that derived diffusivities may be upper limits (chapter 4). If helium loss were dominated by passage from a large vesicle or inclusion through a surrounding glass or mineral "membrane", the release behavior would differ from volume diffusion. Initial releases of helium permeating the membrane would increase with time, and steady-state loss would display a constant release rate, rather than the linear in $t^{1 / 2}$ behavior of volume diffusion (see below). In addition, the initial "transient permeation" of the solid by the vesicle helium can greatly enhance isotopic fractionation (Rama and Hart, 1965). These characteristics were not observed (chapter 4), suggesting the release process and helium distributions were more akin to volume diffusion and an approximately homogeneous initial condition.

Heterogeneous radiogenic helium distributions may be generated by variations in parent uranium and thorium contents, which are known to primarily reside in fine-grained accessory minerals in basaltic minerals (Polve, 1985). However, in glasses (as studied in chapter 3) these elements, and thus radiogenic helium $\left({ }^{\star 4} \mathrm{He}\right)$, are likely to be isotropically distributed. Cosmogenic helium $\left({ }^{3} \mathrm{He}_{c}\right)$ distributions in the rocks studied here should also be homogeneous, because the dominant production mechanism is spallation of major elements (chapter 5). However, in some situations, secondary production through reactions on lithium could lead to ${ }^{3} \mathrm{He}_{\mathrm{c}}$ heterogeneities. The energies associated with ${ }^{* 4} \mathrm{He}$ and ${ }^{3} \mathrm{He}_{\mathrm{c}}$ generation may also redistribute these atoms, however, as discussed in chapter 5, most rocks will not be strongly affected by this process, because ejection losses will generally be balanced by injection gains. Exceptions would be rocks with dramatically varying parent nuclide compositions. Recoil related redistribution problems have been recognized in ${ }^{40} \mathrm{Ar}$ studies (e.g. 
Hunecke, 1976) but are unlikely to significantly affect the results presented here.

The most important departure from homogeneous distribution is the occurrence of prior diffusive loss. This can lead to large errors in diffusivity estimates, if unaccounted for. In particular, D values will be highly underestimated at low fractional losses, because initial release rates are much lower for a grain which has already depleted its near surface helium contents. At higher F, both distributions will suggest similar diffusivities. This can be seen by approximating the series in equation 2.9 by its first term at high F (Huneke, 1976):

$$
C(t)=A_{1} \cdot \exp \left(-1^{2} \pi^{2} / a^{2} D t\right)
$$

which after taking logarithms and differentiating yields, (because $A_{1}$ is independent of $t$ ):

$$
d(\ln C(t)) / d t=1 / C(t) d C(t) / d t=-\pi^{2} D / a^{2}
$$

This equation shows that the fractional release rate $\mathrm{dC} / \mathrm{C}$ at high $\mathrm{F}$ (long time) depends only on $\mathrm{D}$ and a and not the initial distribution, even though the amount of helium remaining is a function of the initial distribution (equation 2.10). Thus, diffusivities obtained at sufficiently high $\mathrm{F}$ will be unaffected by problems associated with unknown initial conditions. Numerical simulations of prior loss effects on apparent diffusivities confirm this behavior, and reveal that $D$ values can be underestimated by more than an order of magnitude at low F, even for relatively small prior losses (figure 2.6). D values approach the correct diffusivity at $\mathrm{F}$ values greater than approximately twice the prior loss amount. The numerical experiments also revealed that diffusive prior loss does not have a large effect on the determination of isotopic diffusivity ratios, essentially because both isotopes have similar initial profiles.

In the glass experiments (chapter 3), prior loss problems were minimized by preparing glass grains from the center of large pieces, but in experiments with fine powders helium was lost between preparation and 
Figure 2.6. Effect of prior diffusive loss on apparent diffusivities. Curves derived by discarding successive amounts of synthetic release data (spherical model with homogeneous initial condititon, $2 \%$ release intervals and $\log \mathrm{D}=-15$ ) and processing the remaining release data ( $\mathrm{F}$ versus time) as if it constituted the entire helium content. Prior loss leads to apparent diffusion coefficients that are strongly underestimated at low $\mathrm{F}$, but which trend toward correct values at high F. This is because helium is initially lost far more slowly from a grain with a smooth, diffusive profile than one with an initially discontinuous (step) profile at the grain surface, yet loss is similar at high $\mathrm{F}$ once both grains have established diffusive profiles. Prior diffusive loss produces only small errors in estimating isotopic diffusivity ratios, and as with absolute diffusivities, only at low F.. For a true $D^{3} \mathrm{He} / \mathrm{D}^{4} \mathrm{He}$ ratio of 1.08 the apparent ratio at low $\mathrm{F}$ is 1.081 for $2 \%$ loss, 1.082 at $5 \%, 1.084$ at $10 \%$ and 1.089 at $20 \%$ loss. 
Figure 2.6

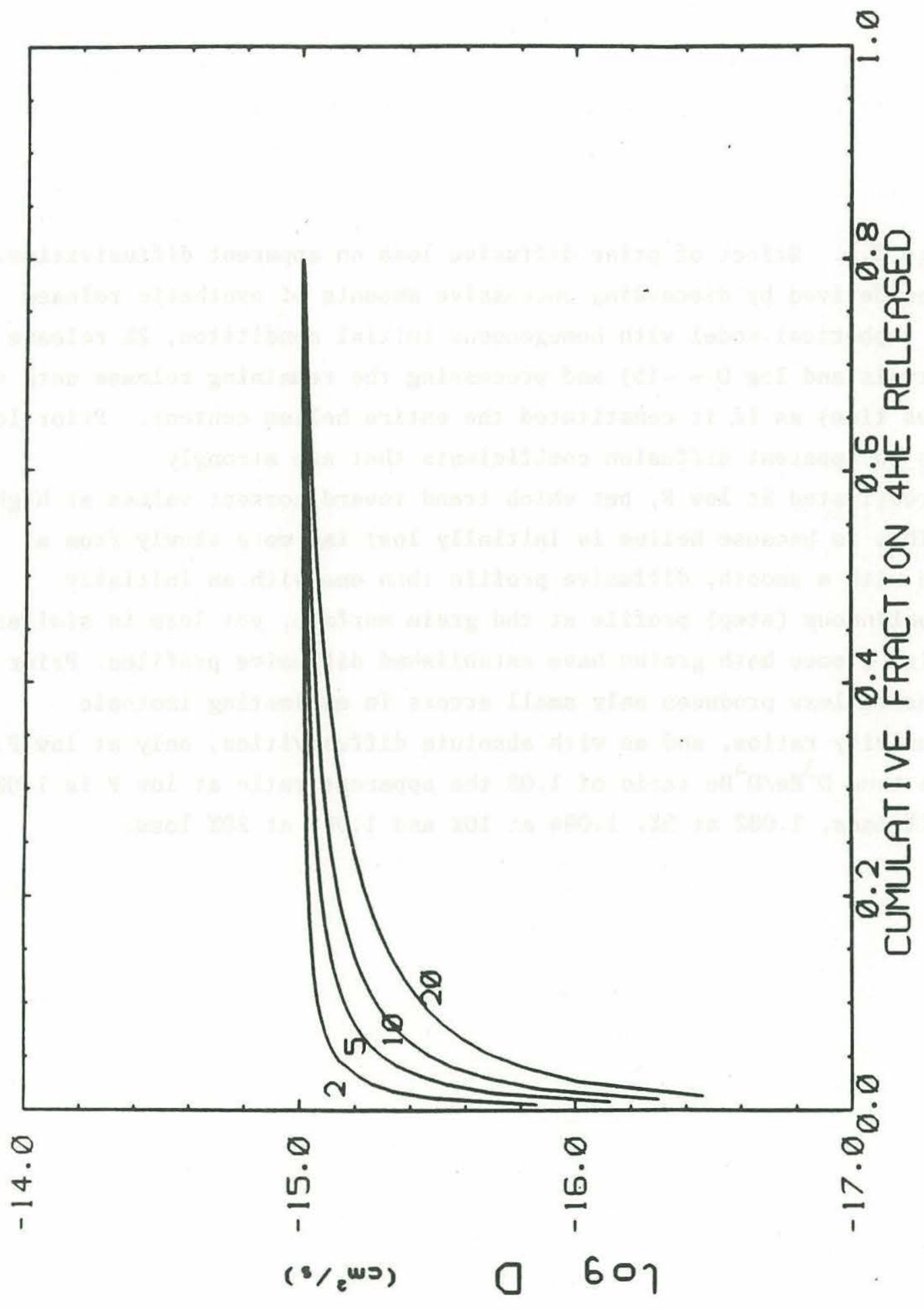


analysis. This problem was easily recognized by increasing trends of $D$ versus $\mathrm{F}$ at constant temperature which did not continue at high $\mathrm{F}$. As discussed in chapter 3, prior loss problems can be corrected for, either by comparison of the $D$ versus $F$ trends to figure 2.6, or more directly when emanation data is obtained at the temperature at which prior loss is likely to have occured. When the extent of prior loss can not be readily determined, $\mathrm{D}$ estimates at higher $\mathrm{F}$ are more reliable than those at lower F, especially if an assymptotic trend can be verified (e.g. data for helium diffusion in olivine, chapter 4). This is one important instance of the advantage of multiple aliquot experiments; release systematics can be used to identify potential errors in estimating diffusivities.

Equation 2.9 is rarely used to obtain diffusion coefficients because it converges slowly at low $\mathrm{F}$ and requires an iterative solution. However, approximation equations have been developed which give diffusion coefficients directly from sequential release data for spherical geometry (Inthoff and Ziemen, 1956; Fechtig and Kalbitzer, 1966, ):

$$
\begin{aligned}
& \text { for } F<.1: D_{i}=\pi a^{2} / 36 /\left(t_{i}-t_{i-1}\right)\left(F_{i}^{2}-F_{i-1}^{2}\right) \\
& \text { for } .1<F<.9: D_{i}=a^{2} / \pi^{2} /\left(t_{i}-t_{i-1}\right) \\
& \quad x\left[-\pi^{2} / 3\left(F_{i}-F_{i-1}\right)-2 \pi\left\{\left(1-\pi / 3 F_{i}\right)^{1 / 2}-\left(1-\pi / 3 F_{i-1}\right)^{1 / 2}\right\}\right] \\
& \text { for } F>.9: D_{i}=a^{2} / \pi^{2} /\left(t_{i}-t_{i-1}\right) \ln \left\{\left(1-F_{i-1}\right) /\left(1-F_{i}\right)\right\}
\end{aligned}
$$

These approximations have small numerical errors when compared to the full series solution for a sphere (figure 2.7). The first equation readily shows the proportionality between $F$ and $(D t)^{1 / 2}$ expected for 
Figure 2.7. Apparent diffusivities obtained by processing exact emanation data for diffusive loss $\left(D=10^{-10}\right.$ ) from a sphere (with homogeneous initial distribution, equation 2.9) with approximation equations $2.12 \mathrm{a}(+), 2.12 \mathrm{~b}(\mathrm{o})$ and $2.12 \mathrm{c}(\nabla)$. The approximations yield numerically accurate D estimates, provided they are used over limited ranges of F. Approximation 2.12a progressively underestimates D. Approximation $2.12 \mathrm{~b}$ is accurate below $\mathrm{F}$ of about .9 , at which point $2.12 \mathrm{c}$ is better until very high $\mathrm{F}$. Typical analytical uncertainties are shown, and dominate the numerical errors. 
Figure 2.7

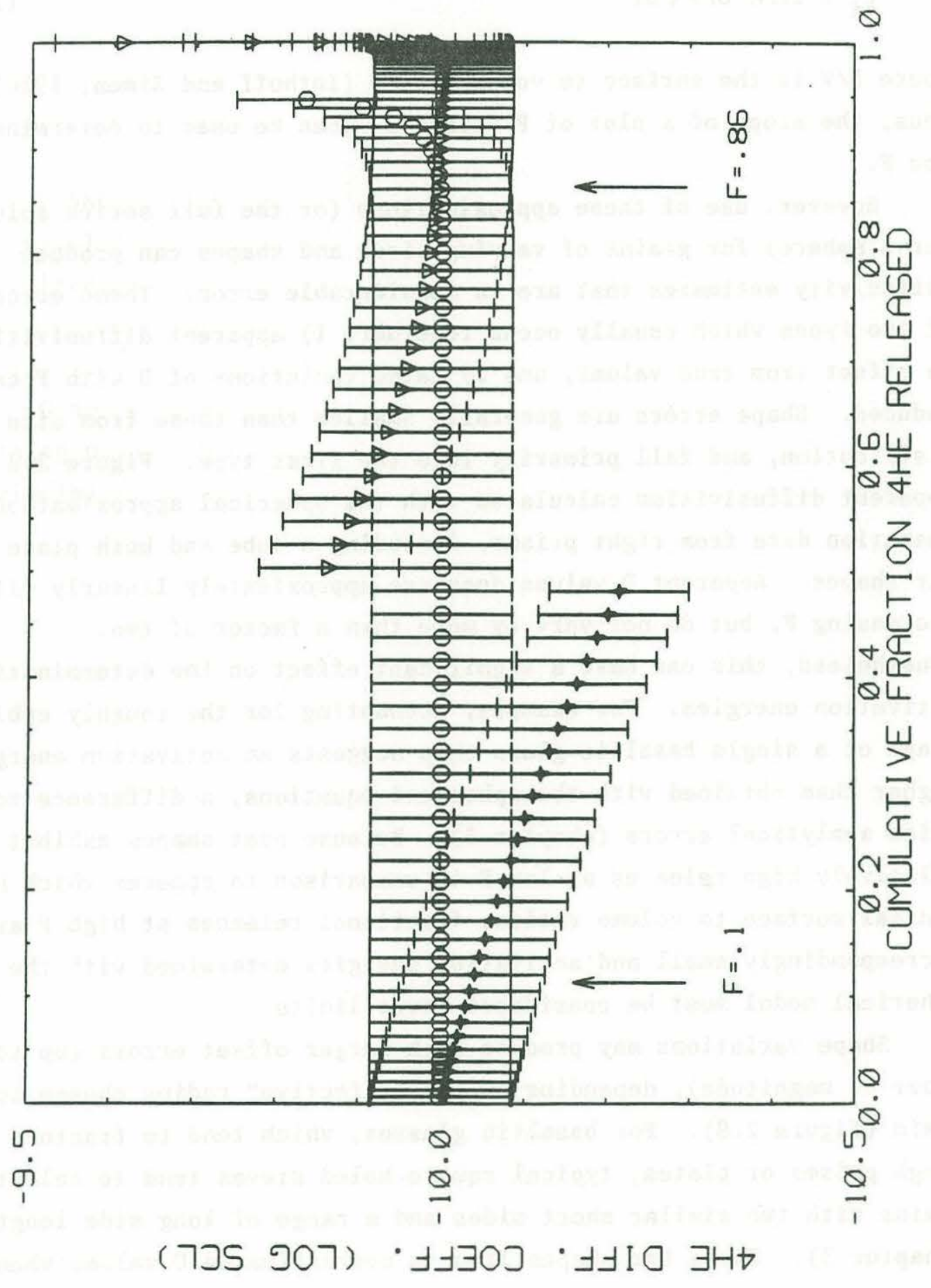


volume diffusion at low $F$. This expression can be written more generally for all geometries at low $\mathrm{F}$ as:

$$
F_{i}=2 / \sqrt{\pi} S / V \sqrt{D t}
$$

where $S / V$ is the surface to volume ratio (Inthoff and Zimen, 1956). Thus, the slope of a plot of $F$ versus $t^{1 / 2}$ can be used to determine $D$ at low $\mathrm{F}$.

However, use of these approximations (or the full series solution for a sphere) for grains of varying sizes and shapes can produce diffusivity estimates that are in considerable error. These errors are of two types which usually occur together, 1) apparent diffusivities may be offset from true values, and 2) false variations of $D$ with $F$ can be induced. Shape errors are generally smaller than those from size distribution, and fall primarily into the first type. Figure 2.8 shows apparent diffusivities calculated with the spherical approximations for emanation data from right prisms, including a cube and both plate and bar shapes. Apparent D values decrease approximately linearly with increasing $F$, but do not vary by more than a factor of two. Nonetheless, this can have a significant effect on the determination of activation energies. For example, accounting for the roughly cubic shape of a single basaltic glass chip suggests an activation energy $4 \%$ higher than obtained with the spherical equations, a difference roughly twice analytical errors (chapter 3 ). Because most shapes exhibit relatively high releases at low $\mathrm{F}$ in comparison to spheres which have minimal surface to volume ratios, fractional releases at high $\mathrm{F}$ are correspondingly small and activation energies determined with the spherical model must be considered lower limits.

Shape variations may produce much larger offset errors (up to an order of magnitude), depending on the "effective" radius chosen for the grain (figure 2.8). For basaltic glasses, which tend to fracture into rough prisms or plates, typical square-holed sieves tend to select grains with two similar short sides and a range of long side lengths (chapter 3 ). These bar shapes lead to overestimated $D$ values when the spherical routines are used and the sieve size is chosen as the 
Figure 2.8. Effect of shape variations on apparent diffusivities. The solid lines show diffusivities calculated from synthetic fractional release data (generated using equation 2.14) for several different parellelpipeds, using the approximation equations for a sphere. The dotted horizontal lines simply provide a reference to make the slopes of the solid lines more visible. Note that using the spherical model can result in large offsets in apparent diffusivities from the correct value of $1 \times 10^{-9} \mathrm{~cm}^{2} / \mathrm{s}$ used to generate the fractional release data, as well as apparent decreases in diffusivities with increasing gas loss of up to a factor of 2 . This means that both absolute $\mathrm{D}$ estimates and activation energies can be in error when grain shapes are not appropriately represented in diffusion calculations. However, as with prior losses, errors in determining the isotopic diffusivity ratio are small, the shapes with the steepest $D$ versus $F$ curves lead to underestimates of $\mathrm{D}^{3} \mathrm{He} / \mathrm{D}^{4} \mathrm{He}$ of only .4 to $.5 \%$. The shapes corresponding to curves 1 to 5 are parallepipeds with side lengths as follows:

Shape Side lengths "Spherical radius"

$\begin{array}{ccccc}1 & 1 & .25 & .25 & 1 \\ 2 & 1 & .5 & .5 & 1 \\ 3 & 1 & 1 & 1 & 1 \\ 4 & 1 & 1.45 & 1.45 & 1 \\ 5 & 1 & 2 & 2 & 1\end{array}$


Figure 2.8

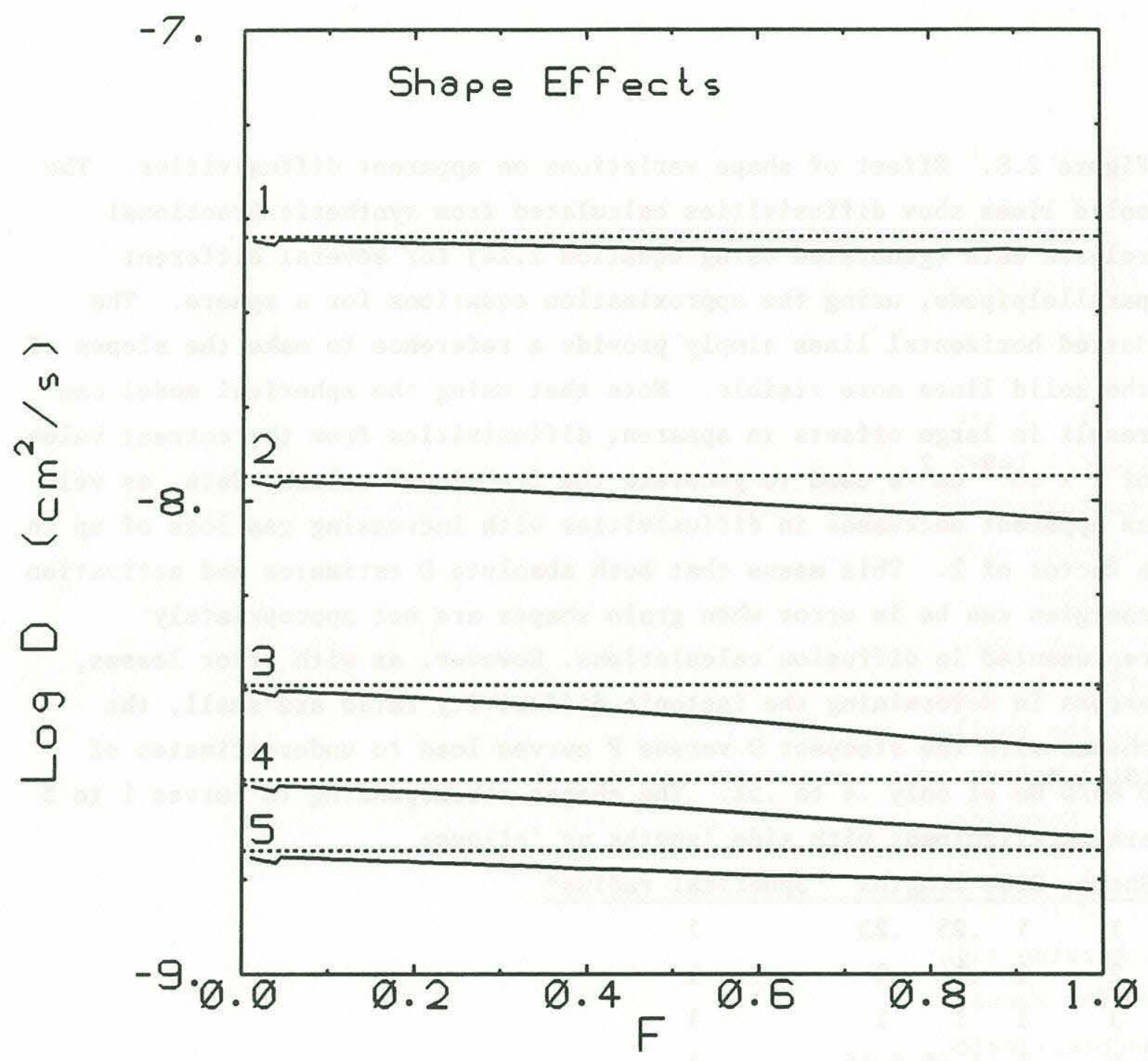


effective diameter (e.g. a factor of 4 overestimate for a cube, figure $2.8)$. Because both isotopes experience the same release geometry, shape errors are not a significant problem in determining isotopic diffusivity ratios. Jagged-edged and porous geometries produce release patterns that are roughly similar to those for a collection of many small and one large particle, and many small particles, respectively.

Size variations in grain assemblies produce offset errors in apparent diffusivities for the same reasons that shape errors do - it is difficult to assign an effective spherical radius. However, size distribution can lead to much larger decreases in D values at high $\mathrm{F}$. This occurs because the larger grains lose their helium far more slowly (loss scales with $\mathrm{a}^{2}$ ) and because relatively few grains contribute helium after smaller grains have released all their gas. Thus, D values at high $\mathrm{F}$ can be underestimated well beyond that expected from the overall range in sizes, because it is the helium mass distribution that controls emanation rates and not just the spread in grain size. Gallager (1965) has discussed this problem for log normal size distributions and developed correction equations for this specific distribution. More generally, Shaked (1965) pointed out that this effect becomes important at $\mathrm{F}$ values that correspond to large releases for the smallest particles which contain significant fractions of the total helium.

Numerical experiments with several simple distributions reveal that errors in relative diffusivities increase with $F$, particularly above $F$ of .8 to .9 (figure 2.9). The presence of a single large grain in an otherwise tightly clustered sample produces particularly large errors. As for shapes, not accounting for size distributions will lead to underestimated activation energies. This problem can be identified by decreasing $\mathrm{D}$ values in multiple release experiments at high $\mathrm{F}$, and can be minimized by avoiding data collection at high $\mathrm{F}$ when possible. In contrast to shape errors, grain size distribution does have significant effects on the determination of isotopic diffusivity ratios, as shown in figure 2.10. This figure also demonstrates that helium release from a rock or mineral sample with a wide range of grain sizes can mimic the presence of components with different diffusivities and isotopic 
Figure 2.9. Effects of grain size distributions on apparent diffusivities. Note scale changes between top and bottom figures! The curves were obtained by processing synthetic release data for $\mathrm{D}=10^{-9} \mathrm{~cm}^{2} / \mathrm{s}$ with the spherical approximation equations. The slope breaks at fractional releases (F) of .04 and .86 are the result of changing approximation equations. Distributions $1-5$ and $A$ were calculated using 7 spherical grain sizes with radii ranging from .7 to $1.3 \mathrm{~cm}$ as follows: .7, .8, .9, 1., 1.1, 1.2, 1.3, with grain distributions among these bins of:

Curve Distribution Type Bins:

\begin{tabular}{|c|c|c|}
\hline 1 & Normal (approx.) & $\begin{array}{lllllll}1 & 2 & 5 & 8 & 5 & 2 & 1\end{array}$ \\
\hline 2 & Single grain & 1 \\
\hline 3 & Decreasing & $\begin{array}{lllllll}7 & 6 & 5 & 4 & 3 & 2 & 1\end{array}$ \\
\hline 4 & Uniform & $\begin{array}{lllllll}1 & 1 & 1 & 1 & 1 & 1 & 1\end{array}$ \\
\hline 5 & Increasing & 1234567 \\
\hline A & Flyer & $\begin{array}{lllllll}100 & 0 & 0 & 0 & 0 & 0 & 1\end{array}$ \\
\hline B & High Flyer & $\begin{array}{l}100 \text { parallepipeds with side lengths of } \\
1, .5, .5 \text { and one parallelpiped with side } \\
\text { lengths of } 4,2,2 \text {. }\end{array}$ \\
\hline
\end{tabular}

Curve B combines the effects of size and shape variations. 
Figure 2.9
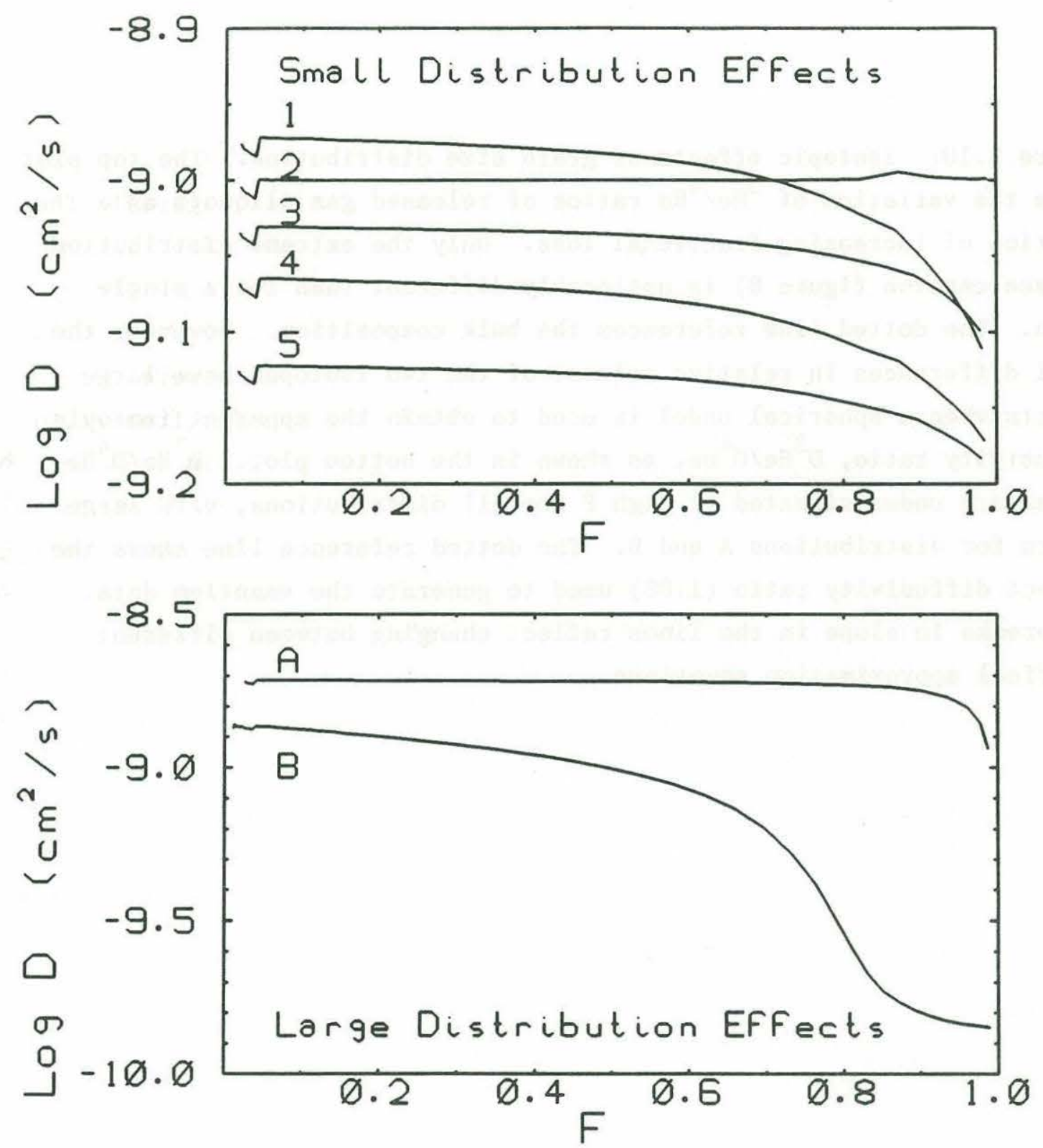
Figure 2.10. Isotopic effects of grain size distribution. The top plot shows the variation of ${ }^{3} \mathrm{He} /{ }^{4} \mathrm{He}$ ratios of released gas aliquots as a function of increasing fractional loss. Only the extreme distribution (B, see caption figure 8 ) is noticeably different than for a single grain. The dotted line references the bulk composition. However, the small differences in relative release of the two isotopes have large effects when a spherical model is used to obtain the apparent isotopic diffusivity ratio, $\mathrm{D}^{3} \mathrm{He} / \mathrm{D}^{4} \mathrm{He}$, as shown in the bottom plot. $\mathrm{D}^{3} \mathrm{He} / \mathrm{D}^{4} \mathrm{He}$ values are underestimated at high $\mathrm{F}$ for all distributions, with large errors for distributions $A$ and $B$. The dotted reference line shows the correct diffusivity ratio (1.08) used to generate the emantion data. The breaks in slope in the lines reflect changing between different spherical approximation equations. 
Figure 2.10
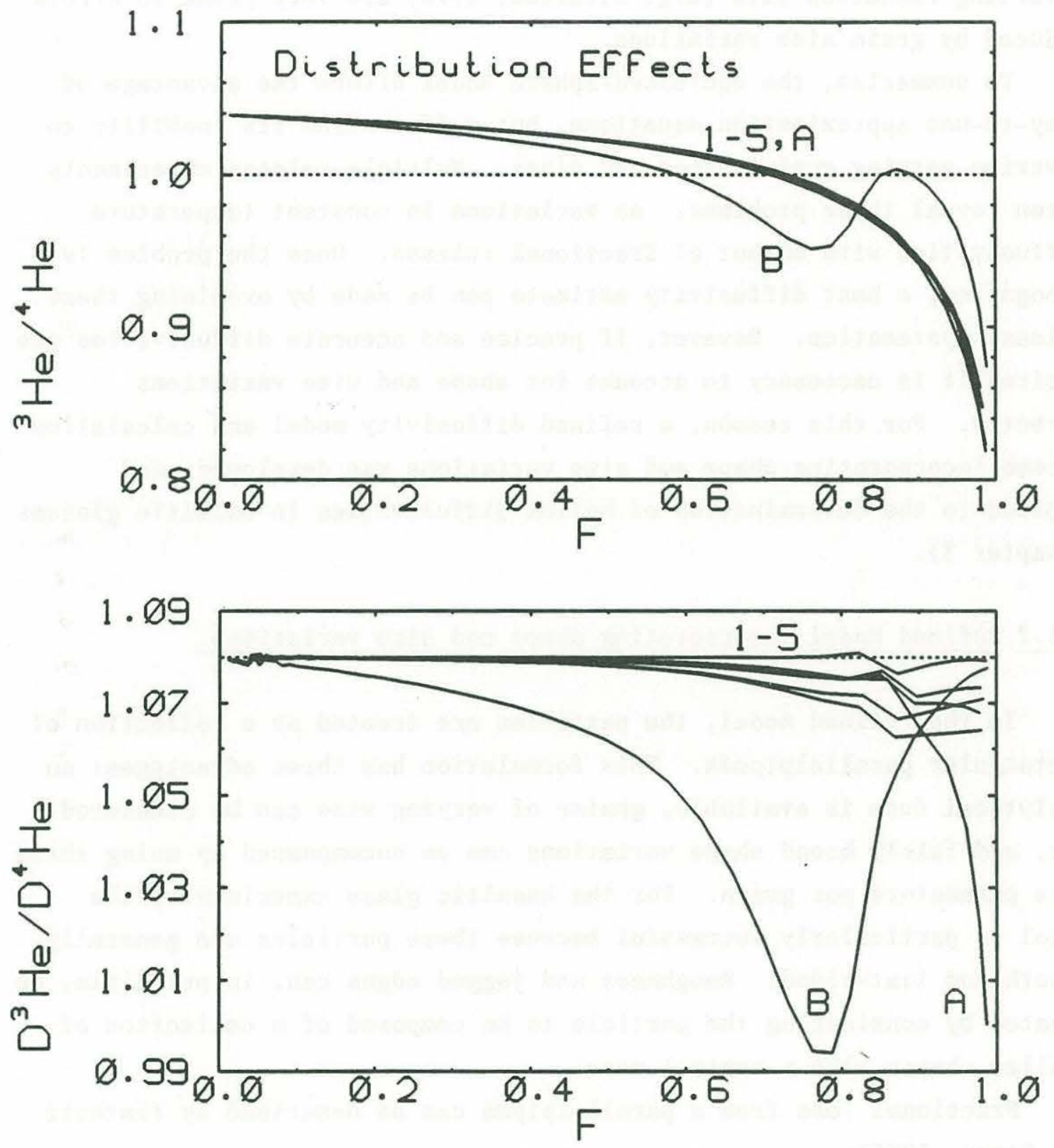
compositions. For this reason, studies which attempt to determine the spatial distribution of isotopically variable noble gas components by inverting emanation data (e.g. Albarede, 1978) are very prone to errors induced by grain size variations.

To summarize, the equisized-sphere model offers the advantage of easy-to-use approximation equations, but suffers from its inability to describe varying grain shapes and sizes. Multiple release experiments often reveal these problems, as variations in constant temperature diffusivities with extent of fractional release. Once the problem is recognized, a best diffusivity estimate can be made by examining these release systematics. However, if precise and accurate diffusivities are desired it is neccesary to account for shape and size variations directly. For this reason, a refined diffusivity model and calculation scheme incorporating shape and size variations was developed, and applied to the determination of helium diffusivities in basaltic glasses (chapter 3).

\section{$\underline{2.4 .2}$ Refined Model incorporating shape and size variations.}

In the refined model, the particles are treated as a collection of rectangular parallelpipeds. This formulation has three advantages: an analytical form is available, grains of varying size can be accounted for, and fairly broad shape variations can be encompassed by using three size parameters per grain. For the basaltic glass experiments, the model is particularly successful because these particles are generally smooth and flat-sided. Roughness and jagged edges can, in principle, be treated by considering the particle to be composed of a collection of smaller shapes plus a central core.

Fractional loss from a parallelpiped can be described by (Inthoff and Zimen, 1956):

$$
F=1-\left\{\sum(a) \cdot \sum(b) \cdot \sum(c)\right\}
$$

where $a, b$ and $c$ are the side lengths, and the summation signs 
represent:

$$
\left.\sum(a)=\sum_{0}^{\infty} 8 / \pi^{2} /(2 n+1)^{2} \exp \left\{-(2 n+1)^{2} \pi^{2} / q^{2} D t\right)\right\}
$$

For step-heating experiments $\mathrm{D}$ is given by equation 2.4. For a collection of particles, this becomes:

$$
1-F=\frac{\sum p\left\{\frac{q=a, b, c}{\mid}\left(\sum(q)\right)\right\}}{\sum p(a \cdot b \cdot c)}
$$

In this expression the denominator represents the volume of each grain summed over all the grains, and the numerator expresses the amount of helium remaining in each grain summed over all the grains.

Solving this expression for $D$ (by iteration) at the points $\left(F_{i}, t_{i}\right)$ and $\left(F_{i-1}, t_{i-1}\right)$ yields mean value diffusivities $(\bar{D})$ for the intervals $\left(t_{i}-t_{0}\right)$ and $\left(t_{i-1}-t_{0}\right)$, respectively, of :

$$
\begin{aligned}
& \text { at } F_{i-1}, \quad \bar{D}_{i-1}=1 /\left(t_{i-1}-t_{0}\right) \int_{t_{0}}^{t_{i-1} D(t) d t} \\
& \text { at } F_{i}, \quad \bar{D}_{i}=1 /\left(t_{i}-t_{0}\right) \int_{t_{0}}^{t_{i}} D(t) d t
\end{aligned}
$$

The effective diffusivity, $D_{\Delta i}$, in the interval $\left(t_{i}-t_{i-1}\right)$ can then be obtained from:

$$
D_{\Delta i}=\left\{\bar{D}_{i}\left(t_{i}-t_{0}\right)-\bar{D}_{i-i}\left(t_{i-1}-t_{0}\right)\right\} /\left(t_{i}-t_{i-1}\right) .
$$

For constant temperature steps, $D_{\Delta i}$ represents an estimate of the true diffusion coefficient, but for temperature ramp aliquots $D_{\Delta i}$ is itself a mean value on the interval.

The problem is computationally intensive for two reasons. The series converges slowly (particularly at low F) so that up to a million 
terms may be required to obtain an absolute error of $1 \times 10^{-6}$ in $\mathrm{F}$ or about $1 \%$ error for the low temperature emanation experiments which measured small fractional releases at low temperature. The second problem is that the precision of $D_{\Delta i}$ estimates depends on small differences between $\bar{D}_{i}$ and $\bar{D}_{i-1}$, so that the iteration must be performed until the $\bar{D}$ are obtained with precisions of $.00001 \%$ for precision of . $1 \%$ in $D_{\Delta i}$.

These tasks were performed by a series of nested subroutines which are provided in appendix A, along with the driver program that calculates fractional releases and their associated errors from aliquot measurements. The programs are written in Fortran 77 , but with some non-ANSI-standard statements, and are well documented (see Appendix A). They were run on a Sun $3 / 260$ computer with floating point acceleration and required a few hours to complete processing for a typical experiment involving two isotopes and a few tens of aliquots.

Use of the refined model with measured grain sizes and shapes (as represented by three side lengths) lead to significantly different helium diffusivity and activation energy estimates for basaltic glasses than initially obtained with the spherical model (chapter 3). The improved grain size description also demonstrated that Arrhenius temperature plots for these glasses were more closely linear than suggested by the spherical model. This lends confidence in the extrapolation of laboratory determined diffusivities to other temperatures using these relations (see chapter 3 ). This model is the first general scheme (to my knowledge) to account for arbitrary size and (limited) shape variations in diffusion experiments, and should be useful in future studies. 


\section{REFERENCES}

ALBAREDE F. (1978) The recovery of spatial isotope distributions from stepwise degassing data. Earth Plan. Sci. Let. 39, 387-397.

CLARKE W.B., JENKINS W.J. and TOP Z. (1976) Determination of tritium by mass spectrometric measurement of ${ }^{3} \mathrm{He}$. Inter. J. Appl. Rad. and Isotopes 27, 515-522.

CRANK J. (1975) The Mathematics of Diffusion, $2^{\text {nd }}$ edition, Oxford Press.

EINSTEIN A. (1905) Ann. Physik 17, 549.

FECHTIG H. and KALBITZER S. (1966) Diffusion of argon in potassium bearing solids. In: Potassium-Argon Dating (ed. 0. SCHAEFFER)

GALLAGHER K.J. (1965) The effect of particle size distribution on the kinetics of diffusion reactions in powders. In Reactivity of Solids. 5th International Symposium on the reactivity of solids. Munich, August 2-8, 1964. (ed. G.M. SCHWAB), Elsevier, New York, 192-203.

GRAHAM D. V. (1987) Helium and lead isotope geochemistry of oceanic volcanic rocks from the East Pacific and South Atlantic. WHOI/MIT Ph.D., 252 p.

HUNEKE J.C. (1976) Diffusion artifacts by dating in stepwise thermal release of rare gases. Earth Plan. Sci. Let. 28, 407-417.

INTHOFF W. and ZIMEN K.E. (1956) Kinetik der Diffusion radioaktiver Edelgase aus festen Stoffen nach Bestrahlung (Edelgasdiffusion in Festkorpern 2). Trans. Chalmers. Univ. Techn. (Goteborg) 176, 16p. JENKINS W.J. (1974) Helium isotope and rare gas ocaenology. PhD. Diss., McMaster Univ.

KURZ M.D. (1982) Helium isotope geochemistry of oceanic volcanic rocks: implications for heterogeneity and degassing. PhD. Diss. MIT/WHOI.

KURZ M.D., GURNEY J.J., JENKINS W.J.and LOTT D.E. (1987) Helium isotopic variability within single diamonds from the Orapa kimberlite pipe. Earth Plan. Sci. Let. 86, 57-68. 
LOTT D.E. and JENKINS W.J. (1984) An automated cryogenic charcoal trap system for helium isotope mass spectromtry. Rev. Sci. Instr. 55, 1982-1988.

POLVE M. (1985) Fission track mapping of $U$ in alkali basalts: implications for U behavior during crystallization. EOS 66, 404.

RAMA S.N.I. and HART S.R. (1965) Neon isotope fractionation during transient permeation. Science 147, 737-738.

SHAKED H. (1965) Simple theory for the release of fission gas from powders. J. Nucl. Materials 17, 136-141.

STAUDACHER T., JESSBERGER E.K., DORFLINGER D.and KIKO J. (1978) A refined ultra-high vacuum furnace for rare gas analysis. J. Phys. E 11, 781-784. 
CHAPTER 3 .

The Diffusivity of ${ }^{3} \mathrm{He}$ and ${ }^{4} \mathrm{He}$ in basaltic glasses. 


\section{$\underline{3.1}$ INTRODUCTION}

Basaltic glasses, which form in submarine, subglacial, and to a lesser extent, subaerial environments are an important sample type for studying the elemental and isotopic variability of helium and other noble gases in igneous systems because they trap magmatic gases (e.g. Fisher, 1971). Thus, it is essential to understand the rates at which helium can be lost from or added to samples of this type. In addition, glass is a reasonable, if limited, analog for the structure of melts (e.g Mysen, Virgo and Scarfe, 1980; Stolper, 1982 and references therein), and studying helium transport in glasses can provide estimates for its behavior in magmas.

Diffusion may lead to the alteration of basaltic glass isotopic compositions by either preferential loss of one isotope or diffusive exhange of helium with the surrounding seawater or atmosphere. The effectiveness of these processes depends on the rate of helium exchange, the extent to which helium isotopes are fractionated by diffusion, and the glass helium concentration at eruption and after equilibration with the surrounding medium. Unfortunately, none of these factors are very well known, and some are in dispute. The rate of helium diffusion has been measured in two previous studies. Kurz and Jenkins (1981) estimated the ${ }^{4} \mathrm{He}$ diffusion coefficient to be very low at seafloor temperatures $\left(1 . \pm .6 \times 10^{-17} \mathrm{~cm}^{2} / \mathrm{s}\right.$ at $0^{\circ} \mathrm{C}$ and $1.6 \times 10^{-16}$ at $\left.20^{\circ} \mathrm{C}\right)$, from extrapolation of emanation measurements at elevated temperatures, and concluded that "diffusion is an insignificant mechanism for helium loss" from mid-ocean-ridge-basalts (MORB) glasses. In contrast, Jambon, Weber and Begemann (1985) observed significant helium loss from powdered and sieved basaltic glass stored in their laboratory, and estimated the diffusion coefficient to be 40 times higher $\left(6 \times 10^{-15} \mathrm{~cm}^{2} / \mathrm{s}\right.$ at $\left.20^{\circ} \mathrm{C}\right)$. The difference in these values has great significance for the interpretation of helium isotope measurements in basaltic glasses. As an example, the timescale to lose $1 / 2$ of the initial helium content of a $1 \mathrm{~cm}$ sphere of basaltic glass decreases from several million years to less than 100,000 years, if the higher diffusivity is correct. This is 
an important difference, because most dredged MORB samples will be less than than a million years old (at a $1 \mathrm{~cm} /$ year plate separation rate this corresponds to samples dredged within $10 \mathrm{~km}$ of the ridge crest), but many could be 100,000 years old. Thus it is essential to determine which of these previous helium diffusivity estimates is correct. Interestingly, the two studies found a similar Arrhenius dependence of the diffusion coefficient on temperature, although only Kurz and Jenkins (1981) obtained an activation energy with reasonable precision $(\mathrm{Ea}=19.9 \pm 1.0 \mathrm{Kcal} / \mathrm{mole})$. Determining the precise nature of the temperature dependence of helium diffusion in basaltic glasses is essential to their study and the work presented here, because measurements made at elevated temperatures in the laboratory are extrapolated to obtain diffusivity estimates at lower environmental temperatures.

No previous study has measured the diffusivity of ${ }^{3} \mathrm{He}$ in basaltic or other natural glasses, so the extent to which diffusion fractionates helium isotopes is unknown. From a theoretical viewpoint, diffusion is expected to fractionate helium isotopes because of their large mass difference. Simple heuristic models, such as pure gas kinetic theory $\left(E=1 / 2 \mathrm{mv}^{2}\right)$ or one-dimensional harmonic oscillation of an isolated atom (frequency proportional to $(m)^{-1 / 2}$ ), suggest that the relative rates of ${ }^{3} \mathrm{He}$ and ${ }^{4} \mathrm{He}$ diffusion will correspond to the square-root of the mass ratio of ${ }^{4} \mathrm{He}$ to ${ }^{3} \mathrm{He}$ (i.e the isotopic diffusivity ratio, $D^{3} \mathrm{He} / \mathrm{D}^{4} \mathrm{He}$ would equal $\left(\mathrm{m}^{4} \mathrm{He} / \mathrm{m}^{3} \mathrm{He}\right)^{1 / 2}$, which is 1.154$)$. This predicts that ${ }^{3} \mathrm{He}$ will be diffuse about $15 \%$ more rapidly than ${ }^{4} \mathrm{He}$. More sophisticated theory reveals that the diffusity ratio for two isotopes depends on properties of the solid in addition to the diffusing species, and allows for the possibility of a larger isotope effect (Prigogine and Bak, 1959; Stoneham and Flynn, 1971), a somewhat smaller diffusivity ratio because of quantum effects (Le Claire, 1966), and even preferential ${ }^{4} \mathrm{He}$ mobility (Ebisuzaki, Kass and 0'Keefe, 1967).

Measurements made in synthetic glasses observed a temperature dependent helium isotopic diffusivity ratio $\left(D^{3} \mathrm{He} / \mathrm{D}^{4} \mathrm{He}\right)$ ranging from 1.12 at $600^{\circ} \mathrm{C}$ down to 1.07 at $300^{\circ} \mathrm{C}$ (Shelby, 1971). However, the applicability of this result to basalt glasses is uncertain because of 
the large compositional differences, which are known to lower the diffusivity for ${ }^{4} \mathrm{He}$ in basalts in comparison to synthetic glasses by several orders of magnitude (Jambon and Shelby, 1980). Unfortunately, network-modifier/network-former theory, which does offer a qualitative explanation for the lower natural glass diffusivities (e.g Jambon and Shelby, 1980; Kurz and Jenkins, 1981), can be used to predict isotopic fractionation variations. Small differences in $D^{3} \mathrm{He} / \mathrm{D}^{4} \mathrm{He}$ are important because this ratio governs the fraction of helium which must be lost by a glass to lower its initial ratio significantly. For example, to reduce an initial ${ }^{3} \mathrm{He} /{ }^{4} \mathrm{He}$ ratio by $10 \%$ requires $80 \%$ gas loss for $\mathrm{D}^{3} \mathrm{He} / \mathrm{D}^{4} \mathrm{He}$ equal to 1.08 , but only $65 \%$ loss (which occurs in half the time) for $D^{3} \mathrm{He} / \mathrm{D}^{4} \mathrm{He}$ of 1.15 . Craig and Lupton (1976) have suggested that preferential diffusive loss of ${ }^{3} \mathrm{He}$ accounts for the low ${ }^{3} \mathrm{He} /{ }^{4} \mathrm{He}$ ratios of two oceanic basalt glasses with low helium contents, but assumed a high diffusivity based on synthetic glass studies and an isotopic diffusivity ratio of 1.15 . Determining the true diffusion rate and $\mathrm{D}^{3} \mathrm{He} / \mathrm{D}^{4} \mathrm{He}$ ratio is essential to understanding whether samples such as these represent primary magma characteristics or contamination effects.

The timescales for loss of helium from a sample also apply to helium exchange with seawater. The effect of this contamination process on observed ${ }^{3} \mathrm{He} / \mathrm{He}$ ratios is obviously most severe for those samples which were erupted with less helium than expected for basaltseawater equilibrium, because helium with atmospheric composition can be added to the sample. Samples with more helium than this level will lose helium until the equilibrium level is obtained. Of course ${ }^{3} \mathrm{He}$ and ${ }^{4} \mathrm{He}$ behave independently in this equilibration process. The equilibrium concentration level can be estimated from the helium partial pressure in seawater $\left(\mathrm{P}_{\mathrm{He}}^{\mathrm{Sw}}\right)$ which is essentially in equilibrium with air at one atmosphere total pressure (Weiss, 1971) and the Henry's law constant for helium solubility in basaltic glass $\left(\mathrm{K}_{\mathrm{He}}\right)$ from the definition:

$$
[\mathrm{He}]_{\mathrm{g}}^{\mathrm{SW}}=\mathrm{K}_{\mathrm{He}} \times \mathrm{P}_{\mathrm{He}}^{\mathrm{SW}}
$$

Henry's law coefficients have been estimated for basaltic melts and glasses in laboratory studies and from natural distributions, and 
average $5 \times 10^{-4} \mathrm{ccSTP} / \mathrm{g}-\mathrm{atm}$ (table 3.1). The temperature dependence is very small, similar to experimental errors in the melt studies, but favors higher solubilities as temperature increases (Jambon, Weber and Braun, 1986; Lux, 1987). No measurements of the ${ }^{3}$ He solubility have been made, but indistinguishable isotopic compositions of helium in vesicles and dissolved in MORB glasses suggest the two isotopes have identical solubilities (Kurz and Jenkins, 1981) although data precision allows a $1 \%$ difference, similar to that observed for seawater, which favors ${ }^{3} \mathrm{He}$ dissolution by $1.7 \%$ (Weiss, 1971). Assuming these Henry's law constants apply to glasses at low temperature on the seafloor, the equilibrium value for helium dissolved in basalt is $2-3 \times 10^{-9} \mathrm{ccSTP} / \mathrm{g}$ (i.e. 4$5 \times 10^{-4} \mathrm{ccSTP} / \mathrm{g}$-atm $\left.\times 5.3 \times 10^{-6} \mathrm{~atm}\right)$. This can be compared to typical seawater concentrations (e.g $2 \times 10^{-8} \mathrm{ccSTP} / \mathrm{g}$ in deepwater in the Galapagos; Jenkins, Edmond and Corliss, 1987) and to basaltic glass concentrations which are on the order of $10^{-5}$ to $10^{-8} \mathrm{ccSTP} / \mathrm{g}$ in MORB (e.g. Lupton and Craig, 1975; Kurz and Jenkins, 1981), but which range as low as $10^{-8}$ to $10^{-10} \mathrm{ccSTP} / \mathrm{g}$ in rocks from seamounts and island arcs (Graham et al, 1987; Trull, Perfit and Kurz, 1989). Therefore, contamination by helium addition on the seafloor is a concern in extending helium isotopic studies to these sample types, particularly if the helium diffusivity is relatively high. In this regard, helium isotopic disequilibrium within basaltic glasses from Loihi seamount has been interpreted as the result of diffusive exchange with seawater (Rison and Craig, 1983) and the low ${ }^{3} \mathrm{He} /{ }^{4} \mathrm{He}$ ratios of some glasses from the Woodlark basin probably are also contaminated by this process (see chapter 6).

The production of radiogenic ${ }^{4} \mathrm{He}$ by alpha-decay of $U$ and Th series nuclides is another mechanism by which the isotopic composition of basaltic glasses can be altered after eruption (e.g. Graham et al, 1987; Trull, Perfit and Kurz, 1989) and which provides a promising dating method for seamount basalts (Graham et al, 1987). Helium loss by diffusion bears directly on the utility of this method, because apparent ages will be lowered in proportion to the fraction of helium lost, and because helium exhange between glass and vesicles affects the correction 
Table 3.1 Henry's law coefficients for Helium solubility in basaltic melts and glasses.

Experiment and Reference Equilibration of tholeitic melts at $1250-1600^{\circ} \mathrm{C}$ followed by analysis of quenched glasses. Jambon, Weber and Braun, 1986. $\mathrm{K}_{\mathrm{He}}$

Equilibration of tholeiitic melt at $1350^{\circ} \mathrm{C}$ followed by analysis of quenched glass.

Lux, 1987.

$6.4 \pm .8$

Comparison of vesicle and glass helium contents of mid-ocean-ridge-basalts.

Kurz and Jenkins, 1981; Kurz, 1982;

Graham et al, 1988.

$$
\left(\begin{array}{cc}
510^{-4} & 5+1.1 \\
\operatorname{ccSTP} / \mathrm{g} & -\mathrm{atm}
\end{array}\right)
$$

Permeability measurement of basaltic glass at $200-300^{\circ} \mathrm{C}$.

Jambon and Shelby, 1980. 
required for inherited helium (Graham et al, 1987). The mobility of insitu produced radiogenic helium (denoted ${ }^{\star 4}$ He here) may differ from inherited helium because of the nature of its generation. Keevil (1940) suggested, on theoretical grounds, that the disruption of bonds associated with alpha-particle ejection and daughter nuclide recoil is insufficient to enhance diffusion of ${ }^{* 4} \mathrm{He}$, at least in silicate crystals. However, many studies have documented elevated losses of radionuclides, including ${ }^{222} \mathrm{Rn}$ from rocks and crystals (e.g. Giletti and Kulp, 1955; Rama and Moore, 1984; Fleischer, 1988) and ${ }^{234} \mathrm{Th}$ and ${ }^{234} \mathrm{U}$ from granites (Fleischer and Raabe, 1978) where release is enhanced by recoil energies. ${ }^{* 4} \mathrm{He}$ has a much higher energy/mass ratio than these elements, resulting in a stopping range of 20-30 microns in basalt (e.g. Graham, 1986), and suggesting that its release may be similarly, and perhaps more strongly affected. Many efforts to use ${ }^{* 4} \mathrm{He}$ acccumulation as a gechronometer have documented helium loss (e.g. Hurley, 1954 for zircon and granitic minerals; Fanale, 1964 for magnetite; Bender, 1970 for corals; Leventhal, 1975 for holocrystalline basalts). However, it is unclear that these losses result from enhanced mobility related to radioactive decay, rather than preferential siting of the parent nuclides on grain boundaries and surfaces.

In summary, because of the potential role of diffusion in releasing and exhanging helium and thereby affecting their isotopic compositions, the work described in this chapter was designed to determine the diffusivity of inherited ${ }^{3} \mathrm{He}$ and ${ }^{4} \mathrm{He}$, and ${ }^{* 4} \mathrm{He}$ in several basaltic glasses. The large discrepancy between previous studies (Kurz and Jenkins, 1981; Jambon, Weber and Begemann, 1985) and the desire to extend helium isotopic analysis to low helium samples from new tectonic terrains, such as island arcs and seamounts, made a reassessment of this problem particularly important.

\section{$\underline{3.2}$ SAMPLES}

All the samples analyzed were basaltic glasses produced by the rapid quenching that occurred when the lavas erupted on the seafloor. The sample numbers, their provenance, rock type, and bulk helium 
contents are listed in table 3.2a. Chemical compositions are given in tables 3.2b and 3.2c. Charco $98-11 \mathrm{~T}$ is a tholeiitic basalt typical of those recovered from normal mid-ocean-ridges (Bougault and Treuil, 1981), and is very similar in chemical composition to another sample from the same dredge studied for He diffusion by Jambon, Weber and Begemann (1985, sample Charco 98-DR11). The letter $\mathrm{T}$ designates the split from the bulk dredge sample prepared in this study from the center of a $3 \mathrm{~cm}$ drop-shaped lobe which appears to have been cooled as it was extruded from a larger lava volume. Chemically, sample Alv892-1a is also in the range of normal mid-ocean-ridge-basalts (MORB) although it has been described as iron and titanium rich, perhaps reflecting its origin in the relatively fast-spreading Galapagos rift (Byerly, Melson and Vogt, 1976). A similar sample from the same dive (Alv892-2) was among those studied by Kurz and Jenkins (1981). The sub-sample prepared for Alv892-1a came from the center of a $20 \mathrm{~cm}$ long, $5 \mathrm{~cm}$ diameter, cylindrically-shaped, entirely vitreous lava tube. These two samples were chosen because they have chemical compositions representive of many mid-ocean-ridge basalts and allow ready comparison with previous work. Aftermath D9 and Alv1389-1854B are alkali basalt glasses recovered from off-axis seamounts (Graham et al 1987, 1988). D9 was selected to examine the compositional dependence of helium diffusion by comparison with the tholeiites. Sample $1854 \mathrm{~B}$ contains predominantly radiogenic ${ }^{4} \mathrm{He}(97 \%$ $\left.{ }^{* 4} \mathrm{He}\right)$, and has been dated at $680( \pm 140)$ thousand years using the U+Th/He method (Graham et al, 1987). It was studied in order to examine the effect of diffusive ${ }^{* 4} \mathrm{He}$ loss on on this dating method. In contrast to the tholeiites, both alkali basalt samples were prepared from the glassy margins of pillow lava fragments.

For all samples, large glass fragments (.5 to $1 \mathrm{~cm}$ chunks) were broken from the rocks using a stainless-steel mortar and in some cases a small steel chisel. The glasses were examined in thin section before crushing to identify sections of the samples that were free from spherulites, varioles, and other devitrification textures. These fragments were then further broken, sieved on a shaker table with repeated acetone washings to disperse electrostatic aggregates and remove fines (ISO/USA standard stainless steel sieves), and handpicked 
Table 3.2a. Sample descriptions and bulk helium contents.

\begin{tabular}{|c|c|c|c|c|c|c|}
\hline SAMPLE & TYPE & PROVENANCE & $\begin{array}{l}\text { VES. } \\
(\%)\end{array}$ & $\begin{array}{l}\text { HELIUM COM } \\
{[\mathrm{He}] \mathrm{cc} / \mathrm{g}}\end{array}$ & $\begin{array}{l}\text { POSITION } \\
\text { R/Ra }\end{array}$ & METHOD \\
\hline ALV892-1A & $\mathrm{TH}$ & $\begin{array}{l}\text { Galapagos } \\
\text { Spreading Center } \\
049^{\prime} \mathrm{N}, 8608^{\prime} \mathrm{W} \\
2750 \mathrm{~m} \text { depth }\end{array}$ & 0 & $\begin{array}{l}3.21 \times 10-7 \\
8.60 \times 10-7 \\
8.92 \times 10-6\end{array}$ & $\begin{array}{l}8.48(.04) \\
8.51(.06) \\
8.32(.07)\end{array}$ & $\begin{array}{l}1 \\
1 \\
2\end{array}$ \\
\hline CHARCO $98-11 \mathrm{~T}$ & $\mathrm{TH}$ & $\begin{array}{l}\text { Mid-Atlantic-Ridge } \\
3041^{\prime} \mathrm{N}, 4149^{\prime} \mathrm{W} \\
3500-3600 \mathrm{~m} \text { depth }\end{array}$ & .5 & $\begin{array}{l}6.93 \times 10-6 \\
9.42 \times 10-6\end{array}$ & $\begin{array}{l}8.57(.07) \\
8.73(.06)\end{array}$ & $\begin{array}{l}1 \\
2\end{array}$ \\
\hline $\begin{array}{l}\text { AFTERMATH D9 } \\
\text { station glass } \\
\# 1\end{array}$ & FAB & $\begin{array}{l}\text { Mathematicians } \\
\text { Rift, Pacific } \\
17 \mathrm{~N}, 110 \mathrm{~W} \\
1800-2000 \mathrm{~m}\end{array}$ & 2 & $\begin{array}{l}2.79 \times 10-6 \\
7.95 \times 10-7 \\
4.97 \times 10-7\end{array}$ & $\begin{array}{l}6.62(.05) \\
7.24(.12) \\
7.14(.06)\end{array}$ & $\begin{array}{l}1 \\
1 \\
2\end{array}$ \\
\hline ALV1389-1854B & FAB & $\begin{array}{l}\text { Seamount } 6 \\
1244^{\prime} \mathrm{N}, 10235^{\prime} \mathrm{W} \\
\text { East Pacific Rise } \\
\text { approx. } 2000 \mathrm{~m}\end{array}$ & 1 & $\begin{array}{l}6.19 \times 10-9 \\
2.16 \times 10-7 \\
2.1 \times 10-7\end{array}$ & $\begin{array}{l}1.67(.03) \\
0.06(.01) \\
\quad * 4 \mathrm{He}\end{array}$ & $\begin{array}{l}1 \\
2 \\
3\end{array}$ \\
\hline
\end{tabular}

a. $\mathrm{FAB}=$ fractionated alkali basalt $\mathrm{TH}=$ tholeiite

b. Ves. denotes the vesicle content estimated visually from thin sections.

c. Method: 1 - helium released by crushing (from vesicles)

2 - release by melting the crushed powder (from within glass)

3 - radiogenic helium generated in the sample since eruption, derived

from: $* 4 \mathrm{He}=[\mathrm{He}] \mathrm{m}(1-\mathrm{Rm} / \mathrm{Rc})$, c denotes crushing and $\mathrm{m}$, melting.

d. $3 \mathrm{He} / 4 \mathrm{He}$ ratios are normalized to the atmospheric ratio, $\mathrm{Ra}=1.38 \times 10-6$.

e. 1 sigma errors for the $3 \mathrm{He} / 4 \mathrm{He}$ ratios are shown in parentheses.

f. Analyses for D9 and 1854B from Graham et al, 1987, 1988, respectively. 
Table 3.2b. Chemical compositions of basaltic glasses.

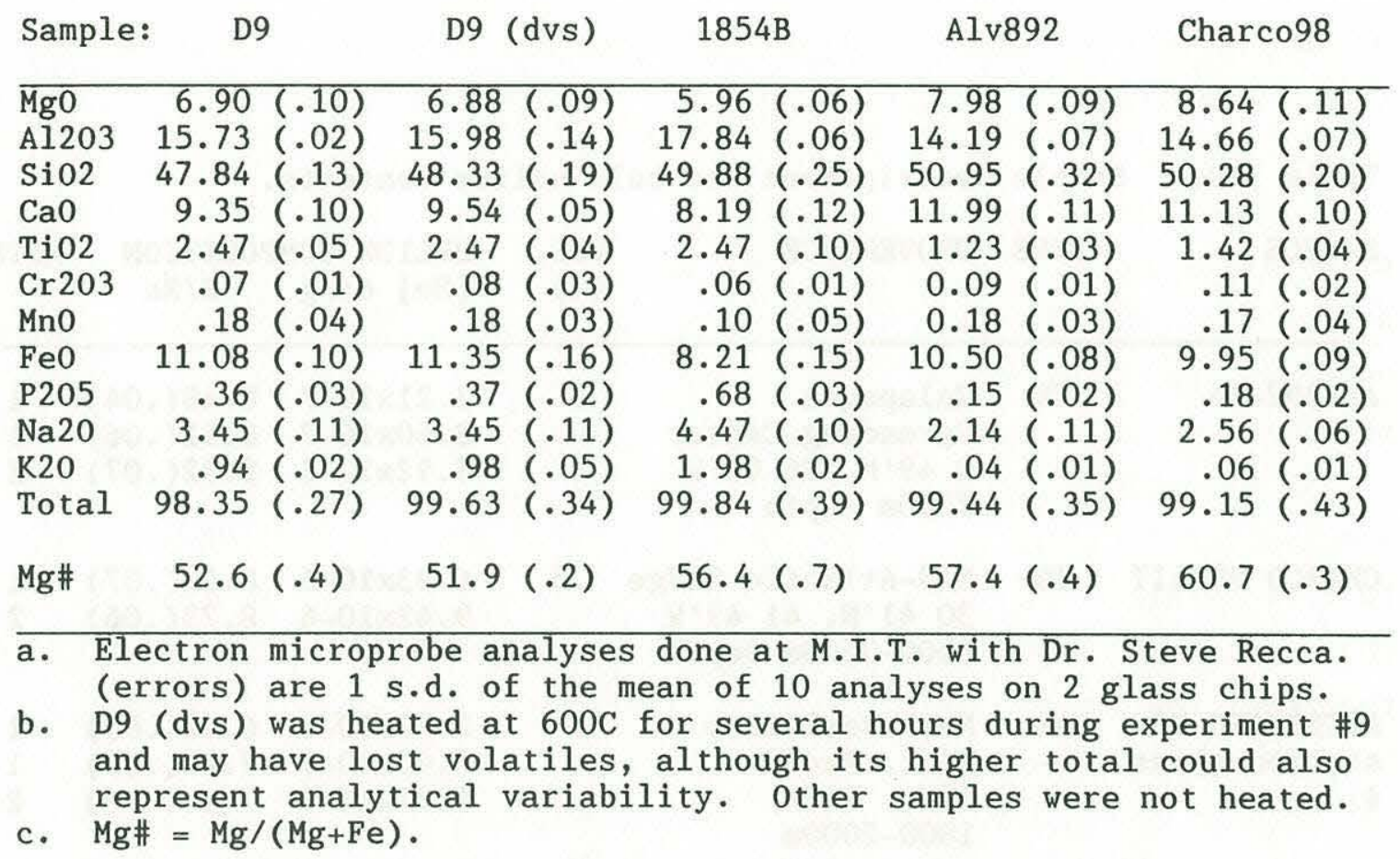

Table 3.2c. Molar compositions of major network formers/modifiers.

\begin{tabular}{|c|c|c|c|c|}
\hline Sample: & $\begin{array}{l}\mathrm{D} 9 \\
\mathrm{AB}\end{array}$ & $\begin{array}{c}1854 \mathrm{~B} \\
\mathrm{AB}\end{array}$ & Alv892 & Charco98 \\
\hline & & & & \\
\hline$\overline{\mathrm{SiO} 2}$ & .536 & .558 & .548 & .541 \\
\hline Al203 & . 104 & . 117 & .090 & .092 \\
\hline Ti02 & .021 & .021 & .010 & .012 \\
\hline $\mathrm{CaO}$ & . 112 & .098 & . 138 & . 129 \\
\hline $\mathrm{Fe} 0$ & .076 & .056 & .069 & .066 \\
\hline $\mathrm{MgO}$ & .115 & .099 & .128 & .138 \\
\hline $\mathrm{Na} 20$ & .030 & .038 & .017 & .021 \\
\hline K20 & .006 & .012 & .0003 & .0004 \\
\hline NF & 66.1 & 69.6 & 64.8 & 64.5 \\
\hline CD & 2.68 & 2.61 & 2.69 & 2.68 \\
\hline \multicolumn{5}{|c|}{$\begin{aligned} \mathrm{AB}= & \text { alkali basalt }, \mathrm{TH}=\text { tholeiite } \\
\mathrm{NF}= & \text { mole percent network formers } \\
& \quad(\text { SiO2+Al203+Ti02) } \\
\mathrm{CD}= & \text { density calculated from compositions } \\
& (\text { Stebbins et al, 1984). }\end{aligned}$} \\
\hline
\end{tabular}


(in both reflected and transmitted light in air and in ethanol, which reveals surface features) to completely eliminate adhering alteration minerals, phenocrysts, and devitrification textures. Grains (200 microns or larger) were individually examined; smaller sizes were obtained by crushing handpicked .5-1mm grains. All samples were cleaned by brief ultrasonic agitation in distilled water, acetone and methanol, followed by drying in air at room temperature. The glass powders were examined extensively in thin section for alteration and devitrification and during electron microprobe analyses for evidence of glass immiscibility, none was found, with the exception of one Alv892-1a subsample which was specifically prepared from a spherulitic glass fragment.

The total helium contents of the MORB glasses (about $10^{-5}$ ccSTP) are typical for this rock type (e.g. Kurz and Jenkins, 1981) and are 3 to 5 times higher than for the alkali basalts (table 3.2a). In general, the relative releases of He by crushing large $(2 \mathrm{~mm})$ chips and melting the resulting powder are consistent with the vesicularities determined in thin section and the partitioning relation found for MORB samples by Kurz and Jenkins (1981). Alkali basalt sample D9 had the highest vesicularity at $2 \%$, which suggests the two-fold variation in vesicle helium concentrations obtained from replicate analysis derives from heterogeneous vesicle distributions. No vesicles were observed in sample Alv892-1a, and with only about $1 \%$ of the helium released by crushing (suggesting $.04 \%$ vesicles by volume) this is consistent with either sub-micron vesicles, or with the presence of rare larger vesicles not intersected by a thin section. Alternatively, it is possible that this small fractional release results from diffusion of He from new surfaces produced by crushing. To investigate this, the fraction of helium that could diffuse from new grains during a typical 1 hour crushing and analysis period was calculated by assuming that the crushing process produces cubic grains of a uniform size. Figure 3.1 shows that the fraction released is much less than one percent for likely room temperature helium diffusivities $\left(10^{-15}\right.$ to $\left.10^{-16} \mathrm{~cm}^{2} / \mathrm{s}\right)$ except when 10um or smaller powders are produced. Because much of the powdered glass samples are in the 20-100um size range, the helium released during crushing sample Alv892-1a probably derives from rare larger vesicles. 
Figure 3.1 Approximate fraction of helium in a glass sample that may be released by diffusion during a crushing analysis, for several values of the diffusion coefficient, given as Log D in $\mathrm{cm}^{2} / \mathrm{s}$ on the curves. The calculation assumes crushing produces cubic particles of uniform size (x-axis), and that diffusve loss occurs over a typical 1 hour crushing and analysis period. For example, if $\mathrm{D}$ is $1 \times 10^{-13} \mathrm{~cm}^{2} / \mathrm{s}$ and the crushed powder size is 20um, approximately $5 \%$ of the helium contained in the glass will be diffusively released, and will contribute to the helium released by fracturing vesicles. 
Figure 3.1

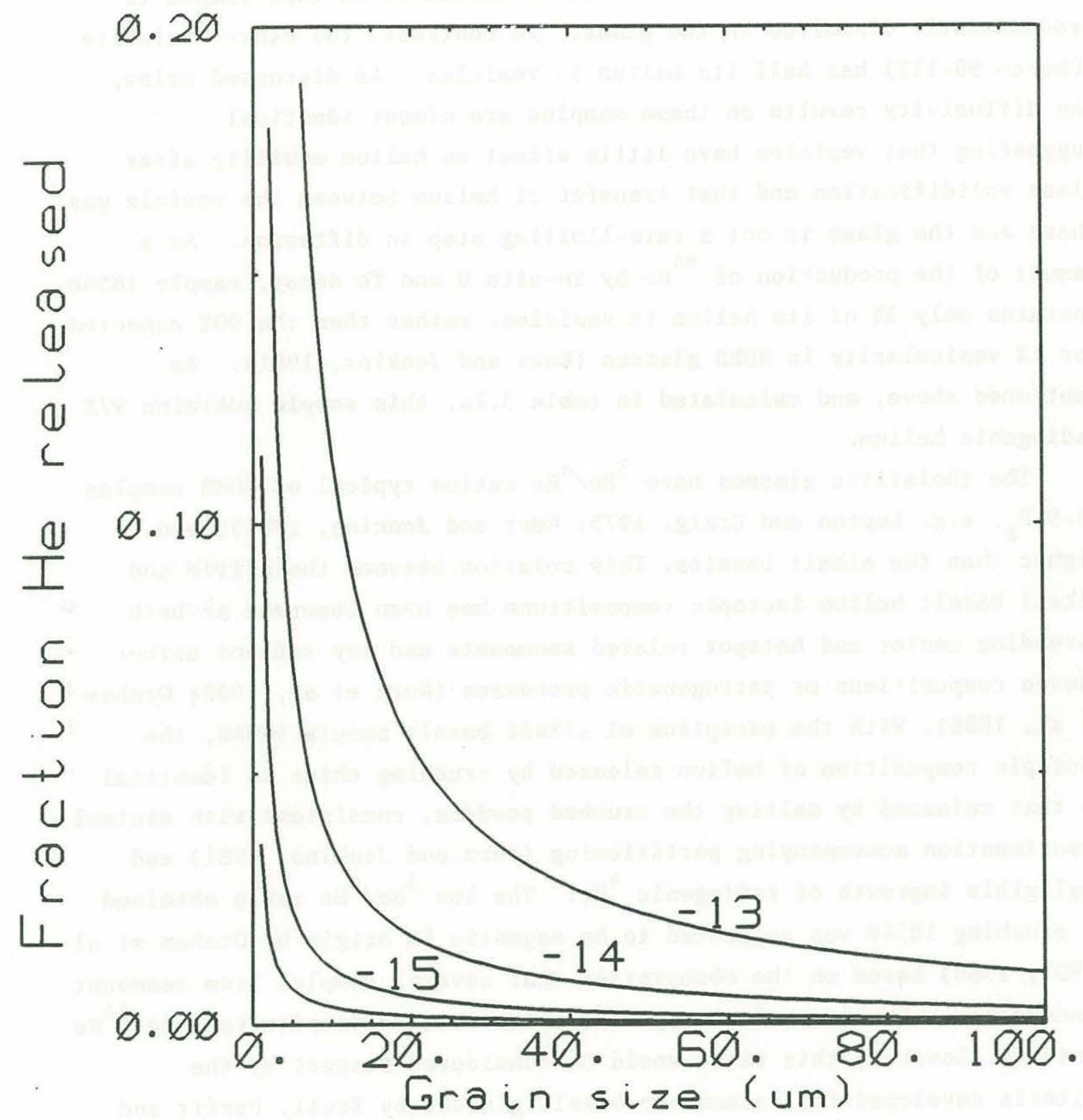


In any case, it is clear that the helium contained in this sample is predominantly dissolved in the glass. In contrast, the other tholeiite (Charco 98-11T) has half its helium in vesicles As discussed below, the diffusivity results on these samples are almost identical, suggesting that vesicles have little effect on helium mobility after glass solidification and that transfer of helium between the vesicle gas phase and the glass is not a rate-limiting step in diffusion. As a result of the production of ${ }^{* 4} \mathrm{He}$ by in-situ $U$ and Th decay, sample 1854B contains only $3 \%$ of $i$ ts helium in vesicles, rather than the $90 \%$ expected for $1 \%$ vesicularity in MORB glasses (Kurz and Jenkins, 1981). As mentioned above, and calculated in table 3.2a, this sample contains $97 \%$ radiogenic helium.

The tholeiitic glasses have ${ }^{3} \mathrm{He} /{ }^{4} \mathrm{He}$ ratios typical of MORB samples (8-9 Ra, e.g. Lupton and Craig, 1975; Kurz and Jenkins, 1981), and higher than the alkali basalts. This relation between tholeiitic and alkali basalt helium isotopic compositions has been observed at both spreading center and hotspot related seamounts and may reflect either source compositions or petrogenetic processes (Kurz et al, 1982; Graham et al, 1988). With the exception of alkali basalt sample 1854B, the isotopic composition of helium released by crushing chips is identical to that released by melting the crushed powders, consistent with minimal fractionation accompanying partitioning (Kurz and Jenkins, 1981) and negligible ingrowth of radiogenic ${ }^{*} \mathrm{He}$. The low ${ }^{3} \mathrm{He} /{ }^{4} \mathrm{He}$ ratio obtained by crushing 1854B was suggested to be magmatic in origin by Graham et al (1987, 1988) based on the observation that several samples from seamount 6 and other submarine volcanos give similar results despite varying ${ }^{*} \mathrm{He}$ contents. However, this ratio would be considered suspect by the criteria developed for island arc basalt glasses by Trull, Perfit and Kurz (1989, chapter 6)) because the large radiogenic helium content of the glass is readily capable of contaminating the 100 -fold smaller vesicle helium content. Graham et al (1987) suggested that the diffusive timescale for this to occur was too long, using the low diffusivity estimate of Kurz and Jenkins (1981), but did not consider higher diffusivity estimates (Jambon, Weber and Begemann, 1985) or release of ${ }^{*} \mathrm{He}$ during crushing (figure 3.1). However, as shown below, the 
diffusivity of ${ }^{* 4} \mathrm{He}$ is quite low in this sample, which supports the interpretation that the vesicle helium represents magmatic helium.

\section{3 $\underline{\text { RESULTS }}$}

Two methods were used to estimate the helium diffusivity. The simplest method was to determine the helium contents of powdered and sieved glasses by total fusion analysis before and after storage for several months in air at room temperature, and to calculate a diffusion coefficient from the fractional loss, the elapsed time and the grain size. This approach has the advantages that the helium diffusivity can be estimated at low temperature because the powder can be stored for a long time, and determining low helium emanation rates is not required. The disadvantage is that only one determination of the extent of loss as a function of time can be made, revealing little about the mechanism of release, and the temperature dependence of the diffusivity is not measured. In principle, these objectives could be achieved by storing powder splits at different temperatures for various lengths of time, but other aspects of the storage technique make the second method, measuring helium emanation in-vacuo directly, more precise. In particular, it is difficult to determine the initial helium content of powdered glasses (before storage), because of heterogeneous vesicle distributions and because helium is lost from fine powders between preparation and analysis.

\subsubsection{Storage experiments.}

The storage experiments were designed to examine and reproduce the work of Jambon, Weber, and Begemann (1985), who found that the helium content of powdered basaltic glass (Charco 98-DR11) decreased with grain size after storage in their laboratory for about a year. After estimating the fractional loss for the powders by comparison to the helium content of larger fragments (circa $1 \mathrm{~mm}$ ) they obtained a helium diffusivity estimate at room temperature of $6 \times 10^{-15} \mathrm{~cm}^{2} / \mathrm{s}$ with an error range estimate of a factor of 3 . To see if part of the dependence of 
helium content on grain size these researchers observed might result from the crushing process, rather than from diffusive loss, a similar sample from the same dredge (Charco 98-11T) was crushed, sieved, and immediately analyzed by melting. Figure 3.2 shows these results superimposed on the diagram of Jambon, Weber and Begemann (1985) for their sample and suggests that part of the helium loss observed in their study occured during powder preparation, because fine powders contain less helium than larger chips even before storage. This suggests that small vesicles may have contributed to the apparent loss in their study and that their diffusion coefficient is an overestimate. Both the Charco 98 sample used by Jambon, Weber and Begemann (1985) and the one studied here contained . $5 \%$ vesicles, primarily.5 $-1.0 \mathrm{~mm}$ in size.

To further examine the problem of crushing losses in powder storage experiments, sample Alv892-1a, which is extemely devoid of vesicles (table 3.2a), was powdered and analysed by melting before and after storage. Again, the powders had lower "initial" helium contents than larger grains, suggesting a loss related to crushing. Diffusive loss during the 3-5 days delay (table 2a) between crushing and analysis can not account for these low helium contents because this would require that the diffusion coefficient (D) be greater than $10^{-14} \mathrm{~cm}^{2} / \mathrm{s}$, which is not compatible with previous studies or the results of the emanation experiments. After storage, the reduced helium contents suggest high diffusivities when fractional loss is calculated by comparison to the helium content of a $2 \mathrm{~mm}$ chip (the proceedure used by Jambon, Weber and Begemann, 1985), but accounting for crushing losses by calculating fractional losses from the initial powder contents determined soon after crushing reduces D estimates by an order of magnitude (figure 3.3). The data do not determine the diffusivity with high precision because of the spread in grain size within a sieve fraction, errors in specifying grain geometry and the low fractional losses, but suggest a mean value of $5 \mathrm{x}$ $10^{-16} \mathrm{~cm}^{2} / \mathrm{s}$, with an error range of a factor of 2 . This is considerably less than the value of $6 \times 10^{-15}$ obtained by Jambon, Weber, and Begemann (1985) but higher than the value of $1 \times 10^{-16} \mathrm{~cm}^{2} / \mathrm{s}$, predicted by the Arrhenius temperature relation of Kurz and Jenkins (1981). 
Figure 3.2 Helium content of basaltic glass as a function of grain size (adapted from Jambon, Weber and Begemann, 1985). The horizontal bars are the helium contents measured by these workers for sieved powders after one year storage, and suggest a room temperature diffusivity of $6 \times 10^{-15} \mathrm{~cm}^{2} / \mathrm{s}$ (the dashed curves are for diffusivities of 3 times lower and higher). The horizontal line at $800 \times 10^{-8} \mathrm{ccSTP} / \mathrm{g}$ He marks the helium content below which their study assumed no helium was contained in vesicles, corresponding to a grain size of $.04 \mathrm{~cm}$. However, analyses of unstored powders (solid circles) reveal that powder preparation releases more helium from small particles than large grains, implying the diffusivity estimate of Jambon, Weber and Begemann (1985) is overestimated. 
Figure 3.2

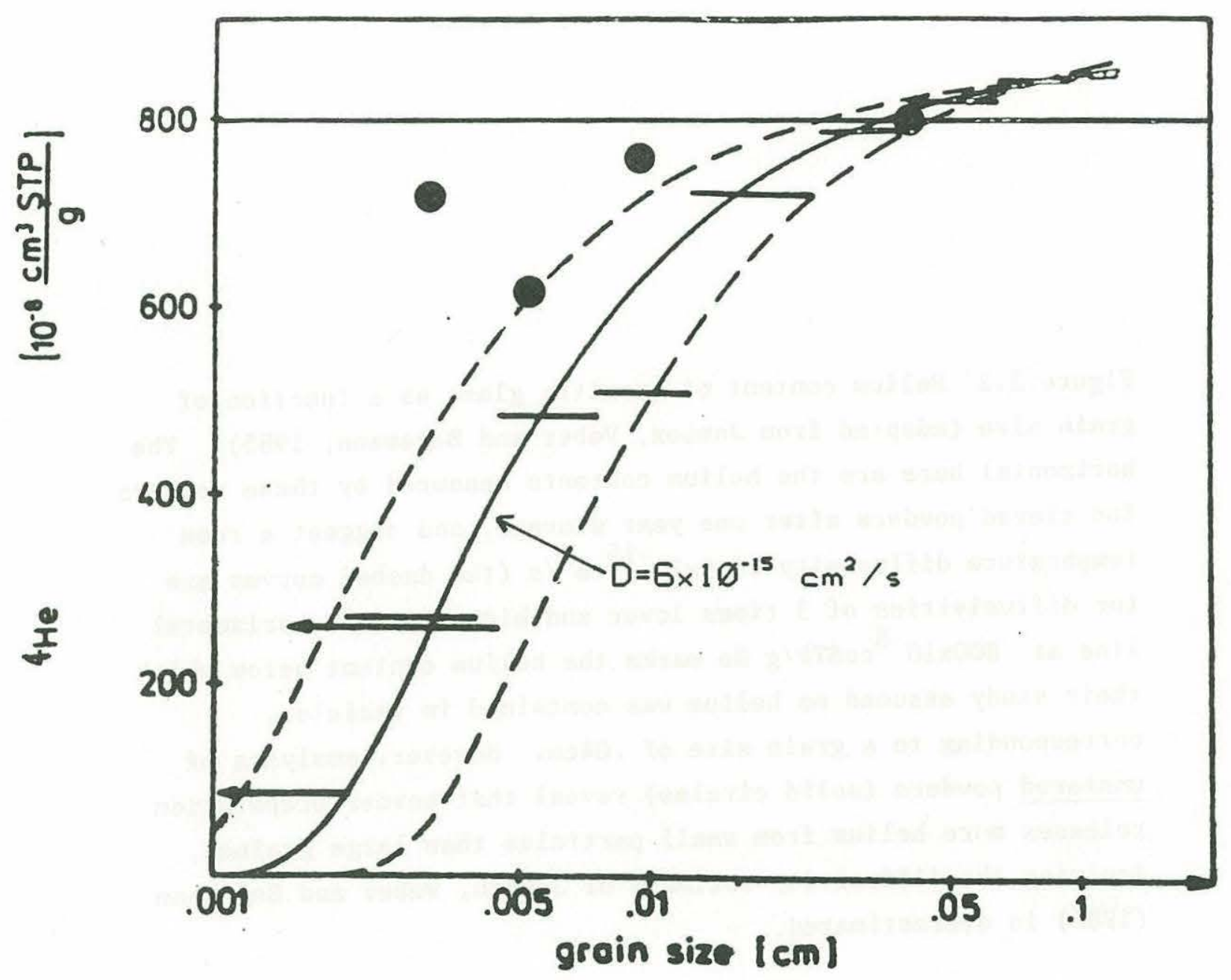


Table 3.3 Helium contents of powders stored at room temperature.

\begin{tabular}{llcccc}
$\begin{array}{l}\text { SAMPLE } \\
\text { (glass) }\end{array}$ & $\begin{array}{c}\text { GRAIN SIZE } \\
\text { (um) }\end{array}$ & $\begin{array}{c}\text { STORAGE TIME } \\
\text { (days) }\end{array}$ & $\begin{array}{c}\text { [He] } \\
(10-6 \mathrm{cc} / \mathrm{g})\end{array}$ & $\begin{array}{c}3 \mathrm{He} / 4 \mathrm{He} \\
(\mathrm{R} / \mathrm{Ra})\end{array}$ & $(1 \mathrm{~s})$ \\
\hline a. Total fusion & analyses & & & & \\
Charco 98-11T & $1 \mathrm{~mm}$ chip & years & 9.42 & 8.73 & $(.06)$ \\
& $38-53$ & 5 & 8.63 & 8.51 & $(.08)$ \\
& $53-75$ & 5 & 8.95 & 8.76 & $(.05)$ \\
Alv892-1A & $2 \mathrm{~mm}$ chip & 3 & 8.92 & 8.32 & $(.07)$ \\
& $25-38$ & 3 & 7.70 & 8.37 & $(.06)$ \\
& $38-53$ & 3 & 6.73 & 8.22 & $(.04)$ \\
& $75-106$ & 3 & 8.51 & 8.31 & $(.04)$ \\
& $25-38$ & 235 & 5.70 & 8.27 & $(.09)$ \\
& $53-75$ & 235 & 7.73 & 8.40 & $(.07)$ \\
& $75-106$ & 235 & 7.86 & 8.26 & $(.07)$ \\
& $106-180$ & 235 & 8.35 & 8.42 & $(.07)$ \\
& $250-300$ & 337 & 7.83 & 8.27 & $(.06)$ \\
& $355-425$ & 337 & 8.06 & 8.39 & $(.06)$ \\
& $425-500$ & 337 & 8.16 & 8.27 & $(.06)$
\end{tabular}

b. Sums of diffusive releases for step-heating expts. in table 3 .

$\begin{array}{llrlrl}\text { Alv892-1A } & 38-53 & 3 & 8.0(8.1) * & 8.31 & (.01) \\ & 75-106 & 55 & 7.7(7.9) * & 8.22 & (.01) \\ & 300-355 & 90 & 8.0(8.2) * & 8.34 & (.01) \\ & \text { 2mm shard } & 8 & 8.3 & 8.56 & (.03) \\ & \text { 2mm chips } & 68 & 8.6 & 8.35 & (.01) \\ \text { Charco 98-11T } & \text { 2mm chips } & 87 & 7.7 \# & 8.54 & (.04) \\ & \text { 1mm chips } & \text { years } & 9.7 & 8.73 & (.04)\end{array}$

a. 1 sigma analytical error in concentration is less than . $3 \%$

b. Storage times are from crushing $\mathrm{cm}$-sized pieces until analysis.

c. * (values) corrected for helium loss prior to analysis - see text.

d. \# variolitic texture may contribute to low helium content. 
Figure 3.3 Comparison of helium contents of stored glass powders with expected loss curves calculated assuming spherical geometry and the diffusivities shown (e.g. the leftmost curve is for $\left.\mathrm{D}=5 \times 10^{-17} \mathrm{~cm}^{2} / \mathrm{s}\right)$. The two plots are for different storage times, given in days. Samples are plotted for their sieve range assuming it approximates the diameter of an equivalent sphere The thin bars represent fractional contents calculated by comparison to a the helium content of a $2 \mathrm{~mm}$ chip and suggest a relatively high diffusivity of $10^{-15}$ to $10^{-14} \mathrm{~cm}^{2} / \mathrm{s}$. In contrast, fractional contents calculated by comparison to the helium contents of unstored powder splits (thick bars) suggest the correct diffusivity is an order of magnitude lower. 
Figure 3.3

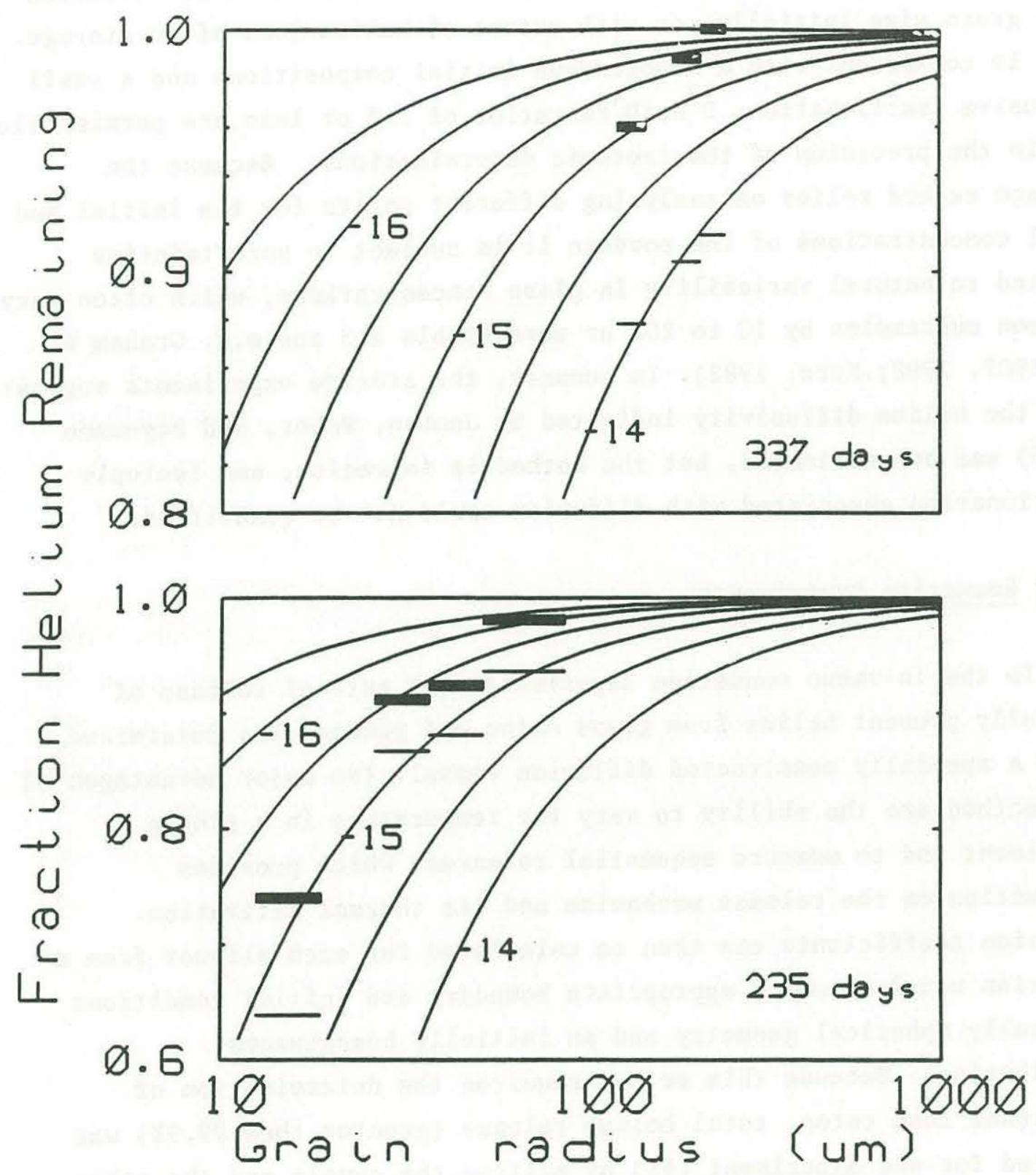


The isotopic composition of the powders showed no clear variation with grain size initially, or with extent of helium loss after storage. This is consistent with a homogeneous initial compositionn and a small diffusive fractionation; $\mathrm{D}^{3} \mathrm{He} / \mathrm{D}^{4} \mathrm{He}$ ratios of 1.2 or less are permissable within the precision of the isotopic determinations. Because the storage method relies on analysing different splits for the initial and final concentrations of the powders it is subject to uncertainties related to natural variability in glass concentrations, which of ten vary between subsamples by 10 to $20 \%$ or more (table 2.3 and e.g. Graham et al, 1987, 1988;.Kurz, 1982). In summary, the storage experiments suggest that the helium diffusivity indicated by Jambon, Weber, and Begemann (1985) was overestimated, but the method is imprecise, and isotopic fractionation associated with diffusion could not be quantified.

\subsubsection{Emanation Experiments.}

In the in-vacuo emanation experiments the rate of release of naturally present helium from glass chips and powders was determined using a specially constructed diffusion vessel. Two major advantages of this method are the ability to vary the temperature in a single experiment and to measure sequential releases, which provides information on the release mechanism and its thermal activation. Diffusion coefficients can then be calculated for each aliquot from a diffusion model assuming appropriate boundary and initial conditions (typically spherical geometry and an initially homogeneous distribution). Because this method requires the determination of fractional loss rates, total helium release (greater than 99.9\%) was verified for one experiment (\#1) by melting the sample and the other samples were heated at $600^{\circ} \mathrm{C}$ until the release rate limited the remaining helium to less than . $1 \%$ of the total. The experimental conditions also ensure that the helium diffuses into a surrounding volume with essentially zero helium content, which simplifies the diffusion model boundary conditions. The temperature range investigated was $20^{\circ} \mathrm{C}$ to $600^{\circ} \mathrm{C}$, essentially limited by safety concerns for heating the vessel at the high end, and by the ability to distinguish small 
helium releases from atmospheric leaks at low temperature. Typically, sample sizes were about $100 \mathrm{mg}$, but one experiment was performed with a single $7 \mathrm{mg}$ chip, and the powder experiments used up to $300 \mathrm{mg}$ of glass. Details of the diffusion vessel construction, temperature control, gas processing, mass spectrometry and the calculation of diffusion coefficients can be found in chapter 2 .

Six experiments were performed using tholeiite sample Alv892-1a to investigate reproducibility and the nature of helium release in detail (table 3.4; expts. \#1-6). This included three experiments with large chips and three with powders. One experiment was done with a single glass fragment to minimize grain size distribution effects (\#1) and another with slightly variolitic glass to examine the effect of glass devitrification on release (\#3). The powder experiments were designed to extend the temperature range from the $100^{\circ} \mathrm{C}$ to $600^{\circ} \mathrm{C}$ range of the chip experiments to room temperature, and to see if the glass crushing induced any high diffusivity paths. Most of the experiments were stepheated to investigate the temperature dependence of the diffusivity, but experiment \#4 was carried out isothermally (except for a few initial aliquots at low temperature). This simplifies examination of the release time dependence in terms of diffusion mechanisms because uncertainties in the temperature dependence of diffusion need not be considered. Experiments were also carried out on another tholeiite (Charco 98-11T, expt. 7 in table 3.4) and two alkali basalts to examine compositional effects (Aftermath D9, expts. 8 and 9) and the release of ${ }^{* 4} \mathrm{He}(1854 \mathrm{~B}$, expts. 10 and 11). The six Alv892-1a experiments are described first, as a guide to understanding the nature of helium mobility and in interpreting results for the other samples.

\subsubsection{Systematics of helium loss.}

Calculation of diffusivities from emanation rates assumes that helium loss is controlled by volume diffusion. By measuring multiple aliquots within each experiment it was possible to investigate this assumption. All the experiments displayed smooth extents of gas release as a function of time, as shown in figure 3.4. Helium loss rates 
Table 3.4 Basaltic Glass Step-heating Helium Diffusivity Results.

\begin{tabular}{|c|c|c|c|}
\hline Experiment & $\begin{array}{l}\text { Simple Model } \\
\text { Ea (kcal) } \\
\text { log Do }\end{array}$ & $\begin{array}{l}\text { Refined Model } \\
\text { Ea (kcal) } \\
\text { log Do }\end{array}$ & $\begin{array}{l}\text { Nominal } \\
\quad \text { Size } \\
\quad \text { (um) }\end{array}$ \\
\hline $\begin{array}{l}\text { AlV892-1A } \\
\begin{array}{l}\text { 1. } \\
\text { chingle tabular } \\
\text { chip }\end{array}\end{array}$ & $\begin{aligned} 17.4(.3) \\
-2.54(.10)\end{aligned}$ & $\begin{aligned} 18.1 & (.3) \\
-2.06 & (.08)\end{aligned}$ & $1400(150)$ \\
\hline $\begin{array}{l}\text { 2. } 13 \text { roughly cubic } \\
\text { chips }\end{array}$ & $\begin{aligned} 16.6 & (.1) \\
-2.79 & (.04)\end{aligned}$ & $\begin{aligned} 17.1 & (.1) \\
-2.30 & (.03)\end{aligned}$ & $1300(230)$ \\
\hline $\begin{array}{l}2 \text { chips with } 5 \% \\
100 \text { um varioles }\end{array}$ & $\begin{aligned} 17.9 & (.4) \\
-2.30 & (.13)\end{aligned}$ & $\begin{array}{r}18.2(.4) \\
-1.85(.1)\end{array}$ & $1620(100)$ \\
\hline $\begin{array}{l}\text { 4. } 300-355 \text { um grains } \\
\text { prior loss: } 2.6 \%\end{array}$ & $\begin{aligned} 16.8 & (.2) \\
-2.57 & (.11)\end{aligned}$ & $\begin{array}{r}17.2(.2) \\
-1.90(.1)\end{array}$ & (28) \\
\hline $\begin{array}{l}\text { 5. } 75-106 \text { um powder } \\
\text { prior loss: } 5.8(.1) \%\end{array}$ & $\begin{aligned} 15.5 & (.2) \\
-3.33 & (.07)\end{aligned}$ & $\begin{aligned} 16.1 & (.1) \\
-2.96 & (.05)\end{aligned}$ & (16) \\
\hline $\begin{array}{l}\text { 6. } 38 \text {-53um powder } \\
\text { prior loss: } 9.3(.4) \%\end{array}$ & $\begin{array}{r}14.0(.4) \\
-3.88(.2)\end{array}$ & $\begin{array}{r}14.9(.3) \\
-3.38(.2)\end{array}$ & (8) \\
\hline $\begin{array}{c}\text { CHARC0 } 98-11 \mathrm{~T} \\
7 . \quad 8 \text { chips }\end{array}$ & $\begin{aligned} 17.2(.5) \\
-2.56(.21)\end{aligned}$ & $\begin{array}{r}17.4(.5) \\
-2.15(.21\end{array}$ & $820(200)$ \\
\hline $\begin{array}{l}\text { AFTERMATH D9 } \\
8 . \quad 17 \text { chips }\end{array}$ & $\begin{aligned} 14.5 & (.6) \\
-3.07 & (.26)\end{aligned}$ & $\begin{aligned} 14.7 & (.6) \\
-2.74 & (.26)\end{aligned}$ & $980(200)$ \\
\hline 9. 26 chips & $\begin{aligned} 13.9 & (.5) \\
-3.87 & (.25)\end{aligned}$ & $\begin{aligned} 14.0 & (.5) \\
-3.75 & (.25)\end{aligned}$ & 990 (180) \\
\hline $\begin{array}{l}\text { ALV1389-1854B } \\
\text { 10. } 9 \text { chips } \\
\text { *4He }\end{array}$ & $\begin{aligned} & 28.2(1.0) \\
+ & 3.0 \quad(.5)\end{aligned}$ & $\begin{aligned} & 28.3(1.1) \\
+ & 3.5 \quad(.5)\end{aligned}$ & $1160(300)$ \\
\hline $\begin{array}{l}\text { 11. } 212-250 \text { um grains } \\
\star 4 \mathrm{He}\end{array}$ & $\begin{aligned} & 25.8(2.2) \\
&+1.0(1.2)\end{aligned}$ & $\begin{aligned} & 26.0(2.4) \\
&+1.2(1.3)\end{aligned}$ & 231 \\
\hline
\end{tabular}

a. The simple (equisized-spheres) model used the nominal size given to estimate Do values. The refined model used measured sizes and shapes. Both models corrected for prior loss in expts. \#4-6, using emanation data for 5 and 6 ( 1 s fitting errors given), and a minimum estimate for \#4 (see text). Expt. 4 measured release at only two temperatures., its Ea is poorly constrained.

b. 1-sigma Arrhenius line-fitting errors are given for Ea and Do values in parentheses. Including grain measurement errors estimated at $10 \%$ suggests 1 -sigma errors in absolute diffusivities and Do values of $20 \%$. 
Figure 3.4 - \#1-6. Fractional release (F) versus time plots for each of the Alv892-1a experiments, numbered as in table 3.4.

Note the changing time scales. Experiments \#1-3 used glass chips at temperatures of $200-600^{\circ} \mathrm{C}$. Experiments \#4-6 used sieved powders at $20-300^{\circ} \mathrm{C}$. Specific temperature steps for each experiment are given in figure 3.7 , here the temperature ramp steps are marked by solid symbols. Experiment \#4 was run isothermally except for the first few aliquots. 
Figure 3.4
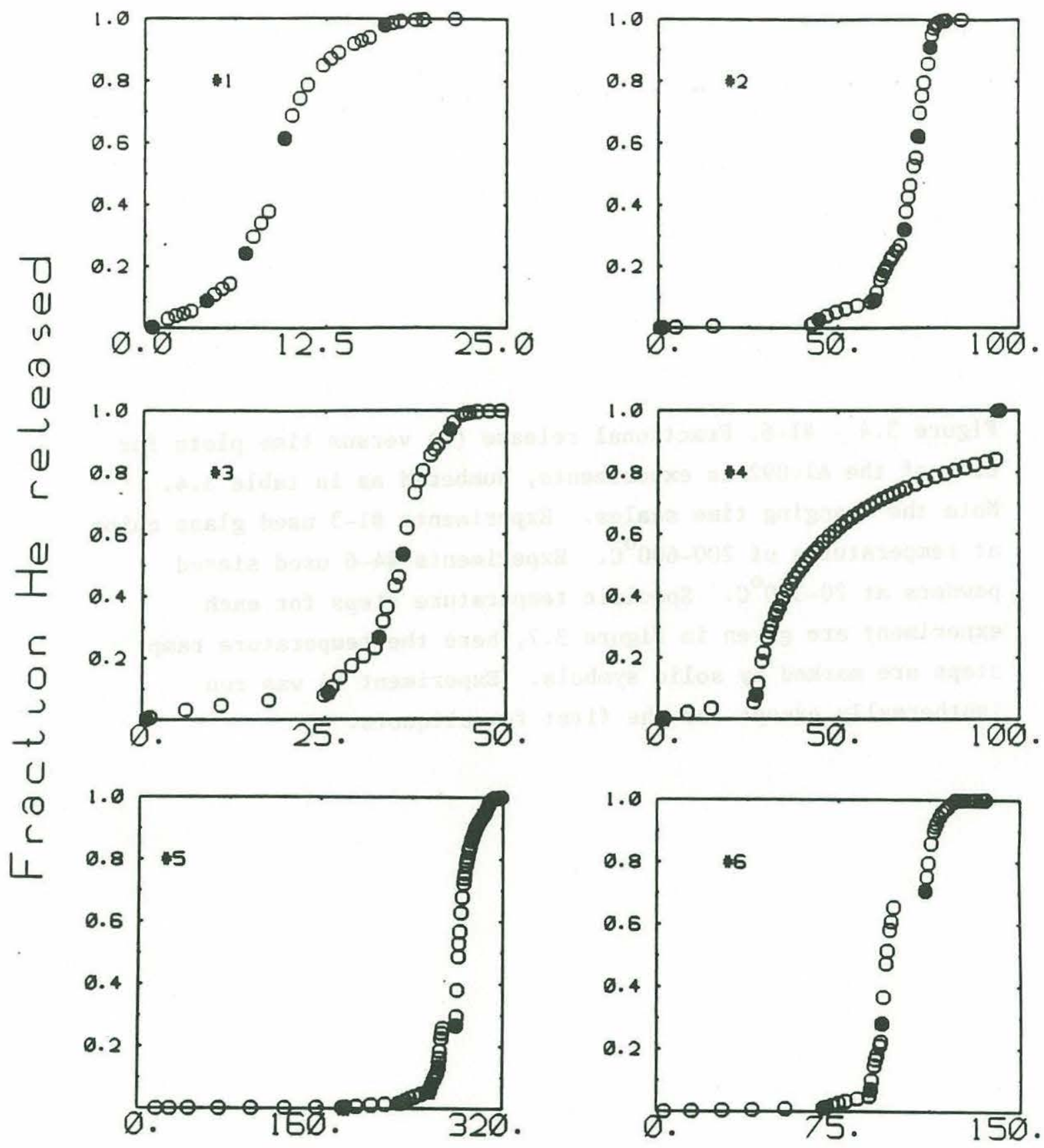

Emanation Time (hours). 
Figure 3.4 - \#7-11. Fractional release (F) versus time plots for tholeiite Charco 98-11T (\#7), alkali basalt Aftermath D9 (\#8 and \#9) and for ${ }^{\star 4} \mathrm{He}$ release from alkali basalt $1854 \mathrm{~B}$ (\#10 and \#11). Specific temperatures for each experiment are given in figure 3.7 (the range is $200-600^{\circ} \mathrm{C}$ ), here the temperature ramp steps are marked by solid symbols. 

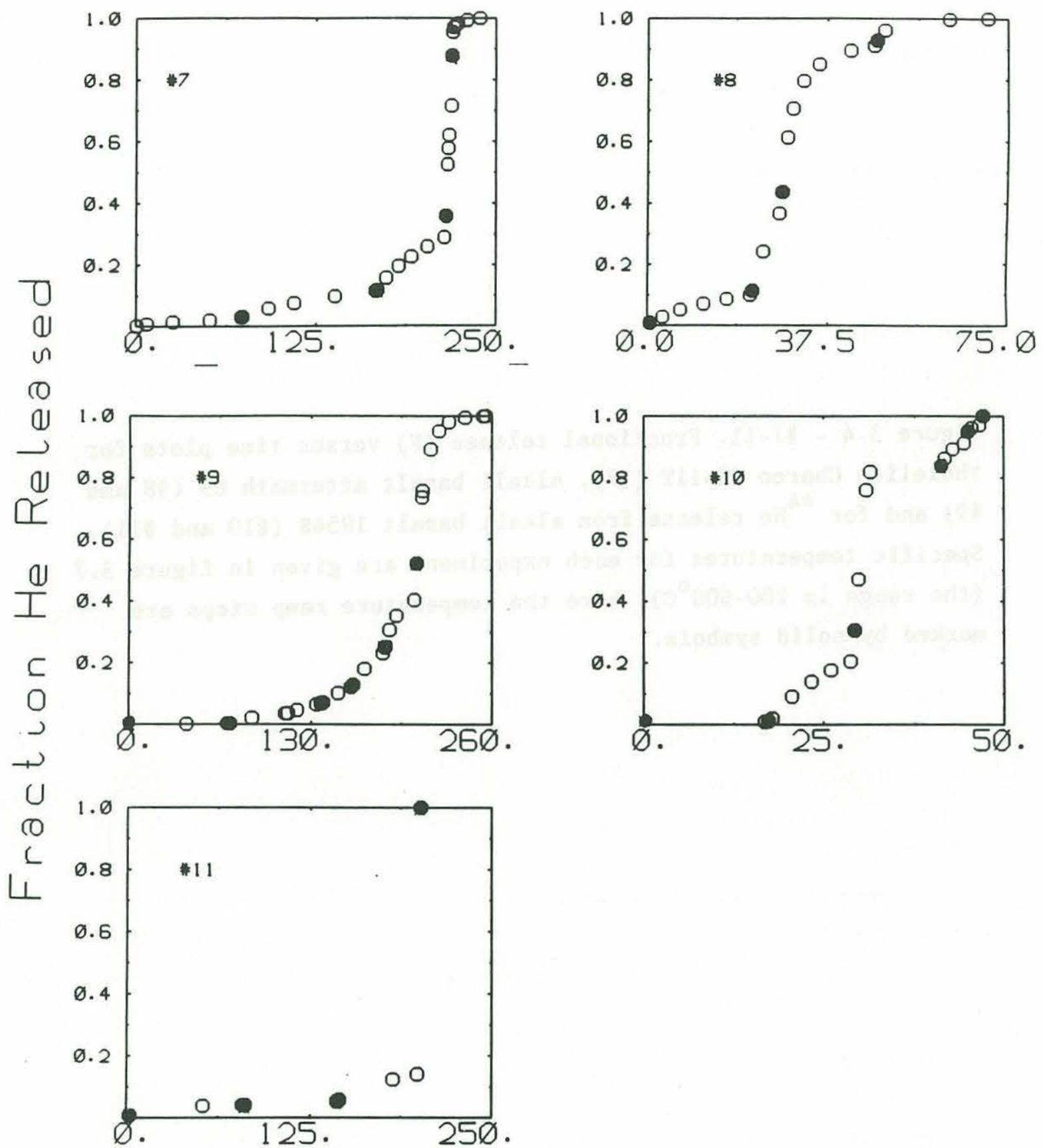

Emanation Time (hours) 
increased when the temperature was raised and leveled off at a new rate without any sudden releases. As discussed below, the isotopic compositions of sequential releases also varied smoothly. These aspects of the releases point towards continuum process such as volume diffusion, rather than events such as vesicle decrepitation or cracking. This is in agreement with examination of the samples in thin section before and after the experiments, which revealed no internal cracks or other changes. The time dependence of release can also aid in distinguishing between different loss mechanisms.

As shown analytically, volume diffusion exhibits a linear dependence between fractional release $(F)$ and the square-root of time $\left(t^{1 / 2}\right.$ ) for low extents of gas loss (e.g less than $20 \%$ for a sphere, Inthoff and Zimen, 1956; Fechtig and Kalbitzer, 1966). The F range over which this relation holds varies with grain geometry and in a sample with a distribution of grain sizes and shapes, it is reduced to that for which the smallest grains (with the largest surface to volume ratio) still retain a major portion of their gas (Shaked, 1965). At higher extents of release, plots of $\mathrm{F}$ versus the square-root of time will remain monotonic but exhibit concave downward curve shape as the gas release rate tapers off. Other time dependences for release are indicative of different loss mechanisms. For instance, steady-state leakage from a vesicle through a glass wall would yield a linear $\mathrm{F}$ versus time relation until the vesicle began to be depleted, and desorption processes exhibit sigmoidal (s-shaped) release curves (Crank, 1975).

All the experiments exhibited release rates consistent with volume diffusion (i.e linear $\mathrm{F}$ vs. $\mathrm{t}^{1 / 2}$ relations). A particularly good example is experiment \#4 which collected isothermal aliquots over a large $\mathrm{F}$ range. Its emanation curve demonstrates that the release is compatible with volume diffusion but not with a steady-state leak or desorption (figure 3.5). Examining the time dependence of release in the finest powders (expts. \# 5 and 6) shows both that they lost helium between preparation and analysis and that the release is consistent with volume diffusion (figure 3.6a). However, the limited $\mathrm{F}$ range examined at room temperature prevented ruling out a linear $\mathrm{F}$ versus time relation (figure 
Figure 3.5 Release systematics for small fractional losses in experiment \#4. The rate of helium loss displays good agreement with volume diffusion (a, linear with $t^{1 / 2}$ ), and shows a distinct departure from a constant emanation rate (b). The break in slope corresponds to an increase in emanation temperature from $123^{\circ} \mathrm{C}$ to $236^{\circ} \mathrm{C}$. 
Figure 3.5
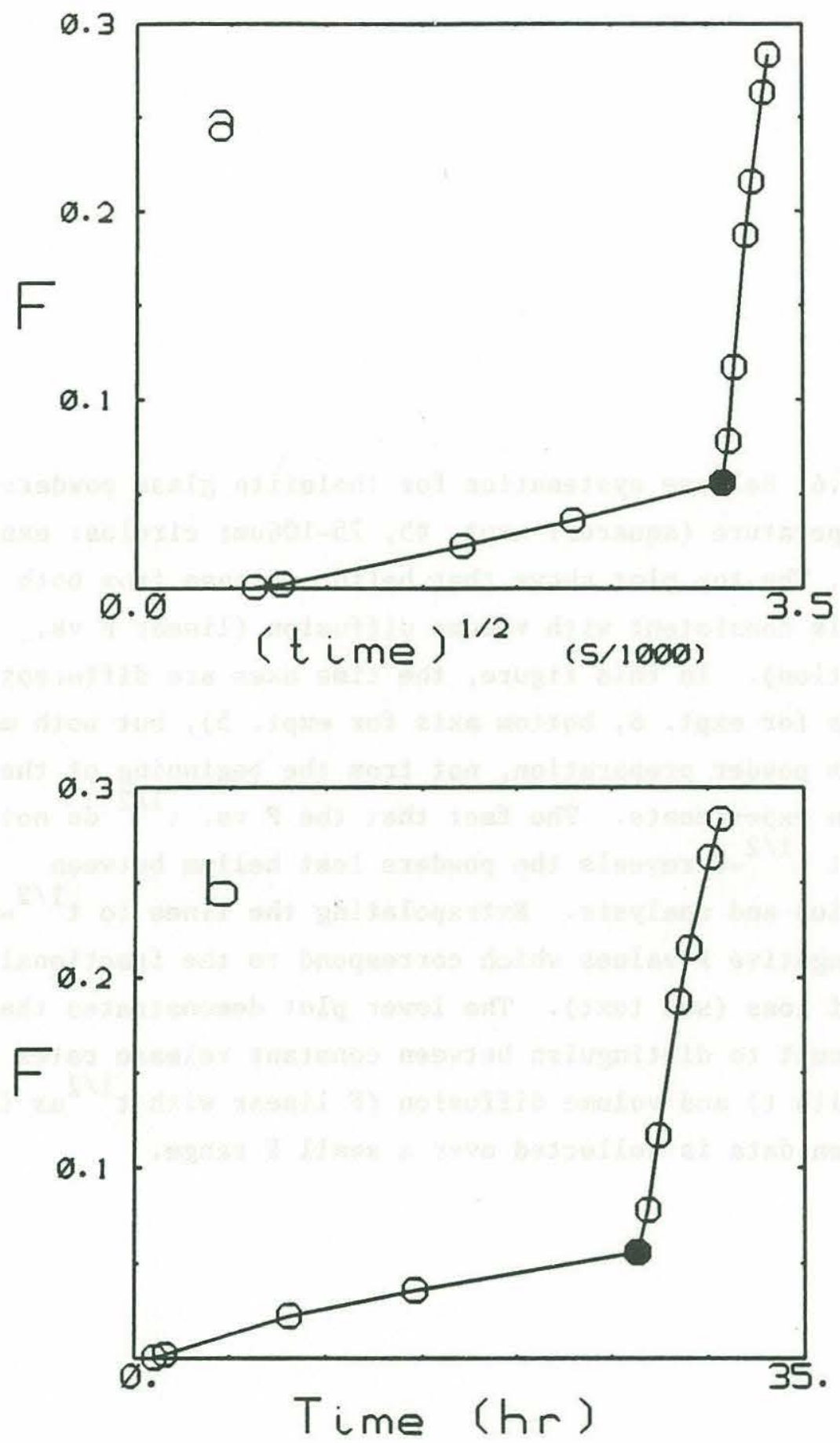
Figure 3.6 Release systematics for tholeiite glass powders at room temperature (squares: expt. \#5, 75-106um; circles: expt. \#6, 38-53um). The top plot shows that helium release from both powders is consistent with volume diffusion (linear $F$ vs. $t^{1 / 2}$ relation). In this figure, the time axes are different (top axis for expt. 6, bottom axis for expt. 5), but both measure time from powder preparation, not from the beginning of the emanation experiments. The fact that the $F$ vs. $t^{1 / 2}$ do not tend to $\mathrm{F}=0$ at $\mathrm{t}^{1 / 2}=0$ reveals the powders lost helium between preparation and analysis. Extrapolating the lines to $\mathrm{t}^{1 / 2}=0$ yields negative $F$ values which correspond to the fractional extent of loss (see text). The lower plot demonstrates that it is difficult to distinguish between constant release rates ( $F$ linear with $\mathrm{t}$ ) and volume diffusion ( $\mathrm{F}$ linear with $\mathrm{t}^{1 / 2}$ as in top plot) when data is collected over a small $\mathrm{F}$ range. 
Figure 3.6
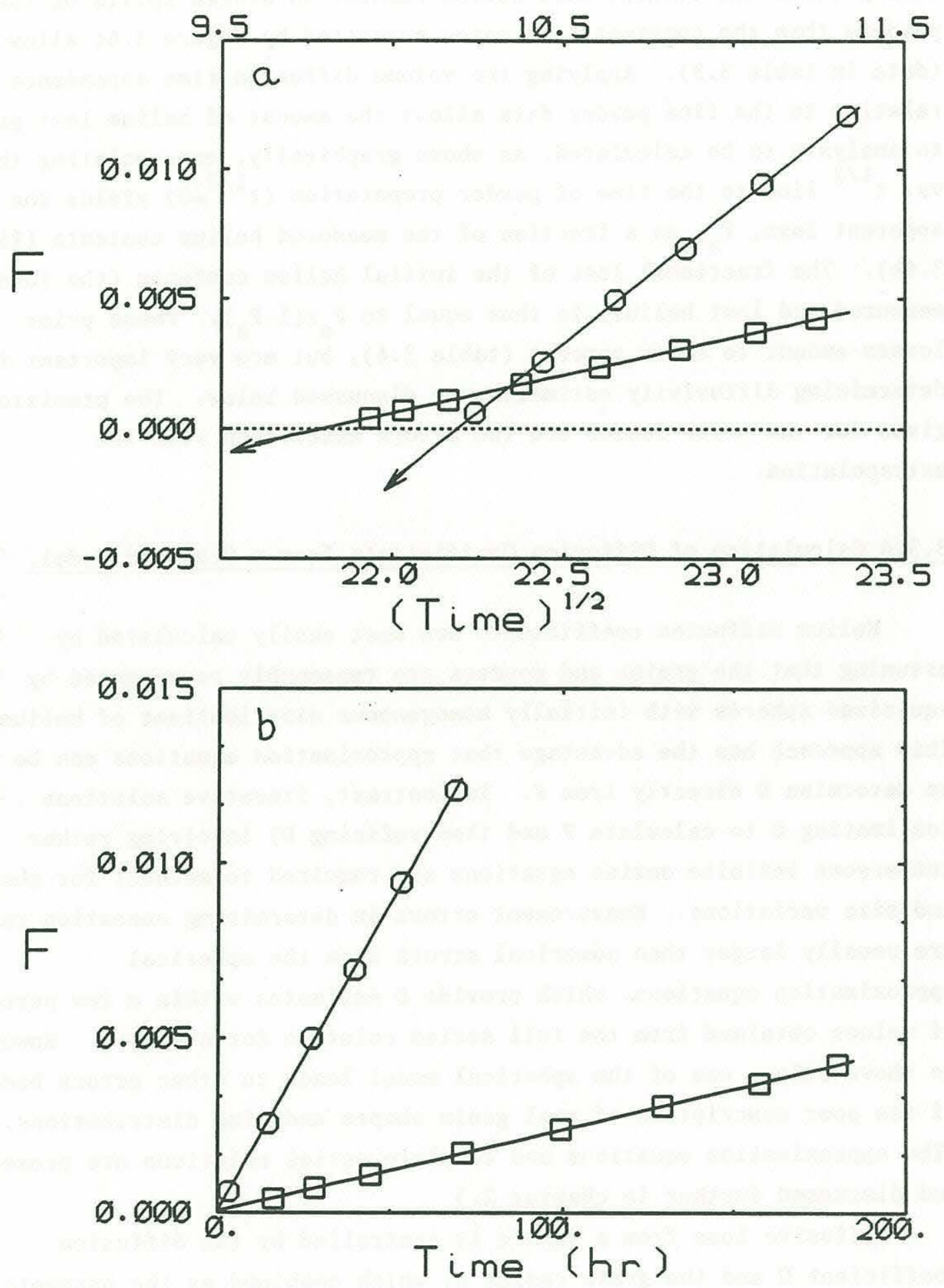
3.6b). Nonetheless, it is clear that the release process is not a steady-state one because more helium remains in stored splits of the powders than the constant loss rates suggested by figure $3.6 \mathrm{c}$ allow (data in table 3.3). Applying the volume diffusion time dependence relation to the fine powder data allows the amount of helium lost prior to analysis to be calculated. As shown graphically, extrapolating the $\mathrm{F}$ vs. $t^{1 / 2}$ line to the time of powder preparation $\left(t^{1 / 2}=0\right)$ yields the apparent loss, $\mathrm{F}_{\mathrm{a}}$, as a fraction of the measured helium contents (figure $3.6 \mathrm{~b}$ ). The fractional loss of the initial helium contents (the total of measured and lost helium) is thus equal to $\mathrm{F}_{\mathrm{a}} \mathrm{x}\left(1-\mathrm{F}_{\mathrm{a}}\right)$. These prior losses amount to a few percent (table 3.4), but are very important in determining diffusivity estimates, as discussed below. The precisions given for the prior losses are the errors associated with the extrapolation.

\subsubsection{Calculation of Diffusion Coefficients from a Standard Model.}

Helium diffusion coefficients are most easily calculated by assuming that the grains and powders are reasonably represented by equisized spheres with initially homogeneous distributions of helium. This approach has the advantage that approximation equations can be used to determine D directly from F. In contrast, iterative solutions (estimating $\mathrm{D}$ to calculate $\mathrm{F}$ and then refining $\mathrm{D}$ ) involving rather cumbersome infinite series equations are required to account for shape and size variations. Measurement errors in determining emanation rates are usually larger than numerical errors from the spherical approximation equations, which provide D estimates within a few percent of values obtained from the full series solution for a sphere. However, as shown below, use of the spherical model leads to other errors because of its poor description of real grain shapes and size distributions. (The approximation equations and complete series solutions are presented and discussed further in chapter 2.)

Diffusive loss from a sphere is controlled by the diffusion coefficient $D$ and the grain radius $a$, which combined as the parameter $\mathrm{D} / \mathrm{a}^{2}$ define the characteristic diffusive timescale (note its units are 
$1 / t) . \quad D / a^{2}$ values can be calculated directly from fractional release and elapsed time data without estimating a representative grain radius. The nature of helium loss can then be considered by examining variations in these values. In general, sequential aliquots taken at constant temperature (T) exhibit constant values of $\mathrm{D} / \mathrm{a}^{2}$ (figure $3.7, \# 1-11$ ). This suggests that the release process is not strongly dependent on concentration or heating history, but only on temperature as expected for trace component diffusion. Experiment \#4, for which helium release was measured isothermally over the greatest range of $\mathrm{F}$, is the best example of this. However, there are exceptions to this behavior. In several experiments $\mathrm{D} / \mathrm{a}^{2}$ increases steadily within the early temperature steps. This effect is particularly pronounced in the fine powder experiments where apparent diffusivities vary by over an order of magnitude (\#5 and \#6 in figure 3.7), but can be seen in the larger grain runs as well (e.g in the first temperature steps of experiments 3 and 4). This feature is more related to low extents of fractional release than to temperature. For example, it is pronounced in the $113^{\circ} \mathrm{C}$ data of experiment \#2 at very low $\mathrm{F}$ (less than .02), present in the $123^{\circ} \mathrm{C}$ data of experiment \#4 at $\mathrm{F}$ less than .06 , weakly visible in experiment \#5 at $112^{\circ} \mathrm{C}$ for $\mathrm{F}$ ranging from .06 to .12 , and absent in experiment \#6 at $118^{\circ} \mathrm{C}$, where $\mathrm{F}$ exceeds $10 \%$. Similarly, the strong trends observed in the fine powders (expts. \#5 and \#6) occur at very low $F$ (less than a few percent). It is clear that these increases of $\mathrm{D} / \mathrm{a}^{2}$ with $\mathrm{F}$ are not the result of slow thermal equilibration or temperature drift problems because they also occur at room temperature, and because measured thermal equilibration times for the diffusion vessel ( $<15$ minutes) were much shorter than the emanation intervals (chapter 2).

A different type of deviation from the expected constant $D$ at constant temperature relation occurs at high $\mathrm{F}$ values, where $\mathrm{D} / \mathrm{a}^{2}$ values exhibit smooth, and in some cases strong, decreases with increasing F. Examples include experiments using both powders and glass chips (e.g.\#6,7,8, and 10). Again, this effect is more correlated with extent of fractional release than with temperature. However, the $\mathrm{F}$ value at which $\mathrm{D} / \mathrm{a}^{2}$ begins to decline varies in these experiments from as low as .6 in \#10 to over .9 in \#6 and \#7. In addition to these large trends, 
Figure 3.7 - \#1-3. Spherical-model helium diffusivities calculated for each release in the Alv892-1a tholeiite glass chip experiments (\#1-3). Filled symbols mark ramp steps between temperature plateaus (in ${ }^{\circ} \mathrm{C}$ ). Also shown are histograms describing the grain assemblies. Viewing the grains as rectangular prisms, the bars represent the short side length (hatched), long side length (open) and mean size (filled). 
Figure 3.7
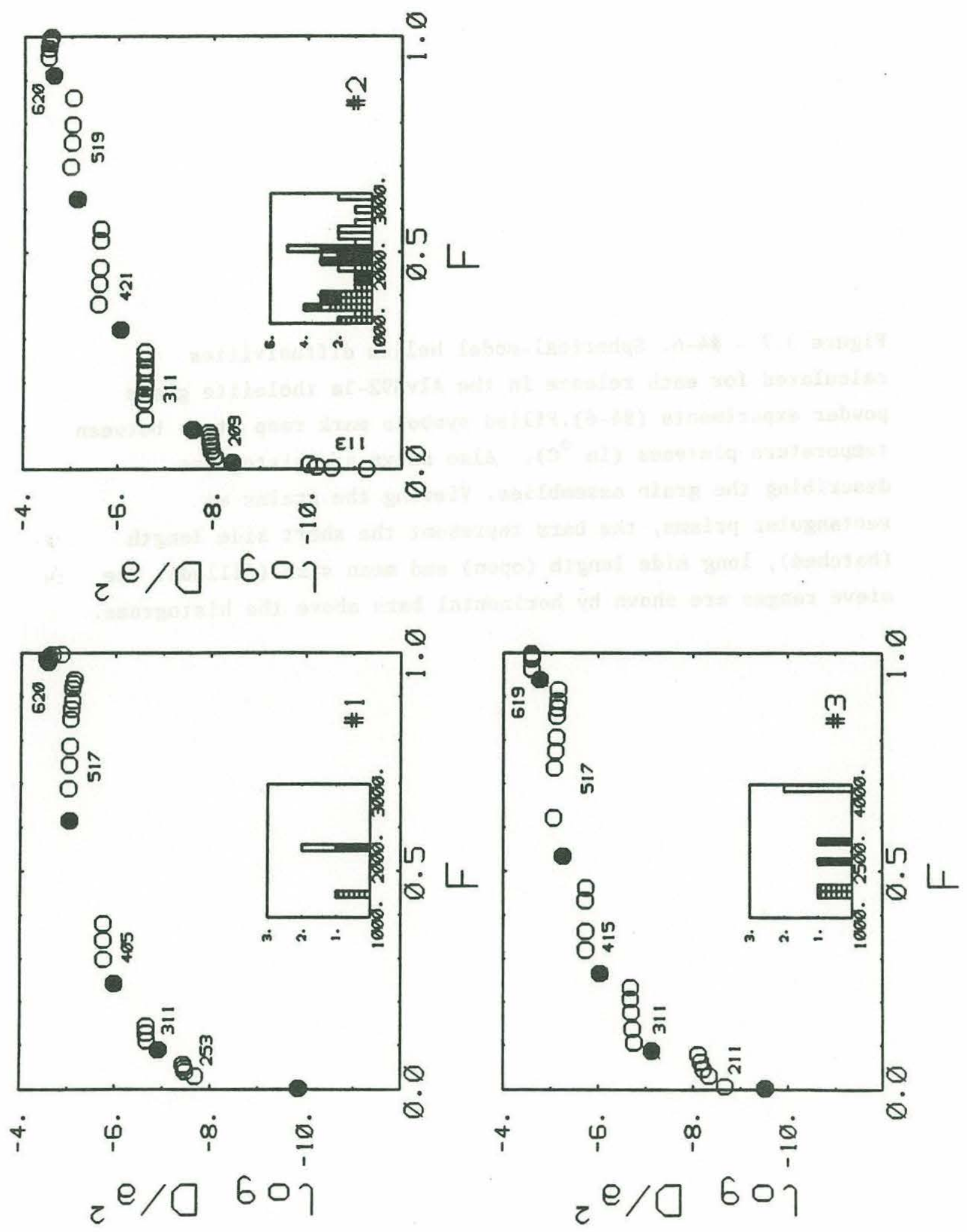
Figure 3.7 - \#4-6. Spherical-model helium diffusivities calculated for each release in the Alv892-1a tholeiite glass powder experiments (\#4-6).Filled symbols mark ramp steps between temperature plateaus (in ${ }^{\circ} \mathrm{C}$ ). Also shown are histograms describing the grain assemblies. Viewing the grains as rectangular prisms, the bars represent the short side length (hatched), long side length (open) and mean size (filled). The sieve ranges are shown by horizontal bars above the histograms. 

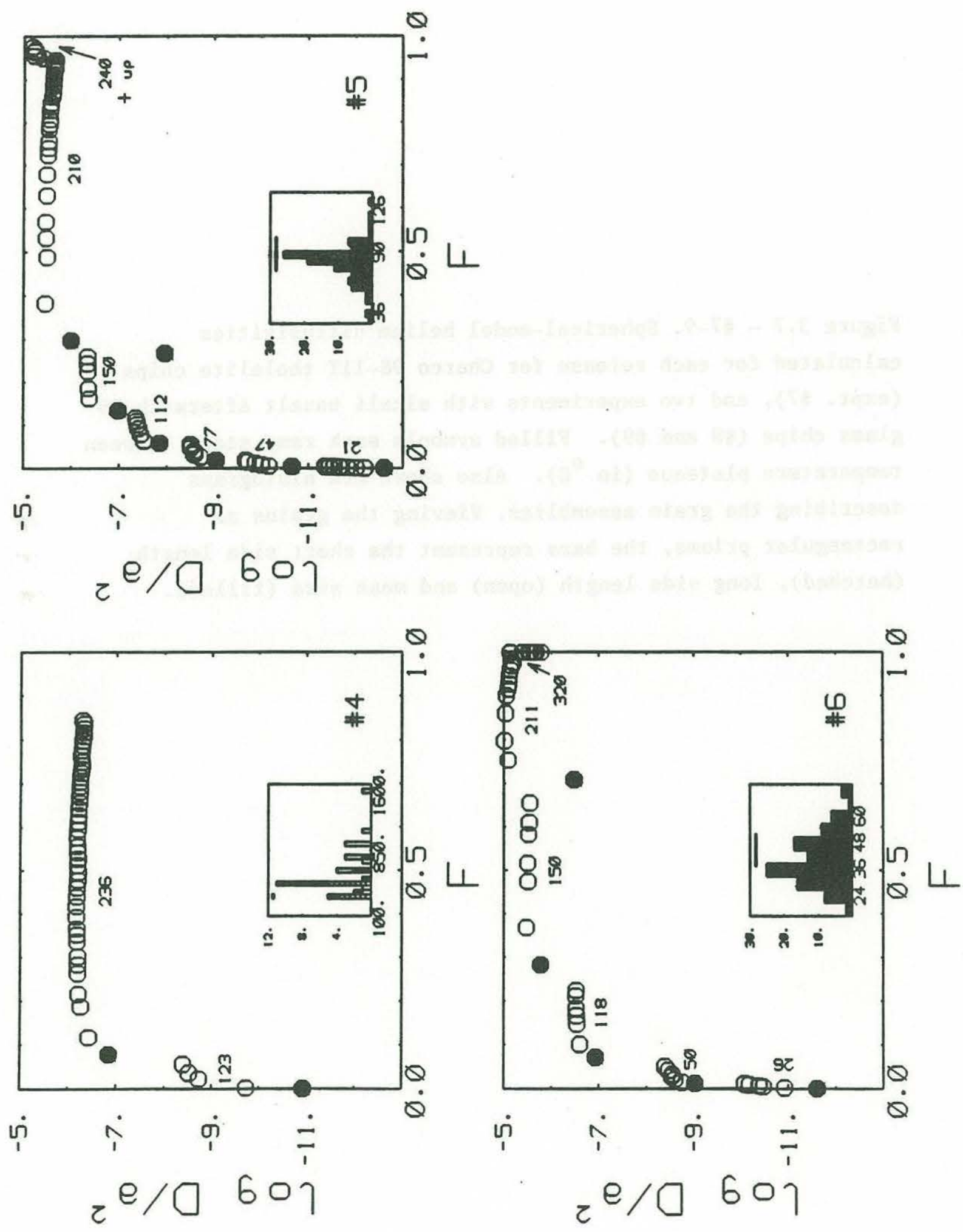
Figure 3.7 - \#7-9. Spherical-model helium diffusivities calculated for each release for Charco 98-11T tholeiite chips (expt. \#7), and two experiments with alkali basalt Aftermath D9 glass chips (\#8 and \#9). Filled symbols mark ramp steps between temperature plateaus (in ${ }^{\circ} \mathrm{C}$ ). Also shown are histograms describing the grain assemblies. Viewing the grains as rectangular prisms, the bars represent the short side length (hatched), long side length (open) and mean size (filled). 


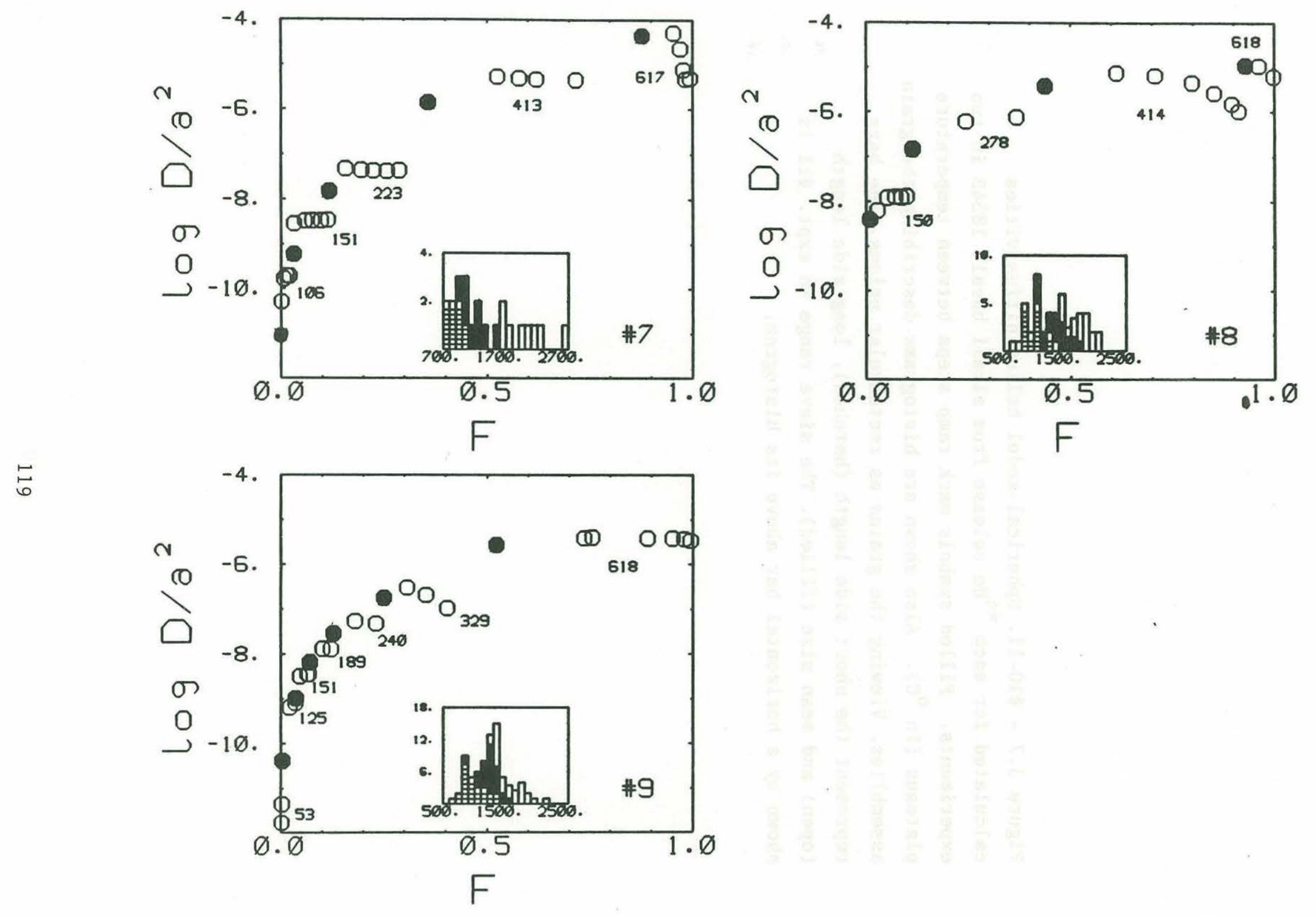


Figure 3.7 - \#10-11. Spherical-model helium diffusivities calculated for each ${ }^{\star 4} \mathrm{He}$ release from alkali basalt $1854 \mathrm{~B}$ in two experiments. Filled symbols mark ramp steps between temperature plateaus (in ${ }^{\circ} \mathrm{C}$ ). Also shown are histograms describing the grain assemblies. Viewing the grains as rectangular prisms, the bars represent the short side length (hatched), long side length (open) and mean size (filled). The sieve range in expt. \#11 is shown by a horizontal bar above its histogram. 

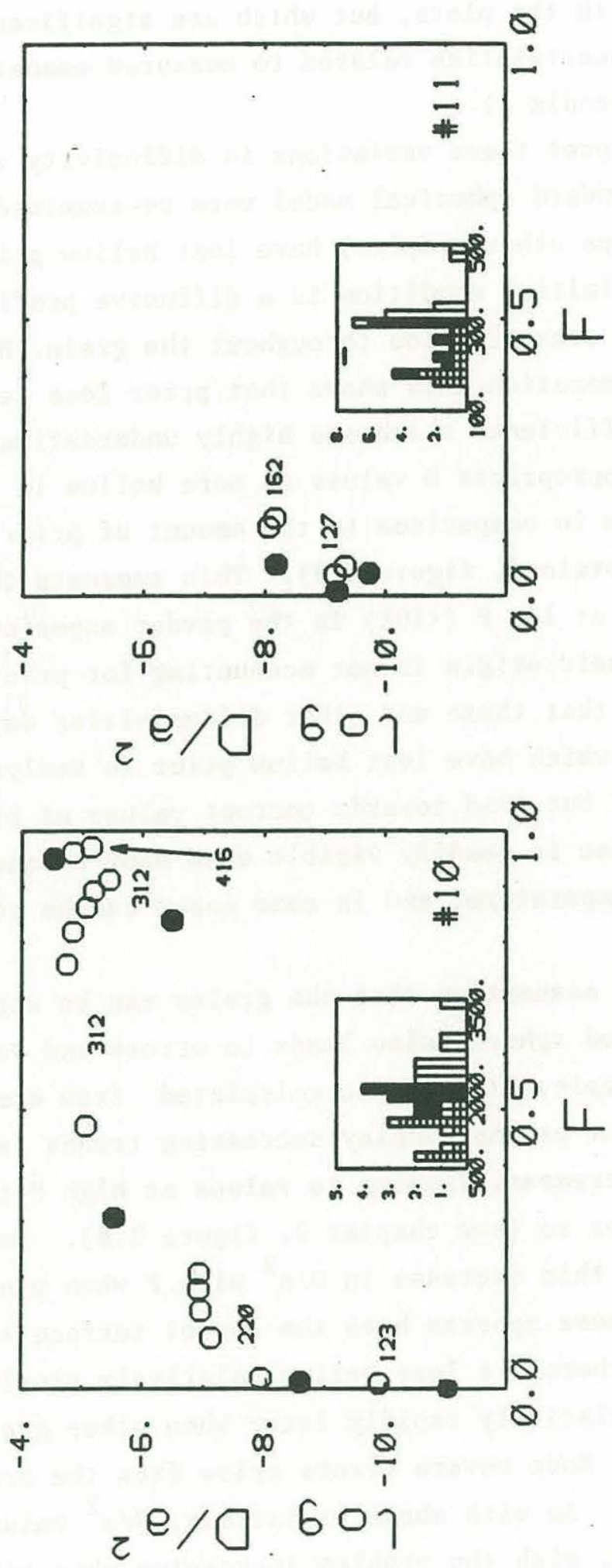
there are many smaller monotonic variations in $\mathrm{D} / \mathrm{a}^{2}$ with $\mathrm{F}$ at constant $\mathrm{T}$ which are not visible in the plots, but which are significantly larger than the analytical uncertainties related to measured emanation rates alone (see data in Appendix A).

In order to interpret these variations in diffusivity values, the assumptions of the standard spherical model were re-examined. Because the powders (and perhaps other samples) have lost helium prior to analysis, the correct initial condition is a diffusive profile rather than a constant helium concentration throughout the grain. Modelling synthetic isothermal emanation data shows that prior loss leads to apparent diffusion coefficients which are highly underestimated at low F but increase towards appropriate $\mathrm{D}$ values as more helium is released (at $F$ values that are large in comparison to the amount of prior loss, correct D values are obtained, figure 3.8). This suggests the increasing $\mathrm{D} / \mathrm{a}^{2}$ values at low $\mathrm{F}(<10 \%)$ in the powder experiments (figure 3.7 , \#5 and \#6) have their origin in not accounting for prior diffusive loss. It also implies that these and other diffusivities determined from grains or powders which have lost helium prior to analysis will be underestimated at low $\mathrm{F}$ but tend towards correct values at high $\mathrm{F}$. Fortunately, this problem is readily visible when many aliquots are measured at constant temperature, and in some cases can be corrected for, as shown below.

The standard model assumption that the grains can be represented as a collection of equisized spheres also leads to errors and variations in $\mathrm{D} / \mathrm{a}^{2}$ values. As an example, $\mathrm{D} / \mathrm{a}^{2}$ values calculated from emanation data for cubes and other right prisms display decreasing trends (with roughly constant slope) as $\mathrm{F}$ increases, leading to values at high $\mathrm{F}$ that are lower by a factor of 2 or so (see chapter 2, figure 2.8). Essentially all shapes will display this decrease in $\mathrm{D} / \mathrm{a}^{2}$ with $\mathrm{F}$ when processed with the spherical model because spheres have the lowest surface to volume ratio of any shape and therefore lose helium relatively slowly as emanation begins, but relatively rapidly later when other grain shapes are becoming exhausted. More severe errors arise from the presence of grain size distribution. As with shape variations, $\mathrm{D} / \mathrm{a}^{2}$ values are underestimated at high $\mathrm{F}$, with the problem increasing when size 
Figure 3.8 Effect of prior diffusive loss on apparent diffusivities. Curves derived by discarding successive amounts of synthetic release data (spherical model with homogeneous initial condititon, $2 \%$ release intervals and $\log D=-15$ ) and processing the remaining release data ( $F$ versus time) as if it constituted the entire helium content. Prior loss leads to apparent diffusion coefficients that are strongly underestimated at low F, but which trend toward correct values at high F. Note that the apparent diffusivity is within a factor of 2 of the correct value for $\mathrm{F}$ greater than twice the prior loss amount. This is because helium is initially lost far more slowly from a grain with a smooth, diffusive profile than one with an initially discontinuous (step) profile at the grain surface, yet loss is similar at high $\mathrm{F}$ once both grains have established diffusive profiles. 


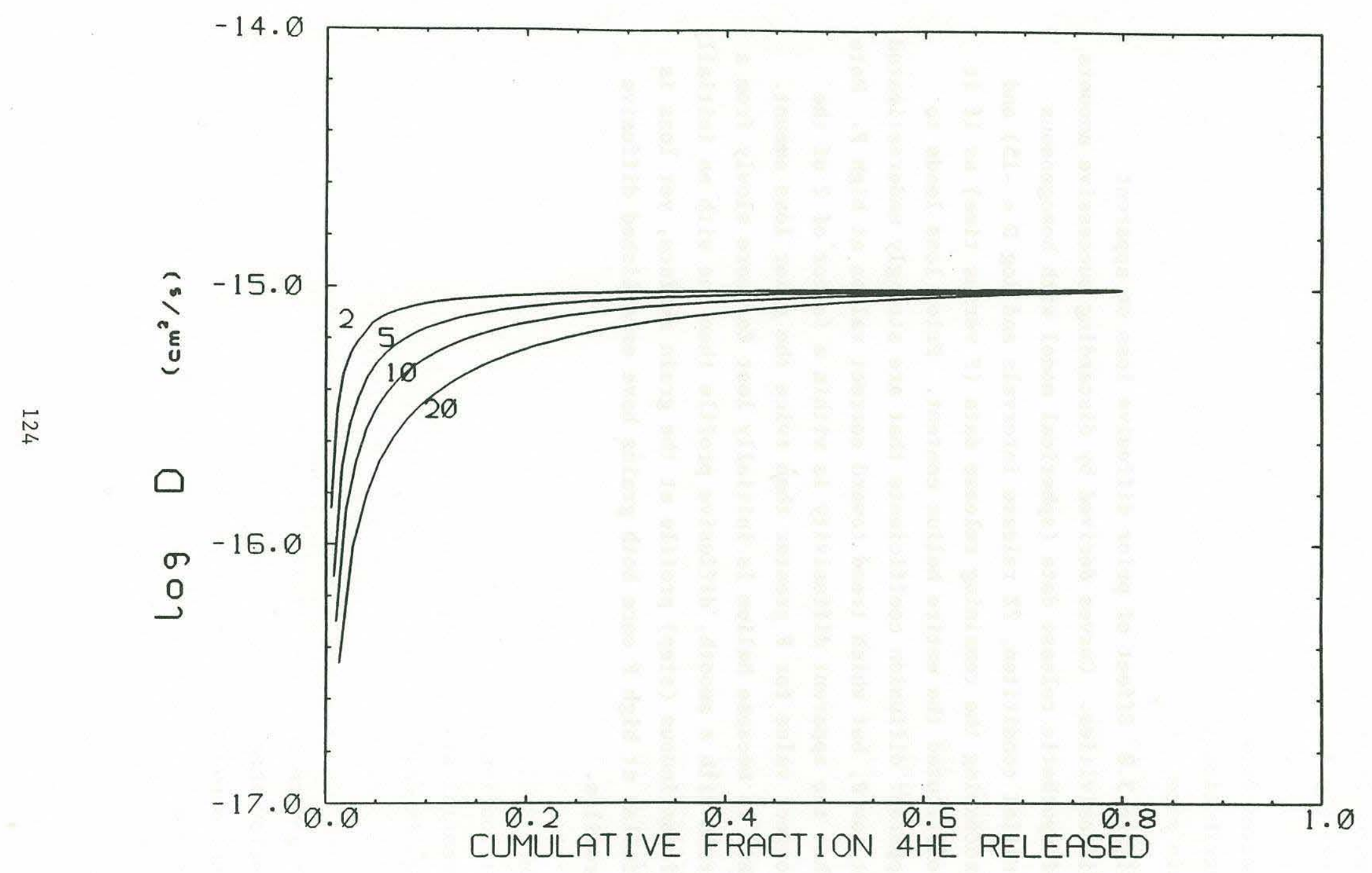

承 
distributions are broad or include rare grains larger than the average size ( $\mathrm{D} / \mathrm{a}^{2}$ vs. $\mathrm{F}$ trends for several model distributions are displayed in chapter 2, figure 2.9). The decrease in apparent diffusivities occurs because the larger grains lose their helium more slowly and because relatively few of them contribute to the gas loss. Thus it is important to realize that $\mathrm{D} / \mathrm{a}^{2}$ values can be underestimated well beyond the error expected from the overall range in sizes, because it is the helium mass distribution that controls emanation rates and not just the spread in grain size. For this reason, conversion of $\mathrm{D} / \mathrm{a}^{2}$ values to $\mathrm{D}$ values involves a choice for "a" that is not clear from the size range alone, and without knowledge of the mass distribution large errors can result since $D$ varies with $a^{2}$. The effects of grain size distribution have been discussed previously in regard to reaction rate determinations (Gallagher, 1965) and noble gas emanation experiments (e.g. Huneke et al, 1969; Abe, Rauch and Brandt, 1971).

Examining grain size histograms for the experiments (figure 3.7) suggests that most of the decreases in $\mathrm{D} / \mathrm{a}^{2}$ values at high $\mathrm{F}(>.8)$ reflect size distribution effects not accounted for by the standard model. The effect is nearly absent in the single grain experiment (figure 3.7, \#1), and in experiment \#3 which used only two glass chips of very similar size and shape. Experiments \#8 and \#10 have broad, decreasing $\mathrm{D} / \mathrm{a}^{2}$ trends at high $\mathrm{F}$ (above .7 or so) which reflect wide distribution in the short side lengths of these grains (three grain size parameters were measured for each grain in the chip experiments - the shortest side, the "average" side, and the longest side, as shown in figure 3.7). Experiment \#8 exemplifies the fact that helium mass distribution governs loss in that $\mathrm{D} / \mathrm{a}^{2}$ drops by an order of magnitude in sequential aliquots at $414^{\circ} \mathrm{C}$ although the overall short side length range is only a factor of 2 . The decrease of $\mathrm{D} / \mathrm{a}^{2}$ in experiment \#7 $\left(617^{\circ} \mathrm{C}\right.$ data) occurs only for $\mathrm{F}$ in excess of $90 \%$, consistent with a tighter clustering of short and average side lengths, which in large part govern loss since they represent more direct routes to the grain exterior than the long side length. The powder experiments (\# 5 and \#6) do not show strong $\mathrm{D} / \mathrm{a}^{2}$ decreases at high $\mathrm{F}$ reflecting their narrow size ranges. However, not all variations agree well with the 
histograms, for example $\mathrm{D} / \mathrm{a}^{2}$ values do not vary strongly in expt. \#2 despite similar size distribution to \#8, and the strongly bimodal distribution required to explain the decrease in $\mathrm{D} / \mathrm{a}^{2}$ at $329^{\circ} \mathrm{C}$ in expt. \#9 which does not continue at higher temperature is not evident in the histogram.

In summary, use of the overly simple standard model can produce large errors in calculated diffusivities at both low and high extents of gas release, because of prior loss and grain size and shape distribution effects. These errors can be recognized if many sequential aliquots are measured in emanation experiments. We now address these problems with a refined model.

\subsubsection{A Refined Model for Diffusion Coefficient Calculation}

Because one of the goals of this research was to accurately determine the low temperature diffusivity of $\mathrm{He}$, a refined model incorporating grain size and shape variations and correcting for prior loss was used. As already discussed, prior helium loss can be recognized in multiple release experiments from the presence of increasing apparent diffusivities at constant temperature, especially when this behavior occurs at low extents of gas loss but does not continue at higher $\mathrm{F}$. When multiple aliquots are collected, a minimum estimate of the extent of prior loss can be made in two ways. If isothermal aliquots were collected over a large $\mathrm{F}$ range, the $\mathrm{F}$ value at which standard model diffusivites stop increasing and remain constant is a rough minimum (and also a rough maximum) estimate for prior loss, as revealed by the model calculations displayed in figure 3.8. Secondly, diffusivities can be recalculated assuming progressively larger extents of loss until constant values are obtained for constant temperature. This approach is illustrated for the data from powder experiment \#5 and suggests a prior loss of about $4.5 \%$ (figure 3.9). Correcting the room temperature diffusivities in this experiment for this loss leads to a $\mathrm{D} / \mathrm{a}^{2}$ value of about $5 \times 10^{-11} \mathrm{~cm}^{2} / \mathrm{s}$ as opposed to values of $5 \times 10^{-13}$ to $5 \times 10^{-12}$ without correction. However, without a maximum constraint on prior loss it is possible the diffusivity is even higher. 
Figure 3.9 Successive corrections (subplots a-f) for prior helium loss in expt. \#5 increase and coalesce apparent diffusion coefficients at low $F$, leading to relatively constant values of $\mathrm{D} / \mathrm{a}^{2}$ within temperature plateaus. Temperature ramp steps are shown by filled symbols. The dotted lines at $D=10^{-11} \mathrm{~cm}^{2} / \mathrm{s}$ are a visual reference to reveal that apparent room temperature diffusivities (RT) increase with the extent of prior loss correction. 
Figure 3.9

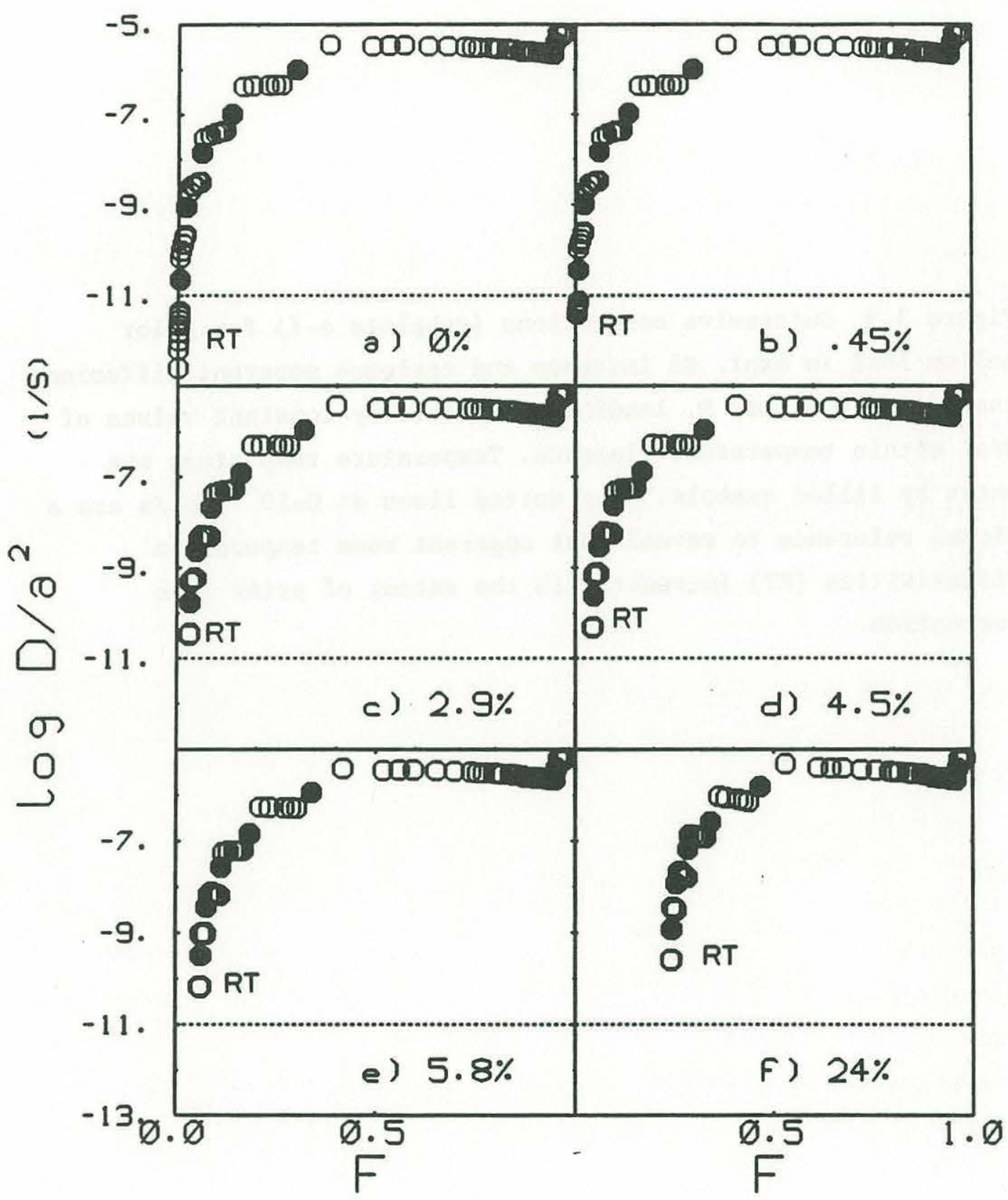


The relationship between extent of prior loss correction and apparent room temperature diffusivity is shown in figure 3.10. This figure also shows that prior loss corrections have an effect on the estimation of thermal activation energies (discussed further below). Fortunately, as discussed earlier, precise estimates of the extent of prior loss can be made for this experiment and for expt.\#6, from the linear relations between $F$ and $t^{1 / 2}$ observed at room temperature (the method is illustrated graphically in figure 3.10). These prior loss values are a few percent higher than suggested by the minimum estimation method (e.g. 5.8\% instead of $4.5 \%$ for expt. \#5). Corrected diffusivities for each aliquot in these experiments were then calculated by incorporating the prior loss amount as a first aliquot. In the other experiments, where it was not possible to accurately correct for prior loss (e.g. \#2 and \#3), aliquots which showed $D / a^{2}$ vs. F trends indicative of prior loss were discounted in obtaining diffusivities and activation energies. Considering the powder experiments as a case study, it is clear that prior loss can lead to highly understimated diffusivites and incorrect activation energies. Thus we believe it is essential to check for this problem by measuring multiple aliquots, particularly in experiments which use natural grain sizes from older rocks where helium loss may have occured over many years.

In addition to making prior loss corrections, the refined model accounts for a distribution of sizes and shapes by treating the grains as collections of parallelpipeds. In the chip experiments, three size parameters were used for each grain, the short side length, "average" grain width, and longest side length, determined by microscopically examining the grains with a size-calibrated grid. For the powders only the average grain width was determined (for 100 randomly selected particles) and the side lengths were assumed to be in the ratio $1,1,1.45$ based on visual examination of about 20 randomly selected grains. The size parameters are given along with the emanation data in appendix A, and are displayed as histograms in figure 3.7. The errors in measuring the lengths were about $10 \%$. The grain shapes are not actually parallelpipeds, of course, but this representation does account for major variations in both sizes and shapes and is particularly reasonable 
Figure 3.10 Effect of unrecognized prior loss on apparent diffusion coefficients and activation energies, calculated for experiment \#5 using the spherical model. The inset shows the method used to determine the extent of prior loss by extrapolating release trends at room temperature to the time of sample preparation (see also figure 3.6). 
Figure 3.10

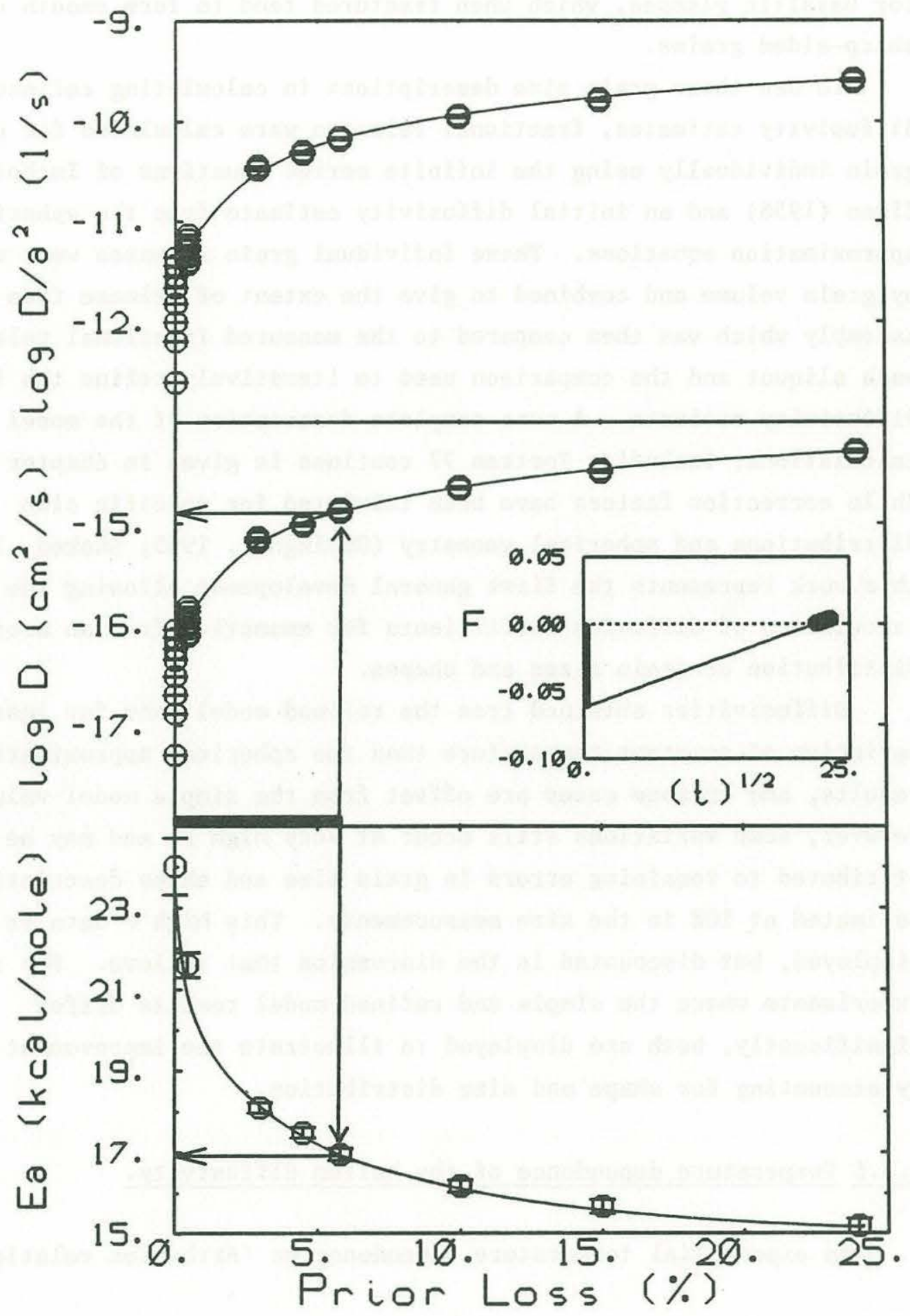


for basaltic glasses, which when fractured tend to form smooth surfaced, sharp-sided grains.

To use these grain size descriptions in calculating refined diffusivity estimates, fractional releases were calculated for each grain individually using the infinite series equations of Inthoff and Zimen (1956) and an initial diffusivity estimate from the spherical approximation equations. These individual grain releases were weighted by grain volume and combined to give the extent of release from the assembly which was then compared to the measured fractional release in each aliquot and the comparison used to iteratively refine the initial diffusivity estimate. A more complete description of the model calculations, including Fortran 77 routines is given in chapter 2 . While correction factors have been tabulated for specific size distributions and spherical geometry (Gallagher, 1965; Shaked, 1965), this work represents the first general development allowing the calculation of diffusion coefficients for emanation from an arbitrary distribution of grain sizes and shapes.

Diffusivities obtained from the refined.model show far less variation at constant temperature than the spherical approximation results, and in some cases are offset from the simple model values. However, some variations still occur at very high $\mathrm{F}$, and may be attributed to remaining errors in grain size and shape description, estimated at $10 \%$ in the size measurements. This high $\mathrm{F}$ data is displayed, but discounted in the discussion that follows. For those experiments where the simple and refined model results differ significantly, both are displayed to illustrate the improvement provided by accounting for shape and size distribution.

\subsubsection{Temperature dependence of the helium diffusivity.}

An exponential temperature dependence or 'Arrhenius relation' :

$$
\mathrm{D}=\mathrm{D}^{\mathrm{O}} \exp \left(-\mathrm{E}_{\mathrm{a}} / \mathrm{RT}\right)
$$

where $\mathrm{D}^{\mathrm{O}}$ and $\mathrm{E}_{\mathrm{a}}$ are constants independent of temperature, is often used to characterize diffusion processes. In some instances this may be an 
oversimplification of the true temperature dependence, but examining the data with an Arrhenius plot of $\log _{10}$ versus $1 / \mathrm{T}\left({ }^{\circ} \mathrm{K}\right)$ is a good way to determine an effective activation energy, Ea, and any deviations from this simple relation.

The three Alv892-1a glass chip experiments at 200 to $600^{\circ} \mathrm{C}$ exhibit high linearity in Arrhenius diagrams (figure 3.11). The slopes are very similar (figure 3.14) and imply activation energies ranging from 17.1 to $18.1 \mathrm{Kcal} / \mathrm{mole}$ with errors of less than $2 \%$ (table 3.4 ). As expected, the refined model suggests greater temperature dependence than the simple model ( 16.6 to $17.9 \mathrm{Kcal} / \mathrm{mole}$; table 3.4 ) because it accounts for the emanation decrease from grain exhaustion at high $F$, where the high temperature data was obtained. Comparing the results of the simple and refined model for experiment \#1 which used a single glass chip (17.4 and $18.1 \mathrm{Kcal} / \mathrm{mole}$, respectively) demonstrates the importance of correct shape description as well as of grain size distribution. The remaining errors in describing exact grain shapes and sizes will have the effect of reducing the apparent activation energy, so that even the refined model values must be considered minima. There was no discernible effect of variolitic texture on activation energy Ea (expt. \#3), suggesting that the presence of unconnected varioles in a glass sample does not enhance helium loss. The variation in $D^{\circ}$ values for these experiments (table 3.4) primarily reflects the different Ea values, as the diffusivities are essentially overlapping (figure 3.14).

Diffusivities and the activation energy $(17.4 \pm .5 \mathrm{Kcal} / \mathrm{mole}$ ) for the other tholeitte, Charco98-11T, are very similar to the Alv892-1a results (figures $3.13 \mathrm{a}, 3.14 \mathrm{~b}$ and table 3.4 ). The agreement between the two tholeiites is strong evidence that the measured helium loss rates are fundamental properties of the material, and not the result of extrinsic factors such as cracking or thermal history. Further, the difference in internal helium distribution between the two tholeiites (table 3.2a) did not affect release, suggesting that dissolution at vesicle walls is not a limiting process.

Arrhenius plots of the Alv892-1a powder experiments are not linear until corrected for prior loss (figure 3.12) and derived activation energies can be in considerable error if this problem goes unrecognized 
Figure 3.11 Arrhenius plots for the Alv892-1a tholeiite glass chip experiments. One sigma errors are smaller than the symbols: circles for the spherical model, and diamonds for the refined model. Activation energies corresponding to these regression lines are given in table 3.4 The lowest temperature data in experiment \#2, obtained at very low $\mathrm{F}$ and affected by prior loss, were not used to obtain the regression line. 
Figure 3.11

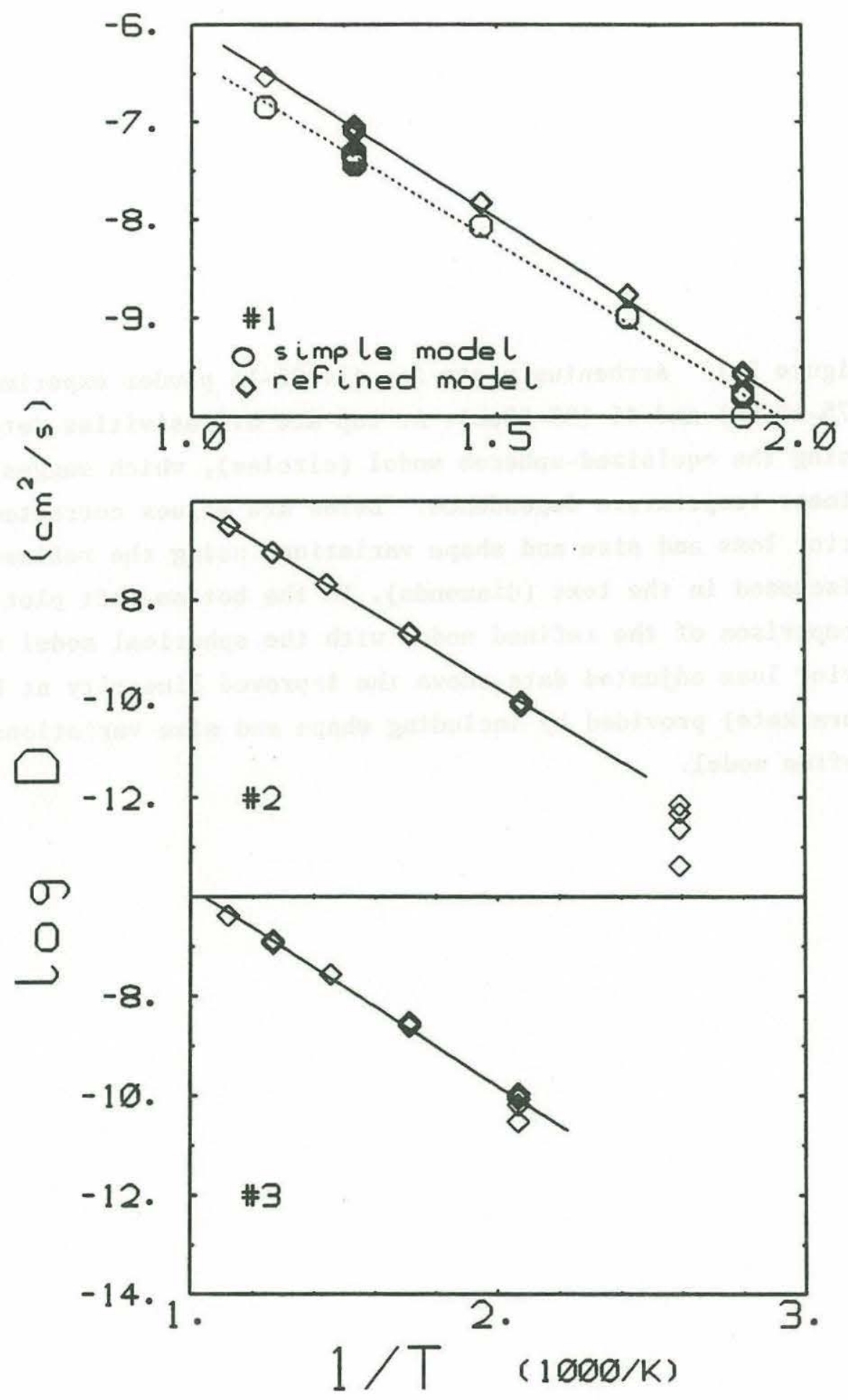


Figure 3.12 Arrhenius plots for Alv892-1a powder experiments \#5 (75-106um) and \#6 (38-53um). At top are diffusivities determined using the equisized-spheres model (circles), which suggest nonlinear temperature dependence. Below are values corrected for prior loss and size and shape variations using the refined model discussed in the text (diamonds). In the bottom-left plot, a comparison of the refined model with the spherical model using prior loss adjusted data shows the improved linearity at high $\mathrm{F}$ (brackets) provided by including shape and size variations in the refine model. 
Figure 3.12

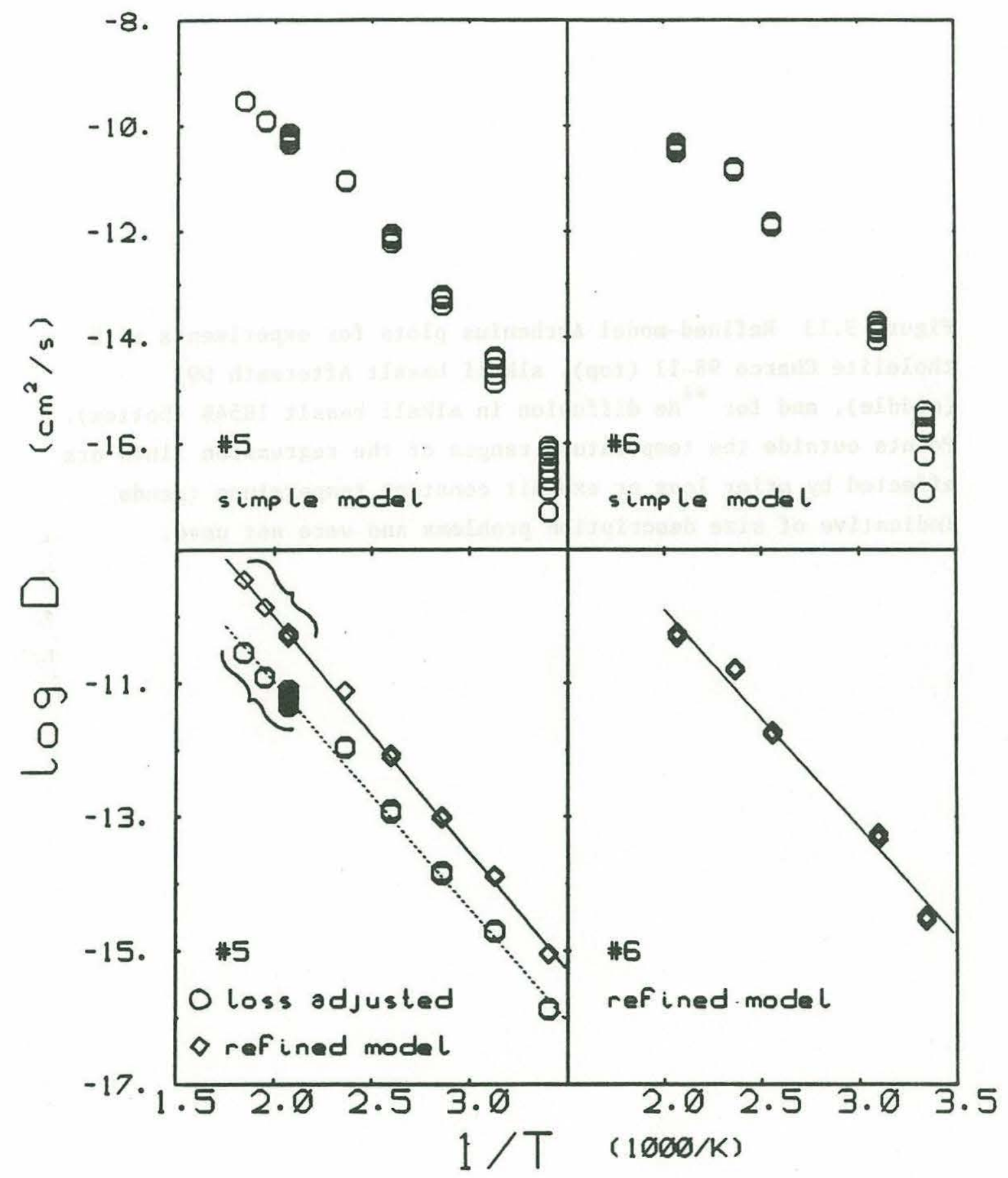


Figure 3.13 Refined-model Arrhenius plots for experiments with tholeiite Charco 98-11 (top), alkali basalt Aftermath D9 (middle), and for ${ }^{* 4}$ He diffusion in alkali basalt $1854 \mathrm{~B}$ (bottom). Points outside the temperature ranges of the regression lines are affected by prior loss or exhibit constant temperature trends indicative of size description problems and were not used. 
Figure 3.13

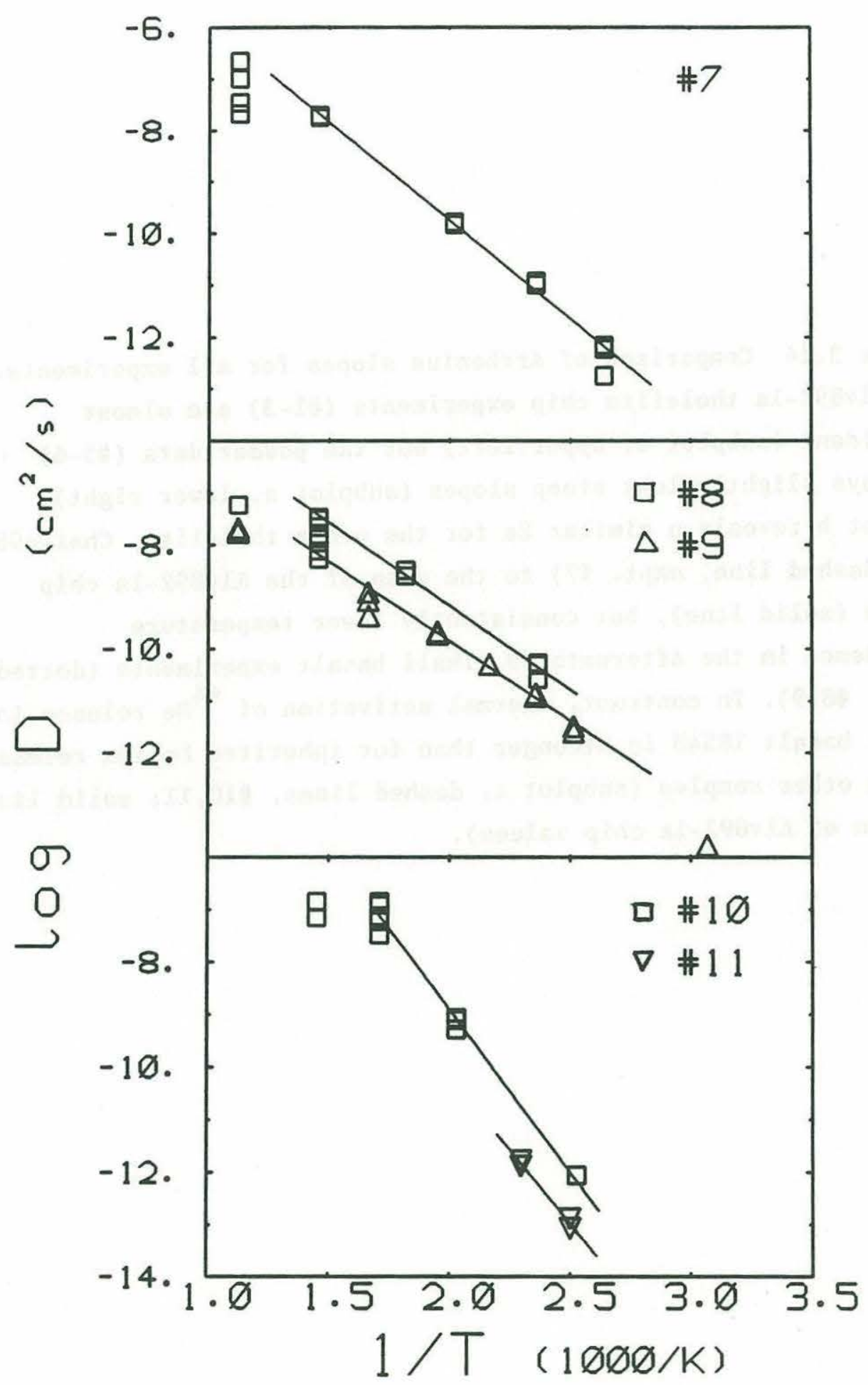


Figure 3.14 Comparison of Arrhenius slopes for all experiments. The Alv892-1a tholeiite chip experiments (\#1-3) are almost coincident (subplot a, upper left) but the powder data (\#5-6) displays slightly less steep slopes (subplot a, lower right). Subplot b reveals a similar Ea for the other tholeiite, Charco98$11 \mathrm{~T}$ (dashed line, expt. \#7) to the mean of the Alv892-1a chip slopes (solid line), but consistently lower temperature dependence in the Aftermath D9 alkali basalt experiments (dotted lines, $\# 8,9)$. In contrast, thermal activation of ${ }^{\star 4} \mathrm{He}$ release in alkali basalt $1854 \mathrm{~B}$ is stronger than for inherited helium release in the other samples (subplot c, dashed lines, \#10,11; solid line is mean of Alv892-1a chip values). 
Figure 3.14

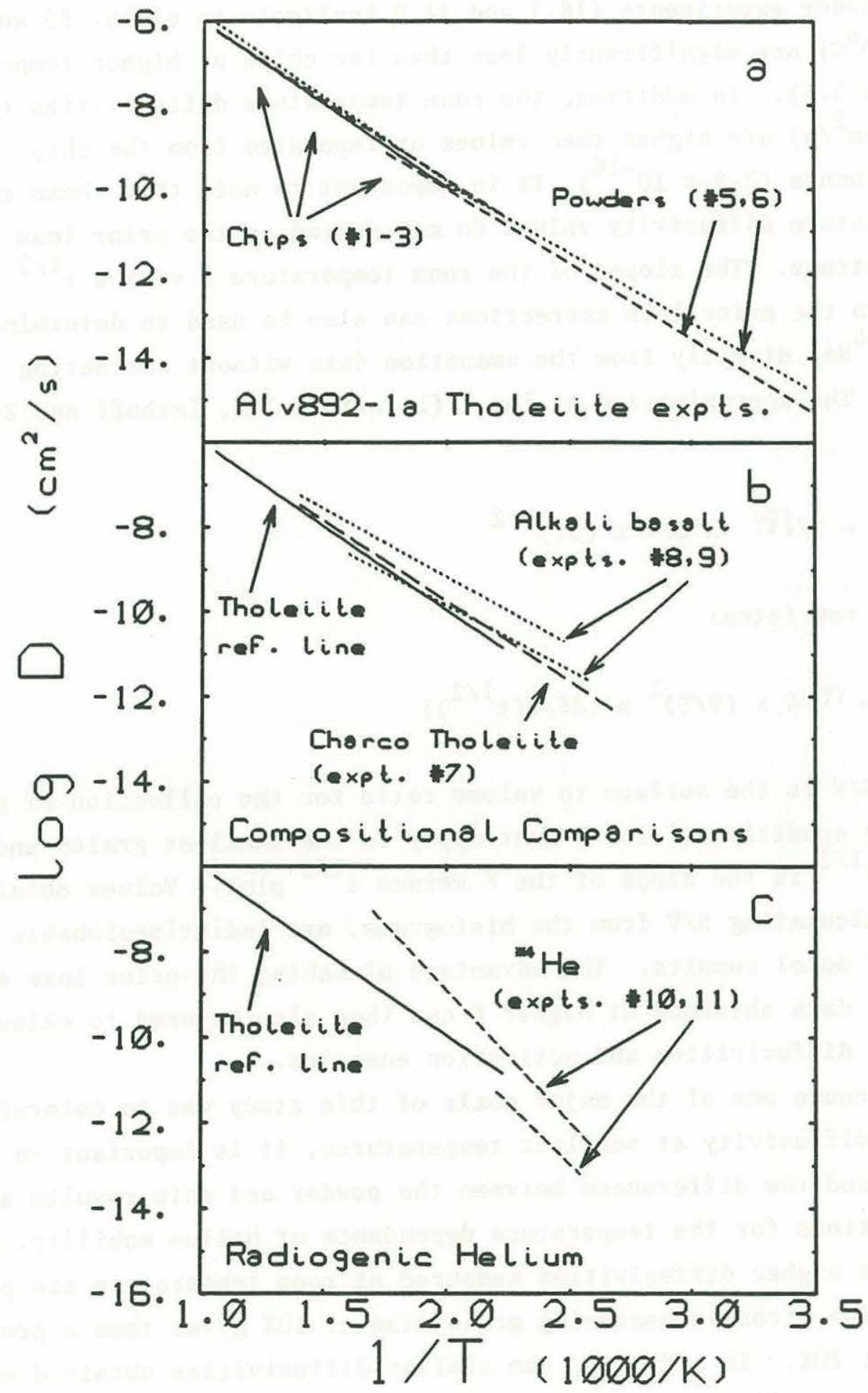


(figure 3.10). When prior loss corrections are made, the Ea values for the powder experiments (16.1 and $14.9 \mathrm{kcal} / \mathrm{mole}$ in expts. \#5 and \#6 at $20-200^{\circ} \mathrm{C}$ ) are significantly less than for chips at higher temperature (table 3.4 ). In addition, the room temperature diffusivities (1-4 $\mathrm{x}$ $10^{-15} \mathrm{~cm}^{2} / \mathrm{s}$ ) are higher than values extrapolated from the chip experiments $\left(3-9 \times 10^{-16}\right)$. It is important to note that these room temperature diffusivity values do not depend on the prior loss corrections. The slopes of the room temperature $F$ versus $t^{1 / 2}$ relations used in the prior loss corrections can also be used to determine D (and $D^{3} \mathrm{He} / \mathrm{D}^{4} \mathrm{He}$ ) directly from the emanation data without correcting for prior loss. The approximation at low $\mathrm{F}$ (less than $10 \%$, Inthoff and Zimen, 1956):

$$
\mathrm{F}=2 / \sqrt{\pi} \times \mathrm{S} / \mathrm{V} \times(\mathrm{Dt})^{1 / 2}
$$

can be rewritten:

$$
D=\pi / 4 \times(V / S)^{2} \times\left\{d F / d\left(t^{1 / 2}\right)\right\}
$$

where $S / V$ is the surface to volume ratio for the collection of grains and the condition of low $\mathrm{F}$ must apply to the smallest grains and $\mathrm{dF} / \mathrm{d}(\mathrm{t})^{1 / 2}$ is the slope of the $\mathrm{F}$ versus $t^{1 / 2}$ plot. Values obtained this way, calculating $\mathrm{S} / \mathrm{V}$ from the histograms, are indistinguishable from the refined model results. The advantage of making the prior loss estimate is that data obtained at higher $\mathrm{F}$ can then also be used to calculate correct diffusivities and activation energies.

Because one of the major goals of this study was to determine the helium diffusivity at seafloor temperatures, it is important to understand the differences between the powder and chip results and their implications for the temperature dependence of helium mobility. First of all, the higher diffusivities measured at room temperature are probably real. The error in measuring grain size of $10 \%$ gives them a precision of about $20 \%$. In addition, the similar diffusivities obtained near $200^{\circ} \mathrm{C}$ for a wide range of grain sizes (experiments $1-7$ ) suggest that the diffusion length scale corresponds to the physical grain size, and thus 
that any effects of powder preparation, such as generation of internal cracks, are small. This is consistent with the optical and SEM appearance of the powder particles which showed no deep fracturing or particles finer than the sieve range, and with surface area measurements using the nitrogen condensation method (Brunnauer, Emmett and Teller, 1938) which gave values in good agreement with measured grain sizes (unpublished results). Therefore, the diffusivities are probably accurate as well, although differences between the powders throughout the $20-200^{\circ} \mathrm{C}$ range, as well as between all experiments in the vicinity of $200^{\circ} \mathrm{C}$, allow variations of a factor of 2 to 3 (see figure 3.15 ). The powder activation energies rely on the prior loss corrections, but these are quite precise (table 3.4) and in accuracy are likely to err on the low side (because of the nature of the $\mathrm{F}$ vs. $\mathrm{t}^{1 / 2}$ approximation) so that the activation energies are effectively maxima. This and the fact that both powder experiments have lower activation energies than the chip experiments suggests the difference in activation energies reflects the different temperature ranges of the experiments, powders below $200^{\circ} \mathrm{C}$ and chips above $200^{\circ} \mathrm{C}$, rather than a dependence of helium mobility on grain size.

Synthetic glass studies have also found that helium diffusion activation energies increase slightly with temperature (Shelby and Keeton, 1974). These workers suggested this could result from the noncrystalline nature of glass structure, and showed that a Gaussian distribution of activation energies could account for the observed temperature-dependent activation according to:

$$
D=D^{0} e^{(-u / R T)} e^{\left(s^{2} / 4 R^{2} T^{2}\right)}
$$

where $s$ is the standard deviation of a normal distribution of activation energies with mean $u$. However, transition-state models assuming a single activation energy also allow for varying temperature dependence of the form:

$$
D=D^{o} T^{n} e^{(-E a / R T)}
$$


Depending on the assumptions made about solute atom motions in the transition state, $\mathrm{n}$ can take on the values of 1 (high frequency vibrations), $1 / 2$ (translational motions), or 0 (low frequency vibrations) corresponding to the Arrhenius relation (e.g. Stearn and Eyring, 1940).

In an effort to investigate these different diffusion models, data from all the tholeiite experiments (\#1-7) were combined (228 points after culling values affected by prior loss or size distribution problems) and examined for a best description of their temperature dependence by $\mathrm{chi}^{2}$ minimization (Bevington, 1969). In terms of minimizing $\mathrm{chi}^{2}$ values and producing temperature-independent scatter of residuals, none of the more complex expressions provided a better fit to the data than a simple Arrhenius exponential with Ea of $16.85 \pm .13$ and $\log \mathrm{D}^{0}$ of $-2.37 \pm .06$. However, this Arrhenius expression cannot incorporate the lower activation energies observed below $200^{\circ} \mathrm{C}$ in the powder experiments (less than $16 \mathrm{Kcal} / \mathrm{mole}$ ). In this regard, the expressions with a non-zero $\mathrm{T}^{\mathrm{n}}$ term also were unable to provide as large a variation in apparent $\mathrm{Ea}$ as observed in the experiments, and therefore offered no particular advantage over the Arrhenius relation. The function incorporating a distribution of activation energies could account for the increase of apparent activation energies (Arrhenius $\mathrm{Ea}$ values) from 15 to $16 \mathrm{Kcal}$ at less than $200^{\circ} \mathrm{C}$ to 17 to $18 \mathrm{kcal}$ above $200^{\circ} \mathrm{C}$ (table 3.4 ), provided the normal distribution was characterized by a mean $(\mathrm{u})$ in the range of $20-21 \mathrm{Kcal} / \mathrm{mole}$ and a standard deviation (s) of 2.6 to $2.8 \mathrm{Kcal} / \mathrm{mole}$. These expressions gave slightly higher $\mathrm{chi}^{2}$ values in fitting the collected data than did the simple Arrhenius line. However, because the fitting errors are dominated by grain size errors, which affect the comparison of absolute diffusivities from different experiments to a far greater extent than they affect activation energies derived within single experiments, the ability to account for Ea values which vary with temperature is an important criterion in selecting a temperature-dependence equation. The distribution of activation energies model does the best job of incorporating this aspect of the data (a curve for $u=20.5$ and $s=2.8$ is shown along with the best Arrhenius fit in figure 3.15). However, it must be emphasized that 
Figure 3.15 Temperature dependence of helium diffusion in tholeiitic glass. Open circles- data combined from experiments \#1-7.; solid hourglass - storage results; solid circles - results of Jambon, Weber and Begemann (1985). The dotted line shows the Arrhenius temperature dependence ( $E a=19.9 \pm 1.0 \mathrm{Kcal} / \mathrm{mole}$ ) determined by Kurz and Jenkins (1981). The solid line is a least-square Arrhenius relation to the combined data from expts. $\# 1-7$ and yields Ea=16.85+.13 Kcal/mole. The dashed line is the temperature dependence predicted for a Gaussian distribution of activation energies with mean of $20.5 \mathrm{Kcal} / \mathrm{mole}$ and $2.8 \mathrm{Kcal} / \mathrm{mole}$ standard deviation. 
Figure 3.15

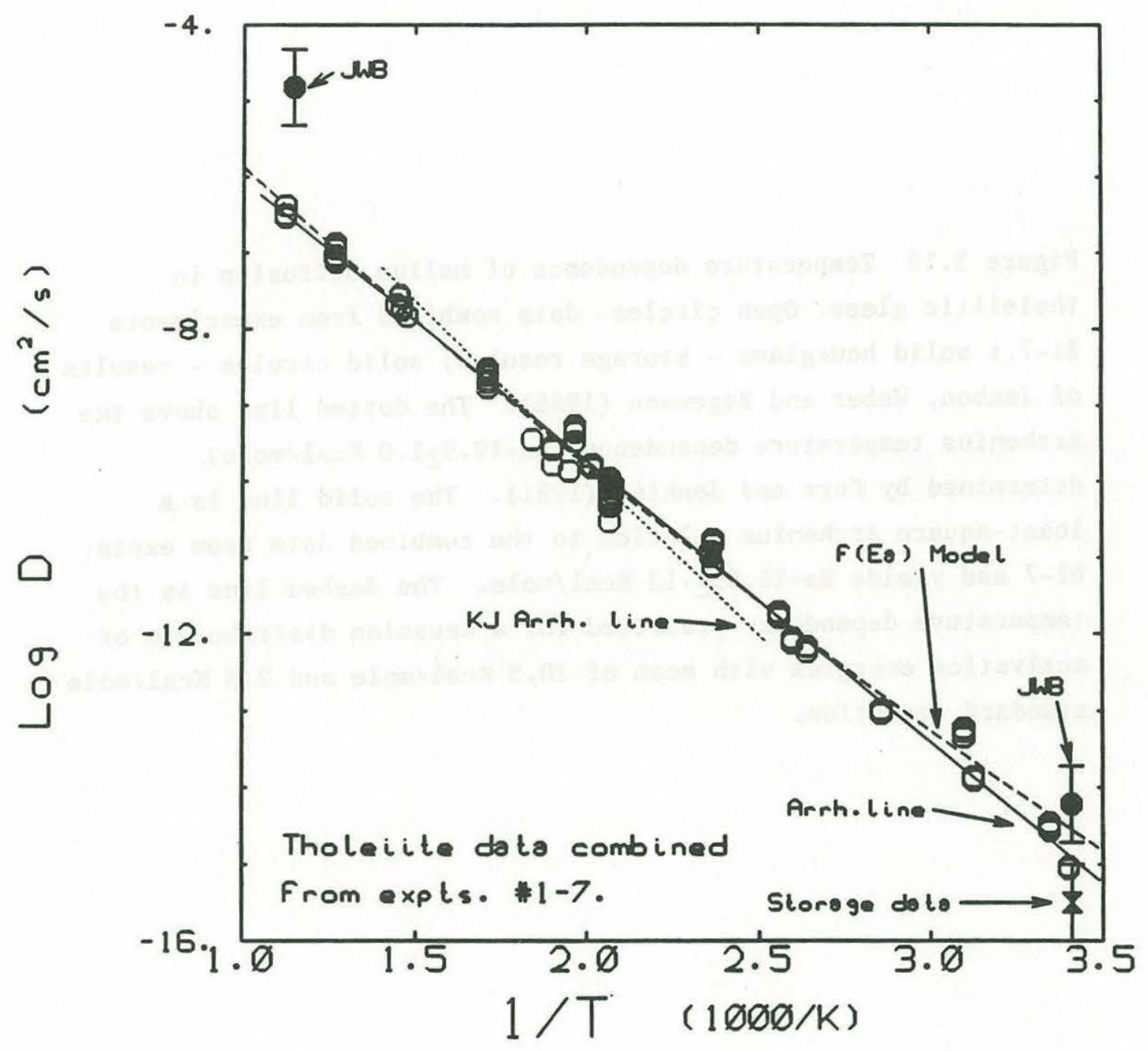


given the experimental errors it cannot be confidantly asserted that helium diffusion in basaltic glass must be characterized by a nonArrhenius temperature dependence.

In principle, the distribution of activation energies model could be investigated further by measurements at higher temperature. It is also possible that the low temperature helium diffusivity departs from Arrhenius behavior for other reasons, such as contributions from more than one atomic mechanism for example extrinsic defects may be relatively important at low temperature but not at high temperature (e.g. Reynolds, 1960). In the discussion that follows, both the bestfit Arrhenius function and the distribution model are considered in extrapolating the measurements to different temperatures.

The results of experiments \#5 and \#6 represent the lowest temperatures, $21^{\circ} \mathrm{C}$ and $26^{\circ} \mathrm{C}$, for which helium emanation from basaltic glass has been measured. The diffusivities at these temperatures are $9.0_{ \pm} .3 \times 10^{-16}$ and $21 \pm 4 \times 10^{-16} \mathrm{~cm}^{2} / \mathrm{s}$ respectively (Appendix A data tables \#5 and \#6). These values are in good agreement with the $20^{\circ} \mathrm{C}$ diffusivities obtained by the overall Arrhenius fit to the 7 tholeiite experiments $\left(12 \pm 2.5 \times 10^{-16} \mathrm{~cm}^{2} / \mathrm{s}\right)$ and the distribution of activation energies model $\left(30 \pm 10 \times 10^{-16} \mathrm{~cm}^{2} / \mathrm{s}\right.$; table 3.5$)$. In comparison to the "room temperature" storage results of Jambon, Weber and Begemann (1985), $60 \times 10^{-16} \mathrm{~cm}^{2} / \mathrm{s}$, the diffusivities obtained here are lower, but within the factor of 3 error bounds reported by those workers. The difference is consistent with the already discussed observation that storage results are likely to be biased toward high values because of experimental shortcomings.

The new diffusivity estimates are much higher than the value of $1.6 \times 10^{-16} \mathrm{~cm}^{2} / \mathrm{s}$ at $20^{\circ} \mathrm{C}$ implied by extrapolating the Arrhenius line obtained above $200{ }^{\circ} \mathrm{C}$ by Kurz and Jenkins (1981). In order to understand this difference, their data (published in Kurz, 1982) was reexamined. Evidence for prior loss was found in two of their 90 and 160um powder experiments which exhibited increasing apparent D values at constant temperature at low F. In addition, these workers combined D values from several experiments with different grain sizes to obtain an activation energy, a procedure which (as already discussed) introduces 
grain size measurement errors into the $\mathrm{E}_{\mathrm{a}}$ determination. Discounting the prior loss compromised data and recalculating activation energies on an individual experiment basis leads to a mean $\mathrm{E}_{\mathrm{a}}$ of $17.8 \pm .9 \mathrm{Kcal} / \mathrm{mole}$ (very similar to the results obtained for chips at elevated temperatures here, table 3.4 ). It should also be pointed out that propagating the reported error of $1 \mathrm{Kcal} / \mathrm{mole}$ in the original work to $20^{\circ} \mathrm{C}$ leads to a factor of 6 error in D, so that the apparently large difference between D values reported by Kurz and Jenkins (1981) and Jambon, Weber and Begemann (1985) was not as significant as initially suggested. To summarize the new low temperature results and their relation to the previous studies, it can be said that unconsidered errors in the previous studies lead to diffusivity estimates which appeared to be radically different but actually were not given the real errors involved, and that the true low temperature helium diffusivity lies between the previous results.

Extending the new results to seafloor temperatures $\left(0^{\circ} \mathrm{C}\right)$ suggests values of a few times $10^{-16} \mathrm{~cm}^{2} / \mathrm{s}$ for tholeitic glass, with the precise value depending on the temperature relation chosen. Extrapolating the powder Arrhenius fits (epxts. 5 and 6 ) suggests a one sigma range of 1 $8 \times 10^{-16}$, the overall Arrhenius fit to experiments $1-7$ suggests $1.4 \pm \cdot 3 \times 10^{-16}$, and the distribution of activation energies model implies $5 \pm 2 \times 10^{-16}$ (table 3.5 ). These variations can be taken as representative of the errors in characterizing the temperature dependence of helium diffusion. Note that all values are an order of magnitude higher than the value $\left(1 \times 10^{-17} \mathrm{~cm}^{2} / \mathrm{s}\right)$ suggested by Kurz and Jenkins (1981). The results can also be extrapolated to magmatic temperatures. The results for a single chip at $200-600^{\circ} \mathrm{C}$ (expt. \#1) extrapolate to $1.2 \times 10^{-5} \mathrm{~cm}^{2} / \mathrm{s}$ at $1100^{\circ} \mathrm{C}$, the overall Arrhenius relation (expts. \#1-7) yields $9.2 \pm .5 \times 10^{-6}$ at $1100^{\circ} \mathrm{C}$ and $2.4 \pm .6 \times 10^{-5} \mathrm{~cm}^{2} / \mathrm{s}$ at $1350^{\circ} \mathrm{C}$. These values are very similar to the diffusivity of $5 \times 10^{-5} \mathrm{~cm}^{2} / \mathrm{s}$ measured for tholeiitic melt at $1350^{\circ} \mathrm{C}$ by Lux (1987). In contrast, the distribution of activation energies model suggests a diffusivity at this temperature of a few times $10^{-2} \mathrm{~cm}^{2} / \mathrm{s}$, implying that this model can not hold at higher temperatures (above the glass transition). 
Table 3.5 Helium diffusivity estimates for basaltic glass.

\begin{tabular}{|c|c|c|c|c|}
\hline Experiment & $\log$ Do & $\mathrm{Ea}$ & $\mathrm{D}\left(20^{\circ} \mathrm{C}\right)$ & $\mathrm{D}\left(0^{\circ} \mathrm{C}\right)$ \\
\hline & $\left(\mathrm{cm}^{2} / \mathrm{s}\right)$ & (Kcal/mole) & $\left(10^{-16}\right.$ & $\left.\mathrm{cm}^{2} / \mathrm{s}\right)$ \\
\hline
\end{tabular}

Tholeiitic basalt:

Emanation experiments at $20-600^{\circ} \mathrm{C}(\# 1-7)$ :

Overall Arrhenius line $\quad-2.37 \pm .06 \quad 16.85_{ \pm} .13 \quad 12 \pm 2.5 \quad 1.4 \pm .3$

Ea Distribution model
$\begin{array}{llll}\text { (mean u, stand. deviation } s \text { ) } & -1.5 \pm .15 \mathrm{u}=20.5 \pm .5 & 30 \pm 10 & 5 \pm 2 \\ \mathrm{~s}=2.8 & \pm .3\end{array}$

Diffusivities measured by

emanation at room temperature: $\left(21^{\circ} \mathrm{C}\right.$, expt. 5) $8-9$

(26 ${ }^{\circ} \mathrm{C}$, expt. 6) 23-35

Diffusivities measured by

storage at room temperature:

Uncorrected

Corrected for crushing losses

$10-100$

$1-10$

Alkali basalt $\left(200-600^{\circ} \mathrm{C}\right) \quad-3.24 \pm .20 \quad 14.4 \pm .5 \quad 150(\mathrm{x} 2)^{\mathrm{a}} \quad 20(\mathrm{x} 2)$

Radiogenic helium in

alkali basalt $\left(200-600^{\circ} \mathrm{C}\right)+2.4 \pm 1.0 \quad 27 \pm 2 \quad .03(x 5) .003(x 10)$

Kurz and Jenkins (1981) $-1.2(.3) \quad 19.9(1.0) \quad 1.6(x 6) \quad 0.1(x 7)$

Tholeitic basalt emanation data at $150-600^{\circ} \mathrm{C}$. The low temperature diffusivities are imprecise because of the large temperature extrapolation. Errors associated with prior loss explain may explain the relatively high Ea (see text).

Jambon, Weber and

$\begin{array}{llllll}\text { Begemann (1985) } & {[+0.1(.8)]} & 19.2[(1)] & 60(\mathrm{x} 3) & {[5(\mathrm{x} 14)]}\end{array}$

Tholeiitic basalt room temperature storage data and a single loss estimate at $600^{\circ} \mathrm{C}$. Values in square brackets estimated from data in original work. Initial losses related to crushing may account for the relatively high diffusivity at room temperature (see text). 


\subsubsection{Compositional effects on helium mobility}

Relative to tholeiitic glass, alkali basalt (Aftermath D9) appears to have significantly lower activation energy (14-15 Kcal/mole vs. 17$18 \mathrm{Kcal} / \mathrm{mole}$ at $200-600^{\circ} \mathrm{C}$ ), although both experiments with this sample exhibited scatter in their Arrhenius plots (figure 13b) and have correspondingly imprecise activation energies (table 3.4). However, the good agreement between the two experiments suggests the difference is indeed real. The alkali basalt results imply considerably higher diffusivities than for tholeiitic basalt glass when extrapolated to room and seafloor temperatures (table 3.5). Understanding which components of basaltic glass contribute to this difference is important to extending the results obtained here to other samples, for example, andesitic glasses in island arc environments (chapter 6).

Large compositional effects on noble gas mobility in synthetic silicate glasses are well recognized (e.g Doremus, 1973). Studies with several binary systems have shown that helium diffusivities generally increase (and activation energies decrease) with increasing mole percent silica (Shelby, 1973). These and other results have been interpreted as the relative effect of network formation by silica (which increases the partial molar volume of the glass, thereby providing passageways for interstitial diffusion), and network modification by other species which depolymerize the structure, thereby increasing glass density and blocking diffusive channels. This model can explain the much lower helium diffusivity in basaltic than synthetic glasses as the result of their lower silica content (Jambon and Shelby 1980, Kurz and Jenkins, 1981; Jambon, Weber and Braun, 1986).

However, the differences determined here can not be explained by silica content alone. The alkali basalt, with lower weight percent silica (and mole fraction $\mathrm{SiO}_{2}, 3.2 \mathrm{~b}$ and $3.2 \mathrm{c}$ ) has a lower Ea than the tholeiites, the opposite relation to that expected. In addition, the difference is larger than predicted from the small silica variation, for a silica difference of $3 \%$ the synthetic glass results suggest an activation energy difference of less than $1 \mathrm{Kcal} / \mathrm{mole}$ (Shelby and Eagan, 1976; Jambon and Shelby, 1980). This suggests, rather unsurprisingly, 
that other components than silica are important in determining glass structure and helium mobility in basalts. To extend the network former/modifier model to multicomponent systems, $\mathrm{TiO}_{2}, \mathrm{P}_{2} \mathrm{O}_{5}$ and some portion of $\mathrm{Al}_{2} \mathrm{O}_{3}$ (which can exhibit both properties) should be considered as polymeric components in addition to $\mathrm{SiO}_{2}$ (e.g. Hess, 1980). For basalts, which have low Al/cation molar ratios (see table $3.2 \mathrm{c}$ ), essentially all the Al is charge balanced and will act as a network former in this simple model, although the true structural behaviour of alumina is more complex (e.g. Mysen, Virgo and Scarfe, 1980). Titania contents vary enough in basalts to warrant their inclusion in the model, but phosphorous contents are too low to be of significance (table $3.2 \mathrm{~b}$ ). The role of other cations involves both their ability to depolymerize the network by complexing bridging oxygen atoms and the extent to which they "fill" the structure. Ionization energies correlate roughly with the proclivity for oxygen bond formation (Hess, 1980) and this data suggests that Ca plays a much larger role than $\mathrm{Fe}$ or $\mathrm{Mg}$ in depolymerization. Ionic radii indicate blocking ability, so that singly charged species such as alkalis may be particularly effective in filling internal spaces. These arguments suggest that helium diffusion in basalts will be enhanced by increased $\mathrm{Si}, \mathrm{Ti}$, and $\mathrm{Al}$ contents and decreased by $\mathrm{Ca}$ and alkali contents for a given total cation content. Unfortunately, it is not possible to evaluate the individual importance of different glass components in limiting helium diffusion using the present data set, so two integrative properties, density and total network-former content were considered as possible indices for the compositional dependence of helium mobility. Helium solubilities in natural melts are known to decrease with melt density (Lux, 1987) and helium diffusivities increase with increasing solubility in many synthetic glasses (Doremus, 1973) so that increasing glass density may be an indicator of decreasing diffusivity. However, densities calculated for the glasses studied here do not differ sufficiently to be of use in predicting helium diffusion (table $3.2 \mathrm{c}$ ). Including $\mathrm{TiO}_{2}$ and $\mathrm{Al}_{2} \mathrm{O}_{3}$ as well as $\mathrm{SiO}_{2}$ as network formers does suggest that helium will be more mobile in alkali basalt than in tholeiite, as observed (table $3.2 c$ ), and thus total-network-formers may be a useful index for helium 
diffusivities. However, the $3 \mathrm{KcaL} / \mathrm{mole}$ difference in Ea remains large in comparison to synthetic results, where silica contents must increase by 10 mole percent to yield a similar change, and small changes in Al/cation ratios or $\mathrm{TiO}_{2}$ contents result in Ea variations of $1 \mathrm{kcal} / \mathrm{mole}$ or less (Shelby and Eagan, 1976; Shelby, 1973). Therefore, other oxide components (e.g. Ca variations) may also be important, and extrapolations using total-network-former contents must be cautious.

The role of glass water contents in influencing helium diffusion is uncertain. Water is known to have large effects on synthetic glass properties, including lowering viscosities drastically, and increasing electrical conductivity and the diffusivity of sodium ions (Doremus, 1973 and references therein). Water may also diffuse relatively rapidly in glasses; estimates at $1000^{\circ} \mathrm{C}$ range from $10^{-9} \mathrm{~cm}^{2} / \mathrm{s}$ to $10^{-6} \mathrm{~cm}^{2} / \mathrm{s}$ for synthetic glasses and obsidians (Doremus, 1973; Karsten, Holloway and Delaney, 1982). Extrapolating to the basalts and temperatures used here suggests water mobilities ranging from .001 to 1 times that for helium. Thus, water could affect helium diffusion by altering glass structure and perhaps also by sweeping helium through the glass structure if its mobility is high. However, direct measurements have not confirmed a role for water in controlling helium diffusion. Shelby (1972) found no difference for He diffusion between anhydrous glasses and those with up to .1 weight percent water, suggesting limited structural effects of water at low concentrations. However, the water content of alkali basalt D9 is higher than this (about $1 \%$ as opposed to only . $1 \%$ for the tholeiites; D. Graham, personal communication), and without specific measurements at these higher water contents, the structural effect of water should be considered an unkown, but possibly important, control on He diffusion. The diffusivity of water in basalts has not been measured, but given the 1000-fold faster diffusivity of helium than water in obsidian (Jambon and Shelby, 1980; Friedman and Long, 1976), dynamic effects of water diffusion on helium mobility in basaltic glass seem unlikely. 


\subsubsection{Diffusion of Radiogenic Helium}

The two experiments (\#10 and \#11) measuring loss of ${ }^{* 4} \mathrm{He}$ from alkali basalt sample $1854 \mathrm{~B}$ displayed higher diffusivities than inherited helium at temperatures of $200-400^{\circ} \mathrm{C}$, and much greater temperature

dependence (figure 3.14). The activation energy for ${ }^{* 4}$ He release $(26-28$ $\mathrm{Kcal} / \mathrm{mole}$, table 3 ) is larger than for inherited He release from the tholeiites (17-18 Kcal/mole) or the other alkali basalt (14-15 $\mathrm{Kcal} / \mathrm{mole}$; table 3.4). It is not likely that this difference is compositional, because network former contents predict that helium release from 1854B would have a lower, not higher, activation energy (table 3.2c).

The high thermal activation required for ${ }^{* 4}$ He release may represent the effect of trapping by site characteristics produced by the decay. That is, alpha-particle damage may actually reduce He mobility at low temperature instead of enhancing it! This seems counterintuitive, but similar effects have been observed for heavier noble gases. Diffusivities of neutron-irradiation produced argon decrease with increasing radiation dose, in both simple ionic crystals (e.g. Felix and Muller, 1976; Spindler and Lindner, 1966) and in lunar silicate minerals (Horn et al, 1975). Similar results were found for neon and helium release from basaltic glass, including an increase in activation energy with irradiation dose (Stettler and Bochsler, 1979). The mechanism responsible for reduced mobility probably involves increased trapping of the helium atoms in vacancies generated by the radiogenic decays (Kalbitzer, Kiko and Zahringer, 1969; Reed, 1977). This explanation implicitly recognizes that helium atoms in interstitial sites generate significant lattice strain, and that when vacancies are available, helium atoms will tend to reside in them. This is borne out by studies in pure metals which have documented that lattice strain energies for interstitial helium atoms ( 1.5 to $4.5 \mathrm{eV}$ (35-100 $\mathrm{Kcal} / \mathrm{mole})$ ) restrict the transfer of helium from lower energy vacancy sites (strain energy of about $1 \mathrm{eV}$ ) to the interstitial positions (e.g. Reed, 1977). 
The activation energy observed for ${ }^{*}$ He suggests that diffusive loss at seafloor temperatures will be very slow $\left(D=3 \times 10^{-19} \mathrm{~cm}^{2} / \mathrm{s}\right.$ at $0^{\circ} \mathrm{C}$; table 3.5), and much lower than for inherited helium (D greater than $\left.10^{-16} \mathrm{~cm}^{2} / \mathrm{s}\right)$. Therefore, the $\mathrm{U} /{ }^{* 4} \mathrm{He}$ geochronology methodology developed for seamount basalt glasses by Graham et al (1987) should not be hampered by diffusive ${ }^{* 4}$ He loss. However, this result should be applied with caution because data was not obtained at low temperature, where it is possible that another diffusion mechanism comes into play, and because the relationship between extent of radiogenic decay and diffusion rate is unknown.

\subsubsection{Isotopic fractionation during diffusion}

Enhanced mobility of ${ }^{3} \mathrm{He}$ is evident in all the emanation experiments. In general, early releases have higher ${ }^{3} \mathrm{He} /{ }^{4} \mathrm{He}$ ratios than the bulk glasses, and the ${ }^{3} \mathrm{He} /{ }^{4} \mathrm{He}$ ratios of subsequent fractions decrease, often to below the bulk value at high F. This trend is well developed in both the Alv892-1a chip and powder experiments (figures 3.16 and 3.17). There are small differences between the experiments, however, including the exact $\mathrm{F}$ values at which the curves of decreasing ${ }^{3} \mathrm{He} /{ }^{4} \mathrm{He}$ ratios cross the bulk values of ${ }^{3} \mathrm{He} /{ }^{4} \mathrm{He}$, and the extent of offset between the ${ }^{3} \mathrm{He} /{ }^{4} \mathrm{He}$ of initial releases and the bulk value. In addition, experiments \#1 and \#3 initially released helium of approximately bulk composition, before displaying a compositional trend similar to the other Alv892-1a experiments. The origin of this behavior is not clear, but the extents of release are too large for a surface origin, and the bulk release rates are not unusual, so that a decrepitation process is unlikely. The other tholeiite (Charco 98-11T) and the alkali basalt (D9) displayed similar trends to the Alv892-1a experiments (figure 3.18), although experiment \#8 showed a return to bulk values at high $\mathrm{F}$, after initial ${ }^{3} \mathrm{He} /{ }^{4} \mathrm{He}$ decrease. This could reflect either release of helium remaining in a large grain (for which the individual $\mathrm{F}$ is small) or activation of helium release from a vesicle at high temperature. Overall, it appears that diffusion is responsible for the preferential release of ${ }^{3}$ He rather than a surface 
Figure 3.16 Isotopic compositions of sequential releases in the Alv892-1a glass chip experiments. Asterisks indicate temperature ramp steps. Horizontal lines mark the bulk ${ }^{3} \mathrm{He} /{ }^{4} \mathrm{He}$ ratio summed from all steps. The curves in subplot \#1 model release from a prismatic grain similar to the real chip shape for $D^{3} \mathrm{He} / \mathrm{D}^{4} \mathrm{He}$ of 1.152 (dotted) and 1.08 (solid). Grain size histograms as in figure 3.7 . 
Figure 3.16

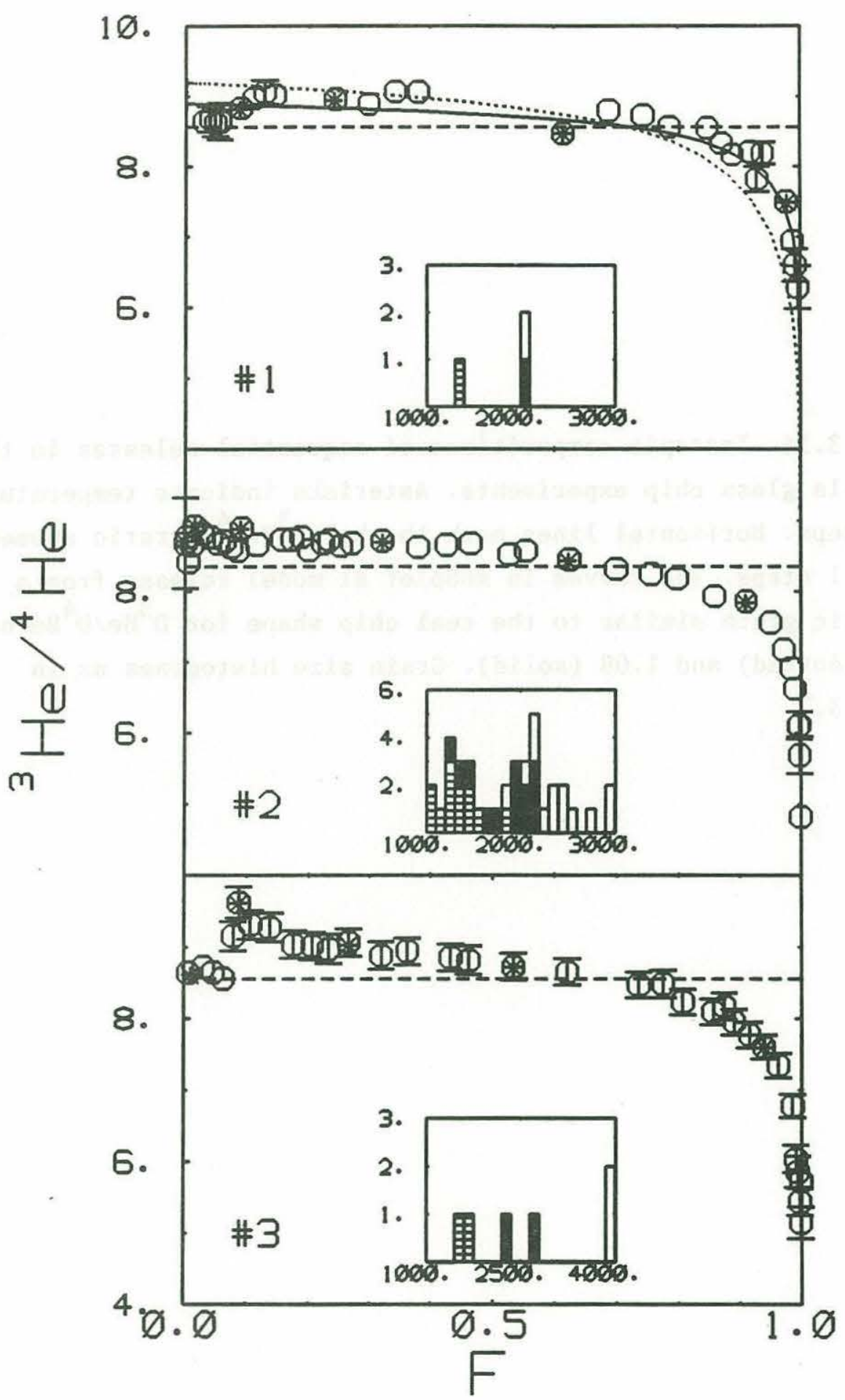


Figure $3.17{ }^{3} \mathrm{He} /{ }^{4} \mathrm{He}$ ratios versus extent of release for the Alv892-1a powder experiments. Symbols as in figure 3.16. The box in subplot \#5 encloses aliquots for which ${ }^{3}$ He releases were larger than the instrumentally calibrated range and may be slightly underestimated. 
Figure 3.17

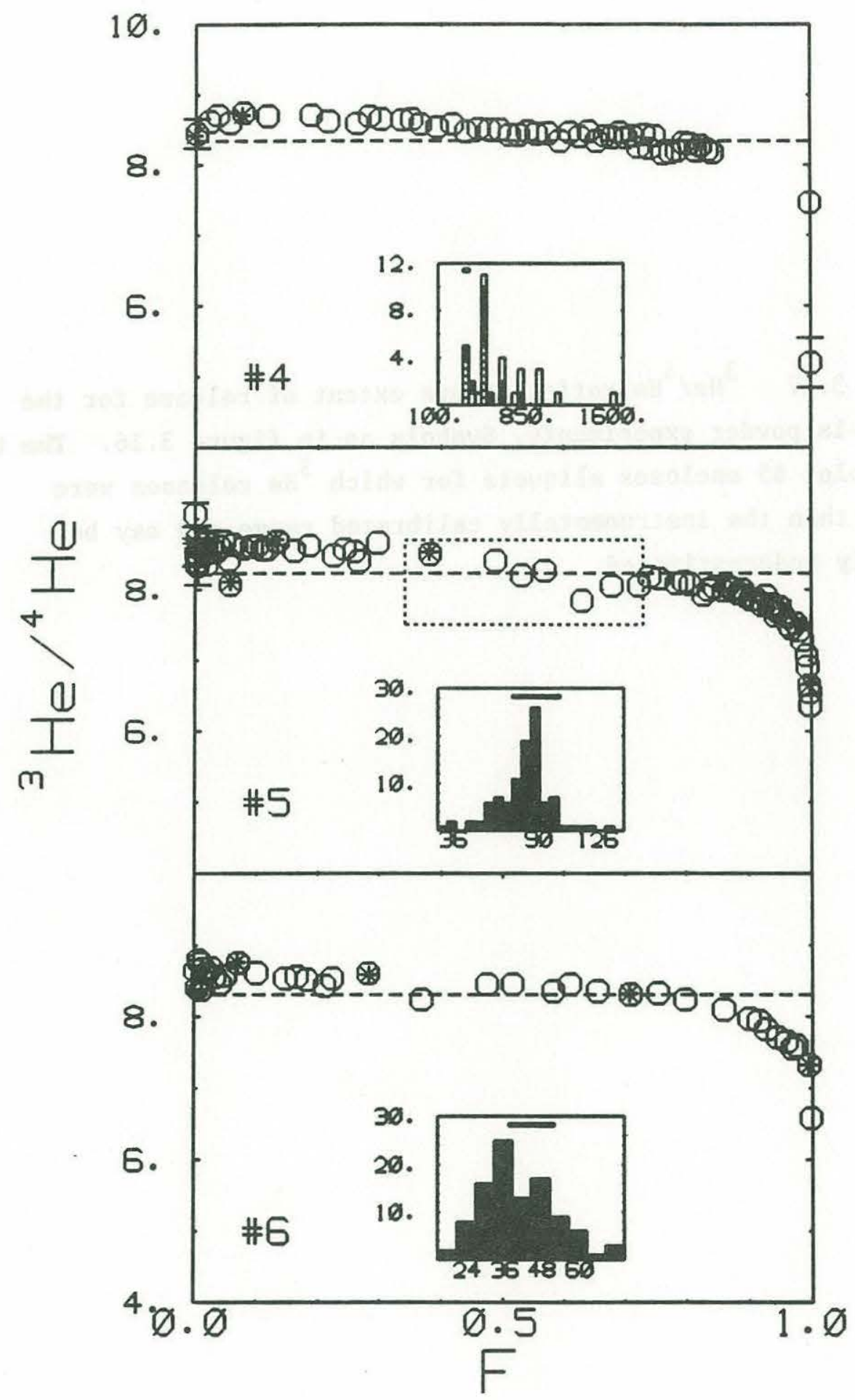


Figure $3.18{ }^{3} \mathrm{He} /{ }^{4} \mathrm{He}$ ratios for tholeiite sample Charco98-11T (top) and alkali basalt Aftermath D9 (middle and bottom). Symbols as in figure 3.16 . 
Figure 3.18

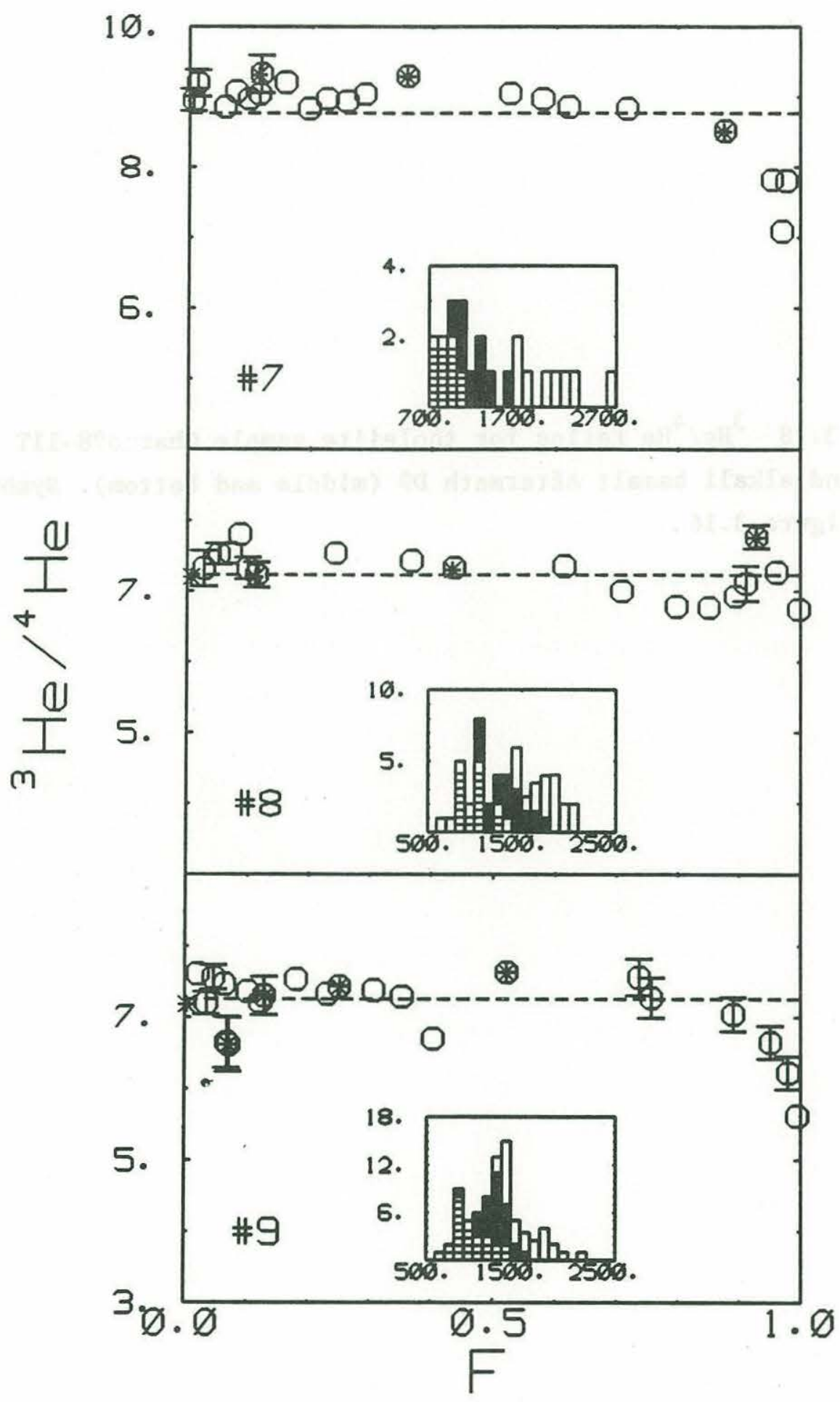


effect, because the decreasing trends continue to high $\mathrm{F}$, and similar trends were observed for a wide range of particle sizes.

For each experiment the isotopic diffusivity ratio, $D^{3} \mathrm{He} / \mathrm{D}^{4} \mathrm{He}$ can be estimated in three related ways. The trends of ratio versus extent of release plots (figures $3.16-3.18$ ) can be compared to model release curves calculated for different $D^{3} \mathrm{He} / \mathrm{D}^{4} \mathrm{He}$ values. This approach provides an estimate of the effective $D^{3} \mathrm{He} / \mathrm{D}^{4} \mathrm{He}$ ratio throughout the experiment and thus cannot be used to constrain any temperature dependence for $D^{3} \mathrm{He} / \mathrm{D}^{4} \mathrm{He}$. Nonetheless, it immediately reveals that $\mathrm{D}^{3} \mathrm{He} / \mathrm{D}^{4} \mathrm{He}$ may be less than 1.15, the value expected for inverse proportionality to the square root of mass (e.g. expt\#1 in figure 3.16). Precise determination of $\mathrm{D}^{3} \mathrm{He} / \mathrm{D}^{4} \mathrm{He}$ by this method must account for grain size distribution. Size distribution keeps the ${ }^{3} \mathrm{He} /{ }^{4} \mathrm{He}$ ratios of sequential aliquots relatively high to large $F$, yielding underestimated $D^{3} \mathrm{He} / \mathrm{D}^{4} \mathrm{He}$ ratios. Differing shapes are a much smaller effect, and are below the analytical resolution of these experiments (model calculations of the effect of size and shape variations on $\mathrm{D}^{3} \mathrm{He} / \mathrm{D}^{4} \mathrm{He}$ values are presented in chapter 2).

The second way to determine the isotopic diffusivity ratio is to compare the ${ }^{3} \mathrm{He} /{ }^{4} \mathrm{He}$ ratios at low $\mathrm{F}$ to the bulk ratio. The initial releases will have ${ }^{3} \mathrm{He} /{ }^{4} \mathrm{He}$ ratios enhanced by a factor of $\left(D^{3} \mathrm{He} / \mathrm{D}^{4} \mathrm{He}\right)^{1 / 2}$, as is readily seen from the approximation equations for $\mathrm{D}$ at low F (see section above). For the Alv892-1a experiments this suggests isotopic diffusivity ratios of 1.05 to 1.1 , centered on 1.08 , and in the powder experiments, where linear relations between $F$ and $t^{1 / 2}$ were established at room temperature (figure 3.6 ), $\mathrm{D}^{3} \mathrm{He} / \mathrm{D}^{4} \mathrm{He}$ is $1.06 \pm .01$. This method has the advantage of being relatively unaffected by grain size or shape variations, but again does not account for temperature variations when applied to step-heated experiments.

The most informative approach is to calculate the diffusivites of ${ }^{3} \mathrm{He}$ and ${ }^{4} \mathrm{He}$ independently using the refined model, and then take their ratio for each aliquot. This allows the investigation of variations during the experiment. Figures $3.19-3.21$ show $D^{3} \mathrm{He} / \mathrm{D}^{4} \mathrm{He}$ values calculated this way. The one sigma uncertainties in these figures are relatively large, and reflect errors in measuring ${ }^{3} \mathrm{He} /{ }^{4} \mathrm{He}$ ratios of 
Figure 3.19 Isotopic diffusivity ratios for the Alv892-1a chip experiments as a function of release, calculated from the refined model. 
Figure 3.19

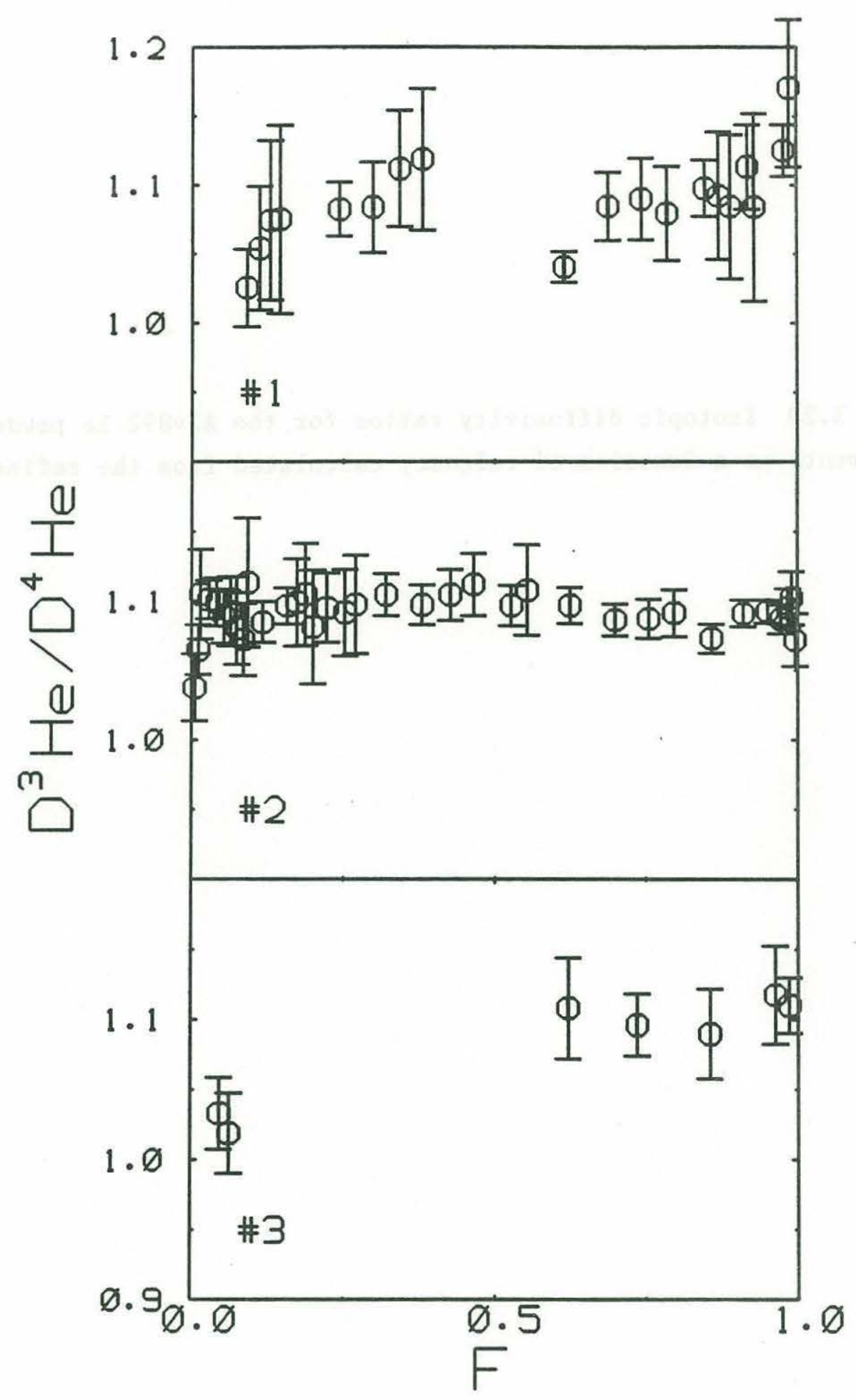


Figure 3.20 Isotopic diffusivity ratios for the Alv892-1a powder experiments as a function of release, calculated from the refined model. 
Figure 3.20

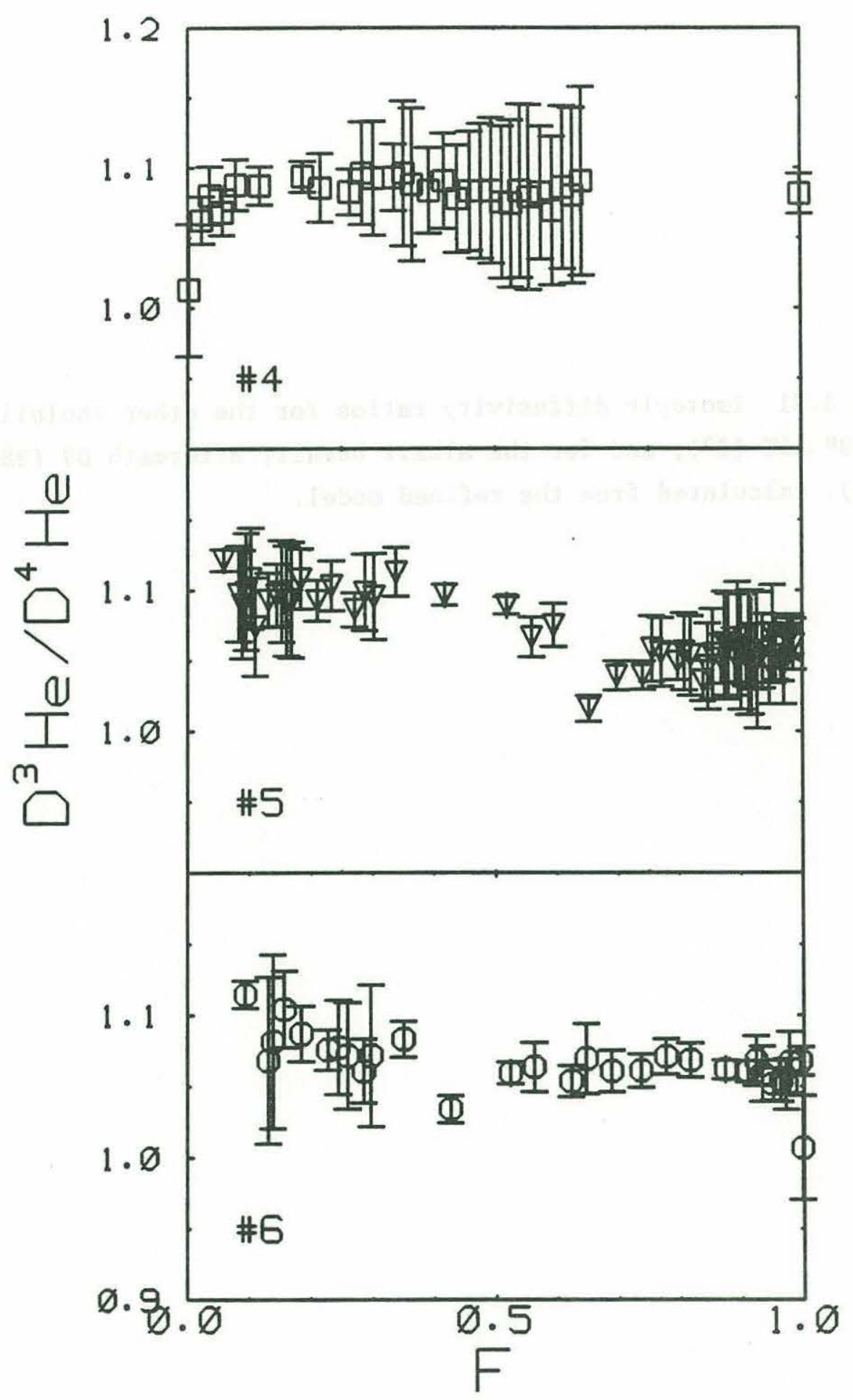


Figure 3.21 Isotopic diffusivity ratios for the other tholeiite, Charco98-11T (\#7), and for the alkali basalt, Aftermath D9 (\#8 and \#9), calculated from the refined model. 
Figure 3.21

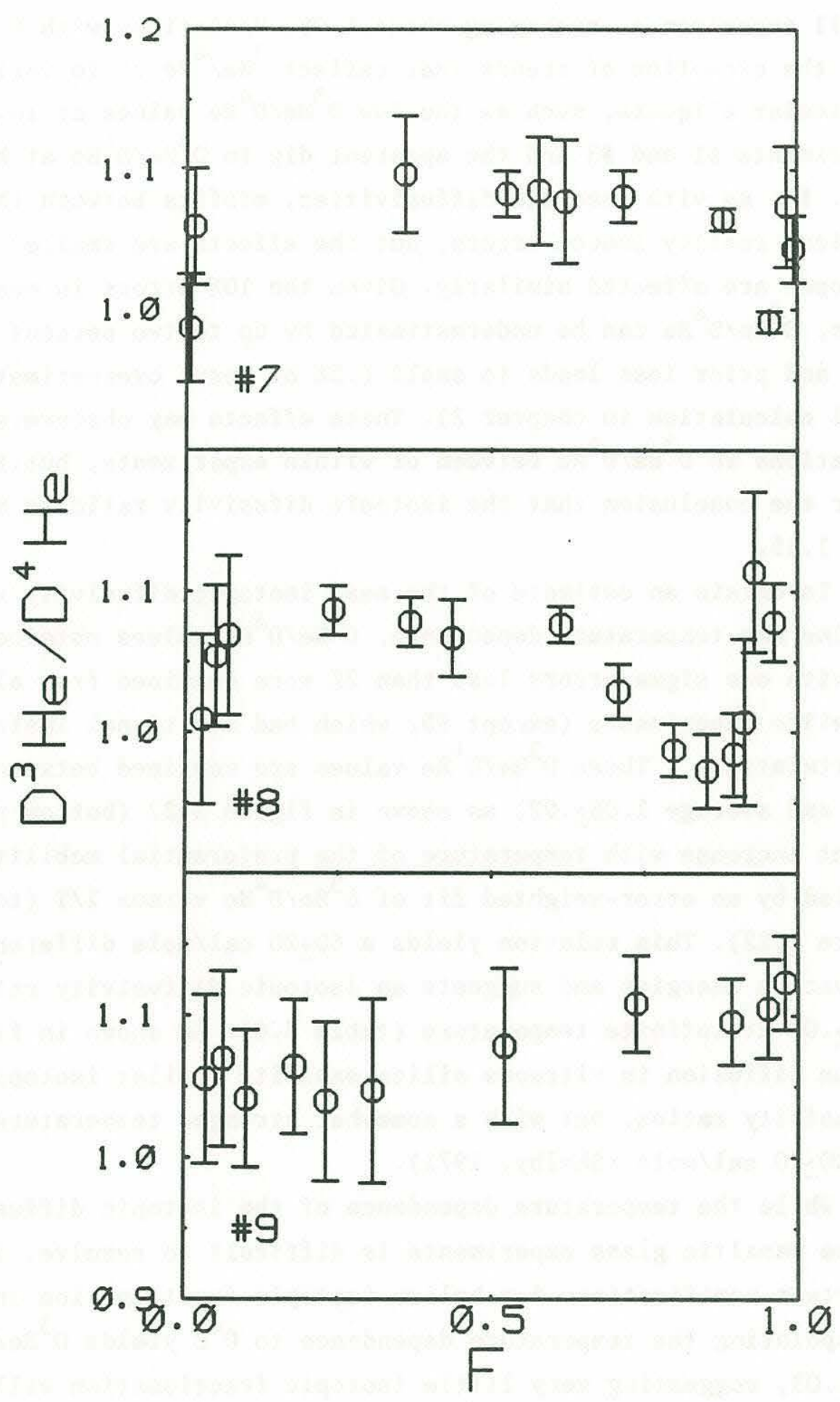


small helium quantities. However, it is clear that $D^{3} \mathrm{He} / \mathrm{D}^{4} \mathrm{He}$ is similar in all experiments, averaging about 1.08. Variations with $\mathrm{F}$ are small, with the exception of trends that reflect ${ }^{3} \mathrm{He} /{ }^{4} \mathrm{He}$ ratio variations in particular aliquots, such as the low $\mathrm{D}^{3} \mathrm{He} / \mathrm{D}^{4} \mathrm{He}$ values at low $\mathrm{F}$ in experiments \#1 and \#3 and the apparent dip in $D^{3} \mathrm{He} / \mathrm{D}^{4} \mathrm{He}$ at high $\mathrm{F}$ in expt. \#8. As with absolute diffusivities, misfits between the model and physical reality induce errors, but the effects are smaller because both isotopes are affected similarly. Given the $10 \%$ errors in measuring grain sizes, $\mathrm{D}^{3} \mathrm{He} / \mathrm{D}^{4} \mathrm{He}$ can be underestimated by up to two percent or so at $\mathrm{F}$ $>.9$ and prior loss leads to small (.5\% or less) overestimates (see model calculation in chapter 2 ). These effects may obscure small variations in $D^{3} \mathrm{He} / \mathrm{D}^{4} \mathrm{He}$ between or within experiments, but they do not alter the conclusion that the isotopic difusivity ratio is much less than 1.15 .

To obtain an estimate of the mean isotopic difusivity ratio and examine any temperature dependence, $D^{3} \mathrm{He} / \mathrm{D}^{4} \mathrm{He}$ values obtained at $\mathrm{F}<.85$ and with one sigma errors less than $2 \%$ were combined from all the tholeiite experiments (except \#5, which had additional instrumental uncertainties). These $\mathrm{D}^{3} \mathrm{He} / \mathrm{D}^{4} \mathrm{He}$ values are confined between 1.0 and 1.15 and average $1.08 \pm .02$, as shown in figure 3.22 (bottom plot). A slight increase with temperature of the preferential mobility of ${ }^{3} \mathrm{He}$ is implied by an error-weighted fit of $D^{3} \mathrm{He} / \mathrm{D}^{4} \mathrm{He}$ versus $1 / \mathrm{T}$ (top plot in figure 3.22). This relation yields a $60 \pm 20 \mathrm{cal} / \mathrm{mole}$ difference in activation energies and suggests an isotopic diffusivity ratio of $1.13_{ \pm} .02$ at infinite temperature (table 3.6). As shown in figure 3.22, helium diffusion in vitreous silica exhibits similar isotopic diffusivity ratios, but with a somewhat stronger temperature dependence of $130 \pm 20 \mathrm{cal} / \mathrm{mole}$ (Shelby, 1971).

While the temperature dependence of the isotopic diffusivity ratio in the basaltic glass experiments is difficult to resolve, it has important ramifications for helium isotopic fractionation in nature. Extrapolating the temperature dependence to $0^{\circ} \mathrm{C}$ yields $D^{3} \mathrm{He} / \mathrm{D}^{4} \mathrm{He}$ of $1.02 \pm .03$, suggesting very little isotopic fractionation will accompany helium loss from seafloor basalts. This result is reinforced by the mean isotopic diffusivity ratio of $1.06 \pm .01$ obtained at room temperature 
Figure 3.22 Examination of the temperature dependence of the isotopic diffusivity ratio, $\mathrm{D}^{3} \mathrm{He} / \mathrm{D}^{4} \mathrm{He}$ for tholeiitic basalt

glass. The data are 137 determinations combined from experiments $\# 1-4$, and \#6-7, with one sigma errors of less than $2 \%$. Errorweighted regression lines are shown to the data (solid line) and for results on vitreous silica (dotted line; Shelby, 1971). The slight temperature dependence suggests the activation energy for $3_{\mathrm{He}}$ is $60 \pm 20 \mathrm{cal} / \mathrm{mole}$ higher than for ${ }^{4} \mathrm{He}$ in basalt, and $130 \pm 20$ cal/mole higher in silica. 
Figure 3.22
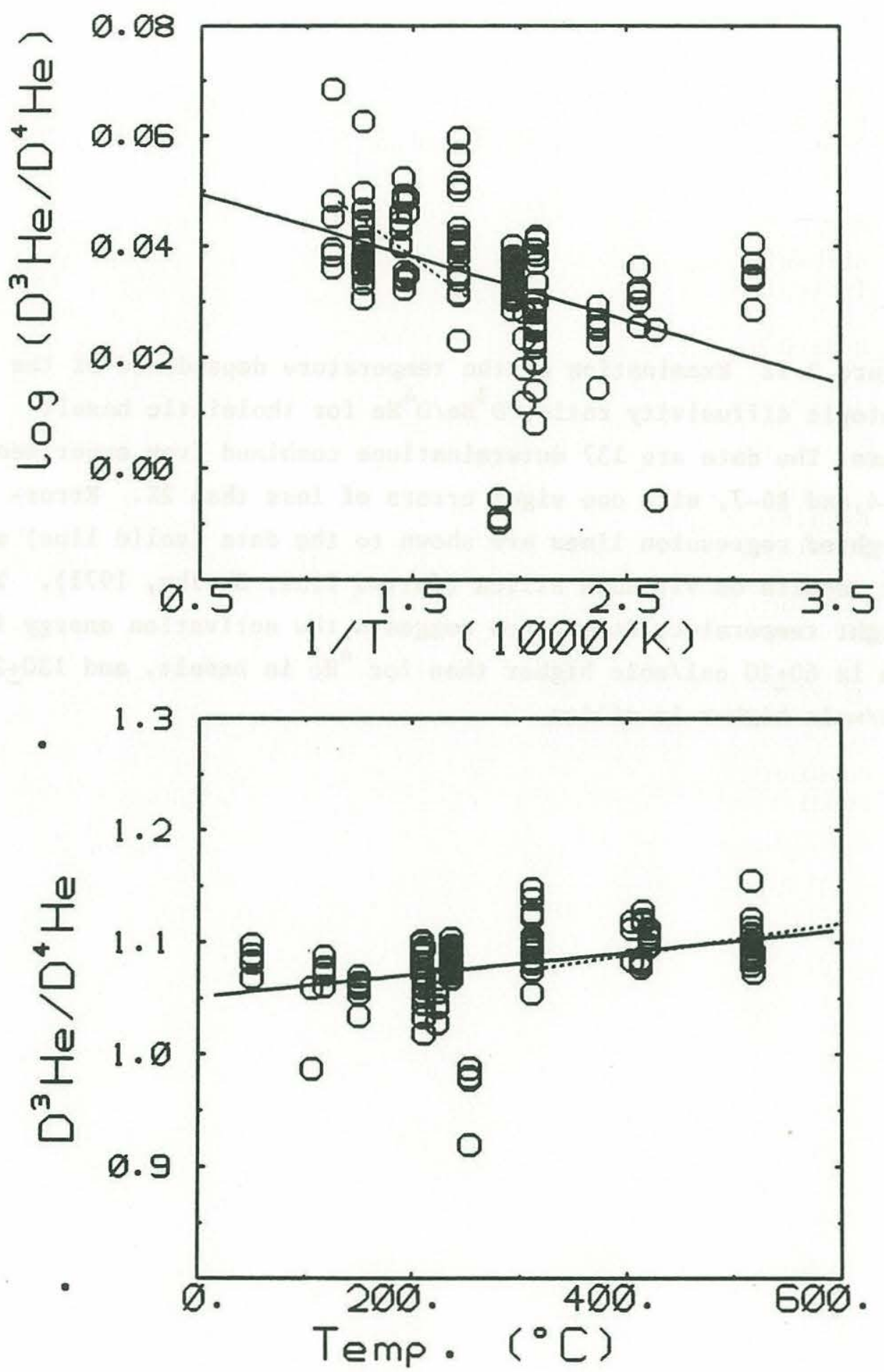
Table 3.6 The Isotopic Diffusivity Ratio of Helium in Tholeiitic Glass.

Arrhenius equation for the temperature dependence of the isotopic diffusivity ratio:

\begin{tabular}{lcc}
$\mathrm{D}^{3} \mathrm{He} / \mathrm{D}^{4} \mathrm{He}=\mathrm{D}_{\mathrm{o}}{ }^{3} \mathrm{He} / \mathrm{D}_{\mathrm{o}}{ }^{4} \mathrm{He} \times \exp \left\{-\left(\mathrm{E}_{\mathrm{a}}^{3} \mathrm{He}-\mathrm{E}_{\mathrm{a}}{ }^{4} \mathrm{He}\right) / \mathrm{RT}\right\}$ \\
\cline { 2 - 2 } & $\mathrm{D}_{\mathrm{o}}{ }^{3} \mathrm{He} / \mathrm{D}_{\mathrm{o}}{ }^{4} \mathrm{He}$ & $\left(\mathrm{E}_{\mathrm{a}}^{3} \mathrm{He}-\mathrm{E}_{\mathrm{a}}{ }^{4} \mathrm{He}\right)$ \\
$\begin{array}{l}\text { Basaltic glass } \\
\text { results }\end{array}$ & $1.13_{ \pm} .02$ & $60 \pm 20 \mathrm{cal} / \mathrm{mole}$ \\
$\begin{array}{l}\text { Vitreous silica } \\
\text { (Shelby,1971) }\end{array}$ & $1.20_{ \pm} .01$ & $130 \pm 30$ \\
$\begin{array}{l}\text { Classical } \\
\text { prediction }\end{array}$ & 1.152 & 0 \\
$\begin{array}{l}\text { Quantum theory } \\
\text { prediction }\end{array}$ & $1.23 \pm 2$ & $125 \pm 25$
\end{tabular}


in the powder experiments (as discussed above). In contrast, diffusive fractionation at magmatic temperatures will be higher, the extrapolated isotopic diffusivity ratio at $1100^{\circ} \mathrm{C}$ is $1.12 \pm .02$.

Both the magnitude of the mean isotopic diffusivity ratio and the temperature effect are departures from the simple relation often quoted in the geochemical literature (e.g. Craig and Lupton, 1976; Hart, 1984; Kaneoka, 1980; Sarda, 1989):

$$
D^{a} / D^{b}=\left(m^{b} / m^{a}\right)^{1 / 2}=1.154 \text { for He }
$$

In addition, the idea that isotopic fractionation increases at higher temperature may seem counterintuitive. For these reasons, solid-state diffusion theory is briefly reviewed with the additional goal of evaluating the experimental results and understanding to what degree they can be extended to other geologic materials. The general expression for diffusion in isotropic, three-dimensional space is (Einstein, 1905; LeClaire, 1970):

$$
D=\left\langle R^{2}\right\rangle / 6 t
$$

where $\left\langle\mathrm{R}^{2}\right\rangle$ is the mean square atomic displacement in time $t$. This can be expressed as a vector sum of $\mathrm{N}$ individual displacements:

$$
\begin{aligned}
\left\langle R^{2}\right\rangle & =\left\langle\left(r_{i}\right)^{2}\right. \\
& =N\left\langle r_{1}^{2}\right\rangle+2 N\left\{\left\langle r_{i} \cdot r_{i+1}\right\rangle+\left\langle r_{i} \cdot r_{i+2}\right\rangle+\ldots\right\} \\
& =N\left\{\left\langle r_{1}^{2}\right\rangle+2\left\langle r_{i}\right\rangle^{2}\left(\left\langle\cos w_{1}\right\rangle+\left\langle\cos w_{2}\right\rangle+\ldots\right)\right\}
\end{aligned}
$$

where the $\left\langle\cos w_{i}\right\rangle$ are the mean values of the cosines of the angles between successive displacements.

Solid-state diffusion theory usually adapts this general expression by assuming that the $r_{i}$ are approximately equal and correspond to atomic 
spacings of lattice atoms in a crystal, leading to (adapted from LeClaire, 1970, Manning, 1971; Wolf, 1981):

$$
D=f a^{2} \mathrm{~N} / 6 \mathrm{t}
$$

where: $f=1+2 \sum_{i}\left\langle\cos w_{i}\right\rangle$ is called the correlation factor, and a is the atomic spacing. $\mathrm{N} / \mathrm{t}$ is related to the availability of sites for the diffusing atom to move to and the rate at which the solute atom moves, often described as the jump rate, $v_{i}$. If lattice atom spacing and site availability is considered to be independent of the mass of the diffusing atom, the isotopic diffusivity ratio (written here for helium) can be expressed as a function of only correlation factors and solute atom jump rates:

$$
D^{3} / D^{4}=v_{3} f^{3} / v_{4} f^{4}=\left(f^{3} / f^{4}\right)\left(v_{3} / v_{4}\right)
$$

Determining the mass dependence of the isotopic diffusivity ratio thus depends on evaluating $f$ and $v$ and their mass dependence.

Diffusion in solids is often divided into different "mechanisms", based on the type of sites the diffusing atom occupies. In interstitial diffusion the solute atom moves between spaces in the crystalline lattice which are not occupied by host atoms. If all these spaces are equally accessible and isotropically distributed the successive jumps of the solute atom will be random and $\mathrm{f}$ will equal 1 for all isotopes. In this case, the mass dependence of diffusivities depends only on the jump rates. Other mechanism classes include vacancy diffusion, in which the diffusing atom moves between lattice sites which are empty because of crystal imperfection; and interstitialcy, in which the diffusing atom moves between interstitial and lattice sites, displacing a host atom to an interstitial site in the process. Because these mechanisms involve the migration of lattice atoms or vacancies, which generally have different jump rates than the diffusing atom, successive solute atom jumps are correlated and f differs from 1 . For example, a diffusing atom may exchange many times with an adjacent vacancy before moving to another vacancy which is opened up by slower host atom movements. Thus, 
in these mechanisms the total population of vacancies, and thus crystal purity, also influences the extent of correlation.

Formulation of correlation factors in terms of host and solute atom jump rates has been done for several simple crystals (e.g. Lidiard, 1955) and reveals that for most vacancy diffusion mechanisms $f$ values will range from 0 to 1 (Schoen, 1958) as shown by a general form proposed for $\mathrm{f}$ by LeClaire (1970):

$$
\mathrm{f}^{\mathrm{i}}=\mathrm{u} /\left(\mathrm{v}_{\mathrm{i}}+\mathrm{u}\right)
$$

where $u$ depends only on host atom jump rates. Combining this expression, written for each isotope, with the previous equation leads to:

$$
\left(D^{3} / D^{4}\right)-1=f^{3}\left(\left(v_{3} / v_{4}\right)-1\right) .
$$

This equation explicitly states that correlation effects reduce diffusive isotopic fractionation. It implicitly recognizes that when correlation occurs there will be an isotopic effect on the extent of correlation, i.e. $\mathrm{f}^{3} / \mathrm{f}^{4}$ in the previous expression for $\mathrm{D}^{3} / \mathrm{D}^{4}$ can only equal 1 if no correlation occurs $(f=1)$. The exact relation between $f^{3} / f^{4}$ and extent of correlation depends on the solid structure and specific atomic movements, but its general form is a slow decrease in $\mathrm{f}^{3} / \mathrm{f}^{4}$ as correlation increases, as shown by the curve in figure 2.23 (calculated from theoretical results for cubic lattices Tharmalingan and Lidiard, 1959). An intuitive understanding of the role of correlation in limiting diffusive fractionation can be had by considering it as the temporal equivalent of a "reduced mass" effect. Because $f$ values calculated for simple crystals are in the range of .6 to .8 , where the isotopic effect on $f$ is small, the superscript on $f$ in the relation between diffusivities and jump frequencies is often dropped (LeClaire, 1966, 1970).

Evaluating the helium diffusion mechanism in basaltic glasses is one way to set limits on the role of correlation in reducing the isotopic diffusivity ratio. There are several experimental observations 
Figure 3.23 Theoretical relation for the isotopic effect on correlation in vacancy diffusion (Tharmalingan and Lidiard, 1959) calculated for helium isotopes. The correlation factors $\mathrm{f}^{3}$ and $\mathrm{f}^{4}$ are only equal at when no correlation occurs $(f=1)$. However, their values remain within $10 \%$ of each other for a large range in f. 
Figure 3.23

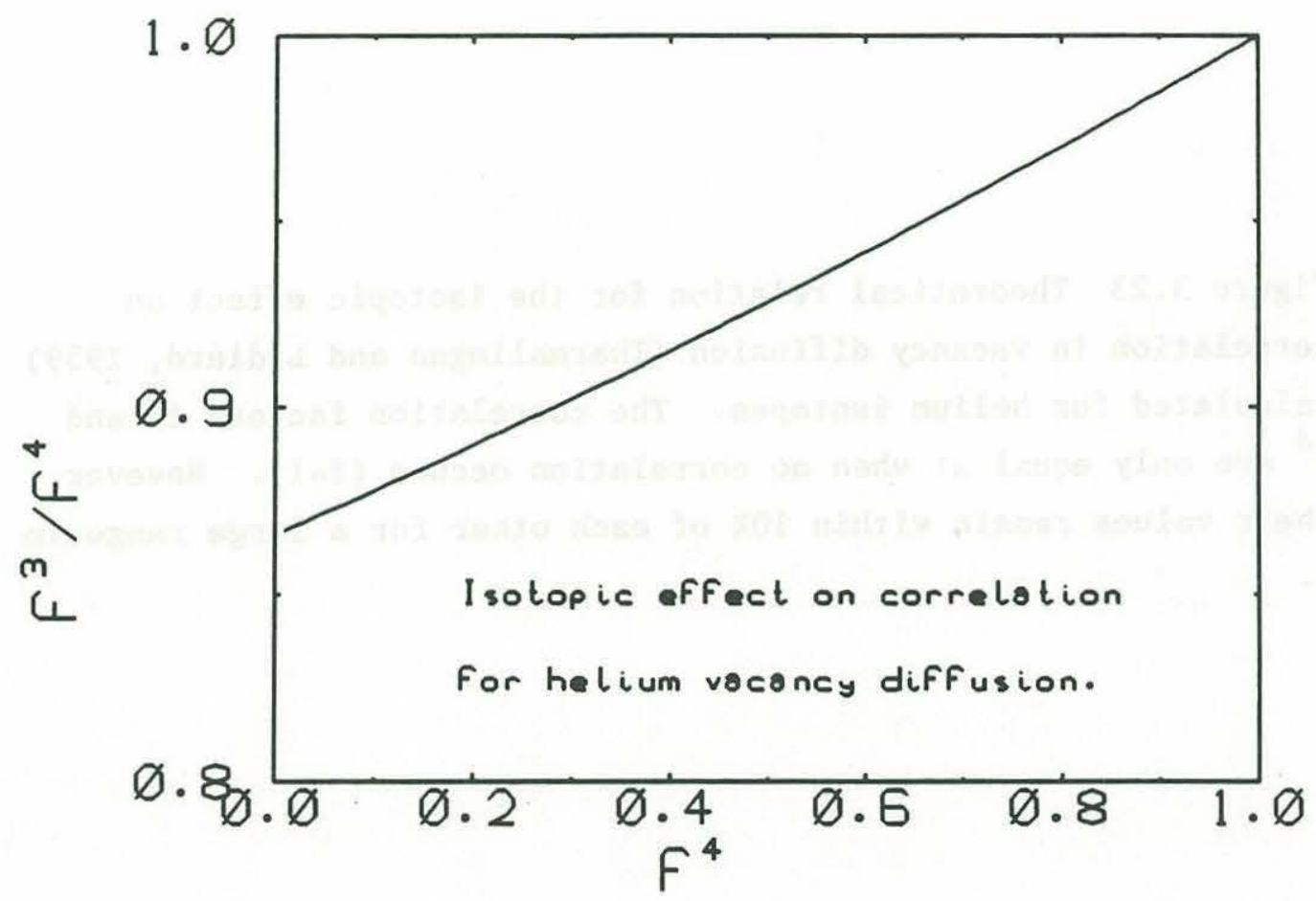


which are most easily explained if helium diffuses in silicate glasses by an interstitial mechanism. (In glasses, which do not have highly ordered lattice sites, interstitial may be taken to mean that passage of the helium atom does not involve displacements of the glass atoms which are large in comparison to vibrational excursions or which are not reversed). An interstitial mechanism can explain the 2-3 orders of magnitude higher diffusivities of helium in comparison to cations in synthetic glasses, obsidians and basalts (see Doremus, 1973; Jambon and Shelby, 1980; this work). The necessity of a fundamentally different mechanism is emphasized by considering the relative size of the helium atom and common cations. Helium atoms are larger than most cations, (Keevil, 1940; figure 3.24), yet diffuse more rapidly. In addition, helium diffusivities vary greatly between obsidian and basalt while many cation diffusivities are relatively unchanged (figure 3.25). This suggests the rates of glass host atom motions limit cation diffusion but not helium diffusion, consistent with interstitial helium diffusion and a vacancy mechanism for cations. In addition, the isotopic diffusivity ratio for neon $\left({ }^{20} \mathrm{Ne} /{ }^{22} \mathrm{Ne}\right)$ in silicate glasses appears to equal the inverse square-root of the isotopic masses (Frank, Swets, and Lee, 1961; Rama and Hart, 1965), suggesting no correlation effects, and thus an interstitial mechanism. Helium and neon have similar atomic sizes in crystals (figure 3.24) and exhibit similar diffusivities in synthetic glasses (Doremus, 1973) and basaltic melts (Lux, 1987) suggesting an identical mechanism and thus that correlation effects are not important for helium either.

The isotopic dependence of jump rates is difficult to determine. A model for the displacement must be constructed and then examined for mass dependence. Most previous studies have adapted transition-state theory developed for molecular reactions to describe a critical "saddle point" or "barrier" in the displacement of the diffusing atom (e.g. Stearn and Eyring, 1940; Wert and Zener, 1949; Vineyard, 1957). Notable exception to this approach is the work of Stoneham and Flynn (1971), who considered the probability of displacement to be governed by the total crystal configurational energies (Hamiltonians) for the initial and final (eigen)states, and made no assumptions about the transition. In 
Figure 3.24 Comparison of effective atomic radii for helium, other noble gases and common ions in crystals (Keevil, 1940).

The ionic radii were determined by $x$-ray diffraction measurements in simple crystals, and the noble gas radii obtained by interpolation between ions of different charges which share the same electronic configuration as the noble gas. This data suggests the atomic radius of helium is about 1.2 Angstrom, and is very similar to the radius for Neon. 
Figure 3.24

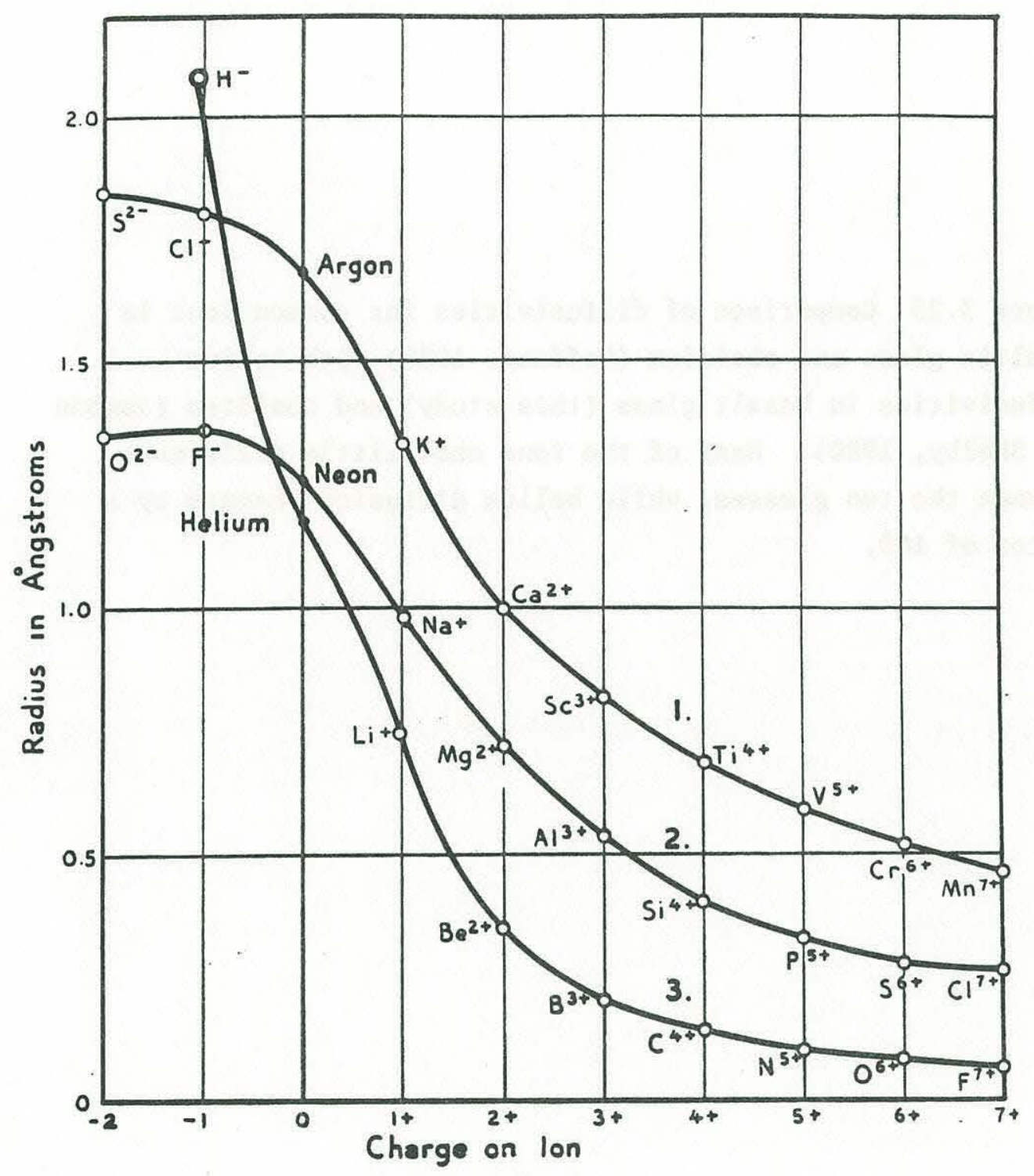


Figure 3.25 Comparison of diffusivities for common ions in basaltic glass and obsidian (Hoffman, 1980) with helium diffusivities in basalt glass (this study) and obsidian (Jambon and Shelby, 1980). Many of the ions show little difference between the two glasses, while helium diffusion changes by a factor of 100 . 
Figure 3.25

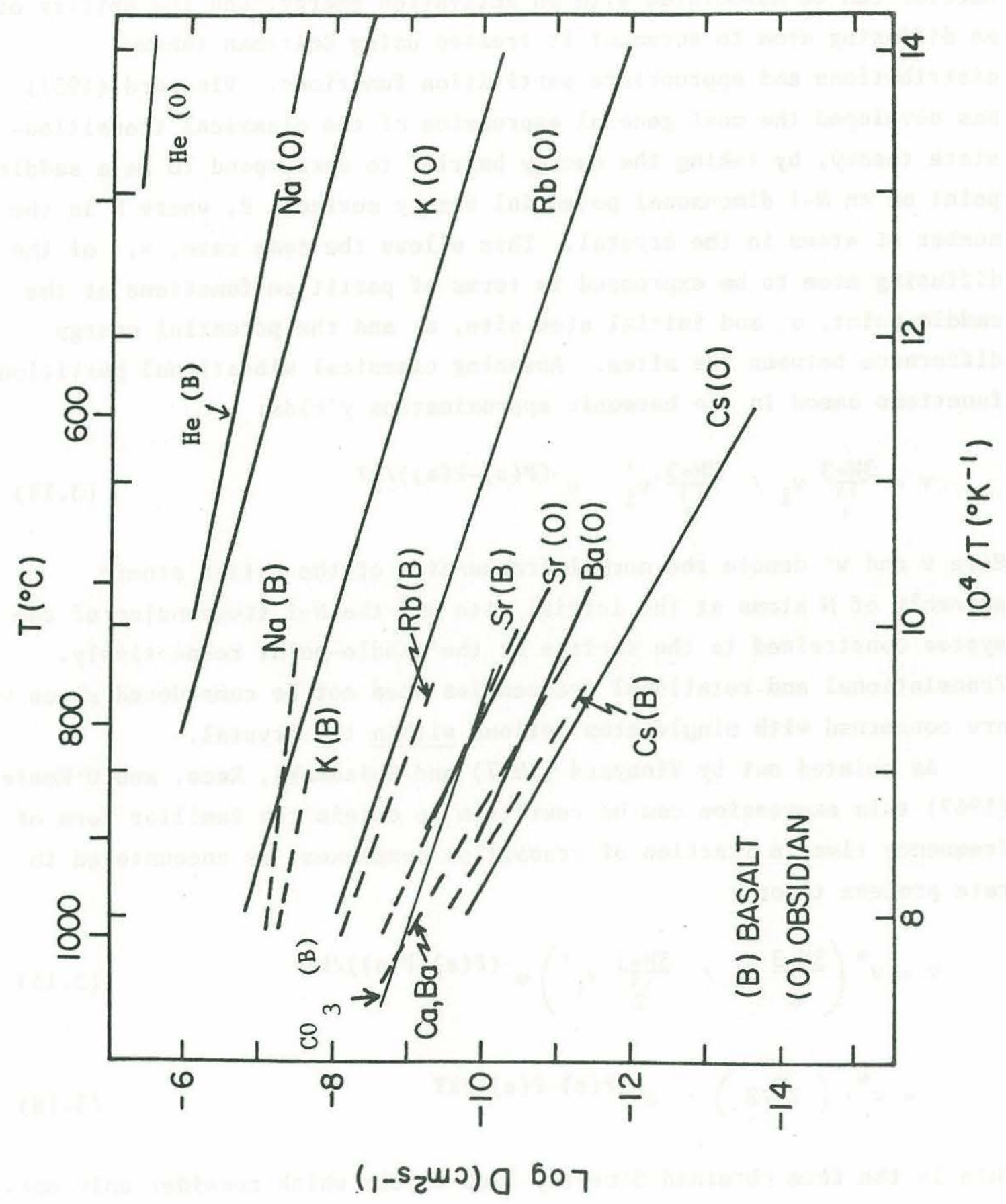


principle this method should better describe solute interactions, but in practice it has not been sufficiently developed to allow a quantitative evaluation of isotopic effects. In the transition state approach the barrier can be associated with an activation energy, and the ability of an diffusing atom to surmount it treated using Boltzman thermal distributions and appropriate partitition functions. Vineyard (1957) has developed the most general expression of the classical transitionstate theory, by taking the energy barrier to correspond to be a saddlepoint on an $\mathrm{N}-1$ dimensonal potential energy surface, $\mathrm{P}$, where $\mathrm{N}$ is the number of atoms in the crystal. This allows the jump rate, $v$, of the diffusing atom to be expressed in terms of partition functions at the saddle-point, $s$, and initial atom site, a, and the potential energy difference between the sites. Assuming classical vibrational partition functions based in the harmonic approximation yields:

$$
v=\prod_{1}^{3 N+3} w_{i} / \prod_{2}^{3 N+3} w_{i}^{\prime} \quad e^{-(P(s)-P(a)) / k T}
$$

Here $w$ and $W^{\prime}$ denote the normal frequencies of the entire atomic assembly of $\mathrm{N}$ atoms at the initial site and the $\mathrm{N}-1$ frequencies of the system constrained to the surface at the saddle-point respectively. Translational and rotational frequencies need not be considered since we are concerned with single atom motions within the crystal.

As pointed out by Vineyard (1957) and Ebisuzaki, Kass, and 0'Keefe (1967) this expression can be rewritten to obtain the familiar form of a frequency times a fraction of transition complexes, as encountered in rate process theory:

$$
\begin{aligned}
v & =w^{*}\left(\frac{3 N+3}{2} w_{i} / \frac{3 N+3}{2} w_{i}^{\prime}\right) e^{-(P(s)-P(a)) / k T} \\
& =w^{*} \cdot\left(z^{*} / Z\right) \cdot e^{-(P(s)-P(a)) / k T}
\end{aligned}
$$

This is the form obtained directly from models which consider only onedimensional solute atom motion, or which allow only the solute atom to vibrate, while keeping the crystal atoms fixed. However, it must be 
pointed out that ${ }^{*}$ has only imaginary significance, because the potential surface at the saddle-point must have negative curvature, and thus can not be equated with vibration of the diffusing atom (Ebisuzaki, Kass, and 0'Keefe, 1967). Determining the mass effect on jump rate thus depends on evaluating the mass effect on the products of normal mode frequencies at both the initial configuration and constrained at the saddle point. Before summarizing those results, it is worth noting that the ratio $Z^{*} / Z$ can be equated with the entropy change $(S)$, and the potential difference, $P(s)-P(a)$, with the activation enthalpy $(H)$ in the semi-empirical diffusion theory presented earlier by Wert and Zener (1949).

The mass dependence of the frequency product in the initial state is readily obtained from the harmonic approximation:

$$
\nu=1 / 2 \pi(\mathrm{k} / \mu)^{1 / 2}
$$

where $\mathrm{k}$ is the force constant for oscillation, and $\mu$ the reduced mass $\mathrm{m}_{1} \mathrm{~m}_{2} / \mathrm{m}_{1}+\mathrm{m}_{2}$ and the Redlich-Teller product rule (e.g. Vinyard, 1957), to obtain:

$$
\prod_{1}^{N} w_{i}=\left(m_{i}\right)^{-3 / 2}
$$

The dependence on mass in the surface-constrained transition state is more difficult to determine, but can be shown to depend on the product of $\left(m_{i}\right)^{-3 / 2}$ and the square-root of the "effective mass", $m_{i}^{*}$, associated with the imaginary $\mathrm{N}^{\text {th }}$ normal mode, ${ }^{*}$ (Vineyard, 1957). Thus, $v_{i}$ is proportional to $\left(m_{i}^{*}\right)^{-1 / 2}$. Therefore the isotope effect for helium atomic jump rates is proportional to $\left(\mathrm{m}_{4}^{*} / \mathrm{m}_{3}^{*}\right)^{1 / 2}$. The $\mathrm{m}^{*}$, which can be thought of in a rough sense as reduced masses, are constrained to lie between the solute and crystal atom masses (Vineyard, 1957), so that the isotopic effect is predicted to vary between a maximum of $\left(\mathrm{m}_{4} / \mathrm{m}_{3}\right)^{1 / 2}$ and a minimum of zero. Mullen (1961) and Leclaire (1966) have extended and clarified this concept to identify ${ }^{*}$ with the 
fraction of kinetic energy possessed by the solute atom in the vibrational mode which leads to a transition $(\Delta \mathrm{K})$, and thus obtained:

$$
\left(w_{3} / w_{4}\right)-1=\Delta K\left(\left(m_{4} / m_{3}\right)^{1 / 2}-1\right)
$$

This can be combined with the previously obtained relation between diffusivities and jump rates (equation 12) to yield:

$$
\left(D^{3} / D^{4}\right)-1=f \Delta K\left(\left(m_{4} / m_{3}\right)^{1 / 2}-1\right)
$$

However, as might be expected, correlation factors and $\Delta \mathrm{K}$ are related so that this expression is only rigorously true when either $f$ or $\Delta K$ equals 1 (LeClaire, 1966).

Equation 3.20 shows that the helium isotopic diffusivity ratio observed in basaltic glasses may be smaller than the rule-of-thumb of 1.15 because more than one atom moves along the reaction coordinate during the transition. However, this appears to be an unsatisfactory explanation given the already discussed results for neon and helium isotopes in synthetic glasses. First, it seems unreasonable that neon isotopes would not also experience a "reduced mass" effect if helium movements involve considerable coupling with glass atom motions and secondly, given the drastic difference in helium diffusivities and atomic compositions between vitreous silica and basaltic glass, how is it that the helium isotopic diffusivity ratio, and thus the reduced mass effect is so similar? This suggests that properties of the helium atoms rather than the solids are responsible for the mass dependence.

In calculating the mass dependence of the jump rates above, classical partition functions were used. However, this may not be appropriate for light helium atoms. Substitution of quantum vibrational partition functions is one way to estimate the effect of quantized vibrational energies on diffusion. LeClair (1966) expanded the crystal quantum vibrational partition function:

$$
\mathrm{q}_{\mathrm{v}}=\mathrm{e}^{-\mathrm{hv} / 2 \mathrm{kT}} /\left(1-\mathrm{e}^{-\mathrm{hv} / \mathrm{kt}}\right)=\mathrm{kT} / \mathrm{hv}\left(1-1 / 24(\mathrm{hv} / \mathrm{kT})^{2}+\ldots\right)
$$


and estimated quantum effects by retaining the first two terms in the series. This yields:

$$
\left(D^{3} / D^{4}-1\right)=\left(\left(m_{4} / m_{3}\right)^{1 / 2}-1\right)\left[1-\left\{.207(h n / k T)^{2}\left(\left(n^{\prime} / n\right)^{2}-3 / 2\right)\right\}\right]
$$

The second term on the right contains the quantum corrections to the classical relation. This equation implicitly assumes that both $f$ and $\mathrm{K}$ are 1 , as in interstitial diffusion, and that the atomic jump is reasonably characterized by oscillations of the diffusing atom alone in the initial state, $n$, and the transition state, $\mathrm{n}^{\prime}$. Examining the quantum correction term shows that the isotopic diffusivity ratio may be larger or smaller than $\left(\mathrm{m}_{4} / \mathrm{m}_{3}\right)^{1 / 2}$ depending on the size of $\mathrm{n}^{\prime} / \mathrm{n}$. It also predicts that as the quantum effect diminishes with increasing temperature ( $\mathrm{hn} / \mathrm{kT}$ becomes small) the isotopic fractionation will approach the classical inverse-square-root-of-mass value.

Thus, quantized vibration is capable of explaining both the low helium isotopic diffusivity ratio and its increase with temperature in silicate glasses, provided quantum effects actually occur for helium at these temperatures. One way to assess this possibility is to estimate $\mathrm{hn} / \mathrm{k}$, which can reasonably be assumed to be at least as large as the vibrational temperature of the glass atoms, $\mathrm{T}_{\mathrm{v}}$. A simple approximation for $\mathrm{T}_{\mathrm{v}}$ in crystals is the Debye temperature, which is generally about .7 times the melting point in metals (LeClaire, 1966). Extending this approximation to glass suggests that $\mathrm{T}_{\mathrm{v}}$ and thus $\mathrm{hn} / \mathrm{k}$ will be on the order of $800^{\circ} \mathrm{C}$, so that quantum effects may reasonably be expected for the laboratory temperatures used $\left(20-600^{\circ} \mathrm{C}\right)$. Another way to assess the vibrational temperatures of basaltic glasses is through heat capacity data. In simple solids, constant volume heat capacities will increase to a high temperature limit of about $6 \mathrm{cal} / \mathrm{mole}-{ }^{\circ} \mathrm{K}$ ( 3 times the gas constant $R$ ) as vibrational energy-level spacing becomes small in comparison to thermal energies (e.g. Nash, 1974). In more complex solids, heat capacities can exceed this value, but values less than $3 R$ at a given temperature still suggest that vibrational energy gaps are large in comparison to thermal energies. Measured heat capacities of many simple silicate glasses as well as a glass of diopside composition 
are less than $3 \mathrm{R}$ until temperatures exceed $800^{\circ} \mathrm{C}$ (Stebbins, Carmichael and Monet, 1984). Thus, quantum effects for helium diffusion can reasonably expected in basaltic glasses up to $800^{\circ} \mathrm{C}$ or so. For comparison, vibrational temperatures for hydrogen in metals are on the order of $1000^{\circ} \mathrm{C}$ (Ebisuzaki, Kass and 0'Keeffe, 1967).

What other ways are there to test the quantum explanation of helium isotopic diffusivity ratios in basaltic glasses? It is not possible to estimate $\mathrm{n}^{\prime / n}$ directly. However, the quantum theory predicts a proportionality between the size of the isotopic diffusivity ratio's departure from the classical value and the steepness of $i$ ts temperature dependence which can be compared with the experimental results (LeClaire, 1966), . Expressing the temperature dependence as the difference in activation energies for the two isotopes, this can be written (Shelby, 1971):

$$
\left(\mathrm{E}_{\mathrm{a}}{ }^{3} \mathrm{He}-\mathrm{E}_{\mathrm{a}}{ }^{4} \mathrm{He}\right)=2 \mathrm{RT}\left\{1-\left(\mathrm{D}^{3} \mathrm{He} / \mathrm{D}^{4} \mathrm{He}\right) /\left(\mathrm{m}^{4} \mathrm{He} / \mathrm{m}^{3} \mathrm{He}\right)^{1 / 2}\right\}
$$

Ea differences calculated from this equation are in excellent agreement with the measured values for synthetic glasses (Shelby, 1971). For the basalts, an activation energy difference of $60 \pm 20 \mathrm{cal} / \mathrm{mole}$ was observed, somewhat less that the predicted value of $125 \pm 25 \mathrm{cal} / \mathrm{mole}$, but within the two sigma error bounds (table 3.6).

Overall, the quantum model is a reasonable interpretation of the experimental results, which has important implications. First of all, the model reinforces the experimental observation that helium isotopic diffusivity ratio is temperature dependent and at low temperature is likely to be very close to 1 , and thus suggests that little fractionation will accompany gas loss on the seafloor. Secondly, the quantum model suggests that this behavior will apply to helium in many other geologic materials which have similar or higher melting points; especially if heat capacities $(\mathrm{Cv})$ in the solids of interest are small $(<3 \mathrm{R})$. This has been confirmed for olivine and pyroxene crystals, which appear to have $D^{3} \mathrm{He} / \mathrm{D}^{4} \mathrm{He}$ ratios of 1.08 to 1.10 at magmatic temperatures (close to their approximate Debye temperature of .7 times the melting point, chapter 4). However, because all diffusion models 
make simplifying assumptions, extending the quantum model for He should be done with caution.

In summary, a brief review of solid-state diffusion theory revealed three major factors which affect isotopic fractionations: correlation $(\mathrm{f})$, energy distribution during the atomic displacement ( $\mathrm{K}$ ), and quantization of vibrational energies. Correlation reduces isotopic fractionations and is most important in diffusion mechanisms involving host atom displacements. K effects can occur for any mechanism, but, as with correlation, are likely to be most important when solute and host atoms have similar masses or vibrational energies. Quantum effects are probably only important for light atoms above room temperature, but can either reduce or enhance isotopic effects. Quantum effects can explain the isotopic diffusivity ratios observed for helium in basaltic glass. In other solids the heat capacity may provide a rough guide to the likliehood of quantum effects. 0verall, the transition-state theory suggests the $(m)^{1 / 2}$ approximation (equation 3.7 ) will often be an upper limit for isotopic effects. However, it must be emphasized that diffusion theories make many assumptions, and that larger isotope effects have been observed in some systems (e.g. Vinyard, 1957).

\section{$\underline{3.4}$ DISCUSSION}

The measured helium diffusivities can be used to estimate timescales for helium loss. Model loss curves calculated for spheres with homogeneous initial concentrations and zero surface concentration, suggest $50 \%$ helium loss can occur about 1 million years for mid-oceanridge tholeiites, given the diffusivity of $5 \times 10^{-16} \mathrm{~cm}^{2} / \mathrm{s}$ at $0^{\circ} \mathrm{C}$ (table $3.5)$, and asssuming $1 \mathrm{~cm}$ as a characteristic pillow lava glass fragment size (figure 3.26). In contrast, the higher helium diffusivity in alkali basalt glass yields approximately 100,000 years. Initial ${ }^{3} \mathrm{He} /{ }^{4} \mathrm{He}$ ratios would be changed very little by $50 \%$ gas 10 ss, less than $2 \%$ given the $\mathrm{D}^{3} \mathrm{He} / \mathrm{D}^{4} \mathrm{He}$ ratio of $1.02 \pm .03$ suggested for $0^{\circ} \mathrm{C}$ by the temperature dependence of the isotopic diffusivity ratio (table 3.6 ). Even the mean value $\mathrm{D}^{3} \mathrm{He} / \mathrm{D}^{4} \mathrm{He}$ value of 1.08 suggests $80 \%$ gas loss must occur to lower the initial ${ }^{3} \mathrm{He} /{ }^{4} \mathrm{He}$ ratio by $10 \%$ and this would require 
Figure 3.26 Timescales for He loss or exchange for basaltic glasses. The model curves are calculated for spheres of radius a and zero surface concentration. For example, an alkali basalt glass with natural grain size of $1 \mathrm{~cm}$ and diffusion coefficient of $2 \times 10^{-15} \mathrm{~cm}^{2} / \mathrm{s}$ at $0^{\circ} \mathrm{C}$ has $\log \mathrm{D} / \mathrm{a}^{2}=-14.1$ and can lose $50 \%$ of its initial helium in about 100,000 years. 
Figure 3.26

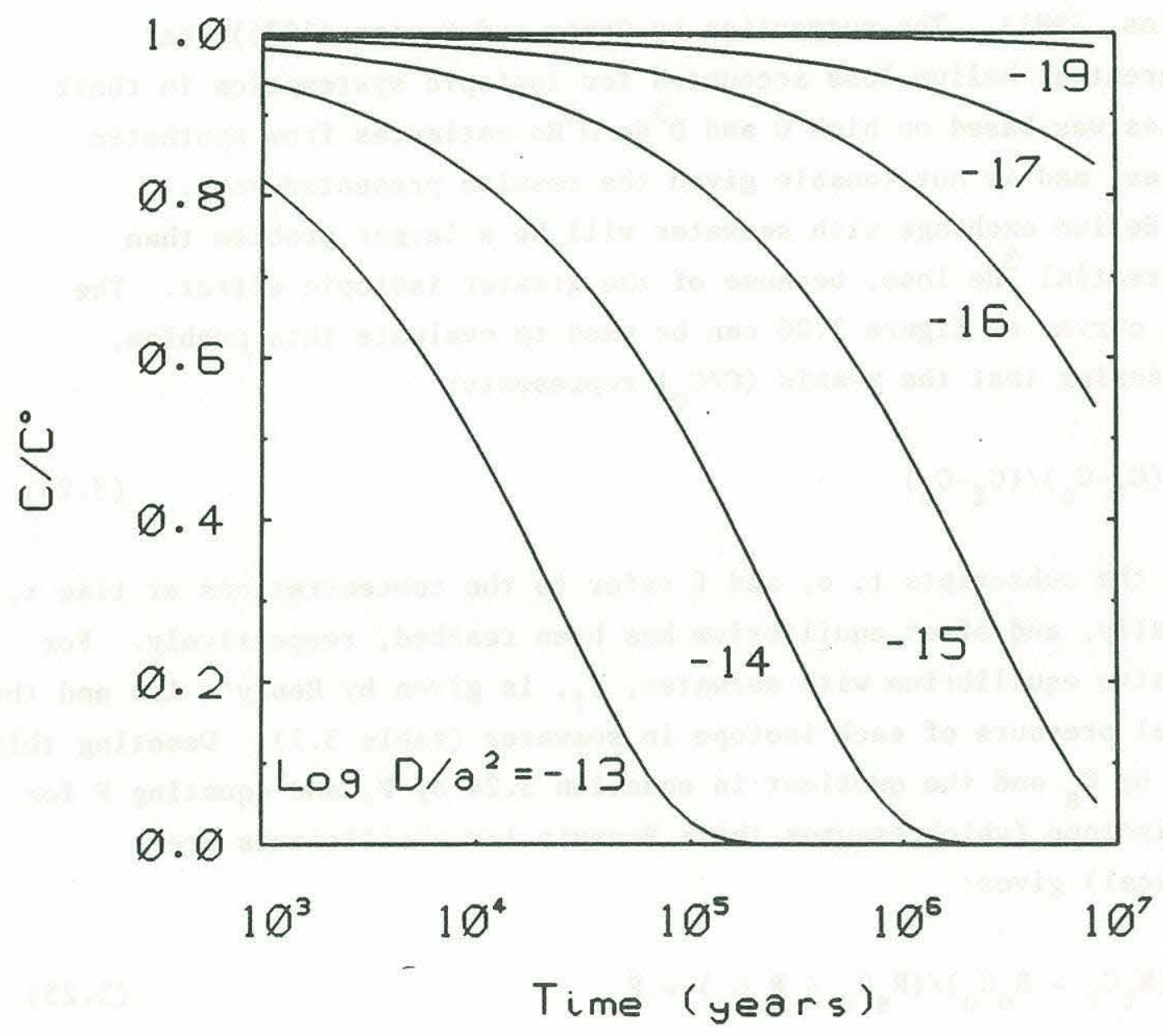


more than 5 times as long a loss period. (Model curves for the change in initial ratio as a function of loss are given in figure 4.8 for different values of the isotopic diffusivity ratio.) Thus, it is very unlikely that a significant fraction of the range of ${ }^{3} \mathrm{He} /{ }^{4} \mathrm{He}$ ratios observed in MORB (7-9 $R_{a}$ ) is produced by preferential loss of ${ }^{3} \mathrm{He}$ after eruption. This result is also clear from the lack of correspondence between helium concentration and isotopic composition in MORB (Kurz and Jenkins, 1981). The suggestion by Craig and Lupton (1976) that preferential helium loss accounted for isotopic systematics in their samples was based on high $D$ and $D^{3} H e / D^{4} H e$ estimates from synthetic glasses, and is not tenable given the results presented here.

Helium exchange with seawater will be a larger problem than preferential ${ }^{3}$ He loss, because of the greater isotopic effect. The model curves of figure 3.26 can be used to evaluate this problem, considering that the $\mathrm{y}$-axis $\left(\mathrm{C} / \mathrm{C}_{0}\right)$ represents:

$$
\left(\mathrm{C}_{\mathrm{t}}-\mathrm{C}_{\mathrm{o}}\right) /\left(\mathrm{C}_{\mathrm{f}}-\mathrm{C}_{\mathrm{o}}\right)
$$

where the subscripts $t, o$, and $f$ refer to the concentrations at time $t$, initially, and after equilibrium has been reached, respectively. For diffusive equilibrium with seawater, $\mathrm{C}_{\mathrm{f}}$, is given by Henry's law and the partial pressure of each isotope in seawater (table 3.1). Denoting this value by $\mathrm{C}_{\mathrm{S}}$ and the quotient in equation 3.24 by $\mathrm{F}$, and equating $\mathrm{F}$ for each isotope (which assumes their Henry's law coefficients are identical) gives:

$$
\left(R_{t} C_{t}-R_{0} C_{0}\right) /\left(R_{s} C_{s}-R_{0} C_{0}\right)=F
$$

where $\mathrm{R}$ refers to the respective ${ }^{3} \mathrm{He} /{ }^{4} \mathrm{He}$ ratios. This can be rewritten to obtain an expression for the fractional change in the initial ratio of the sample in terms of $\mathrm{F}$ :

$$
R_{t} / R_{0}=\left\{F\left(\left[R_{S} C_{s} / R_{0} C_{0}\right)-1\right]+1\right\} /\left\{F\left(\left[C_{s} / C_{0}\right)-1\right]+1\right\}
$$


which shows that the fractional change depends on both the concentration and isotopic ratios between the glass and seawater, as expected.

Following the example of $50 \%$ loss for diffusion above, and considering $\mathrm{R}_{0} / \mathrm{R}_{\mathrm{s}}=8$, and $\mathrm{C}_{\mathrm{o}} / \mathrm{C}_{\mathrm{s}}=100 \mathrm{yields} \mathrm{R}_{\mathrm{t}} / \mathrm{R}_{\mathrm{o}}=.991$. Thus, exchange of helium with seawater is similarly ineffective to preferential ${ }^{3} \mathrm{He}$ loss in altering isotopic compositions of helium rich mid-ocean-ridge basalts. However, for samples with low helium contents it becomes very important, for example, if $\mathrm{C}_{0}$ equal to the seawater equilibrium value $\left(2-3 \times 10^{-9} \mathrm{ccSTP} / \mathrm{g}\right.$; see introduction), the initial ratio will be lowered by $44 \%$ for an $\mathrm{F}$ value of .5. Thus, glasses from island arc regions which have helium contents in this range, and relatively high diffusivities because of their more silicic compositions, can exhibit significantly altered isotopic compositions after relatively short residence on the seafloor (e.g. for a $2 \mathrm{~mm}$ glass rind, $F$ is about .5 after 10,000 years, considering $\left.\mathrm{D}=2 \times 10^{-15} \mathrm{~cm}^{2} / \mathrm{s}\right)$. This prediction is supported by the observation that Woodlark Basin andesites helium contents at or below the seawater equilibrium value exhibit atmospheric isotopic compositions (chapter 6 ). Curves showing the change in initial ratio as a function of $F$ (equation 3.26) are given for several glass helium concentrations in figure 3.27.

The model curves and calculations presented above apply in a rough sense to radiogenic ${ }^{4} \mathrm{He}$ loss, given the appropriate $\mathrm{D}$ and a values. However, the solution for $\mathrm{F}$ in the case of simultaneous production and diffusion is developed in chapter 5 and provides more precise estimates of radiogenic helium loss rates. Discussion of the relative diffusivities of helium and other cations at high temperatures is presented in chapter 4 . 
Figure 3.27 Fractional change in the initial ${ }^{3} \mathrm{He} /{ }^{4} \mathrm{He}$ ratio (Ro) with extent of glass-seawater helium exchange (F). The solid curves are for different glass (C) to glass in equilibrium with seawater (Cs) helium concentration ratios, with an initial glass ratio of $8 \mathrm{Ra}$. The dotted line is for a concentration ratio of 10 , but initial ratio of $4 \mathrm{Ra}$. 
Figure 3.27

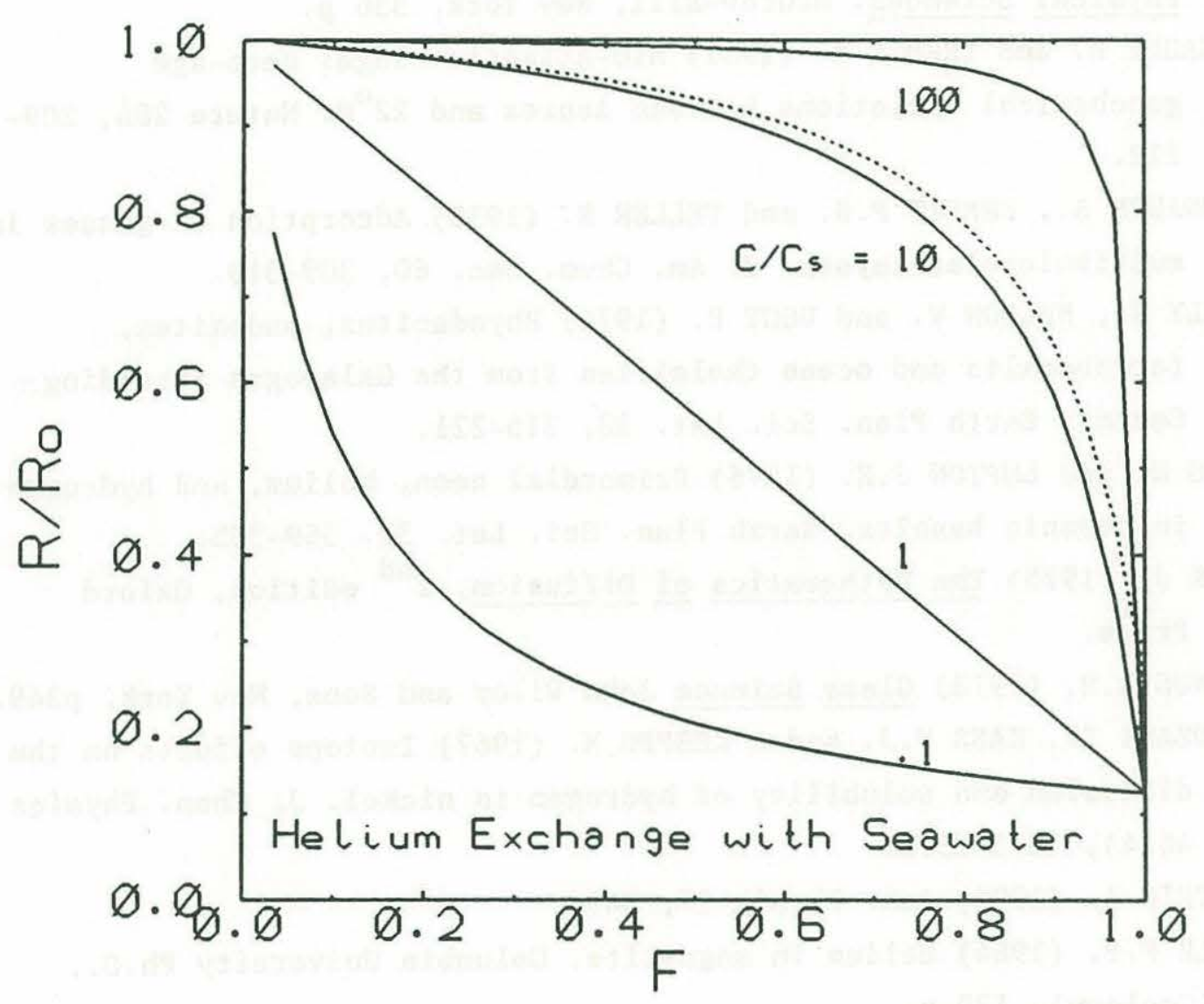




\section{REFERENCES}

ABE M., RAUCH B. and BRANDT W.W. (1971) Nonisothermal diffusion measurements on granular solids: Noble gas diffusion in fused silica. Zeitschrift Naturforsch. 26, 997-1004.

BENDER M.L. (1970) Helium-uranium dating of corals. Columbia University, Ph.D. (Pure Science), 149p.

BEVINGTON, P.R. (1969) Data Reduction and Error Analysis for the Physical Sciences. McGraw-Hill, New York, 336 p.

BOUGAULT H. and TREUIL M. (1981) Mid-Atlantic ridge: zero-age geochemical variations between Azores and $22^{\circ} \mathrm{N}$. Nature 286, 209212 .

BRUNNAUER S., EMMETT P.H. and TELLER E. (1938) Adsorption of gasses in multimolecular layers. J. Am. Chem. Soc. 60, 309-319.

BYERLY G., MELSON W. and VOGT P. (1976) Rhyodacites, andesites, ferrobasalts and ocean tholeiites from the Galapagos Spreading Center. Earth Plan. Sci. Let. 30, 215-221.

CRAIG H. and LUPTON J.E. (1976) Primordial neon, helium, and hydrogen in oceanic basalts. Earth Plan. Sci. Let. 31, 369-385.

CRANK J. (1975) The Mathematics of Diffusion, $2^{\text {nd }}$ edition, 0xford Press.

DOREMUS R.H. (1973) Glass Science John Wiley and Sons, New York, p349. EBISUZAKI Y., KASS W.J. and 0'KEEFFE M. (1967) Isotope effects on the diffusion and solubility of hydrogen in nickel. J. Chem. Physics 46(4), 1373-1378.

EINSTEIN A. (1905) Ann. Physik 17, 549.

FANALE F.P. (1964) Helium in magnetite. Columbia University Ph.D., (Geology), 135 p.

FECHTIG H. and KALBITZER S. (1966) Diffusion of argon in potassium bearing solids. In: Potassium-Argon Dating (ed. 0. SCHAEFFER) p68-106

FELIX F.W. and MULLER M. (1976) Radioactive rare gas atoms as a probe for lattice defects in ionic crystals. Indian J. Pure and Appl. Physics 14, 247-252. 
FISHER D.E. (1971) Incorporation of Ar in east Pacific basalts. Earth Plan. Sci. Let. 12, 321-324.

FLEISCHER R. L. (1988) Alpha-recoil damage: relation to isotopic disequilibrium and leaching of radionuclides. Geochim. Cosmo. Acta 52, 1459-1466.

FLEISCHER R.L. and RAABE 0.G. (1978) Recoiling alpha-emitting nuclei: mechanisms for uranium-series disequilibrium. Geochim. Cosmo. Acta 42(7), 973-978.

FRANK R.C., SWETS D.E. and LEE R.W. (1961) Diffusion of neon isotopes in fused quartz. J. Chem. Physics 35(4), 1451-1459.

FRIEDMAN I. and LONG W. (1976) Hydration rate of obsidian. Science $191,347-352$.

GALLAGHER K.J. (1965) The effect of particle size distribution on the kinetics of diffusion reactions in powders. In Reactivity of Solids. 5th International Symposium on the reactivity of solids. Munich, August 2-8, 1964. (ed. G.M. SCHWAB), Elsevier, New York, 192-203.

GILETTI B.J. and KULP J.L. (1955) Radon leakage from radioactive minerals. Am. Mineralogist 40, 481-496.

GRAHAM D. V. (1987) Helium and lead isotope geochemistry of oceanic volcanic rocks from the East Pacific and South Atlantic. WHOI/MIT Ph.D., 252 p.

GRAHAM D.V., JENKINS W.J., KURZ M.D. and BATIZA R. (1987) Helium isotope disequilibrium and geochronology of glassy submarine basalts. Nature 326(6111), 384-386.

GRAHAM D.W., ZINDLER A., KURZ M.D., JENKINS W.J., BATIZA R. and STAUDIGEL H. (1988) Helium, lead, strontium and neodymium isotope constraints on magma genesis and mantle heterogeneity beneath young Pacific seamounts. In press.

HART S.R. (1984) He diffusion in olivine. Earth Plan. Sci. Let. 70, 297-302.HESS P.C. (1980) Polymerization model for silicate melts. In Physics of Magmatic Processes (ed. R.B. HARGRAVES) pp.3-44. Princeton Univ. Press, Princeton, N.J. 
HOFMANN, A.W. (1980) Diffusion in natural silicate melts: a critical review, in Physics of Magmatic Processes (ed. R.B. HARGRAVES) pp.3-44. Princeton Univ. Press, Princeton, N.J.

HORN P., JESSBERGER T., KIRSTEN T. and RICHTER H. (1975) ${ }^{39} \mathrm{Ar}-{ }^{40} \mathrm{Ar}$ dating of lunar rocks: Effects of grain size and neutron irradiation, Proc. Lunar Sci. Conf. 6 p 1563-1591.

HUNEKE J.C., NYQUIST L.E., FUNK H., KOPPEL V. and SIGNER p. (1969) The thermal release of rare gases from separated minerals of the Mocs meteorite. In Meteorite Research (ed. MILLMAN) pp 901-921. D. Reidel, Dordrecht, Holland.

HURLEY P.M. (1954) The helium age method and the distribution and migration of helium in rocks. In Nuclear Geology (ed. H. FAUL) pp. 301-329. J. Wiley and Sons, New York.

INTHOFF W. and ZIMEN K.E. (1956) Kinetik der Diffusion radioaktiver Edelgase aus festen Stoffen nach Bestrahlung (Edelgasdiffusion in Festkorpern 2). Trans. Chalmers. Univ. Techn. (Goteborg) 176, $16 \mathrm{p}$.

JAMBON A. and SHELBY J.E. (1980) Helium diffusion and solubility in obsidians and basaltic glass in the range $200-300^{\circ} \mathrm{C}$. Earth Plan. Sci. Let. 51, 206-214.

JAMBON A., WEBER H.W. AND BEGEMANN F. (1985) Helium and Argon from an Atlantic MORB glass: concentration, distribution and isotopic composition. Earth Plan. Sci. Let. 73 255-267.

JAMBON A., WEBER H. and BRAUN 0. (1986) Solubility of $\mathrm{He}, \mathrm{Ne}, \mathrm{Ar}, \mathrm{Kr}$ and $\mathrm{Xe}$ in a basalt melt in the range $1250-1600^{\circ} \mathrm{C}$. Geochemical implications. Geochim. Cosmo. Acta 50, 401-408.

JENKINS W.J., EDMOND J.M. and CORLISS J.B. (1978) Excess ${ }^{3} \mathrm{He}$ and ${ }^{4} \mathrm{He}$ in Galapagos submarine hydrothermal waters. Nature 272, 156-158. KALBITZER S., KIKO J. and ZAHRINGER J. (1969) The diffusion of ${ }^{3} \mathrm{He}$ and ${ }^{4} \mathrm{He}$ in LiF. Zeitschrift. Naturforsch. 24a, 1996-2000.

KANEOKA I. (1980) Rare gas isotopes and mass fractionation: an indicator of gas transport into or from a magma. Earth Plan. Sci. Let. 48, 284-292. 
KARSTEN J., HOLLOWAY J.R. and DELANEY J.R. (1982) Ion microprobe studies of water in silicate melts: temperature-dependent water diffusion in obsidian. Earth Plan. Sci. Let. 59, 420-428.

KEEVIL N.B. (1940) Interatomic forces and helium in rocks. Proc. Amer. Acad. Arts and Sci. 73, 311-359.

KURZ M.D. and JENKINS W.J. (1981) The distribution of helium in oceanic basalt glasses. Earth Plan. Sci. Let. 53, 41-54.

KURZ M. D., JENKINS W. J., SCHILLING J. G. and HART S. R. (1982) Helium isotopic variations in the mantle beneath the central North Atlantic Ocean. Earth Plan. Sci. Let. 58, 1-14.

LE CLAIRE A.D. (1966) Some comments on the mass effect in diffusion. Phil. Mag. 14, 1271-1284.

LE CLAIRE A. D. (1970) Isotope effects in self-diffusion. In Diffusion Processes (ed. J.N. SHERWO0D, A.V. CHADWICK, W.M. MUIR and F.L. SWINTON) pp. 153-171. Gordon and Breach Sci. Pub., New York.

LEVENTHAL J.S. (1975) An evaluation of the U-Th-He method for dating young basalts. J. Geophys. Res. 80, 1911-1914.

LIDIARD A.B. (1955) Phil. Mag. 46, 1218.

LUPTON J.E. and CRAIG H. (1975) Excess ${ }^{3}$ He in oceanic basalts:

evidence for terrestrial primordial helium. Earth Plan. Sci. Let. 26, 133-139.

LUX G (1987) The behavior of noble gases in silicate liquids: solution, diffusion, and surface effects, with applications to natural samples. Geochim. Cosmo. Acta 51, 1549-1560.

MANNING J.R. (1968) Diffusion kinetics for atoms in crystals. p. 115, D. van Nostrand, Princeton.

MULLEN J.G. (1961) Phys. Rev. 121, 1649.

MYSEN B.0., VIRGO D. and SCARFE C.M. (1980) Relations between the anionic structure and viscosity of silicate melts - a Raman spectroscopic study. Am. Mineralogist 65, 690-710.

NASH L.K. (1974) Elements of Statistical Thermodynamics. AddisonWesley Pub. Co., Inc., Reading, MA, 138 p. 
PRIGOGINE I. and BAK T.A. (1959) Diffusion and chemical reaction in a one-dimensional condensed system. Jour. Chem. Physics 31(5), 1368-1370.

RAMA S.N.I. and HART S.R. (1965) Neon isotope fractionation during transient permeation. Science 147, 737-738.

RAMA and MOORE, W.S. (1984) Mechanism of transport of U-Th series radioisotopes from solids into ground water. Geochim. Cosmo. Acta 48, 395-399.

REED D.J. (1977) A review of recent theoretical devlopments in the understanding of the migration of helium in metals and its interaction with lattice defects. Radiation Effects 31, 129-147.

REYNOLDS J.H. (1960) Rare gases in tektites. Geochim. Cosmo. Acta 20, 101-114.

RISON, W. and CRAIG, H. (1983) Helium isotopes and mantle volatiles in Loihi seamount and Hawaiian Island basalts and xenoliths. Earth Plan. Sci. Let. 66, 407-426.

SARDA P., STAUDACHER T. and ALLGRE C.J. (1989) Neon isotopes in basalts. In press.

SCHOEN, A.H. (1958) Correlation and the isotope effect for diffusion in crystalline solids. Phys. Rev. Let. 1(4), 138-140.

SHAKED H. (1965) Simple theory for the release of fission gas from powders. J. Nucl. Materials 17, 136-141.

SHELBY J.E. (1971) Diffusion of helium isotopes in vitreous silica. Physical Review B 4(8), 2681-2686.

SHELBY J.E. (1972) Helium migration in natural and synthetic vitreous silica. J. Am. Ceram. Soc. 55(2), 61-64.

SHELBY J.E. (1973) Effect of phase separation on helium migration in sodium silcate glasses. J. Am. Ceram. Soc. 56(5), 263-266.

SHELBY J.E. (1973) Neon Migration in $\mathrm{TiO}_{2}-\mathrm{SiO}_{2}$ glasses. J. Am. Cer. Soc. 56(6), 340-341.

SHELBY J.E. and KEETON S.C. (1974) Temperature dependence of gas diffusion in glass. J. App. Physics 45(3), 1458-1460.

SHELBY J.E. and EAGAN R.J. (1976) Helium migration in sodium aluminosilicate glasses. J. Am. Cer. Soc. 59(9-10), 420-425. 
SPINDLER P. and LINDNER R. (1966) Diffusion of xenon-133 in $\mathrm{U}_{2}$ single crystals. Zeitschrift Naturforsch. 21, 1723-1725.

STEARN A.E. and EYRING H. (1940) Absolute rates of solid reactions: diffusion. J. Phys. Chem. 44, 955-980.

STEBBINS J.F., CARMICHAEL I.S.E. and MORET L.K. (1984) Heat capacities and entropies of silicate liquids and glasses. Contrib. Mineral Petrol. 86, 131-148.

STETTLER A. and BOCHSLER P. (1979) He, Ne and Ar composition in a neutron activated sea-floor basalt glass. Geochim. Cosmo. Acta 43, 157-169.

STOLPER E. (1982) The speciation of water in silicate melts. Geochim. Cosmo. Acta 46, 2609-2620.

STONEHAM A.M. and FLYNN C.P. (1971) Quantum theory of interstitial diffusion. In Atomic Transport in Solids and Liquids (eds. A. LODDING and T. LAGERWALL) pp.365-376. Verlag der Zeitschrift fur Naturforschung, Tubingen.

THARMALINGAM K. and LIDIARD A.B. (1959) Isotope effect in vacancy diffusion. Phil. Mag. Series 8, 899-906.

TRULL T.W., PERFIT M. R. and KURZ M.D. (1988) Helium and strontium isotopic constraints on the origin of island arc magmas in the Woodlark Basin. In press.

VINYARD, G.H. (1957) Frequency factors and isotope effects in solid state rate processes. J. Phys. Chem. Solids 3, 121-127.

WEISS R.F. (1971) The solubility of helium and neon in water and seawater. J. Chem. Eng. 16, 235.

WERT C. and ZENER C. (1949) Interstitial atomic diffusion coefficients. Phys. Rev. 76, 1169-1175.

WOLF D. (1981) Theory of correlation effects in diffusion. In Mass Transport in Solids (eds. F. BENIERE and C.R.A. CATLOW) pp. 149168. Plenum Press, New York. 


\section{Chapter 4 .}

Diffusivity of ${ }^{3} \mathrm{He}$ and ${ }^{4} \mathrm{He}$ in olivine and clinopyroxene at magmatic and mantle temperatures. 


\section{$\underline{4.1} \underline{\text { INTRODUCTION }}$}

Helium measurements in crystalline phases have many applications in igneous geochemistry, including $\mathrm{U}+\mathrm{Th} / \mathrm{He}$ geochronology, the identification of mantle components in ultramafic xenoliths, and the characterization of magmatic volatile sources via measurements on phenocrysts in volcanic rocks. Many of these studies require estimates of the extent of equilibration of helium between different phases within the source region, the magma, or the rocks after eruption. For example, if the helium isotopic composition of xenolith minerals is to be considered a signature of the mantle source region it must be clear that the host magma has not exchanged helium with the xenolith. Similarly, the relation between helium isotopic compositions and the source of other magma components is unclear if it is possible that helium is transported rapidly in the mantle. Another example is the significance of helium isotopic disequilibrium between fluid inclusions and helium dissolved in crystal lattices (e.g. data in chapter 6; Polve and Kurz, 1989). 0ther studies of helium isotopic variations as indicators of magma sources rely on assumptions of minimal gas loss or that loss has not affected helium isotopic compositions. Zindler and Hart (1986) have pointed out that this may not be true for the sources of certain oceanic island basalts or magma chambers in general, both because helium loss leads to accelerated change of the residual ${ }^{3} \mathrm{He} /{ }^{4} \mathrm{He}$ ratio by radioactive decay and because ${ }^{3}$ He may be preferentially lost.

In general, the degree to which helium isotopic compositions of crystalline phases (or glasses) have exchanged or lost helium depends on the helium transport rate in comparison to the rates of the important geologic processes in the system, such as magma migration rates, degassing rates, and cooling rates. Little is known about helium mobility in crystalline phases at high temperature. Gramlich and Naughton (1972) measured single estimates of helium diffusion coefficients for olivine and pyroxene at about $1100^{\circ} \mathrm{C}$, but did not investigate the temperature dependence. Hart (1984) measured the helium diffusivity in olivine at three temperatures and obtained a rough 
Arrhenius relation, which suggested He diffusion was strongly temperature dependent and several orders of magnitude higher than for many cations of geologic importance. No measurements of the relative diffusivity of ${ }^{3} \mathrm{He}$ and ${ }^{4} \mathrm{He}$ in basaltic minerals have been made. Because of the small amount of previous work, and the importance of helium isotopic studies to basaltic petrogenesis studies, this chapter focuses on the measurement of ${ }^{3} \mathrm{He}$ and ${ }^{4} \mathrm{He}$ diffusion in two common igneous minerals (olivine and clinopyroxene) at magmatic and mantle temperatures, with the goal of defining rates of isotopic equilibration between basaltic magmas and minerals, and setting limits on the extent of isotopic fractionation accompanying helium loss from these minerals as magma chambers age and lavas cool.

\section{$\underline{4.2}$ METHODS}

Diffusivities were estimated for olivine and pyroxene by measuring ${ }^{3} \mathrm{He}$ and ${ }^{4} \mathrm{He}$ degassed from mineral separates in vacuo in a high temperature furnace at $700-1400^{\circ} \mathrm{C}$. After obtaining several sequential release aliquots over a period of hours to days, the samples were melted to determine their total helium contents, allowing the calculation of fractional release rates. The experiments were done primarily at constant temperature, but in two cases (expts. 4 and 8 ) the temperature was increased during the experiment and more aliquots were collected at higher temperature before melting. Diffusion coefficients for each release aliquot were then calculated using model equations which assume equisized, spherical grains with initially homogeneous helium concentrations. The furnace, helium mass spectrometry, and diffusivity calculations are described in detail in chapter 2 , and are discussed below as neccesary in interpreting the experimental results.

The mineral separate samples were loaded as loose grains into a sample holder mounted above the furnace crucible and maintained at room temperature. This device, "the 4-shooter", is pictured in figure 2.1 (chapter 2), and allowed the furnace crucible to be baked out $\left(1600^{\circ} \mathrm{C}\right)$, equilibrated at the run temperature, and a blank analyzed before introducing the sample. In addition, each experiment used a new 
tantalum crucible insert cup to avoid the potential problem of reaction with previously melted samples. The furnace blanks were identical to typical processing line blanks of $4-5 \times 10^{-15}$ ccSTP He, with atmospheric isotopic composition. However, some diffusion experiments extended to nearly 30 hours between aliquots, so high temperature long-time "closeups" were also analyzed. The mean leak rate was $1.5 \times 10^{-16}$ $\mathrm{cc}^{4} \mathrm{He} / \mathrm{s}$ with a high of $2.8 \times 10^{-16} \mathrm{cc} / \mathrm{s}$ and a low of $.45 \times 10^{-16} \mathrm{cc} / \mathrm{s}$, or always less than $3 \times 10^{-11} \mathrm{cc} /$ day. For all experiments this leak contributed less than $2 \%$ of the helium in any aliquot, and for most aliquots significantly less. The leak was corrected for, assuming an atmospheric isotopic composition. Less than 4 parts per thousand of any release aliquot was lost during sample processing, as verified by analyzing the helium left after melting a sample, and by analyzing an air standard expanded into the furnace volume. Combined with frequent calibration by comparison to air standards (see chapter 2), the low blanks and losses allowed the fractional releases ( $F$ values) to be calculated with high precision (1 sigma error of .2 to .3\%) and accuracy (1 sigma error <.5\%).

Furnace temperatures were measured using an optical pyrometer (Micro-optical model, Pyro. Instr. Co.). The pyrometer was focused through the sapphire viewing port and onto the smooth floor of the tantalum crucible insert, i.e. the surface on which the mineral grains rest. The measured pyrometric temperatures were corrected for the emissivity of this surface (using data for polished Ta metal provided by the instrument manufacturer) and for transmission losses through the sapphire window, which reduced apparent temperatures by 20 to $40^{\circ} \mathrm{C}$ over the range of 800 to $1700^{\circ} \mathrm{C}$ (as measured by focusing the pyrometer on the source filament of a thermal ionization mass spectrometer, with and without the window in the line of sight). Succesful use of the pyrometer required considerable care, because small variations in the azimuth angle to the window produced deviations of up to $20^{\circ} \mathrm{C}$, and different observers percieved temperatures differing by $10^{\circ} \mathrm{C}$ or so. However, repeated measurements by a single observer with constant pyrometer allignment were reproducible within $5-8^{\circ} \mathrm{C}$, and established 
that the furnace maintained constant temperature at constant power for long periods (up to several days).

Using a freshly cleaned window and empty crucible, a pyrometric temperature versus power calibration curve was determined ( $\mathrm{J}$ symbols in figure 4.1a). This figure also shows that in some experiments, particularly those of long duration, progressive coating of the sapphire window reduced apparent pyrometric temperatures by as much as $200^{\circ} \mathrm{C}$. Fortunately, this effect was made obvious by temporal decreases in apparent temperatures at constant furnace power. In these experiments, the power-pyrometric temperature calibration curve was used to obtain sample temperatures. Figure $4.1 \mathrm{~b}$ shows that throughout the experiments (using 2 crucibles and 9 inserts over the course of 5 months) the powercurrent relation was essentially constant: the maximum power difference for a given currrent is 20 watts which corresponds to less than $10^{\circ} \mathrm{C}$. This suggests that the furnace resistance element did not oxidize or otherwise age during the experiments, and lends credence to relying on constant power as an indicator of constant temperature when the window becamed coated.

Unfortunately, the pyrometric temperature measured for different crucible inserts during the experiments did not always agree with the pyrometric temperature versus power calibration curve, even when the window remained clean. For example, measurements in one experiment suggested temperatures up to $100^{\circ} \mathrm{C}$ above the calibration curve (V symbols in figure 4.1a). In order to better understand this variation, and to determine the accuracy of the pyrometric temperatures, the furnace power-temperature relation was also calibrated by melting pure metals ( $\mathrm{Ag}, \mathrm{Au}, \mathrm{Mn}, \mathrm{Ni}, \mathrm{Pd}$; less than 1 ppm impurities; Johnson-Matthey Inc.). In general, the metals were observed to melt at a sharp temperature to form a small pool of liquid. An exception was nickel, which appeared to melt over a large temperature range and was not used in the calibration. The currents at which silver and manganese melted were reproducible to within about 5 amps (equivalent to $15^{\circ} \mathrm{C}$ for $\mathrm{Ag}$ and $10^{\circ} \mathrm{C}$ for $\mathrm{Mn}$; figure 4.2). Reproducibility for palladium was poorer, about 15 amps $\left(45^{\circ} \mathrm{C}\right)$, in part reflecting the difficulting in stepping the furnace temperature by small amounts (less than 5 amps) at high 
Figure 4.1 Apparent pyrometric temperature (uncorrected for emissivity) as a function of furnace power (a, at top). The low values (e.g. H and D symbols) are the result of coating of the sapphire window. Symbols correspond to different emanation experiments and calibrations $(\mathrm{J})$. The reproducible current power relation for the furnace during all the experiments is shown at the bottom (b). 
Figure 4.1

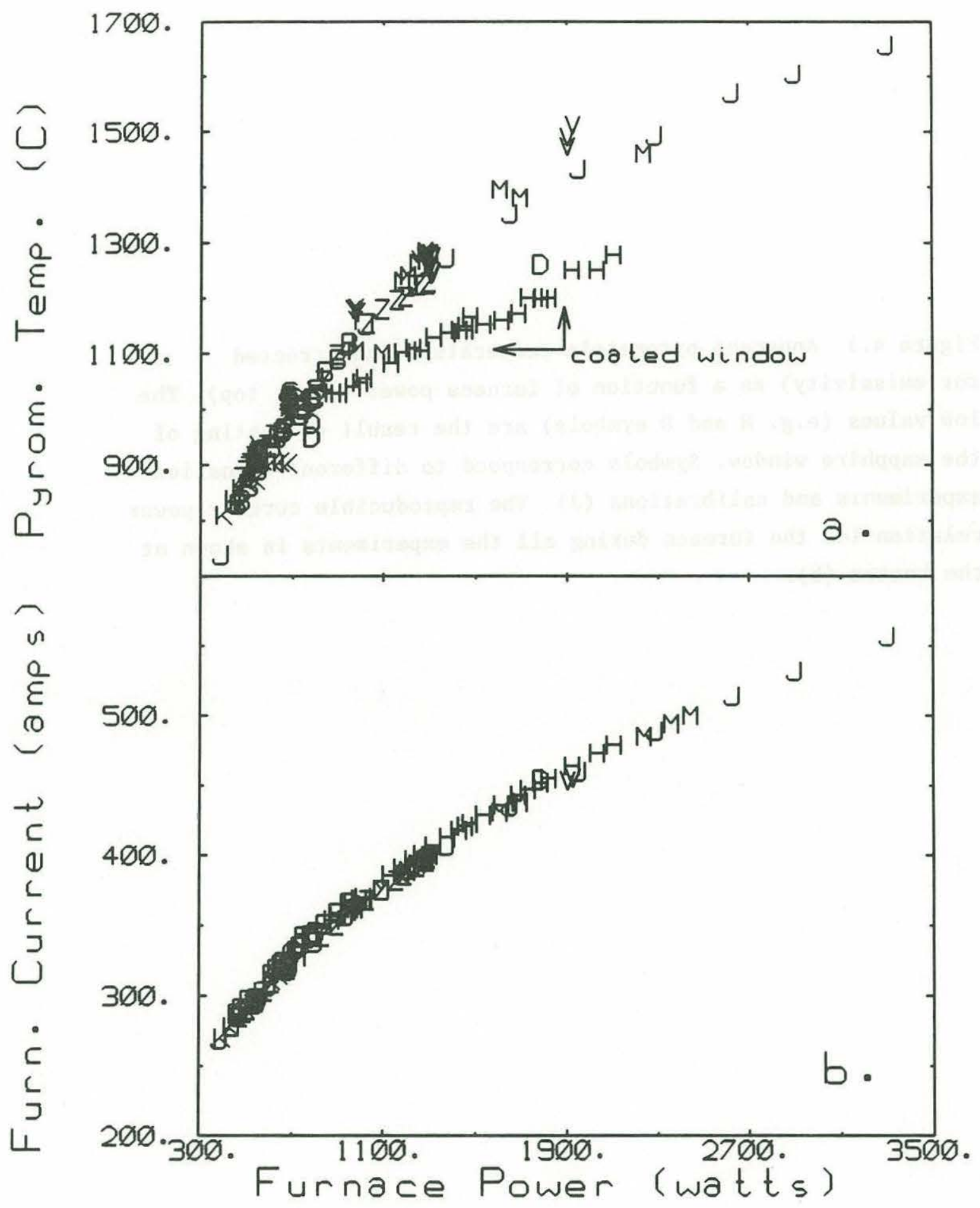


Figure 4.2 Comparison of pyrometric temperature calibration (curves) with fusion data for pure metals placed directly in the crucibles and for one gold sample in a tantalum foil boat. Two gold calibrations were performed in a different crucible than was used for most of the experiments (solid dots). The raw pyrometric temperature data has uncertainties of $5-8^{\circ} \mathrm{C}$, and the melting point current uncertainties are about 10 amps. The corrected pyrometric curve accounts for emissivity changes for $\mathrm{Ta}$ with temperature and for the measured attenuation of the sapphire window. The metal fusion data suggests corrected pyrometric temperatures are overestimated above $1200^{\circ} \mathrm{C}$. 
Figure 4.2

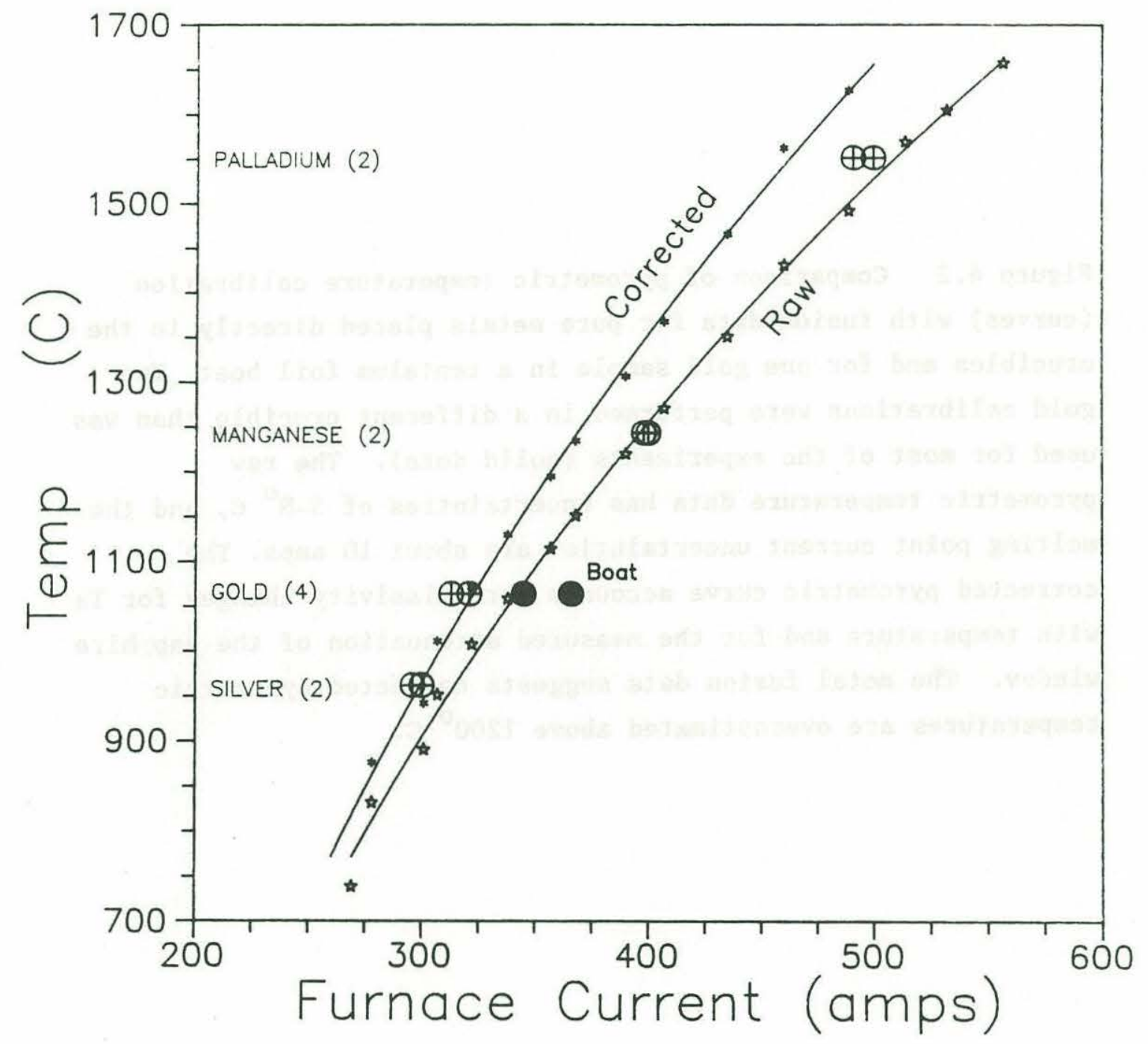


power. The current required to melt gold varied by 15 amps $\left(50^{\circ} \mathrm{C}\right)$ for two calibration runs with different inserts within the same crucible. However, an even larger difference was observed for different outer crucibles. Gold melted at a greater current (equivalent to almost $100^{\circ} \mathrm{C}$ higher temperature) in the first crucible used (C6) for experiments \#4 and \#8, than in the second crucible (C8) used for all the other (isothermal) experiments. In addition, melting a gold sample loaded in a small tantalum boat (crucible C6) required a higher current than any of the loose samples.

The variations in the furnace currents required to melt the standard metals may be best explained as a combination of different placements of the two outer crucibles with respect to the heating element and variations in the heat shielding effect of the loose-fitting crucible inserts (and tantalum boat). This explanation also accounts for the fact that the observed pyrometric temperature tended to increase with the amount of current required to melt a standard, as follows. The pyrometer, in some sense, integrates the light emitted by the crucible insert bottom and the light emitted from the gap between the insert and crucible wall, which appeared to be at higher temperature, by up to $40^{\circ} \mathrm{C}$. Thus, when the insert is a relatively poor fit the inner cup temperature is relatively cool and more power is required to melt a standard metal, but the "temperature" measured by pyrometry appears higher, because more high temperature radiation is emitted from the insert-crucible gap then when the insert fits well. The currents at which the metal standards melted agreed reasonably well with the corrected pyrometric temperatures at low temperature ( $\mathrm{Ag}$ and $\mathrm{Au}$ ), but at higher temperatures ( $\mathrm{Mn}$ and $\mathrm{Pd}$ ) the metal melting data suggests the furnace is approximately $100^{\circ} \mathrm{C}$ cooler than the corrected pyrometry data (figure 4.2 ). This suggests the emissivity correction may be overestimated at higher temperatures.

Because of the uncertainties in calibrating the power-temperature relation and in using the pyrometer most of the diffusion experiments were performed isothermally at furnace currents very close to the melting points of the metals. Temperatures and associated uncertainties (which range from 10 to $30^{\circ} \mathrm{C}$, see data tables, Appendix C) for these 
experiments were then obtained by comparing both the current and pyrometric temperature for the experiment with the closest fusion calibrations in the same crucible, to correct for both differences in furnace power and differing extents of heat shielding by the inserts. The shape of the pyrometric curve obtained in the same crucible (and where possible, the same insert) was used to extrapolate from the melting calibration current to the diffusion experiment current. In some cases, e.g. experiments 1 and 5, the current and pyrometry for the experiment and for melting the nearest standard ( $\mathrm{Ag}$ ) were in excellent agreement and these temperatures are reasonably certain (within $10^{\circ} \mathrm{C}$ ). For other experiments, the agreement was poorer, and the uncertainty therefore larger (up to $30^{\circ} \mathrm{C}$ ).

\section{$\underline{4.3}$ SAMPLES}

Diffusion measurements were made on clinopyroxene and olivine mineral separates from Hawaiian mafic xenoliths. These samples were chosen because of the ease of preparation of pure phase separates, and their relatively high helium contents. The mineral separates were prepared by Dr. Mire Polve using previously described methods (Polve and Kurz, 1989).

The olivine mineral separate was prepared from a dunite xenolith (USNM 113987-107) from the 1801 alkali basalt flow of Hualalai Volcano, Hawaii. The grains used in the experiments (500-710um) are intermediate in the size spectrum present in the porphyroclastic rock $(.1-2 \mathrm{~mm})$. As observed previously, (Kurz et al, 1983), the grains contain abundant arrays of small (approximately 5 microns in diameter) fluid inclusions, and only rare larger inclusions in this grain size. Comparing the relative release of helium by in vacuo crushing and by melting the grains suggests that most ( $90 \%$ or more) of the helium is contained in these inclusions (table 4.1). The isotopic compositions of helium released by these methods are indistinguishable. The grains handpicked for the diffusion measurements were roughly spherical, completely devoid of adhering matrix or other phases, and showed no signs of internal cracking or crystal domains in thin-section. 
The clinopyroxene sample was prepared from a spinel-lherzolite nodule (69SAL219) from Salt Lake Crater, Oahu, which has been extensively studied for the distribution and isotopic composition of helium (Polve and Kurz, 1989). Approximately 50\% of the helium in these crystals is released by crushing, the remainder is presumably dissolved in the crystal lattice, rather than trapped in fluid inclusions. These two components have identical helium isotopic compositions (table 4.1). In thin section, the pyroxene grains were not zoned, and were generally free from internal cracks. Fifteen separated pyroxene grains were examined by back-scattered electron imaging and no evidence of extensive pyroxene exsolution was found at the resolution of this technique (a micron or so). However, exsolved, lamellar chrome spinel inclusion on the order of $5-15$ by $1-5$ microns were found in abundances ranging up to 10 or slightly more per 500-1000um grain. In addition, in 3 of the 15 grains some evidence of slight compositional variability (Wollastonite 46-48) was found in diffuse zones on the order of 100 um in size. In general, the appearance of the grains did not suggest the presence of any features which might make the diffusive length scale differ greatly from the physical grain size.

The 500-710um sieve fraction used in all but one experiment was composed primarily of broken grains from the porphyroclastic whole rock. The larger grain separate $(.5-1 \mathrm{~mm}$, expt. \#8) was more representative of the original grain sizes. Both size fractions had small (1\% or less by volume) amounts of adhering neoblasts of olivine and orthopyroxene. These phases contribute an even smaller fraction of the total helium because of their low helium contents (Polve and Kurz, 1989). In contrast to all the other samples, the larger grain size separate also had small amounts ( $2 \%$ by volume) of adhering basaltic matrix. (The experiment with this separate, designated \#8 here, was performed first as a trial.) All the mineral separates were cleaned by ultrasonic agitation in distilled water, acetone, and methanol prior to analysis. 
Table 4.1 Sample compositions and bulk helium contents.

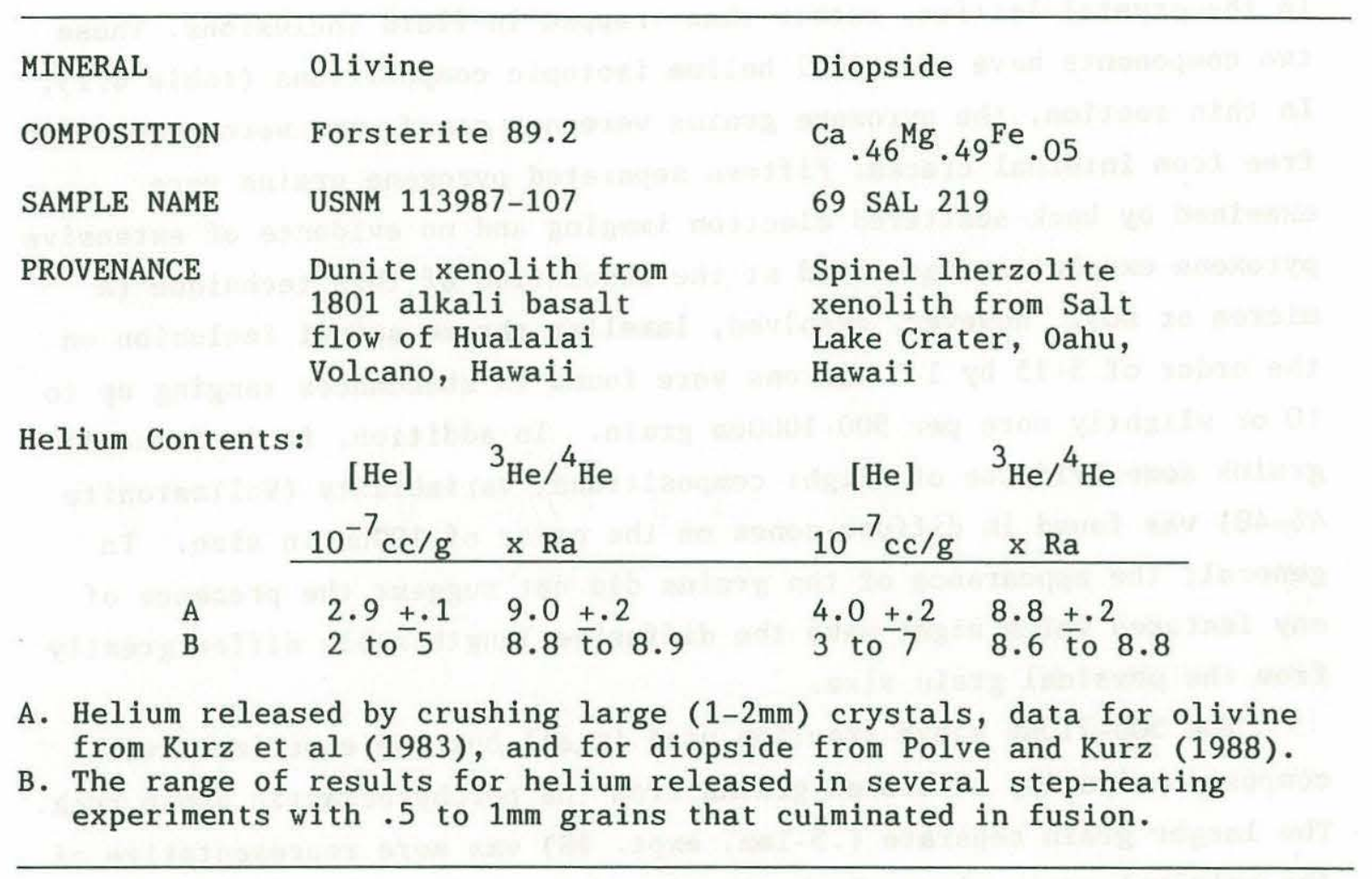


For both olivine and diopside, three isothermal multiple-release experiments were performed in the temperature range $960-1260^{\circ} \mathrm{C}$, using 500-710 um grains. These experiments (designated \#1-3 for olivine and \#5-7 for diopside) allowed the nature of the helium release mechanism to be considered from the time dependence of sequential releases and provided a three point temperature trend. In addition, for both phases, experiments were run in which the temperature was increased after partial release, to obtain an estimate of the temperature dependence from a single sample, which minimizes grain size effects (experiments 4 and 8 ). These experiments also extended the temperature range to 750 to $1400^{\circ} \mathrm{C}$. Figure 4.3 shows that for low extents of fractional release (F less than .4 or so) the time dependence of helium release varied linearly with the square root of time, as is consistent with volume diffusion. In contrast, loss by leaks along cracks from a large internal reservoir such as a fluid inclusion would be expected to display a linear $F$ versus time relation at low to medium $F$, and this was not observed (figure 4.3). (A more complete discussion of helium release systematics and their relation to release mechanisms appears in chapter 3, section 3.3.3) The general smoothness of the releases also argues against a loss process involving disruption of fluid inclusions or crystal fracture. This is consistent with the visual examination $(80 \mathrm{x})$ of pyroxene grains after heating at $770^{\circ} \mathrm{C}$ in experiment \#8, which revealed no changes, and with the unchanging appearance of the grains as observed in the crucible through the sapphire window. In summary, it appears helium release from both olivine and pyroxene was. controlled by diffusion during the experiments.

Diffusion coefficients calculated using the spherical model (designated Dsm in the data tables, Appendix C) are generally constant for constant temperature, as shown in figure 4.4. However, there are several exceptions, including trends of increasing $D$ values at low $F$ in experiments 2 and 4 with olivine, and decreasing $D$ values for pyroxene at both low and high $\mathrm{F}$ in experiment 8 . Understanding the cause of these trends is important to obtaining best estimates for the 
Figure 4.3 Fractional release of He versus time and versus the square root of time. The top plots are for 0livine ( 0 expt. 1 , 口 expt. 2, $\Delta$ epxt. $3, \diamond$ expt. 4$)$ and the bottom plots for pyroxene ( 0 expt. 5, 口 expt. 6, $\Delta$ epxt. 7, $\diamond$ expt. 8). Filled symbols designate Load, Ramp, or Cool steps. The time dependence of helium release is more consistent with volume diffusion (linear in $\mathrm{t}^{1 / 2}$ at low $\mathrm{F}$ ) than with leaks from fluid inclusions (expected to be linear in $t$, perhaps with excursions). 
Figure 4.3
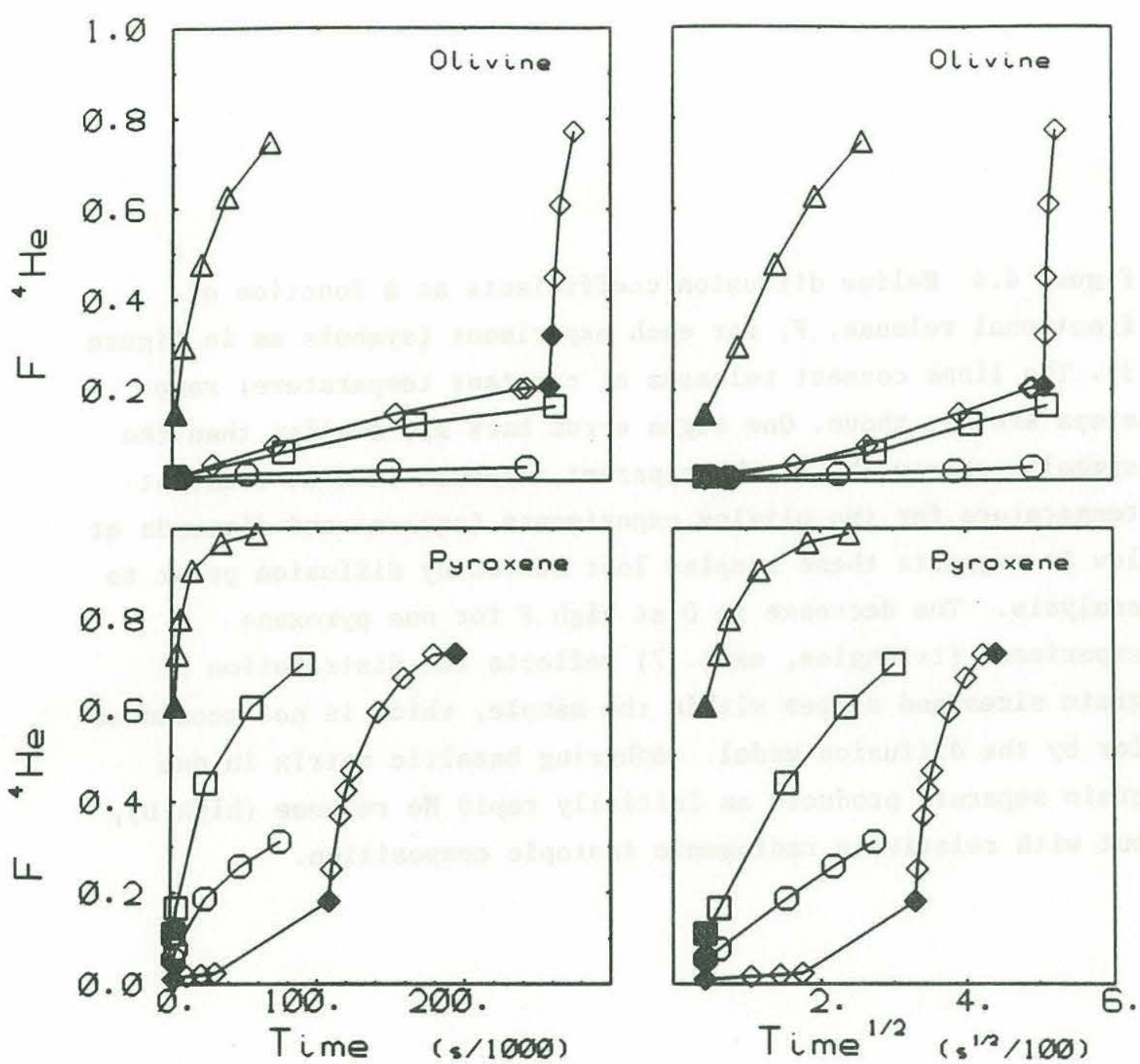
Figure 4.4 Helium diffusion coefficients as a function of fractional release, $F$, for each experiment (symbols as in figure 3). The lines connect releases at constant temperature; ramp steps are not shown. One sigma error bars are smaller than the symbols, unless shown. The apparent increase in $\mathrm{D}$ at constant temperature for two olivine experiments (squares and diamonds at low F) suggests these samples lost helium by diffusion prior to analysis. The decrease in $\mathrm{D}$ at high $\mathrm{F}$ for one pyroxene experiment (triangles, expt. 7) reflects the distribution of grain sizes and shapes within the sample, which is not accounted for by the diffusion model. Adhering basaltic matrix in one grain separate produced an initially rapid He release (high D), but with relatively radiogenic isotopic composition. 
Figure 4.4

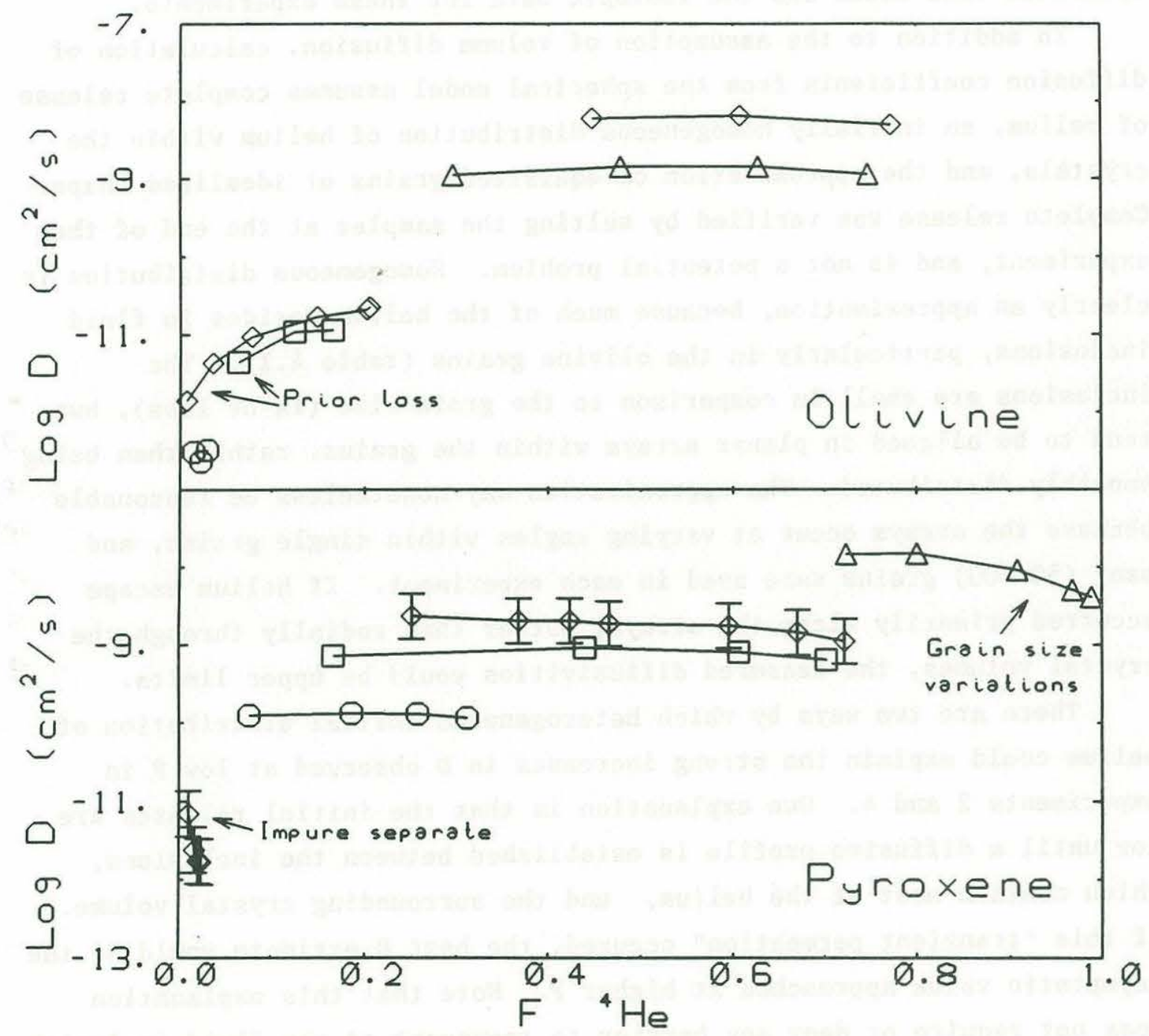


diffusivity of helium in these minerals. These trends are not the result of temperature variations, because pyrometric temperatures and furnace currents were constant (within $10^{\circ} \mathrm{C}$ ) during the experiments and the long emanation times (hours to days) suggest thermal equilibrium. Instead, the explanation lies in the nature of the helium distribution in the samples, as becomes apparent on examining the assumptions of the spherical loss model and the isotopic data for these experiments.

In addition to the assumption of volume diffusion, calculation of diffusion coefficients from the spherical model assumes complete release of helium, an initially homogeneous distribution of helium within the crystals, and the approximation of equisized grains of idealized shape. Complete release was verified by melting the samples at the end of the experiment, and is not a potential problem. Homogeneous distribution is clearly an approximation, because much of the helium resides in fluid inclusions, particularly in the olivine grains (table 4.1). The inclusions are small in comparison to the grain size ( $1 \%$ or less), but tend to be aligned in planar arrays within the grains, rather than being smoothly distributed. The approximation may nonetheless be reasonable because the arrays occur at varying angles within single grains, and many (50-100) grains were used in each experiment. If helium escape occurred primarily along the arrays, rather than radially through the crystal volumes, the measured diffusivities would be upper limits.

There are two ways by which heterogeneous initial distribution of helium could explain the strong increases in $\mathrm{D}$ observed at low $\mathrm{F}$ in experiments 2 and 4 . One explanation is that the initial releases are low until a diffusive profile is established between the inclusions, which contain most of the helium, and the surrounding crystal volume. If this "transient permeation" occured, the best $D$ estimate would be the asymptotic value approached at higher $\mathrm{F}$. Note that this explanation does not require or deny any barrier to transport at the fluid inclusion - crystal interface, but just reflects departures between the model and true initial distributions. However, this mechanism would predict that release rates increase over the $\mathrm{F}$ ranges in question ( 0 to $20 \%$, figure 4.4), and they do not. Instead, the release rates drop by about a factor of 2. (Increasing $D$ values still result because the spherical 
model predicts release rates should drop by a factor of 8 over this $F$ range.) A better explanation is that the grains have already established diffusive profiles from helium loss prior to analysis, as could have occurred during transport in the host magma or in the cooling lava flow (Hart, 1984; discussion below). As discussed in chapters 2 and 3 , this condition leads to underestimated $\mathrm{D}$ values that increase assymptotically with $\mathrm{F}$ to true diffusivities (e.g. figure 2.6). This behavior is consistent with the $\mathrm{D}$ versus $\mathrm{F}$ trends observed, assuming prior losses of 8 to $10 \%$, and suggests the best diffusivity estimates in these experiments are the highest values.

It is also worth noting that, in principle, apparent diffusivity variations related to transient permeation may be distinguished from prior loss problems by differing isotopic effects. Transient permeation from the fluid inclusions to the crystal surfaces would significantly increase preferential release of ${ }^{3} \mathrm{He}$ in comparison to loss from a solid with homogeneous distribution. This effect was demonstrated for neon isotopes in synthetic glass, and discussed theoretically, by Rama and Hart (1965). The extent of isotopic fractionation depends only on the ratio of release rate during the transient to the rate at steady state, for a given isotopic diffusivity ratio. In contrast, prior loss slightly reduces the ${ }^{3} \mathrm{He} /{ }^{4} \mathrm{He}$ ratios measured in later releases, because some preferential ${ }^{3} \mathrm{He}$ loss has already occured. Unfortunately, the helium isotopic ratios in these experiments could not be determined precisely enough to distinguish these possibilities, as is discussed further below.

Violation of the single grain size assumption is almost certainly responsible for the decrease in $D$ values at high $F(>.8)$ for pyroxene in experiment 7 . The apparent $D$ values decrease because smaller grains have released all their helium, as shown in model calculations in chapter 2 and for real distributions of glass particle sizes in chapter 3. The values of $\mathrm{D}$ obtained at lower $\mathrm{F}$ are most reliable, because they are least affected by the misfit between the model assumptions and the true helium distribution among the different grains. Finally, the decrease in $\mathrm{D}$ values at low $\mathrm{F}\left(770^{\circ} \mathrm{C}\right)$ in experiment 8 reflects early enhanced helium loss from adhering basaltic matrix (present in this 
experiment only). This is apparent from the low ${ }^{3} \mathrm{He} /{ }^{4} \mathrm{He}$ ratios of the first releases (data table 8, Appendix C). Thus, the lower D values obtained at higher $\mathrm{F}$ at this temperature are most reliable for diffusion in the pyroxene itself.

In summary, many of the $D$ variations can be understood in light of the model assumptions, and this understanding allows best estimates to be made for the diffusivities. While a refined grain size model could be applied (as in chapter 3 ), the extra effort is not warranted given other uncertainties, such as the internal helium distribution and temperature control. It is important to recognize that calculated diffusivities have inherent uncertainties, and potentially systematic inaccuracies, that derive from model simplifications, in addition to analytical uncertainties. In these experiments, potential errors from model misfits are estimated to be a factor of 2 or so based on experience with the refined model of chapter 3 , and possibly more in experiment 8 which used a larger range of grain sizes.

The temperature dependence of helium diffusion displays simple exponential (Arrhenius) relations for both olivine and diopside (figure 4.5). The lines $\left(\log D=\log D^{O}-E_{a} / R T\right)$ in this figure were estimated considering the already discussed systematics within individual experiments and, as is readily apparent, are not best fits to all the data. Considering the Arrhenius slopes reasonably accomodated by the data, the activation energies are distinctly different for the two phases, $100 \pm 5 \mathrm{Kcal} / \mathrm{mole}$ in olivine and $70 \pm 10 \mathrm{Kcal} / \mathrm{mole}$ in diopside (table 4.2). The diffusivities and activation energy determined for olivine are in good agreement with measurements by Hart (1984), but are somewhat higher than the results determined by Gramlich and Naughton (1972; see figure 4.6). Gramlich and Naughton also found a lower diffusivity for pyroxene than measured here (Gramlich and Naughton, 1972), although this could reflect the mix of orthopyroxene and clinopyroxene used in their experiment, if the orthopyroxene contained a significant fraction of the helium and had a lower helium diffusivity. In contrast, extrapolating lower temperature results for radiogenic ${ }^{4} \mathrm{He}$ diffusion in augite (Lippolt and Weigel, 1988) suggests much higher diffusivities than observed here (figure 4.6 ). The difference may 
Figure 4.5 Arrhenius diagrams for helium diffusion in olivine and pyroxene. Symbols identify individual experiments as in figure 3. The error symbol, lower left, denotes the upper range of 1 sigma errors. Lines are best estimates based on diffusivity systematics within and between individual experiments and are not regressions to all the data. The highest $\mathrm{D}$ values obtained in experiments affected by prior loss or size distribution at high $\mathrm{F}$ were judged most reliable, and the lowest $\mathrm{D}$ values in one experiment with an impure mineral separate (see text). Note that the temperature trends determined in the step-heating experiments (diamonds) are similar to the isothermal results (all other points). 
Figure 4.5

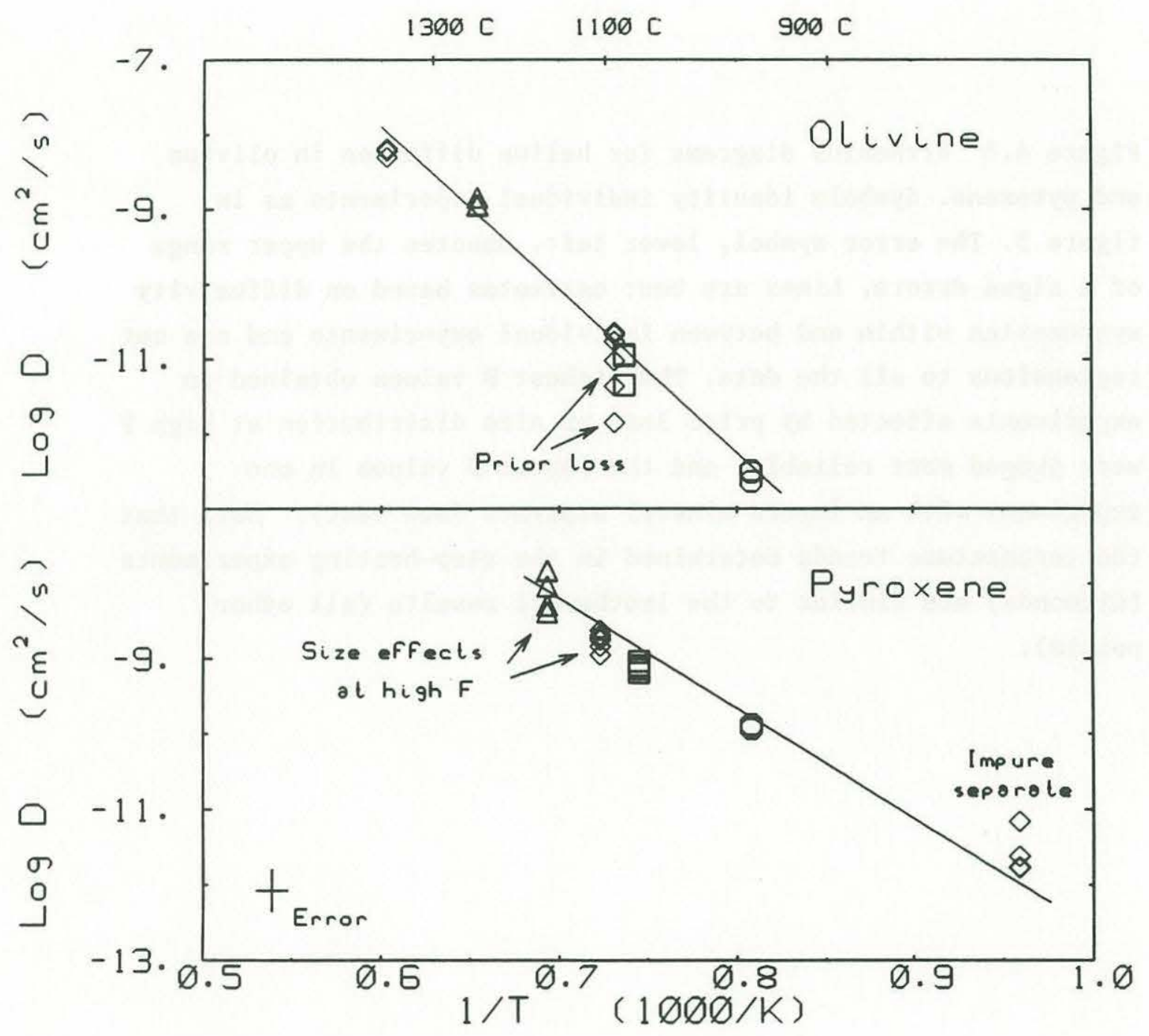


Table 4.2 Helium Diffusivity Results

\begin{tabular}{lcccc} 
Mineral & $\begin{array}{c}\text { Temp. Range } \\
\left({ }^{\circ} \mathrm{C}\right)\end{array}$ & $\begin{array}{c}\mathrm{E}_{\mathrm{a}} \\
(\mathrm{kcal})\end{array}$ & $\begin{array}{c}\log \mathrm{D}^{\mathrm{O}} \\
\left(\mathrm{cm}^{2} / \mathrm{s}\right)\end{array}$ & $\begin{array}{c}\mathrm{D} \text { at } 1100{ }^{\circ} \mathrm{C} \\
\left(\mathrm{cm}^{2} / \mathrm{s}\right)\end{array}$ \\
\hline Olivine & $965-1385$ & $100 \pm 5$ & ${ }_{5} 5.1 \pm .7$ & $2 \times 10^{-11}$ \\
Diopside & $770-1170$ & $70 \pm 10$ & ${ }^{+} 2.1 \pm 1.2$ & $1 \times 10^{-9}$
\end{tabular}

a. Approximate 1 sigma errors estimated as described in the text are given for $E$ and $D^{\circ}$ values.

b. D estimates at $1100{ }^{\circ} \mathrm{C}$ have 1 sigma errors of a factor of 2, as estimated from data scatter, temperature uncertainty, and particle size range. 
Figure 4.6 Comparison of diffusivity results for pyroxene and olivine. Also shown are literature values, dotted line and anvil symbols for olivine (Hart, 1985); cross for olivine and triangle for a mix of clino- and ortho- pyroxene grains (Gramlich and Naughton, 1972). The dotted line at the upper right is an extrapolation of radiogenic helium diffusivities obtained below $800^{\circ} \mathrm{C}$ in augite (Lippolt and Weigel, 1988). The higher diffusivity in their study may reflect differences between inherited and radiogenic helium, or perhaps compositional effects, and is a cautionary note in the application of the results presented here to other rock types. 
Figure 4.6

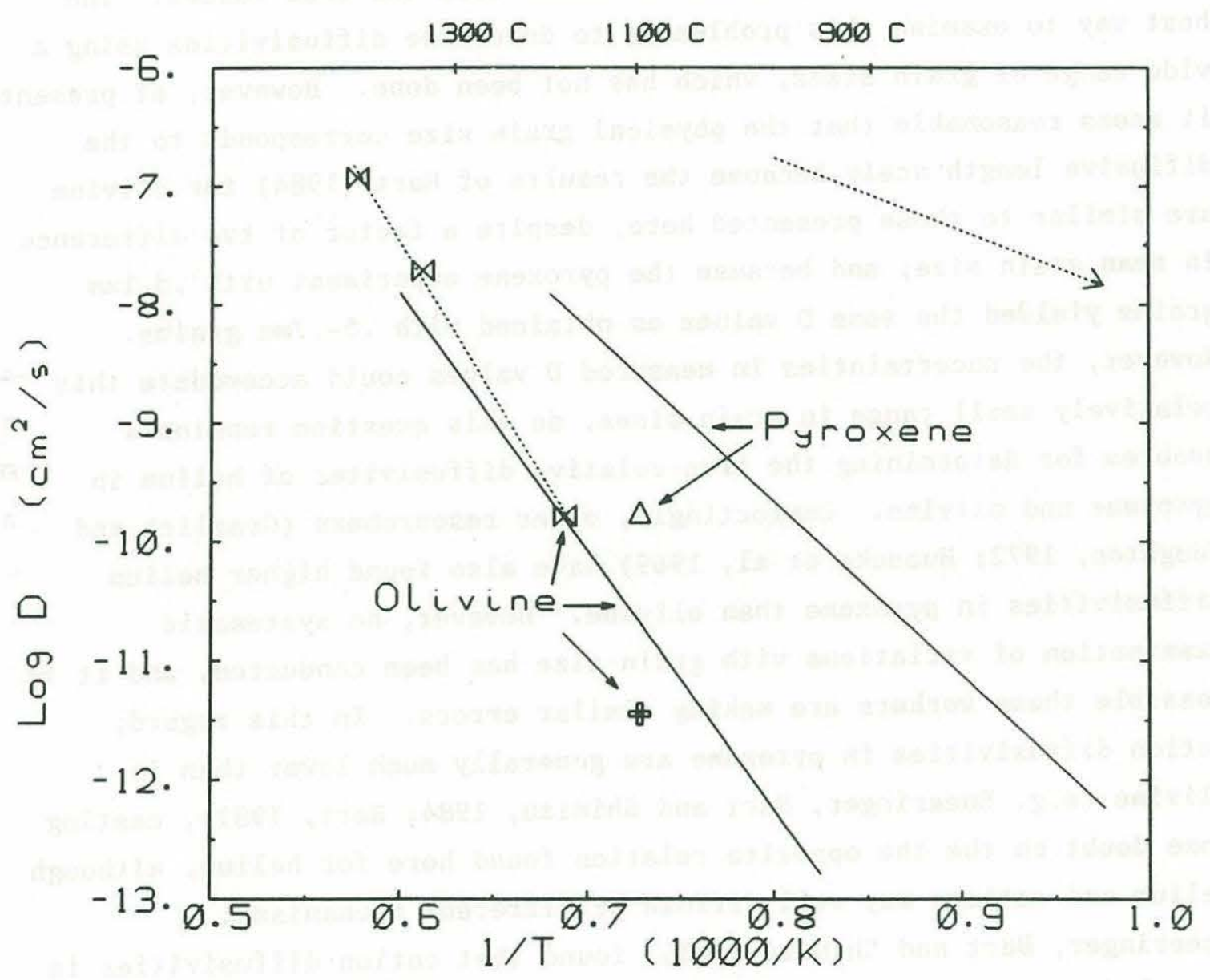


reflect decay-enhanced release, compositional effects, or the possibility that the physical grain size in this experiments is larger than the effective diffusive length scale.

While the activation energy difference between pyroxene and olivine is undoubtedly real, the relative helium diffusivities in these phases are less certain. It is possible that the diffusive length scale differs from the physical grain size in either or both minerals, and thus that the D estimates could be offset from the true values. The best way to examine this problem is to determine diffusivities using a wide range of grain sizes, which has not been done. However, at present it seems reasonable that the physical grain size corresponds to the diffusive length scale because the results of Hart (1984) for olivine are similar to those presented here, despite a factor of two difference in mean grain size; and because the pyroxene experiment with $.5-1 \mathrm{~mm}$ grains yielded the same D values as obtained with .5-.7mm grains. However, the uncertainties in measured D values could accomodate this relatively small range in grain sizes, so this question remains a problem for determining the true relative diffusivites of helium in pyroxene and olivine. Comfortingly, other researchers (Gramlich and Naughton, 1972; Hunecke et al, 1969) have also found higher helium diffusivities in pyroxene than olivine. However, no systematic examination of variations with grain size has been conducted, and it is possible these workers are making similar errors. In this regard, cation diffusivities in pyroxene are generally much lower than in olivine (e.g. Sneeringer, Hart and Shimizu, 1984; Hart, 1981), casting some doubt on the the opposite relation found here for helium, although helium and cations may well diffuse by different mechanisms. Sneeringer, Hart and Shimizu (1984) found that cation diffusivities in annealed synthetic pyroxenes were much lower than in natural samples (and varied with crystallographic direction). This may reflect a difference between physical and diffusive grain sizes in the natural samples, or perhaps enhanced mobility in response to higher defect populations. To summarize, until the relation between physical and diffusive length scales in the laboratory is better understood (and the role of defects in governing diffusion is documented), the suggestion 
that helium diffuses more rapidly in pyroxene than olivine (and more rapidly than cations) must be viewed with caution. Given this caveat, in the discussion that follows the helium diffusivities are taken at face value.

The isotopic compositions of sequential releases in the emanation experiments are consistent with a small preferential loss of ${ }^{3} \mathrm{He}$, although the analytical uncertainties are large (figure 4.7). These trends of ${ }^{3} \mathrm{He} /{ }^{4} \mathrm{He}$ versus extent of release (F) and the isotopic diffusivity ratios calculated for individual aliquots (experimental data tables 1-8, Appendix $C$ ) suggest a $D^{3} \mathrm{He} / \mathrm{D}^{4} \mathrm{He}$ value between 1.0 and 1.15 , with the possibility of a lower value in pyroxene $(1.04 \pm .04)$ than in olivine $(1.08 \pm .04)$. Both phases suggest values somewhat lower than 1.15, corresponding to the inverse square-root of the ratio of the masses, $\left(\mathrm{m}_{4} / \mathrm{m}_{3}\right)^{1 / 2}$. As discussed in detail in chapter 3 , there are several reasons for departures from this relationship, including diffusion mechanisms which involve correlation of successive atomic jumps, coupled motions of the diffusing atom and host crystal atoms, and vibrational energy quantization for light atoms like helium. The quantum explanation was found to best account for the low isotopic diffusivity ratio in basaltic glasses and is probably also tenable for the crystals studied here at higher temperature (see chapter 3 ) The small isotopic diffusivity ratio, $\mathrm{D}^{3} \mathrm{He} / \mathrm{D}^{4} \mathrm{He}$, implies large amounts of helium loss must occur to significantly affect the residual ${ }^{3} \mathrm{He} /{ }^{4} \mathrm{He}$ ratio (figure 4.8 ).

In summary, helium release from olivine and diopside mineral separates in laboratory heating experiments appears to be dominated by volume diffusion at magmatic and mantle temperatures. The temperature dependence of helium mobility is different for the two phases with loss from pyroxene occuring about 50 times faster than in olivine at $1100^{\circ} \mathrm{C}$ but with similar loss rates at higher temperatures, near $1550^{\circ} \mathrm{C}$. The measured helium diffusivities are higher than most cation mobilities in pyroxenes and olivine. Figure 4.9 shows the helium results in comparison to a compilation of pyroxene diffusivities (Sneeringer, Hart and Shimizu, 1984). He is more mobile than all cations at $1100^{\circ} \mathrm{C}$, except $\mathrm{Pb}$, and has a similar diffusivity to argon. In olivine, helium 
Figure 4.7 Helium isotopic data for emanation experiments. The average ${ }^{3} \mathrm{He} /{ }^{4} \mathrm{He}$ obtained by summing the incremental losses are shown for each phase by solid lines, along with the expected fractionation trends for isotopic diffusivity ratios of 1.15 (dotted) and 1.04 (dashed), assuming spherical geometry. Overall, the data suggest a somewhat lower isotopic diffusivity ratio for pyroxene $(1.04+.04)$ than for olivine $(1.09+.04)$. 
Figure 4.7

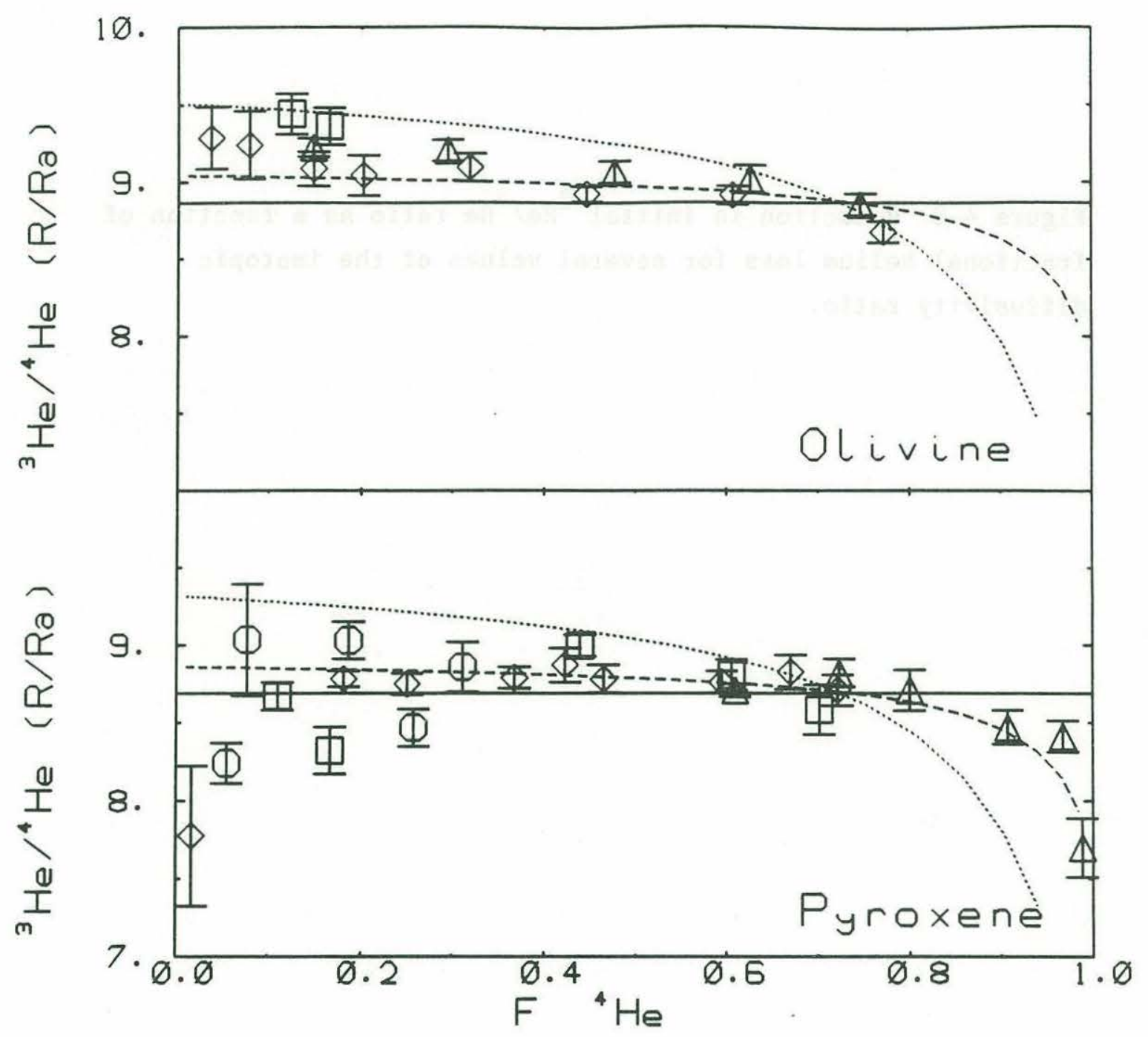


Figure 4.8 Reduction in initial ${ }^{3} \mathrm{He} /{ }^{4} \mathrm{He}$ ratio as a function of fractional helium loss for several values of the isotopic diffusivity ratio. 
Figure 4.8

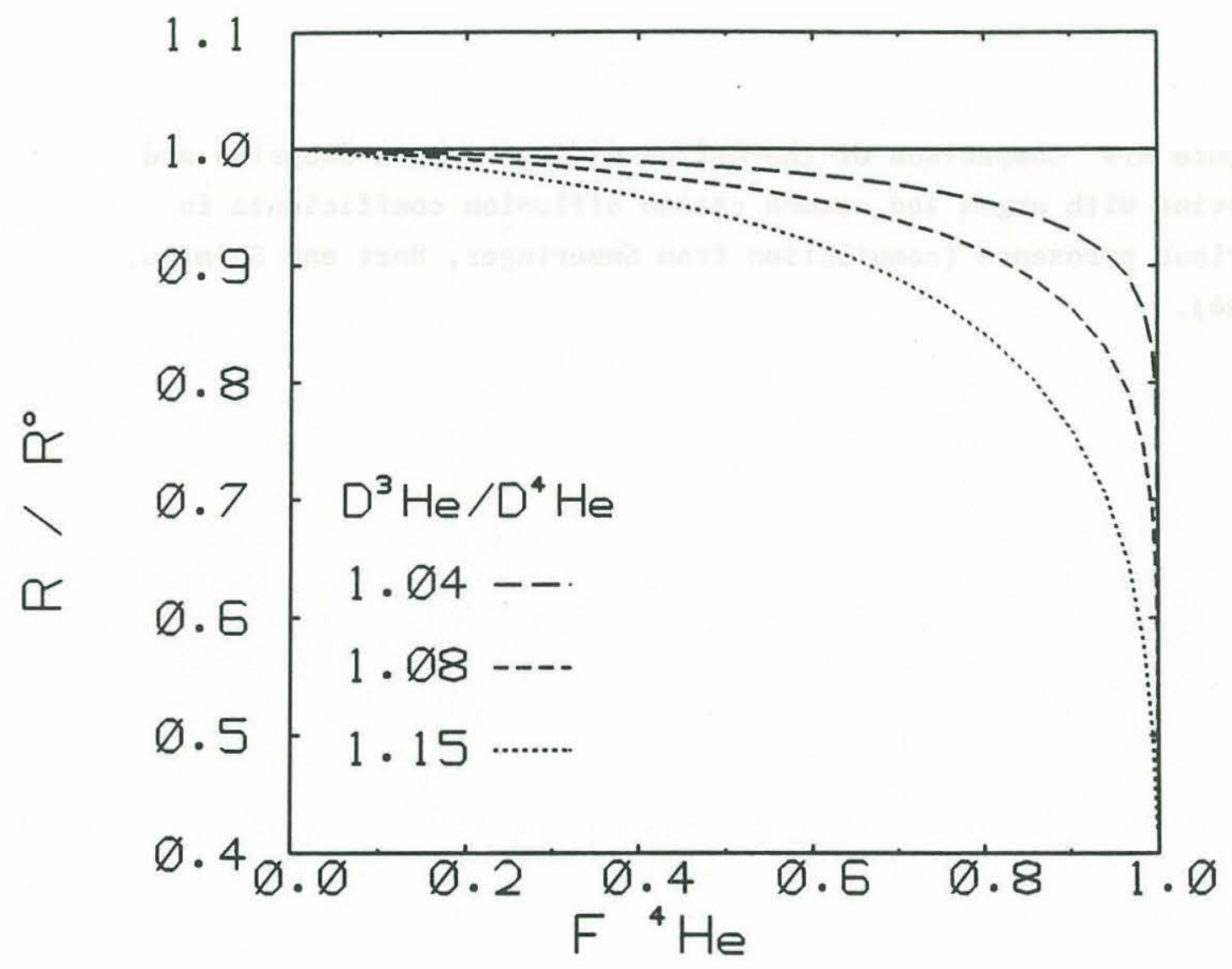


Figure 4.9 Comparison of the helium diffusivity in diopside and olivine with argon and common cation diffusion coefficients in various pyroxenes (compilation from Sneeringer, Hart and Shimizu, 1984). 
Figure 4.9

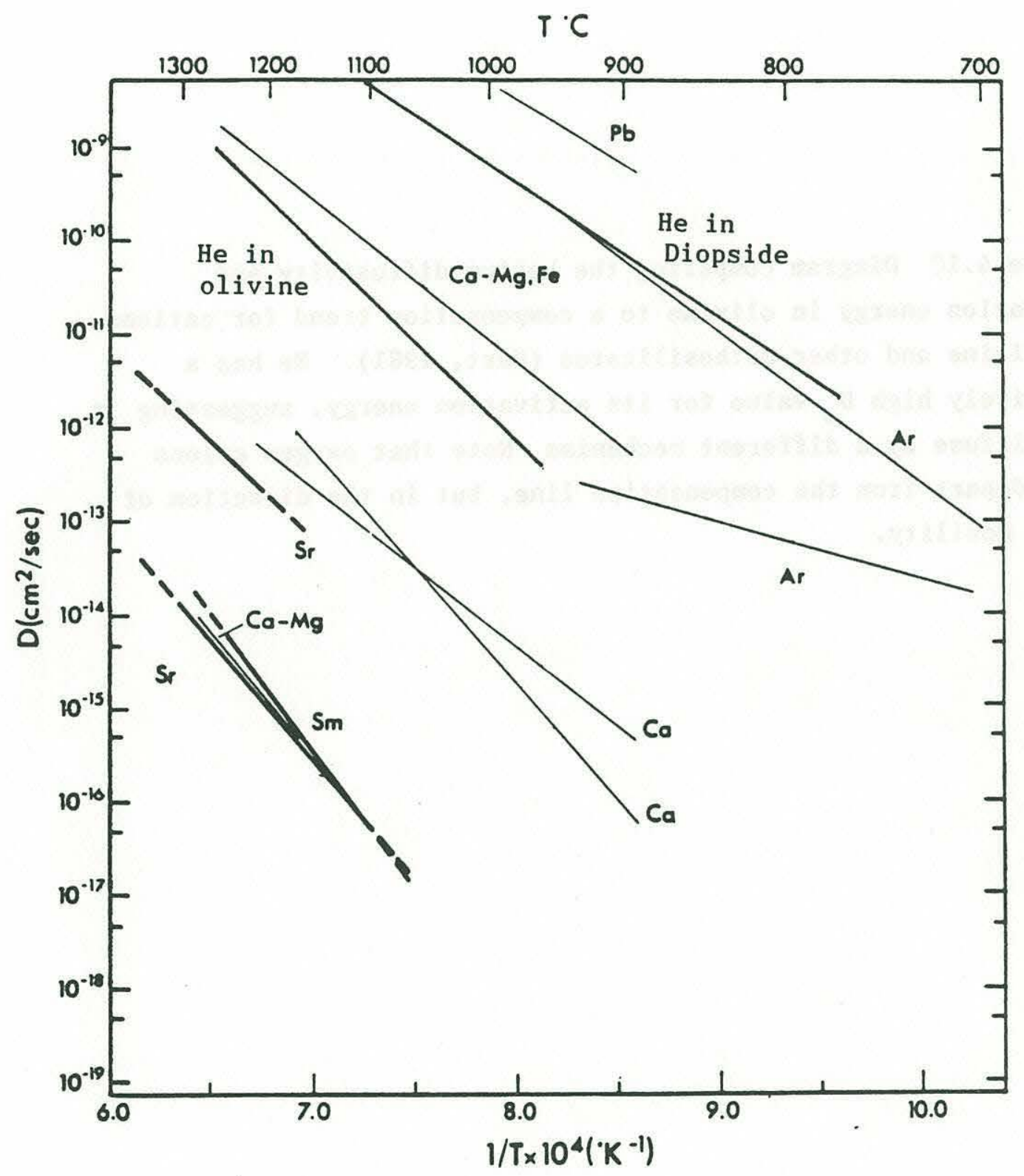


Figure 4.10 Diagram comparing the helium diffusivity and activation energy in olivine to a compensation trend for cations in olivine and other orthosilicates (Hart, 1981). He has a relatively high $D_{0}$ value for $i$ ts activation energy, suggesting it may diffuse by a different mechanism. Note that oxygen anions also depart from the compensation line, but in the direction of lower mobility. 
Figure 4.10

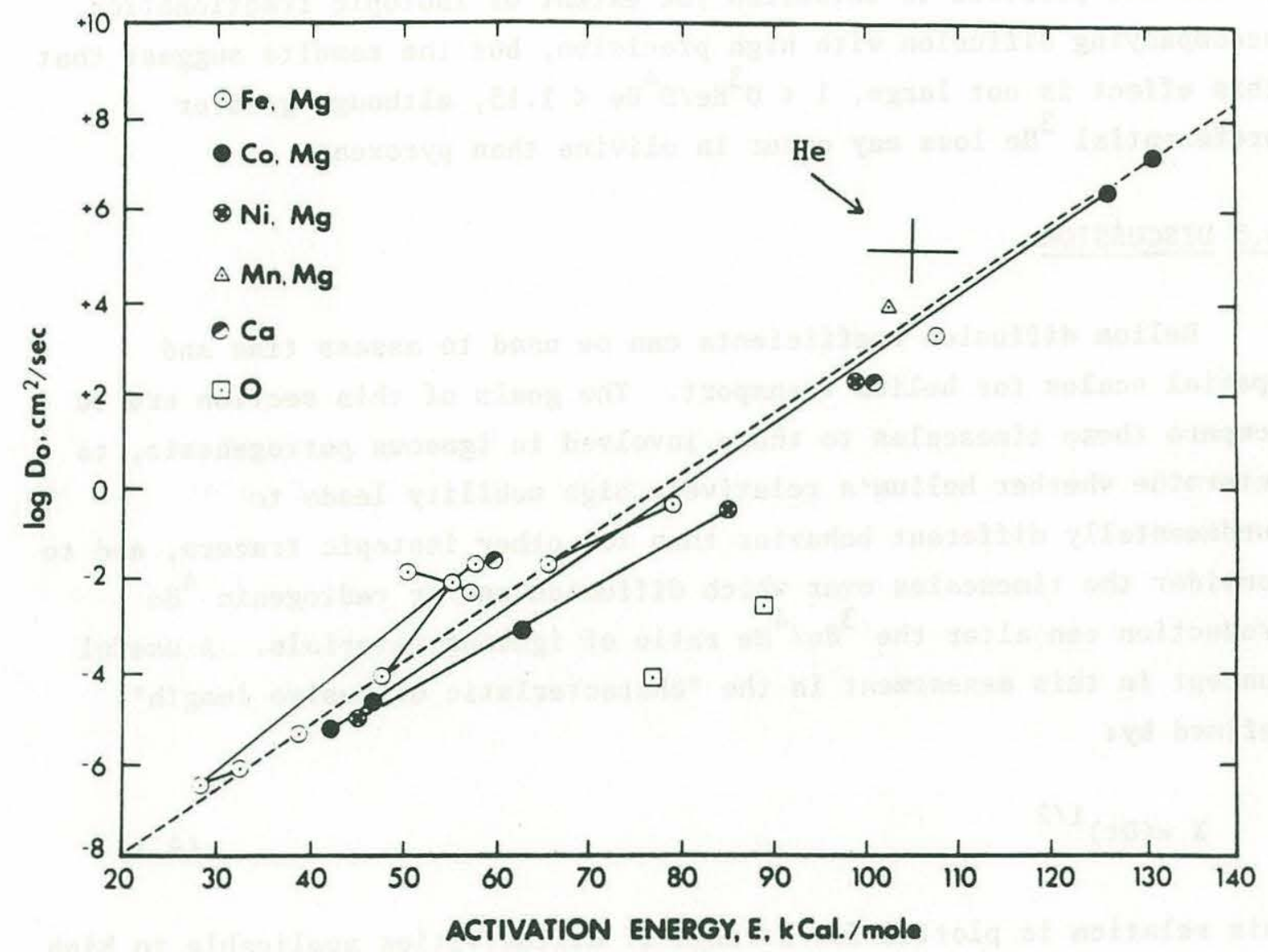


diffusion is also enhanced over cations, and along with oxygen anions departs from the compensation trend observed for many elements (figure 4.10; Hart, 1981). These differences are reasonably explained by an interstitial mechanism for helium and argon diffusion, in contrast to vacancy diffusion for the cations and larger, close-packed oxygen ions. It was not possible to establish the extent of isotopic fractionation accompanying diffusion with high precision, but the results suggest that this effect is not large, $1<\mathrm{D}^{3} \mathrm{He} / \mathrm{D}^{4} \mathrm{He}<1.15$, al though greater preferential ${ }^{3} \mathrm{He}$ loss may occur in olivine than pyroxene.

\subsection{DISCUSSION}

Helium diffusion coefficients can be used to assess time and spatial scales for helium transport. The goals of this section are to compare these timescales to those involved in igneous petrogenesis, to determine whether helium's relatively high mobility leads to fundamentally different behavior than for other isotopic tracers, and to consider the timescales over which diffusion and/or radiogenic ${ }^{4} \mathrm{He}$ production can alter the ${ }^{3} \mathrm{He} /{ }^{4} \mathrm{He}$ ratio of igneous materials. A useful concept in this assessment is the "characteristic diffusive length", defined by:

$$
X=(D t)^{1 / 2}
$$

This relation is plotted for a range of diffusivities applicable to high temperature transport in olivine, pyroxene, and tholeiitic melt in figure 4.11. The meaning of the characteristic length is clarified by considering that $99 \%$ of diffusive loss, gain or exchange of helium will occur for a spherical volume of diameter $\mathrm{X}$ in time $t$, and that $50 \%$ transfer occurs in about $1 / 5$ this time, as shown by the curves (scaled to phenocryst sizes) given in figure 4.12.

Proceeding sequentially from the mantle source region to melt generation, magma transport, chamber storage, and eruption; the effect of diffusive helium transport on natural variations in helium isotopic compositions is considered. Two general principles are worth noting. 
Figure 4.11 Characteristic diffusive length scales $\left(x^{2}=D t\right)$ for helium diffusion in olivine (dashed lines) and diopside (solid lines) at magnetic and mantle temperatures. The dotted line is for $D=5 \times 10^{-5} \mathrm{~cm}^{2} / \mathrm{s}$ in tholeitic melt at $1350^{\circ} \mathrm{C}$ (Lux, 1987). 
Figure 4.11

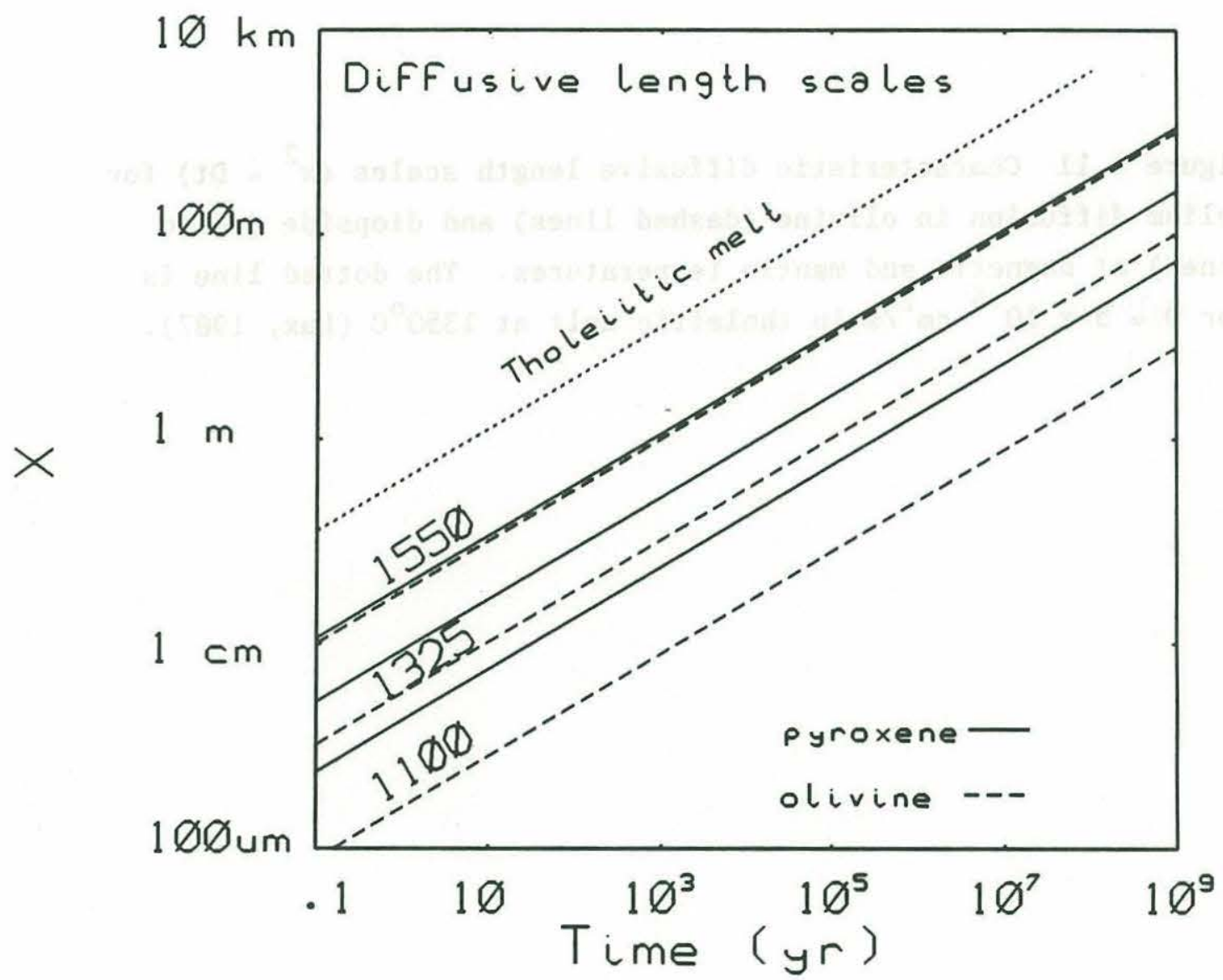


Figure 4.12 Fractional loss or equilibration curves for helium diffusion from a sphere for several values of the diffusion coefficient and grain radius (a). For example, $2 \mathrm{~mm}$ diameter diopside grains with a diffusivity of $1 \times 10^{-9} \mathrm{~cm}^{2} / \mathrm{s}$ at $1100^{\circ} \mathrm{C}$ have a $\log \mathrm{D} / \mathrm{a}^{2}$ value of -7 and will lose $60 \%$ of their initial helium in a few days, provided the surrounding magma or rock contains no helium. These curves also apply to the timescales required to exchange helium with the surrounding medium. 
Figure 4.12

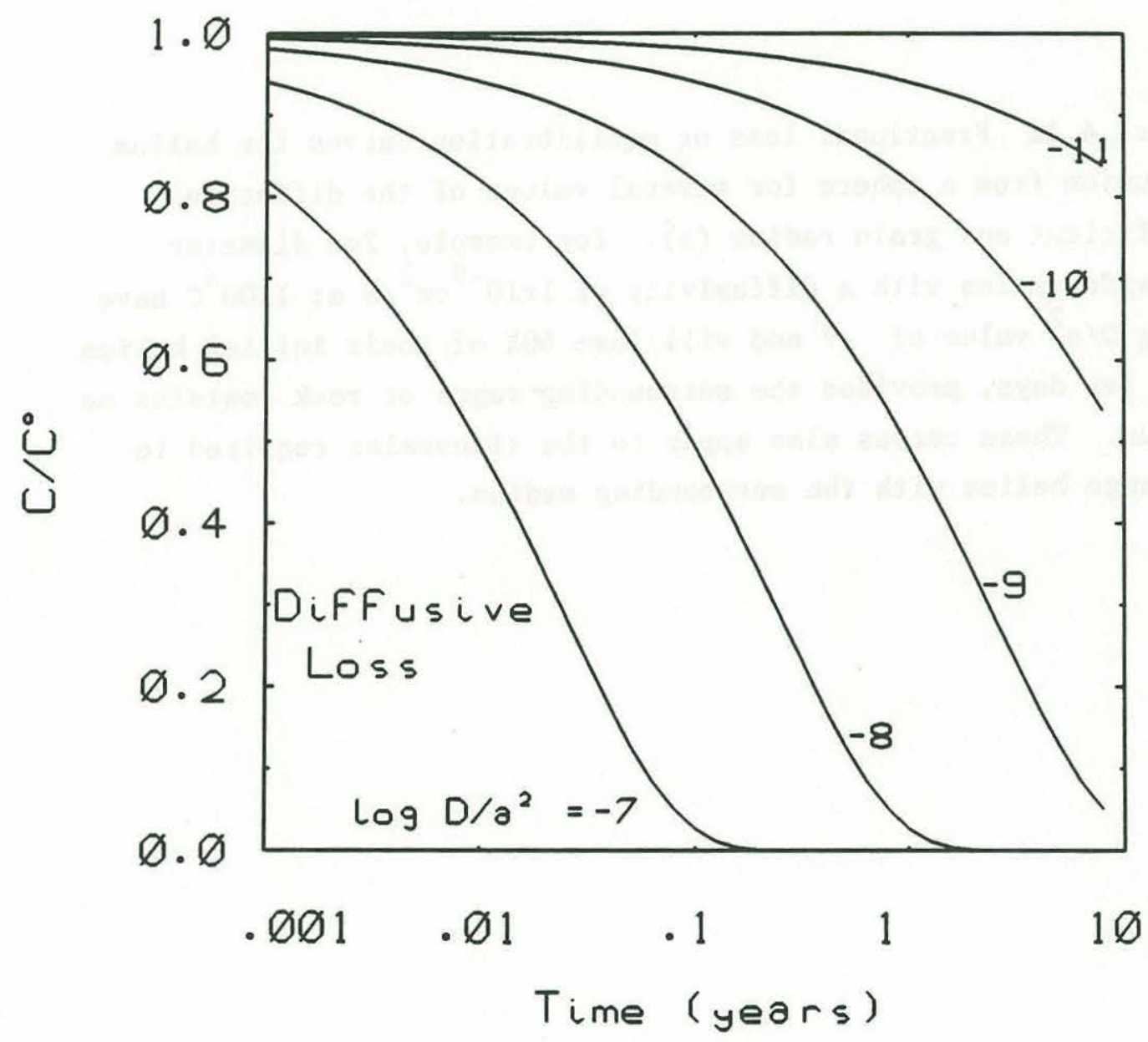


Figure 4.11 emphasizes that helium diffusion in the solid state proceeds very slowly in comparison to transport in magmas, where the helium diffusivity is about $5 \times 10^{-5} \mathrm{~cm}^{2} / \mathrm{s}$ (Lux, 1987). This is also true for isotopic tracers such as $\mathrm{Pb}, \mathrm{Sr}$ and $\mathrm{Pb}$ and cations in general which have melt diffusivities on the order of $10^{-6}$ to $10^{-7} \mathrm{~cm}^{2} / \mathrm{s}$ (e.g. Hoffman, 1980 and figure 3.25, chapter 3 ). Thus, when melt is present it will dominate diffusive mass transport. Secondly, conductive heat transport occurs far more rapidly than diffusive elemental migration (thermal diffusivities are on the order of $10^{-2} \mathrm{~cm}^{2} / \mathrm{s}$; e.g. Spera, 1980), suggesting cooling may limit diffusive transport in igneous systems which are not maintained at high temperature by insulating boundaries or heat inputs. In this regard, heat flow is a good analogy for diffusive mass transport, because it emphasizes the dependence on both the diffusivity (conductivity), and the difference in chemical potential (temperature) between the exchanging reservoirs. For example, a high helium diffusivity will not lead to gas loss from a phenocryst if the surrounding magma or cooling lava is helium rich.

Diffusion is not an effective means of transporting helium in the mantle on a large scale. At $1550^{\circ} \mathrm{C}$, the characteristic diffusive length scale is only $1 \mathrm{~km}$ after 1 billion years (figure 4.11). The presence of melt enhances transport, but not greatly, $\mathrm{X}$ is about $13 \mathrm{~km}$ considering the diffusivity in magma of about $5 \times 10^{-5} \mathrm{~cm}^{2} / \mathrm{s}$ to apply to the mantle. The presence of a carbon-oxygen-hydrogen fluid in the mantle is likely to lead to a similar estimate, because helium diffusion in water is similar to the melt value (Jahne, Heinz and Dietrich, 1987). Grain boundary diffusion may enhance helium transport, but will occur more slowly than tranport in a melt or fluid phase (e.g. Hoffman and Hart, 1978). Pressure effects may alter these length scales to some degree, but not greatly given the less than an order of magnitude variations in diffusivities (factor of 3 in $\mathrm{X}$ ) observed in experiments up to $10^{\prime} \mathrm{s}$ of kilobars (e.g. Watson, 1981; Shimizu and Kushiro, 1984; Sneeringer, Hart and Shimizu, 1984).

Therefore, helium can not "permeate" the mantle, and large scale helium transport must occur by convection, as it does for other elements. This means that the relatively narrow range of ${ }^{3} \mathrm{He} /{ }^{4} \mathrm{He}$ ratios 
of mid-ocean-ridge basalts (e.g. Kurz, 1982; Mamyrin and Tolstikhin, 1984) is related to mantle mixing processes, rather than properties of helium transport. In addition, different regions of the mantle can build up varying helium contents and isotopic compositions by radioactive decay, depending on their uranium and thorium contents. This process may explain the low ${ }^{3} \mathrm{He} /{ }^{4} \mathrm{He}$ region of the Mid-Atlanticridge adjacent to the Azores which was suggested to derive from uranium enriched, subducted lithosphere by Kurz et al (1982).

On a mineral scale diffusion becomes important as a control on helium isotopic compositions and the separation of helium from other elements. Mineral grains will equilibrate rapidly with a melt or other crystals. For example, for $2 \mathrm{~mm}$ grains the characteristic timescale is 63 years for olivine and $1.3 \mathrm{yr}$ for pyroxene at $1100^{\circ} \mathrm{C}$ (figure 4.11). Therefore, equilibrium will govern helium separation during most melt generation and fractional crystallization events. This means that helium isotopic zoning in crystals is not to be expected, a contrast with some other cationic tracers. In addition, the generation of different ${ }^{3} \mathrm{He} /{ }^{4} \mathrm{He}$ ratios in minerals with varying $\mathrm{U} / \mathrm{He}$ ratios will tend to be homogenized by diffusion within the mantle and lower lithosphere. The conclusion that the mantle is isotopically homogeneous on a mineral scale and melts to yield isotopic compositions characteristic of the bulk source region, have been previously championed by Hofmann and Hart (1978) who considered more detailed inter-mineral exchange constraints, and the lower diffusivities of $\mathrm{Sr}$ and other cations. However, the possibility of dramatically different grain sizes and defect populations (and thus effective diffusivities) in the mantle than in laboratory experiments makes this conclusion less than absolute, as pointed out in the more recent discussion by Sneeringer, Hart and Shimizu (1984).

Interaction of migrating melts with mantle solids or lithospheric and crustal wall rocks may be enhanced for helium in comparison to other trace elements, because of its higher mobility. The specific extents of exchange depend on the magma migration rate, flow geometry (e.g. conduit or porous flow), temperature, and partitioning of helium between the solids and the melt (e.g. Navon and Stolper, 1987). Kurz et al, 1987 have suggested that this process could explain the fact that temporal 
helium isotopic changes in Hawaiian basalts are more pronounced than for strontium isotopes. It is difficult to quantify models of this type and as pointed out by these workers, differing partition coefficients and degrees of melting also produce elemental abundance variations, which affect the degree to which wall-rock interaction alters isotopic compositions. However, enhanced exchange for helium will clearly be more important in the lithosphere than at crustal depths where magma transport is likely to occur in larger conduits driven by crustal fracture (Spera, 1980).

Diffusive transport of helium in magma is slow enough that stratified magma chambers derived from sequential episodes of injection, assimilation and fractional crystallization can maintain different ${ }^{3} \mathrm{He} /{ }^{4} \mathrm{He}$ ratios for long times. For example, over a million years is required to homogenize a $1 \mathrm{~km}$ magma body, considering the diffusivity in tholeiitic melt (figure 4.11). In order for diffusive degassing to change initial magmatic ${ }^{3} \mathrm{He} /{ }^{4} \mathrm{He}$ ratios by preferential ${ }^{3} \mathrm{He}$ transport large gas losses must occur. For example, a $10 \%$ lowering of the initial ratio requires $65 \%$ gas loss for an isotopic diffusivity ratio of 1.15 (figure 4.8), which would require several hundred thousand years for the example of a $1 \mathrm{~km}$ sphere. Maintaining the magma volume above the solidus for this long would require a thermal contrast between magma and wall rock of less than $10^{\circ} \mathrm{C}$ (e.g. Hart and Zindler, 1987). Many aspects of magma degassing in real volcanos make these conductive and diffusive timescales heuristic at best. However, given the much greater diffusivity of heat than helium, it seems likely that magma bodies, whatever their geometry or rate of convection will tend to cool before significant helium loss occurs, unless they are deep-seated and thermally insulated.

Another way in which magmatic helium isotopic compositions can be lowered during chamber residence is by radiogenic ingrowth of ${ }^{4} \mathrm{He}$. Zindler and Hart (1986) have suggested that exhalative helium degassing accompanying saturation of carbon dioxide may increase the $\mathrm{U} / \mathrm{He}$ ratio of a magma, leading to decreases in its ${ }^{3} \mathrm{He} /{ }^{4} \mathrm{He}$ ratio over times ranging $10^{5}$ to $10^{6}$ years. These workers find suggestive evidence for this process in a correlation of increasing $\mathrm{U} / \mathrm{He}$ ratios with decreasing $\mathrm{Mg}$ 
number (an index of increased fractional crystallization). However, it is not clear that the lower helium contents in the more evolved rocks derive from earlier magma chamber degassing rather than processes associated with eruption. As pointed out by Zindler and Hart (1986), if this process is responsible for significant lowering of mantle ${ }^{3} \mathrm{He} /{ }^{4} \mathrm{He}$ ratios, for example as observed at Hawaiian volcanos over time (Kurz et al, 1987), magma chambers must remain at high temperature for long periods at relatively shallow depths where $\mathrm{CO}_{2}$ degassing can occur. This requires periodic chamber replenishment, and leads to a rapid decrease in ${ }^{3} \mathrm{He} /{ }^{4} \mathrm{He}$ ratios after a long period without much change (Zindler and Hart, 1986). This pattern of ${ }^{3} \mathrm{He} /{ }^{4} \mathrm{He}$ change is similar to that for preferential diffusive loss of ${ }^{3}$ He (figure 4.8 ), and both processes would tend to produce large differences in $3 \mathrm{He} /{ }^{4} \mathrm{He}$ ratios of magma batches which had undergone only small differences in degassing and cooling histories.

To date, temporal studies of individual volcanos have neither documented nor excluded this process. However, helium isotopic variations in Hawaian volcanos are generally slow and smooth and are reasonably described by changes in source compositions related to tectonic plate motions (Kurz et al, 1987). Further study of the role of shallow degassing in altering ${ }^{3} \mathrm{He} /{ }^{4} \mathrm{He}$ ratios is warranted, and may provide a way to study magma chamber residence times. Coupling helium measurements with carbon isotopic determinations may prove useful, because phase separation of $\mathrm{CO}_{2}$ leads to decreases in ${ }^{13} \mathrm{C} /{ }^{12} \mathrm{C}$ ratios (e.g. Javoy, Pineau and Delorme, 1986) which should correlate with helium changes. However, helium diffusion in phenocrysts is too rapid for zoned phenocrysts to record magmatic ${ }^{3} \mathrm{He} /{ }^{4} \mathrm{He}$ changes.

The high diffusivities of helium in olivine and pyroxene also constrain the origins of ultramafic xenoliths. Rapid helium exchange between xenoliths and host magmas is predicted by the characteristic time scales in figure 4.11. However, several studies have found that xenoliths and magmas have different helium isotopic compositions (e.g. Kurz et al, 1983; Polve and Kurz, 1989), an observation made for other isotopic systems as well (e.g. Hofmann and Hart, 1978). This means that xenoliths must spend very short times in magmas (weeks at most for $1 \mathrm{~mm}$ 
grains, figure 4.12). For a depth of origin of $30 \mathrm{~km}$ this suggests ascent rates are on the order of .05 meters per second. Similar velocities have been suggested from phase equilibrium constraints and transport kinematics (e.g. Spera, 1984). In addition to helium isotopic disequilibrium between magmas and xenoliths, small isotopic differences have been observed between different minerals in Hawaiian ultramafic xenoliths (Polve and Kurz, 1989). One means for producing the differences would be preferential ${ }^{4}$ He production in the phases with low ${ }^{3} \mathrm{He} /{ }^{4} \mathrm{He}$ ratios. Given the 500,000 year timescale required to produce the observed differences (op. cit.) the diffusivity of helium must be less than $6 \times 10^{-16} \mathrm{~cm}^{2} / \mathrm{s}$ to avoid homogenization. This suggests the xenoliths must come from lithospheric depths where the temperature is on the order of 550 to $750^{\circ} \mathrm{C}$, given the Arrhenius relations for pyroxene and olivine, respectively.

Finally, consider helium loss from phenocrysts erupted in lavas, as are often used for helium isotopic studies of basaltic petrogenesis. As pointed out by Hart (1984) the combination of high diffusivities and observable helium contents in these samples suggests that they do not lose all their helium during eruption because the magma has a high helium fugacity (chemical potential). As pointed out for olivine (op. cit.), the closure temperatures (Dodson, 1973) for helium in pyroxene are below the solidus for basalts, except for very high cooling rates. For example, a $1 \mathrm{~mm}$ pyroxene has a closure temperature of about $800^{\circ} \mathrm{C}$ for a cooling rate of $10^{7} \mathrm{C} / \mathrm{million}$ years, a typical rate for the interior of a thick basalt flow (Hart, 1984). Thus, some helium loss may occur as the basalt fugacity drops during cooling after eruption. However, this is unlikely to be important for most extrusive rocks, because they cool too quickly, but will become important in studying intrusive rocks. The large differences in helium diffusivities between pyroxene and olivine place strong contraints on the importance of preferential ${ }^{3} \mathrm{He}$ loss in altering the isotopic composition of phenocrysts. Because He will be lost much faster from pyroxene, its ${ }^{3} \mathrm{He} /{ }^{4} \mathrm{He}$ ratio will be significantly lower than cogenetic olivine whenever diffusion has contributed to significant gas loss. As an example, lowering the ratio of $1 \mathrm{~mm}$ spherical olivine grains from 8.4 to $6.9 \mathrm{R}_{\mathrm{a}}$ in this manner would 
yield a ratio of less than $5 \mathrm{Ra}$ in pyroxene grains of similar size, for an isotopic diffusivity ratio of 1.08 and 100-fold faster diffusion in pyroxene at $1000^{\circ} \mathrm{C}$. This difference will be larger at lower

temperatures. Similarly, crystals of different sizes would be expected to be differentially depleted in ${ }^{3} \mathrm{He}$. When disequilibrium of this nature is observed (i.e between cogenetic minerals), it also provides an indication of the integrated thermal history (for rocks of known ages) or rough ages for defined cooling rates by backtracking diffusive loss to helium isotopic equilibrium at emplacement (as demonstrated earlier by Gramlich and Naughton, 1972). Helium will be separated from other volatile elements if diffusion does occur, so that elemental abundances in phenocrysts (or basaltic glasses) may differ from those of the primary system.

In conclusion, diffusion is an important mechanism for equilibrating the helium isotopic compositions of minerals and melts, but not on mantle scales. Diffusion is slow in comparison to many volcanic cooling and transport rates so that measurements on glasses and phenocrysts can be used to characterize magmas, however caution must be exercised in systems which remain at high temperature for longer times. Finally, diffusive separation of helium from other elements may be important in processes such as melt percolation, and wall-rock interaction. The importance of diffusion in altering isotopic compositions may be examined by comparing cogenetic olivine and pyroxene crystals, because these minerals have dramatically different helium diffusivities. 


\section{REFERENCES}

DODSON M.H. (1973) Closure temperature in cooling geochronological and petrological systems. Contrib. Mineral. and Petrol. 40, 259274.

GRAMLICH J.W. and NAUGHTON J.J. (1972) Nature of source material for ultramafic minerals from Salt Lake Crater, Hawaii, from measurment of helium and argon diffusion. J. Geophys. Res. 77-17, 3032-3042.

HART S.R. (1981) Diffusion compensation in natural silicates. Geochim. Cosmo. Acta 45, 279-291.

HART S.R. (1984) He diffusion in olivine. Earth Plan. Sci. Let.70, 297-302.

HART S.R. and ZINDLER A., Constraints on the nature and development of chemical heterogeneities in the mantle, in Mantle Convection $\mathrm{R}$. Peltier, ed. in press.

HOFMANN A.W. (1980) Diffusion in natural silicate melts: a critical review, in Physics of Magmatic Processes (ed. R.B. HARGRAVES) pp.3-44. Princeton Univ. Press, Princeton, N.J.

HOFMANN A.W. and HART S.R. (1978) An assessment of local and regional isotopic equilibrium in the mantle. Earth Plan. Sci.Let. 38, 44-62.

HUNECKE J.C., NYQUIST L.E., FUNK H., KOPPEL V. AND P. SIGNER (1969) The thermal release of rare gases from separated minerals of the Mocs meteorite, in: Meteorite Research, Reidel, Dordrecht, p901. JAHNE B., HEINZ G. and DIETRICH W. (1987) Measurement of the diffusion coefficients of sparingly soluble gases in water JGR 92, 1076710776.

JAVOY M., PINEAU F. AND H. DELORME, (1986) Carbon and nitrogen isotopes in the mantle, Chemical Geology 57 p41-62.

KURZ M.D. (1982) Helium isotope geochemistry of oceanic volcanic rocks: implications for mantle heterogeneity and degassing. PhD. Diss., MIT/WHOI. 
KURZ M. D., JENKINS W. J., SCHILLING J. G. and HART S. R. (1982) Helium isotopic variations in the mantle beneath the central North Atlantic 0cean. Earth Plan. Sci. Let. 58, 1-14.

KURZ M.D., JENKINS W.J., HART S.R. and CLAGUE D. (1983) Helium isotopic variations in volcanic rocks from Loihi Seamount and the Island of Hawaii. Earth Plan. Sci. Let. 66, 388-406.

KURZ M.D., GARCIA M.0., FREY F.A. and 0'BRIAN P.A. (1987) Temporal helium isotopic variations within Hawaiian volcanoes: basalts from Mauna Loa and Haleakala. Geochim Cosmo. Acta 51, 2905-2914. LIPPOLT H.J. and WEIGEL E. (1988) ${ }^{4} \mathrm{He}$ diffusion in ${ }^{40} \mathrm{Ar}$-retentive minerals. Geochim. Cosmo. Acta 52, 1449-1458.

LUX G (1987) The behavior of noble gases in silicate liquids: solution, diffusion, and surface effects, with applications to natural samples. Geochim. Cosmo. Acta 51, 1549-1560.

MAMYRIN B.A. and TOLSTIKHIN I.N. (1984) Helium Isotopes in Nature. Elsevier, Amsterdam.

NAVON 0. and STOLPER E. (1987) Geochemical consequences of melt percolation: the upper mantle as a chromatographic column. J. Geol. 95, 285-307

POLVE M. and KURZ M. (1989) ${ }^{3} \mathrm{He} /{ }^{4} \mathrm{He}$ systematics in spinel-lherzolite nodules. In press.

RAMA S.N.I. and HART S.R. (1965) Neon isotope fractionation during transient permeation. Science 147, 737-738.

SHIMIZU N. and I. KUSHIRO (1984) Diffusivity of oxygen in jadeite and diopside melts at high pressures, Geochim. Cosmochim. Acta 48 p1295-1303.

SNEERINGER M., HART S.R. and SHIMIZU N. (1984) Strontium and samarium diffusion in diopside. Geochim. Cosmo. Acta 48, 1589-1608.

SPERA F.J. (1980) Aspects of magma transport. In: Physics of Magmatic Processes (ed. R.B. HARGRAVES) pp.265-323. Princeton Univ. Press, Princeton, N.J. 
SPERA F.J. (1984) Carbon dioxide in petrogenesis III: role of volatiles in the ascent of alkaline magma with special reference to xenolith-bearing mafic lavas. Contrib. Mineral. Petrol. 88, 217-232.

WATSON E.B.(1984) Diffusion in magmas at depth in the earth: the effects of pressure and dissolved $\mathrm{H}_{2}$ 0. Earth Plan. Sci. Let. 52, 291-301.

ZINDLER A. and HART S. (1986) Helium: problematic primordial signals. Earth Plan. Sci. Let. 79, 1-8. 


\section{Chapter 5.}

\section{Diffusion \\ of cosmogenic ${ }^{3} \mathrm{He}$}

in olivine and quartz:

Implications for surface exposure dating. 


\section{$\underline{5.1} \underline{\text { INTRODUCTION }}$}

The recently identified production of ${ }^{3}$ He in Hawaiian basalts by in-situ interaction with cosmic rays offers great promise for studies of surface exposure ages, erosion rates, and ancient cosmic ray fluxes (Kurz et al, 1985; Kurz, 1986a,b; Craig and Poreda, 1986; Marti and Craig, 1987). Before this discovery can be widely applied as a geochemical tool several fundamental aspects of the behavior of cosmogenic helium $\left({ }^{3} \mathrm{He}_{\mathrm{c}}\right)$ must be better understood, including its production rate, the extent to which it is retained in rocks, and how it can be distinguished from inherited or radiogenic helium. This chapter focuses on quantifying losses related to the formation mechanism of ${ }^{3} \mathrm{He}_{\mathrm{C}}$ or which occur by diffusion. To this end, the diffusivity of ${ }^{3} \mathrm{He}{ }_{\mathrm{c}}$ was measured in two minerals important to present and future cosmogenic helium studies, olivine and quartz. The measurements also suggest ways by which inherited and cosmogenic helium can be separated.

Several nuclear reactions contribute to ${ }^{3} \mathrm{He}_{\mathrm{c}}$ production in surface rocks. Most important is spallation of major element target atoms by high energy neutrons which are produced as secondary particles in the atmosphere when the primary cosmic rays (predominantly protons, but containing some neutrons and multi-nucleonic particles) collide with atmospheric atoms (Lal and Peters, 1967; Kurz, 1986b; Lal, 1987). These endothermic reactions produce ${ }^{3} \mathrm{H},{ }^{3} \mathrm{He}$ and ${ }^{4} \mathrm{He}$, leading to an overall ${ }^{3} \mathrm{He} /{ }^{4} \mathrm{He}$ production ratio of about .1 (Kurz, 1986a). The great difference between this ratio and that of unexposed terrestrial rocks $\left(10^{-8}\right.$ to $10^{-5}$; e.g. Mamyrin and Tolstikhin, 1984) is essential to the detection of ${ }^{3} \mathrm{He}_{\mathrm{c}}$. Because the energy spectrum of cosmic rays falls off very steeply with increasing energy, most of these reactions occur at or near the threshold interaction energies for the target elements, which are in the range of 10-50 MeV (Lal and Peters, 1967; Lal, 1987). As discussed further below, these energies may contribute to ${ }^{3} \mathrm{He}_{\mathrm{c}}$ mobility. The attenuation half-length for the cosmic neutron flux, and thus spallation production of ${ }^{3} \mathrm{He}_{\mathrm{c}}$, is approximately $160 \mathrm{~g} / \mathrm{cm}^{2}$, or about 60 $\mathrm{cm}$ in dense rock (Kurz, 1986b and references therein). 
Another production reaction is the interaction of thermalized cosmogenic secondary neutrons with ${ }^{6} \mathrm{Li}$ to yield equal amounts of ${ }^{3} \mathrm{He}$ and ${ }^{4} \mathrm{He}$. For rocks with lithium contents of a few parts per million this mechanism is only about $1 \%$ as effective as spallation, but it becomes roughly equivalent in importance for $\mathrm{Li}$ contents of about 100 ppm (Kurz, 1986b; Simmons, 1986). This exothermic reaction releases similar energies to uranium and thorium series decays, about $5 \mathrm{MeV}$ (Lal, 1987). Its depth dependence is essentially the same as for spallation, because the thermal neutron population derives from the fast neutrons. An exception occurs at the atmosphere-rock density boundary, where thermal neutron production can be enhanced several times by thermalization of atmospheric fast neutrons (e.g. Kurz, 1986a). It is also worth pointing out that the ${ }^{6} \mathrm{Li}$ reaction is responsible for some production of ${ }^{3} \mathrm{He}$ even in the absence of cosmic irradiation, from neutrons supplied predominantly by $X(a l p h a, n) Y$ reactions on major rock elements instigated by radiodecay of ${ }^{238} \mathrm{U}$ and ${ }^{232} \mathrm{Th}$ (Morrison and Pine, 1955). Because only a small fraction of these alpha particles produce neutrons, the ${ }^{3} \mathrm{He} /{ }^{4} \mathrm{He}$ radiogenic production ratio is very low in most unexposed rocks, on the order of $10^{-7}$ to $10^{-9}$ depending on lithium content (op. cit.; Andrews, 1985), and thus is readily distinguished from cosmogenic helium.

A third mechanism produces much less ${ }^{3} \mathrm{He}_{\mathrm{c}}$ and only becomes relatively important at depths below where the secondary neutrons have been largely absorbed (a meter or more). This is the interaction of cosmogenic muons with major element nuclei to produce neutrons which then interact with ${ }^{6} \mathrm{Li}$ by the low-energy reaction already described, yielding a ${ }^{3} \mathrm{He} /{ }^{4} \mathrm{He}$ production ratio of 1 (Lal and Peters, 1967; Kurz, 1986b; Lal, 1987). The half-depth for ${ }^{3} \mathrm{He}$ c production is basalt by this process is on the order of $1000 \mathrm{~g} / \mathrm{cm}^{2}$ (Kurz, 1986b).

It is important to keep these production mechanisms and their associated energies in mind when assessing helium mobility. Elevated losses of alpha-decay produced nuclides are known to occur, particularly in weathering processes, but also from rock interiors (e.g. Giletti and Kulp, 1955; Rama and Moore, 1984; Fleischer, 1988). For this reason, it may not be appropriate to apply diffusivities determined for inherited 
helium to cosmogenic helium problems. However, it should be noted that even in ancient rocks the low cosmogenic neutron flux (order $10^{-4} / \mathrm{g} / \mathrm{s}$; Kurz, 1986a) and ${ }^{3} \mathrm{He}_{\mathrm{c}}$ production rate (order 100 atoms/g/yr; Kurz, 1986b) will lead to much lower radiation doses than experienced by irradiated basalts (circa $10^{18}$ neutrons/g) or alpha-decay damaged metamict zircons (circa $10^{13}$ decays/g) which display elevated noble gas mobilities (Stettler and Bochsler, 1979; Damon and Kulp, 1957). To preface the results, ${ }^{3} \mathrm{He}_{\mathrm{c}}$ diffusivities in quartz and olivine were found to be compatible with both these observations, in that release was enhanced over inherited helium, but was still much slower than from damaged crystals or glasses (analogous to the metamict state).

Finally, it should be pointed out that the in-situ ${ }^{3} \mathrm{He}_{\mathrm{c}}$ production rate and its possible variation are not yet well known. To date, the production rate has only been measured in Hawaiian basalts (using olivine phenocrysts and calibrating to ${ }^{14} \mathrm{C}$ ages; Kurz, $1986 \mathrm{~b}$; Kurz et al, 1989). It is about $125( \pm 25)$ atoms/g/yr at sea-level at that geomagnetic lattitude. This rate is expected to very significantly with altitude (e.g. 2-fold increase at $1000 \mathrm{~m}$ ) and lattitude (2 to 3 -fold increase from the equator to the poles depending on altitude) because cosmic rays are deflected by Earth's magnetic field and attenuated by the atmosphere (for a recent review see Lal, 1988). Theoretical estimates of the dependence of production rates on these factors, as well as with depth in the rock, are available (e.g. Lal and Peters, 1967; Lal, 1987, 1988; Yokoyama, Reyss, and Guichard, 1977). However, the theory does not fully explain variations in apparent production rate of $25 \%$ or so observed at Hawaii (Kurz et al, 1989), and thus may only be applied in an approximate way in extending the Hawaiian results to other locations. Measurements of the compositional dependence of ${ }^{3}$ He production in meteorites (Bogard and Cressy, 1973) and theoretical estimates (Lal, 1987) do agree in suggesting this effect will be small ( 5 to $10 \%$ for most silicate rocks). More measurements from other locales and improved theory are both required, if ${ }^{3} \mathrm{He}{ }_{c}$ exposure geochronology is to become a precise and widely applicable method. 


\subsection{METHODS AND SAMPLES}

Diffusivities were estimated for olivine and quartz by measuring ${ }^{3} \mathrm{He}$ and ${ }^{4} \mathrm{He}$ degassed from mineral separates in vacuo during incremental heating in a specially designed vessel (figure 2.2). The samples were then melted in a furnace to obtain total helium contents, allowing the calculation of fractional release rates. Diffusion coefficients were calculated for each sequential release using model equations which assume equisized, spherical grains with initially homogeneous helium concentrations. The diffusion vessel, furnace, analytical methods and model equations are described in detail in chapter 2 , and are discussed below as neccesary in interpreting the experimental results.

Basaltic olivine crystals were chosen for study because of their importance in determining the production rate for ${ }^{3} \mathrm{He}_{\mathrm{c}}$ in Hawaiian rocks (Kurz et al, 1988). The olivine mineral separate was prepared from sample HA6 of the Kula formation ankaramite flow (approximately 500800,000 years old McDougall, 1964; Naughton et al, 1980) which forms White Hill near the summit of Haleakala Volcano, Maui. This extremely fresh, porphyritic rock has been previously described and contains abundant cosmogenic helium predominantly produced by spallation, rather than by reaction with ${ }^{6} \mathrm{Li}$ (Kurz, 1986a). The rock was crushed in a stainless steel mortar and large (1-2mm) olivine grains were handpicked to be free of adhering basalt matrix, alteration, or other mineral phases, although grains with a few small spinel inclusions were accepted. These grains were then crushed and sieved (in methanol to disrupt aggregates and remove fines), dried in air and handpicked again to provide the 212-250um particles used in the diffusion experiment.

Crushing removed most of the helium contained in fluid inclusions, as revealed by comparing the total ${ }^{4} \mathrm{He}$ contents of the sieved separates $\left(1.7 \times 10^{-10} \mathrm{cc} / \mathrm{g}\right.$ on fusion) with the contents of whole olivine grains $\left(1.3 \times 10^{-8} \mathrm{cc} / \mathrm{g}\right.$ as measured in a $1-2 \mathrm{~mm}$ olivine separate from the same rock by Kurz, 1986b). In addition to removing magmatic helium, crushing the phenocrysts should produce subgrains with relatively homogeneous ${ }^{3} \mathrm{He}_{\mathrm{c}}$ distributions, which is an assumption of the diffusion model. Microscopic examination of these particles revealed no pervasive 
internal cracking, suggesting that using particle size as the effective length scale in calculating diffusivities was also valid. Electron microprobe analysis of 10 olivine grains yielded identical chemical compositions (Forsterite-78) within analytical errors (table 5.1).

Quartz was chosen for study for several reasons. It is a common mineral in glacial terrains in the Dry Valleys of the Transantarctic Mountains, an area for which efforts are underway in this laboratory to obtain ${ }^{3} \mathrm{He}_{\mathrm{c}}$ exposure ages, in order to constrain the history of the Antarctic ice cap. The issue of helium retention on several thousand to several million year timescales is important to this research. In addition, quartz is an ideal phase for the measurement of another cosmogenic nuclide, ${ }^{26} \mathrm{Al}_{\mathrm{c}}$, because of its low natural $\mathrm{Al}$ background (Lal and Arnold, 1985; Nishiizumi et al, 1986). Combined measurement of ${ }^{26} \mathrm{Al}_{\mathrm{c}}$, with a half-life of 705,000 years and stable ${ }^{3} \mathrm{He}$ offers promise for determining prior surface exposures of rocks that are now buried, if both nuclides are effectively retained. Finally, quartz is a ubiquitous mineral and understanding ${ }^{3} \mathrm{He}_{\mathrm{c}}$ mobility in it will be valuable to future cosmogenic helium studies in other environments.

The quartz separate was prepared by crumbling a friable orthoquartzite rock (BW84-105) recovered from high altitude (1300 meters) in Arena Valley in the Transantartic Mountains (provided by G. Denton, Univ. of Maine). This rock is from the Taylor IVb moraine, which is estimated to be 2-4 Myr old based on field relations with $\mathrm{K}-\mathrm{Ar}$ dated lava flows (personal communication from G. Denton to M. Kurz). As in the olivine sample, spallation dominates (>90\%) the ${ }^{3}{ }^{3 e}$ C production in this rock, because lithium contents are relatively low (about 10 ppm, D. Colodner, unpublished results). The quartz grains were sieved, handpicked to avoid discolored grains and adhering iron hydroxide phases, and cleaned by ultrasonic agitation in distilled water, acetone and methanol. The grains used in the diffusion experiment (500-710um sieve fraction) are typical of the size in the rock, and thus may have inhomogeneous internal helium distributions if diffusive loss has already occurred. Several grains were examined by electron microprobe. They were almost exclusively silica, but a few (1-5 per grain) 5 to 15 micron in size inclusions were found and were tentatively identified 
Table 5.1 HA6 Olivine Composition

\begin{tabular}{cl} 
Oxide & Weight \% \\
\hline Mg0 & 41.7 \\
$\mathrm{Al}^{0}{ }_{3}$ & 0.1 \\
$\mathrm{SiO}^{2}$ & 38.8 \\
$\mathrm{CaO}$ & 0.2 \\
$\mathrm{TiO}{ }_{2}$ & 0.1 \\
$\mathrm{Cr}_{2}{ }_{3}$ & 0.1 \\
$\mathrm{MnO}$ & 0.3 \\
$\mathrm{Fe} 0$ & 20.3 \\
$\mathrm{NiO}$ & 0.1 \\
$\mathrm{TOTAL}$ & 101.7 \\
$\mathrm{Ca} / \mathrm{Mg} / \mathrm{Fe}$ & $0.003 / 0.784 / 0.213$ \\
$\mathrm{Mg} /(\mathrm{Mg}+\mathrm{Fe})$ & 0.786 \\
\hline
\end{tabular}

a) Average of $10 \mathrm{microprobe}$ determinations.

b) $\mathrm{Ca} / \mathrm{Mg} / \mathrm{Fe}$ normalized to 1.0 
(from energy-dispersive x-ray spectra) as iron oxides (hematite?), phosphate minerals (apatite and fluoroapatite, some of which contained elevated rare earth or actinide element contents), zircon, barite, and sulfide minerals (pyrite and chalcopyrite). The zircons and phosphate inclusions may contain significant fractions of the $U$ and $T h$, which could be important for radiogenic helium production, but this could not be measured. No phases enriched in lithium (above the detection limit of about $30 \mathrm{ppm}$ ) were found. More work is needed to determine typical abundances and compositions of these inclusions.

\subsection{RESULTS}

5.3.1 Olivine

A single step-heating experiment was performed for olivine over the temperature range of $200-600{ }^{\circ} \mathrm{C}$ (table 5.2). Only ${ }^{3} \mathrm{He}_{\mathrm{c}}$ could be measured in the released aliquots as ${ }^{4} \mathrm{He}$ release was two small to be distinguished above background, except on fusion. The calculated diffusivities define a linear trend in an Arrhenius diagram (figure 5.1) as is often observed for volume diffusion governed by a single activation energy, here found to be $25 \pm 4 \mathrm{Kcal} / \mathrm{mole}\left(\log \mathrm{D}^{\circ}=-3.7 \pm .8\right.$ $\left.\mathrm{cm}^{2} / \mathrm{s}\right)$. This result must be considered preliminary until it is reproduced because of the narrow temperature range. However, the data demonstrate that ${ }^{3} \mathrm{He}_{\mathrm{c}}$ release is greatly enhanced in comparison to inherited helium in dunitic olivine (figure 5.2). The activation energy is greatly different than for inherited helium diffusion in olivine (100 $\mathrm{Kcal} / \mathrm{mole}$, chapter 4), and may reflect a different mechanism for ${ }^{3} \mathrm{He}{ }_{\mathrm{c}}$ release, perhaps involving increased vacancy populations associated with the cosmic neutron flux.

Extrapolating the ${ }^{3} \mathrm{He}_{\mathrm{c}}$ diffusivity results to $40^{\circ} \mathrm{C}$, as a reasonable upper limit to the mean annual temperature of a Hawaiian lava flow, suggests a ${ }^{3} \mathrm{He}$ difffusivity of $7 \times 10^{-22} \mathrm{~cm}^{2} / \mathrm{s}$ with an upper limit of $1.5 \times 10^{-17} \mathrm{~cm}^{2} / \mathrm{s}$ based on two-sigma uncertainty of the Arrhenius leastsquares line. Equivalent values at $20^{\circ} \mathrm{C}$ are $5 \times 10^{-23}$ and $1 \mathrm{x}$ $10^{-18} \mathrm{~cm}^{2} / \mathrm{s}$ respectively. Errors in these values from the model assumption of equisized spherical grains should not be larger than a 
Table 5.2. Step-heated Release of Cosmogenic ${ }^{3}$ He from Olivine.

Sample: HA 6 0livine, 212-250um sieve fraction.

$44.9 \mathrm{mg}$, model grain radius $=116 \pm 19 \mathrm{um}$.

\# Temp. Time ${ }^{3}$ He $1 \mathrm{~s} \quad \mathrm{~F} \quad 1 \mathrm{~s} \quad \mathrm{D} \quad 1 \mathrm{~s} \quad \mathrm{E}$

$\left({ }^{\mathrm{O}} \mathrm{C}\right) \quad(\mathrm{hr}) \quad\left(10^{-16} \mathrm{cc}\right) \quad$ (fraction) $\quad\left(\mathrm{cm}^{2} / \mathrm{s} \times 10^{\mathrm{E}}\right)$

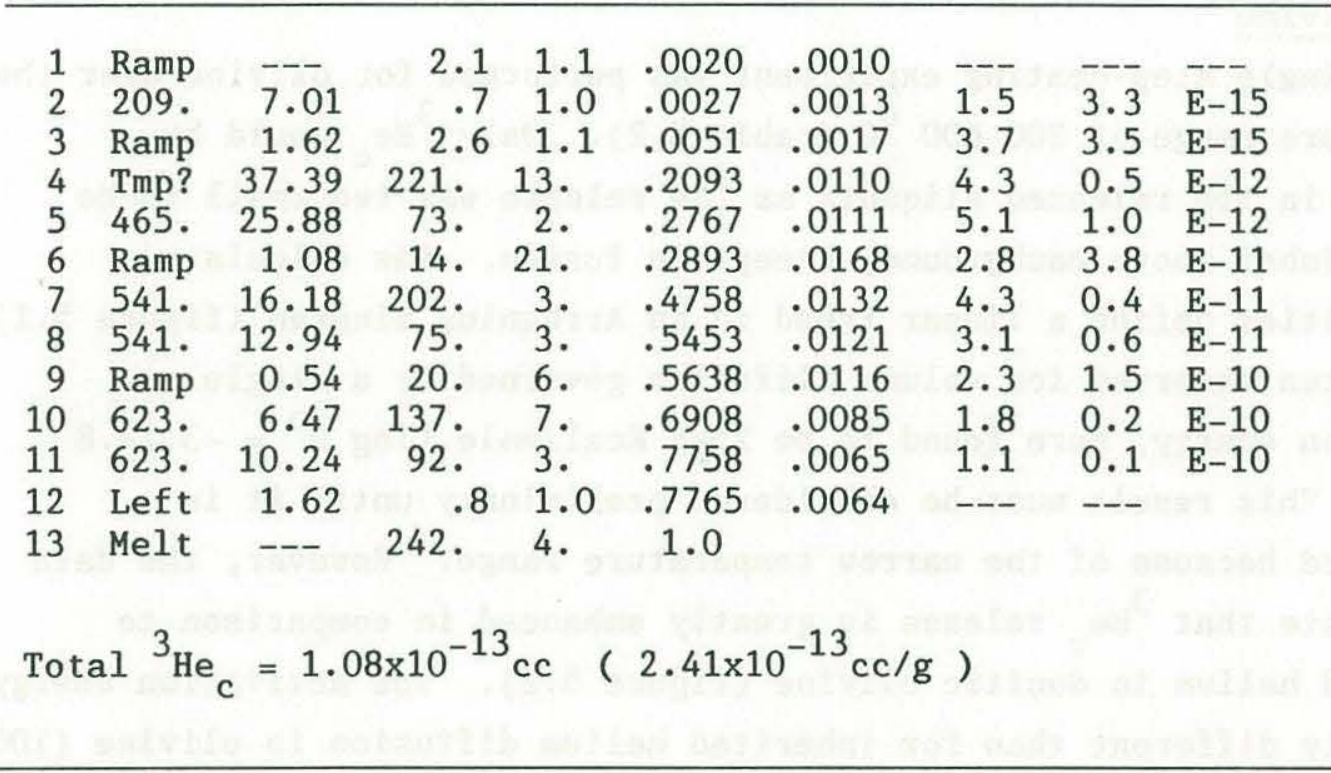

a. Step 4 varied between 300 and $450^{\circ} \mathrm{C}$ because of instrumental difficulties.

b. The amount of ${ }^{3} \mathrm{He}$ released was within one sigma of the detection limit in aliquots 2,6 and 12. The diffusivities for these steps are within error of zero. This data is included because these releases affect the determination of $F$ values for other steps, and because the low releases set upper limits on diffusivities within these steps. 
Figure 5.1. Arrhenius relation for the release of cosmogenic ${ }^{3} \mathrm{He}_{\mathrm{c}}$ from olivine crystals. One sigma errors are close to the symbol size, except for the point near $200^{\circ} \mathrm{C}$ which released so little helium that only an upper bound could be obtained. The best fit line is not significantly changed if this point is not included. 
Figure 5.1

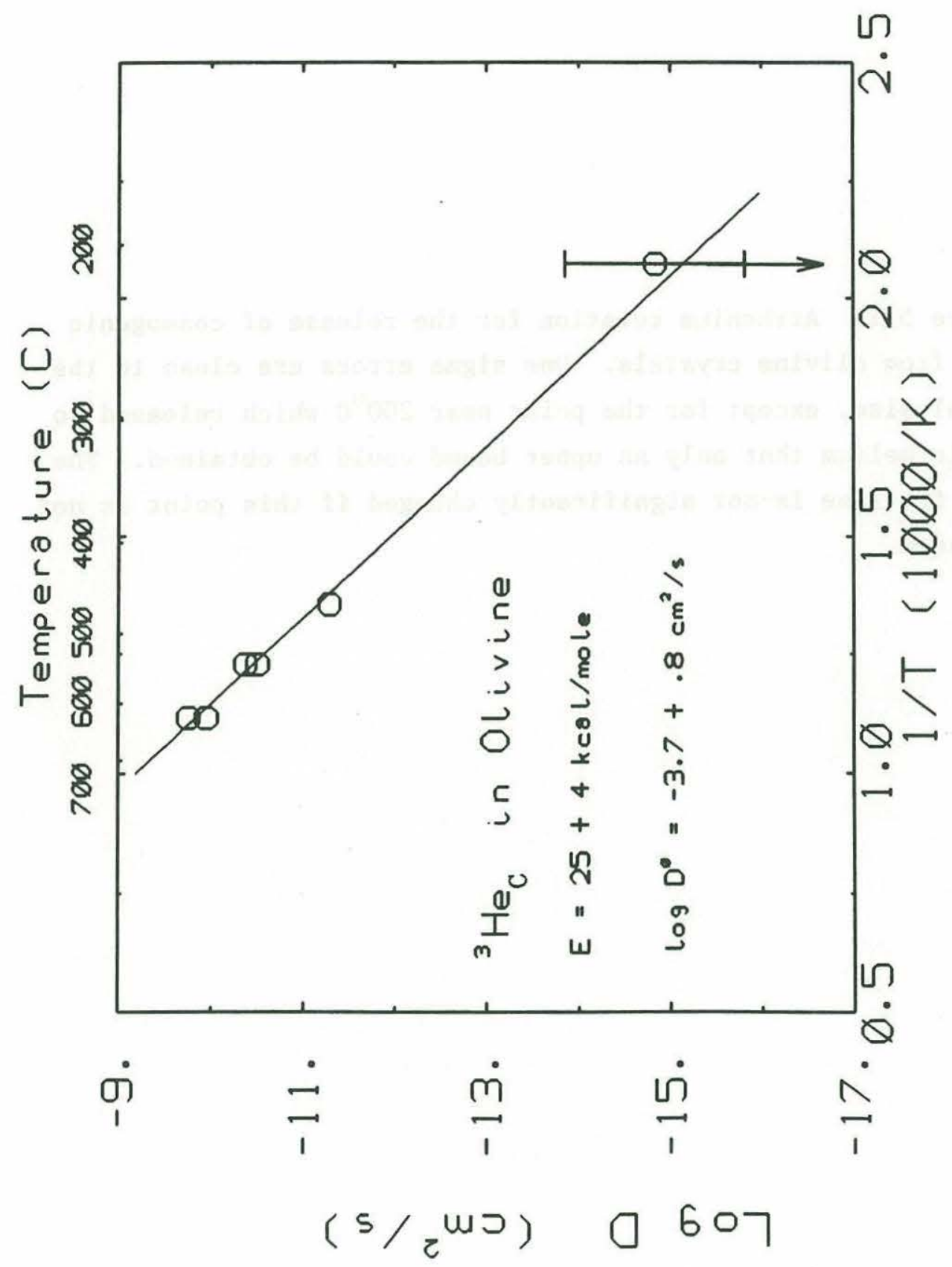


Figure 5.2. Diffusive release of cosmogenic ${ }^{3}{ }^{3 e}{ }_{c}$ appears to be both considerably enhanced and less thermally activated that the release of inherited ${ }^{3} \mathrm{He}$ from olivine. However, because the results were obtained on different samples over different temperature ranges, these conclusions must be viewed with caution. Arrhenius line for inherited ${ }^{3}$ He from chapter 4. 
Figure 5.2

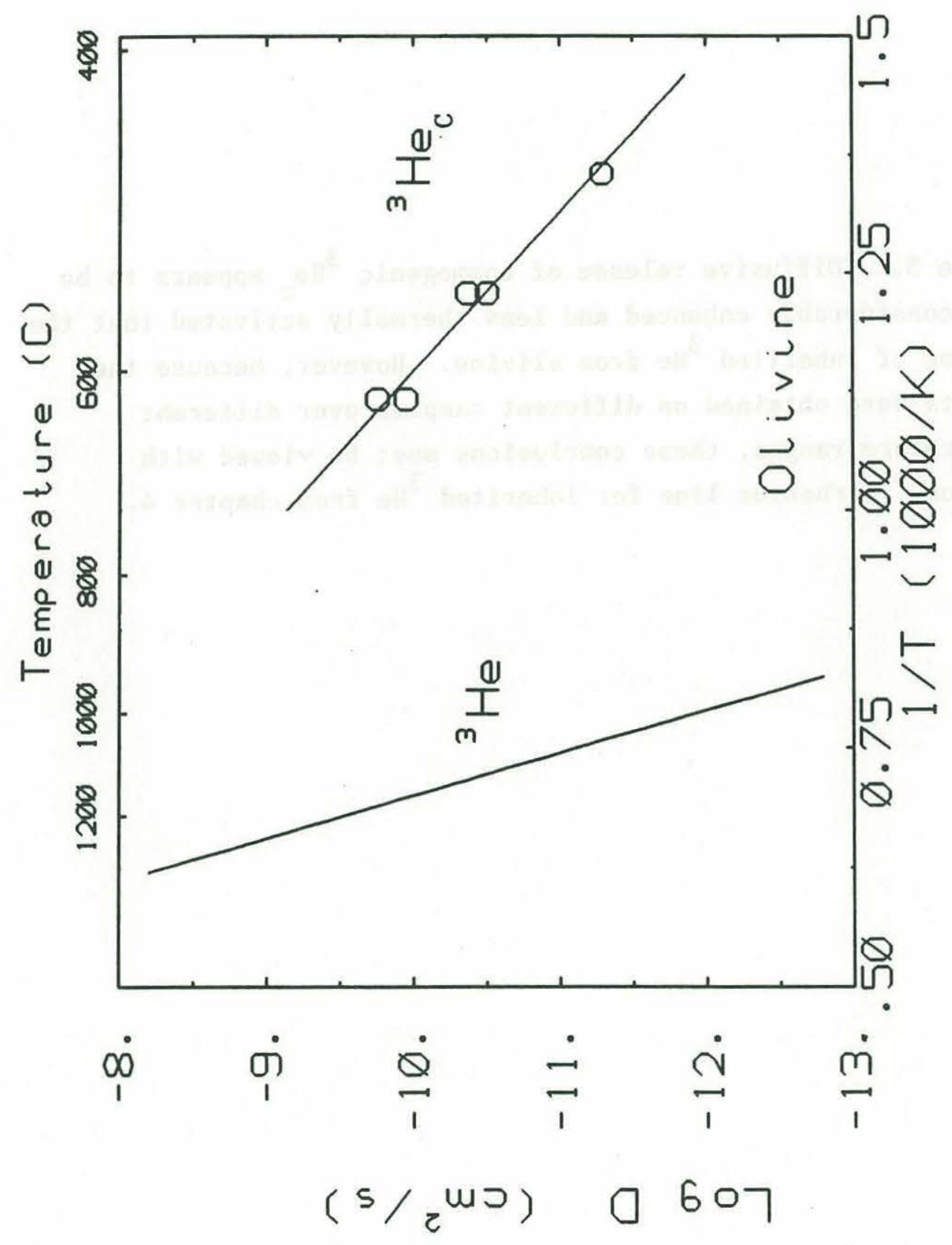


factor of two given the narrow particle size range used, provided that the ${ }^{3} \mathrm{He}_{\mathrm{c}}$ content does not vary strongly with size in this range. This is a safe assumption because the grains were prepared by crushing larger grains, thereby homogenising any initial variability. The greatest uncertainty in determining a low temperature diffusivity is the extent of extrapolation required, because it is possible the temperature dependence of ${ }^{3} \mathrm{He}_{\mathrm{c}}$ changes at lower temperature. Lower temperature emanation experiments with larger samples of smaller particles could reduce this problem somewhat, although not a lot because the strong decrease in diffusivity with temperature would make it difficult to obtain release rates much above typical atmospheric ${ }^{3}$ He leak rates into the vessel and mass spectrometer. Measurement of helium remaining after storage of a powder separate may be a more appropriate way to refine these preliminary results.

\subsubsection{Quartz}

It was possible to measure both ${ }^{3} \mathrm{He}$ and ${ }^{4} \mathrm{He}$ in the quartz diffusion experiment, which extended from 150 to $600{ }^{\circ} \mathrm{C}$ and involved more than forty steps before melting the grains (table 5.3). ${ }^{3}$ He was released far more rapidly and at lower temperature than ${ }^{4} \mathrm{He}$ (figure 5.3). The smooth character of these releases suggest a volume diffusion process rather than disruption of vesicles or the grains themselves. Examination of the grains under a binocular microscope at $80 \mathrm{x}$ before and after heating revealed no changes, also implying a diffusive mechanism. The early dominant release of ${ }^{3} \mathrm{He}$ is reflected in the isotopic compositions of the aliquots (figure 5.4). With the exception of a few heating steps which released very little helium, the early releases had greatly elevated ${ }^{3} \mathrm{He} /{ }^{4} \mathrm{He}$ ratios, near $120 \mathrm{R}$, in comparison to the bulk helium composition of $11.3 \mathrm{R}_{\mathrm{a}}$ (table 5.3). These ratios steadily decreased to values of .01 to $.02 \mathrm{R}_{\mathrm{a}}$, at which point approximately $70 \%$ of the total ${ }^{4}$ He still remained, although more than $99 \%$ of the ${ }^{3}$ He had been released. This strong separation suggests that essentially all the ${ }^{4} \mathrm{He}$ is radiogenic, and all the ${ }^{3} \mathrm{He}$ of cosmogenic origin. Thus, the ${ }^{3} \mathrm{He}$ release rates can be used to obtain diffusivities for cosmogenic ${ }^{3} \mathrm{He}{ }_{\mathrm{c}}$ without any correction for inherited helium. In addition, this means 
Table 5.3 Step-heated Release of Cosmogenic 3 He and Radiogenic 4 He from Quartz.

Sample: BW84-105 Quartz, 300-355um sieve fraction, $115.6 \mathrm{mg}$, model radius $=164 \pm 28$ um. The values shown for step 39 were estimated from those for adjacent steps.

\begin{tabular}{|c|c|c|c|c|c|c|c|c|c|c|c|c|c|c|c|c|}
\hline \# & $\begin{array}{l}\text { Temp. } \\
\text { C }\end{array}$ & $\begin{array}{c}\text { Time } \\
\text { hr }\end{array}$ & $\begin{array}{l}4 \mathrm{He} \\
\mathrm{cc} .\end{array}$ & $\begin{array}{r}1 \mathrm{~s} \\
-10\end{array}$ & $\begin{array}{l}3 \mathrm{He} \\
\mathrm{CC} . \mathrm{E}-\end{array}$ & $\begin{array}{l}1 \mathrm{~s} \\
-14\end{array}$ & $\begin{array}{r}3 \mathrm{He} / 4 \mathrm{He} \\
\mathrm{R} / \mathrm{Ra}\end{array}$ & $1 \mathrm{~s}$ & $F(4 \mathrm{He})$ & $\mathrm{F}(3 \mathrm{He})$ & $\begin{array}{l}D(4 \mathrm{H} \epsilon \\
\mathrm{cm} 2 / \mathrm{s}\end{array}$ & $\begin{array}{r}15 \\
\times \quad 10\end{array}$ & & $\begin{array}{l}D(3 H \\
\operatorname{cm} 2\end{array}$ & $x$ & $0^{\wedge} \mathrm{E}$ \\
\hline 1 & Ramp & 0.53 & .162 & .005 & .02 & .01 & 9. & 5. & .0002 & .0002 & 5.5 & .3 & $E-16$ & 3. & 4. & $E-16$ \\
\hline 2 & 146. & 1.62 & .279 & .006 & .25 & .03 & 64. & 6. & .0006 & .0022 & 1.16 & .05 & $E-15$ & 2.0 & .5 & E-14 \\
\hline 3 & 147. & 9.74 & .776 & .010 & 1.10 & .03 & 102 . & 2. & .0016 & .0115 & 1.49 & .03 & $E-15$ & 8.6 & .5 & E-14 \\
\hline 4 & 148. & 9.33 & .475 & .004 & .72 & .02 & 109 . & 2. & .0022 & .0176 & 1.67 & .05 & $E-15$ & 1.2 & .1 & E-13 \\
\hline 5 & Ramp & 0.54 & .281 & .003 & .38 & .02 & 99. & 5. & .0026 & .0208 & 2.1 & .1 & E-14 & 1.5 & .2 & $E-12$ \\
\hline 6 & $210^{\circ}$ & 1.63 & 1.25 & .004 & 1.92 & .03 & 111. & 1. & .0042 & .0371 & 4.52 & .06 & E-14 & 3.8 & .1 & $E-12$ \\
\hline 7 & 210 . & 1.62 & .930 & .004 & 1.57 & .02 & 122. & 2 . & .0055 & .0503 & 4.77 & .09 & $E-14$ & 4.8 & .2 & $E-12$ \\
\hline 8 & 210. & 1.62 & .800 & .003 & 1.35 & .03 & 123. & 3. & .0065 & .0618 & 5.1 & .1 & $E-14$ & 5.4 & .3 & E-12 \\
\hline 9 & Ramp & 0.54 & .693 & .003 & 1.14 & .02 & 119. & 2. & .0074 & .0714 & 1.53 & .04 & $E-13$ & 1.6 & .1 & $E-11$ \\
\hline 10 & $235^{\circ}$ & 0.54 & 1.10 & .003 & 1.80 & .02 & 118. & 1. & .0089 & .0866 & 2.85 & .05 & E-13 & 3.1 & .1 & $E-11$ \\
\hline 11 & 235. & 1.08 & 2.22 & .004 & 3.66 & .05 & 119. & 2. & .0118 & .1176 & 3.64 & .03 & E-13 & 4.2 & .1 & $E-11$ \\
\hline 12 & 235. & 1.08 & 1.84 & .004 & 2.92 & .04 & 115. & 2. & .01 & .14 & 3.79 & .04 & $E-13$ & 4.3 & .2 & -11 \\
\hline 13 & 235. & 1.08 & 1.58 & .004 & 2.59 & .03 & 119. & 2. & .01 & .16 & 3.8 & .05 & $E-13$ & 4.6 & .2 & -11 \\
\hline 14 & 235. & 1.08 & 1.40 & .003 & 2.26 & .03 & 117. & 2 . & .0181 & .1833 & 3.83 & .06 & $E-13$ & 4.7 & .2 & $E-11$ \\
\hline 15 & 235. & 1.08 & 1.33 & .004 & 1.97 & .02 & 107. & 1. & .0199 & .2000 & 4.01 & .07 & $E-13$ & 4.6 & .3 & -11 \\
\hline 16 & 235. & 1.62 & 1.57 & .005 & 2.33 & .04 & 107. & 2. & .0220 & .2197 & 3.47 & .06 & E-13 & 4.0 & .2 & -11 \\
\hline 17 & Ramp & 0.54 & 1.49 & .003 & 1.96 & .03 & 95. & 2. & .02 & .236 & 1.09 & .02 & $E-12$ & 1.11 & .07 & $E-10$ \\
\hline 18 & 280. & 0.54 & 2.34 & .004 & 3.1 & .04 & 97. & 1. & .02 & .26 & 1.89 & .02 & E-12 & & .08 & -10 \\
\hline 19 & 280. & 0.54 & 2.18 & .003 & 2.81 & .03 & 93. & 1. & .0299 & .286 & 1.97 & .03 & $E-12$ & 2.02 & .09 & -10 \\
\hline 20 & 280. & 1.08 & 4.25 & .005 & 5.12 & .06 & 87. & 1. & .0355 & .3299 & 2.20 & .02 & $E-12$ & 2.14 & .06 & $E-10$ \\
\hline 21 & 280. & 1.08 & 3.71 & .005 & 4.27 & .04 & 83. & 1. & .0403 & .3660 & 2.30 & .02 & $E-12$ & 2.11 & .07 & $E-10$ \\
\hline 22 & 280. & 1.08 & 3.29 & .005 & 3.66 & .04 & 81. & 1. & .0447 & .3969 & 2.29 & .03 & $E-12$ & 2.07 & .08 & $E-10$ \\
\hline 23 & 280. & 1.08 & 2.85 & .009 & 3.12 & .05 & 79. & 1. & .0484 & .4233 & 2.18 & .03 & $E-12$ & 2.0 & .1 & $E-10$ \\
\hline 24 & 279. & 0.54 & 1.23 & .005 & 1.38 & .04 & 81. & 2 . & .0500 & .43 & 1.99 & .07 & $E-12$ & 1.8 & .2 & $E-10$ \\
\hline 25 & Ramp & 0.54 & 5.33 & .01 & 5.18 & .07 & 71. & 1. & .0570 & .4788 & 9.43 & .09 & $E-12$ & 7.8 & .2 & $E-10$ \\
\hline 26 & 342 . & 1.08 & 11.7 & .03 & 9.69 & .09 & 60. & .6 & .0724 & .5607 & 1.26 & .01 & $E-11$ & 9.1 & .2 & $E-10$ \\
\hline 27 & 342 . & 0.54 & 17.4 & .04 & 12.7 & .1 & 53 & .5 & .09 & .6684 & 4.94 & .03 & E-11 & 3.37 & .05 & E-09 \\
\hline 28 & Ramp & 0.54 & 27.1 & .07 & 15. & .1 & 42.3 & .3 & .1309 & .8020 & 1.07 & .01 & $E-10$ & 6.82 & .07 & $E-09$ \\
\hline 29 & 413. & 0.54 & 25.3 & .06 & 11.0 & .1 & 31.5 & .3 & .1642 & .8951 & 1.34 & .01 & $E-10$ & 8.9 & .1 & $E-09$ \\
\hline 30 & 413. & 0.54 & 14.2 & .04 & & .06 & 25.2 & .3 & .1828 & .9369 & 9.1 & .1 & E-11 & 7.1 & .1 & E-09 \\
\hline 31 & 413. & 1.08 & 16.0 & .04 & 4.20 & .06 & 19.0 & .3 & .2039 & .9724 & 5.79 & .07 & $E-11$ & 5.8 & .1 & E-09 \\
\hline 32 & Ramp & 1.08 & 44.2 & .1 & 2.9 & .03 & 4.89 & .05 & & & 2.01 & .01 & $E-10$ & 1.7 & .1 & E-08 \\
\hline 33 & 515. & 0.54 & 23.5 & .06 & .13 & .02 & .40 & .06 & .2928 & .9987 & 2.66 & .03 & E-10 & 8. & 4. & E-09 \\
\hline 34 & 515. & 0.54 & 14.9 & .04 & .05 & .01 & .24 & .05 & .31 & .99 & 1.89 & .03 & E-10 & 5. & 5. & $E-09$ \\
\hline 35 & 515. & 0.54 & 11.2 & .03 & .01 & .01 & .09 & .06 & .3272 & .99 & 1.53 & .04 & $E-10$ & 2 . & 6. & E-09 \\
\hline 36 & 515. & 1.0 & 17.3 & .06 & .01 & .02 & .04 & .07 & .3500 & .9993 & 1.28 & .02 & $E-10$ & .7 & 3. & E-09 \\
\hline 37 & Ramp & 0.54 & 63.9 & .2 & .02 & .01 & .02 & .01 & .4340 & .9994 & 1.17 & .01 & $E-09$ & 3. & 8. & E-09 \\
\hline 38 & 622. & & & .2 & .02 & .01 & .02 & .01 & .5336 & .9996 & 9.75 & .06 & E-10 & 3. & 6. & E-09 \\
\hline 39 & Los & 3.2 & $(85)$. & -- & $(.02)$ & -- & $(.02)$ & -- & .6453 & .9998 & 5.32 & -- & E-10 & 2 . & -- & E-09 \\
\hline 40 & 622. & 7.59 & 73.0 & .2 & -- & -- & -- & -- & & -- & 2.89 & .02 & E-10 & -- & -- & -- \\
\hline 41 & 622. & 1.08 & 47 & .01 & -- & - & -- & -- & .74 & -- & 1.9 & .2 & E-10 & -- & -- & $-\cdots$ \\
\hline 42 & 622. & 3.25 & 14.3 & .01 & -- & -- & -- & -- & .7673 & -- & 1.74 & .06 & $E-10$ & - & -- & -- \\
\hline 43 & 622 . & 3.79 & 8.92 & .006 & -- & -- & - & -- & .7790 & -- & 10.0 & .6 & $E-11$ & - & -- & -- \\
\hline 44 & Cool & 8.67 & .941 & .003 & -- & -- & -- & -- & .7802 & -- & 4.8 & 2. & E-12 & -- & -- & -- \\
\hline 45 & Melt & 6.90 & 167. & .6 & .03 & .01 & $\begin{array}{l}.011 \\
11.27\end{array}$ & $\begin{array}{l}.004 \\
.03\end{array}$ & 1.0 & 1.0 & -- & -- & ---- & -- & -- & --- \\
\hline & & & & & & & & & & & & & & & & \\
\hline
\end{tabular}


Figure 5.3. Comparison of ${ }^{3} \mathrm{He}$ and ${ }^{4} \mathrm{He}$ release from quartz grains during the step-heating experiment. ${ }^{3}$ He was released more rapidly (top) and at lower temperature (bottom) than ${ }^{4} \mathrm{He}$. The ${ }^{4} \mathrm{He}$ remaining after 75 hours was released by melting the sample. Temperature ramp steps are not shown in the lower plot. 
Figure 5.3
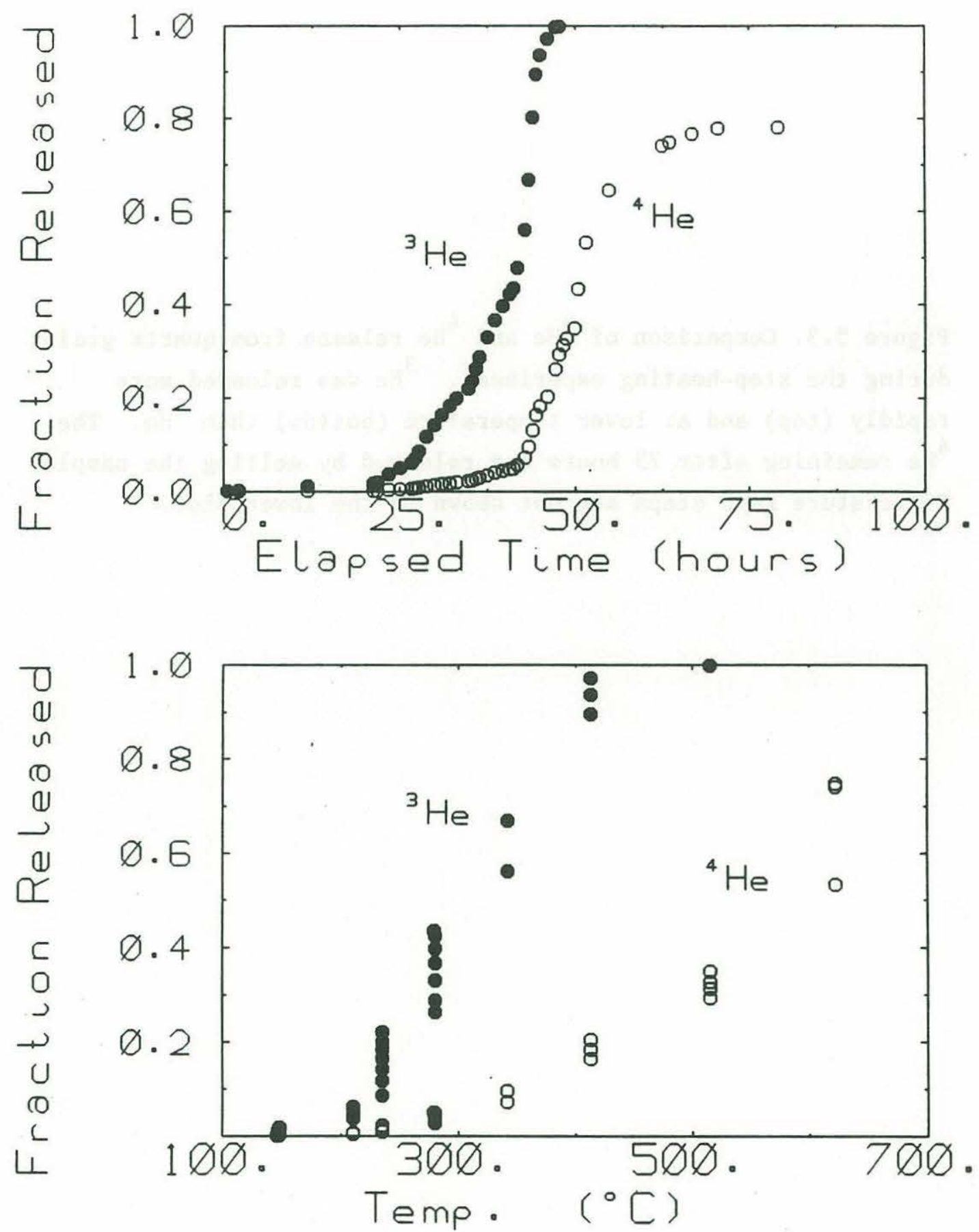
Figure 5.4 Isotopic compositions of sequential releases from BW84-105 quartz grains (500-710 um). The overall pattern is highly preferential release of ${ }^{3} \mathrm{He}_{\mathrm{c}}$, in comparison to the total ${ }^{3} \mathrm{He} /{ }^{4} \mathrm{He}$ ratio in the sample (dotted lines). The few initial aliquots with relatively low ${ }^{3} \mathrm{He} /{ }^{4} \mathrm{He}$ ratios contained very little helium $\left(0.2 \%\right.$ of $\left.{ }^{4} \mathrm{He}\right)$ and probably reflect loss of ${ }^{4} \mathrm{He}$ from surface sites. After $30 \%$ release of ${ }^{4} \mathrm{He}$, no measurable ${ }^{3} \mathrm{He}$ remained ( $x$-axis, top plot) and the isotopic composition of the remaining helium approached the radiogenic production ratio (.01 $\mathrm{Ra})$. 
Figure 5.4
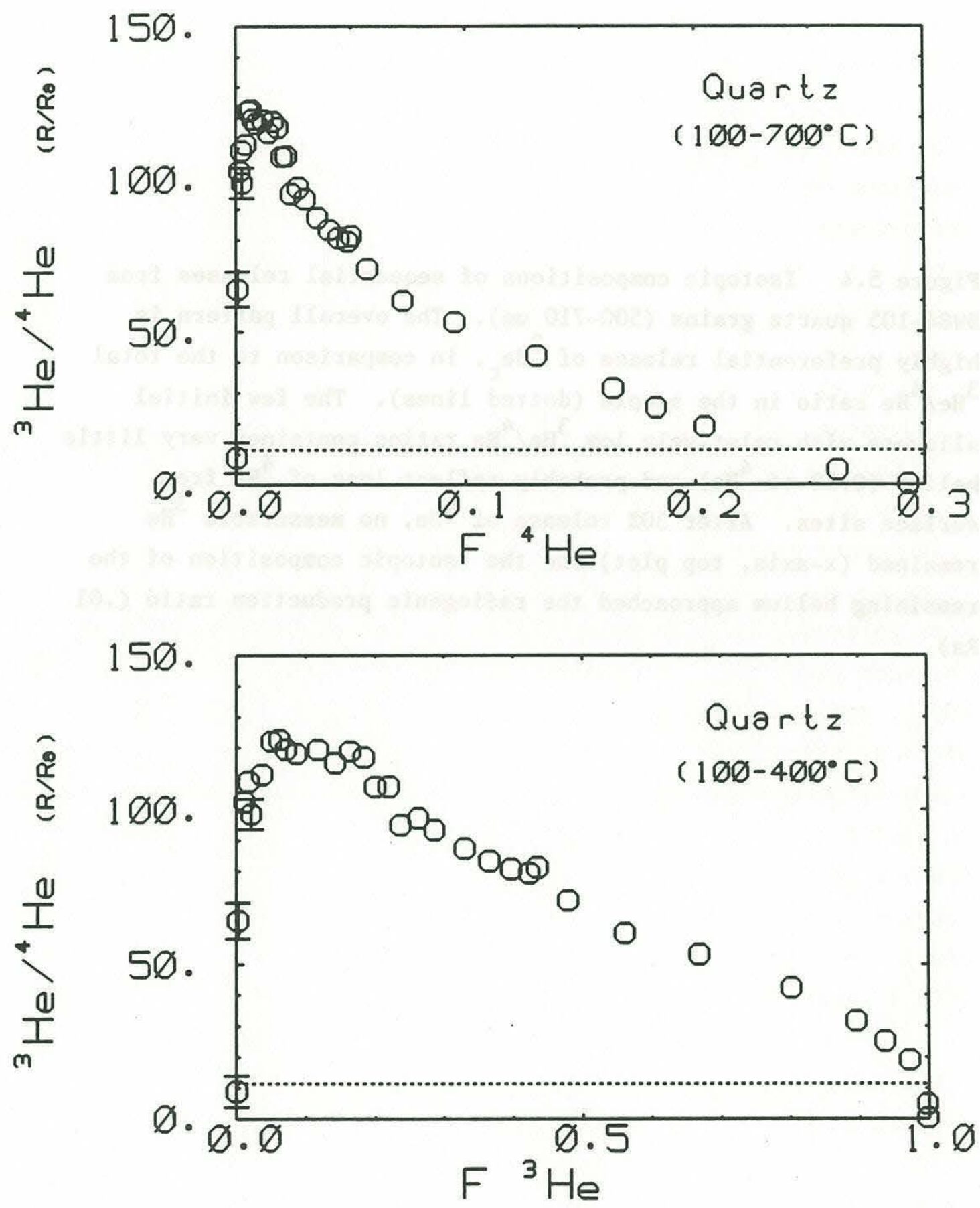
that the bulk ${ }^{3} \mathrm{He} /{ }^{4} \mathrm{He}$ ratio reflects the integrated production of cosmogenic and radiogenic helium since formation and exposure respectively, rather than characterizing a source component.

The ${ }^{3} \mathrm{He} /{ }^{4} \mathrm{He}$ ratio of the helium released by fusion, after driving off all the ${ }^{3} \mathrm{He}_{c}$, was $.011_{ \pm} .004 \mathrm{R}_{\mathrm{a}}$ (table 5.3). This represents the radiogenic production ratio, $R_{p}$, in the quartz grains in the absence of cosmogenic effects. The small amount of radiogenic ${ }^{3} \mathrm{He}$ is produced from the reaction ${ }^{6} \mathrm{Li}(\mathrm{n}, \alpha) \mathrm{T}{ }^{3} \mathrm{He}$, with the neutrons supplied by $\mathrm{X}(\alpha, \mathrm{n}) \mathrm{Y}$ reactions on major elements $(\mathrm{X})$, which are caused by uranium and thorium radio-decay (Morrison and Pine, 1955). In common silicate rocks, $R_{p}$ is about .01 to .02 $\mathrm{R}_{\mathrm{a}}$, but its exact value depends on $\mathrm{Li}$ contents, and to a lesser extent on the major element composition of the rock. For quartz, the observed ratio of $.011 \pm .004 \mathrm{R}_{\mathrm{a}}$ corresponds to a Li content of 10 to $25 \mathrm{ppm}$ (Andrews, 1985), in good agreement with preliminary measurements of $\mathrm{Li}$ in the sample (10 ppm, D. Colodner unpublished data).

Calculated helium diffusivities for each isotope are shown as a funtion of release in figure 5.5. Multiple steps at constant temperature exhibited constant diffusivities for most of the ${ }^{3} \mathrm{He}_{\mathrm{c}}$ releases, consistent with the volume diffusion model. In contrast, ${ }^{*} \mathrm{He}$ release exhibited this behavior in the early low temperature releases (most easily seen from table 5.3) but at temperatures above $400^{\circ} \mathrm{C}$, diffusivities tended to decrease within constant temperature plateaus (figure 5.5). This behavior was encountered in the basaltic glass diffusion experiments described in chapter 3 , and was demonstrated to derive from the presence of a range of grain sizes in those samples. However, this explanation is not reasonable here because of the wide range of $\mathrm{F}\left({ }^{4} \mathrm{He}\right)$ values over which it occurs $(.15-.8)$ and because similar behavior was not observed in the ${ }^{3} \mathrm{He}_{\mathrm{c}}$ releases over this $\mathrm{F}$ $\left({ }^{3} \mathrm{He}_{\mathrm{c}}\right)$ range.

In general, changes in slope in Arrhenius temperature-dependence plots are attributable to three classes of phenomena: a change in diffusion mechanism of a single specie, a change in the specie that is diffusing, or a change in the solid through which diffusion is occuring. If more than one diffusion mechanism applies, the one with higher activation energy will dominate at higher temperature. Thus, this is 
Figure 5.5. Examination of ${ }^{3} \mathrm{He}_{\mathrm{c}}$ and ${ }^{4} \mathrm{He}$ diffusivities in quartz. Solid dots are heating steps between temperature plateaus $\left({ }^{\circ} \mathrm{C}\right)$. ${ }^{3} \mathrm{He}$ release is characterized by generally constant $\mathrm{D}$ values at constant temperature, except at very low F (perhaps because of prior loss) and at very high $\mathrm{F}$ (where the calculation model is poor). In contrast, ${ }^{4} \mathrm{He}$ exhibits decreasing $\mathrm{D}$ values within several temperature plateaus. Most of these releases occurred after all ${ }^{3} \mathrm{He}$ was released (shown by hatched box in lower figure) and at higher temperature. This behavior may reflect a phase change in the mineral grains - see text. One aliquot was lost to valve failure, and the release shown is an estimate. Uncertainty in this estimate is insignificant for ${ }^{3}$ He diffusivities, but contributes $10 \%$ error to the ${ }^{4} \mathrm{He}$ diffusivities. 
Figure 5.5
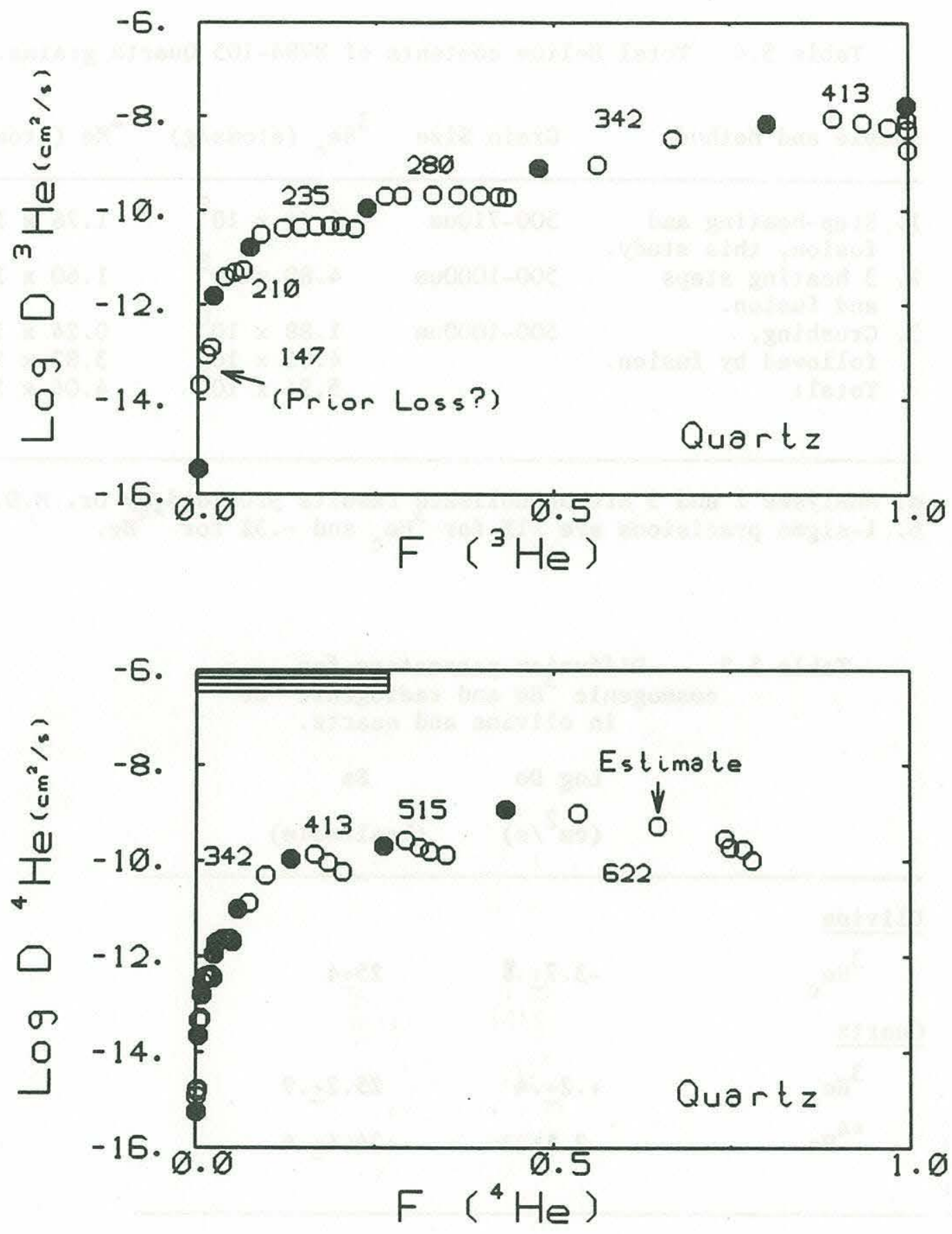
Table 5.4 Total Helium contents of BW84-105 Quartz grains.

Sample and Method Grain Size ${ }^{3} \mathrm{He}_{\mathrm{c}}$ (atoms/g) ${ }^{* 4} \mathrm{He}$ (atoms/g)

1. Step-heating and $\quad 500-710$ um $\quad 2.74 \times 10^{8} \quad 1.76 \times 10^{13}$

fusion, this study. $500-1000$ um $4.80 \times 10^{8} \quad 1.60 \times 10^{13}$

2. 3 heating steps $\quad 500-1000 u m \quad 4.80 \times 10^{8} \quad 1.60 \times 10^{13}$

3. Crushing, 500-1000um $1.88 \times 10_{8}^{8} \quad 0.24 \times 10_{13}^{13}$

$\begin{array}{lll}\text { followed by fusion. } & 4.06 \times 10_{8}^{8} & 3.82 \times 10^{13} \\ \text { Total: } & 5.94 \times 10^{8} & 4.06 \times 10^{13}\end{array}$

a. Analyses 2 and 3 are unpublished results provided $\star$ by Dr. M.D. Kurz.

b. 1-sigma precisions are $\sim 1 \%$ for ${ }^{3 e}{ }_{c}$ and $\sim .3 \%$ for ${ }^{4} \mathrm{He}$.

Table 5.5 Diffusion parameters for
cosmogenic ${ }^{4} \mathrm{He}$ and radiogenic ${ }^{4} \mathrm{He}$ in olivine and quartz.

$\begin{array}{lc}\text { Log Do } & \text { Ea } \\ \left(\mathrm{cm}^{2} / \mathrm{s}\right) & \text { (kcal/mole })\end{array}$

Olivine

${ }^{3} \mathrm{He}_{\mathrm{C}} \quad-3.7 \pm .8 \quad 25 \pm 4$

Quartz

$\begin{array}{lll}{ }^{3} \mathrm{He}_{\mathrm{C}} & +.2 \pm .4 & 25.2 \pm .9 \\ { }^{* 4} \mathrm{He} & -2.1 \pm .3 & 24.4 \pm .6\end{array}$


not a possible explanation for the ${ }^{* 4}$ He behavior which involves a decrease in temperature dependence at higher temperature. Similarly, it is unlikely that the change in slope represents a difference between the release of inherited ${ }^{4} \mathrm{He}$ residing in fluid inclusions and in-situ generated ${ }^{* 4} \mathrm{He}$, because 1 ) crushing the sample released only $6 \%$ of the total ${ }^{4} \mathrm{He}$ (table 5.4), and the change in release behavior occurs at $\mathrm{F}$ of about $20 \%$ and 2 ), if two components of ${ }^{4} \mathrm{He}$ were involved and governed by separate activation energies, the change in Arrhenius slope would be towards higher temperature dependence at higher temperature, as with a change in mechanisms.

The preferred explanation is that the change in ${ }^{* 4}$ He release reflects a change in the quartz matrix. A phase change occurs in quartz near $570^{\circ} \mathrm{C}$ for conversion of the low temperature stable form, trigonal alpha-quartz, to a more ordered hexagonal form, hexagonal beta-quartz (Deer, Howie, and Zussman, 1966). However, this conversion occurs quite sharply (over $2-3^{\circ} \mathrm{C}$ ), and it is unlikely to affect diffusion at temperatures below $500^{\circ} \mathrm{C}$. In contrast, rapid annealing of defects in quartz occurs in the temperature range of $300-400{ }^{\circ} \mathrm{C}$, as shown by studies of electron spin resonance associated with Schottkey-Frenkel defects generated by alpha-decay (e.g. Odom and Rink, 1988). This process is the likely source of the decrease in ${ }^{* 4} \mathrm{He}$ release rate, which occurs in this temperature range (figure 5.6). Helium diffusion in a well-annealed hexagonal silica polymorph, tridymite, exhibits a similarly low activation energy to the ${ }^{* 4}$ He results obtained at annealing temperatures here (figure 5.7), although caution must be used in comparing the release of trace quantities of natural helium with these results, which were obtained by experimentally equilibrating heated grains with large amounts of helium (Barrer and Vaughan, 1967). The annealing explanation could be verified by reproducing the break in Arrhenius slope, while simultaneously measuring electron spin resonance changes: In general, understanding the role of lattice-damage and its annealing rate will be important to the use of ${ }^{* 4} \mathrm{He},{ }^{3} \mathrm{He}{ }_{c}$, or electron spin resonance (Odom and Rink, 1988) as quartz geochronometers, because diffusivities and annealing rates may depend on radiation doses. In the 
present work, it appears both ${ }^{* 4} \mathrm{He}$ and ${ }^{3} \mathrm{He}{ }_{\mathrm{C}}$ releases were governed by Arrhenius behavior at the low temperatures of interest.

Below $400^{\circ} \mathrm{C}$, cosmogenic ${ }^{3} \mathrm{He}_{\mathrm{C}}$ diffusion proceeds almost 100 times as fast as radiogenic ${ }^{* 4}$ He diffusion, but the two species are governed by indistinguishable activation energies, $25.2 \pm .9$ and $24.4 \pm .6 \mathrm{kcal} / \mathrm{mole}$ respectively $\left(\log D^{0}\right.$ values are $+.2 \pm .4$ and $-2.1_{ \pm} .3$; figure 5.6$)$. This suggests that thermal activation is controlled by properties of the quartz matrix (e.g. lattice vibrational moments and the extent of thermal expansion), and that the rapid ${ }^{3} \mathrm{He}$ c release may reflect greater access to spallation related lattice damage that enhances loss. Further experiments with different grain sizes of different exposure ages may help to establish the extent to which lattice damage controls ${ }^{3} \mathrm{He}_{\mathrm{c}}$ and ${ }^{* 4} \mathrm{He}$ release.

Because the activation energies for ${ }^{\star 4} \mathrm{He}$ and ${ }^{3} \mathrm{He}{ }_{\mathrm{c}}$ release are large, the diffusivity for a natural sample may vary considerably seasonally, for example $\mathrm{D}$ is 45 times higher at $25^{\circ} \mathrm{C}$ than at $0^{\circ} \mathrm{C}$. The long-term effective diffusivity is thus the mean value associated with the time integral of the temperature-dependent diffusivity (see equation 2.4 , chapter 2). Because of the strong increase in $\mathrm{D}$ with temperature this effective diffusivity will generally be higher than the diffusivity implied by the mean annual temperature. Extrapolating the temperature trends in figure 5.6 to $0^{\circ} \mathrm{C}$ implies very low diffusivities for both isotopes, $2 \times 10^{-20} \mathrm{~cm}^{2} / \mathrm{s}$ for ${ }^{3} \mathrm{He}_{\mathrm{c}}$ and $1 \times 10^{-22} \mathrm{~cm}^{2} / \mathrm{s}$ for ${ }^{* 4} \mathrm{He}$ with 1 sigma errors of a factor of two (figure 5.7). As elaborated below, these low diffusivities suggest helium loss will not greatly impede surface exposure dating in the Antartic Dry Valleys, where mean annual temperatures are close to $-20^{\circ} \mathrm{C}$ and rarely much above freezing ( $\mathrm{C}$. Hendy, personal experience). As always in experiments of this type, temperature extrapolations assume that the diffusion mechanisms which dominate in the laboratory also control release at lower temperature, and that the temperature dependence is indeed a simple exponential. However, there is no evidence to suggest this is a bad assumption and the results are in reasonable agreement with the value of $10^{-20} \mathrm{~cm}^{2} / \mathrm{s}$ suggested by Tolstikhin et al (1974) to explain the presence of internal isotopic disequilibrium in geologically old quartz grains. 
Figure 5.6 Arrhenius relations for cosmogenic ${ }^{3} \mathrm{He}$ and radiogenic ${ }^{4} \mathrm{He}$ diffusion in BW84-105 quartz grains have identical activation energies $(24.4 \pm .6$ and $25.2 \pm .9 \mathrm{kcal}$ per mole, respectively) but ${ }^{3} \mathrm{He}_{\mathrm{c}}$ diffusivities are 200 times higher (Log $\mathrm{D}^{\mathrm{O}}$ values $=-2.1 \pm .3$ and $+.2 \pm .4)$. 1 sigma errors are similar or smaller than the symbol size. The departure from linearity for ${ }^{4} \mathrm{He}$ at high temperature may reflect annealing of defects within the quartz grains (see text). 
Figure 5.6

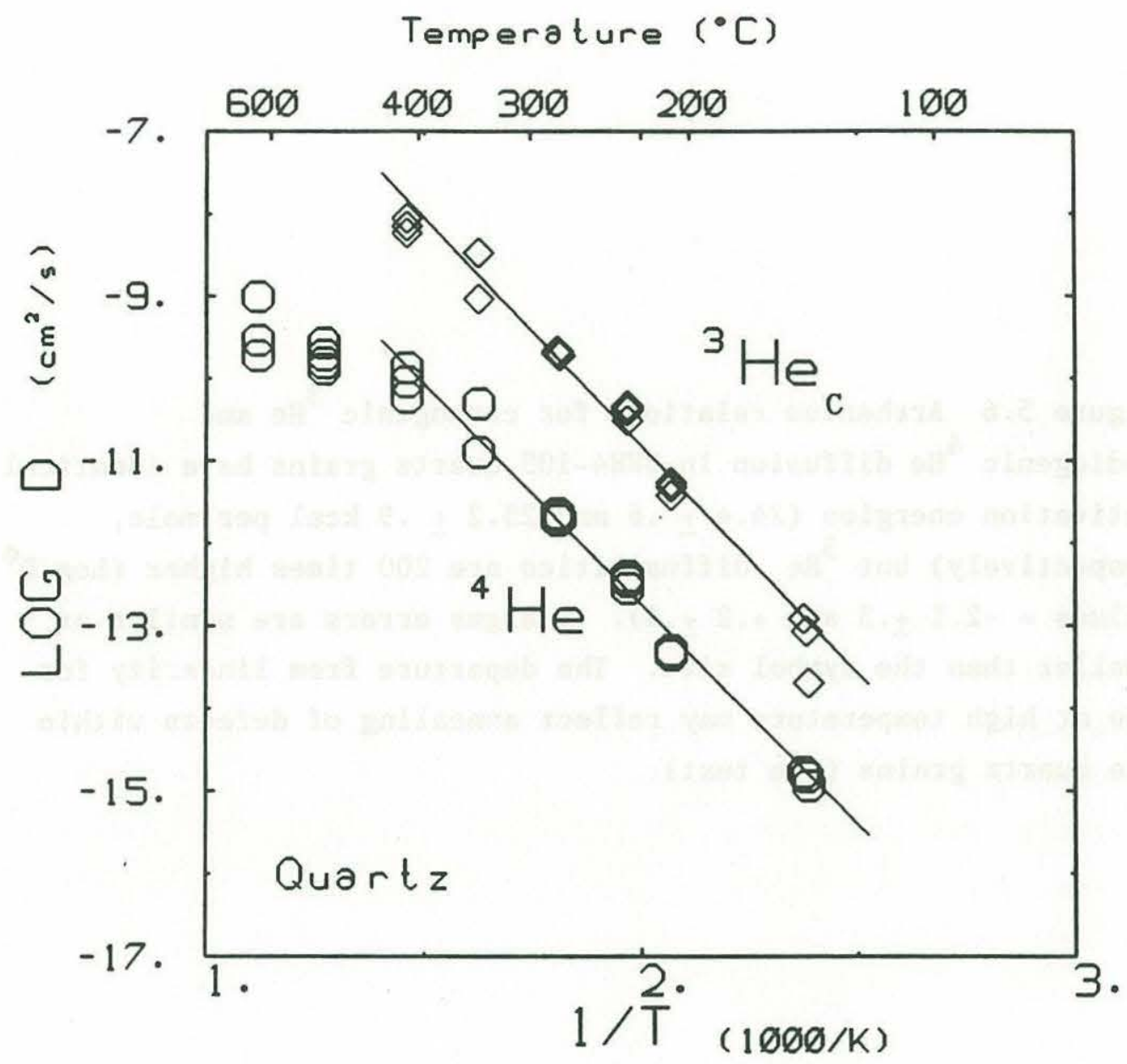


Figure 5.7 Comparison of Arrhenius relations for ${ }^{3} \mathrm{He}$, and ${ }^{* 4} \mathrm{He}$ in quartz with release of ${ }^{4} \mathrm{He}$ from tridymite (Barrer and Vaughan, 1967). The dashes extrapolate the experimental results to low temperature, suggesting D $=2 \times 10^{-20}$ and $1 \times 10^{-22}{ }^{3} \mathrm{He}_{\mathrm{c}}$ and ${ }^{4} \mathrm{He}$ at $0^{\circ} \mathrm{C}$. The break in slope in ${ }^{* 4} \mathrm{He}$ diffusion above $400^{\circ} \mathrm{C}$ may reflect annealing of defects in the quartz sample. The activation energy (slope) above $400^{\circ} \mathrm{C}$ is similar to the value determined in an annealed quartz polymorph, tridymite, at lower temperature by Barrer and Vaughan, 1967). 
Figure 5.7

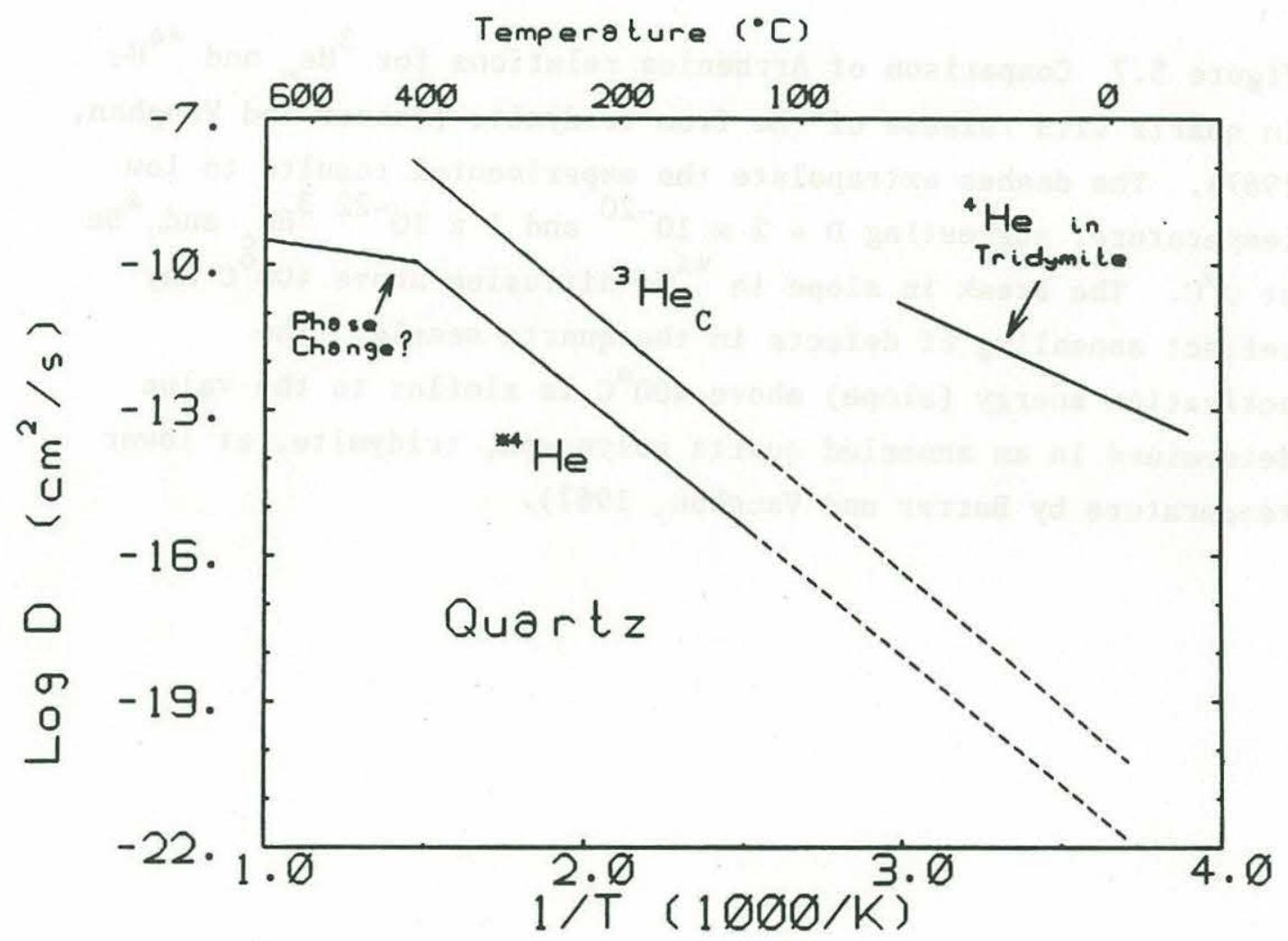


For those who feel that a low mass neutral atom like helium must be more mobile than this, it is worth repeating the words of Keevil (1940) - "Statements are sometimes made that helium should be able to diffuse through certain kinds of crystals because of their open structure, but these appear to be guesses without any quantitative picture of the situation". Keevil went on to discuss the interatomic potentials governing lattice spacings in silicate crystals including quartz, and concluded that helium atomic dimensions were relatively large (effective radius of .95 to 1.3 Angstrom) in comparison to any "holes", so that its mobility was likely to be low, and would require local distortions, coupled vibrations, and the crossing of significant energy barriers. Refining the estimates obtained here through experimentation at lower temperatures will face the same difficulties as for olivine, although somewhat alleviated by the roughly 5-fold higher ${ }^{3} \mathrm{He}$ contents in the quartz sample. An alternative method of assessing ${ }^{3} \mathrm{He}$ mobility at low temperature would be to compare the cosmogenic helium content of a wide range of natural grain sizes from a sample of known age.

\section{$\underline{5.4} \underline{\text { DISCUSSION }}$}

The measured diffusivities can be used to place limits on helium loss problems in surface exposure dating. For a mineral grain undergoing both diffusive loss and constant production by cosmic ray spallation the helium concentration is governed by:

$$
\mathrm{dC} / \mathrm{dt}=\mathrm{D} \nabla^{2} \mathrm{C}+\mathrm{P}
$$

where $\mathrm{P}$ is the production rate (per unit volume). This equation assumes that the helium diffusion coefficient, $D$, is independent of concentration, which is probably true for typical trace helium contents, but may not hold in crystals which have undergone extreme bombardment, e.g. lunar materials. The fraction of produced helium which remains at time $t$ can then be written:

$$
\mathrm{F}_{\mathrm{t}}=\mathrm{C} / \mathrm{Pt}=\left\{\mathrm{Pt}-\int_{0}^{\mathrm{t}}(\mathrm{dC} / \mathrm{dt}) \mathrm{dt}\right\} / \mathrm{Pt}
$$


This fraction represents the underestimation of an exposure age produced by diffusive loss. For example, if $\mathrm{F}_{\mathrm{t}}$ is .2 , the model age

$$
t^{\prime}=C / P
$$

will be underestimated by a factor of five.

$\mathrm{F}_{\mathrm{t}}$ values calculated numerically for spherical grains (see Appendix D) are plotted for several diffusivities in figure 5.8. Compared with diffusive loss in the absence of production, the fractional loss of cosmogenic helium is slower, but both scale with the ratio $\mathrm{D} / \mathrm{a}^{2}$, where $\mathrm{D}$ is the diffusion coefficient and a the spherical grain radius. (The infinite-series solution used to calculate the curves for diffusion alone is given in chapter 2.) Similarly, radial concentration profiles for the two solutions have generally similar shapes, but the profile for production and diffusion evolves more slowly and reaches a steady state quadratic profile (figure 5.9). Both the effect of loss on apparent ages and the approach to steady state are very clear in figure 5.10, which shows the relationship between apparent $(\mathrm{C} / \mathrm{P})$ and true ages for different $\mathrm{D} / \mathrm{a}^{2}$ values. For example, at a $\mathrm{D} / \mathrm{a}^{2}$ value of $1 \times 10^{-15} \mathrm{~cm}^{2} / \mathrm{s}$ model ages will begin to underestimate true ages at about 1 million years, and will become meaningless in rocks over 10 million years once the helium content has reached steady-state. These curves provide a way to correct model ages using measured grain sizes and diffusivities. For this purpose, an expanded plot is given, which, while drawn for the time interval of .1 to 1 million years, can be used for any order of magnitude age range by scaling the diffusivities (figure 5.11).

For olivine, diffusive loss of cosmogenic helium will not significantly affect surface exposuring dating except in the smallest grain sizes. Given the extrapolated diffusivity of $7 \times 10^{-22} \mathrm{~cm}^{2} / \mathrm{s}$ at $40^{\circ} \mathrm{C}$ (as an upper limit for Hawaiian lavas), $\log \mathrm{D} / \mathrm{a}^{2}$ values are less than -17 for grains greater than $.25 \mathrm{~mm}$ in diameter. Thus, exposure dates will not be in error for ages less than 100 million years (figure $5.10) !$ This result is apparent from the diffusive length scale $\left(x^{2}=D t\right)$ of 1 um for this $\mathrm{D}$ value (22um is the 2-sigma upper limit at $40^{\circ} \mathrm{C}$ ). This low predicted mobility of ${ }^{3} \mathrm{He}_{\mathrm{c}}$ in olivine is consistent with the 
observation that inherited helium released from fluid inclusions by crushing Hawaiian olivine phenocrysts is never contaminated by ${ }^{3} \mathrm{He}_{\mathrm{c}}$ produced in the crystal matrices, at least for rocks less than 1 million years old (Kurz, 1986a,b). Therefore, cosmogenic dating of basaltic rocks using olivine mineral separates is more likely to be limited by geologic problems such as shielding and erosion than by helium loss problems.

Diffusive effects on exposure ages are somewhat greater in quartz than olivine, but are still relatively small. For the Antarctic sample studied here, the grain size of $.5 \mathrm{~mm}$ and the approximation of the $0^{\circ} \mathrm{C}$ diffusivity for the seasonal average, $\mathrm{D} / \mathrm{a}^{2}=3.2 \times 10^{-17}$, so that apparent exposure ages begin to be significantly underestimated (factor of 2) for samples older than about 10 million years (figure 5.10). The ${ }^{3}{ }{ }_{c}$ content of Bw84-105 quartz (3-6 $\times 10^{8}$ atoms/g; table 5.4) suggests an age of 800,000 to 1.7 million years, given a production rate of 360 atoms/g/yr. (The production rate has not been measured directly and was estimated from a Hawaiian sea-level rate of 100 atoms/g/yr (Kurz, 1986b) adjusting for lattitude (factor of 1.4) and altitude (factor of 2.55 at $1300 \mathrm{~m})$; the error should be less than a factor of 2). Correcting these model ages for diffusive loss suggests the corresponding true ages are 850,000 and 2.1 million years (figure 5.11), i.e a 5-20\% correction. At present, this is well within other uncertainties such as the precise production rate, and the origin of the factor of two variability in ${ }^{3} \mathrm{He}_{\mathrm{c}}$ contents of replicate analyses (table 5.4). However, diffusive effects will be more important at higher temperatures, for example at an environmental temperature of $30^{\circ} \mathrm{C}$, the quartz ${ }^{3} \mathrm{He}_{\mathrm{c}}$ diffusivity is about 140 times higher than at $0^{\circ} \mathrm{C}$, so that model ages for $.5 \mathrm{~mm}$ grains will err by a factor of two at ages of about 100,000 years (figure 5.10). The $\mathrm{F}_{\mathrm{t}}$ curves can also be used to consider diffusive loss problems for $\mathrm{U}+\mathrm{Th} / \mathrm{He}$ geochronology, since ${ }^{*} \mathrm{He}$ production is essentially constant for ages much less the mean life of its radioactive parents (billions of years). The measured ${ }^{* 4}$ He diffusivities in quartz are 100 -fold smaller than ${ }^{3} \mathrm{He}_{\mathrm{c}}$ values, so that radiogenic helium is likely to be quantitatively retained for ages less than 100 million years even in 
Figure 5.8. Comparison of diffusive helium loss with and without helium production. The model curves are for spherical grains with constant isotropic helium production and homogeneous initial helium distribution, respectively. The y-axis represents the remaining fraction of helium produced $\left(\mathrm{F}_{\mathrm{t}}\right)$ or initially present $\left(\mathrm{C} / \mathrm{C}^{\mathrm{O}}\right)$. Fractional loss occurs more rapidly in the absence of production, but both loss problems scale with the ratio of the diffusion coefficient (D) to the square of the grain radius, a. 
Figure 5.8

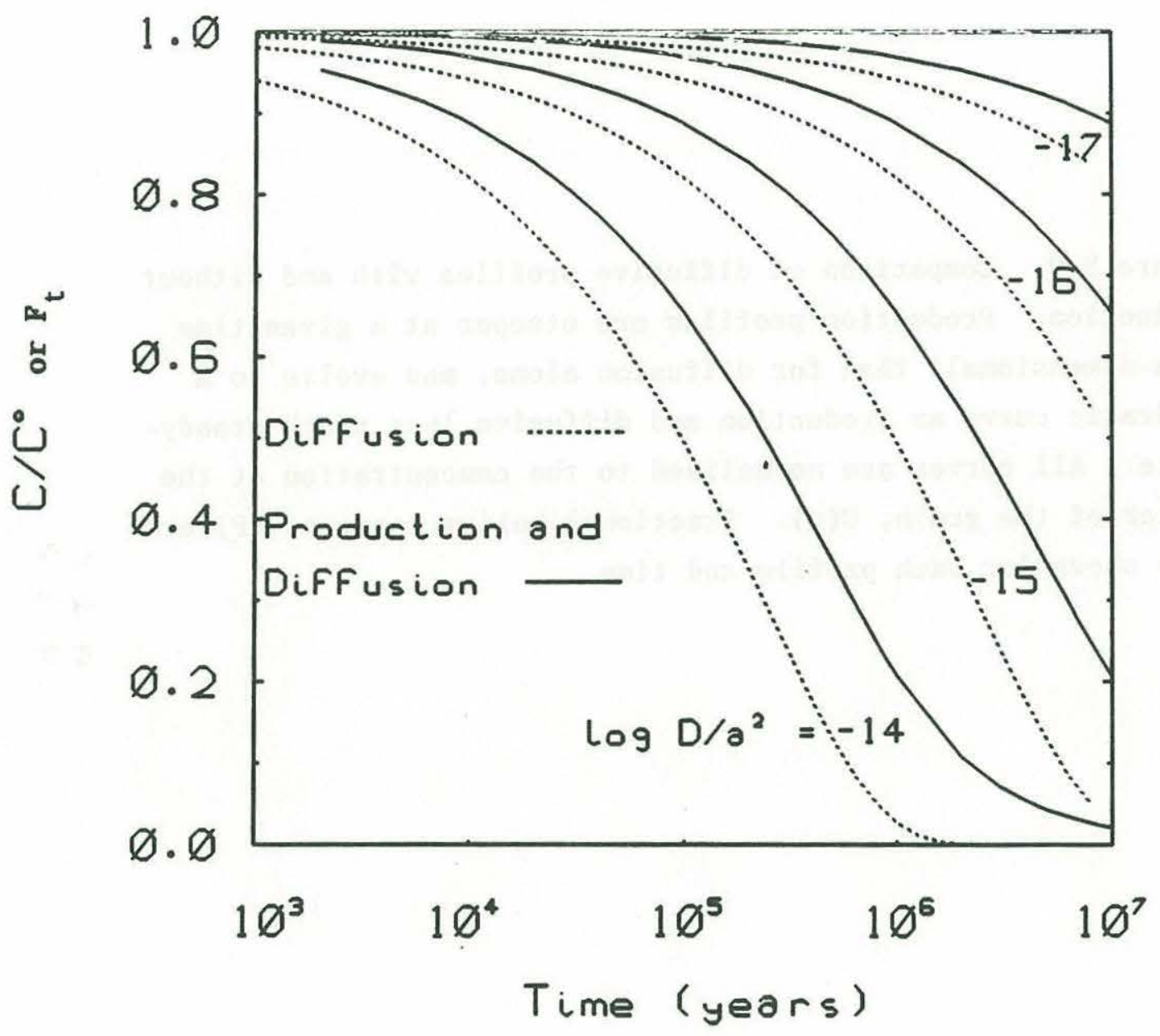


Figure 5.9. Comparison of diffusive profiles with and without production. Production profiles are steeper at a given time (non-dimensional) than for diffusion alone, and evolve to a quadratic curve as production and diffusive loss reach steadystate. All curves are normalized to the concentration at the center of the grain, $\mathrm{C}(\mathrm{o})$. Fractional helium contents (F) are also shown for each profile and time. 
Figure 5.9

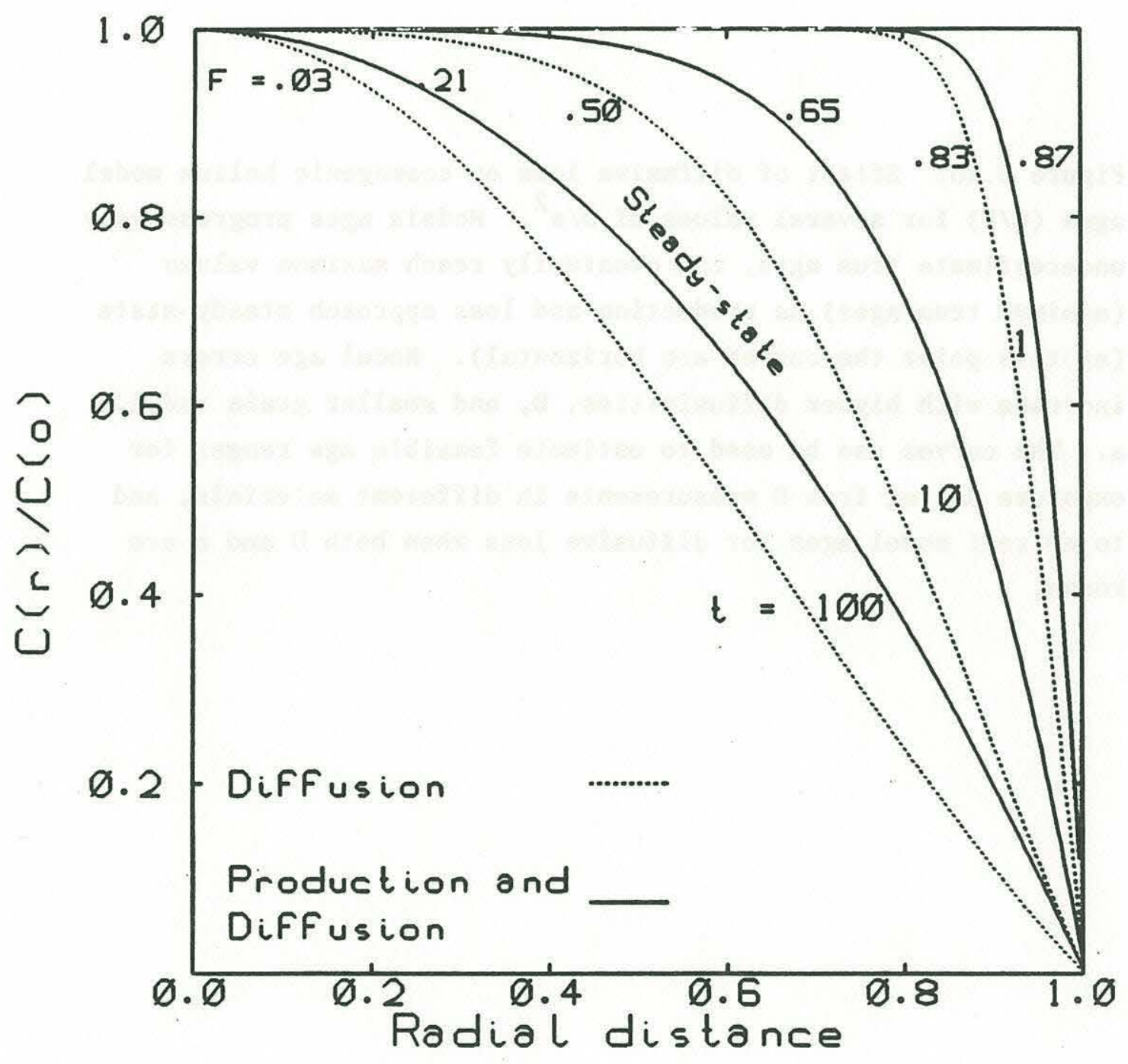


Figure 5.10. Effect of diffusive loss on cosmogenic helium model ages $(\mathrm{C} / \mathrm{P})$ for several values of $\mathrm{D} / \mathrm{a}^{2}$. Models ages progressively underestimate true ages, and eventually reach maximum values (minimum true ages) as production and loss approach steady-state (at this point the curves are horizontal). Model age errors increase with higher diffusivities, D, and smaller grain radii, a. The curves can be used to estimate feasible age ranges for exposure dating from $D$ measurements in different materials, and to correct model ages for diffusive loss when both $\mathrm{D}$ and a are known. 
Figure 5.10

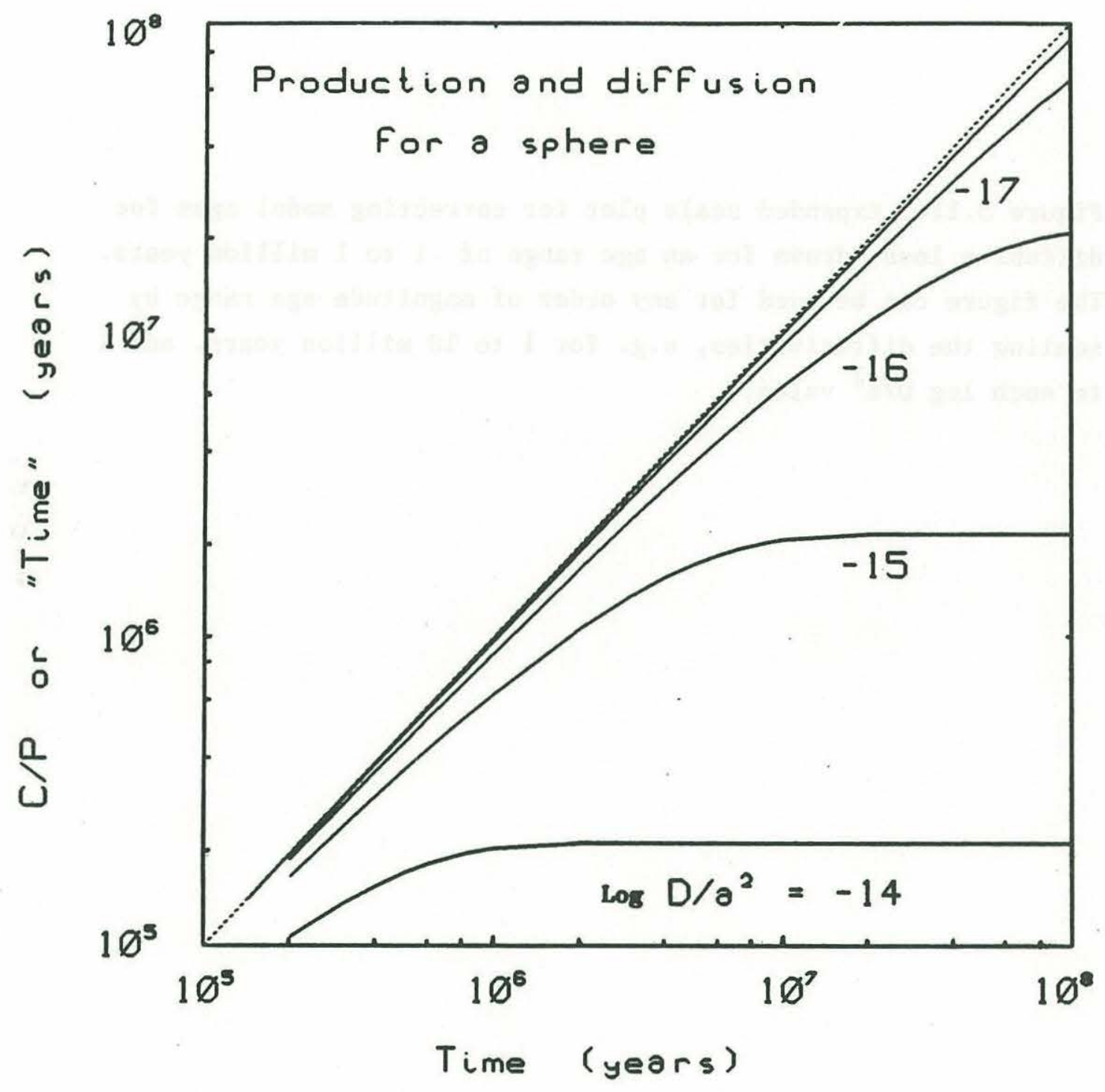


Figure 5.11. Expanded scale plot for correcting model ages for diffusive loss, drawn for an age range of .1 to 1 million years. The figure can be used for any order of magnitude age range by scaling the diffusivities, e.g. for 1 to 10 million years, add 1 to each $\log \mathrm{D} / \mathrm{a}^{2}$ value. 
Figure 5.11

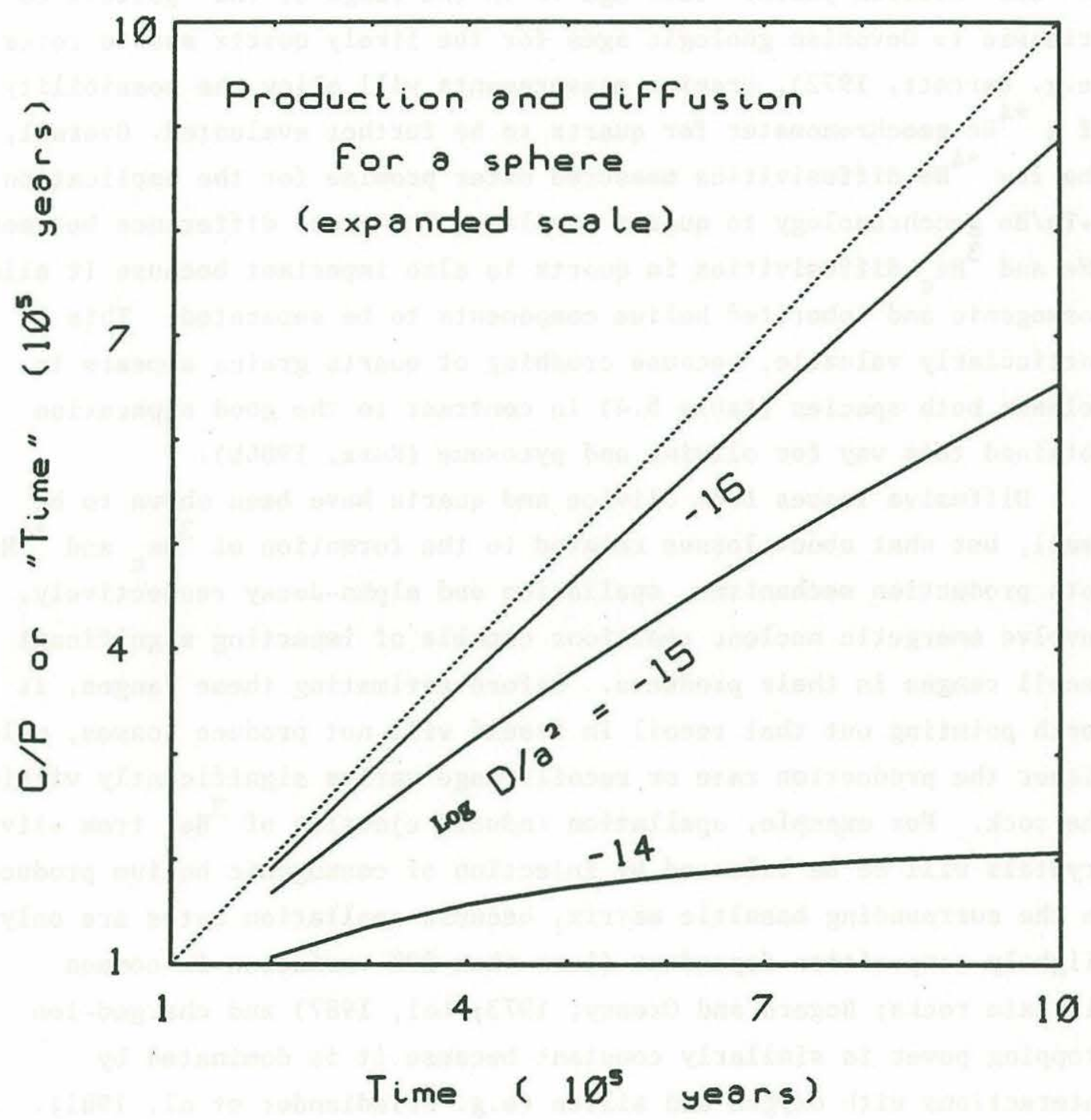


. $1 \mathrm{~mm}$ diameter grains. Given the ${ }^{* 4}$ He content of BW84-105 quartz (2$4 \times 10^{13}$ atoms/gr; table 5.4), a very rough $U$ content estimate of less than $10 \mathrm{ppb}$ (based on a microprobe $\mathrm{K}$ estimate of less than $.01 \%$, and $\mathrm{K} / \mathrm{U}$ of about $2 \times 10^{4}$ in orthoquartzites, Murray and Adams, 1958), and a Th/U ratio of $3-4$ (op.cit.), the age for this sample is on the order of 200 to 600 million years. This age is in the range of the postulated Triassic to Devonian geologic ages for the likely quartz source rocks (e.g. Barrett, 1972). Uranium measurements will allow the possibility of a ${ }^{* 4} \mathrm{He}$ geochronometer for quartz to be further evaluated. Overall, the low ${ }^{\star 4}$ He diffusivities measured offer promise for the application of $\mathrm{U}+\mathrm{Th} / \mathrm{He}$ geochronology to quartz samples. The great difference between ${ }^{4} \mathrm{He}$ and ${ }^{3} \mathrm{He}_{\mathrm{c}}$ diffusivities in quartz is also important because it allows cosmogenic and inherited helium components to be separated. This is particularly valuable, because crushing of quartz grains appears to release both species (table 5.4) in contrast to the good separation obtained this way for olivine and pyroxene (Kurz, 1986b).

Diffusive losses from olivine and quartz have been shown to be small, but what about losses related to the formation of ${ }^{3} \mathrm{He}_{\mathrm{c}}$ and ${ }^{* 4} \mathrm{He}$ ? Both production mechanisms, spallation and alpha-decay respectively, involve energetic nuclear reactions capable of imparting significant recoil ranges in their products. Before estimating these ranges, it is worth pointing out that recoil in itself will not produce losses, unless either the production rate or recoil range varies significantly within the rock. For example, spallation induced ejection of ${ }^{3} \mathrm{He}_{\mathrm{c}}$ from olivine crystals will be be balanced by injection of cosmogenic helium produced in the surrounding basaltic matrix, because spallation rates are only slightly composition dependent (less than $20 \%$ variation in common silicate rocks; Bogard and Cressy, 1973; Lal, 1987) and charged-ion stopping power is similarly constant because it is dominated by interactions with oxygen and silica (e.g. Friedlander et al, 1981). This balance will even hold for rocks with significant porosity, because stopping power is roughly proportional to density (op. cit.) so that particles ejected from grains will tend to cross pore spaces and enter other grains. For example, the range of alpha-particles in air is several centimeters (Friedlander et al, 1981). However, production of 
${ }^{* 4} \mathrm{He}$ and the component of ${ }^{3} \mathrm{He}_{\mathrm{c}}$ formed by neutron reactions on ${ }^{6} \mathrm{Li}$ may vary within rocks because of heterogeneous distribution of $\mathrm{U}$, Th or Li. Thus, in basalts more radiogenic helium will be injected into olivine and other phenocrysts than is ejected because $U$ and $T h$, as incompatible elements, are relatively enriched in the ground mass. A similar situation may hold for ${ }^{6} \mathrm{Li}$ produced ${ }^{3} \mathrm{He}_{\mathrm{c}}$.

The fraction of helium injected or ejected from any location within a spherical grain can be calculated from the fraction of particles whose recoil paths reach the grain boundary. Making the approximations of a constant stopping range $\left(r^{*}\right)$ and that the distribution of recoil directions is isotropic, the loss can be integrated over the spherical volume for a homogeneous spatial distribution of production to give:

$$
F=3 / 4\left(r^{*} / r_{0}\right)-1 / 16\left(r^{*} / r_{0}\right)^{3}
$$

where $r_{0}$ is the grain radius (Flugge and Zimens, 1939; Giletti and Kulp, 1955). This formula assumes $r *$ is less than $2 r_{0}$. The stopping range of alpha-particles $\left({ }^{\star 4} \mathrm{He}\right)$ in basalt and quartz can be calculated from experimental data for major element targets. For the 3-6 Mev alpha-decay energies of the $U$ and Th series, the mean stopping distance is 25um (range 20-45um) in basalt and somewhat less in quartz (Graham, 1987). For this range, the ${ }^{* 4} \mathrm{He}$ fraction lost or added to a $1 \mathrm{~mm}$ diameter grain is only $4 \%$ ( $8 \%$ for $.5 \mathrm{~mm}$ ) of the production in the grain or surrounding matrix, respectively. This loss would be insignificant for $U+T h / H e$ geochronology studies in minerals, but the injection could be important to mineral separate helium isotopic studies of petrogenesis in uranium rich, older rocks. The injection of ${ }^{*} \mathrm{He}$ also has a small, indirect effect on the determination of phenocryst ${ }^{3}{ }_{\mathrm{He}}$ c contents, because the ${ }^{4} \mathrm{He}$ content of the crystals is used to correct for inherited (magmatic) ${ }^{3} \mathrm{He}$ (Kurz, 1986a,b). Injection of ${ }^{\star 4}$ He produced in the surrounding, relatively $U$ and Th rich matrix will lead to overcorrection and thus low ${ }^{3} \mathrm{He}_{\mathrm{c}}$ ages. However, this error will generally be insignificant in comparison to other uncertainties, e.g. less than $4 \%$ in the approximately 500,000 yr old Kula basalt samples studied by Kurz (op. cit.). The small component of ${ }^{3} \mathrm{He}_{\mathrm{c}}$ produced by the reaction of 
thermalized neutrons with ${ }^{6} \mathrm{Li}$ will also have a short recoil range (about 30um), because the energy of this reaction is less than $5 \mathrm{MeV}$ (Lal, 1987), so that injection and ejection processes for this component are insignificant.

In contrast to alpha-particles and ${ }^{6} \mathrm{Li}$ produced ${ }^{3} \mathrm{He}$, the recoil range of spallation produced helium is not well known. The track lengths of spallation produced alpha-particles in film emulsions exposed to cosmic rays at high altitude range from 50-250um (Rossi, 1952). However, most spallations in surface rocks will occur at lower energies than these events. Cosmic-ray neutrons are responsible for the vast majority of spallation events in surface rocks (e.g. Lal and Peters, 1967; Kurz, 1986b). Because the energy spectrum for these particles at sea level drops off extremely rapidly with increasing energy (e.g. Wolfendale, 1973) and spallation cross-sections for the important target elements are relatively invariant with energy above a threshold value (10-50 MeV), essentially all ${ }^{3} \mathrm{He}_{\mathrm{c}}$ production will occur at energies close to the thresholds (Lal and Peters, 1967). A maximum energy for a spalled nuclide can be estimated from the threshold energies by considering the reaction to be a two-body collision (between the neutron, $\mathrm{n}$, and a target atom, $\mathrm{x}$ ) which yields only two products (a daughter nuclide, $d$, and the spalled nuclide, $s$ ). Conserving momentum, kinetic energy, and mass in this reaction gives the approximation:

$$
E_{s}=E_{n} \cdot m_{x}\left(m_{x}-m_{s}\right) /\left(m_{n}+m_{x}\right)^{2}
$$

Which can be written, for $m_{x} \gg m_{s}$, as:

$$
E_{S}=E_{n}\left(1-m_{S} / m_{x}\right)
$$

which shows that the energy of the spalled particle approaches that of the incoming cosmic neutron as the target mass increases. This large energy transfer occurs because the products must equipartition the initial momentum of the cosmic neutron (in the center-of-mass coordinate system), so that the velocity and therefore the energy share of the small spallation nuclide is relatively large. For target elements of 
mass $16(0)$ to $28(\mathrm{Si})$, the approximate energy of a spalled ${ }^{3} \mathrm{He}$ will be 70 to $85 \%$ of the incoming neutron energy .

This estimate is a maximum for two reasons. First, the approximation ignores the conversion of kinetic energy into nuclear binding energy, that is, mass is not actually conserved. Considering the curve for binding energy per nucleon as a function of nuclear mass shows that $10-20 \mathrm{MeV}$ of the incoming energy will be lost to increased mass of the products ( $s$ and $d$ ), for typical target masses of less than $50 \mathrm{amu}$. Secondly, more than two products are generally produced in spallation reactions. Three to five or more particles are common (Rossi, 1952; Lal, 1988 figure 2). The partitioning of energy between these particles depends on their masses, binding energies and directions of recoil and can not be readily calculated, but in a rough sense the total energy will be equipartitioned because the spectrum of particle masses produced in low energy spallation reactions is very narrow and centered on low mass particles (Friedlander et al, 1981) which have similar binding energy deficits in comparison to target atoms. This suggests that many spallation reactions will produce ${ }^{3} \mathrm{He}_{\mathrm{c}}$ particles with quite low energies. For example, $10 \mathrm{MeV}$ of the incoming energy (say 50 $\mathrm{MeV}$ ) may be lost to mass increase in each of three products, and the remaining $20 \mathrm{MeV}$ of kinetic energy split among them to give an average energy of 6-7 MeV.

The stopping range for these energies can be estimated by comparison with empirical results for alpha-particles in aluminum (an element with similar stopping power to Si, and less power than oxygen). At 6-7 MeV, a range of 20 to $30 \mathrm{um}$ is predicted (Friedlander et al, 1981). However, it must be emphasized that individual ${ }^{3} \mathrm{He}_{\mathrm{c}}$ atoms may have higher energies, and that the stopping range has never been measured for geologic materials. As a note of caution - the predicted stopping range at $50 \mathrm{MeV}$ is approximately 600um. If the stopping range estimate of 20-30 um is correct, ejection will clearly be an insignificant problem in most surface exposue dating efforts. For larger stopping distances this will still tend to be true, because of the general balance between injection and ejection. Therefore, it appears ${ }^{3} \mathrm{He}_{\mathrm{c}}$ retention is unlikely to limit ${ }^{3} \mathrm{He}_{\mathrm{c}}$ geochronology. 


\section{$\underline{5.5}$ SUMMARY}

Measured diffusivities for cosmogenic ${ }^{3} \mathrm{He}$ in quartz and olivine were enhanced by several orders of magnitude over those for inherited helium, but were still very low. Extrapolating the approximately 25 $\mathrm{Kcal} / \mathrm{mole}$ Arrhenius temperature relations, observed at $150-400^{\circ} \mathrm{C}$ in both minerals, suggests $20^{\circ} \mathrm{C}$ diffusivities of less than $10^{-18}$ and $10^{-22} \mathrm{~cm}^{2} / \mathrm{s}$ for quartz and olivine, respectively. Comparing these low mobilities to model calculations for production and diffusive loss suggests surface exposure dating using ${ }^{3} \mathrm{He}_{\mathrm{c}}$ will not be limited by losses for timescales up to 10 million years in quartz and 100 million years in olivine (for $1 \mathrm{~mm}$ grains). However, this conclusion depends strongly on the mineral grain sizes used, for smaller grains (e.g. 250um) sample ages will begin to be underestimated at 100,000 to 1 million years. When significant loss occurs, the model curves can be used to correct the apparent ages, whenever the ${ }^{3} \mathrm{He}_{\mathrm{c}}$ diffusion coefficient and grain size are known or measured.

Consideration of ${ }^{3} \mathrm{He}_{\mathrm{c}}$ formation processes also suggests that ${ }^{3} \mathrm{He}_{\mathrm{c}}$ is likely to be highly retained. Recoil energy estimates and thus stopping ranges are relatively low (20-30um, similar to radiogenic alpha-particles). Moreover, in most rocks any ejection losses are likely to be balanced by injection. Combined with the low diffusivities, this suggests helium losses will not hamper most ${ }^{3} \mathrm{He}_{\mathrm{c}}$ studies, at least in olivine and quartz. In addition, it appears cosmogenic helium can be operationally distinguished from inherited helium in these sample types, because crushing olivine releases only magmatic helium, and incremental heating of quartz releases ${ }^{3} \mathrm{He}{ }_{\mathrm{c}}$ more rapidly and at lower temperatures than inherited or radiogenic helium. $\mathrm{U}+\mathrm{Th} / \mathrm{He}$ geochronology may be possible in quartz because of this low ${ }^{*} \mathrm{He}$ diffusivity, as well. In summary, the measurements presented here suggest the potential problems of accounting for inherited helium and helium loss in ${ }^{3} \mathrm{He}_{\mathrm{c}}$ studies are readily surmountable, which is good news for future applications of cosmogenic helium geochronology. 


\section{REFERENCES}

ANDREWS J.N. (1885) The isotopic composition of radiogenic helium and its use to study groundwater movement in confined aquifers. Chem. Geol. 49, 339-351.

BARRER R.M. and VAUGHAN D. E. W. (1967) Solution and diffusion of helium and neon in tridymite and cristobalite. Trans. Farad. Soc. $63,2225-2290$.

BARRETT P.J. (1972) Stratigrapgy and petrology of the mainly fluviatile Permian and Triassic part of the Beacon Supergroup, Beardsmore Glacier area. In: Antarctic Geology and Geophysics (ed. R.J. ADIE), Scandinavian University Books.

BOGARD D.D. and CRESSY P.J. (1973) Spallation production of ${ }^{3} \mathrm{He},{ }^{21} \mathrm{Ne}$, and ${ }^{38} \mathrm{Ar}$ from target elements in the Bruderheim chondrite. Geochim. Cosmo. Acta 37, 527-546.

CRAIG H. and POREDA R.J. (1986) Cosmogenic ${ }^{3}$ He in terrestrial rocks: the summit lavas of Maui. Proc. Natl. Acad. Sci. 83, 1970-1974. DAMON P.E. and KULP J.L. (1957) Determination of radiogenic helium in zircon by stable isotope dilution technique. Trans. Am. Geophys. Union 38, 945-953.

DEER, HOWIE and ZUSSMAN (1966) Rock Forming Minerals. Longmens, London, 528 pp..

FLEISCHER R. L. (1988) Alpha-recoil damage: relation to isotopic disequilibrium and leaching of radionuclides. Geochim. Cosmo. Acta 52, 1459-1466.

FLUGGE S. and ZIMENS K.E. (1939) Die Bestimmung von Korngrossen und von Diffusionkonstanten aus dem Emaniervermogen. Z. Physik. Chem.42, 179-220.

FRIEDLANDER G., KENNEDY J.W., MACIAS E.S. and MILLER J.M. (1981) Nuclear and Radiochemistry. John Wiley and Sons, Inc., New York. GILETTI B.J. and KULP J.L. (1955) Radon leakage from radioactive minerals. Am. Mineralogist 40, 481-496.

GRAHAM D. V. (1987) Helium and lead isotope geochemistry of oceanic volcanic rocks from the East Pacific and South Atlantic. WHOI/MIT Ph.D., 252 p. 
INTHOFF W. and ZIMEN K.E. (1956) Kinetik der Diffusion radioaktiver Edelgase aus festen Stoffen nach Bestrahlung (Edelgasdiffusion in Festkorpern 2). Trans. Chalmers. Univ. Techn. (Goteborg)176, 16p. KEEVIL N.B. (1940) Interatomic forces and helium in rocks. Proc. Amer. Acad. Arts and Sci. 73, 311-359.

KURZ M.D., 0'BRIAN P., GARCIA M. and FREY F.A. (1985) Isotopic evolution of Haleakala volcano: Primordial, radiogenic, and cosmogenic helium. E0S 66, 1120.

KURZ M.D. (1986a) Cosmogenic helium in a terrestrial igneous rock. Nature $320,435-439$.

KURZ M.D. (1986b) In situ production of terrestrial cosmogenic helium and some applications to geochronology. Geochim. Cosmo. Acta 50, 2855-2862.

KURZ M.D., COLODNER D., TRULL T.W., SAMPSON D., MOORE R.B. and O'BRIAN K. (1989, in revision) Exposure-age dating with cosmogenic ${ }^{3} \mathrm{He}$.

LAL D. and PETERS B. (1967) Cosmic-ray-produced radioactivity on the earth. In: Handbuch der Physik XLVI/2 (ed. S. FLUGGE), pp. 551612. Springer-Verlag, Berlin.

LAL D. and ARNOLD J.R. (1985) Tracing quartz through the environment. Proc. Indian Acad. Sci. (Earth Planet. Sci.) 94, 1-5.

LAL D. (1987) Production of ${ }^{3} \mathrm{He}$ in terrestrial rocks. Chemical Geology (Isotope Geoscience Section) 66, 89-98.

LAL D. (1988) In situ-produced cosmogenic isotopes in terrestrial rocks. Ann. Rev. Earth Planet. Sci. 16, 355-388.

MAMYRIN B.A. and TOLSTIKHIN I.N. (1984) Helium Isotopes in Nature. Elsevier, Amsterdam.

MARTI K. and CRAIG H. (1987) Cosmic-ray-produced neon and helium in the summit lavas of Maui. Nature 325, 335-337.

MCDOUGALL I. (1964) GSA 75, 107-128.

MORRISON P. and PINE J. (1955) Radiogenic origin of the helium isotopes in rock. Ann. N.Y. Acad. Sci. 62, 71-92.

MURRAY E.G. and ADAMS J.A.S. (1958) Thorium, uranium and potassium in some sandstones. Geochim. Cosmo. Acta 13, 260-269. 
NAUGHTON J.J., MACDONALD G.A. and GREENBERG V.A. (1980) J. Volcan.

Geotherm. Res. 7, 335-339.

NISHIIZUMI K., LAL D, KLEIN J, MIDDLETON R and ARNOLD J.R. (1986) Production of ${ }^{10} \mathrm{Be}$ and ${ }^{26} \mathrm{Al}$ by cosmic rays in terrestrial quartz in situ and implications for erosion rates. Nature 319, 134-136.

ODOM A.L. and RINK, W.J. (1988) Natural accumulation of SchottkeyFrenkel defects: Implications for a quartz geochronometer. Geology 17, p55-58.

RAMA and MOORE, W.S. (1984) Mechanism of transport of U-Th series radioisotopes from solids into ground water. Geochim. Cosmo. Acta 48, 395-399.

ROSSI B. (1952) High Energy Particles. Prentice-Hall, Inc., Englewood Cliffs, N.J.

SIMMONS S.F. (1986) Physio-chemical nature of the mineralizing solutions for the St. Nino Vein; results from fluid inclusion, deuterium, oxygen and helium studies in the Fresnillo District, Zacatecas, Mexico. University of Minnesota PhD., 192 pp.

STETTLER A. and BOCHSLER P. (1979) He, Ne and Ar composition in a neutron activated sea-floor basalt glass. Geochim. Cosmo. Acta 43, 157-169.

TOLSTIKHIN I.N., PRASOLOV E.M., KHABARIN L.V. and AZBEL I.Y. (1974) Estimation of the helium diffusion coefficient in crystalline quartz. In: Geokhimiya radioactivnykh i radiogennykh isotopov (eds. Y.A. SHUKOLYUKOV and E.K.GERLING), pp 19-90. Nauka, Lenningrad.

WEED R. (1985) Chronology of chemical and biological weathering of cold desert sandstones in the Dry Valleys, Antarctica. University of Maine at Orono Master's Thesis, $138 \mathrm{pp}$.

WOLFENDALE A.W. (1973) Cosmic Rays At Ground Level. The Institute of Physics, London.

YOKOYAMA Y., REYSS J. and GUICHARD F. (1977) Production of radionuclides by cosmic rays at mountain altitudes. Earth Plan. Sci. Let. 36, 44-50. 
CHAPTER 6

Helium and strontium isotopic constraints on the origin of island arc magmas in the Woodlark Basin - Solomon Islands region.

T.W. Trull ${ }^{1}$, M.R. Perfit ${ }^{2}$, and M.D. Kurz ${ }^{1}$

1. Dept. of Chemistry

Woods Hole Oceanographic Institution

Woods Hole, MA 02543

2. Dept. of Geology

University of Florida

Gainesville, FL 32611

Accepted for publication, Geochimica et Cosmochimica Acta. 


\section{ABSTRACT}

In an effort to better understand volatile sources and petrogenesis in island arcs, helium and strontium isotopes were measured in dredged volcanic rocks from the Woodlark Basin (western Pacific), the site of subduction of the Woodlark spreading center (WSC) beneath the Solomon Islands. Helium isotope ratios $\left({ }^{3} \mathrm{He} /{ }^{4} \mathrm{He}\right)$ range from 0.01 to 9 times the atmospheric ratio $\left(R_{a}\right)$. However, low concentrations in whole rocks and some glasses, and helium isotopic disequilibrium observed by crushing and melting phenocrysts in vacuo, suggest that post-eruptive addition of radiogenic and seawater helium produces part of this variability. ${ }^{3} \mathrm{He} /{ }^{4} \mathrm{He}$ ratios in wSC tholeiites and NaTi basalts are indistinguishable from mid-ocean-ridge-basalt (MORB) values ( $8-9 \mathrm{R}_{\mathrm{a}}$ ) despite the small size of the Woodlark Basin and extensive subduction in the surrounding area. Samples from Kavachi submarine volcano (Solomon Islands forearc), exhibit significantly lower magmatic ${ }^{3} \mathrm{He} /{ }^{4} \mathrm{He}$ ratios $\left(6.9+\frac{4}{4} \cdot 2 \mathrm{R}_{\mathrm{a}}\right)$, which may derive from the source region, or from degassing and ${ }^{4} \mathrm{He}$ ingrowth during long (>10,000 yr.) magma transport times.

Strontium isotope ratios $\left({ }^{87} \mathrm{Sr} /{ }^{86} \mathrm{Sr}\right.$ ) range from 0.7026 (typical of MORB) in tholeiites and NaTi basalts from the WSC and triple-junction region to a high of 0.7040 in a calcalkaline dacite in the forearc, with intermediate values in transitional rock types. Systematic increases in Sr contents and ${ }^{87} \mathrm{Sr} /{ }^{86} \mathrm{Sr}$ from the central Woodlark Basin toward the active arc suggest a mixing origin for the lower ${ }^{3} \mathrm{He} /{ }^{4} \mathrm{He}$ and higher ${ }^{87} \mathrm{Sr} /{ }^{86} \mathrm{Sr}$ ratios (approximately .7036) observed in Kavachi lavas. Contributions to the sub-arc mantle from Pacific lithosphere subducted during the Miocene may best explain these compositions, but fluids introduced by subduction do not appear to be directly involved in the current magmagenesis, because predominantly radiogenic ${ }^{3} \mathrm{He} /{ }^{4} \mathrm{He}$ ratios (1 $\mathrm{R}_{\mathrm{a}}$ or less) were not observed. 


\subsection{INTRODUCTION}

Isotopic and elemental compositions of island arc basalts (IAB) require a more complex petrogenesis than closed system melting of an upper mantle similar in composition to the source of mid-ocean-ridgebasalts (MORB), (e.g. Perfit et al,1980; Morris and Hart, 1983; Davidson, 1987). Geophysical models suggest that release of C-O-H fluids from hydrated minerals of the ocean crust, in response to increasing pressure and temperature during subduction, may play an important role in arc volcanism (e.g. Ringwood, 1974; Kay, 1980; Gill, 1981; Wyllie, 1982). These fluids appear capable of strong inter-element fractionation and may serve as a flux in melting the overlying mantle wedge (e.g. Kushiro, 1975; Flynn and Burnham, 1978). However, the location of fluid release, and therefore the role of this metasomatic process in controlling arc petrogenesis, is uncertain (e.g. Wyllie, 1984).

In this study helium and strontium isotopes were measured in island arc basalts and andesites, and nearby spreading center basalts, in an effort to examine the role of slab-derived fluids in arc petrogenesis. The He isotopic composition of subducted lithosphere beneath arcs depends essentially on its age and uranium and thorium contents. It is expected to have ${ }^{3} \mathrm{He} /{ }^{4} \mathrm{He}$ significantly lower than the ratio of $8.4 \pm .3$ $\mathrm{R}_{\mathrm{a}}(\mathrm{Ra}=$ atmospheric ratio) observed in normal MORB and thought to characterize the upper mantle (Kurz et al, 1982). As a noble gas, helium partitions strongly into fluids relative to crystals, glasses, and melts (Kurz and Jenkins, 1981; Kurz, 1982) and may therefore act as a tracer of fluids derived from subducted lithosphere. Thus, fluids released from subducted oceanic lithosphere may contribute radiogenic ${ }^{4} \mathrm{He}$ to magmas that erupt at convergent plate boundaries, thereby lowering their ${ }^{3} \mathrm{He} /{ }^{4} \mathrm{He}$ ratios. Similarly, Sr acts as an incompatible element during melting and may be concentrated in melts or fluids derived from subducted lithospere along with other large ion lithophile elements (LIL) that are characteristically enriched in IAB. Positive correlations between ${ }^{87} \mathrm{Sr} /{ }^{86} \mathrm{Sr}$ ratios and important geochemical parameters (e.g. $\mathrm{Sr} / \mathrm{Zr}, \mathrm{La} / \mathrm{Yb}$, and $\mathrm{Ba} / \mathrm{La}$ ) in MORB and IAB from western Melanesia (Perfit, McCulloch and Johnson, 1982; and Perfit, unpublished 
data) indicate $\mathrm{Sr}$ isotopes are sensitive indicators of variations in source chemical compositions as well as of sample contamination on the seafloor. Combining $\mathrm{He}$ and $\mathrm{Sr}$ isotopic studies may improve our understanding of the behavior of Sr during dehydration of subducted lithosphere, allowing further discrimination of the components involved in arc petrogenesis.

Previous efforts to exploit He as a slab-derived-fluid tracer have focused on the measurement of volcanic gases and fluids (Craig, Lupton, and Horibe, 1978; Poreda, 1983; Sano and Wakita, 1985; Sano, Wakita, and Giggenbach, 1987). Hydrothermal fluid samples have ${ }^{3} \mathrm{He} /{ }^{4} \mathrm{He}$ ratios ranging from about $0.1 \mathrm{R}_{\mathrm{a}}$ to nearly $9 \mathrm{Ra}$. However, fumarole gases, especially those from volcanic summits, exhibit a narrower range, roughly $6-9 \mathrm{R}_{\mathrm{a}}$, suggesting that radiogenic He from crustal rocks contributes to at least some gas samples. Direct measurement of helium isotopes in volcanic rocks may better characterize volatiles from magmatic source regions. The few rocks previously analysed exhibit a wide range in ${ }^{3} \mathrm{He} /{ }^{4} \mathrm{He}$ ratios; from extremely radiogenic ratios (approximately .06 $\mathrm{R}_{\mathrm{a}}$ ) to normal MORB ratios and higher (Poreda, 1983; 1985; Mamyrin and Tolstikhin, 1984 and references therein).

Unfortunately, these samples were of uncertain age and part of the range in ${ }^{3} \mathrm{He} /{ }^{4} \mathrm{He}$ ratios may be produced by ingrowth of ${ }^{4} \mathrm{He}$ from the decay of $\mathrm{U}$ and Th after eruption. Because of the scarcity of helium data from arc rocks, and the unknown extent to which the helium isotopic composition of a sample reflects source characteristics, a major goal of this study is to understand how helium may be used as a tracer in arc environments.

\subsubsection{Petrogenesis in the Woodlark Basin - Solomon Islands region.}

The Solomon Island-Woodlark Basin region was chosen for study because fresh basaltic samples were available from both the overriding and downgoing plates, and many samples have glassy rims, which have been shown to trap magmatic gases (e.g Funkhouser, Fisher, and Bonatti, 1968). This area in the western Pacific can be divided into three tectonic regions: the Woodlark Spreading Center (WSC) which is a normal accretionary ridge in the middle of the Woodlark Basin, the forearc 
slope of the Solomon Islands arc to the northeast (including the active Kavachi submarine volcano), and the complex triple junction region (encompassing Simbo and Ghizo ridges) where the WSC subducts into the trenches along the western edge of the Solomon Islands. Figure 6.1 shows the tectonic relations and dredge sites. An excellent overview of the tectonic history and regional geology of the arc was presented by Dunkley (1984), and the triple junction area has been described in detail (Taylor, 1987).

The rocks dredged from the Woodlark Basin and studied here are geochemically diverse (Johnson et al, 1987; Perfit et al, 1987; Staudigel et al, 1987) but can be grouped into three general petrologic associations: basalts with compositions similar to MORB, rocks ranging from basalt to dacite with typical island arc basalt (IAB) compositions, and a few basalts and basaltic andesites with chemical compositions that are transitional between the MORB and IAB compositions. These groupings correspond roughly to the three tectonic regions mentioned above.

Predominantly normal MORB were recovered from the WSC (dredges 26 and 29). Some basalts from dredge 29 have slight back-arc-basin-basalt (BABB) characteristics (e.g. increased $\mathrm{Sr} / \mathrm{Zr}, \mathrm{La} / \mathrm{Yb}$, and ${ }^{87} \mathrm{Sr} /{ }^{86} \mathrm{Sr}$ ). In addition, a unique basalt type with trace element and isotopic characteristics similar to MORB but exceptionally high $\mathrm{Na}_{2} \mathrm{O}$ and $\mathrm{TiO}_{2}$ was recovered at dredge site 32 where the WSC meets Simbo ridge. These NaTi basalts (samples $32-8,9,10$ ) have depleted large ion lithophile and rare earth element abundances despite being nepheline normative (Perfit et al, 1987). The MORB from dredges 29 and 26 probably best represent the composition of the ocean floor that is subducted beneath the Solomon Islands.

Both medium- and high- $\mathrm{K}_{2} 0$ island arc lavas were recovered in the forearc slope region (dredges 24 and 25). However, at the active submarine volcano, Kavachi, only low- $\mathrm{K}_{2} 0$ island arc tholeiites were recovered (dredges 34,35 ). These rock types are very similar to those erupted on the New Georgia Islands and in the nearby New Hebrides and New Britain arcs (Johnson et al, 1985). They represent typical eruptive products of subduction zone magmagenesis. 
Figure 6.1 Tectonic map for the Woodlark Basin and Solomon Islands region, modified from Taylor and Exon, 1984). The Woodlark spreading center (WSC) has been opening at $7 \mathrm{~cm} /$ year for about $5 \mathrm{Ma}$. This new seafloor is being subducted orthogonally into the New Britain (NBT) and San Cristobal (SCT) trenches at about $10 \mathrm{~cm} / \mathrm{yr}$. The trenches shoal from their typical depths of $4000 \mathrm{~m}$ or more to less than $2000 \mathrm{~m}$ near their intersection with the WSC. The complex triple-junction region encompasses Simbo ridge, thought to be an extinct ridge transform fault and Ghizo ridge, which may represent a deformed spreading segment. Dredge 33 marks the location of Kana Keoki seamount near the end of Simbo-Ghizo Ridge. Historical volcanism has occurred on most of the Western Islands - along the line between Guadalcanel (GC) and Vella Lavella (VL). Kavachi submarine volcano is presently active. Santa Isabel (SL) and the other Eastern Islands were volcanically active in the Miocene, when subduction occurred from the northeast, at the now extinct Vitiaz Trench (VT). The WSC is propagating westwards at approximately $12 \mathrm{~cm} / \mathrm{yr}$ into the submarine extension of Papua New Guinea, as marked by the $2000 \mathrm{~m}$ rise contours. Dredge locations are numbered correspond to the sample numbers in Table 6.1 


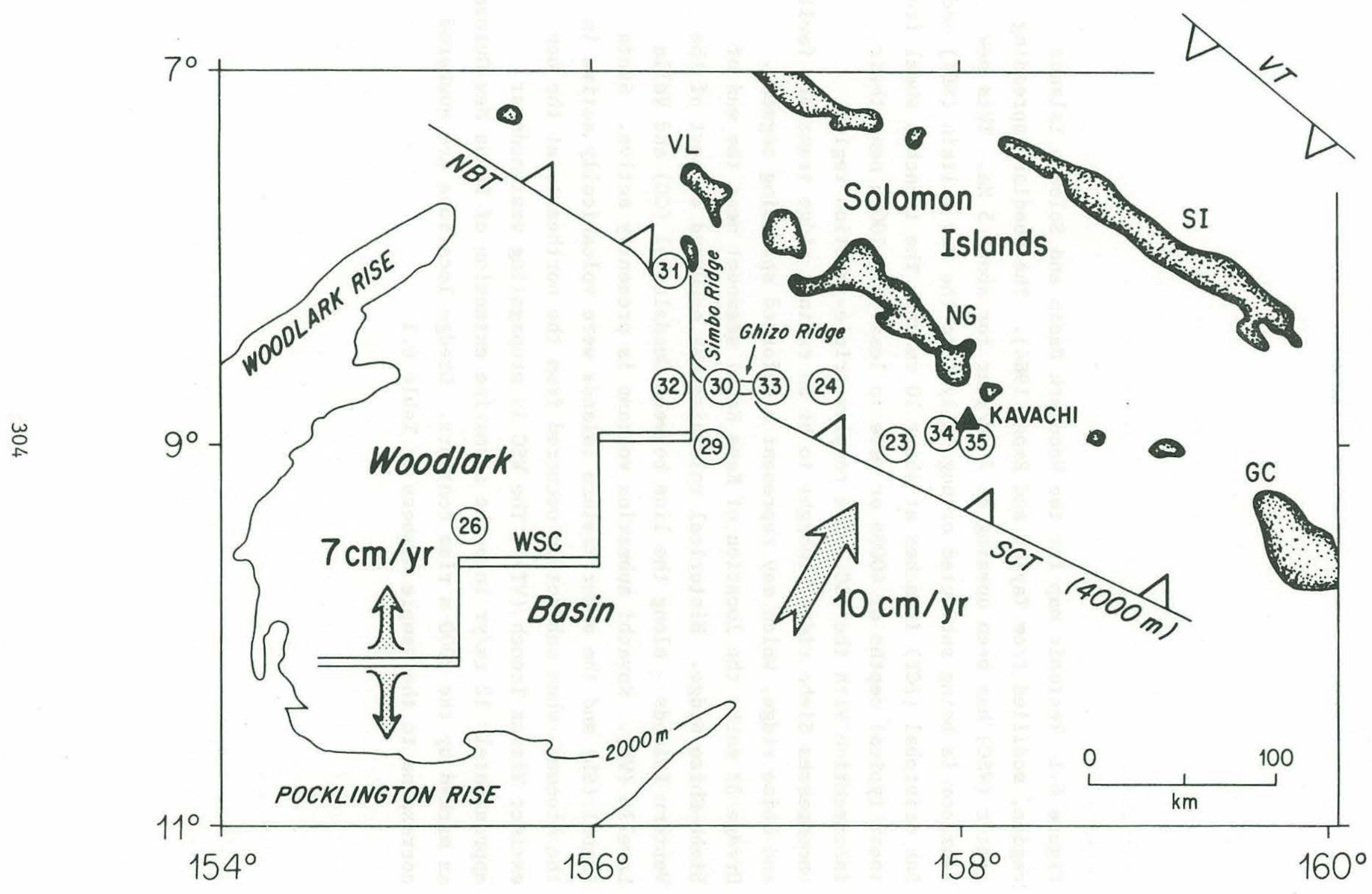


Rocks ranging in composition from high-Mg basalts ("boninites") to low $\mathrm{K}_{2} \mathrm{O}$ tholeiites, to normal calcalkaline dacites and rhyolites were recovered from the complex triple junction region encompassing the Simbo and Ghizo ridges (dredges 30,31, and 32) and Kana Keoki Seamount (dredge 33). The more silicic rocks (dredge 33), including glassy dacites, are similar to highly fractionated arc rocks found in New Britain and Bougainville (Johnson et al, 1987). Perfit et al (1987) have shown that the tholeiitic basalts and basaltic andesites from dredges 30 and 32 are chemically transitional between the WSC MORB and the forearc IAB. Thus, these samples span the compositional range of rock types seen in island arcs, including extreme compositions.

Overall, the trace element and isotopic variability of the samples from the Woodlark Basin-Solomon Islands region is less extreme than the diversity of petrologic types. For example, both the unusual NaTi basalts and the more normal spreading-ridge environment basalts found along the WSC have rare earth element patterns and $\mathrm{Sr}$ isotopic compositions similar to MORB (Johnson et al, 1987; Perfit et al, 1987; this study). Moreover, Perfit et al (1987) and Staudigel et al (1987) have shown that isotopic compositions and trace element ratios that indicate relative source enrichments, vary smoothly spatially in the Woodlark Basin-Solomon Islands region.

These researchers have proposed that the observed variation represents an increase toward the arc in the petrogenetic involvement of upper mantle previously modified by subduction of lithosphere along the Vitiaz Trench (figure 6.1). This was the locus of convergence and volcanism until Miocene time (Dunkley, 1984 and references therein). In this view, subduction of the WSC acts as a "thermal trigger" to melt the modified mantle. The thermal anomaly associated with the WSC is reflected in the shoaling of both the bathymetric expression of the trench and the loci of subduction-related earthquakes by several kilometers near the triple junction (Cooper and Taylor, 1985), in the rapid uplift of the islands of the New Georgia Group on the overriding Pacific plate (Taylor, 1987), and in the unusually small gap between the trench and the volcanic front (e.g. just $30 \mathrm{~km}$ at Kavachi submarine volcano). 
Alternatively, Abbot and Fisk (1986) have suggested that relict Pacific lithosphere continues to release volatiles into the mantle beneath the Woodlark Basin, and that this process is actively involved in determining the compositions of erupted rocks. The extension of ancient Pacific lithosphere under the Solomon Islands is suggested by the spatial distribution of deep earthquakes in the region (Cooper and Taylor, 1985). If this model is correct, correlation between source composition indicators (such as isotopic and incompatible element ratios) and the nature of petrogenesis (as reflected in rock type) would be expected to be stronger than for tectonically controlled melting of a previously modified and laterally variable mantle. For example, island arc basalts (IAB) should have similar, radiogenic, helium and strontium isotope compositions, whether erupted in the forearc or the triplejunction region. In either case, one probable petrogenetic effect of ridge subduction is the unusual exposure of voluminous mafic lavas, including picrites in the New Georgia island group of the Solomon Islands. These compositions appear to result from accumulation of mafic phenocrysts and the lavas have trace element and isotopic signatures similar to more typical arc basalts from elsewhere in the Solomon Islands and other island arcs (Cox and Bell, 1972; Ramsay, Crawford and Foden, 1984, Staudigel et al, 1987; Perfit et al, in preparation). Unfortunately, we were unable to obtain samples of these rocks as part of this study.

\subsection{METHODS}

\subsubsection{Helium}

In light of the diversity of rock types erupted at arcs, an important aspect of this study was determining the best way to obtain the magmatic helium isotopic composition from arc samples. Whole rocks, mineral separates (olivine, pyroxenes, and plagioclase), and glass fragments were analysed to determine which phases contained measurable amounts of helium, and which were reliably free from air or seawater contamination. In addition, the generally low He concentrations 
observed and the indeterminate age of the dredge samples required developing analytical criteria to distinguish samples affected by posteruptive radiogenic ${ }^{4} \mathrm{He}$ contamination. These criteria are discussed briefly here and in more detail below. Brief sample descriptions are given in Table 6.1, detailed petrographic descriptions of the whole rocks can be found in Johnson et al (1987) and Perfit et al (1987).

Glass samples analyzed were chips greater than $1 \mathrm{~mm}$ in their largest dimension, free of visual alteration in reflected light under $40 \mathrm{x}$ magnification. For most samples, with the notable exception of those from dredge 30 , this was much larger than the mean vesicle diameters. From $20 \mathrm{mg}$ to 1 gram of clean glass was analyzed, depending on the sample's helium concentration. Some glass samples (see Table 6.1) were pretreated by ultrasonic cleaning in $8 \mathrm{~N}$ nitric acid as a means of removing altered material. Optically clean fragments were then chosen from the leached material. Experiments on leached and unleached glass suggest that concentrations and isotopic compositions are not significantly affected by this process. However, this treatment was performed only on samples with slight alteration - visible as hazing or discoloration of surfaces.

Mineral separates were handpicked from the 1-2 $\mathrm{mm}$ size fraction (or smaller in some cases) of jaw-crushed samples and contained as little adhering matrix and/or other mineral phases as possible (less than $1 \%$ ). Inclusions of devitrified melt were often present, particularly in clinopyroxene separates and many olivine samples contained small spinel inclusions. Fluid inclusions were often visible in these crystal types, both in thin section and by viewing the grains in transmitted light. Depending on helium concentrations, from ten to a few hundred milligrams of crystals were analyzed, generally consisting of 10 - 50 individual grains.

Groundmass samples were picked from the $.8-1.0 \mathrm{~mm}$ size fraction to be free of obvious crystals, and were cleaned ultrasonically in $\mathrm{H}_{2} \mathrm{O}$ for approximately an hour. These samples were dried in air overnight, at $40-80^{\circ} \mathrm{C}$; this had no effect on helium concentrations or compositions, based on duplicate analyses. 
In order to assess the contribution of radiogenic ${ }^{4} \mathrm{He}$ added after eruption to the observed isotopic compositions, samples were analyzed first by crushing the grains in vacuo and then by melting the resulting powder in the vacuum furnace. Because crushing selectively releases gases trapped in vesicles and inclusions, this helium should represent the isotopic composition of the magma at eruption. In contrast, helium released by melting includes radiogenic ${ }^{4} \mathrm{He}$ from $U$ and $\mathrm{Th}$ series alphaparticle decay in the solid phases. These procedures and the instrumentation for helium extraction have been described recently (Kurz, Gurney, Jenkins and Lott, 1987). The mechanical efficiency of crushing varies according to sample type and weight, and concentrations of helium released by crushing are thus operationally defined. All powders analyzed by melting passed through a 100 um sieve, with the exception of a few mineral separate samples for which a $200 \mathrm{um}$ separation was used in order to have enough material to analyze (see Table 6.1). Therefore, inclusions smaller than these dimensions contribute helium to the melt analysis. The degree to which this (and incomplete operational separation of inherited and ingrown helium, in general) affects the determination of the ${ }^{3} \mathrm{He} /{ }^{4} \mathrm{He}$ ratio of the magma at eruption is discussed in the results section below.

Glasses and groundmass were melted at $1300^{\circ} \mathrm{C}$, and mineral separates at approximately $1500^{\circ} \mathrm{C}$. The estimated uncertainty in these temperatures is $30^{\circ} \mathrm{C}$. Powders were loaded in aluminum foil boats (approx. $20 \mathrm{mg}$ ) for melting in the furnace; melting of the samples was verified by examination of the silicate slag after heating, and by the absence of additional helium in procedural blanks following the analyses.

Procedural blanks for crushing and melting were identical and ranged from 3 to $6 \times 10^{-11} \mathrm{ccSTP} H e$. The isotopic ratio of the blank was always within 2 sigma of the atmospheric ratio. Blank corrections for the samples reported in Table 6.1 were generally much less than $1 \%$, and rarely exceeded $10 \%$. The detection limit for ${ }^{3} \mathrm{He}$ ( 2 sigma on the ${ }^{3} \mathrm{He}$ blank) was approximately $3 \times 10^{-16}$ ccSTP. Samples with less ${ }^{3} \mathrm{He}$ than this are reported as having ${ }^{3} \mathrm{He} /{ }^{4} \mathrm{He}$ ratios less than or equal to 
the ratio of the detection limit for ${ }^{3} \mathrm{He}$ to the observed ${ }^{4} \mathrm{He}$ concentration (see Table 6.1).

\section{$\underline{6.2 .2}$ Strontium.}

Strontium isotopes were measured using a VG Isomass 354 thermal ionization mass spectrometer at the University of Florida. Measurements of ${ }^{87} \mathrm{Sr} /{ }^{86} \mathrm{Sr}$ were made by simultaneous collection into three Faraday cups and normalized to ${ }^{86} \mathrm{Sr} /{ }^{88} \mathrm{Sr}=0.1194$. The value of NBS SRM 987 during the time period of these analyses averaged 0.710240 (12\%, 2 sigma). Total procedural blanks were $1 \mathrm{ng} \mathrm{Sr}$. A few samples were spiked prior to dissolution and $\mathrm{Sr}$ and $\mathrm{Rb}$ were determined by isotope dilution.

Where possible fresh hand-picked glasses were selected for analysis. Even the optically freshest samples were ultrasonically cleaned in $1 \mathrm{~N} \mathrm{HCl}$ for 20 minutes and triple-distilled $\mathrm{H}_{2} \mathrm{O}$ for up to an hour. Glasses and glassy samples with slightly oxidized or discolored surfaces were leached in $2 \mathrm{~N} \mathrm{HCl}$ for up to 12 hours before being washed in clean $\mathrm{H}_{2} \mathrm{O}$. Whole rocks were trimmed of macroscopic alteration prior to crushing and hand-picking. The freshest fragments containing both groundmass and crystals were leached in $2 \mathrm{~N} \mathrm{HCl}$ and washed in clean $\mathrm{H}_{2} 0$. All samples were dried under heat lamps for more than 12 hours before being powdered in a tungsten-carbide ball-mill (most glasses were dissolved without pulverization). Between 50 and $100 \mathrm{mg}$ of sample were dissolved in $3-5 \mathrm{ml}$ of $50 \% \mathrm{HF}$ and 3 drops of $14 \mathrm{~N} \mathrm{HNO}_{3}$. Samples were sealed in teflon reaction vessels and kept at $100^{\circ} \mathrm{C}$ for 48 hours. They were converted to chloride form before using standard cation exchange columns to separate $\mathrm{Rb}, \mathrm{Sr}$, and the rare earth elements. Samples were loaded as a nitrate on oxidized Ta filaments.

A few experiments with the cleaning and leaching techniques showed that even the freshest looking samples may have minor amounts of alteration that contain radiogenic $\mathrm{Sr}$ from seawater. In most cases cleaning with $1 \mathrm{~N} \mathrm{HCl}$ was sufficient to remove this material, but in others, noteably the crystalline samples, leaching in $2 \mathrm{~N} \mathrm{HCl}$ was 
Table 1. Helium Isotope Data

\begin{tabular}{|c|c|c|c|c|c|c|c|c|c|c|c|c|c|c|}
\hline \multirow[b]{2}{*}{$\begin{array}{l}\text { LATITUDE } \\
\text { (South) }\end{array}$} & \multicolumn{3}{|l|}{$\perp 0$ CA T } & \multicolumn{4}{|c|}{$S A M P L E D$} & \\
\hline & $\begin{array}{l}\text { LONGITUDE } \\
\text { (East) }\end{array}$ & $\begin{array}{l}\text { DEPTH } \\
\text { (meters) }\end{array}$ & DREDGE & SAMP & $\begin{array}{ll}\text { PLE } & \text { ROCK } \\
\text { TYPE }\end{array}$ & PHASE & CORMENTS & "METHOD & $\begin{array}{l}\text { WGT; } \\
\text { (mg) }\end{array}$ & $\begin{array}{l}{ }^{3} \mathrm{He} /{ }^{4} \mathrm{He} \\
(\mathrm{R} / \mathrm{Ra})\end{array}$ & & $\begin{array}{c}{[\mathrm{He}]} \\
\left.10^{-7} \mathrm{cc} / \mathrm{g}\right)\end{array}$ & $\begin{array}{l}{ }^{x} \mathrm{He}_{1} \\
\left(10^{-10}\right.\end{array}$ & $\begin{array}{l}{ }^{*} \mathrm{He}_{2} \\
\mathrm{cc} / \mathrm{g})\end{array}$ \\
\hline \multirow{5}{*}{$\begin{array}{l}9^{\circ} 29.94^{\prime} \\
\text { WSC axis, } \\
\text { trench. }\end{array}$} & $155^{\circ} 25.15^{\prime}$ & $3830-3460$ & 26 & 11 & MORB & glass & $\sim 3 \%$ vesicles & 1 & 448 & 8.44 & .05 & 87 & $310 \pm 80$ & $310 \pm 80$ \\
\hline & . $180 \mathrm{~km}$ west & $t$ of the & & & & glass & & 2 & 115 & 7.84 & .03 & 4.3 & & \\
\hline & & & & & & glass & & 1 & 28 & 8.42 & .05 & 3.0 & & \\
\hline & & & & & & glass & 40. leach $50 \% \mathrm{HNO}_{3}$ & 1 & 35 & 8.43 & .05 & .85 & & \\
\hline & & & & & MORB & glass & & 1 & 35 & 8.48 & .05 & 4.6 & & \\
\hline \multirow{6}{*}{$\begin{array}{l}9^{\circ} 05.44 \\
\text { WSC axis. } \\
\text { trench. }\end{array}$} & & $3973-3570$ & 29 & A & MORB & glass & & 1 & 28 & 9.14 & .04 & 6.1 & & \\
\hline & $80 \mathrm{~km}$ west & of the & & B & MORB & glass & & i & 32 & 9.30 & .05 & 3.9 & & \\
\hline & & & & c & MORB & glass & & 1 & 37 & 8.44 & .05 & 1.7 & & \\
\hline & & & & D & MORB & glass & & i & 155 & 8.18 & .03 & 21 & & \\
\hline & & & & & BABB? & glass & & 1 & 113 & 8.95 & .05 & .75 & & \\
\hline & & & & & & glass & & 3 & 129 & 8.96 & .06 & & & \\
\hline \multirow{15}{*}{$\begin{array}{l}8^{\circ} 45.39^{\prime} \\
\text { West flank } \\
\text { Simbo Rids } \\
\text { section wi } \\
\text { the triple }\end{array}$} & & $2830-2050$ & 32 & 8 & NaTi Basalt & glass & & 1 & 335 & 9.2 & .1 & .027 & & \\
\hline & k of souther & $\mathrm{rn}$ end of & & & & glass & & 2 & 75 & 9.0 & .1 & .49 & & \\
\hline & dge, near its & $s$ inter- & & & & glass & & 3 & 213 & 7.4 & 1.0 & .009 & & \\
\hline & & idge, in & & 9 & NaTf Basalt & glass & $10 \%$ dulled surfaces & 1 & 290 & 5.0 & .4 & .023 & & \\
\hline & Te-junction $r$ & region. & & & & glass & $10 \%$ dulled surfaces & 3 & 135 & 7.8 & .1 & .17 & & \\
\hline & & & & & & glass & $40^{\circ}$ Leach $8 \mathrm{NHO}_{3}$ & 1 & 290 & 6.4 & .4 & .019 & & \\
\hline & & & & & & glass & $40^{\circ}$ Leach $8 \mathrm{~N} \mathrm{HNO}_{3}$ & 1 & 429 & 5.7 & .2 & .011 & & \\
\hline & & & & & & glass & >200um powder & 2 & 213 & 8.2 & .1 & .19 & & \\
\hline & & & & & & glass & <200um powder & 2 & 147 & 8.3 & .1 & .15 & & \\
\hline & & & & & & $\begin{array}{l}\text { glass } \\
\text { glas }\end{array}$ & step-heating sum & 2 & 92 & 7.7 & .7 & .12 & & \\
\hline & & & & 10 & NaTi Basalt & glass & & 1 & 170 & 8.73 & .04 & 3.1 & & \\
\hline & & & & 16 & Basaltic andesite & glass & & $i$ & 194 & 1.3 & .6 & .11 & & \\
\hline & & & & & & glass & & 2 & 174 & 1.0 & .4 & .015 & & \\
\hline & & & & & & glass & & 3 & 280 & .8 & .2 & .026 & & \\
\hline & & & & & & altered & glass, $10 \%$ palagonite & 1 & 303 & .5 & .4 & .009 & & \\
\hline \multirow{6}{*}{$\begin{array}{l}8^{\circ} 47 \cdot 19^{\prime \prime} 1 \\
\text { South flan } \\
\text { the triple }\end{array}$} & & $3830-3460$ & 30 & 5 & Basaltic andesite & glass & $10 \%$ of surfaces altered & $i$ & 168 & 1.1 & .2 & .16 & & \\
\hline & ank of Ghizo & Ridge in & & & & glass & & 2 & & 1.2 & .1 & .13 & & \\
\hline & & region. & & & & glass & $20^{\prime}$ Leach $50 \% \mathrm{HNO}_{3}$ & $i$ & 144 & 1.6 & .2 & .10 & & \\
\hline & & & & & & glass & 40. Leach $50 \% \mathrm{HNO}_{3}$ & 1 & 171 & 1.1 & .2 & .13 & & \\
\hline & & & & & & glass & <100um powder & 2 & 95 & 2.1 & .4 & .015 & & \\
\hline & & & & & Basaltic andesite & glass & 2200um powder & 2 & $\begin{array}{r}60 \\
175\end{array}$ & 1.22 & .6 & .040 & & \\
\hline \multirow{13}{*}{\multicolumn{2}{|c|}{$\begin{array}{l}8^{\circ} 12^{\prime} 43^{\prime \prime} 156^{\circ} 27^{\prime} 56^{\prime \prime} \\
\text { West flank of norther } \\
\text { Simbo Ridge in the tr } \\
\text { Junction regiton. }\end{array}$}} & $2045-1635$ & 31 & 1 & $\mathrm{High}-\mathrm{Mg}$ andesite & $\begin{array}{l}\text { glass } \\
\text { glassy }\end{array}$ & groundmass & 1 & 114 & 1.3 & .10 & .076 & $40 \pm 10$ & $560 \pm 50$ \\
\hline & & $\mathrm{rn}$ end of & & & ("boninite") & glassy & groundmass & 2 & 95 & .35 & .2 & .058 & & \\
\hline & & & & & & bronzit & e $\left(\mathrm{Mg}_{8} \mathrm{Fe}_{14} \mathrm{Ca}_{04}\right)$ & 1 & 167 & 5.4 & .2 & .028 & $11+2$ & $10+1$ \\
\hline & & & & & & bronzit & & & 143 & 5.07 & & .011 & & \\
\hline & & & & 10 & $\begin{array}{l}\mathrm{High}-\mathrm{Mg} \text { andesite } \\
\text { ("boninite") }\end{array}$ & $\begin{array}{l}\text { glassy } \\
\text { glassy }\end{array}$ & $\begin{array}{l}\text { groundmass } \\
\text { groundmass }\end{array}$ & $\begin{array}{l}1 \\
2\end{array}$ & $\begin{array}{l}251 \\
128\end{array}$ & .6 & .2 & .024 & $240 \pm 30$ & $270 \pm 30$ \\
\hline & & & & & ("boninite") & $\begin{array}{l}\text { glassy } \\
\text { bronzit }\end{array}$ & $e^{\text {groundmass }}$ partial crush & $\stackrel{2}{1}$ & 260 & 2.7 & .4 & .012 & $9 \pm 2$ & $13 \pm 1$ \\
\hline & & & & & & $\left(\mathrm{Mg}_{83} \mathrm{Fe}\right.$ & $\left.{ }_{14} \mathrm{Ca}_{04}\right)$ recrush & 1 & 260 & 2.1 & .3 & .009 & & \\
\hline & & & & & & bronzit & & 2 & 220 & .9 & .3 & .014 & & \\
\hline & & & & & & bronzit & e & 3 & 221 & 2.0 & .3 & .02 & & \\
\hline & & & & 13 & $\mathrm{High}-\mathrm{SiO}_{2}$ andesite & augite & $\left(\mathrm{Mg}_{50} \mathrm{Fe} \mathrm{Fe}_{1}, \mathrm{Ca}_{3} \mathrm{~g}\right)$ & 1 & 230 & 6 & 3 & .002 & $5+2$ & $6+1$ \\
\hline & & & & & & augite & & 2 & 191 & 1.5 & .7 & .007 & & \\
\hline & & & & & & $\begin{array}{l}\text { hyperst } \\
\text { hyperst }\end{array}$ & $\begin{array}{l}\text { hene } \\
\text { hene }\end{array}$ & $\begin{array}{l}1 \\
2\end{array}$ & $\begin{array}{l}170 \\
141\end{array}$ & $\begin{array}{l}2.2 \\
5.08\end{array}$ & $\begin{array}{l}.5 \\
.04\end{array}$ & $\begin{array}{l}.008 \\
.009\end{array}$ & $9 \pm^{3}$ & $9 \pm 1$ \\
\hline & & & & & & & & & & & & & & \\
\hline
\end{tabular}

* Method codes: 1 = crushing. 2 = melting of powder (1ess than 100 um unless otherwise indicated), $3=$ melting of whole grains or shards. Rock types are from Johnson et al (1987) and Perfit et al (1987).

Phenocryst identifications are based on micropros Recca.

. Errors for ${ }^{*} \mathrm{He}$ in the ${ }^{3} \mathrm{He} /{ }^{4} \mathrm{He}$ ratios are based on a single mass spectrometer analysis.

e. Maximum values of ${ }^{3} \mathrm{He} /{ }^{4} \mathrm{He}$ ratios are reported for samples with ${ }^{3} \mathrm{He}$ contents below the detection 1 imit. 
Table 1. (cont.) Helium Isotope Data

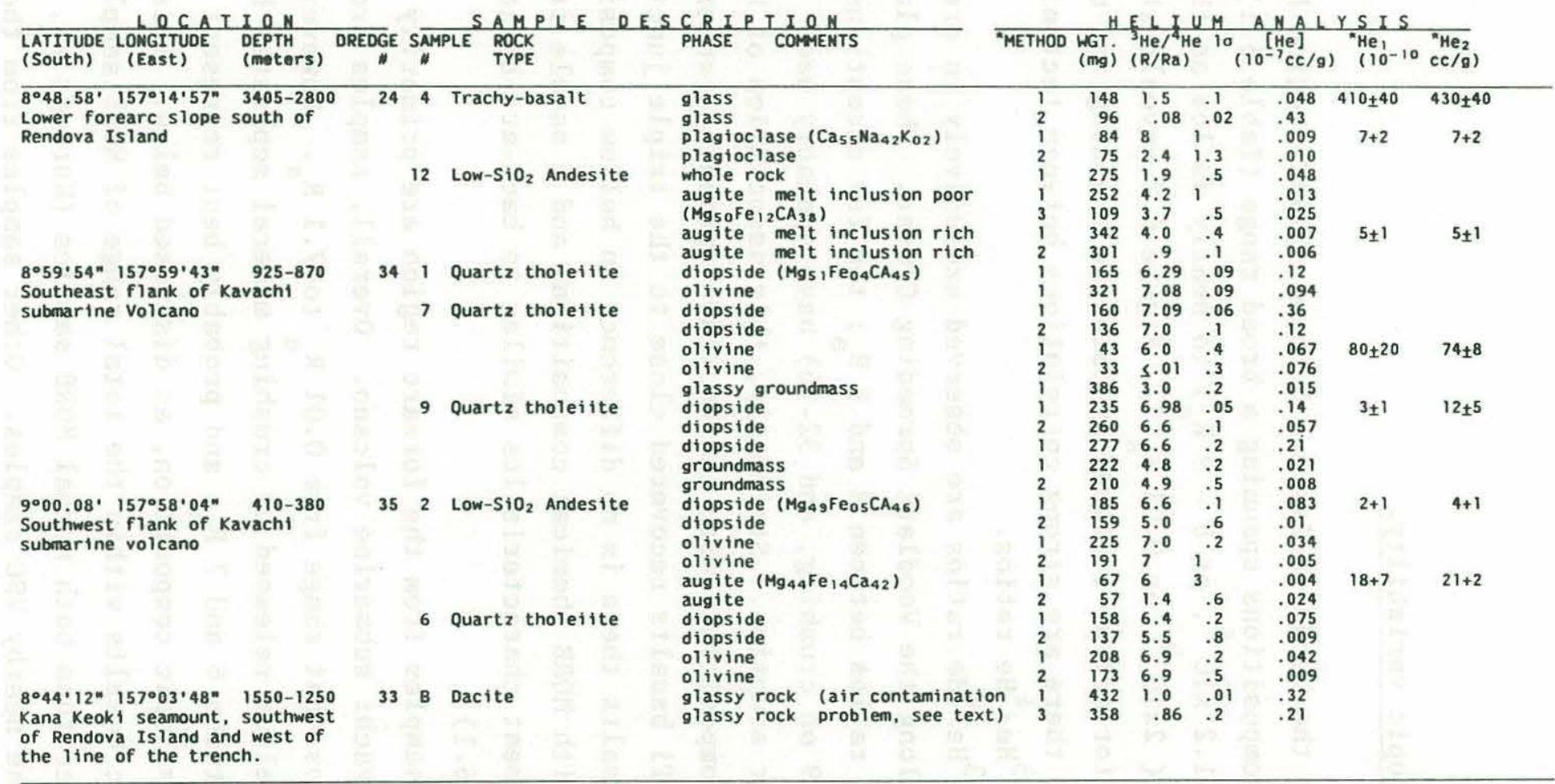

- Method codes: 1 = crushing, 2 = melting of powder (less than 100 um unless otherwise indicated), $3=$ melting of whole grains or shards.

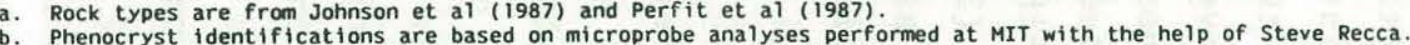

c. I sigma errors in the ${ }^{3} \mathrm{He} /{ }^{4} \mathrm{He}$ ratios are based on a single mass spectrometer analysis.

d. Errors for ${ }^{*} \mathrm{He}$ contents are 1 sigma calculated from analytical errors in the ${ }^{3} \mathrm{He} /{ }^{4} \mathrm{He}$ ratios.
e. Maximum values of ${ }^{3} \mathrm{He} /{ }^{4} \mathrm{He}$ ratios are reported for samples with ${ }^{3} \mathrm{He}$ contents below the detection limit. 
neccesary. Leaching in stronger acid (see Staudigel et al, 1987) did not significantly improve the results.

\subsection{RESULTS}

\subsubsection{Helium isotopic variabilty.}

Samples from the Woodlark Basin - Solomon Islands region have helium isotopic compositions spanning a broad range (Table 6.1), from MORB values $\left(1.1-1.2 \times 10^{-5}\right.$, or $8-9 \mathrm{R}_{\mathrm{a}}$ ) to nearly as low as the ${ }^{3} \mathrm{He} /{ }^{4} \mathrm{He}$ production ratio $\left(2 \times 10^{-8}\right.$, or $\left.0.01 \mathrm{R}_{\mathrm{a}}\right)$. Figure 6.2 reveals that this diversity exists for helium released by both the crushing and melting methods, and that there are strong correlations between tectonic regions and the observed ${ }^{3} \mathrm{He} /{ }^{4} \mathrm{He}$ ratios.

The highest ${ }^{3} \mathrm{He} /{ }^{4} \mathrm{He}$ ratios are observed exclusively in dredged samples erupted along the Woodlark Spreading Center. These glasses generally exhibit ratios between 8 and $9 \mathrm{R}_{\mathrm{a}}$; the few exceptions with lower ratios (32-9 on crushing, and 32-16) have probably been contaminated after eruption. Strikingly, this association of MORB helium isotopic compositions with the Woodlark spreading center extends to the unusual NaTi basalts recovered close to the triple junction. Within the WSC basalts there is no difference in helium compositions between samples with MORB chemical compositions and a sample from dredge 29 with trace element characteristics similar to back-arc-basin-basalts (BABB, see Table 6.1).

Analyses of samples from the forearc region are primarily mineral separates from Kavachi submarine volcano. Overall, samples from Kavachi have ${ }^{3} \mathrm{He} /{ }^{4} \mathrm{He}$ ratios that range from $0.01 \mathrm{R}_{\mathrm{a}}$ to $7.1 \mathrm{R}_{\mathrm{a}}$. However, measurements on helium released by crushing mineral separates have ${ }^{3} \mathrm{He} /{ }^{4} \mathrm{He}$ ratios between 6 and $7 \mathrm{R}_{\mathrm{a}}$, and probably best represent the magmatic helium isotopic composition, as discussed below. This places Kavachi island arc basalts within the total range of MORB samples, but significantly lower than both normal MORB samples (Kurz et al, 1982; Kurz, 1982) and the nearby WSC samples. Other samples from the forearc 
Figure 6.2 Histograms of ${ }^{3} \mathrm{He} /{ }^{4} \mathrm{He}$ compositions ( normalized to the atmospheric ratio, $R_{a}=1.38 \times 10^{6}$ ) of gas released by powder melting (a) and crushing (b) of Woodlark Basin dredge samples. Symbols denote tectonic regions: WSC (squares), triple-junction region (circles), Kavachi submarine volcano (diamonds), forearc (triangles). Samples plotted in the first column at left have ${ }^{3} \mathrm{He} /{ }^{4} \mathrm{He}$ ratios less than 0.5 $\mathrm{R}_{\mathrm{a}}$. Duplicate analyses are not displayed. 
Figure 6.2
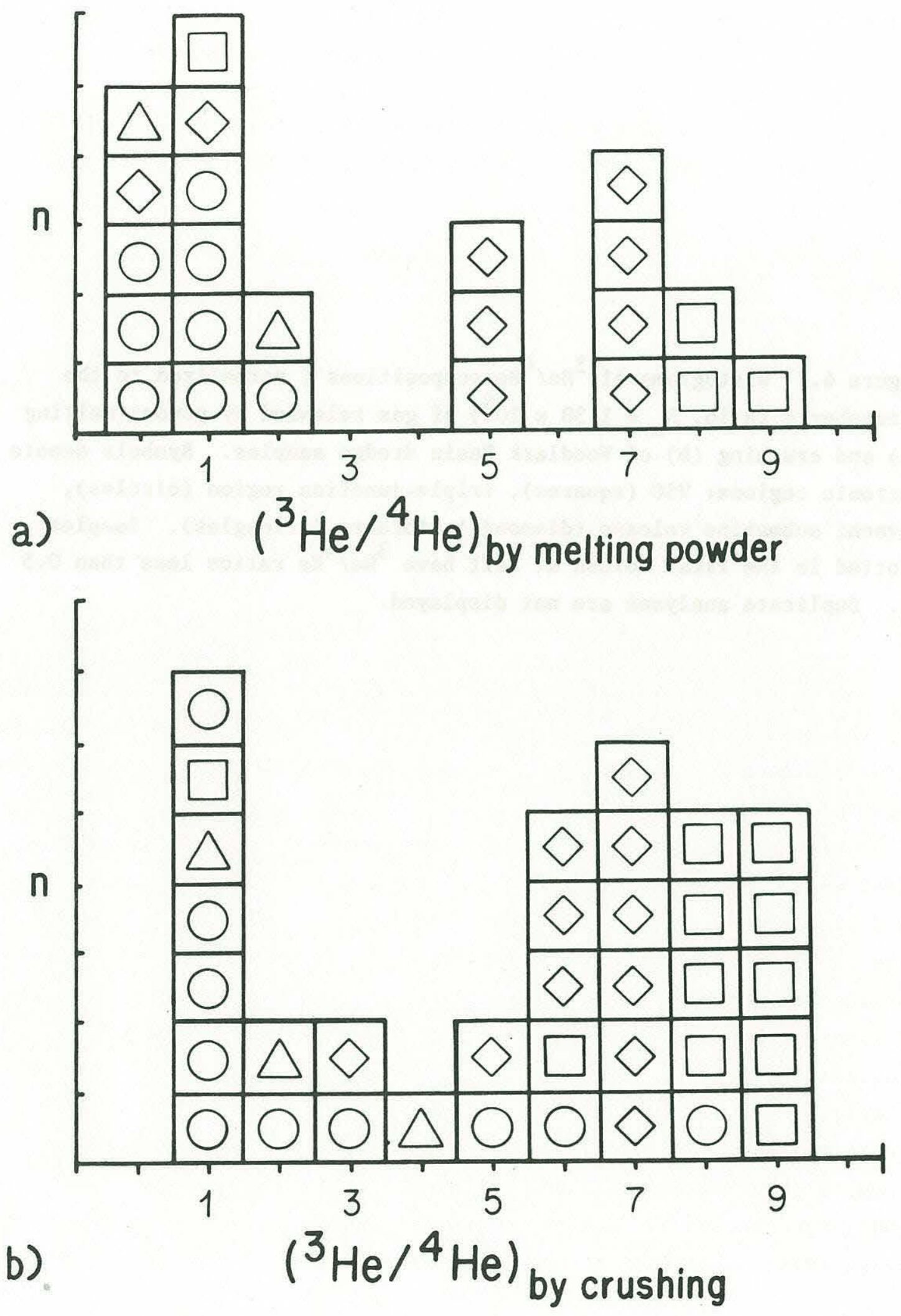
generally exhibit lower ${ }^{3} \mathrm{He} /{ }^{4} \mathrm{He}$ ratios than the Kavachi samples, extending to very radiogenic ratios lower than the atmospheric ratio. Samples from the triple junction area generally have much lower ${ }^{3} \mathrm{He} /{ }^{4} \mathrm{He}$ ratios than rocks from either the spreading center or overriding plate, but also include values as high as $8 \mathrm{R}_{\mathrm{a}}$. Among the low ratio samples are the high Mg0 andesites ("boninites") of dredge 31, a rock type thought to involve slab-derived hydrous fluids in its petrogenesis ( e.g. Cameron, McCulloch, and Walker, 1983). Triple junction sample types included mineral separates, glasses, and groundmass (Table 6.1).

In summary, samples from the volcanic forearc and nearby triple junction area have distinctly lower ${ }^{3} \mathrm{He} /{ }^{4} \mathrm{He}$ ratios than do rocks from the Woodlark Spreading Center. However, in order to relate the regional helium isotopic variations to differences in source materials, or petrogenetic variables, it is neccesary to establish that the measured ${ }^{3} \mathrm{He} /{ }^{4} \mathrm{He}$ ratios on rocks, glasses, and mineral separates represent magmatic isotopic compositions.

a) Alteration of magmatic ${ }^{3} \mathrm{He} /{ }^{4} \mathrm{He}$ ratios after eruption.

Contamination of the helium isotopic compositions of rocks erupted on the seafloor may occur by either the exchange of helium with seawater or the addition of radiogenic ${ }^{4} \mathrm{He}$ within the sample. Both processes are time dependant and affect samples with low initial helium contents most severely.

High helium contents ( $10^{-7} \mathrm{ccSTP} / \mathrm{g}$ or more) are essentially limited to glasses from the WSC basalts from dredges 26 and 29. Addition of ${ }^{4} \mathrm{He}$ by either process will not have significantly altered the isotopic composition of helium released by crushing these samples, because of their high helium contents and geologically young ages (see discussion below). Consistent with this conclusion is the lack of any correlation between ${ }^{3} \mathrm{He} /{ }^{4} \mathrm{He}$ ratios and helium concentrations for these samples. The helium contents of these samples are approximately one tenth that of mid-Atlantic-ridge basalts, but are very similar to results from the Marianas and Lau back-arc basins (Kurz and Jenkins, 1981; Poreda, 1985). Helium concentrations in glasses from other rock types (NaTi basalts of dredge 32, basaltic andesites of dredge 30 in the 
triple junction region, and the forearc andesite 24-4) are much lower $\left(10^{-8}\right.$ to $\left.10^{-9} \mathrm{ccSTP} / \mathrm{g}\right)$. As shown below, some of these samples may have been contaminated by helium addition after eruption.

Low He concentrations (order $10^{-8} \mathrm{ccSTP} \mathrm{He} / \mathrm{g}$ ) were found in all mineral separate samples, similar to the amounts observed in phenocrysts from Hawaiian and other oceanic island basalts by Kurz, Jenkins, and Hart (1982), and Kurz et al (1983). Figure 6.3 shows that a large fraction (50-99\%) of the helium is released by crushing. Clinopyroxenes contained the most He and released a larger percentage by crushing than olivines and orthopyroxenes. Samples with high gas contents in one phase tend to have elevated levels in other phases as well, although this is not a strong correlation. These results are in agreement with the qualitative abundances of melt and fluid inclusions in the crystals, and suggest that the He primarily resides in the inclusions. Similar helium distributions were found by Polve and Kurz (1988) for mineral separates from ultramafic xenoliths. Note that while mineral separate helium contents are lower than those of glasses, crystals are not necessarily more prone to contamination because they also have lower uranium and thorium contents and slower diffusive exchange for $\mathrm{He}$ (Polve, 1985; Trull, 1988).

Groundmass (holocrystalline) samples generally contain on the order of $10^{-9} \mathrm{ccSTP} \mathrm{He} / \mathrm{g}$. Such low concentrations, coupled with high uranium and thorium contents in comparison to mineral separates, and short paths for diffusive exchange (i.e. small grain size) make this sample type readily susceptible to contamination. In addition, groundmass samples may contain atmospheric helium. Analysis by crushing and melting of a glassy dacite sample, 33B, released 2 and $3 \times 10^{-8} \mathrm{ccSTP} \mathrm{He} / \mathrm{g}$ respectively, each time with atmospheric isotopic composition. This is probably helium from air trapped in microscopic intergranular spaces within the sample, which is also consistent with the elevated preextraction blanks observed for this sample. One percent pore space is required, in good agreement with the sample's appearance in thin section. This process may affect groundmass samples in general, yet it would go undetected for samples with more typical helium contents of $10^{-9} \mathrm{ccSTP} / \mathrm{g}$. Based on this, and other information discussed below, we 
Figure 6.3 Comparison of the quantity of helium released by crushing versus melting powdered mineral separates. Tielines connect observed values (open symbols) with points corrected for radiogenic helium ( ${ }^{*} \mathrm{He}_{1}$ in table 6.1 ; filled symbols). Errors are about $1 \%$ and are smaller than the symbols. 
Figure 6.3

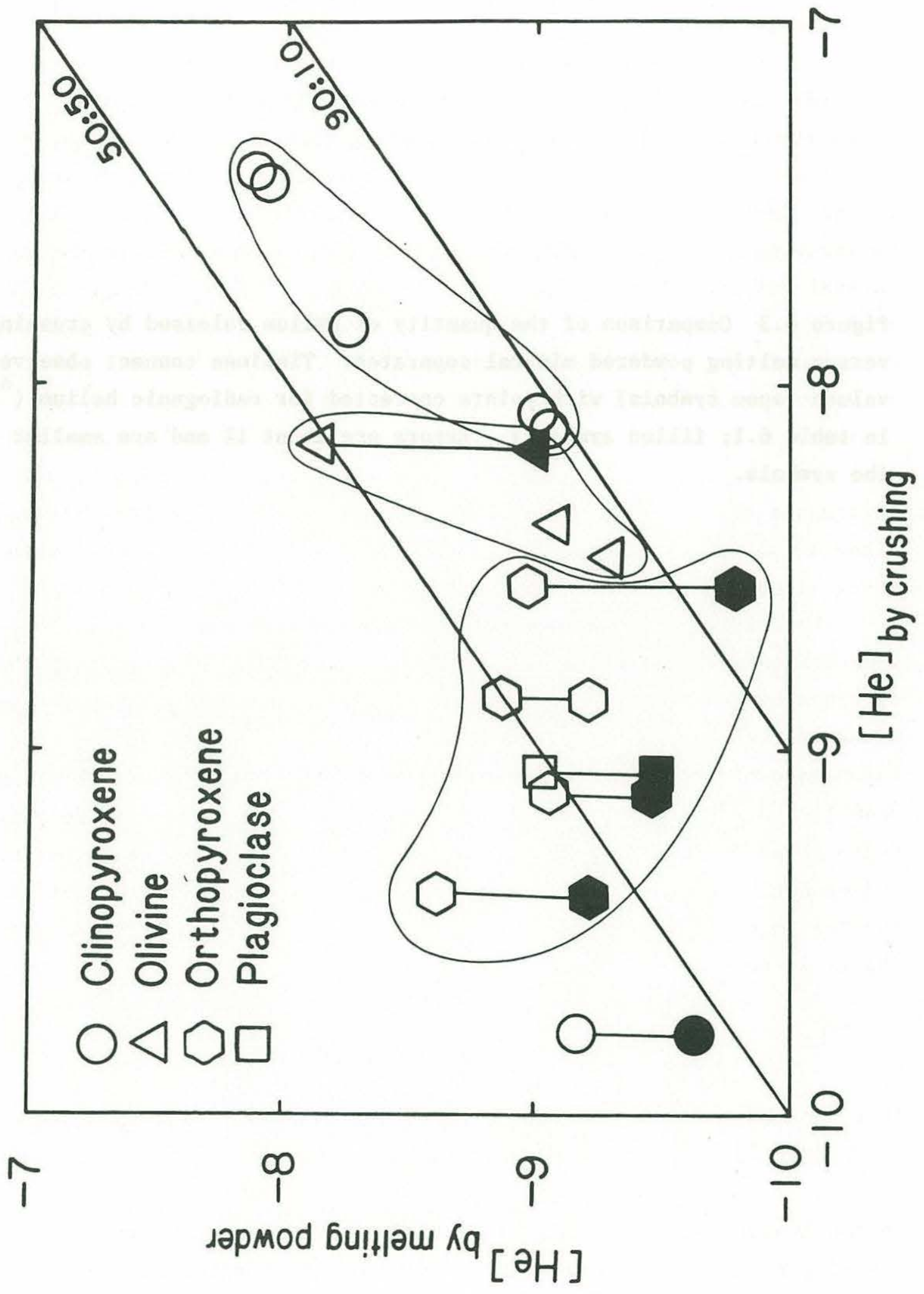


consider groundmass samples unreliable in determining magmatic helium isotopic compositions.

One way to assess the addition of radiogenic He to samples of uncertain age, such as these, is by comparison of the isotopic composition of helium released by crushing with that released by melting the crushed powder. Figure 6.4 reveals that many samples from the forearc region and triple junction exhibit significantly lower ${ }^{3} \mathrm{He} /{ }^{4} \mathrm{He}$ ratios when melted. In contrast, most samples from the WSC and from Kavachi Volcano lie along the line representing isotopic equilibrium in this figure. Lower ${ }^{3} \mathrm{He} /{ }^{4} \mathrm{He}$ ratios on melting are consistent with the post-eruptive addition of radiogenic ${ }^{4} \mathrm{He}$ in the solid phase. Note that significant disequilibrium is observed in both glasses and mineral separates. The only sample to exhibit a higher ${ }^{3} \mathrm{He} /{ }^{4} \mathrm{He}$ ratio on melting than crushing (32-9) was probably affected by helium from seawater (discussed below). It is possible that small amounts of basaltic matrix adhering to mineral separates contribute significantly to the observed disequilibrium because of their higher $U$ and Th contents.

The possibility of radiogenic helium addition to these samples suggests that magmatic ${ }^{3} \mathrm{He} /{ }^{4} \mathrm{He}$ ratios of arc rocks cannot reliably be obtained by total fusion analysis, at least for samples of unknown age. However, combined crushing and melting analyses allow an estimate of the importance of radiogenic helium and may allow the determination of the magmatic ${ }^{3} \mathrm{He} /{ }^{4} \mathrm{He}$ ratio, even if disequilibrium is observed. The ${ }^{3} \mathrm{He} /{ }^{4} \mathrm{He}$ ratio obtained by crushing will not be disturbed unless the amount of radiogenic He generated in the solid phase is a significant fraction of the helium content released by crushing. The total amount of radiogenic ${ }^{4} \mathrm{He}$ is given by:

$$
{ }^{*} \mathrm{He}=[\mathrm{He}]_{m} \times\left[\mathrm{R}_{i}-\mathrm{R}_{\mathrm{m}}\right] /\left[\mathrm{R}_{i}-\mathrm{R}_{\mathrm{p}}\right]
$$

$\left(\mathrm{R}={ }^{3} \mathrm{He} /{ }^{4} \mathrm{He}\right.$ ratio, $\mathrm{m}=$ melted powder, $\mathrm{i}=$ inherited ratio at eruption, $\mathrm{p}=$ production ratio accompanying radiodecay.)

We can approximate $R_{i}$ by $R_{c}$, the ratio obtained by crushing the sample, yielding a minimum estimate for ${ }^{*} \mathrm{He}$, since any transfer of radiogenic 
Figure 6.4 Internal isotopic disequilibrium is shown in this plot of the ${ }^{3} \mathrm{He} /{ }^{4} \mathrm{He}$ ratios measured by crushing, versus melting the resultant powder. Samples below the equilibrium line have been affected by ingrowth of ${ }^{4} \mathrm{He}$ after eruption. WSC sample $32-9$ is the only sample to plot significantly above the line and is discussed in the text. Error bars are 1 sigma. Symbols denote tectonic regions as in figure 6.2. 
Figure 6.4

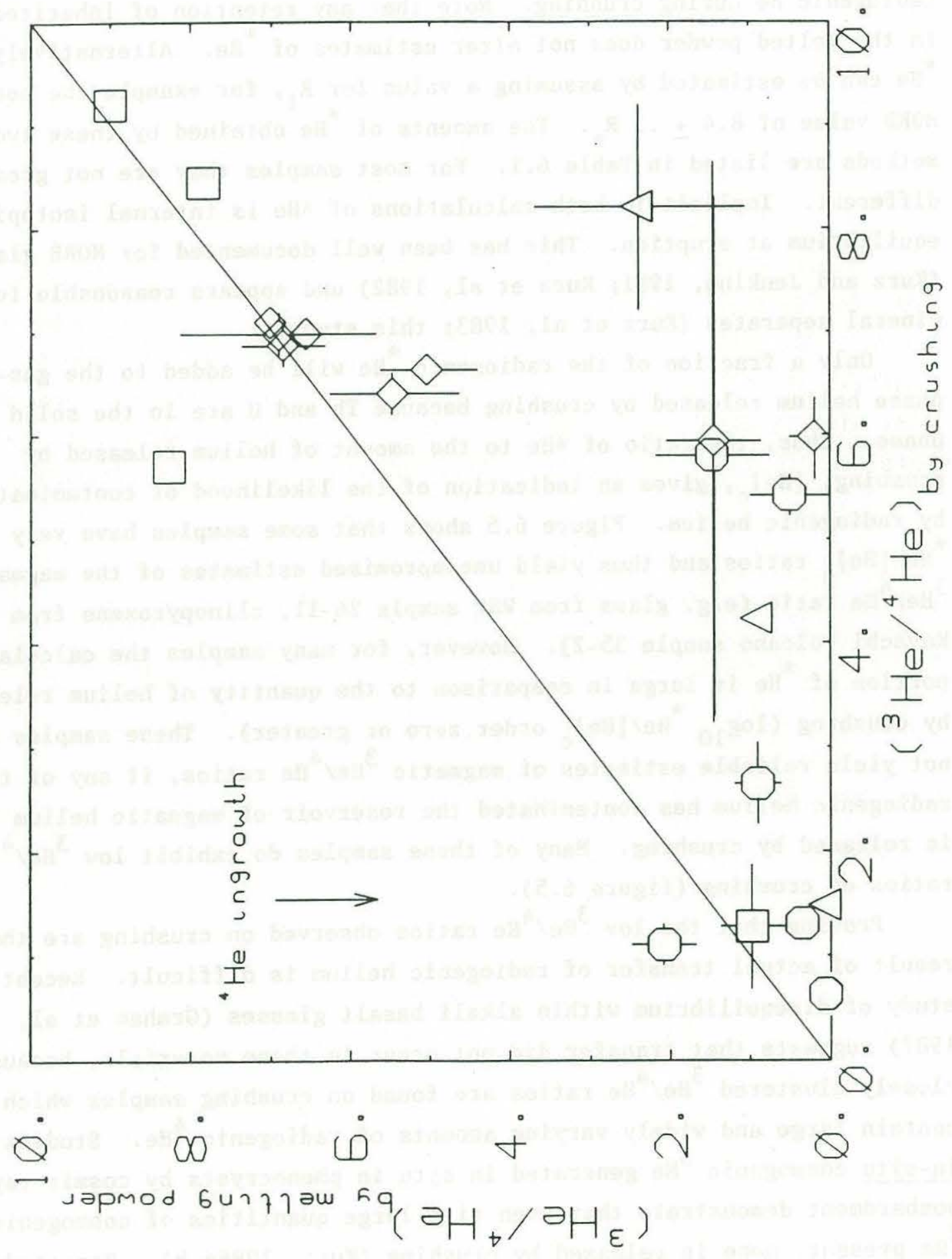


helium from the solid phase to the helium released by crushing will reduce the observed disequilibrium. This includes alpha-particle ejection, diffusive exchange on the seafloor, and any release of radiogenic He during crushing. Note that any retention of inherited He in the melted powder does not alter estimates of ${ }^{*} \mathrm{He}$. Alternatively, *He can be estimated by assuming a value for $R_{i}$, for example the normal MORB value of $8.4 \pm .3 \mathrm{R}_{\mathrm{a}}$. The amounts of ${ }^{*} \mathrm{He}$ obtained by these two methods are listed in Table 6.1. For most samples they are not greatly different. Implicit in both calculations of $\star_{\mathrm{He}}$ is internal isotopic equilibrium at eruption. This has been well documented for MORB glasses (Kurz and Jenkins, 1981; Kurz et al, 1982) and appears reasonable for mineral separates (Kurz et al, 1983; this study).

Only a fraction of the radiogenic * He will be added to the gasphase helium released by crushing because Th and $U$ are in the solid phase. Thus, the ratio of *He to the amount of helium released by crushing, [He] ${ }_{c}$, gives an indication of the likelihood of contamination by radiogenic helium. Figure 6.5 shows that some samples have very low ${ }^{*} \mathrm{He} /[\mathrm{He}]_{\mathrm{C}}$ ratios and thus yield uncompromised estimates of the magmatic ${ }^{3} \mathrm{He} /{ }^{4} \mathrm{He}$ ratio (e.g. glass from WSC sample 26-11, clinopyroxene from Kavachi volcano sample 35-2). However, for many samples the calculated portion of ${ }^{*} \mathrm{He}$ is large in comparison to the quantity of helium released by crushing $\left(\log _{10}{ }^{\star} \mathrm{He} /[\mathrm{He}]_{c}\right.$ order zero or greater). These samples may not yield reliable estimates of magmatic ${ }^{3} \mathrm{He} /{ }^{4} \mathrm{He}$ ratios, if any of the radiogenic helium has contaminated the reservoir of magmatic helium that is released by crushing. Many of these samples do exhibit low ${ }^{3} \mathrm{He} /{ }^{4} \mathrm{He}$ ratios on crushing (figure 6.5 ).

Proving that the low ${ }^{3} \mathrm{He} /{ }^{4} \mathrm{He}$ ratios observed on crushing are the result of actual transfer of radiogenic helium is difficult. Recent study of disequilibrium within alkali basalt glasses (Graham et al, 1987) suggests that transfer did not occur in these materials, because closely clustered ${ }^{3} \mathrm{He} /{ }^{4} \mathrm{He}$ ratios are found on crushing samples which contain large and widely varying amounts of radiogenic ${ }^{4} \mathrm{He}$. Studies of in-situ cosmogenic ${ }^{3}$ He generated in situ in phenocrysts by cosmic ray bombardment demonstrate that even with large quantities of cosmogenic ${ }^{3}$ He present, none is released by crushing (Kurz, 1986a,b). Repeated 
Figure 6.5 Comparison of the ${ }^{3} \mathrm{He} /{ }^{4} \mathrm{He}$ ratio measured by crushing versus the model quantity, $\log \left({ }^{\star} \mathrm{He}_{1} /[\mathrm{He}]_{\text {Crushing }}\right)$, for samples that exhibit significant isotopic disequilibrium. ${ }^{*} \mathrm{He}_{1}$ represents the quantity of radiogenic ${ }^{4} \mathrm{He}$ required to produce the observed disequilibrium (in ccSTP/g see Table 6.1). Error bars are 1 sigma. Symbols denote tectonic regions as in figure 6.2. All samples are phenocrysts with the exceptions of glass from sample 26-11, which has the highest ${ }^{3} \mathrm{He} /{ }^{4} \mathrm{He}$ ratio plotted, and a glass and two glassy groundmass samples from the forearc and triple-junction respectively, which have the lowest ${ }^{3} \mathrm{He} /{ }^{4} \mathrm{He}$ ratios plotted. 
Figure 6.5

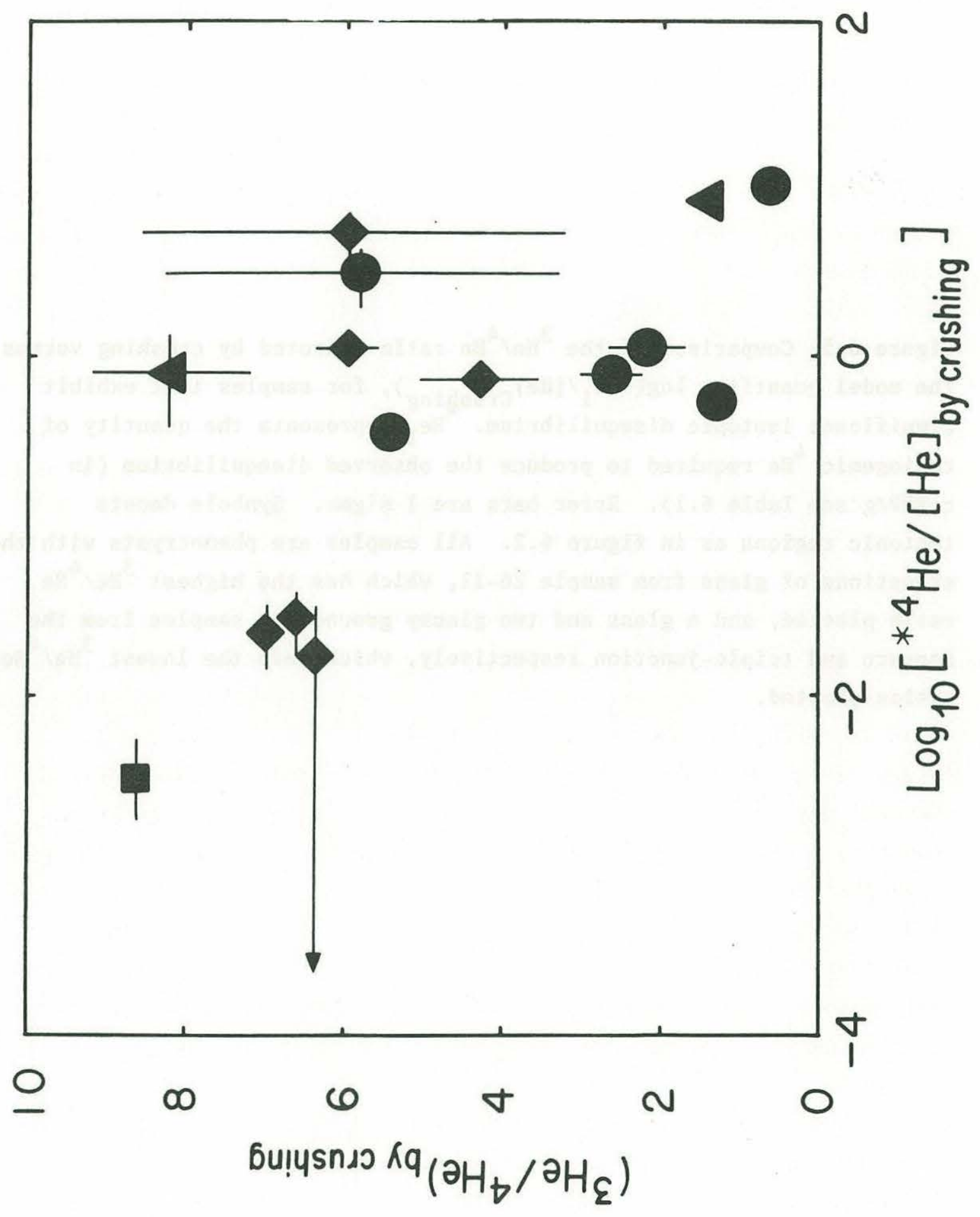


crushing of bronzite from sample 31-10 (Table 6.1) yielded

indistinguishable isotopic compositions, suggesting limited release of radiogenic He during crushing ( 0 to at most $20 \%$ ). The production of new surfaces by crushing can release at most $1-2 \%$ of any radiogenic helium present.

However, transfer of radiogenic helium may occur in mineral grains by alpha - particle ejection, because stopping ranges are tens of microns and uranium mainly resides in melt inclusions (Polve, 1985; and unpublished fission track maps of these samples) which are of ten closely associated with the helium containing fluid inclusions. For this reason, and the suggestive evidence in figure 6.5, we consider samples exhibiting high ${ }^{*} \mathrm{He} /[\mathrm{He}]_{c}$ ratios to be unreliable in determining magmatic helium isotopic compositions. This criterion also discredits any phenocryst samples which may have large amounts of radiogenic helium "shot in" from $U$ and Th in the surrounding matrix.

In summary, radiogenic helium addition (revealed by internal isotopic disequilibrium) prevents the determination of magmatic ${ }^{3} \mathrm{He} /{ }^{4} \mathrm{He}$ ratios for many samples from the forearc and triple junction regions. In contrast, internal equilibrium for many Kavachi volcano samples (as well as identical helium isotope compositions obtained by crushing more than one mineral phase from the same sample, Table 6.1) strongly suggests that the observed helium isotopic compositions are those of Kavachi magmas at eruption. No single mineral phase emerged as most commonly free of contamination by radiogenic He. However, clinopyroxenes consistently release the most helium on crushing (figure 6.3), making them a good choice for analysis. Groundmass samples consistently exhibited lower ${ }^{3} \mathrm{He} /{ }^{4} \mathrm{He}$ when crushed (and larger radiogenic helium contents) than did mineral separates from the same sample, confirming their likely contamination (Table 6.1; samples 24-4, 31-1, and $31-10$ ).

Comparison of calculated radiogenic helium contents with production rates by uranium and thorium decay suggests that samples from the triple-junction region, forearc, Kavachi submarine volcano, and the Woodlark Spreading Center are on the order of $10^{4}$ to $10^{5}$ years old (figure 6.6). These ages appear reasonable within the geologic setting, 
Figure 6.6 The amount of He added by radiodecay is shown for different uranium contents (in ppm) as a function of time (solid lines), assuming a Th/U ratio of 3.0 , and secular equilibrium. Samples are plotted based on their calculated radiogenic ${ }^{4} \mathrm{He}$ content $\left({ }^{*} \mathrm{He}_{1}\right.$ in Table 6.1$)$, and an estimate of their uranium content. The age estimate error bar corresponds to 1 sigma in the ${ }^{*}$ He content, but does not include the error in estimating uranium contents. The uranium estimates were derived from whole rock $U$ analyses for the sample or a similar rock (Johnson et al, 1987) and, for the phenocrysts, a bulk partitioning coefficient derived from examination of induced-fission-track maps of uranium distributions made from thin-sections of the sample or a similar rock. This estimate is very uncertain because the track studies were performed on whole rocks rather than on splits of the mineral separates used for helium analyses. However, the track maps did uniformly suggest that phenocryst contents of uranium are fairly high, and derive from included melt fractions of generally a few percent (Trull, unpublished results; see also Polve, 1985). Another complication in deriving an age estimate from the amount of radiogenic helium observed in a sample is the injection of * He from the surrounding rock matrix. For a spherical phenocryst $.5 \mathrm{~mm}$ in diameter this injection contributes * He equivalent to an internal uranium concentration of $10 \%$ of that of the whole rock (for a $1 \mathrm{~mm}$ crystal the contribution drops to a little less than $5 \%$ ). Similarly, even small amounts of adhering basaltic matrix will increase the apparent disequilibium, and therefore the age estimate. These effects were considered in estimating the rather high effective uranium contents used to plot the points in the figure. For example, the Kavachi sample with the greatest *He content (olivine from 34-7) is plotted at relatively high $U$ contents because it was composed of small crystals with some adhering basalt matrix. Because of the uncertainties in both uranium contents and the calculation of ${ }^{*}$ He the ages should be considered only as an order of magnitude estimate. Symbols: WSC glass sample (square), forearc minerals (triangles), triple junction minerals (octagon), Kavachi submarine volcano minerals (diamonds). 
Figure 6.6

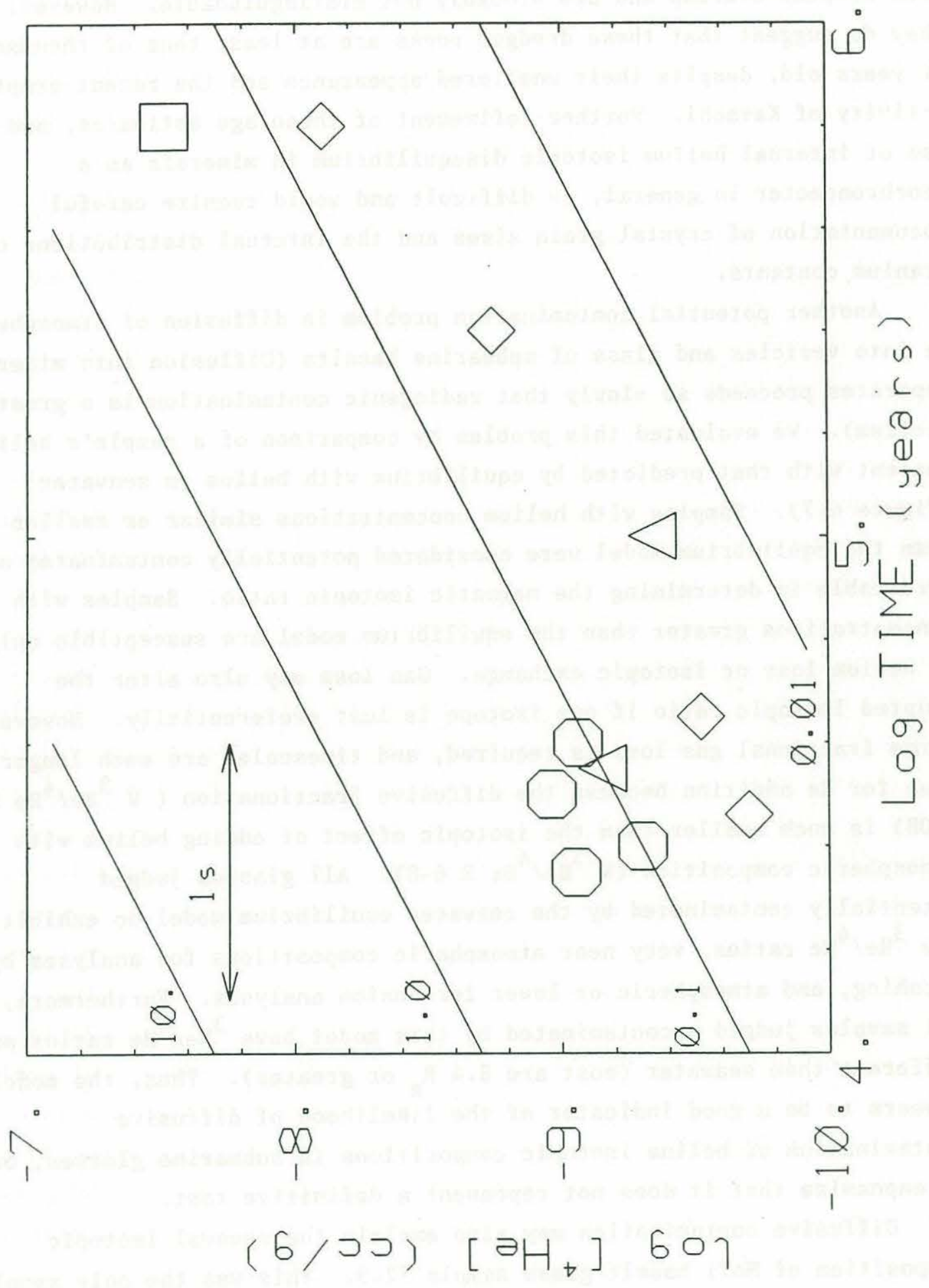


given the large uncertainties. The apparent ages of different samples from Kavachi overlap and are probably not distinguishable. However, they do suggest that these dredged rocks are at least tens of thousands of years old, despite their unaltered appearance and the recent eruptive activity of Kavachi. Further refinement of these age estimates, and the use of internal helium isotopic disequilibrium in minerals as a geochronometer in general, is difficult and would require careful documentation of crystal grain sizes and the internal distributions of uranium contents.

Another potential contamination problem is diffusion of atmospheric He into vesicles and glass of submarine basalts (Diffusion into mineral separates proceeds so slowly that radiogenic contamination is a greater problem). We evaluated this problem by comparison of a sample's helium content with that predicted by equilibrium with helium in seawater (figure 6.7). Samples with helium concentrations similar or smaller than the equilibrium model were considered potentially contaminated and unreliable in determining the magmatic isotopic ratio. Samples with concentrations greater than the equilibrium model are susceptible only to helium loss or isotopic exchange. Gas loss may also alter the erupted isotopic ratio if one isotope is lost preferentially. However, large fractional gas loss is required, and timescales are much longer than for He addition because the diffusive fractionation ( $W^{3} \mathrm{He} /{ }^{4} \mathrm{He} \mathrm{Z}$ 1.08) is much smaller than the isotopic effect of adding helium with atmospheric composition ( $W^{3} \mathrm{He} /{ }^{4} \mathrm{He} \mathrm{Z} 6-8$ ). All glasses judged potentially contaminated by the seawater equilibrium model do exhibit low ${ }^{3} \mathrm{He} /{ }^{4} \mathrm{He}$ ratios, very near atmospheric compositions for analyses by crushing, and atmospheric or lower for fusion analyses. Furthermore, all samples judged uncontaminated by this model have ${ }^{3} \mathrm{He} /{ }^{4} \mathrm{He}$ ratios much different than seawater (most are $8.4 \mathrm{R}_{\mathrm{a}}$ or greater). Thus, the model appears to be a good indicator of the likelihood of diffusive contamination of helium isotopic compositions in submarine glasses, but we emphasize that it does not represent a definitive test.

Diffusive contamination may also explain the unusual isotopic composition of NaTi basalt glass sample 32-9. This was the only sample to exhibit a higher ratio when the powder was melted than on crushing 
Figure 6.7 Comparison of observed helium concentrations with expected values for equilibrium with seawater. The curves model concentration equilibrium of helium dissolved in seawater with helium in basaltic glass (G-SW, at left) and vesicles (G-V, at right). The model assumes that helium behaves ideally in the vesicle gas, and that the Henry's law coefficients for glass - vesicle partitioning during eruption (Kurz and Jenkins, 1981) and seawater - atmosphere equilibration apply during diffusive reequilibration on the seafloor. Samples are plotted according to their measured helium concentrations by crushing and powder melting. Symbol shapes denote tectonic regions or rock types: WSC (squares), triple-junction region (octagons), Kavachi submarine volcano (diamonds), forearc (triangles), NaTi basalt glasses (crosses). Filled symbols are samples with ${ }^{3} \mathrm{He} /{ }^{4} \mathrm{He}$ ratios significantly greater than $1 \mathrm{R}_{\mathrm{a}}$, open symbols are samples with ratios near $1 \mathrm{R}_{\mathrm{a}}$ or less, the half-filled symbol (sample 32-9) may be partially contaminated, see text. 
Figure 6.7
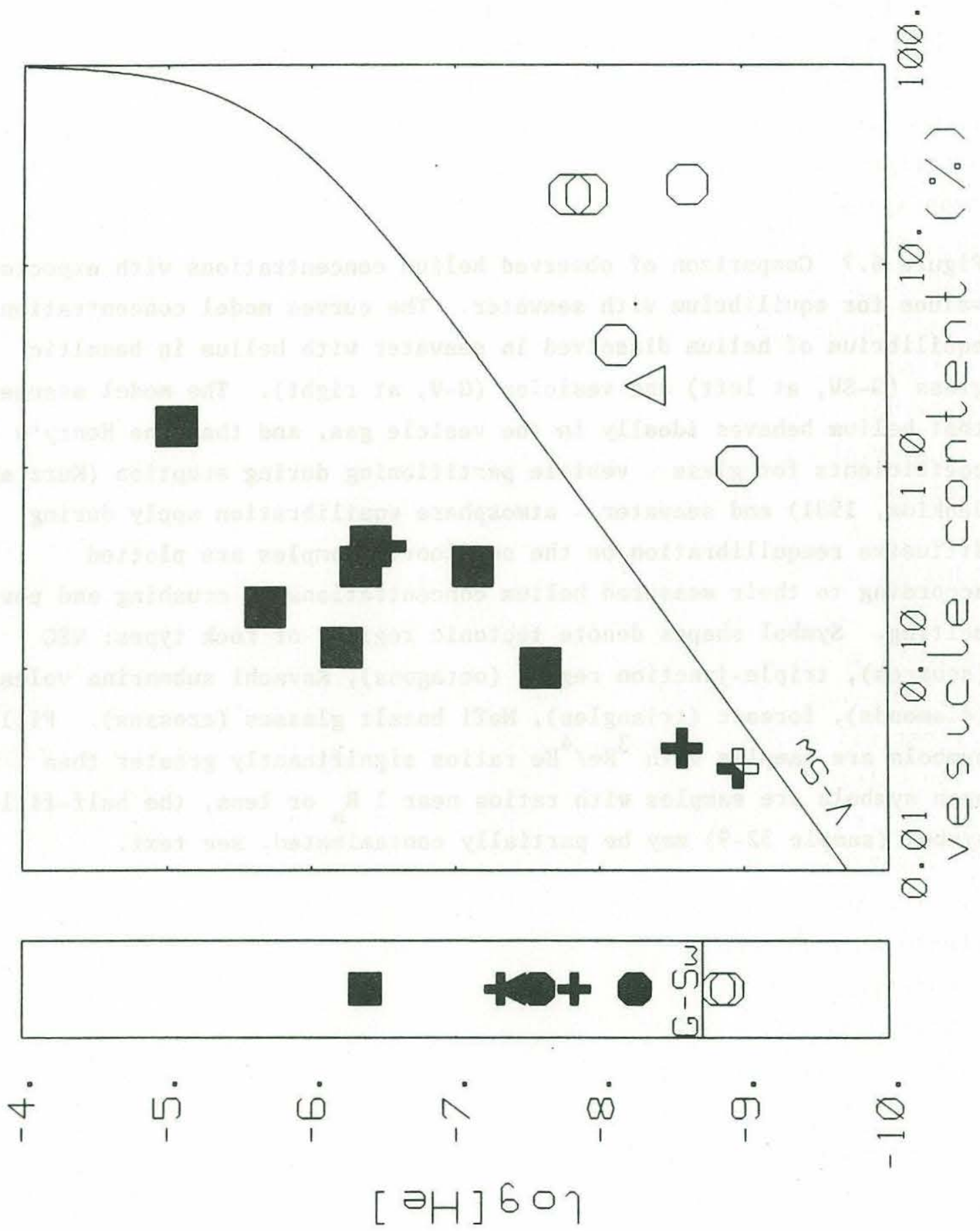
(figure 6.4). The amount of helium released by crushing was very small and close to the seawater equilibrium curve for the sample's vesicle content (figure 6.7), suggesting possible contamination. Step-heating of the powder released helium of three distinct isotopic compositions (figure 6.8). A small amount of helium released at high temperature has a ${ }^{3} \mathrm{He} /{ }^{4} \mathrm{He}$ ratio higher than (but within 2 sigma) of the isotopic compositions of other NaTi samples and may be released from microphenocrysts of pyroxene within the glass. Intermediate temperatures released most of the helium with an isotopic composition like that released by bulk melting. At low temperature the helium isotopic composition is similar to that obtained by crushing: both methods probably release helium from spherulitic zones (visible in thin section) that have been contaminated by helium from seawater. Diffusive contamination by helium in this situation appears to be much rapider than alteration because $\mathrm{Sr}$ and oxygen isotope compositions were not affected.

The rate of addition of helium to glasses and vesicles by diffusion is highly dependent on grain geometry and the size and distribution of vesicles. Applying a membrane diffusion model parameterized to represent glasses with abundant $(10 \%)$, large $(1 \mathrm{~mm})$, thin-walled (100um) vesicles, suggests an influx of helium to vesicles on the order of $10^{-8}$ $\mathrm{cc} / \mathrm{g}$ per million years. This estimate assumes equilibrium with seawater at the external boundary, as defined by [He] $=\mathrm{K}_{\mathrm{H}} \times \mathrm{P}^{\mathrm{SW}} \mathrm{He}$, where $\mathrm{K}_{\mathrm{H}}$ is the Henry's law coefficient derived from vesicle - glass partitioning in MORB (Kurz and Jenkins, 1981), and a diffussion coefficient of $10^{-15}$ $\mathrm{cm}^{2} / \mathrm{s}$ (Jambon, Weber and Begemann, 1985; Trull, 1988). The model is probably an upper limit based on the small membrane thickness, the high vesicularity, and the assumption of no helium in the vesicle even as diffusive contamination proceeds, and because lower estimates of the helium diffusivity have been made (Kurz and Jenkins, 1981). At this rate, diffusive contamination of the samples with low ratios in figure 6.7 requires $10^{5}$ to $10^{6}$ years. 
Figure 6.8 Histograms show the amounts and isotopic compositions of helium released by crushing and melting (a.), and step-heating experiments (b.) for spherulitic glass sample 32-9. One sigma errors are given. See discussion in text. 


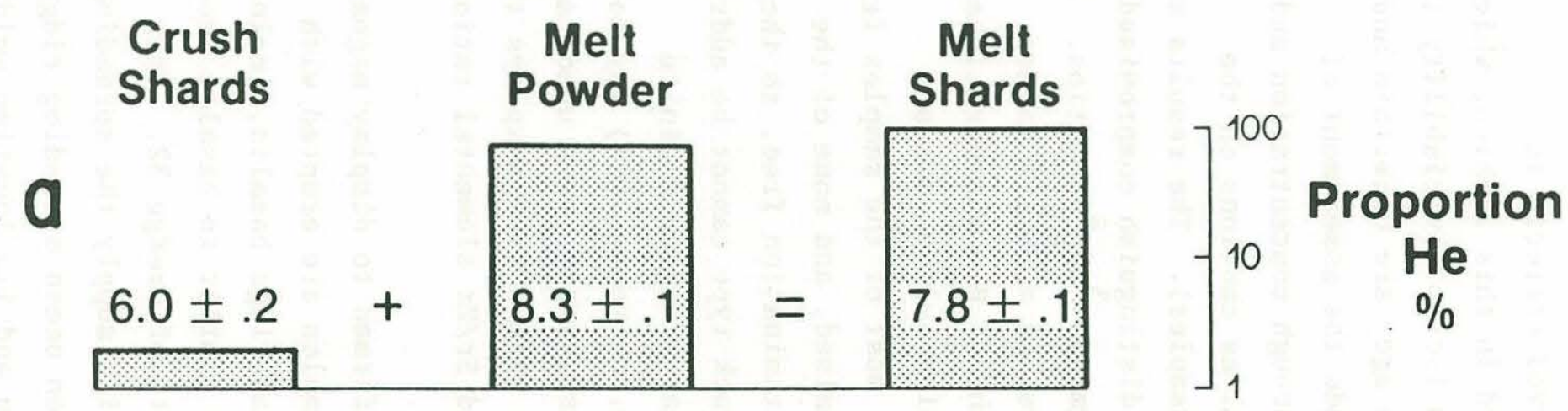

\section{Stepheat Powder}
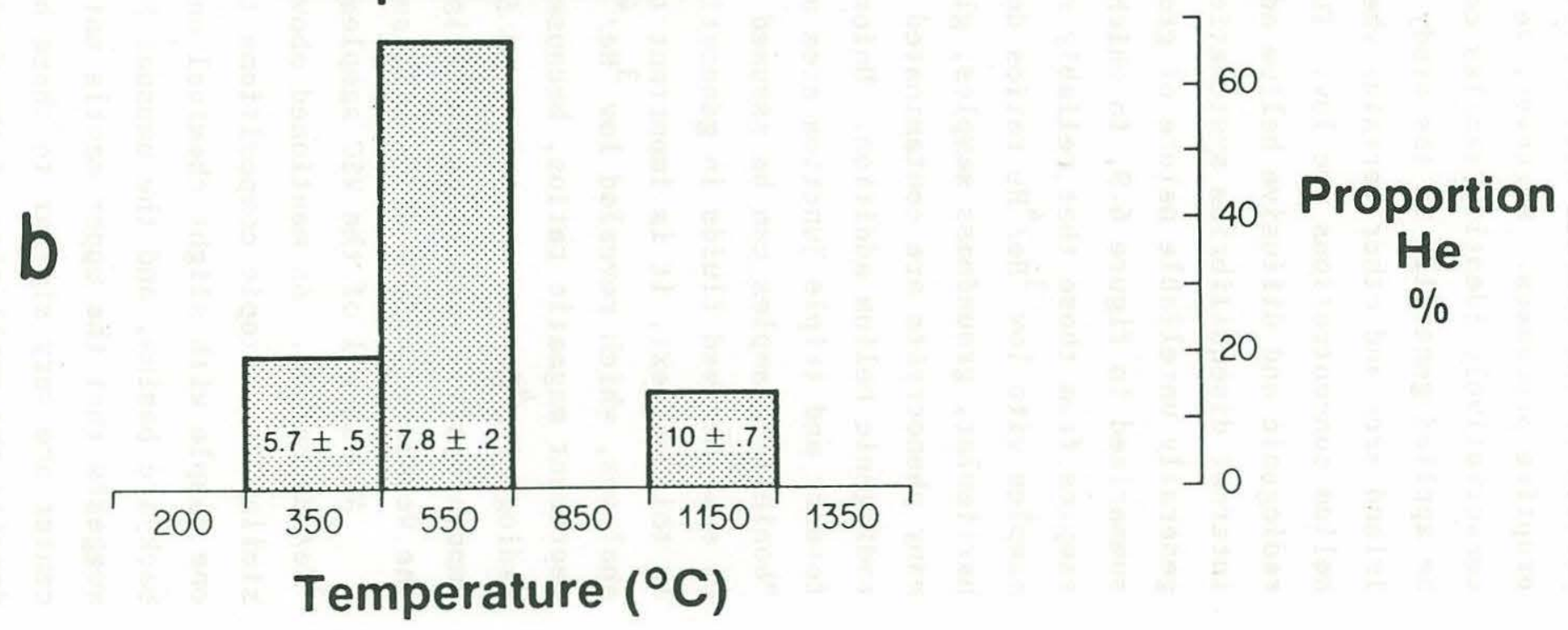
b) Summary of the origins of He isotopic variabilty.

Combined analyses by crushing and melting revealed that a large portion of the overall helium isotopic variability is produced by posteruptive processes. Moreover, we have derived criteria to conservatively identify samples contaminated in this fashion, which may be applied generally in the study of helium isotopic variability in island arcs and other terrains where sample ages are uncertain and helium concentrations are low. These include the assessment of radiogenic and diffusive helium addition through concentration and internal disequilibrium systematics (as well as cautions on the generally unreliable nature of groundmass samples). The results are summarized in figure 6.9, in which symbols distinguish compromised samples from those that reliably reveal magmatic ${ }^{3} \mathrm{He} /{ }^{4} \mathrm{He}$ ratios. Many samples with low ${ }^{3} \mathrm{He} /{ }^{4} \mathrm{He}$ ratios do not represent magmatic values. In particular, groundmass samples, glasses with low He concentrations, and many phenocrysts are contaminated by diffusive helium loss and radiogenic helium addition. Unfortunately, most of the samples from the forearc and triple junction area are compromised, and none of the "boninite" samples can be assumed to be contamination free, so the role of slab-derived fluids in generating this rock type cannot be addressed. In this context, it is important to note that previous boninite analyses, which revealed low ${ }^{3} \mathrm{He} /{ }^{4} \mathrm{He}$ ratios, (Poreda, 1983) may not represent magmatic ratios, because groundmass samples were used, and radiogenic ${ }^{4} \mathrm{He}$ was not accounted for. This figure also displays the smooth spatial variations in $\mathrm{Sr}$ isotopic and $\mathrm{Sr} / \mathrm{Zr}$ elemental ratios in the Woodlark Basin - Solomon Islands region.

Almost all of the WSC samples were confirmed to display magmatic ${ }^{3} \mathrm{He} /{ }^{4} \mathrm{He}$ ratios. As mentioned above, wSC samples are erupted with similar He isotopic compositions to mid-ocean-ridge basalts, including one sample with slight chemical enrichments similar to basalts from back-arc basins, and the unusual NaTi basalts of dredge 32 . This suggests that the upper mantle materials which supply the spreading center are very similar to those beneath open ocean spreading ridges, despite the small size of the Woodlark Basin and its location amidst arc and continental terrains. 
While some samples from Kavachi Volcano have been compromised by radiogenic He addition, the magmatic ${ }^{3} \mathrm{He} /{ }^{4} \mathrm{He}$ ratio can be recovered for most samples, and is uniform at $6.9 \pm .2 R_{a}$. This is significantly lower than ${ }^{3} \mathrm{He} /{ }^{4} \mathrm{He}$ ratios observed in normal MORB and in the basalts from the nearby WSC, although it is within the total range observed in MORB (Kurz, 1982; Kurz et al, 1982). This result is free of the ambiguity associated with hot spring and fumarole samples. That is, the observation of lowered ${ }^{3} \mathrm{He} /{ }^{4} \mathrm{He}$ ratios in arcs can be confidently attributed to the magmas themselves, rather than processes affecting the exsolved gases.

It is important to note that the WSC data are derived from glass analyses, while the Kavachi analyses were on mineral separates. While isotopic exchange between crystals and liquid is rapid (decades), closure temperatures for phenocrysts may be below the solidus if magma cooling is slow, allowing helium loss from phenocrysts and perhaps isotopic fractionation to occur (Hart, 1984). For arc rocks, which often show signs of multiple magmatic episodes (in general: Gill, 1981; for Kavachi rocks: Johnson et al, 1987) it is necessary to assess whether long residence at elevated temperatures contributes to the lower ${ }^{3} \mathrm{He} /{ }^{4} \mathrm{He}$ ratios observed in Kavachi phenocrysts. Two simple tests can be used to assess the relevance of this process. If it were significant, mineral separates should exhibit positive correlations between ${ }^{3} \mathrm{He} /{ }^{4} \mathrm{He}$ ratios and grain sizes, since smaller crystals will have lost more helium. Similarly, pyroxenes should be more affected than olivine, since He diffusion in pyroxene is roughly 100 times faster at magmatic temperatures (Gramlich and Naughton, 1972; Trull, 1988). Our data for olivine/pyroxene pairs display isotopic equilibrium and are therefore not consistent with helium loss by this process. Lowering the ${ }^{3} \mathrm{He} /{ }^{4} \mathrm{He}$ ratio of $1 \mathrm{~mm}$ spherical olivine grains from 8.4 to $6.9 \mathrm{R}_{\mathrm{a}}$ in this manner would yield a ratio of less than $5 \mathrm{R}_{\mathrm{a}}$ in pyroxenes grains of similar size, for a ${ }^{3} \mathrm{He} /{ }^{4} \mathrm{He}$ isotopic diffusivity ratio of 1.08 in both olivine and pyroxene (Trull, 1988), but the result is not highly sensitive to the choice of isotopic diffusivity ratio, and the classical approximation of 1.15 (the inverse ratio of the square roots of the atomic masses) predicts a similar result. We did not systematically 
investigate correlations with grain size, but Kavachi mineral separates ranging from .4 to $1 \mathrm{~mm}$ in grain size yielded indistinguishable ${ }^{3} \mathrm{He} /{ }^{4} \mathrm{He}$ ratios. While this process does not appear important here, it deserves more study, and in some cases, such as the study of xenoliths, could perhaps be used to constrain cooling histories (compare Gramlich and Naughton, 1972).

\subsubsection{Strontium Isotopic Variability.}

The rocks recovered from the Woodlark Basin - Solomon Islands region can be divided into three groups based on their ${ }^{87} \mathrm{Sr} /{ }^{86} \mathrm{Sr}$ values: MORB with ratios from 0.702580 to 0.702910 , island arc lavas with ratios from 0.703468 to 0.704038 (ratios up to .705186 have been measured, Perfit et al, in preparation, but are not reported here) and basaltic andesites with ratios between 0.7031 and 0.7034 and transitional chemical characteristics (Table 6.2 and unpublished data). Incompatible element depleted tholeiitic basalts (n-type MORB) from the WSC have low ${ }^{87} \mathrm{Sr} /{ }^{86} \mathrm{Sr}$ ratios and $\mathrm{Sr}$ concentrations typical of MORB from the major oceanic ridges. A few samples with back-arc-basin-basalt (BABB) chemical characteristics have slightly higher $\mathrm{Sr}$ contents and ${ }^{87} \mathrm{Sr} /{ }^{86} \mathrm{Sr}$ ratios (greater than 0.7027 ) that could not be lowered by acid leaching. The unusual NaTi basalts have high $\mathrm{Sr}$ contents (. 230ppm) but $\mathrm{Rb} / \mathrm{Sr}$ and ${ }^{87} \mathrm{Sr} /{ }^{86} \mathrm{Sr}$ ratios typical of MORB (less than 0.7030 ; Table 6.2 and Staudigel et al, 1987).

Island arc basalts and related lavas from the forearc region, Kana Keoki seamount, and Kavachi submarine volcano exhibit the greatest $\mathrm{Sr}$ isotopic variability and have the highest $\mathrm{Sr}$ contents. These values (Table 6.2) are typical of a wide variety of island arc volcanic rocks from the western Melanesian arcs (DePaolo and Johnson, 1979; Perfit et al, 1982; Gill, 1984; Johnson et al, 1985). The highest isotopic ratios (approximately 0.7045) tend to be in the most alkalic and large-ionlithophile-(LIL)-enriched rocks resulting in positive correlations between ${ }^{87} \mathrm{Sr} /{ }^{86} \mathrm{Sr}$ ratios and LIL abundances. The $\mathrm{Sr}$ isotopic heterogeneity of the samples is not unusual for arcs and corresponds to that measured in lavas from the Solomon Islands (0.70352 - 0.70417; 
Table 2. Sr-isotope and trace element data

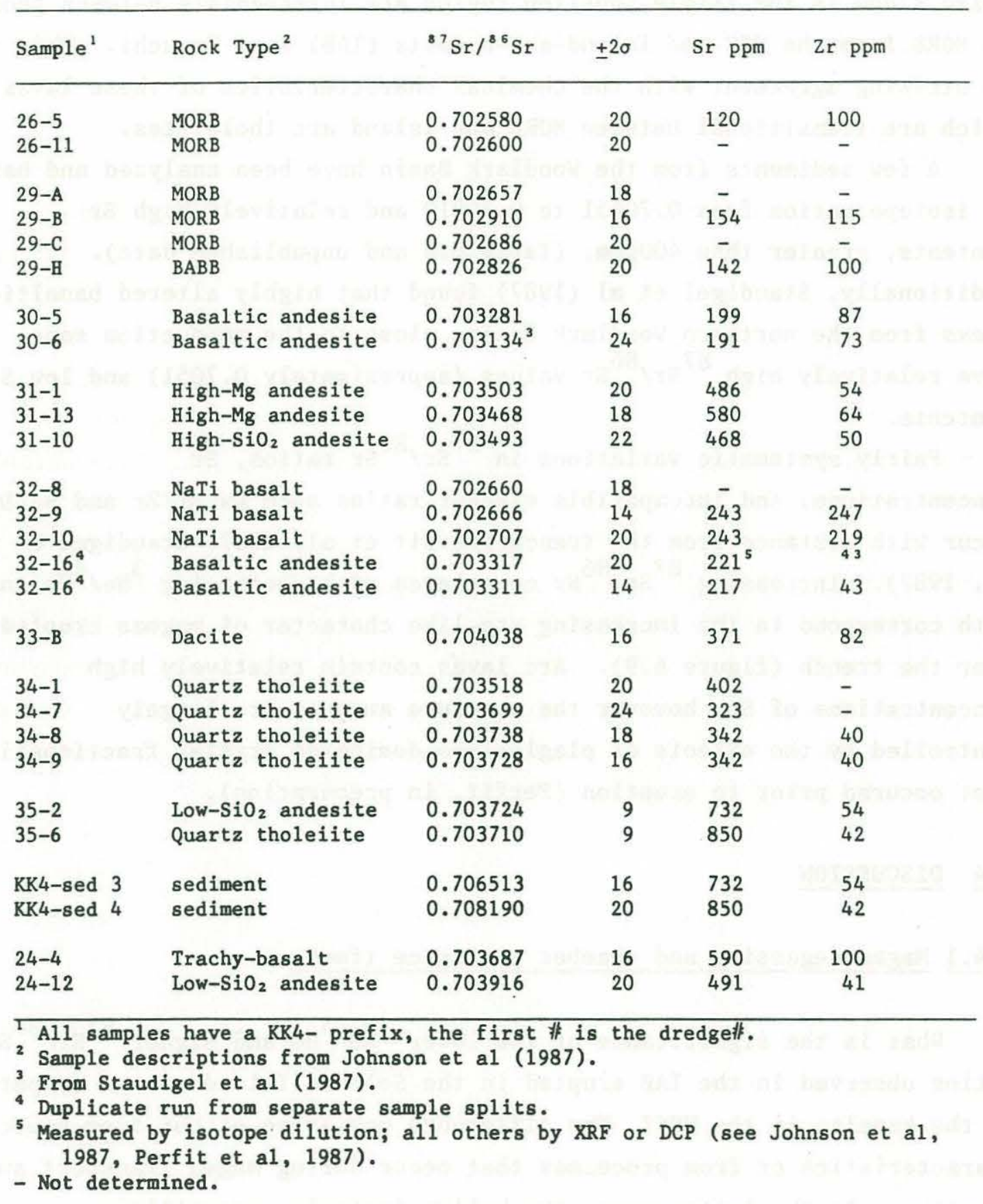


Staudigel et al, 1987; Perfit et al, in preparation). In this study the highest ${ }^{87} \mathrm{Sr} /{ }^{86} \mathrm{Sr}$ ratio was measured in a glassy, LIL-enriched dacite from Kana Keoki seamount in the complex triple-junction area.

The ${ }^{87} \mathrm{Sr} /{ }^{86} \mathrm{Sr}$ values of low-K basaltic andesites from the SimboGhizo Ridge in the triple junction region are intermediate between those of MORB from the WSC and island-arc-basalts (IAB) from Kavachi. This is in striking agreement with the chemical characteristics of these lavas, which are transitional between MORB and island arc tholeiites.

A few sediments from the Woodlark Basin have been analyzed and have $\mathrm{Sr}$ isotope ratios from 0.70651 to 0.70819 and relatively high $\mathrm{Sr}$ contents, greater than 400ppm, (Table 6.2 and unpublished data). Additionally, Staudigel et al (1987) found that highly altered basaltic rocks from the northern Woodlark Basin, close to the subduction zone, have relatively high ${ }^{87} \mathrm{Sr} /{ }^{86} \mathrm{Sr}$ values (approximately 0.7051) and low $\mathrm{Sr}$ contents.

Fairly systematic variations in ${ }^{87} \mathrm{Sr} /{ }^{86} \mathrm{Sr}$ ratios, $\mathrm{Sr}$ concentrations, and incompatible element ratios such as $\mathrm{Sr} / \mathrm{Zr}$ and $\mathrm{Ba} / \mathrm{La}$ occur with distance from the trench (Perfit et al, 1987; Staudigel et al, 1987). Increasing ${ }^{87} \mathrm{Sr} /{ }^{86} \mathrm{Sr}$ correlates with decreasing ${ }^{3} \mathrm{He} /{ }^{4} \mathrm{He}$ and both correspond to the increasing arc-like character of magmas erupted near the trench (figure 6.9). Arc lavas contain relatively high concentrations of $\mathrm{Sr}$, however the absolute amounts are largely controlled by the extents of plagioclase-dominated crystal fractionation that occured prior to eruption (Perfit, in preparation).

\subsection{DISCUSSION}

\subsubsection{Magma degassing and chamber residence times.}

What is the significance of the lower ${ }^{3} \mathrm{He} /{ }^{4} \mathrm{He}$ and higher ${ }^{87} \mathrm{Sr} /{ }^{86} \mathrm{Sr}$ ratios observed in the IAB erupted in the Solomon Island arc as compared to the basalts in the WSC? The difference may arise either from source characteristics or from processes that occur during magma transport and eruption. In the latter case, the helium isotopic composition difference may reflect the different degassing processes between arc and 
Figure $6.9{ }^{3} \mathrm{He} /{ }^{4} \mathrm{He}$ (by crushing), ${ }^{87} \mathrm{Sr} /{ }^{86} \mathrm{Sr}$, and $\mathrm{Sr} / \mathrm{Zr}$ versus distance from the trench. Dredge numbers are shown at the top of the figure. To the left are forearc dredges, and to the right the dredges from further west in the Woodlark Basin. Filled symbols represent samples for which the observed ${ }^{3} \mathrm{He} /{ }^{4} \mathrm{He}$ ratio represents the isotopic composition of the erupted magma. Open triangles indicate that the observed ${ }^{3} \mathrm{He} /{ }^{4} \mathrm{He}$ ratios are considered suspect because of the presence of significant radiogenic He. Crosses indicate samples that are probably contaminated by helium from seawater. Asterixes mark groundmass samples. Error bars are shown only for filled symbols. 


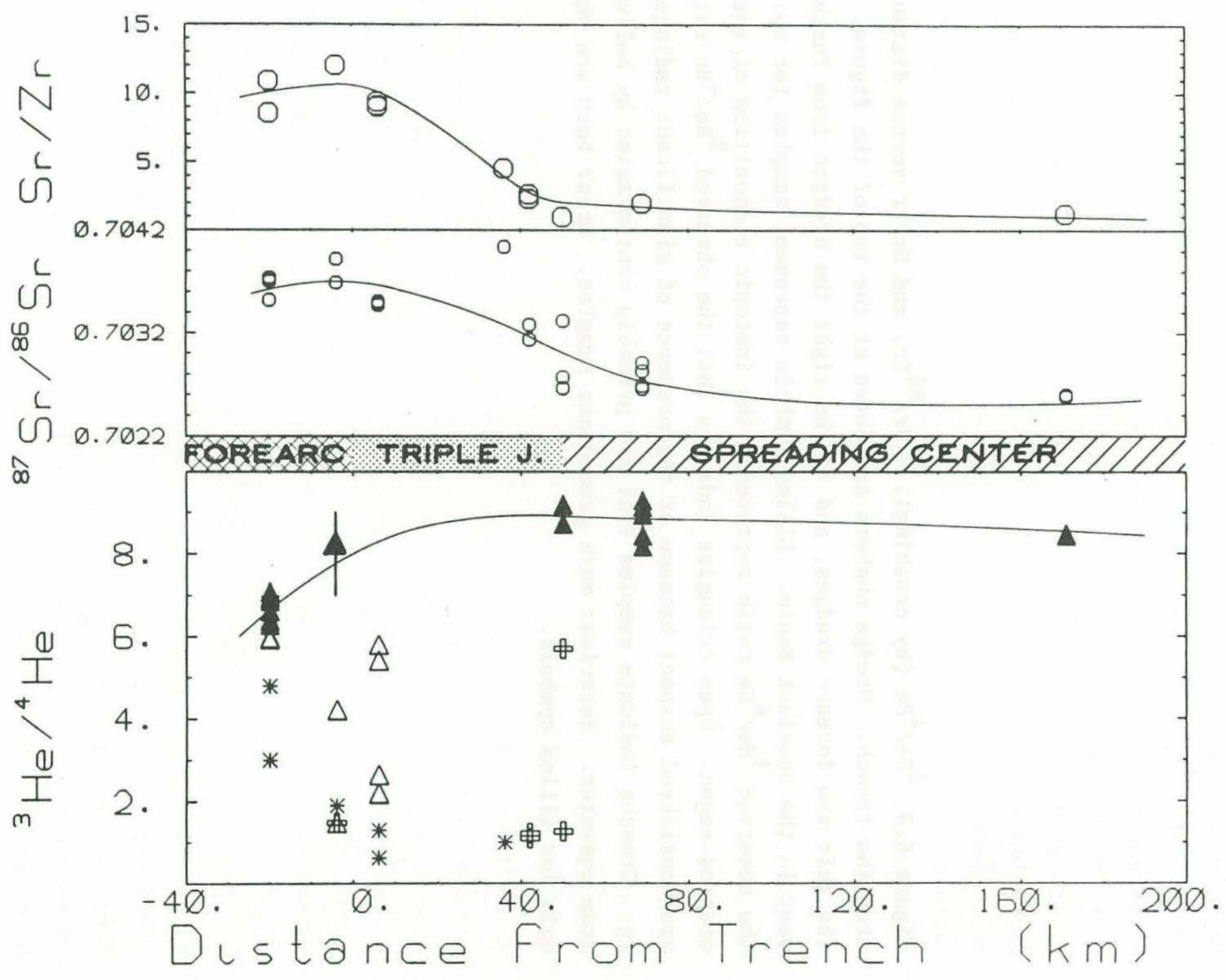


spreading ridge environments. Degassing may lead to lowered ${ }^{3} \mathrm{He} /{ }^{4} \mathrm{He}$ ratios in two ways. First, if He is lost by diffusion, mass fractionation may occur. Limits on this effect can be obtained from a Rayleigh fractionation model (e.g. Kaneoka, 1980; Condomines et al, 1983). Isotopic diffusion rates have been demonstrated to be roughly inversely proportional to the square root of the isotopic masses for neon (Frank, Swets, and Lee, 1961; Rama and Hart, 1965) and helium in silica glasses (Shelby, 1971). This $15 \%$ difference for ${ }^{3} \mathrm{He}$ vs. ${ }^{4} \mathrm{He}$ diffusion is probably a maximum for basaltic magma (Trull, 1988). Using this model, 90\% helium loss is required to reduce a magma's initial ratio by $25 \%$ (Hart, 1984). This corresponds to reduction of a parental magma composition of $9.2 \mathrm{R}_{\mathrm{a}}$, the high value in WSC basalts, to $6.9 \mathrm{R}_{\mathrm{a}}$, the Kavachi Volcano average.

How long would this take? Lux (1987) has measured the diffusion coefficient for helium in tholeitic melt at $1350{ }^{0} \mathrm{C}$ to be $5 \times 10^{-5}$ $\mathrm{cm}^{2} / \mathrm{s}$, in good agreement with extrapolation of glass data measured at lower temperatures (Kurz and Jenkins, 1981). Combining this diffusion coefficient with the diffusive loss equation for spherical bodies (Crank, 1975) yields estimates of longer than 150,000 years for a $1 \mathrm{~km}$ diameter magma body, or 50,000 years for a $.5 \mathrm{~km}$ body, to achieve such large helium losses. Other magma geometries or magma convection will reduce these times, however boundary processes, such as helium loss to surrounding rock, may be rate limiting. Exhalative degassing and/or magma solidification probably occur on much shorter timescales. Phase separation of $\mathrm{CO}_{2}$ or $\mathrm{H}_{2} \mathrm{O}$ fractionates helium isotopes only minimally, if at all, at least in MORB magmas where isotopic equilibrium is observed between helium in vesicles and dissolved in glass (Kurz and Jenkins, 1981).

The second effect of degassing is increased $\mathrm{U}, \mathrm{Th} / \mathrm{He}$ elemental ratios, allowing radiogenic helium to lower a magma's ${ }^{3} \mathrm{He} /{ }^{4} \mathrm{He}$ ratio over relatively "short" times (Condomines et al, 1983; Zindler and Hart, 1986). Using the helium content of Kavachi phenocrysts ( $\left.1 \times 10^{-8} \mathrm{cc} / \mathrm{g}\right)$ and measured whole rock uranium contents $(0.08 \mathrm{ppm}$, Johnson et al, 1987) as representative of magmatic concentrations to calculate radiogenic helium production suggests that greater than $10^{5}$ years are required to 
lower a parental magmatic ${ }^{3} \mathrm{He} /{ }^{4} \mathrm{He}$ ratio from $9.2 \mathrm{R}_{\mathrm{a}}$ to $6.9 \mathrm{R}_{\mathrm{a}}$. Since glass/phenocryst partition coefficients for He are much less than 1 (Hiyagon and Ozima, 1986; Kurz, 1982), this is undoubtably a minimum estimate.

In summary, if lower ${ }^{3} \mathrm{He} /{ }^{4} \mathrm{He}$ ratios in island arc magmas reflect differing degassing processes in arcs as compared to spreading ridge environments, then magma residence timescales must be quite long (approximately $10^{5}$ yrs). Thermal models show that such long times require low temperature contrasts, as might characterize magma chambers at mantle depths or replenished chambers (compare Zindler and Hart, 1986; Graham et al, 1987; Usselman and Hodge, 1978). It is possible that these processes could lead to temporal trends in the helium isotopic compositions of Kavachi lavas in the future. Changes in ${ }^{3} \mathrm{He} /{ }^{4} \mathrm{He}$ ratios with time have been observed at single volcanos in Hawaii, and appear to be related to plate motion across a mantle plume (Kurz et al, 1983).

\subsubsection{Sources of radiogenic Sr and He to Woodlark Basin - Solomon}

Islands magmas.

Addition of ${ }^{4} \mathrm{He}$ to arc magmas or their source regions could also be responsible for the lower ${ }^{3} \mathrm{He} /{ }^{4} \mathrm{He}$ ratios observed in many arc gas samples and most clearly in the rock samples from Kavachi volcano. Possible radiogenic helium sources include subducted materials (seawater, sediments, oceanic crust and lithosphere) as well as older arc basement rocks. The helium content of these materials is poorly known, but is essentially governed by age, uranium and thorium content, and the extent of radiogenic helium retention (Kurz, 1982).

We have investigated the role of these potential radiogenic He sources in the petrogenesis of Kavachi volcano lavas through ${ }^{3} \mathrm{He} /{ }^{4} \mathrm{He}-$ ${ }^{87} \mathrm{Sr} /{ }^{86} \mathrm{Sr}$ systematics. The correlation observed between increasing ${ }^{87} \mathrm{Sr} /{ }^{86} \mathrm{Sr}$ and LIL elemental abundances in rocks from the Solomon islands and the Woodlark Basin suggests mixing between long-term depleted mantle sources like those beneath the Woodlark Basin and some LIL enriched source or contaminant. This hypothesis is further supported by good 
correlation between $\mathrm{Sr}, \mathrm{Nd}$, and $\mathrm{Pb}$ isotopic compositions (Staudigel et al, 1987; Perfit et al, in preparation). However, the nature of this enriched component is most problematical and elusive to determine. Petrogenetic mixing calculations (see Staudigel et al, 1987) point out the difficulties in producing the observed isotope and elemental ratios by simple binary mixing of a MORB source with sediments. These results indicate that although minor amounts of sediment involvement are possible, multiple components and more complex processes (e.g. metasomatism) must be involved in island arc magmagenesis.

If the Kavachi magmas are produced by mixing of WSC type source materials with a more radiogenic "contaminant", then their He-Sr compositions should lie along mixing lines between these isotopic endmembers. Figure 6.10 shows that such an origin requires that the contaminant have a lower $\mathrm{He} / \mathrm{Sr}$ elemental ratio ( $\mathrm{R}$ less than 1 ) than the WSC endmember, at least for closed system mixing with materials such as evolved arc rocks or altered oceanic crust of 10 to $100 \mathrm{Ma}$ age. Melts, fluids, and solid materials from old oceanic crust probably all fulfill this requirement because of the comparatively high He contents of the WSC rocks and their MORB-type source. It is difficult to compare these possible contaminants more quantitatively without experimental study of fluids and melts of altered crust for $\mathrm{He}$ and $\mathrm{Sr}$ contents. This would be in keeping with contamination in the form of solids or melts, but perhaps not by $\mathrm{C}-\mathrm{O}-\mathrm{H}$ fluids which might have relatively high $\mathrm{He} / \mathrm{Sr}$ ratios. Also shown are mixing lines $(R=1)$ for contamination by seawater or an average sediment composition. Contamination by seawater or sediments is unlikely because this would require large contributions that are not consistent with other trace element and isotopic compositions (Perfit et al, 1987; Staudigel et al, 1987).

For any of the endmembers, mixing requires that large fractions of the $\mathrm{Sr}$ be contributed by the contaminant. This suggests that contamination probably occurs in the source region, where addition of small amounts of a melt or fluid derived from subducted materials can produce large changes in incompatible element compositions of subsequently derived magmas, rather than by contamination of magmas at crustal depths, which would require much larger bulk contamination. 
Figure $6.10{ }^{3} \mathrm{He} /{ }^{4} \mathrm{He}$ versus ${ }^{87} \mathrm{Sr} /{ }^{86} \mathrm{Sr}$ for Woodlark Basin samples. Only those samples for which magmatic ${ }^{3} \mathrm{He} /{ }^{4} \mathrm{He}$ ratios were reliably determined are shown. Fields for normal and enriched MORB are from Kurz, Jenkins, and Hart (1982). Two component mixing curves are shown between WSC basalts $\left({ }^{87} \mathrm{Sr} /{ }^{86} \mathrm{Sr}=.7027,{ }^{3} \mathrm{He} /{ }^{4} \mathrm{He}=8.8\right)$ and young arc rocks (YAR: ${ }^{87} \mathrm{Sr} /{ }^{86} \mathrm{Sr}=.7045,{ }^{3} \mathrm{He} /{ }^{4} \mathrm{He}=3 \mathrm{R}$ ). $\mathrm{R}$ values on these curves represent the ratio $(\mathrm{He} / \mathrm{Sr})^{\mathrm{YAR}} /(\mathrm{He} / \mathrm{Sr})^{\text {WSC }}$. Tick marks represent the fraction of Sr contributed to the mix by the 'contaminant'. Other possible contaminants are marked: $O A R=$ old arc rocks, YOC = young oceanic crust, $0 O C=$ old oceanic crust. Also shown are $\mathrm{R}=1$ mixing lines between WSC and seawater and sediments $\left({ }^{87} \mathrm{Sr} /{ }^{86} \mathrm{Sr}=.7065,{ }^{3} \mathrm{He} /{ }^{4} \mathrm{He}=3.0\right)$. Symbols: open circles - WSC, (dredges 26, 29 and NaTi basalts from dredge 32), filled squares - Kavachi volcano (dredges 34 and 35), open triangle - forearc sample 24-4. 


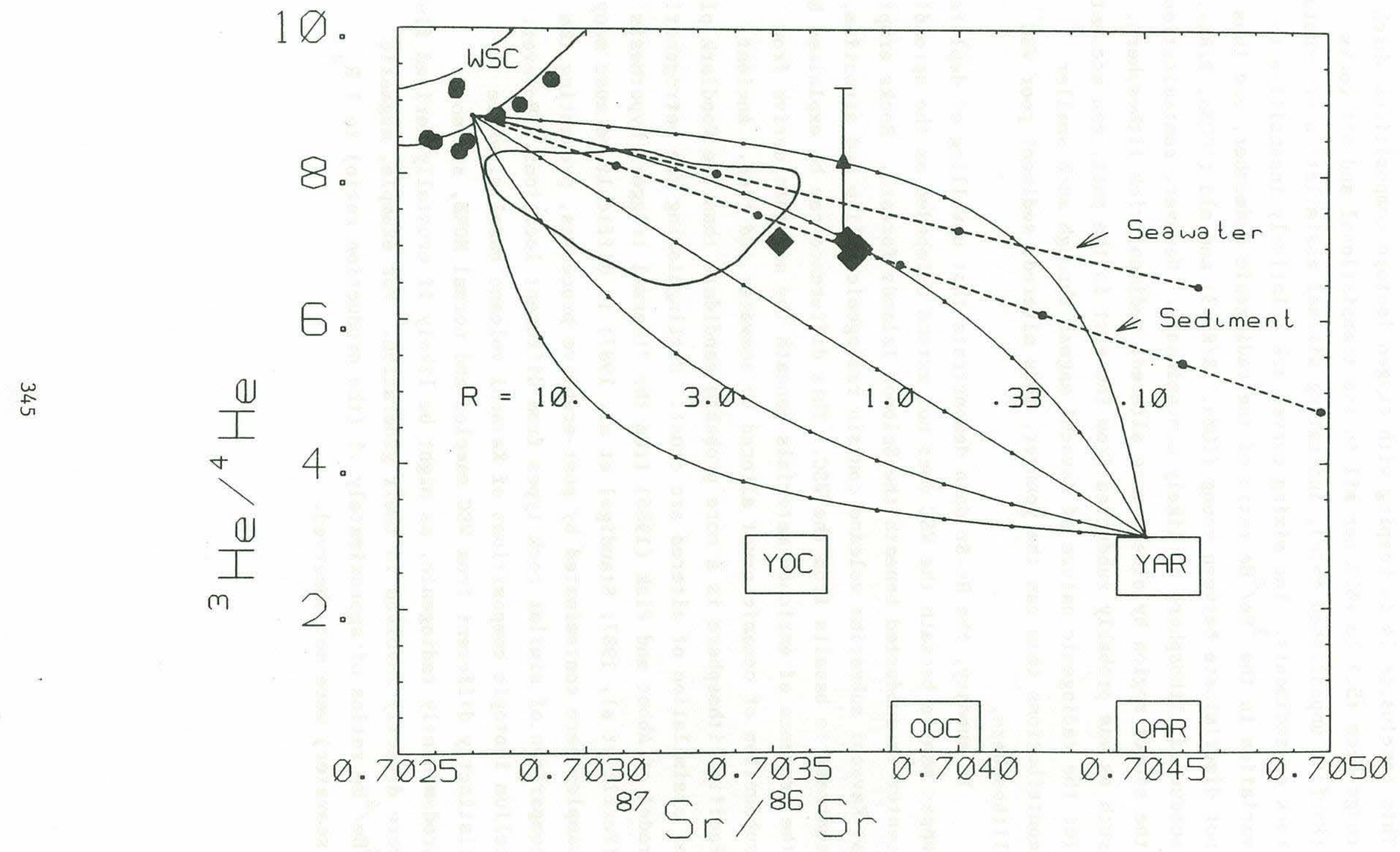


This conclusion is in keeping with oxygen isotope compositions which range from +5.7 to +6.3 per mil in the transistional and arc rocks (Perfit, unpublished data), indicating minimal assimilation of crustal rock or sediments. The mixing curves are relatively insensitive to variation in the ${ }^{3} \mathrm{He} /{ }^{4} \mathrm{He}$ ratio of the radiogenic endmember, and thus do not discriminate between young (10Ma, $\mathrm{R} / \mathrm{Ra}=3$ ) and old (100Ma, $\mathrm{R} / \mathrm{Ra}=.2$ ) subducted lithosphere as likely contaminants. However, contamination of the source region by older, more altered, sediment rich lithosphere, such as was probably subducted from the east in the past, can account for the radiogenic nature of Kavachi magmas through much smaller contributions than can the younger, less altered, sediment poor WSC lithosphere.

In summary, the He-Sr data demonstrate that upwelling of depleted upper mantle beneath the WSC does not extend unimpeded as the spreading center is subducted beneath the Solomon Islands forearc. Rocks erupted at Kavachi submarine volcano contain radiogenic helium and strontium, in contrast to basalts from the WSC. This difference can be explained by the presence of enriched materials beneath the arc that derive from subduction of oceanic crust altered by seawater and time. Ancient Pacific lithosphere is a more probable candidate than the Woodlark plate or assimilation of altered arc crust. Distinguishing the petrogenetic model of Abbot and Fisk (1986) from the "thermal trigger" hypothesis (Perfit et al, 1987; Staudigel et al, 1987) is difficult because many samples were contaminated by post-eruptive processes, preventing the comparison of similar rock types from different locations. However, the helium isotopic compositions of Kavachi volcano basalts, while distinctly different from WSC samples and normal MORB, are not predominantly radiogenic, as might be likely if crustally-derived fluids were directly involved in their generation. For example, magmatic ${ }^{3} \mathrm{He} /{ }^{4} \mathrm{He}$ ratios of approximately .1 (the production ratio) to $1 \mathrm{R}_{\mathrm{a}}$ (seawater) were not observed. 


\subsection{CONCLUSIONS}

In conclusion, three points should be emphasized.

1. Extension of helium isotopic analyses to island arc environments requires careful examination of contamination processes. Groundmass samples and vesicular glasses with low helium contents are particularly susceptible to diffusive and radiogenic contamination, and phenocrysts cannot necessarily be relied upon to yield magmatic ${ }^{3} \mathrm{He} /{ }^{4} \mathrm{He}$ ratios without detailed study involving gas extraction by in vacuo crushing and melting.

2. The Woodlark Spreading Center erupts lavas with helium and strontium isotopic compositions typical of mid-ocean-ridges, despite its location in a region marked by extremely high returns of crustal materials to the mantle (Parsons, 1981). This suggests vigorous mantle convection, or that the WSC basalts derive from a mantle diapir originating at depths unaffected by these lithospheric inputs.

3. Island arc magmas, as represented by Kavachi submarine volcano samples, were confirmed to exhibit ${ }^{3} \mathrm{He} /{ }^{4} \mathrm{He}$ ratios significantly lower than most basalts from spreading centers and mid-ocean-ridges. This solidifies the results of island arc fumarole and hot-spring studies, and suggests that the radiogenic helium (and strontium) contributions derive from sub-crustal sources related to subduction. The He and $\mathrm{Sr}$ isotopic compositions, however, are not predominantly radiogenic and probably derive in large part from upper mantle materials similar to those beneath ocean ridges. Involvement of a fluid phase distinct from silicate melt in their formation may occur in the source region, but does not appear to be directly related to magmagenesis.

\section{Acknowledgements}

We thank D. E. Lott III, R. Shuster, and M.K. Smith for analytical assistance, and C. Langmuir and R.W. Johnson for providing samples and for their scientific input. This research was supported by the National Science Foundation (OCE 83-15725 to MRP; OCE 85-16082 and OCE 87-16970 to MDK). TWT thanks the WHOI Education Office for support, and Dave Graham for advice and encouragement. WHOI contribution \#6720. 


\section{REFERENCES}

ABBOT D. and FISK M. (1986) Tectonically controlled origin of three unusual rock suites in the Woodlark Basin. Tectonics $\underline{5}, 1145-1160$. CAMERON W. E., McCULLOCH M. T. and WALKER D. A. (1983) Boninite petrogenesis: chemical and $\mathrm{Nd}-\mathrm{Sr}$ isotopic constraints. Earth Planet. Sci. Lett. 65, 75-89. CONDOMINES M., GRNVOLD K., HOOKER P. J., MUEHLENBACK K., O'NIONS R. K., 0'SKARSON N. and OXBURGH E. R. (1983) Helium, oxygen, strontium and neodymium isotopic relationships in Icelandic volcanics. Earth Planet. Sci. Lett. 66, 125-136.

COOPER P. A. and TAYLOR B. (1985) Polarity reversal in the Solomon Islands arc. Nature $314,428-430$.

COX K. G. and BELL J. D. (1972) A crystal fractionation model for the basaltic rocks of the New Georgia Group, British Solomon Islands. Contrib. Mineral. Petrol. 37, 1-13.

CRAIG H., LUPTON J. W. and HORIBE Y. (1978) A mantle helium component in circum-Pacific volcanic glasses: Hakone, Marianas, Mt. Lassen. In

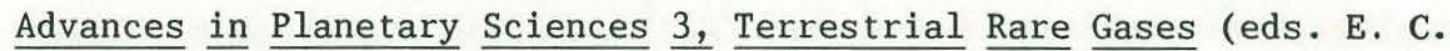
ALEXANDER and M. OZIMA) pp. 3-16. Centre for Academic Publications, Japan Scientific Press, Tokyo,

CRANK J. (1975) The Mathematics of Diffusion, $2^{\text {nd }}$ edition, 0xford Press. DAVIDSON J. P. (1987) Crustal contamination versus subduction zone enrichment: Examples from the Lesser Antilles and implications for mantle source compositions of island arc rocks. Geochim. Cosmochim. Acta 51 , 2185-2198.

DePAOLO D. and JOHNSON R. W. (1979) Magma genesis in the New Britain island-arc: constraints from $\mathrm{Nd}$ and $\mathrm{Sr}$ isotopes and trace element patterns. Contrib. Mineral. Petrol. 70, 367-379.

DUNKLEY P. N. (1984) Volcanism and evolution of the ensimatic Solomon Islands Arc. In Arc Volcanism: Physics and Tectonics, proceedings of a 1981 IAVCEI symposium, Tokyo and Hakone (eds. D. SHIMOZURU and I. YOKOYAMA) Advances in Earth and Planetary Sciences, TERRAPUB/REIDEL. 
FLYNN R. T. and BURNHAM C. W. (1978) An experimental determination of rare earth partition coefficients between a chloride containing vapor phase and silicate melts. Geochim. Cosmochim. Acta 42, 685702.

FRANK R. C., SWETS D. E. and LEE R. W. (1961) Diffusion of neon isotopes in fused quartz. J. Chem. Phys. 35, 1451-1459.

FUNKHOUSER J. G., FISHER D. E. and BONATTI E. (1968) Excess argon in deep sea rocks. Earth Planet. Sci. Lett. ㄱ, 95-100.

GILL J. B. (1981) Orogenic Andesites and Plate Tectonics, Minerals and Rocks series \#16, Springer Verlag.

GILL J. B. (1984) Sr-Pb-Nd isotopic evidence that both MORB and OIB sources contribute to oceanic island arc magmas in Fiji. Earth Planet. Sci. Lett. 68, 443-458.

GRAHAM D., JENKINS W. J., KURZ M. D. and BATIZA R. (1987) Helium isotope disequilibrium and geochronology of glassy submarine basalts. Nature $326,384-386$.

GRAMLICH J. W. and NAUGHTON J. J. (1972) Nature of source material for ultra-mafic minerals from Salt Lake Crater, Hawaii, from measurement of helium and argon diffusion. J. Geophys. Res. $\underline{77}$, 3032-3042.

HART S. R. (1984) He diffusion in olivine. Earth Planet. Sci. Lett. 70, 297-302.

HIYAGON and OZIMA (1986) Partition of noble gases between olivine and basalt melt. Geochim. Cosmochim. Acta 50, 2045-57.

JAMBON A., WEBER H. W. and BEGEMANN F. (1985) Helium and argon from an Atlantic MORB glass: concentration, distribution and isotopic composition. Earth Planet. Sci. Lett. 73, 255-267.

JOHNSON R. W., JACQUES A. L., LANGMUIR C. H., PERFIT M. R., STAUDIGEL H., DUNKLEY P. N., CHAPPEL B. W., TAYLOR S. R and BAEKISAPA M. (1987) Ridge subduction and forearc volcanism: petrology and geochemistry of rocks dredged from the western Solomon Arc and Woodlark Basin. In Circum-Pacific Council for Energy and Mineral

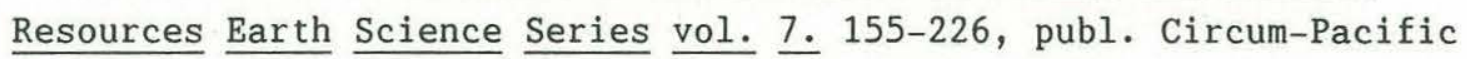
Council for Energy and Mineral Resources, Houston, Texas. 
JOHNSON R. W., JAQUES A. L., HICKEY R. L., MCKEE C. 0. and CHAPPELL B.

W. (1985) Manum Island, Papua New Guinea: petrology and geochemistry of a low-TiO 2 basaltic island-arc volcano. J. Petrol. 26, 283-323.

KANEOKA I. (1980) Rare gas isotopes and mass fractionation: an indicator of gas transport into or from a magma. Earth Planet. Sci. Lett. 48, 284-292.

KAY R. W. (1980) Volcanic arc magmas: Implications of a melting-mixing model for element recycling in the crust-upper mantle system. J. Geol. 88, 497-522.

KURZ M. D. (1982) Helium isotope geochemistry of oceanic volcanic rocks implications for mantle heterogeneity and degassing, Ph.D. dissertation, WHOI/MIT Joint Program in Oceanography.

KURZ M. D. (1986a) Cosmogenic helium in a terrestrial igneous rock. Nature $320,435-439$.

KURZ M. D. (1986b) In situ production of terrestrial cosmogenic helium and some applications to geochronology. Geochim. Cosmochim. Acta 50, 2855- 2862 .

KURZ M. D. and JENKINS W. J. Jenkins (1981) The distribution of helium in oceanic basalt glasses. Earth Planet. Sci. Lett. 53, 41-54.

KURZ M. D., JENKINS W. J. and HART S. R. (1982) Helium isotopic systematics of oceanic islands and mantle hererogeneity. Nature 29, 43-47.

KURZ M. D., JENKINS W. J., SCHILLING J. G. and HART S. R. (1982) Helium isotopic variations in the mantle beneath the central North

Atlantic 0cean. Earth Planet. Sci. Lett. 58, 1-14.

KURZ M. D., JENKINS W. J., HART S. R. and CLAGUE D. (1983) Helium isotopic variations in volcanic rocks from Loihi Seamount and the island of Hawaii. Earth Planet. Sci. Lett. 66, 388-406.

KURZ M. D., GURNEY J. J., JENKINS W. J. and LOTT III D. E. (1987) Helium isotopic variability within single diamonds from the 0rapa kimberlite pipe. Earth Planet. Sci. Lett. 86, 57-68. 
KUSHIRO I. (1975) On the nature of silicate melt and its significance in magma genesis: regularities in the shift of the liquidus boundaries involving olivine, pyroxene, and silica minerals. Amer. Jour. Sci. 275, 411-431.

LUX G (1987) The behavior of noble gases in silicate liquids: solution, diffusion, and surface effects, with applications to natural samples. Geochim. Cosmochim. Acta 51, 1549-1560.

MORRIS J. D. and HART S. R. (1983) Isotopic and incompatible element constraints on the genesis of island arc volcanics from Cold Bay and Amok Islands, Aleutians, and implications for mantle structure. Geochim. Cosmochim. Acta 47, 2015-2030.

PARSONS B. (1981) The rates of plate creation and consumption. Geophys. J. R. astr. Soc. 67, 437-448.

PERFIT M., LANGMUIR C. M., BAEKISAPA M., CHAPPELL B., JOHNSON R. W., STAUDIGEL H. and TAYLOR S. R. (1987) Geochemistry and Petrology of rocks fron the Woodlark Basin: addressing questions of ridge subduction. In Circum-Pacific Council for Energy and Mineral

Resources Earth Science Series vol. 7. 113-154, publ. Circum-Pacific Council for Energy and Mineral Resources, Houston, Texas.

PERFIT M. R., GUST D. A., BENCE A. E., ARCULUS R. T. and TAYLOR S. R. (1980) Chemical characteristics of island-arc basalts: implications for mantle sources. Chem. Geol. 30, 227-256.

PERFIT M. R., McCULLOCH M. T. and JOHNSON R. W. (1982) Isotopic and trace element differences in late Cenozoic volcanic rocks from west Melanesia. Geol. Soc. Amer. Abs. 14, 586.

POLVE M. and KURZ M. D. (1988) ${ }^{3} /^{4} \mathrm{He}$ systematics in spinel-lherzolite nodules, in preparation.

POLVE M. (1985) Fission track mapping of $\mathrm{U}$ in alkali basalts: Implications for U behavior during crystallization. E0S 66, 404. POREDA R. (1983) Helium, neon, water, and carbon in volcanic rocks and gases. Ph.D. dissertation, Scripps Institution of 0ceanography POREDA R. (1985) Helium-3 and deuterium in back-arc basalts: Lau basin and the Mariana Trough. Earth Planet. Sci. Lett. 73, 244-254. 
RAMA S. N. I. and HART S. R. (1965) Neon isotope fractionation during transient permeation. Science 147, 737-738.

RAMSAY W. R. H., CRAWFORD A. J. and FODEN J. D. (1984) Field setting, mineralogy, chemistry, and genesis of arc picrites, New Georgia,

Solomon Islands. Contrib. Mineral. Petrol. 88, 386-402.

RINGWOOD A. E. (1974) The petrological evolution of island arc systems.

J. Geol. Soc. Lond. 130, 183-204.

SANO Y. and WAKITA H. (1985) Geographical distribution of ${ }^{3} \mathrm{He} /{ }^{4} \mathrm{He}$ ratios in Japan: implications for arc tectonics and incipient magmatism.

J. Geophys. Res. 90, 8729-8741.

SANO Y., WAKITA H. and GIGGENBACH W. F. (1987) Island arc tectonics of New Zealand manifested in helium isotope ratios. Geochim.

Cosmochim. Acta 51, 1855-1860.

SHELBY J. E (1971) Diffusion of helium isotopes in vitreous silica. Phys. Rev.B. 4, 2681-2686.

STAUdigel H., McCULLOCH M., ZINDLER A. and PERFIT M.R. (1987) Complex ridge subduction and island arc magmatism: an isotopic study of the New Georgia forearc and the Woodlark Basin. In Circum-Pacific

Council for Energy and Mineral Resources Earth Science Series vol. 7. 227-240, publ. Circum-Pacific Council for Energy and Mineral Resources, Houston, Texas.

TAYLOR B. (1987) Geophysical survey of the Woodlark-Solomon region. In Circum-Pacific Council for Energy and Mineral Resources Earth Science Series vol. 7. 25-48, publ. Circum-Pacific Council for Energy and Mineral Resources, Houston, Texas.

TRULL T. W. (1988) Diffusion of helium isotopes in silicate glasses and minerals: Implications for igneous petrogenesis and geochronolgy. Ph.D. dissertation, WHOI/MIT Joint Program in Oceanography, in preparation. 
USSELMAN T. M. and HODGE D. S. (1978) Thermal control of low pressurefractionation processes. J. Volc. Geotherm. Res. 4, 265281.

WYLLIE P. (1982) Subduction products according to experimental prediction. Geol. Soc. Am. Bull. 93, 468-476.

ZINDLER and HART (1986) Helium: problematic primordial signals. Earth Planet. Sci. Lett. 79, 1-8. 
APPENDIX A: Fortran77 programs for calculating diffusion coefficients from helium emanation data using the refined model presented in chapter 2 . 


\section{Main program:}

\section{DIFPRO}

Calculates fractional release data from aliquot measurements. The errors in $\mathrm{F}$ were derived as follows, where $\mathrm{x}$ and $\mathrm{s}$ are aliquot helium contents and associated errors and $\mathrm{N}$ is the number of aliquots:

$$
F_{i}=\sum_{1}^{i} x_{i} \pm s_{i} / \sum_{1}^{N} x_{i} \pm s_{i}
$$

which when differentiated with respect to $x_{i}$, gives for the variance in

$$
F: \quad\left(1 / \sum_{1}^{N} x_{i}\right)^{4}\left[\left(\sum_{i+1}^{N} x_{i}\right)^{2} \sum_{1}^{i}\left(s_{i}\right)^{2}+\left(\sum_{1}^{i} x_{i}\right)^{2} \sum_{i+1}^{N}\left(s_{i}\right)^{2}\right]
$$

\section{Subroutines:}

DIFPRO calls SPHAPRX to obtain diffusion coefficient estimates from the spherical approximation equations $(2.9 a, b, c)$, which are then refined by calling the subroutine DADJST.

DADJST :

Calculates $\mathrm{D}_{i}$ and $\mathrm{D}_{\mathrm{i}-1}$ by successively calling subroutine DPIPED, calculates $\mathrm{D}_{\Delta \mathrm{i}}$ from equation 2.15 .

DPIPED:

Calculates D by iterative calls to FPIPED. D is first bracketed by a factor of 10 , and then the desired precision is obtained by bisection of the bounded interval.

FPIPED:

Calculates the fractional release from a collection of pipeds using equation 2.11, given a diffusion coefficient, elapsed time and grain size parameters. 
PROGRAM DIFPRO

Tom Trull July 8, 1988

modified from DIFNEW. for for improved $\mathrm{I} / 0$ and to allow the

use of measured grain size distributions in calculating

diffusion coefficients.

Purpose:

Calculates diffusion coefficients from gas release data

Requires:

Subroutines: SPAPRX (contains SPH1, SPH3, SPH3)

DADJST, DPIPED, FPIPED

Input Parameters:

V4 - CC 4He measured in aliquot

V4B - corrected form daily blank

sV4B - 1 sigma error

RAT - 3He/4He ratio normalized to atmospheric ratio (RA)

SRAT - 1 sigma error estimated in program HEMAN5. for

DIFTIM - time ( $s$ from noon) at which the aliquotting valve closed.

TEMPC - temperature of gas release during the aliquot.

SAMNAM - sample name

NANAL, IHR, IMIN - number and time of analysis.

grainR - radius of equivalent sphere characterizing grain size

sigmaR - 1 sigma

Internal Parameters: ( $x$ is either 3 or 4 )

ET - elapsed time in step $i$

TET - total elapsed time to and through step $i$

V3B - volume of $3 \mathrm{He}$ in aliquot, calculated from ratio and $4 \mathrm{He}$

sV3B - 1 sigma

VxL - gas volume corrected for background leak

sVxL - 1 sigma on leak corrected gas volume

VARx - variance of gas volume $=s V x l * \star 2$

RATL - leak corrected ratio

SRATL - 1 sigma

SUMx - total of gas release through aliquot $i$

TVARx - variance on Sumx

RTOT - ratio of total $3 \mathrm{He}$ to total $4 \mathrm{He}$

SRTOT - 1 sigma

FRACx - fraction of gas released in aliquot $i$

TFRACx - fraction of gas in all steps up to and through $i$

VTFRCx - variance on Tfracx (CAUTION: VTFRCx(I) are not independent!)

rDSx - diffusion coefficient from spherical approximations ( $1 / \mathrm{s})$

rsDSx - 1 sigma

DSx - spherical D converted to $\mathrm{cm} 2 / \mathrm{s}$ units

sDSx - 1 sigma from fractional loss error only

TsDSx - 1 sigma error including equivalent sphere size uncertainty

DPx - Refined D value using size distribution of parallelpipeds.

SDPx - 1 sigma

QS - isotopic diffusivity ratio (DS3/DS4)

SQS - 1 sigma

QP - isotopic diffusivity ratio (DP3/DP4)

SQP - 1 sigma

sTEMPC - error in heating temp - specified file by file

RTEMPK - reciprocal kelvin temperature

sRTMPK - 1 sigma

Note: the $\log$ (base 10 ) of a variable is denoted by the suffix L or Log.

I/0 file names: 


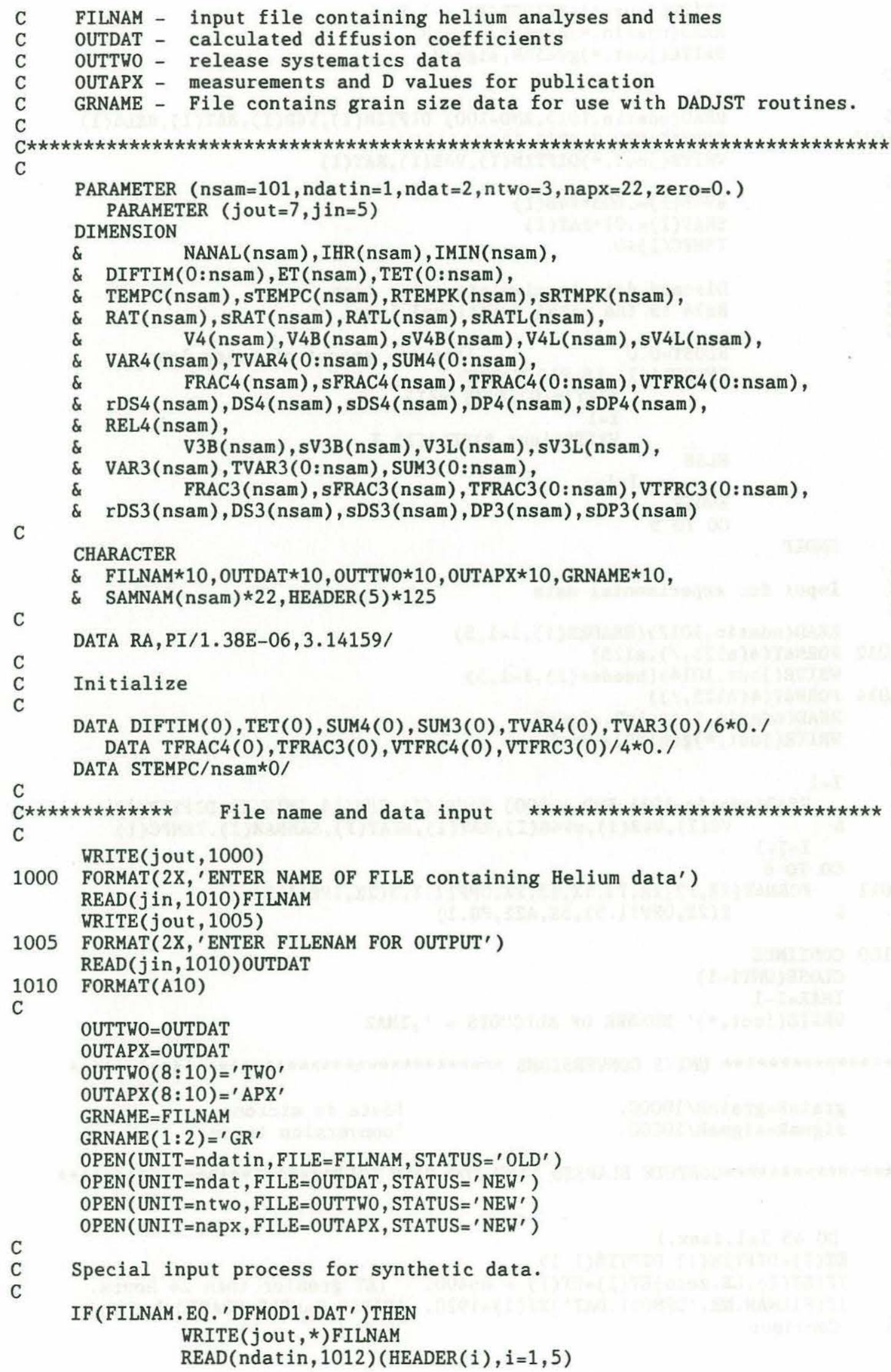




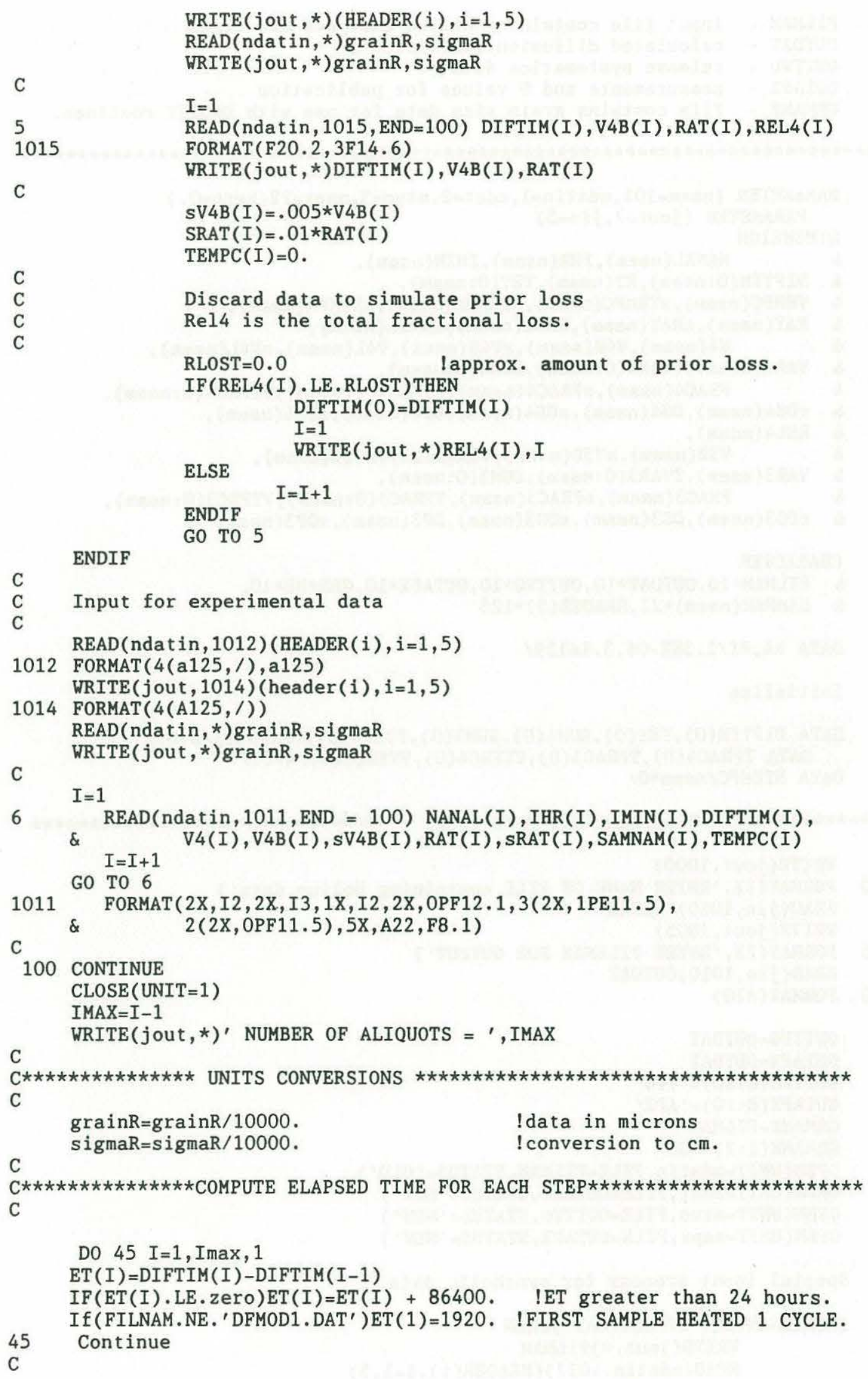


C

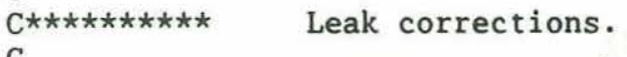

!leak rate in $\mathrm{cc} / \mathrm{s}$

Rleak $4=8 \cdot 3 \mathrm{E}-16$

Rleak3=leak $4 * \mathrm{Ra}$

latmospheric leak

C

IF (FILNAM.EQ. 'DFMOD1.DAT') THEN

RLEAK4=ZER0

ENDIF

RLEAK3=ZER0

C

DO $55 \mathrm{I}=1$, IMAX, 1

$\operatorname{V3} B(I)=\operatorname{RAT}(I) * V 4 B(I) * R A$

$\operatorname{sV} 3 B(I)=(\operatorname{sRAT}(I) * V 4 B(I) \star R A) * \star 2+(\operatorname{RAT}(I) * \operatorname{sV} 4 B(I) * R A) * \star 2 \quad$ !variance $3 \mathrm{He}$

$\operatorname{sV} 3 B(I)=\operatorname{SV} 3 B(I) * \star .5$

$\mathrm{V} 4 \mathrm{~L}(\mathrm{I})=\mathrm{V} 4 \mathrm{~B}(\mathrm{I})-\mathrm{ET}(\mathrm{I}) * \mathrm{Rleak} 4$

$\mathrm{V} 3 \mathrm{~L}(\mathrm{I})=\mathrm{V} 3 \mathrm{~B}(\mathrm{I})-\mathrm{ET}(\mathrm{I}) * \mathrm{Rleak} 3$

$\operatorname{sV} 4 \mathrm{~L}(\mathrm{I})=\mathrm{SV} 4 \mathrm{~B}(\mathrm{I})$

$\operatorname{sV} 3 L(I)=\operatorname{sV} 3 B(I)$

$\operatorname{SRATL}(I)=\operatorname{SRAT}(I)$

IF (V4L(I).LE.zero)THEN

$\mathrm{V} 4 \mathrm{~L}(\mathrm{I})=0$.

ENDIF

$\operatorname{RATL}(\mathrm{I})=0$

IF (V3L(I).LE. zero)THEN

$\mathrm{V} 3 \mathrm{~L}(\mathrm{I})=0$.

ENDIF

$\operatorname{RATL}(I)=0$

$\operatorname{IF}(V 4 L(I)$. GT . zero) RATL (I) $=$ V3L (I) /V4L(I)/RA

55 CONTINUE

$\mathrm{C}$

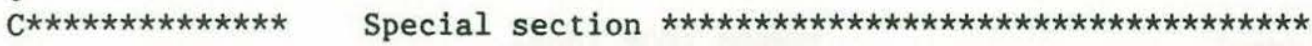

$\mathrm{C}$

C Powder release systematics can be back-corrected to the time of

C sample crushing (rather than the start of analysis) to examine

C the time dependence of loss.

C

C

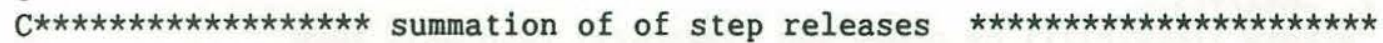

$\mathrm{C}$

IF (FILNAM.EQ.' TV0525. DAT' )THEN write (jout, *)filnam

ENDIF $\operatorname{TET}(0)=1036800$.

!time since crushing

IF(FILNAM.EQ.' TV0715. DAT')THEN write (jout, *)filnam $\operatorname{TET}(0)=4752000$.

!Time since crushing

ENDIF

D0 $65, I=1$, IMAX, 1

$\operatorname{TET}(I)=\operatorname{TET}(\mathrm{I}-1)+\operatorname{ET}(\mathrm{I})$

$\operatorname{SUM} 4(I)=\operatorname{SUM} 4(I-1)+V 4 L(I)$

$\operatorname{SUM} 3(I)=\operatorname{SUM} 3(I-1)+V 3 L(I)$

$\operatorname{VAR} 4(I)=\operatorname{sV} 4 L(I) \star \star 2$

$\operatorname{TVAR} 4(I)=\operatorname{TVAR} 4(I-1)+\operatorname{VAR} 4$ (I)

$\operatorname{VAR} 3(I)=\operatorname{sV} 3 \mathrm{~L}(I) \star * 2$

$\operatorname{TVAR3}(I)=\operatorname{TVAR} 3(I-1)+\operatorname{VAR} 3(I)$

65 CONTINUE

C

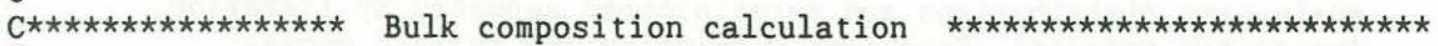

C

RTOT=SUM3 ( IMAX)/SUM4 (IMAX)/RA

SRTOT $=1 . / R A *($ TVAR3 (IMAX) / SUM 4 (IMAX) $* * 2$

\& $\quad$ TVAR4 (IMAX) $)(\operatorname{SUM} 3($ IMAX) $/ \operatorname{SUM} 4(\operatorname{IMAX}) * * 2) * * 2) * * .5$ 


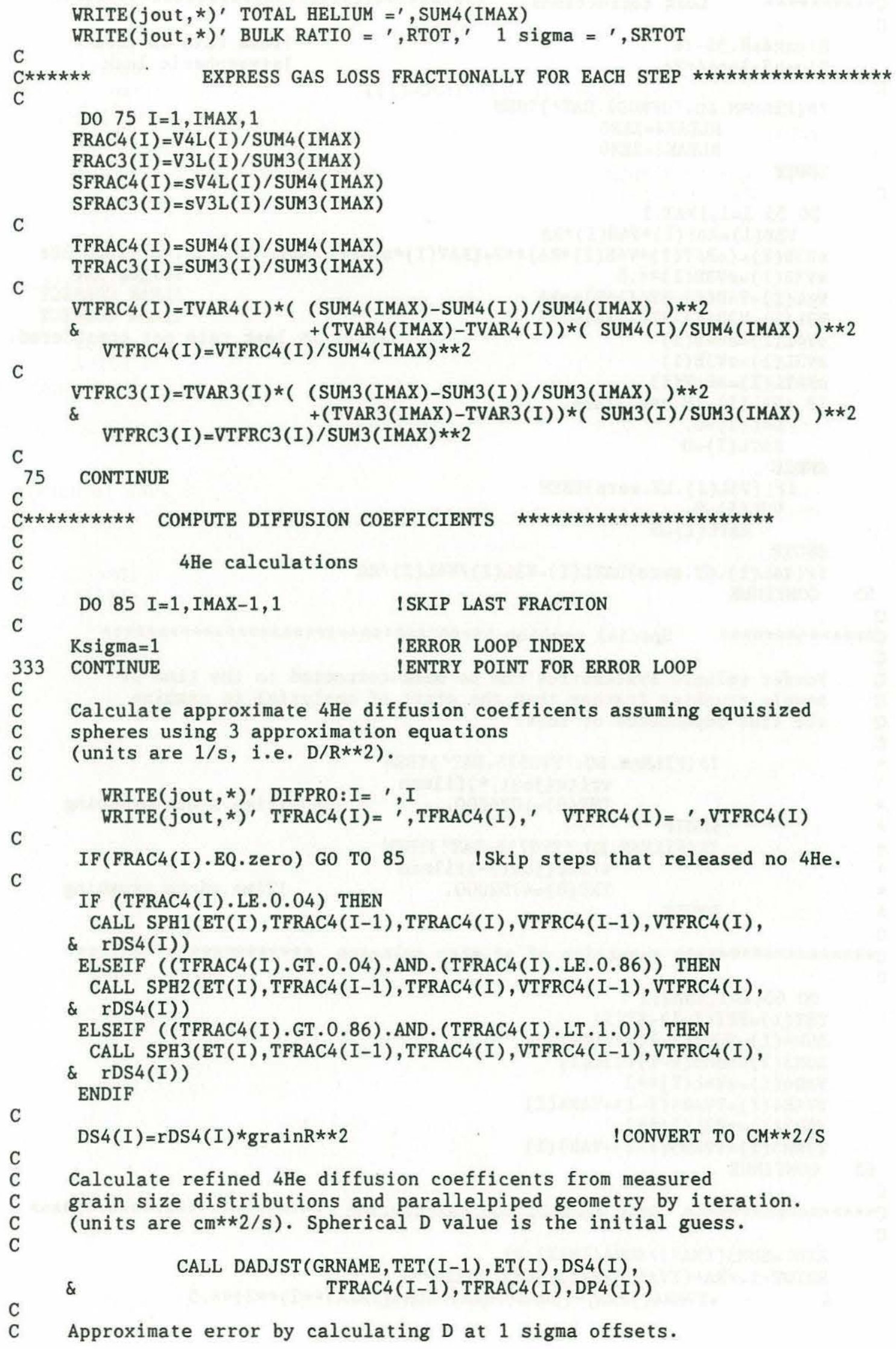


C

IF(Ksigma.EQ.1)THEN

Ksigma=Ksigma+1

DS4M=DS4(I)

$\mathrm{DP} 4 \mathrm{M}=\mathrm{DP} 4(\mathrm{I})$

$\operatorname{TFRAC4}(\mathrm{I})=\operatorname{TFRAC4}(\mathrm{I})+\operatorname{SORT}(\operatorname{VTFRC}(\mathrm{I})$ ) loffset 1 s above

ENDIF GO T0 333

C

IF (Ksigma.EQ.2)THEN

Ksigma $=$ Ksigma +1

$\mathrm{DS} 4 \mathrm{~A}=\mathrm{DS} 4(\mathrm{I})$

DP 4A=DP 4(I)

$\operatorname{TFRAC4}(I)=\operatorname{TFRAC4}(\mathrm{I})-2 * \operatorname{SORT}(\operatorname{VTFRC} 4(\mathrm{I}))$

ENDIF

GO TO 333

!1s above
!1s above
loffset 1s below
!recalculate

I1s below

I1s below

thalve offset value

t to obtain error

C

$\operatorname{TFRAC4}(\mathrm{I})=\operatorname{TFRAC4}(\mathrm{I})+\operatorname{SORT}(\operatorname{VTFRC} 4(\mathrm{I})) \quad$ !return to midpoint
DS4 $(\mathrm{I})=\mathrm{DS} 4 \mathrm{M}$

C

$\mathrm{DP} 4(\mathrm{I})=\mathrm{DP} 4 \mathrm{M}$

$\operatorname{WRITE}($ jout, *)' $I=$ ', I

WRITE (jout, $*$ )' SPHERE APPROX: D4 = ',DS4(I),' $s D 4(I)=', s D S 4(I)$

C

WRITE (jout, $*$ )' PIPEDS APPROX: D4 $=$ ',DP4(I),' $s D 4(I)=', s D P 4(I)$

85 CONTINUE

C

C

C

3He calculations

IF (FILNAM.EQ.' TV0519. DAT') THEN

VRITE $($ jout, *)FILNAM

GO TO 500 !skip 3He section for $\star 4$ He expts.

ENDIF

IF (FILNAM.EQ.'TV1109.DAT' )THEN

WRITE $($ jout, *) FILNAM

ENDIF

GO TO 500 !skip $3 \mathrm{He}$ section for $* 4 \mathrm{He}$ expts.

C

C

D0 95 I=1, IMAX-1, 1

!SKIP LAST FRACTION

C

IF ( (FILNAM.EQ. 'TV0901.DAT') . AND. (J.GT . 33)) THEN

VRITE $(j$ out,$\star)$ FILNAM

GO TO 500

!skip 3He section for aliquots

ENDIF

!with no remaining 3 He.

C

Ksigma $=1$

!ERROR LOOP INDEX

444 CONTINUE

!ENTRY POINT FOR ERROR LOOP

Calculate approximate 3 He diffusion coefficents assuming equisized spheres using 3 approximation equations

(units are $1 / \mathrm{s}$, i.e. $D / R * * 2$ ).

$\operatorname{VRITE}\left(\right.$ jout, $*$ )' DIFPRO: $I={ }^{\prime}, I$

$\operatorname{VRITE}($ jout,$*)$ ' TFRAC3(I) $={ }^{\prime}, \operatorname{TFRAC3}(I),{ }^{\prime} \operatorname{VTFRC3}(I)={ }^{\prime}, \operatorname{VTFRC3}(I)$ 
C

IF(FRAC3(I).EQ.zero) G0 T0 95 !Skip steps that released no 3He.

IF (TFRAC4(I).LE.0.04) THEN

CALL SPH1(ET(I), TFRAC3(I-1), TFRAC3(I), VTFRC3(I-1), $\operatorname{VTFRC3(I),~}$

\& $\operatorname{rDS} 3(I))$

ELSEIF ((TFRAC4(I).GT.0.04).AND. (TFRAC4(I).LE.0.86)) THEN

CALL SPH2(ET(I), TFRAC3(I-1), TFRAC3(I), VTFRC3(I-1), VTFRC3(I),

\& $\operatorname{rDS3}(\mathrm{I}))$

ELSEIF ((TFRAC4(I) . GT .0.86).AND. (TFRAC4(I) . LT .1.0)) THEN

CALL SPH3(ET(I), TFRAC3(I-1), TFRAC3(I), VTFRC3(I-1), VTFRC3(I),

\& $\operatorname{rDS} 3(I))$

C ENDIF

$$
\operatorname{DS} 3(I)=r D S 3(I) \star \operatorname{grainR} * \star 2
$$

! CONVERT TO $\mathrm{CM} * \star 2 / \mathrm{S}$

C

C

Calculate refined 3 He diffusion coefficents from measured grain size distributions and parallelpiped geometry by iteration. (units are $\mathrm{cm} * \star 2 / \mathrm{s}$ ). Spherical $D$ value is the initial guess.

$\&$

CALL DADJST(GRNAME,TET(I-1),ET(I), DS3(I),

$$
\operatorname{TrRAC3}(I-1), \operatorname{TPRAC3}(I), \operatorname{DP} 3(I))
$$

C

Approximate error by calculating $D$ at 1 sigma offsets.

IF (Ksigma.EQ.1)THEN

Ksigma $=$ Ksigma +1

DS $3 M=D S 3(I)$

DP3M=DP3(I)

$\operatorname{TFRAC3}(\mathrm{I})=\operatorname{TFRAC3}(\mathrm{I})+\operatorname{SQRT}(\operatorname{VTFRC} 3(\mathrm{I}))$

ENDIF GO TO 444

Imidpoint

!midpoint

loffset 1s above

Irecalculate

C

IF(Ksigma.EQ.2)THEN Ksigma $=$ Ksigma +1

$\mathrm{DS} 3 \mathrm{~A}=\mathrm{DS} 3(\mathrm{I})$

DP $3 A=D P 3(I)$

$\operatorname{TFRAC3}(I)=\operatorname{TFRAC} 3(I)-2 * \operatorname{SQRT}(\operatorname{VTFRC} 3(I))$

ENDIF GO TO 444

C

$\mathrm{DS} 3 \mathrm{~B}=\mathrm{DS} 3(\mathrm{I})$

$\mathrm{DP} 3 \mathrm{~B}=\mathrm{DP} 3(\mathrm{I})$

$\operatorname{sDS} 3(I)=(D S 3 A-D S 3 B) / 2$.

s

C

$\operatorname{sDP} 3(I)=(D P 3 A-D P 3 B) / 2$.

$\operatorname{TFRAC3}(I)=\operatorname{TFRAC} 3(I)+\operatorname{SQRT}(\operatorname{VTFRC3}(I))$ !return to midpoint

$\operatorname{DS} 3(I)=D S 3 M$

C

$\operatorname{DP} 3(I)=D P 3 M$

!retain values in arrays

$\operatorname{WRITE}(\text { jout }, \star)^{\prime} I={ }^{\prime}, I$

WRITE (jout, $*)^{\prime}$ SPHERE APPROX: D3 $\left.={ }^{\prime}, \mathrm{DS} 3(\mathrm{I})\right)^{\prime} \operatorname{sD3}(\mathrm{I})={ }^{\prime}, \mathrm{sDS} 3(\mathrm{I})$

C

$\operatorname{WRITE}($ jout,$*) \prime$ PIPEDS APPROX: D3 $={ }^{\prime, D P 3}(I),{ }^{\prime} \operatorname{sD3}(I)={ }^{\prime}, \operatorname{sDP} 3(I)$

95 CONTINUE

C

C

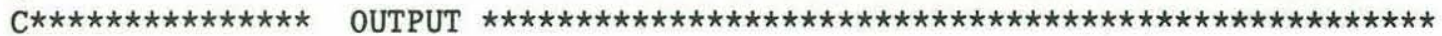

C

C Write headers in files 


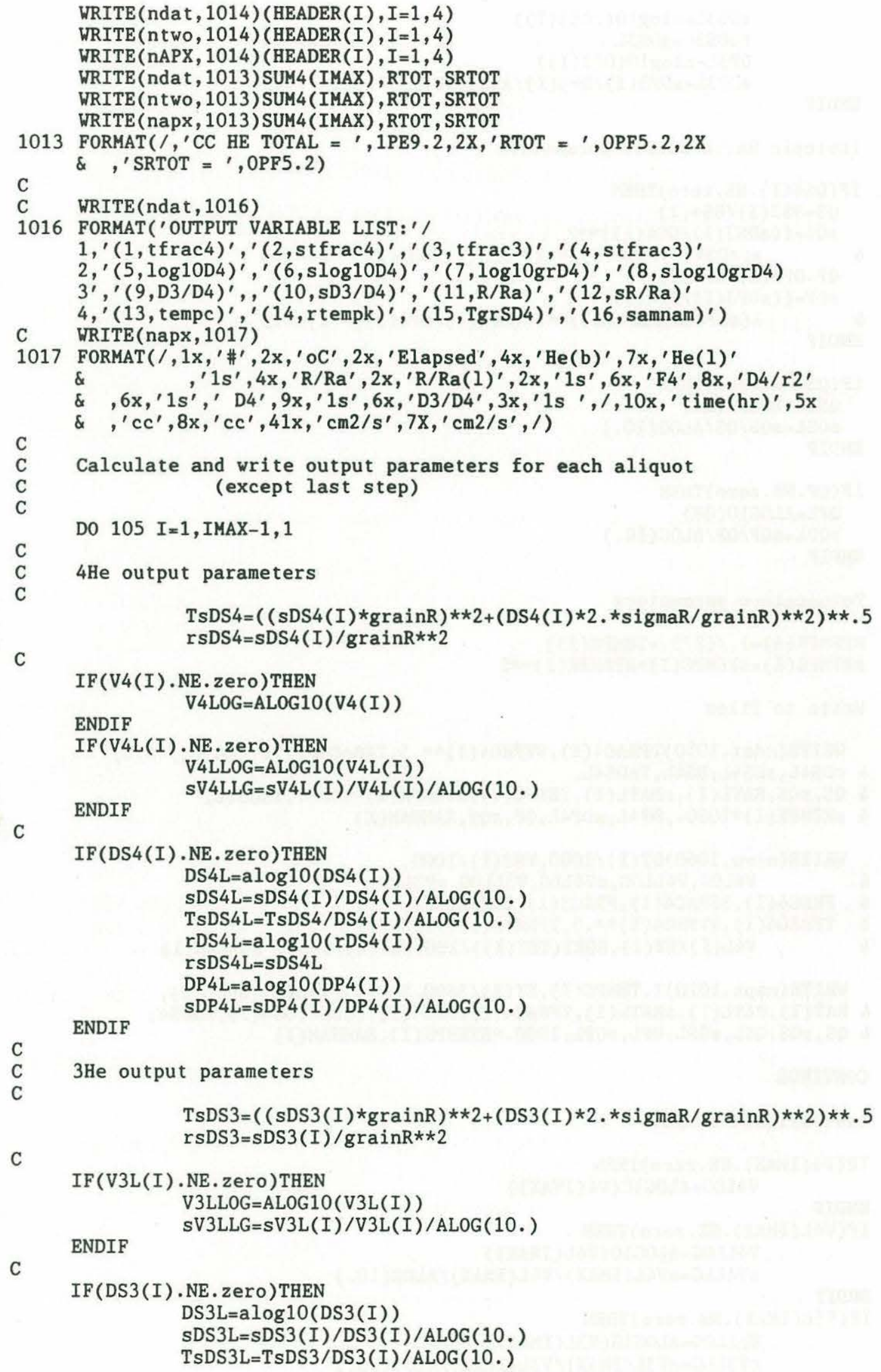




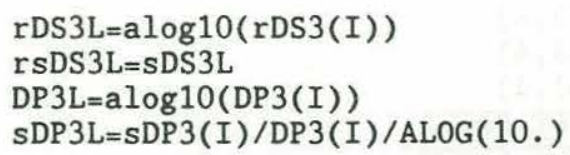

C

ENDIF

IF(QS.NE. zero)THEN

$Q S L=A L O G 10$ (QS) $\mathrm{SQSL}=\mathrm{SQS} / \mathrm{QS} / \mathrm{ALOG}(10$.

C ENDIF

IF (QP.NE. zero)THEN

$Q P L=A L O G 10$ (QP) $s Q P L=S Q P / Q P / A L O G(10$.

ENDIF

C

Temperature parameters

$\operatorname{RTEMPK}(I)=1 . /(273 .+\operatorname{TEMPC}(I))$

$\operatorname{sRTMPK}(I)=\operatorname{STEMPC}(I) * \operatorname{RTEMPK}(I) * \star 2$

Write to files

VRITE (ndat , 1050)TFRAC4 (I), VTFRC4(I) **.5, TFRAC3(I), VTFRC3(I) **.5, \& $\mathrm{rDS} 4 \mathrm{~L}, \mathrm{sDS} 4 \mathrm{~L}, \mathrm{DS} 4 \mathrm{~L}, \mathrm{TsDS} 4 \mathrm{~L}$,

\& QS, SQS, RATL(I) , SRATL(I), TEMPC(I), RTEMPK(I)*1000. ,TsDS4L,

\& $\operatorname{sRTMPK}(\mathrm{I}) * 1000$. DP4L, SDP4L, QP , SQP, SAMNAM(I)

C

$\operatorname{VRITE}(\mathrm{n}$ two, 1060)ET(I)/1000, TET(I)/1000, \& V4LOG, V4LLOG, sV4LLG, V3LLOG, sV3LLG,

\& $\operatorname{FRAC4}(I), \operatorname{SFRAC4}(I), \operatorname{FRAC3}(I), \operatorname{SFRAC3}(I)$,

\& TFRAC4 (I), VTFRC4 (I) $* * .5, \operatorname{TFRAC3}(I), \operatorname{VTFRC} 3(I) * * .5$,

C $\&$ V4L(I)/ET(I), SQRT(TET(I))/100,TET(I)/3600, SAMNAM(I)

VRITE (napx, 1070)I, TEMPC(I), ET(I)/3600,V4B(I),V4L(I), SV4L(I), \& $\operatorname{RAT}(I), \operatorname{RATL}(I), \operatorname{sRATL}(I), \operatorname{TFRAC} 4(I), \operatorname{rDS} 4(I), \operatorname{rsDS} 4, \mathrm{DS} 4(\mathrm{I}), \mathrm{TsDS} 4$,

C

$\&$ QS , SQS, QSL, SQSL, QPL, SQPL, 1000. *RTEMPK(I), SAMNAM(I)

105 CONTINUE

C

C

Last aliquot output

IF (V4 (IMAX). NE. zero) THEN

ENDIF V4LOG=ALOG10 (V4(IMAX))

IF (V4L(IMAX).NE. zero)THEN V4LLOG=ALOG10 (V4L (IMAX)) sV4LLG $=$ sV4L (IMAX)/V4L (IMAX)/ALOG (10.)

ENDIF

IF (V3L(IMAX).NE. zero)THEN

V3LLOG $=A L O G 10($ V3L (IMAX))

sV3LLG $=s V 3 L($ IMAX)/V3L(IMAX)/ALOG (10.) 


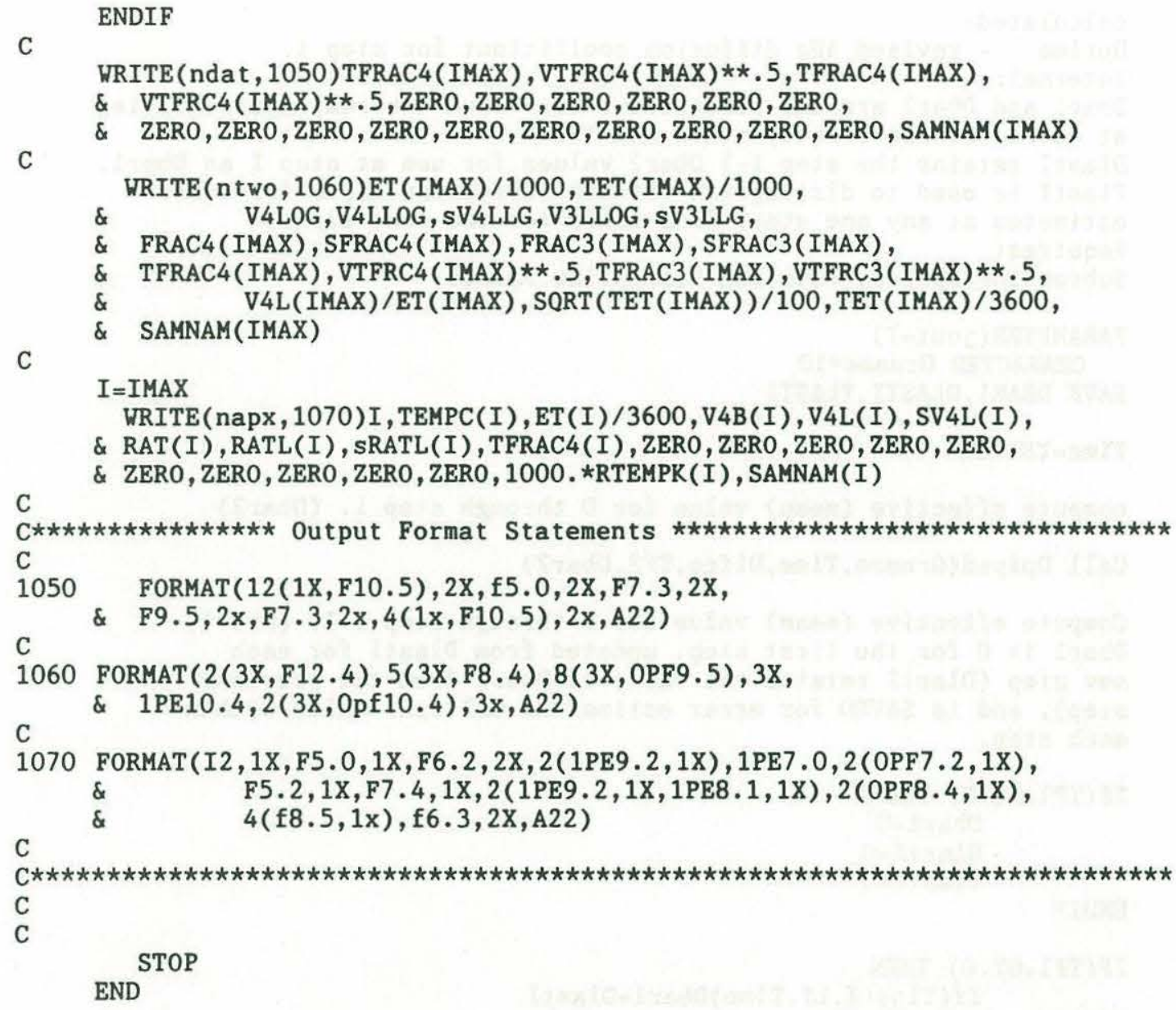




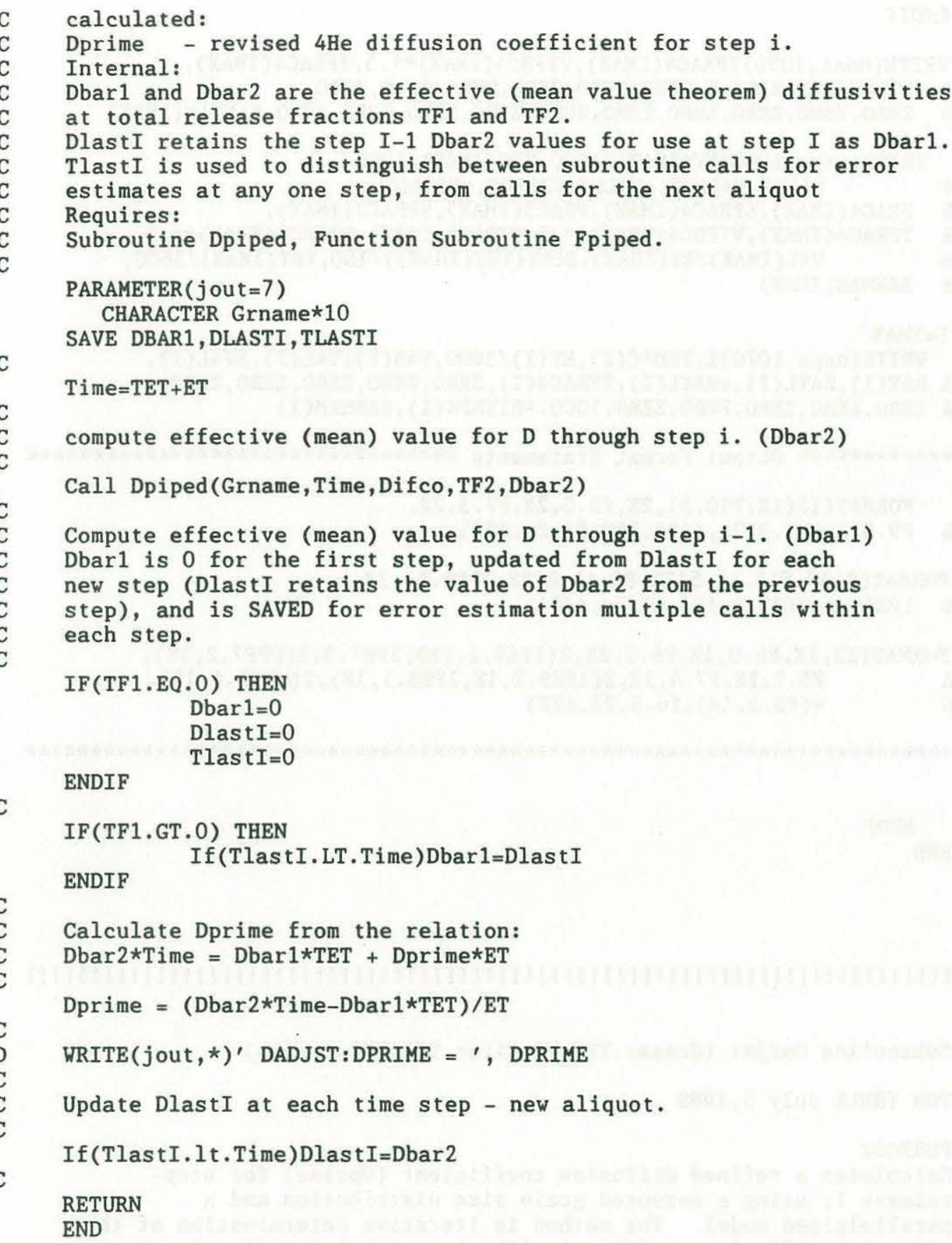




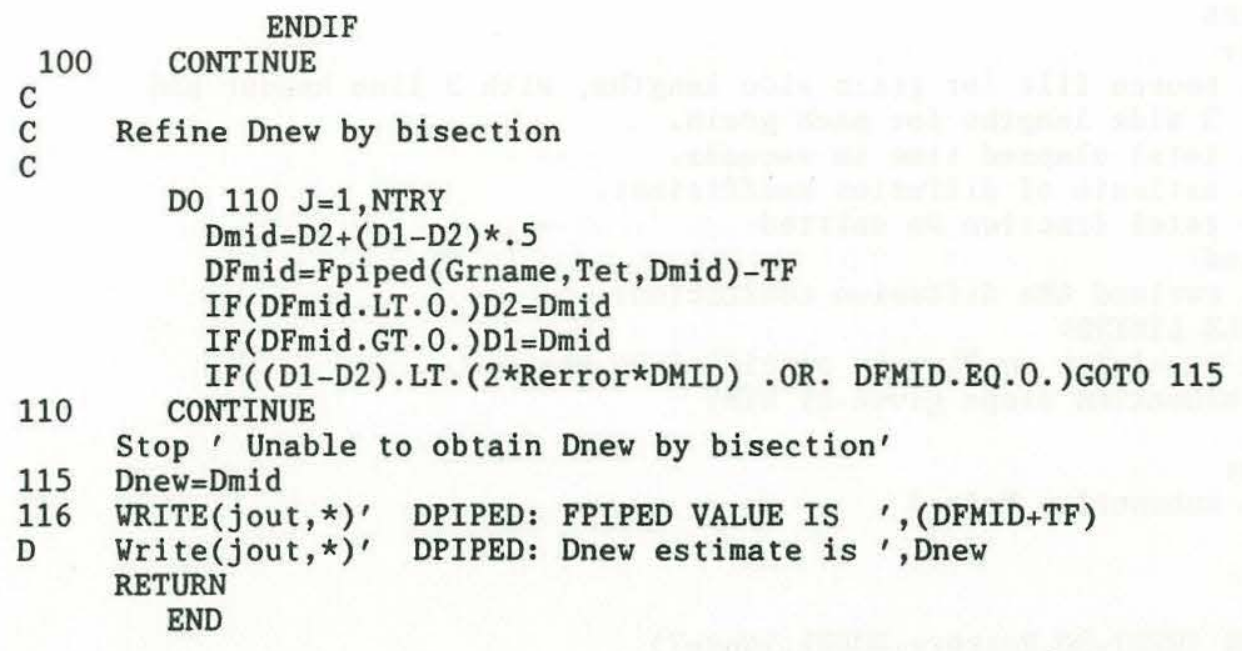

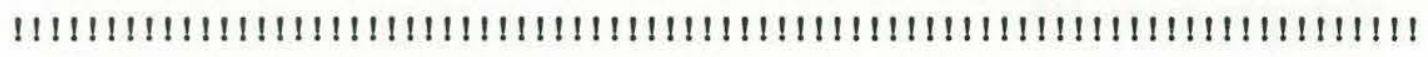

Function Fpiped (Grname,Tet,Difco)

TOM TRULL July 5,1988

PURPOSE

Calculates total fractional loss by diffusion from a collection of parallelpipeds.

ARGUMENTS

supplied:

Grname - source file for grain side lengths, with 3 line header and 3 side lengths for each grain (in um).

Tet - total elapsed time in seconds

Difco - Diffusion coefficient

calculated:

Fpiped - total fraction released

Adjustable limits:

Ngrmax - maximum \# of grains

nGrdat - unit \# for data input from Grname.dat

Cmax - maximum terms in series is $2 * \star C \max$

Error - absolute error allowed for series convergence.

Notes: REM(J) contains the fractional loss for each individual grain.

Each time Fpiped is called, the result is written to a status file for aid in debugging and tracking detached jobs. Alternative grain description input allowed (one bin size and number of grains in bin) - search on $d(j)$. Grain geometry can be specified in this routine as desired.

Parameter $($ Ngrmax $=101$, nGrdat $=1$, ns tat $=6$, jout $=7$, Cmax $=40)$

C

DOUBLE PRECISION TERMR, TERMB, TERMC, SUM, SUMR, SUMB, SUMC

1, CONV (Cmax), REALT, REALI, RMIN, RMAX, PI , R, B , C, DIFCO, TLOSS

2, TOTVOL, VOL, Ax (Ngrmax), Bx(Ngrmax), Cx(Ngrmax), REM (Ngrmax)

C

3, Q, ERROR, ONE , TWO, EIGHT, d(Ngrmax)

INTEGER COUNTR

SAVE COUNTR, SAVEF

DATA COUNTR/0./ 


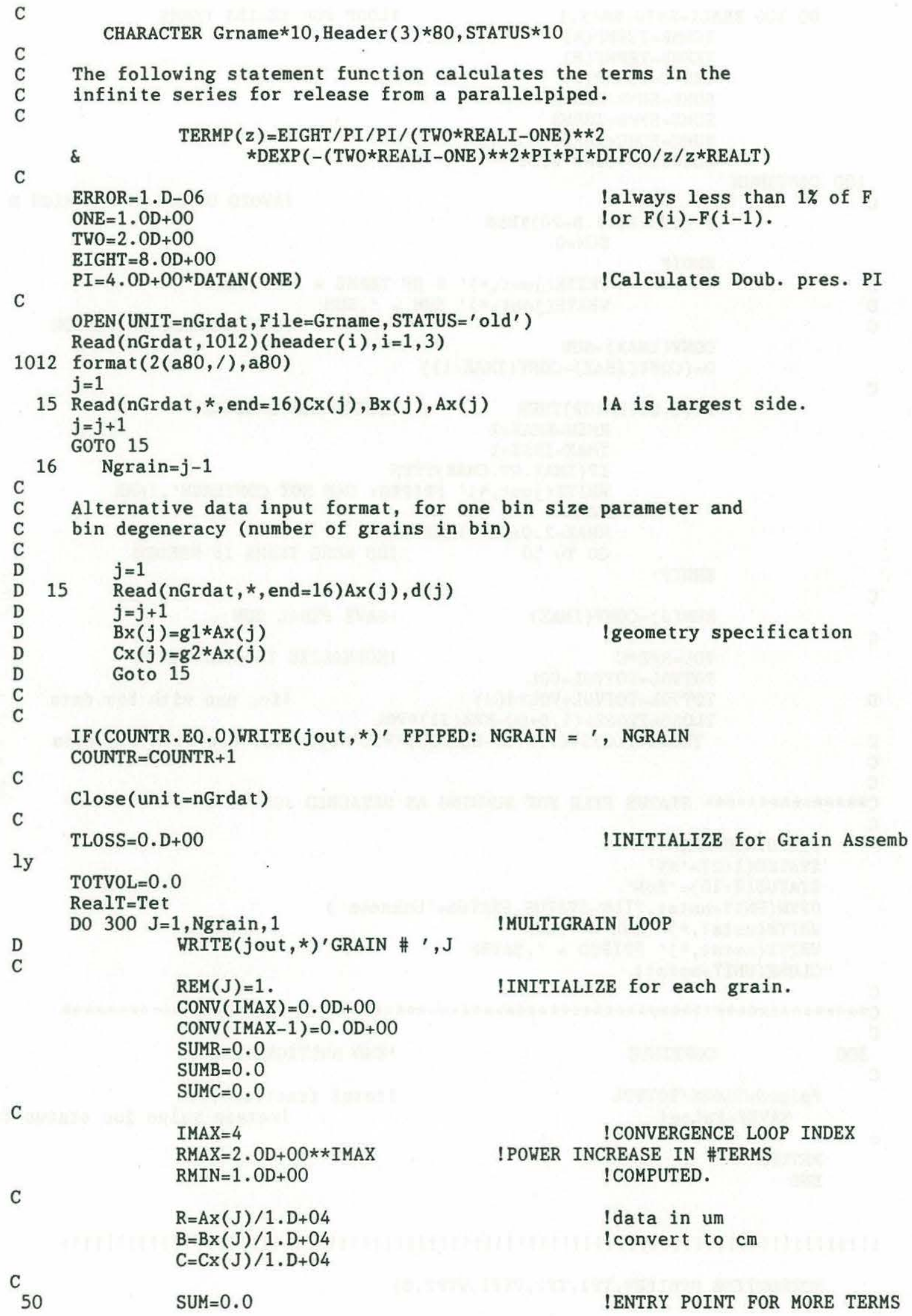




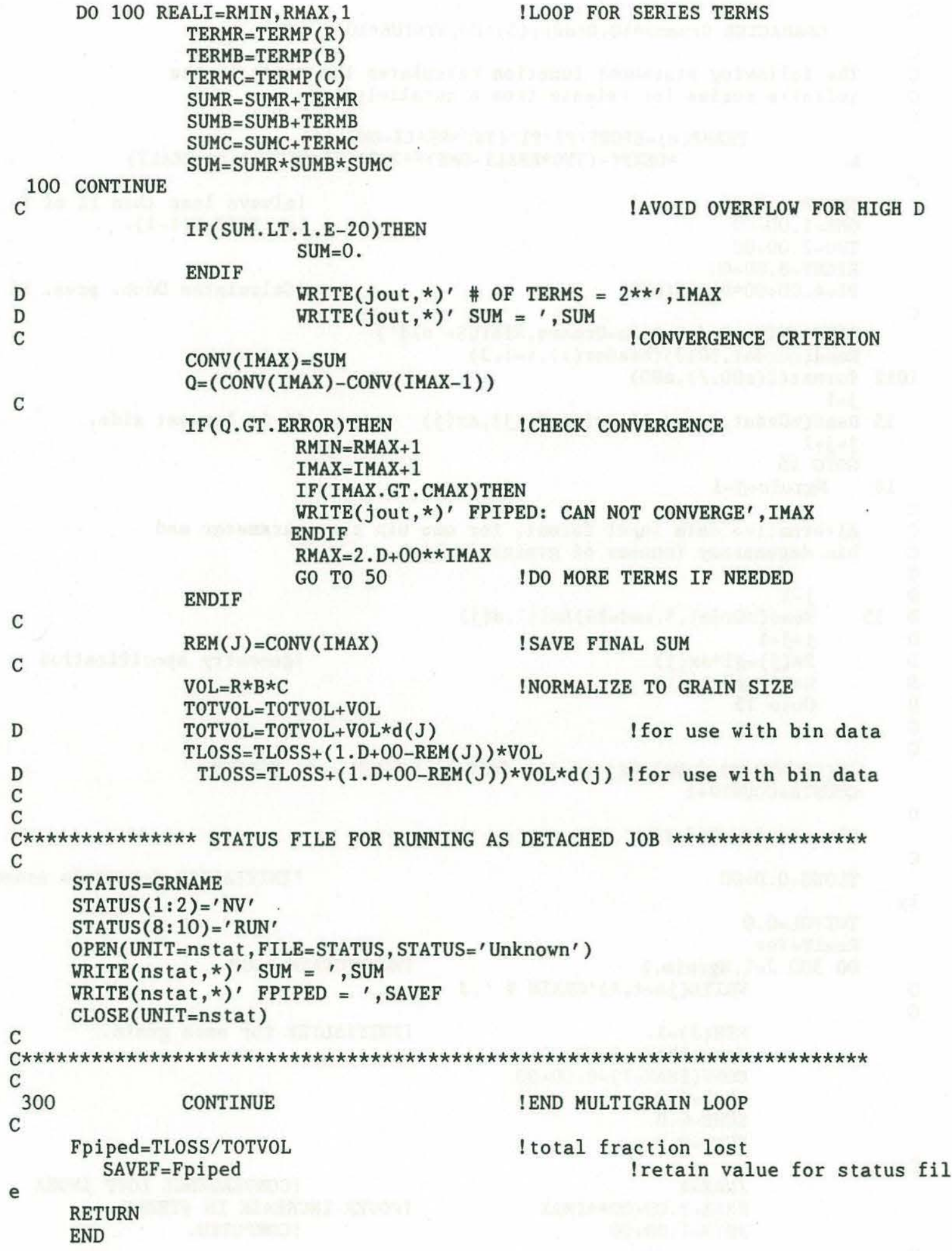




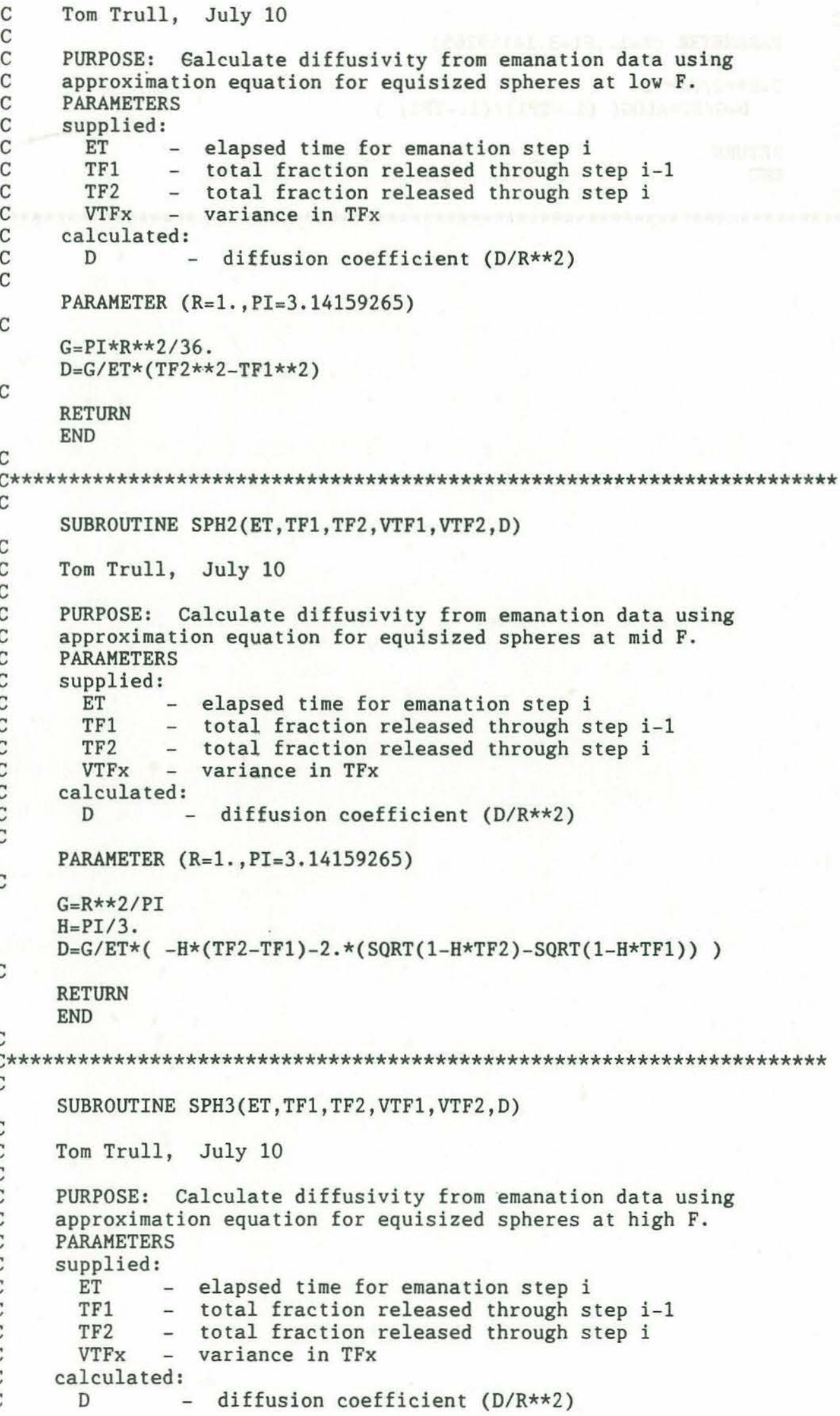




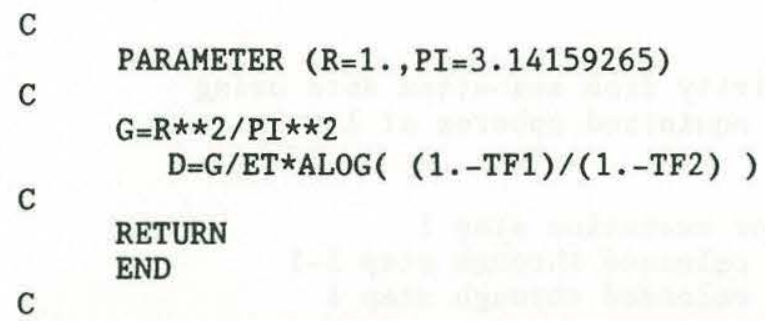


APPENDIX B: Helium emanation data and diffusivity results for basaltic glasses (experiments \#1-11, chap. 3). 
Key to the column headings:

1. The error in the measured ${ }^{4} \mathrm{He}$ amount is given in units of parts per thousand. A value of 0 does not imply no error, but less than $.5 \%$ at 1 sigma.

2. Sm designates values calculated using the equisized sphere model and Rm the refined model results.

3. The errors in $\mathrm{F}$ are not given but were generally less than $.1 \%$ for ${ }^{4} \mathrm{He}$ and a few tenths of a percent for ${ }^{3} \mathrm{He}$. 
Experiment \#1

Alv892-1a MORB tholeite glass

1 tabular grain, $7.26 \mathrm{mg}$

Grain size data (um): short side, "visual average", long side.

$1397 \quad 2032 \quad 2057$

Bulk helium contents: He $=6.02 \mathrm{E}-08 \mathrm{ccSTP} 3 \mathrm{He} / 4 \mathrm{He}=8.56+0.03 \times \mathrm{Ra}$

Sequential release data:

\begin{tabular}{|c|c|c|c|c|c|c|c|c|c|c|c|c|c|}
\hline \# & $\begin{array}{l}\text { Temp. } \\
\text { C }\end{array}$ & $\begin{array}{c}\text { Time } \\
\text { hr }\end{array}$ & $\begin{array}{l}4 \mathrm{He} \\
\operatorname{cosTP}\end{array}$ & $\begin{array}{l}1 \mathrm{~s} \\
0 / 00\end{array}$ & $\begin{array}{c}3 \mathrm{He} / 4 \mathrm{He} \\
\mathrm{Ra}\end{array}$ & $\begin{array}{l}1 \mathrm{~s} \\
\mathrm{Ra}\end{array}$ & $\begin{array}{l}F(4 \mathrm{He}) \\
(\operatorname{total})\end{array}$ & $\begin{array}{c}\text { Ds m } \\
\operatorname{cm} 2 / s\end{array}$ & $\begin{array}{l}\text { Drm } \\
\operatorname{con} 2 / s\end{array}$ & $\begin{array}{l}1 \mathrm{~s} \\
\mathrm{~cm} 2 / \mathrm{s}\end{array}$ & $\begin{array}{l}\mathrm{D} 3 / \mathrm{D} 4 \\
\mathrm{sm}\end{array}$ & $\begin{array}{c}\mathrm{D} 3 / \mathrm{D} 4 \\
\mathrm{rm}\end{array}$ & $\begin{array}{l}1 \mathrm{~s} \\
\mathrm{rm}\end{array}$ \\
\hline 1 & $R a m p$ & 0.53 & $1.05 \mathrm{E}-10$ & 10. & 1.17 & 0.41 & 0.0018 & $6.83 E-13$ & $1.09 \mathrm{E}-12$ & 2. $3 \mathrm{E}-14$ & 0.019 & 0.019 & 0.013 \\
\hline 2 & 253. & 1.07 & 1. $71 \mathrm{E}-09$ & 6. & 8.64 & 0.10 & 0.0302 & $1.01 \mathrm{E}-10$ & $1.65 \mathrm{E}-10$ & 2. $2 \mathrm{E}-12$ & 0.920 & 0.919 & 0.028 \\
\hline 3 & 253 . & 0.53 & $5.96 \mathrm{E}-10$ & 7. & 8.68 & 0.18 & 0.0401 & $1.59 \mathrm{E}-10$ & $2.57 \mathrm{E}-10$ & $6.5 \mathrm{E}-12$ & 0.978 & 0.978 & 0.056 \\
\hline 4 & 253. & 0.53 & $4.99 \mathrm{E}-10$ & 8. & 8.62 & 0.19 & 0.0484 & $1.69 \mathrm{E}-10$ & $2.73 \mathrm{E}-10$ & 8. $4 \mathrm{E}-12$ & 0.980 & 0.980 & 0.070 \\
\hline 5 & 253. & 0.53 & $4.38 \mathrm{E}-10$ & 7. & 8.64 & 0.26 & 0.0557 & 1. $76 \mathrm{E}-10$ & $2.84 \mathrm{E}-10$ & 1. $.0 \mathrm{E}-11$ & 0.988 & 0.987 & 0.086 \\
\hline 6 & Ramp & 1.07 & $2.04 \mathrm{E}-09$ & 5. & 8.84 & 0.08 & 0.0896 & $5.84 \mathrm{E}-10$ & $9.48 \mathrm{E}-10$ & 1. $2 \mathrm{E}-11$ & 1.026 & 1.026 & 0.028 \\
\hline 7 & 311. & 0.53 & $1.27 \mathrm{E}-09$ & 7. & 8.97 & 0.11 & 0.1107 & $1.02 \mathrm{E}-09$ & $1.67 \mathrm{E}-09$ & 3. $4 \mathrm{E}-11$ & 1.054 & 1.054 & 0.045 \\
\hline 8 & 311. & 0.53 & $1.07 \mathrm{E}-09$ & 7. & 9.06 & 0.17 & 0.1285 & $1.05 \mathrm{E}-09$ & $1.71 \mathrm{E}-09$ & 4. $4 \mathrm{E}-11$ & 1.074 & 1.074 & 0.058 \\
\hline 9 & 311. & 0.53 & $9.36 E-10$ & 7. & 9.02 & 0.12 & 0.1440 & $1.06 \mathrm{E}-09$ & $1.74 \mathrm{E}-09$ & 5. $3 E-11$ & 1.075 & 1.075 & 0.068 \\
\hline 10 & Ramp & 1.07 & $5.91 \mathrm{E}-09$ & 7. & 8.96 & 0.05 & 0.2422 & $5.02 \mathrm{E}-09$ & $8.38 \mathrm{E}-09$ & 8. $4 \mathrm{E}-11$ & 1.081 & 1.082 & 0.020 \\
\hline 11 & 405. & 0.53 & $3.34 \mathrm{E}-09$ & 6. & 8.89 & 0.07 & 0.2977 & $8.55 E-09$ & $1.45 \mathrm{E}-08$ & 2. $4 \mathrm{E}-10$ & 1.082 & 1.084 & 0.033 \\
\hline 12 & 405. & 0.53 & $2.69 \mathrm{E}-09$ & 7. & 9.07 & 0.09 & 0.3425 & $8.62 \mathrm{E}-09$ & $1.48 \mathrm{E}-08$ & 3. $2 \mathrm{E}-10$ & 1.109 & 1.112 & 0.042 \\
\hline 13 & 405. & 0.53 & $2.27 \mathrm{E}-09$ & 9. & 9.06 & 0.09 & 0.3802 & $8.62 \mathrm{E}-09$ & $1.50 E-08$ & $3.9 \mathrm{E}-10$ & 1.115 & 1.119 & 0.051 \\
\hline 14 & Ramp & 1.07 & $1.41 \mathrm{E}-08$ & 6. & 8.47 & 0.05 & 0.6145 & $4.55 E-08$ & $8.26 E-08$ & 4. $8 \mathrm{E}-10$ & 1.037 & 1.041 & 0.011 \\
\hline 15 & 517. & 0.53 & $4.42 \mathrm{E}-09$ & 7. & 8.80 & 0.07 & 0.6879 & $4.85 E-08$ & $9.18 \mathrm{E}-08$ & $1.1 \mathrm{E}-09$ & 1.080 & 1.085 & 0.025 \\
\hline 16 & 518. & 0.53 & $3.31 \mathrm{E}-09$ & 6. & 8.73 & 0.07 & 0.7429 & $4.68 \mathrm{E}-08$ & $9.02 \mathrm{E}-08$ & 1. $3 E-09$ & 1.085 & 1.090 & 0.030 \\
\hline 17 & 517. & 0.53 & $2.57 \mathrm{E}-09$ & 8. & 8.56 & 0.08 & 0.7856 & $4.51 E-08$ & $8.83 E-08$ & 1. $4 \mathrm{E}-09$ & 1.075 & 1.079 & 0.034 \\
\hline 18 & 517. & 1.07 & $3.77 \mathrm{E}-09$ & 8. & 8.55 & 0.08 & 0.8481 & $4.38 E-08$ & $8.70 E-08$ & 7. $9 \mathrm{E}-10$ & 1.095 & 1.098 & 0.020 \\
\hline 19 & 517. & 0.53 & 1. $38 \mathrm{E}-09$ & 7. & 8.33 & 0.10 & 0.8710 & $4.22 \mathrm{E}-08$ & $8.42 \mathrm{E}-08$ & 1. $7 \mathrm{E}-09$ & 1.086 & 1.092 & 0.046 \\
\hline 20 & 517. & 0.53 & $1.13 \mathrm{E}-09$ & 7. & 8.17 & 0.12 & 0.8897 & $4.05 E-08$ & $8.15 E-08$ & $1.9 \mathrm{E}-09$ & 1.079 & 1.085 & 0.052 \\
\hline 21 & 517. & 1.07 & $1.77 \mathrm{E}-09$ & 6. & 8.21 & 0.10 & 0.9192 & $4.02 \mathrm{E}-08$ & $8.15 \mathrm{E}-08$ & $1.0 \mathrm{E}-09$ & 1.109 & 1.113 & 0.031 \\
\hline 22 & 517. & 0.53 & $6.75 E-10$ & 7. & 7.82 & 0.18 & 0.9304 & $3.86 E-08$ & $7.88 \mathrm{E}-08$ & 2. $3 E-09$ & 1.081 & 1.084 & 0.068 \\
\hline 23 & 517. & 0.53 & $5.53 \mathrm{E}-10$ & 7. & 8.19 & 0.16 & 0.9396 & $3.66 \mathrm{E}-08$ & $7.48 \mathrm{E}-08$ & $2.6 \mathrm{E}-09$ & 1.152 & 1.155 & 0.080 \\
\hline 24 & Ramp & 1.07 & $2.34 \mathrm{E}-09$ & 9. & 7.50 & 0.07 & 0.9785 & $1.34 \mathrm{E}-07$ & $2.75 E-07$ & 1. $3 \mathrm{E}-09$ & 1.124 & 1.125 & 0.019 \\
\hline 25 & 620. & 0.53 & $5.44 \mathrm{E}-10$ & 7. & 6.94 & 0.13 & 0.9876 & $1.41 \mathrm{E}-07$ & $2.91 \mathrm{E}-07$ & $3.1 \mathrm{E}-09$ & 1.170 & 1.171 & 0.058 \\
\hline 26 & 620. & 0.53 & $2.90 \mathrm{E}-10$ & 7. & 6.60 & 0.23 & 0.9924 & $1.27 \mathrm{E}-07$ & $2.62 \mathrm{E}-07$ & 4. $0 \mathrm{E}-09$ & 1.233 & 1.233 & 0.099 \\
\hline 27 & 620. & 1.07 & $2.60 \mathrm{E}-10$ & 8. & 6.29 & 0.30 & 0.9967 & $1.09 \mathrm{E}-07$ & $2.24 E-07$ & $3.4 \mathrm{E}-09$ & 1.479 & 1.479 & 0.138 \\
\hline 28 & 620. & 0.53 & $4.71 \mathrm{E}-11$ & 15. & 5.57 & 1.12 & 0.9975 & $7.05 E-08$ & $1.45 E-07$ & $8.6 \mathrm{E}-09$ & 1.850 & 1.849 & 0.492 \\
\hline 29 & 620. & 2.22 & 1. $50 \mathrm{E}-10$ & 13. & 2.66 & 0.33 & 1.0000 & & & & & & \\
\hline
\end{tabular}


Experiment \#2

Alv892-1a MORB tholeite glass

13 chips, $90.1 \mathrm{mg}$

Grain size data (um): short side, "visual average", long side.

$\begin{array}{lllllllllllll}1461 & 1270 & 1346 & 1372 & 1448 & 1092 & 965 & 1016 & 1295 & 1219 & 1143 & 1524 & 1829\end{array}$

$\begin{array}{lllllllllllllll}1715 & 2159 & 2057 & 1930 & 1676 & 2134 & 1270 & 1499 & 1321 & 1981 & 2032 & 1981 & 2134\end{array}$

$\begin{array}{lllllllllllll}2159 & 2489 & 2946 & 2769 & 1880 & 2438 & 2946 & 2388 & 2591 & 2184 & 2388 & 2083 & 2235\end{array}$

Bulk helium contents: He $=7.75 \mathrm{E}-07$ ccSTP $3 \mathrm{He} / 4 \mathrm{He}=8.35+0.01 \times \mathrm{Ra}$

Sequential release data:

\begin{tabular}{|c|c|c|c|c|c|c|c|c|c|c|c|c|c|}
\hline & $\begin{array}{l}\text { Temp. } \\
\text { c }\end{array}$ & $\underset{\mathrm{hr}}{\mathrm{Time}}$ & $\begin{array}{l}4 \mathrm{He} \\
\text { CCSTP }\end{array}$ & $\begin{array}{c}1 \mathrm{~s} \\
0 / 00\end{array}$ & $\begin{array}{c}3 \mathrm{He} / 4 \mathrm{He} \\
\mathrm{Ra}\end{array}$ & $\begin{array}{l}1 \mathrm{~s} \\
\mathrm{Ra}\end{array}$ & $\begin{array}{l}F(4 \mathrm{He}) \\
(\operatorname{total})\end{array}$ & $\begin{array}{c}\text { Dsm } \\
\operatorname{cm} 2 / s\end{array}$ & $\begin{array}{l}\text { Drm } \\
\operatorname{cm} 2 / s\end{array}$ & $\begin{array}{l}1 \mathrm{~s} \\
\mathrm{~cm} 2 / \mathrm{s}\end{array}$ & $\begin{array}{c}\mathrm{D} 3 / \mathrm{D} 4 \\
\mathrm{~s} \cap\end{array}$ & $\begin{array}{c}\mathrm{D} 3 / \mathrm{D} 4 \\
\mathrm{r}\end{array}$ & $\begin{array}{l}1 \mathrm{~s} \\
\mathrm{rm}\end{array}$ \\
\hline 1 & Ramp & 0.53 & $5.49 \mathrm{E}-11$ & 9. & 9.00 & 2.04 & 0.0001 & $9.62 \mathrm{E}-16$ & $1.73 \mathrm{E}-15$ & 2. $9 \mathrm{E}-17$ & 1.161 & 1.160 & 0.542 \\
\hline 2 & 113. & 0.54 & $.25 E-10$ & & 8.68 & 0.64 & 0.0004 & $2.37 E-14$ & $4.26 \mathrm{E}-14$ & 3. $4 \mathrm{E}-16$ & 1.092 & 1.092 & \\
\hline 3 & 113. & 3.78 & $.45 \mathrm{E}-09$ & 3. & 8.76 & 0.13 & 0.0022 & 1. $32 \mathrm{E}-13$ & $2.37 \mathrm{E}-13$ & 1. $4 \mathrm{E}-15$ & 1.100 & 1.100 & 0.040 \\
\hline 4 & 113. & 10.25 & $2.66 \mathrm{E}-09$ & 2. & 8.41 & 0.09 & 0.0057 & $2.71 \mathrm{E}-13$ & $4.88 E-13$ & 1. $7 E-15$ & 1.038 & 1.038 & 0.024 \\
\hline 5 & 113. & 27.35 & $4.69 \mathrm{E}-09$ & 2 . & 8.65 & 0.07 & 0.0117 & $3.94 \mathrm{E}-13$ & $7.15 \mathrm{E}-13$ & 2. $5 E-15$ & 1.064 & 1.065 & 0.018 \\
\hline 6 & Ramp & 0.54 & $2.51 \mathrm{E}-09$ & 2. & 8.92 & 0.10 & 0.0150 & $1.64 \mathrm{E}-11$ & $2.98 \mathrm{E}-11$ & $1.8 \mathrm{E}-13$ & 1.104 & 1.105 & 0.032 \\
\hline 7 & 209. & 1.62 & $1.07 E-08$ & 2 . & 8.81 & 0.06 & 0.0287 & $3.81 \mathrm{E}-11$ & $6.99 E-11$ & 2. $2 E-13$ & 1.100 & 1.101 & 0.014 \\
\hline 8 & 209. & 2.16 & $9.89 \mathrm{E}-09$ & 2. & 8.76 & 0.06 & 0.0415 & $4.37 \mathrm{E}$ & 7.91 & $3.1 \mathrm{E}$ & 1.099 & 1.099 & 0.017 \\
\hline 9 & 209. & 2.70 & $9.41 \mathrm{E}-09$ & 2. & 8.68 & 0.05 & 0.05 & 4.56 & 8.26 & & 1.089 & 1.089 & 0.020 \\
\hline 10 & 209. & 2. & $7.91 \mathrm{E}-09$ & 3. & 8.71 & 0.07 & 0.0638 & 4.78 & $8.68 \mathrm{E}-11$ & 4. $9 \mathrm{E}-13$ & 1.092 & 1.092 & 0.025 \\
\hline 11 & 209. & 3.2 & $7.97 \mathrm{E}-09$ & 3. & .62 & 0.05 & 0.0741 & $4.75 \mathrm{E}-11$ & $8.64 E-11$ & 5. $2 \mathrm{E}-13$ & 1.079 & 1.080 & 0.026 \\
\hline 12 & 209. & 3.7 & $8.37 \mathrm{E}-09$ & 2. & 8.57 & 0.06 & 0.0849 & & 9.06 & & 1.071 & & 0.026 \\
\hline 13 & Ramp & 1.08 & $4.76 \mathrm{E}-09$ & 2. & 8.89 & 0.08 & 0.0911 & 1.10 & $2.01 \mathrm{E}-10$ & $2.1 \mathrm{E}$ & 1.113 & 1.113 & 0.046 \\
\hline 14 & 311. & 0.54 & $1.97 \mathrm{E}-08$ & 2 . & 8.66 & 0.05 & 0.1165 & 1.091 & $2.00 \mathrm{E}-09$ & & 1.084 & 1.085 & 0.015 \\
\hline 15 & 311. & 1.08 & $3.13 \mathrm{E}-08$ & 1. & 8.73 & 0.06 & 568 & $1.17 \mathrm{E}$ & $2.17 \mathrm{E}-09$ & 5.7 & 1.095 & 1.096 & 0.013 \\
\hline 16 & 311. & 0.54 & 1. $23 \mathrm{E}-08$ & 2. & 8.73 & 0.07 & 0.1727 & $1.14 \mathrm{E}-09$ & $2.12 \mathrm{E}-09$ & 1. $3 \mathrm{E}-11$ & 1.097 & 1.099 & 0.031 \\
\hline 17 & 311. & 0.5 & -08 & 1. & & 0.07 & & & $2.08 \mathrm{E}-09$ & & 1.103 & 1.105 & 0.037 \\
\hline 18 & 311. & 0.54 & $9.90 \mathrm{E}-09$ & 1. & 8.58 & 0.07 & 0.1995 & $1.11 \mathrm{E}-09$ & $2.07 \mathrm{E}-09$ & 1. $6 \mathrm{E}-11$ & 1.079 & 1.081 & 0.041 \\
\hline 19 & 311. & 1.08 & $1.78 \mathrm{E}-08$ & 1. & 8.68 & 0.07 & 0.2224 & $1.10 \mathrm{E}-09$ & $2.07 \mathrm{E}-09$ & 9. $5 E-12$ & 1.093 & 1.095 & 0.025 \\
\hline 20 & 311. & 0.54 & $8.04 \mathrm{E}-09$ & 1. & 8.72 & 0.08 & 0.2328 & $1.10 \mathrm{E}-09$ & $2.06 \mathrm{E}-09$ & $2.0 \mathrm{E}-11$ & 1.099 & 1.101 & 0.055 \\
\hline 21 & 311. & 1.08 & $1.50 \mathrm{E}-08$ & 1. & 8.64 & 0.06 & 0.2521 & $1.10 \mathrm{E}-09$ & $2.08 \mathrm{E}-09$ & 1. $2 \mathrm{E}-11$ & 1.089 & 1.092 & 0.031 \\
\hline 22 & 311. & 1.08 & $1.36 \mathrm{E}-08$ & 1. & & 0.06 & & $1.10 \mathrm{E}-09$ & $2.08 \mathrm{E}-09$ & & 1.095 & 1.097 & 0.035 \\
\hline 23 & $\operatorname{Ramp}$ & 1.10 & $3.93 E-08$ & 1. & 8.71 & 0.06 & 0.3203 & $3.67 \mathrm{E}-09$ & $7.01 \mathrm{E}-09$ & 1. $8 \mathrm{E}-11$ & 1.101 & 1.104 & 0.015 \\
\hline 24 & 421. & 0.54 & $4.56 \mathrm{E}-08$ & 1. & 8.62 & 0.06 & 0.3791 & $1.09 \mathrm{E}-08$ & $2.11 \mathrm{E}-08$ & 5. $2 \mathrm{E}-11$ & 1.093 & & 0.014 \\
\hline 25 & 421. & 0.54 & $3.61 \mathrm{E}-08$ & 1. & 8.65 & 0.06 & 0.4256 & $1.06 \mathrm{E}-08$ & $2.08 \mathrm{E}-08$ & $6.4 E-11$ & 1.100 & 1.104 & 0.018 \\
\hline 26 & 421. & 0.54 & $2.99 E-08$ & 1. & 8.67 & 0.06 & 0.4642 & $1.03 \mathrm{E}-08$ & $2.04 E-08$ & 7. $5 \mathrm{E}-11$ & 1.107 & 1.112 & 0.022 \\
\hline 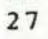 & 421. & 1.08 & $4.89 \mathrm{E}-08$ & 1. & 8.52 & 0.06 & 0.5273 & $1.01 \mathrm{E}-08$ & & & & & \\
\hline & & & $2.04 \mathrm{E}-08$ & & 8.57 & 0.06 & 0.5537 & $9.91 \mathrm{E}-09$ & $2.01 \mathrm{E}-08$ & 1. $1 \mathrm{E}-10$ & 1.102 & 1.108 & 0.033 \\
\hline
\end{tabular}

continued 
Experiment \#2 (continued)

\begin{tabular}{|c|c|c|c|c|c|c|c|c|c|c|c|c|c|}
\hline \# & $\begin{array}{c}\text { Temp. } \\
\text { C }\end{array}$ & $\begin{array}{c}\mathrm{Time} \\
\mathrm{hr}\end{array}$ & $\begin{array}{l}4 \mathrm{He} \\
\operatorname{coSTP}\end{array}$ & $\begin{array}{l}1 \mathrm{~s} \\
0 / 00\end{array}$ & $\begin{array}{c}3 \mathrm{He} / 4 \mathrm{He} \\
\mathrm{Ra}\end{array}$ & $\begin{array}{l}1 \mathrm{~s} \\
\mathrm{Ra}\end{array}$ & $\begin{array}{l}F(4 \mathrm{He}) \\
(\operatorname{total})\end{array}$ & $\begin{array}{c}\text { Ds m } \\
\mathrm{Cm} 2 / \mathrm{s}\end{array}$ & $\begin{array}{l}\text { Drm } \\
\operatorname{cm} 2 / s\end{array}$ & $\begin{array}{l}1 \mathrm{~s} \\
\mathrm{~cm} 2 / \mathrm{s}\end{array}$ & $\begin{array}{l}\mathrm{D} 3 / \mathrm{D} 4 \\
\mathrm{~s} \text { 开 }\end{array}$ & $\begin{array}{c}\mathrm{D} 3 / \mathrm{D} 4 \\
\mathrm{rm}\end{array}$ & $\begin{array}{l}1 \mathrm{~s} \\
\mathrm{rm}\end{array}$ \\
\hline 29 & Ramp & 0.54 & $5.46 \mathrm{E}-08$ & 1. & 8.45 & 0.06 & 0.6241 & $3.15 \mathrm{E}-08$ & $6.48 \mathrm{E}-08$ & 1. $4 \mathrm{E}-10$ & 1.090 & 1.097 & 0.013 \\
\hline 30 & 519. & 0.54 & $5.84 E-08$ & 1. & 8.31 & 0.06 & 0.6994 & $4.41 \mathrm{E}-08$ & $9.28 E-08$ & 1. $8 \mathrm{E}-10$ & 1.079 & 1.086 & 0.012 \\
\hline 31 & 519. & 0.54 & $4.27 E-08$ & 1. & 8.24 & 0.06 & 0.7545 & $4.19 E-08$ & $9.01 \mathrm{E}-08$ & 2. $1 \mathrm{E}-10$ & 1.079 & 1.087 & 0.014 \\
\hline 32 & 519. & 0.54 & 3. $29 \mathrm{E}-08$ & 1. & 8.18 & 0.06 & 0.7969 & $4.03 E-08$ & $8.83 E-08$ & 2. $5 E-10$ & 1.082 & 1.091 & 0.017 \\
\hline 33 & 519. & 1.08 & $4.74 \mathrm{E}-08$ & 1. & 7.93 & 0.05 & 0.8580 & $3.89 \mathrm{E}-08$ & $8.71 \mathrm{E}-08$ & 1. $5 \mathrm{E}-10$ & 1.066 & 1.073 & 0.010 \\
\hline 34 & Ramp & 0.54 & $4.07 E-08$ & 1. & 7.86 & 0.05 & 0.9105 & $1.02 \mathrm{E}-07$ & $2.35 \mathrm{E}-07$ & 3. $5 \mathrm{E}-10$ & 1.079 & 1.091 & 0.009 \\
\hline 35 & 620. & 0.54 & $3.16 \mathrm{E}-08$ & 1. & 7.56 & 0.05 & 0.9514 & $1.34 \mathrm{E}-07$ & $3.22 \mathrm{E}-07$ & 3. $7 \mathrm{E}-10$ & 1.081 & 1.092 & 0.008 \\
\hline 36 & 620. & 0.54 & $1.66 \mathrm{E}-08$ & 1. & 7.17 & 0.05 & 0.9728 & $1.28 \mathrm{E}-07$ & $3.17 \mathrm{E}-07$ & $3.9 \mathrm{E}-10$ & 1.076 & 1.087 & 0.011 \\
\hline 37 & 620. & 0.54 & $9.03 E-09$ & 1. & 6.91 & 0.07 & 0.9845 & $1.23 E-07$ & $3.13 \mathrm{E}-07$ & 4. $3 \mathrm{E}-10$ & 1.084 & 1.095 & 0.014 \\
\hline 38 & 620. & 0.54 & $5.06 \mathrm{E}-09$ & 1. & 6.62 & 0.07 & 0.9910 & $1.20 E-07$ & $3.12 \mathrm{E}-07$ & 5. $3 E-10$ & 1.090 & 1.102 & 0.019 \\
\hline 39 & 620. & 1.08 & $4.60 \mathrm{E}-09$ & 1. & 6.10 & 0.07 & 0.9969 & $1.18 \mathrm{E}-07$ & $3.16 E-07$ & $2.6 \mathrm{E}-10$ & 1.059 & 1.072 & 0.019 \\
\hline 40 & 620. & 0.54 & $9.78 \mathrm{E}-10$ & 1. & 6.11 & 0.19 & 0.9982 & $1.16 \mathrm{E}-07$ & $3.18 \mathrm{E}-07$ & $6.3 \mathrm{E}-10$ & 1.137 & 1.152 & 0.050 \\
\hline 41 & 620. & 0.54 & $5.61 \mathrm{E}-10$ & 1. & 5.69 & 0.26 & 0.9989 & $1.12 \mathrm{E}-07$ & $3.12 \mathrm{E}-07$ & $9.3 \mathrm{E}-10$ & 1.137 & 1.153 & 0.060 \\
\hline 42 & 620. & 4.32 & $8.46 E-10$ & 1. & 4.82 & 0.14 & 1.0000 & & & & & & \\
\hline
\end{tabular}


Experiment \#3

Alv892-1a MORB variolitic glass

2 chips, $28.2 \mathrm{mg}$

Grain size data (um): short side, "visual average", long side.

$15752692 \quad 3962$

$1676 \quad 2235 \quad 3988$

Bulk helium contents: He $=2.17 \mathrm{E}-07 \mathrm{ccSTP} 3 \mathrm{He} / 4 \mathrm{He}=8.55+0.04 \times \mathrm{Ra}$

Sequential release data:

\begin{tabular}{|c|c|c|c|c|c|c|c|c|c|c|c|c|c|}
\hline \# & $\begin{array}{l}\text { Temp. } \\
\text { C }\end{array}$ & $\begin{array}{c}\text { Time } \\
\mathrm{hr}\end{array}$ & $\begin{array}{l}4 \mathrm{He} \\
\operatorname{ccSTP}\end{array}$ & $\begin{array}{l}1 \mathrm{~s} \\
0 / 00\end{array}$ & $\begin{array}{c}3 \mathrm{He} / 4 \mathrm{He} \\
\mathrm{Ra}\end{array}$ & $\begin{array}{l}1 \mathrm{~s} \\
\mathrm{Ra}\end{array}$ & $\begin{array}{l}F(4 \mathrm{He}) \\
(\text { total) }\end{array}$ & $\begin{array}{c}\text { Dsm } \\
\operatorname{cm} 2 / s\end{array}$ & $\begin{array}{l}\text { Drm } \\
\operatorname{cm} 2 / s\end{array}$ & $\begin{array}{l}1 \mathrm{~s} \\
\operatorname{cm} 2 / \mathrm{s}\end{array}$ & $\begin{array}{l}\mathrm{D} 3 / \mathrm{D} 4 \\
\text { Sn }\end{array}$ & $\underset{r m}{\mathrm{D} 3 / \mathrm{D} 4}$ & $\begin{array}{l}1 \mathrm{~s} \\
\mathrm{rm}\end{array}$ \\
\hline 1 & Ramp & 0.53 & $5.62 \mathrm{E}-10$ & 1. & 9.03 & 0.22 & 0.0026 & $2.00 \mathrm{E}-12$ & $4.21 \mathrm{E}-12$ & $6.8 \mathrm{E}-15$ & 1.116 & 1.116 & 0.057 \\
\hline 2 & 211. & 0.54 & $1.04 \mathrm{E}-09$ & 0 . & 8.64 & 0.14 & 0.0074 & $1.41 \mathrm{E}-11$ & 2. $96 \mathrm{E}-11$ & $4.4 E-14$ & 1.045 & 1.045 & 0.035 \\
\hline 3 & 211. & 4.87 & $5.20 \mathrm{E}-09$ & 0 . & 8.72 & 0.06 & 0.0313 & $3.03 \mathrm{E}-11$ & $6.49 \mathrm{E}-11$ & 8. $4 E-14$ & 1.044 & 1.045 & 0.017 \\
\hline 4 & 211. & 4.88 & $3.35 \mathrm{E}-09$ & 0 . & 8.65 & 0.07 & 0.0467 & $4.07 \mathrm{E}-11$ & 8. $58 E-11$ & 1. $8 \mathrm{E}-13$ & 1.033 & 1.033 & 0.026 \\
\hline 5 & 211. & 6.50 & $3.55 \mathrm{E}-09$ & 0 . & 8.56 & 0.06 & 0.0630 & $4.60 \mathrm{E}-11$ & $9.73 \mathrm{E}-11$ & 2. $5 E-13$ & 1.019 & 1.019 & 0.028 \\
\hline 6 & 211. & 7.58 & $3.53 \mathrm{E}-09$ & 3. & 9.15 & 0.20 & 0.0793 & $5.16 \mathrm{E}-11$ & $1.09 \mathrm{E}-10$ & 4. $3 E-13$ & 1.095 & 1.095 & 0.041 \\
\hline 7 & Ramp & 0.54 & $2.00 \mathrm{E}-09$ & 3. & 9.62 & 0.22 & 0.0884 & $4.87 E-10$ & $1.04 \mathrm{E}-09$ & $7.6 \mathrm{E}-12$ & 1.164 & 1.165 & 0.078 \\
\hline 8 & 311. & 0.54 & $3.98 \mathrm{E}-09$ & 3. & 9.31 & 0.20 & 0.1068 & $1.14 \mathrm{E}-09$ & $2.44 \mathrm{E}-09$ & 1. $2 \mathrm{E}-11$ & 1.138 & 1.139 & 0.051 \\
\hline 9 & 311. & 1.08 & $6.80 \mathrm{E}-09$ & 3. & 9.27 & 0.20 & 0.1380 & 1. $25 \mathrm{E}-09$ & $2.68 \mathrm{E}-09$ & 1. $1 \mathrm{E}-11$ & 1.146 & 1.147 & 0.043 \\
\hline 10 & 311. & 1.63 & $8.14 \mathrm{E}-09$ & 2. & 9.03 & 0.19 & 0.1755 & $1.32 \mathrm{E}-09$ & $2.84 \mathrm{E}-09$ & 1. $2 \mathrm{E}-11$ & 1.124 & 1.126 & 0.045 \\
\hline 11 & 311. & 1.63 & $6.69 \mathrm{E}-09$ & 3. & 9.01 & 0.19 & 0.2062 & 1. $36 \mathrm{E}-09$ & $2.95 \mathrm{E}-09$ & 1. $7 E-11$ & 1.124 & 1.126 & 0.059 \\
\hline 12 & 311. & 1.63 & $5.72 \mathrm{E}-09$ & 2. & 8.97 & 0.20 & 0.2325 & $1.38 \mathrm{E}-09$ & $2.99 \mathrm{E}-09$ & $2.1 \mathrm{E}-11$ & 1.120 & 1.122 & 0.073 \\
\hline 13 & Ramp & 0.54 & $7.17 \mathrm{E}-09$ & 3. & 9.06 & 0.19 & 0.2655 & $6.06 \mathrm{E}-09$ & 1. $32 \mathrm{E}-08$ & 8. $3 E-11$ & 1.134 & 1.137 & 0.064 \\
\hline 14 & 415. & 0.54 & $1.17 \mathrm{E}-08$ & 3. & 8.88 & 0.19 & 0.3192 & 1. $21 \mathrm{E}-08$ & $2.67 E-08$ & 1. $2 \mathrm{E}-10$ & 1.114 & 1.117 & 0.047 \\
\hline 15 & 415. & 0.54 & $9.44 \mathrm{E}-09$ & 2 . & 8.94 & 0.19 & 0.3625 & $1.21 \mathrm{E}-08$ & $2.68 \mathrm{E}-08$ & 1. $6 \mathrm{E}-10$ & 1.124 & 1.128 & 0.060 \\
\hline 16 & 415. & 1.08 & $1.53 \mathrm{E}-08$ & 3. & 8.85 & 0.19 & 0.4329 & $1.23 E-08$ & $2.75 E-08$ & 1. $2 \mathrm{E}-10$ & 1.116 & 1.120 & 0.042 \\
\hline 17 & 415. & 0.54 & $6.25 \mathrm{E}-09$ & 3. & 8.81 & 0.20 & 0.4616 & 1. $20 \mathrm{E}-08$ & $2.72 \mathrm{E}-08$ & $2.6 \mathrm{E}-10$ & 1.115 & 1.119 & 0.098 \\
\hline 18 & Ramp & 0.54 & $1.58 \mathrm{E}-08$ & 3. & 8.72 & 0.19 & 0.5342 & $3.65 E-08$ & $8.33 E-08$ & 3. $5 \mathrm{E}-10$ & 1.107 & 1.111 & 0.043 \\
\hline 19 & 517. & 0.54 & 1. $89 \mathrm{E}-08$ & 3. & 8.65 & 0.18 & 0.6210 & $5.80 \mathrm{E}-08$ & $1.34 \mathrm{E}-07$ & 4. $8 \mathrm{E}-10$ & 1.104 & 1.108 & 0.036 \\
\hline 20 & 517. & 1.08 & $2.48 \mathrm{E}-08$ & 2. & 8.47 & 0.18 & 0.7350 & $5.57 \varepsilon-08$ & 1. $30 \mathrm{E}-07$ & 2. $9 \mathrm{E}-10$ & 1.093 & 1.096 & 0.022 \\
\hline 21 & 517. & 0.54 & $8.45 E-09$ & 2 . & 8.48 & 0.19 & 0.7739 & $5.19 \mathrm{E}-08$ & $1.22 \mathrm{E}-07$ & $6.5 E-10$ & 1.110 & 1.113 & 0.052 \\
\hline 22 & 517. & 0.54 & $6.90 \mathrm{E}-09$ & 3. & 8.23 & 0.18 & 0.8056 & $5.03 E-08$ & $1.19 \mathrm{E}-07$ & $7.1 \mathrm{E}-10$ & 1.090 & 1.092 & 0.059 \\
\hline 23 & 517. & 1.08 & $1.08 \mathrm{E}-08$ & 3. & 8.09 & 0.18 & 0.8551 & $4.97 \mathrm{E}-08$ & $1.18 \mathrm{E}-07$ & 3. $9 \mathrm{E}-10$ & 1.090 & 1.089 & 0.032 \\
\hline 24 & 517. & 0.54 & $4.09 \mathrm{E}-09$ & 2. & 8.17 & 0.19 & 0.8740 & $4.77 \mathrm{E}-08$ & $1.13 \mathrm{E}-07$ & 8. $3 E-10$ & 1.113 & 1.121 & 0.072 \\
\hline 25 & 517. & 0.54 & $3.49 \mathrm{E}-09$ & 3 . & 7.95 & 0.18 & 0.8900 & $4.67 \mathrm{E}-08$ & $1.12 \mathrm{E}-07$ & $9.0 \mathrm{E}-10$ & 1.100 & 1.106 & 0.080 \\
\hline 26 & 517. & 1.08 & $5.69 \mathrm{E}-09$ & 2. & 7.77 & 0.18 & 0.9162 & $4.65 E-08$ & $1.12 \mathrm{E}-07$ & $5.1 \mathrm{E}-10$ & 1.095 & 1.101 & 0.045 \\
\hline 27 & Ramp & 0.54 & $5.13 \varepsilon-09$ & 2. & 7.60 & 0.17 & 0.9398 & $1.13 \mathrm{E}-07$ & $2.75 \mathrm{E}-07$ & $1.1 \mathrm{E}-09$ & 1.104 & 1.108 & 0.043 \\
\hline 28 & 619. & 0.54 & $5.09 \mathrm{E}-09$ & 2. & 7.34 & 0.17 & 0.9632 & $1.68 E-07$ & $4.12 \mathrm{E}-07$ & 1. $4 \mathrm{E}-09$ & 1.114 & 1.117 & 0.035 \\
\hline 29 & 619. & 1.08 & $5.04 \mathrm{E}-09$ & 2. & 6.78 & 0.16 & 0.9863 & $1.70 \mathrm{E}-07$ & $4.19 \mathrm{E}-07$ & $6.3 \mathrm{E}-10$ & 1.108 & 1.110 & 0.020 \\
\hline 30 & 619. & 0.54 & $1.14 \mathrm{E}-09$ & 3. & 6.04 & 0.19 & 0.9916 & $1.66 \mathrm{E}-07$ & $4.10 \mathrm{E}-07$ & 1. $4 \mathrm{E}-09$ & 1.067 & 1.068 & 0.049 \\
\hline 31 & 619. & 0.54 & $6.96 \mathrm{E}-10$ & 3. & 5.86 & 0.22 & 0.9948 & $1.64 \mathrm{E}-07$ & $4.05 E-07$ & 1. $7 \mathrm{E}-09$ & 1.071 & 1.072 & 0.063 \\
\hline 32 & 619. & 1.08 & $7.15 \mathrm{E}-10$ & 3. & 5.44 & 0.19 & 0.9981 & $1.70 E-07$ & 4. $22 \mathrm{E}-07$ & $1.1 \mathrm{E}-09$ & 1.022 & 1.022 & 0.054 \\
\hline 33 & 619. & 1.63 & $3.42 \mathrm{E}-10$ & 3. & 5.15 & 0.22 & 0.9996 & $1.92 \mathrm{E}-07$ & $4.75 E-07$ & $1.1 \mathrm{E}-09$ & 0.949 & 0.949 & 0.121 \\
\hline 34 & 619. & 1.63 & $7.85 \mathrm{E}-11$ & 4. & 5.73 & 1.08 & 1.0000 & & & & & & \\
\hline
\end{tabular}


Experiment \#4

Alv892-1a MORB tholeite glass

$300-355$ um sieve fraction, $55.8 \mathrm{mg}$

Grain size data (um): short side, "visual average", long side.

$493 \quad 462385 \quad 431 \quad 462339462308 \quad 308 \quad 462308$

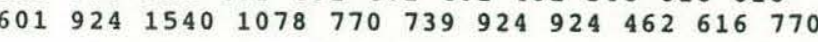

Bulk helium contents: He $=4.48 \mathrm{E}-07 \mathrm{ccSTP} \quad 3 \mathrm{He} / 4 \mathrm{He}=8.32+0.02 \times \mathrm{Ra}$ prior loss corrected: He $=4.61 \mathrm{E}-07$ ccSTP $3 \mathrm{He} / 4 \mathrm{He}=8.34+0.02 \times \mathrm{Ra}$

$2.6 \%$ with $3 \mathrm{He} / 4 \mathrm{He}$ release ratio of 1.056 .

Bulk helium contents:

Sequential release data:

\begin{tabular}{|c|c|c|c|c|c|c|c|c|c|c|c|c|c|}
\hline & $\begin{array}{l}\text { Temp. } \\
\text { C }\end{array}$ & $\begin{array}{c}\mathrm{Time} \\
\mathrm{hr}\end{array}$ & $\begin{array}{l}4 \mathrm{He} \\
\operatorname{ccSTP}\end{array}$ & $\begin{array}{c}1 \mathrm{~s} \\
0 / 00\end{array}$ & $\begin{array}{c}3 \mathrm{He} / 4 \mathrm{He} \\
\mathrm{Ra}\end{array}$ & $\begin{array}{l}\text { Is } \\
\text { Ra }\end{array}$ & $\begin{array}{l}F(4 \mathrm{He}) \\
(\text { total })\end{array}$ & $\begin{array}{c}\text { Dsm } \\
\text { Cm } 2 / s\end{array}$ & $\begin{array}{l}\text { Drm } \\
\operatorname{cm} 2 / s\end{array}$ & $\begin{array}{l}1 \mathrm{~s} \\
\operatorname{cm} 2 / \mathrm{s}\end{array}$ & $\begin{array}{c}\mathrm{D} 3 / \mathrm{D} 4 \\
\mathrm{~s} \text { 列 }\end{array}$ & $\begin{array}{c}\mathrm{D} 3 / \mathrm{D} 4 \\
\mathrm{rm}\end{array}$ & $\begin{array}{l}1 \mathrm{~s} \\
\mathrm{rm}\end{array}$ \\
\hline 1 & Ramp & 1.08 & $3.25 \mathrm{E}-10$ & 1. & & 0.29 & & (1) & & $6=a^{+1}$ & al $r e 1$ & & \\
\hline 2 & 123. & & $45 \mathrm{E}-10$ & & 8.45 & 0.21 & .0293 & $9.63 \mathrm{E}-13$ & 1. $33 \mathrm{E}-13$ & 2. $2 \mathrm{E}-16$ & 0.993 & & .047 \\
\hline 3 & 123. & 6.51 & $.09 \mathrm{E}-09$ & 0 . & .62 & 0.07 & .0490 & $.60 \mathrm{E}-12$ & 1. $34 \mathrm{E}-12$ & 1. $3 E-15$ & 1.027 & & \\
\hline 4 & 123. & 6.50 & -09 & 0 . & .71 & 0.08 & .0623 & $.54 E-12$ & -12 & 2. $9 E-15$ & 1.052 & & 0.021 \\
\hline 5 & 23. & 11.6 & & 1. & .60 & & & & & & & & \\
\hline 6 & Ramp & 0.5 & 09 & 1. & & 0.08 & & & & 1. $5 E-13$ & 1.070 & 1.088 & 0.018 \\
\hline 7 & 236. & 0 & & 1. & & 0. & & & & & & & \\
\hline 8 & & & & 1. & & & & & & & & & \\
\hline 9 & & & & 1. & & & & & & & & & \\
\hline 10 & & & & 0 . & 9 & & & & & & & & \\
\hline 1 & 23 & & & 1. & & 0. & & & & & & & \\
\hline & & & & 1. & & & & & & & & & \\
\hline & 236. & 1 & & 1. & 8.64 & 0.06 & & & & & & & \\
\hline & 236. & 0 & 09 & 1. & 65 & 0.06 & & & & & & & \\
\hline & & & & & & & & & & & & & \\
\hline 6 & 236. & 1. & 08 & 1. & 8.54 & 0.06 & & & & & 1.0 & 1.084 & 0.031 \\
\hline & 236. & & & 1. & & & & & & & & & \\
\hline & & & & 1. & & & & & & & & 1. & \\
\hline 9 & 36. & 1 & & 1. & 8.51 & 0.07 & & & & & 1.0 & & 0.043 \\
\hline & & & & 1 & & & & & & & & & \\
\hline & 236. & & & 1. & & & & & & & 1.077 & 1.084 & 0.052 \\
\hline 2 & 236. & .08 & & 1. & & & & & & & & & \\
\hline & 236 . & & & 1. & & & & & & & & 15 & \\
\hline & & & & 1. & 8.48 & 0.06 & 0.5541 & $8-10$ & 5.1 & $8-12$ & 1.077 & & \\
\hline 5 & 236. & & & 1. & & & & & & & & & \\
\hline & 236 . & & & 1. & & & & & & & 1.0 & 1.0 & 0.048 \\
\hline & 236. & 1.63 & $.16 \mathrm{E}-09$ & 1. & 8.34 & 0.04 & 0.6049 & & & & & & \\
\hline & 236. & 1.63 & $7.52 \mathrm{E}-09$ & 1. & 8.46 & 0.08 & 0.6213 & $1.68 \mathrm{E}-10$ & $5.03 \mathrm{E}-10$ & $4 \cdot 5 \mathrm{E}-12$ & 1.079 & 1.086 & 0.058 \\
\hline
\end{tabular}

continued 


\section{Experiment \#4}

\begin{tabular}{|c|c|c|c|c|c|c|c|c|c|c|c|c|c|}
\hline \# & $\begin{array}{c}\text { Temp. } \\
\text { C }\end{array}$ & $\begin{array}{c}\mathrm{Time} \\
\mathrm{hr}\end{array}$ & $\begin{array}{l}4 \mathrm{He} \\
\text { CCSTP }\end{array}$ & $\begin{array}{l}1 \mathrm{~s} \\
0 / 00\end{array}$ & $\begin{array}{c}3 \mathrm{He} / 4 \mathrm{He} \\
\mathrm{Ra}\end{array}$ & $\begin{array}{l}1 \mathrm{~s} \\
\mathrm{Ra}\end{array}$ & $\begin{array}{l}F(4 \mathrm{He}) \\
(\operatorname{total})\end{array}$ & $\begin{array}{c}\text { Dsm } \\
\operatorname{cm} 2 / \mathrm{s}\end{array}$ & $\begin{array}{l}\text { Drm } \\
\operatorname{cm} 2 / s\end{array}$ & $\begin{array}{l}1 \mathrm{~s} \\
\mathrm{~cm} 2 / \mathrm{s}\end{array}$ & $\begin{array}{l}\mathrm{D} 3 / \mathrm{D} 4 \\
\text { s m }\end{array}$ & $\underset{\mathrm{rm}}{\mathrm{D} 3 / \mathrm{D} 4}$ & $\begin{array}{l}1 \mathrm{~s} \\
\mathrm{rm}\end{array}$ \\
\hline 29 & 236 . & 1.63 & $7.06 \mathrm{E}-09$ & 1. & 8.40 & 0.07 & 0.6366 & $1.67 \mathrm{E}-10$ & $5.04 \mathrm{E}-10$ & $4.9 \mathrm{E}-12$ & 1.074 & 1.080 & 0.063 \\
\hline 30 & 236 . & 1.63 & $6.64 \mathrm{E}-09$ & 1. & 8.47 & 0.06 & 0.6510 & $1.66 \mathrm{E}-10$ & $5.03 \mathrm{E}-10$ & $5.5 \mathrm{E}-12$ & 1.084 & 1.091 & 0.068 \\
\hline 31 & 236 . & 1.63 & $6.13 \mathrm{E}-09$ & 1. & 8.34 & 0.05 & 0.6643 & $1.61 \mathrm{E}-10$ & $4.92 \mathrm{E}-10$ & $5.8 E-12$ & 1.069 & 1.076 & 0.074 \\
\hline 32 & 236. & 1.63 & $5.83 E-09$ & 1. & 8.41 & 0.07 & 0.6769 & $1.61 \mathrm{E}-10$ & $4.94 \mathrm{E}-10$ & 6. $2 \mathrm{E}-12$ & 1.079 & 1.087 & 0.079 \\
\hline 33 & 236 . & 1.63 & $5.47 \mathrm{E}-09$ & 1. & 8.39 & 0.06 & 0.6888 & 1. $59 \mathrm{E}-10$ & $4.89 \mathrm{E}-10$ & $6.7 E-12$ & 1.079 & 1.087 & 0.084 \\
\hline 34 & 236 . & 1.63 & $5.15 \mathrm{E}-09$ & 1. & 8.46 & 0.08 & 0.6999 & $1.57 \mathrm{E}-10$ & 4. $84 E-10$ & $7.0 \mathrm{E}-12$ & 1.090 & 1.098 & 0.091 \\
\hline 35 & 236 . & 1.63 & $4.91 \mathrm{E}-09$ & 1. & 8.38 & 0.06 & 0.7106 & 1. $56 \mathrm{E}-10$ & $4.84 E-10$ & $7.4 \mathrm{E}-12$ & 1.082 & 1.090 & 0.096 \\
\hline 36 & 236. & 1.63 & $4.64 \mathrm{E}-09$ & 1. & 8.41 & 0.06 & 0.7207 & $1.54 E-10$ & $4.80 E-10$ & $8.0 \mathrm{E}-12$ & 1.087 & 1.095 & 0.103 \\
\hline 37 & 236. & 1.63 & $4.35 \mathrm{E}-09$ & 1. & 8.25 & 0.07 & 0.7301 & $1.50 \mathrm{E}-10$ & $4.70 \mathrm{E}-10$ & $8.6 \mathrm{E}-12$ & 1.068 & 1.076 & 0.110 \\
\hline 38 & 236. & 1.63 & $4.09 \mathrm{E}-09$ & 1. & 8.43 & 0.07 & 0.7390 & $1.47 \mathrm{E}-10$ & $4.61 \mathrm{E}-10$ & $9.1 \mathrm{E}-12$ & 1.095 & 1.103 & 0.119 \\
\hline 39 & 236. & 1.63 & $3.82 \mathrm{E}-09$ & 1. & 8.23 & 0.07 & 0.7473 & $1.42 \mathrm{E}-10$ & $4.49 \mathrm{E}-10$ & $9.4 \mathrm{E}-12$ & 1.071 & 1.080 & 0.127 \\
\hline 40 & 236 . & 1.63 & $3.72 \mathrm{E}-09$ & 1. & 8.41 & 0.08 & 0.7553 & 1. $44 \mathrm{E}-10$ & $4.56 \mathrm{E}-10$ & $9.9 \mathrm{E}-12$ & 1.095 & 1.103 & 0.132 \\
\hline 41 & 236. & 2.69 & $6.04 \mathrm{E}-09$ & 0 . & 8.16 & 0.05 & 0.7684 & $1.49 \mathrm{E}-10$ & $4.73 \mathrm{E}-10$ & $6.6 \mathrm{E}-12$ & 1.066 & 1.075 & 0.084 \\
\hline 42 & 236 . & 2.71 & $5.56 E-09$ & 0 . & 8.17 & 0.06 & 0.7805 & $1.44 \mathrm{E}-10$ & $4.61 \mathrm{E}-10$ & $7.0 \mathrm{E}-12$ & 1.069 & 1.077 & 0.092 \\
\hline 43 & 236 . & 2.71 & $5.19 \mathrm{E}-09$ & 0 . & 8.20 & 0.07 & 0.7917 & $1.43 E-10$ & $4.59 \mathrm{E}-10$ & $7.7 E-12$ & 1.076 & 1.085 & 0.100 \\
\hline 44 & 236 . & 2.71 & $4.80 \mathrm{E}-09$ & 0 . & 8.31 & 0.06 & 0.8022 & 1. $39 \mathrm{E}-10$ & $4.51 \mathrm{E}-10$ & $8.1 E-12$ & 1.095 & 1.104 & 0.109 \\
\hline 45 & 236. & 2.71 & $4.36 \mathrm{E}-09$ & 0 . & 8.30 & 0.07 & 0.8116 & 1. $34 \mathrm{E}-10$ & 4. $34 \mathrm{E}-10$ & $8.6 \mathrm{E}-12$ & 1.098 & 1.107 & 0.121 \\
\hline 46 & 236 . & 2.71 & $4.18 \mathrm{E}-09$ & 0 . & 8.20 & 0.06 & 0.8207 & 1. $35 E-10$ & $4.41 \mathrm{E}-10$ & $9.4 \mathrm{E}-12$ & 1.090 & 1.098 & 0.128 \\
\hline 47 & 236 . & 2.71 & $3.94 \mathrm{E}-09$ & 1. & 8.27 & 0.07 & 0.8292 & $1.35 \mathrm{E}-10$ & $4.41 \mathrm{E}-10$ & $1.0 \mathrm{E}-11$ & 1.104 & 1.113 & 0.137 \\
\hline 48 & 236 . & 3.25 & $4.41 \mathrm{E}-09$ & 0 . & 8.21 & 0.07 & 0.8388 & 1. $32 \mathrm{E}-10$ & 4. $36 \mathrm{E}-10$ & $9.0 \mathrm{E}-12$ & 1.102 & 1.110 & 0.125 \\
\hline 49 & 236 . & 2.71 & $3.65 \mathrm{E}-09$ & 1. & 8.17 & 0.09 & 0.8467 & 1. $40 \mathrm{E}-10$ & $4.61 \mathrm{E}-10$ & 1. $2 \mathrm{E}-11$ & 1.103 & 1.109 & 0.152 \\
\hline 50 & Ramp & 0.54 & $7.03 \mathrm{E}-08$ & 1. & 7.46 & 0.06 & 0.9993 & $7.51 \mathrm{E}-08$ & $2.97 \mathrm{E}-07$ & $1.0 \mathrm{E}-10$ & 1.067 & 1.081 & 0.014 \\
\hline 51 & 620. & 0.54 & 3. $24 \mathrm{E}-10$ & 2. & 5.20 & 0.35 & 1.0000 & & & & & & \\
\hline
\end{tabular}


Experiment \#5

Alv892-1a MORB tholeite glass

$75-106$ um sieve fraction, $259.2 \mathrm{mg}$

Grain size data (um):

100 "mean diameter" measurements grouped by size (um)

side lengths approximated from inspection as : $x, .75 x, .75 x$

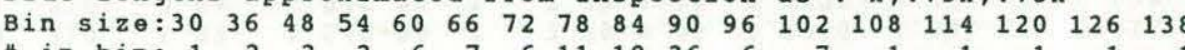

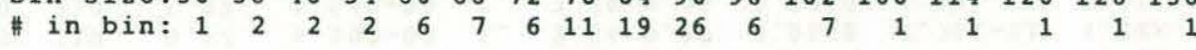

Bulk helium contents: He $=2.00 \mathrm{E}-06$ ccSTP $3 \mathrm{He} / 4 \mathrm{He}=8.23+0.01 \times \mathrm{Ra}$ prior loss corrected: $\mathrm{He}=2.13 \mathrm{E}-06 \mathrm{ccSTP} \quad 3 \mathrm{He} / 4 \mathrm{He}=8.26+0.01 \times \mathrm{Ra}$ 5.8 with $3 \mathrm{He} / 4 \mathrm{He}$ diffusivity ratio of 1.056 .

Sequential release data:

\begin{tabular}{|c|c|c|c|c|c|c|c|c|c|c|c|c|c|}
\hline \# & $\begin{array}{l}\text { Temp. } \\
\text { C }\end{array}$ & $\begin{array}{c}\mathrm{Time} \\
\mathrm{hr}\end{array}$ & $\begin{array}{l}4 \mathrm{He} \\
\text { CсSTP }\end{array}$ & $\begin{array}{l}1 \mathrm{~s} 3 \\
100\end{array}$ & $\begin{array}{c}3 \mathrm{He} / 4 \mathrm{He} \\
\mathrm{Ra}\end{array}$ & $\begin{array}{l}1 \mathrm{~s} \\
\mathrm{Ra}\end{array}$ & $\begin{array}{l}F(4 \mathrm{He}) \\
(\text { total })\end{array}$ & $\begin{array}{c}\text { Dsm } \\
\operatorname{cm} 2 / \mathrm{s}\end{array}$ & $\begin{array}{l}\operatorname{Drm} \\
\operatorname{cm} 2 / \mathrm{s}\end{array}$ & $\begin{array}{l}1 \mathrm{~s} \\
\mathrm{~cm} 2 / \mathrm{s}\end{array}$ & $\begin{array}{l}\mathrm{D} 3 / \mathrm{D} 4 \\
\mathrm{~s} \text { m }\end{array}$ & $\begin{array}{c}\mathrm{D} 3 / \mathrm{D} 4 \\
\mathrm{r} \approx\end{array}$ & $\begin{array}{l}1 \mathrm{~s} \\
\mathrm{rm}\end{array}$ \\
\hline 1 & 21. & 16.22 & $8.22 \mathrm{E}-10$ & 1. & 7.97 & 0.18 & 0.0582 & PL101 105S & & & 21 & & \\
\hline 2 & 21. & 11.90 & $5.72 \mathrm{E}-10$ & 1 . & 9.06 & 0.17 & 0.0585 & 1. $35 \mathrm{E}-15$ & $9.08 \mathrm{E}-16$ & 2. $2 \mathrm{E}-17$ & 1.172 & 1.165 & 0.820 \\
\hline 3 & 21. & 16.22 & 7. $52 \mathrm{E}-10$ & 1 . & 8.52 & 0.20 & 0.0589 & 1. $31 \mathrm{E}-15$ & $8.80 E-16$ & 1. $6 \mathrm{E}-17$ & 1.102 & 1.095 & 0.626 \\
\hline 4 & 21. & 26.50 & $1.21 \mathrm{E}-09$ & 0 . & 8.50 & 0.15 & 0.0594 & $1.31 \mathrm{E}-15$ & $8.72 \mathrm{E}-16$ & $9.8 \mathrm{E}-18$ & 1.087 & 1.093 & 0.391 \\
\hline 5 & 21. & 28.66 & 1. $30 \mathrm{E}-09$ & 0 . & 8.37 & 0.12 & 0.0600 & 1. $32 \mathrm{E}-15$ & $8.81 \mathrm{E}-16$ & $9.8 E-18$ & 1.079 & 1.076 & 0.362 \\
\hline 6 & 21. & 29.78 & 1. $32 \mathrm{E}-09$ & 1 . & 8.39 & 0.14 & 0.0607 & 1. $29 \mathrm{E}-15$ & $8.68 \mathrm{E}-16$ & $9.5 \mathrm{E}-18$ & 1.082 & 1.078 & 0.358 \\
\hline 7 & 21. & 28.13 & 1. $29 \mathrm{E}-09$ & 1. & 8.33 & 0.11 & 0.0613 & 1. $35 E-15$ & $9.05 E-16$ & 1. $0 \mathrm{E}-17$ & 1.063 & 1.068 & 0.367 \\
\hline 8 & 21. & 22.72 & $1.06 \mathrm{E}-09$ & 1. & 8.51 & 0.13 & 0.0618 & 1. $39 \mathrm{E}-15$ & $9.31 E-16$ & 1. $3 \mathrm{E}-17$ & 1.092 & 1.091 & 0.447 \\
\hline 9 & Ramp & 2.16 & $4.64 \mathrm{E}-10$ & 1 . & 8.72 & 0.18 & 0.0620 & $6.46 \mathrm{E}-15$ & $4.32 \mathrm{E}-15$ & 1. $3 E-16$ & 1.117 & 1.116 & 1.016 \\
\hline 10 & 47. & 0.54 & 3. $36 \mathrm{E}-10$ & 1 . & 8.39 & 0.32 & 0.0621 & $1.89 \mathrm{E}-14$ & $1.25 \mathrm{E}-14$ & 5. $2 \mathrm{E}-16$ & 1.066 & 1.082 & 1.250 \\
\hline 11 & 47. & 9.74 & $6.34 \mathrm{E}-09$ & 0. & 8.56 & 0.05 & 0.0651 & $2.01 \mathrm{E}-14$ & $1.35 \mathrm{E}$ & $3.3 \mathrm{E}$ & 1.097 & 1.098 & 0.076 \\
\hline 12 & 47. & 12.98 & $7.48 \mathrm{E}-09$ & 0 . & 8.55 & 0.06 & 0.0686 & $1.88 \mathrm{E}-14$ & 1. $26 \mathrm{E}-14$ & $2.8 \mathrm{E}-17$ & 1.096 & 1.096 & 0.066 \\
\hline 13 & 47. & 11.90 & $7.04 \mathrm{E}-09$ & 1. & 8.50 & 0.07 & 0.0720 & $2.03 \mathrm{E}-14$ & 1. $36 \mathrm{E}-14$ & 3. $5 E-17$ & 1.087 & 1.089 & 0.070 \\
\hline 14 & 47 & 12.98 & $6.83 \mathrm{E}-09$ & 1. & 8.36 & 0.07 & 0.0752 & $1.90 \mathrm{E}-14$ & $1.27 E-14$ & 3. $7 E-17$ & 1.070 & 1.070 & 0.072 \\
\hline 15 & Ramp & 0.54 & $1.07 \mathrm{E}-09$ & 1 . & 8.65 & 0.19 & 0.0757 & 7. $38 \mathrm{E}-14$ & $4.94 \mathrm{E}-14$ & 8. $9 E-16$ & 1.100 & 1.105 & 0.452 \\
\hline 16 & 77. & 4.33 & 1. $52 \mathrm{E}-08$ & 0 . & 8.59 & 0.05 & 0.0828 & $1.37 \mathrm{E}-13$ & $9.20 \mathrm{E}-14$ & 1. $4 \mathrm{E}-16$ & 1.097 & 1.098 & 0.034 \\
\hline 17 & 77. & 3.79 & $1.36 \mathrm{E}-08$ & 0. & 8.53 & 0.05 & 0.0892 & 1. $53 E-13$ & $1.03 E-13$ & $1.9 \mathrm{E}-16$ & 1.088 & 1.090 & 0.038 \\
\hline 18 & 77. & 4.33 & $1.28 \mathrm{E}-08$ & 0 . & 8.61 & 0.05 & 0.0952 & 1. $36 \mathrm{E}-13$ & $9.18 \mathrm{E}-14$ & $1.8 \mathrm{E}-16$ & 1.098 & 1.099 & 0.041 \\
\hline 19 & 77. & 5.41 & $1.52 \mathrm{E}-08$ & 0 . & 8.68 & 0.05 & 0.1024 & 1. $39 \mathrm{E}-13$ & $9.42 \mathrm{E}-14$ & $1.8 \mathrm{E}-16$ & 1.107 & 1.108 & 0.036 \\
\hline 20 & 77. & 6.49 & $1.58 \mathrm{E}-08$ & 0 . & 8.42 & 0.04 & 0.1098 & 1. $30 \mathrm{E}-13$ & $8.81 \mathrm{E}-14$ & 1. $7 \mathrm{E}-16$ & 1.072 & 1.074 & 0.035 \\
\hline 21 & $R a m p$ & 0.53 & $5.15 \mathrm{E}-09$ & 0 . & 8.07 & 0.07 & 0.1122 & $5.47 \mathrm{E}-13$ & $3.71 \mathrm{E}-13$ & 2. $2 \mathrm{E}-15$ & 1.027 & 1.028 & 0.105 \\
\hline 22 & 112. & 0.54 & $1.04 \mathrm{E}-08$ & 0. & 8.63 & 0.04 & 0.1171 & $1.12 \mathrm{E}-12$ & $7.57 E-13$ & 2. $3 E-15$ & 1.097 & 1.099 & 0.053 \\
\hline 23 & 112. & 1.62 & $3.09 \mathrm{E}-08$ & 0. & 8.58 & 0.03 & 0.1317 & 1. $21 \mathrm{E}-12$ & $8.26 \mathrm{E}-13$ & $1.0 \mathrm{E}-15$ & 1.091 & 1.093 & 0.019 \\
\hline 24 & 112. & 1.62 & 2. $80 \mathrm{E}-08$ & 1. & 8.61 & 0.05 & 0.1448 & 1. $24 \mathrm{E}-12$ & $8.46 \mathrm{E}-13$ & 1. $3 E-15$ & 1.095 & 1.097 & 0.022 \\
\hline 25 & 112. & 1.08 & $1.73 E-08$ & 1. & 8.62 & 0.06 & 0.1530 & 1. $25 \mathrm{E}-12$ & $8.55 E-13$ & $2.1 \mathrm{E}-15$ & 1.097 & 1.099 & 0.036 \\
\hline 26 & 112. & 1.08 & $1.63 \mathrm{E}-08$ & 1. & 8.56 & 0.06 & & 1. $24 \mathrm{E}-12$ & $8.53 \mathrm{E}-13$ & $2.4 E-15$ & 1.090 & 1.092 & 0.039 \\
\hline 27 & 112. & 1.08 & $1.58 \mathrm{E}-08$ & 1. & 8.57 & 0.06 & 0.1681 & $1.27 \mathrm{E}-12$ & $8.77 E-13$ & $2.6 \mathrm{E}-15$ & 1.091 & 1.093 & 0.041 \\
\hline
\end{tabular}
continued 
Experiment \#5 (continued)

\begin{tabular}{|c|c|c|c|c|c|c|c|c|c|c|c|c|c|}
\hline & $\begin{array}{c}\text { Temp. } \\
\mathrm{C}\end{array}$ & $\begin{array}{c}\text { Time } \\
\text { hr }\end{array}$ & $\begin{array}{l}4 \mathrm{He} \\
\text { CCSTP }\end{array}$ & $\begin{array}{l}1 \mathrm{~s} \\
100\end{array}$ & $\begin{array}{c}3 \mathrm{He} / 4 \mathrm{He} \\
\mathrm{Ra}\end{array}$ & $\begin{array}{l}1 \mathrm{~s} \\
\mathrm{Ra}\end{array}$ & $\begin{array}{l}F(4 \mathrm{He}) \\
(\text { total })\end{array}$ & $\begin{array}{c}\text { Dsm } \\
\operatorname{cm} 2 / s\end{array}$ & $\begin{array}{l}\text { Drm } \\
\operatorname{cm} 2 / s\end{array}$ & $\begin{array}{l}1 \mathrm{~s} \\
\mathrm{~cm} 2 / \mathrm{s}\end{array}$ & $\begin{array}{c}\mathrm{D} 3 / \mathrm{D} 4 \\
\mathrm{~s} \text { m }\end{array}$ & $\begin{array}{c}\mathrm{D} 3 / \mathrm{D} 4 \\
\mathrm{rm}\end{array}$ & $\begin{array}{l}1 \mathrm{~s} \\
\mathrm{rm}\end{array}$ \\
\hline 28 & $\operatorname{Ramp}$ & 1.08 & $.29 E-08$ & 1. & 8.68 & 0.06 & .1835 & $2.86 \mathrm{E}-12$ & $1.98 \mathrm{E}-12$ & 3. $3 \mathrm{E}-15$ & 1.105 & 1.108 & 0.021 \\
\hline 29 & 150. & 0.54 & $5.60 \mathrm{E}-08$ & 1. & 8.55 & 0.05 & & & $7.76 \mathrm{E}-12$ & $9.1 \mathrm{E}-15$ & 1.090 & 1.093 & 0.015 \\
\hline 30 & 150. & 0.54 & $4.82 \mathrm{E}-08$ & 1. & 8.62 & 0.05 & 0.2325 & $1.11 \mathrm{E}-11$ & 7. $73 \mathrm{E}-12$ & 1. $1 \mathrm{E}-14$ & 1.100 & 1.103 & 0.018 \\
\hline 31 & 150. & 1.08 & $8.05 E-08$ & 1. & 8.48 & 0.05 & 0.2704 & $1.08 \mathrm{E}-11$ & $7.65 \mathrm{E}-12$ & $8.1 E-15$ & 1.083 & 1.086 & 0.012 \\
\hline 32 & 150. & 0.54 & $3.49 \mathrm{E}-08$ & 1. & 8.57 & 0.05 & 0.2868 & $1.07 \mathrm{E}-11$ & $7.62 \mathrm{E}-12$ & & 1.094 & & 0.027 \\
\hline 33 & 150. & 0.54 & $3.19 \mathrm{E}-08$ & 1. & 8.54 & 0.05 & 0.3018 & $1.05 \mathrm{E}-11$ & $7.53 \mathrm{E}-12$ & 2. $0 \mathrm{E}-14$ & 1.091 & 1.095 & 0.030 \\
\hline 34 & Cool & 11.90 & $.60 E-08$ & 1. & 8.40 & 0.06 & 0.3093 & $2.52 \mathrm{E}-13$ & $1.81 \mathrm{E}-13$ & $9.9 \mathrm{E}-16$ & 1.073 & 1.078 & 0.060 \\
\hline 35 & Ramp & 0.53 & $.00 E-08$ & 0 . & 8.66 & 0.05 & 0.3375 & $2.30 \mathrm{E}-11$ & $1.66 \mathrm{E}-11$ & 2. $6 E-14$ & 1.108 & 1.113 & 0.017 \\
\hline 36 & 210. & .54 & $.69 \mathrm{E}-07$ & 1. & 8.50 & 0.04 & 0.4168 & $7.87 E-11$ & & $4.5 \mathrm{E}$ & 1.091 & 1.097 & 0.008 \\
\hline 37 & 210. & 1.08 & & 0 . & 8.40 & 0.03 & & & & & & & 0.006 \\
\hline 38 & 210. & 0.54 & $8.60 \mathrm{E}-08$ & 1. & 8.20 & 0.05 & 0.5582 & $7.20 \mathrm{E}-11$ & $5.66 \mathrm{E}-11$ & 7. $9 \mathrm{E}-14$ & 1.058 & 1.067 & 0.014 \\
\hline 39 & 210. & 0.54 & -08 & 1. & 8.26 & 0.05 & .59 & & & & .066 & 1.075 & 0.016 \\
\hline 40 & 210. & $1 . c$ & .22 & 1. & 7.84 & 0.05 & & 6. & & & & & 0.009 \\
\hline 41 & 210. & 1.08 & .82 & 1. & 8.05 & 0.05 & . & & 5.7 & & 1.0 & 1. & 0.010 \\
\hline 42 & 210. & 1. & 08 & 1. & & & & & & & & & \\
\hline 43 & 210. & 0.54 & $3.39 \mathrm{E}-08$ & 1. & .18 & 0.05 & 0.7549 & 6.311 & $5.56 \mathrm{~B}$ & $1.3 \mathrm{E}$ & 1.049 & 1.058 & 0.023 \\
\hline 44 & 210. & 0. & -08 & 1. & & 0.06 & & & & & & & 0.024 \\
\hline 45 & 210. & 1.08 & .56 & 1. & 8.08 & 0.05 & 8 & 6.14 & 5.5 & 14 & 1. & 49 & 0.013 \\
\hline 46 & 210. & 0.5 & 08 & 1. & & 0.05 & & & & & & & 0.027 \\
\hline 47 & 210. & 0 & & 0 . & .07 & 0.05 & & 5.84 & 5.35 & $1.4 !$ & 1.045 & 1.05 & 0.028 \\
\hline 48 & 210. & 1.08 & $4.13 E-08$ & 1. & .92 & 0.05 & 0.8373 & 5.86 & $5.47 \mathrm{E}$ & $7.2 \mathrm{E}$ & 1.027 & 1.036 & 0.014 \\
\hline 49 & 210. & & -08 & 1. & 7.99 & 0.06 & & & 5.31 & & 1.039 & 046 & 0.030 \\
\hline 50 & 210. & 0.54 & $.68 \mathrm{E}-08$ & 1. & 8.04 & 0.06 & 0.8537 & 5.511 & 5.22 & $1.5 \mathrm{E}$ & 1.048 & 1.055 & 0.032 \\
\hline 51 & 210. & 08 & -08 & 1. & & & 0.8676 & & & & & & 0.017 \\
\hline 52 & 210. & 0 & -08 & 1. & 5 & 0.06 & & 4.99 & 4.82 & 1.6 & 1.051 & 1.062 & 0.037 \\
\hline 53 & 210. & 0.54 & $1.23 E-08$ & 1. & 8.01 & 0.07 & 0.8795 & $4.95 \mathrm{E}$ & $4.82 \mathrm{E}$ & $1.7 \mathrm{E}-13$ & 1.048 & 1.060 & 0.037 \\
\hline 54 & 210. & & & 0 . & & 0.05 & & & & & 1.042 & 1.054 & 0.019 \\
\hline 55 & 210. & 0.5 & $.06 E-08$ & 1. & 8.00 & 0.06 & 0.8954 & 4.89 & $4.86 \mathrm{E}-11$ & $1.7 \mathrm{E}-13$ & 1.053 & 1.066 & 0.040 \\
\hline 56 & 210. & 0.54 & $9.97 \mathrm{E}-09$ & 1. & 7.91 & 0.07 & & & 4.83 & & & & \\
\hline 57 & & & $88 \mathrm{E}-08$ & & 7.86 & 0.05 & 0.9089 & -11 & 4.93 & & 1.040 & 1.052 & 0.021 \\
\hline 58 & 210. & 0.54 & $.56 E-09$ & 1. & 7.85 & 0.07 & 0.9130 & $4.76 \mathrm{E}-11$ & $4.85 \mathrm{E}-11$ & 1. $7 \mathrm{E}-13$ & 1.043 & 1.055 & 0.044 \\
\hline 59 & 210. & 0.54 & & 1. & & & & & & & & & 0.044 \\
\hline 60 & 210. & & $1.54 \mathrm{E}-08$ & 1. & & & & $4.80 \mathrm{E}-11$ & $4.95 \mathrm{E}-11$ & $7.9 \mathrm{E}-14$ & 1.039 & 1.053 & 0.023 \\
\hline 61 & 210. & 0.54 & $6.94 \mathrm{E}-09$ & 1. & 7.77 & 0.08 & 0.9273 & $4.63 \mathrm{E}-11$ & & & 1.039 & & \\
\hline 62 & 210. & & & 1. & & & & & & & 1.059 & 1.073 & 0.051 \\
\hline 63 & 210. & 1.08 & & 1. & 7.76 & 0.06 & 0.9361 & $4.56 \mathrm{E}-11$ & $4.81 \mathrm{E}-11$ & $8.8 \mathrm{E}-14$ & 1.043 & 1.057 & 0.026 \\
\hline 64 & 210. & 0.54 & $5.61 \mathrm{E}-09$ & 1. & 7.74 & 0.07 & 0.9388 & & & & & 1.057 & 0.055 \\
\hline 65 & 210. & & & 1. & & & & & $4.82 \mathrm{E}-11$ & 1. $9 E-13$ & 1.052 & 1.065 & 0.056 \\
\hline 66 & & 1.08 & $1.03 \mathrm{E}-08$ & 1. & 7.63 & 0.06 & 0.9462 & $4.56 \mathrm{E}-11$ & $4.91 \mathrm{E}-11$ & 1. $2 \mathrm{E}-13$ & & & \\
\hline 67 & 210. & 0.54 & $4.63 E-09$ & 1. & 7.69 & 0.06 & 0.9484 & $4.36 \mathrm{E}-11$ & $4.73 E-11$ & $2.0 \mathrm{E}-13$ & 1.044 & 1.059 & 0.061 \\
\hline
\end{tabular}


Experiment \#5 (continued)

\begin{tabular}{|c|c|c|c|c|c|c|c|c|c|c|c|c|c|}
\hline \# & $\begin{array}{c}\text { Temp. } \\
\text { C }\end{array}$ & $\begin{array}{c}\mathrm{Time} \\
\mathrm{hr}\end{array}$ & $\begin{array}{l}4 \mathrm{He} \\
\operatorname{ccSTP}\end{array}$ & $\begin{array}{c}1 \mathrm{~s} \\
0 / 00\end{array}$ & $\begin{array}{c}3 \mathrm{He} / 4 \mathrm{He} \\
\mathrm{Ra}\end{array}$ & $\begin{array}{l}1 \mathrm{~s} \\
\mathrm{Ra}\end{array}$ & $\begin{array}{l}F(4 \mathrm{He}) \\
(\operatorname{total})\end{array}$ & $\begin{array}{c}\text { Dsm } \\
\mathrm{Cm} 2 / \mathrm{s}\end{array}$ & $\begin{array}{l}\text { Drm } \\
\mathrm{cm} 2 / \mathrm{s}\end{array}$ & $\begin{array}{l}1 \mathrm{~s} \\
\mathrm{~cm} 2 / \mathrm{s}\end{array}$ & $\begin{array}{c}\mathrm{D} 3 / \mathrm{D} 4 \\
\mathrm{sm}\end{array}$ & $\begin{array}{c}\mathrm{D} 3 / \mathrm{D} 4 \\
\mathrm{r} \mathbf{m}\end{array}$ & $\begin{array}{l}1 \mathrm{~s} \\
\mathrm{rm}\end{array}$ \\
\hline 68 & Ramp & 0.54 & $7.96 \mathrm{E}-09$ & 1. & 7.75 & 0.08 & 0.9521 & $7.92 \mathrm{E}-11$ & $8.67 \mathrm{E}-11$ & $2.4 \mathrm{E}-13$ & 1.055 & 1.069 & 0.035 \\
\hline 69 & 240. & 0.54 & $1.13 \mathrm{E}-08$ & 1. & 7.63 & 0.06 & 0.9574 & $1.24 \mathrm{E}-10$ & $1.37 \mathrm{E}-10$ & 2. $3 E-13$ & 1.044 & 1.060 & 0.023 \\
\hline 70 & 240 . & 1.08 & $1.88 \mathrm{E}-08$ & 1. & 7.50 & 0.05 & 0.9663 & 1. $23 \mathrm{E}-10$ & 1. $39 \mathrm{E}-10$ & 1. $1 \mathrm{E}-13$ & 1.033 & 1.048 & 0.013 \\
\hline 71 & 240. & 0.54 & $7.60 \mathrm{E}-09$ & 1. & 7.45 & 0.06 & 0.9699 & $1.18 \mathrm{E}-10$ & $1.36 \mathrm{E}-10$ & $2.3 \mathrm{E}-13$ & 1.031 & 1.047 & 0.028 \\
\hline 72 & Ramp & 1.08 & $1.42 \mathrm{E}-08$ & 0 . & 7.52 & 0.04 & 0.9766 & 1. $32 \mathrm{E}-10$ & 1. $56 \mathrm{E}-10$ & $1.1 \mathrm{E}-13$ & 1.048 & 1.066 & 0.015 \\
\hline 73 & 270 . & 0.54 & 1. $21 \mathrm{E}-08$ & 0 . & 7.42 & 0.06 & 0.9822 & $2.93 \mathrm{E}-10$ & $3.57 \mathrm{E}-10$ & $2.4 \mathrm{E}-13$ & 1.048 & 1.069 & 0.014 \\
\hline 74 & 270 . & 0.54 & $8.86 \mathrm{E}-09$ & 0 . & 7.28 & 0.04 & 0.9864 & $2.82 \mathrm{E}-10$ & $3.55 \mathrm{E}-10$ & $2.5 E-13$ & 1.040 & 1.062 & 0.018 \\
\hline 75 & 270 . & 1.62 & $1.53 \mathrm{E}-08$ & 0. & 7.07 & 0.05 & 0.9936 & $2.64 E-10$ & $3.54 \mathrm{E}-10$ & $9.8 \mathrm{E}-14$ & 1.024 & 1.047 & 0.009 \\
\hline 76 & 270 . & 0.54 & $2.72 \mathrm{E}-09$ & 0. & 6.91 & 0.10 & 0.9949 & $2.35 \mathrm{E}-10$ & $3.33 \mathrm{E}-10$ & $3.5 \mathrm{E}-13$ & 1.013 & 1.036 & 0.033 \\
\hline 77 & Ramp & 1.08 & $4.49 \mathrm{E}-09$ & 0. & 6.65 & 0.07 & 0.9970 & $2.80 \mathrm{E}-10$ & $4.15 \mathrm{E}-10$ & 1. $9 \mathrm{E}-13$ & 0.970 & 0.989 & 0.019 \\
\hline 78 & 299. & 0.54 & $2.01 \mathrm{E}-09$ & 0 . & 6.55 & 0.10 & 0.9979 & $3.96 \mathrm{E}-10$ & $6.16 \mathrm{E}-10$ & $4.7 \mathrm{E}-13$ & 0.935 & 0.950 & 0.032 \\
\hline 79 & 299. & 0.54 & $1.18 \mathrm{E}-09$ & 1. & 6.36 & 0.12 & 0.9985 & $3.29 \mathrm{E}-10$ & $5.29 \mathrm{E}-10$ & $5.8 \mathrm{E}-13$ & 0.881 & 0.891 & 0.046 \\
\hline 80 & 299. & 1.08 & $1.17 \mathrm{E}-09$ & 0 . & 6.37 & 0.14 & 0.9990 & $2.38 \mathrm{E}-10$ & $3.96 \mathrm{E}-10$ & $4.0 \mathrm{E}-13$ & 0.836 & 0.841 & 0.040 \\
\hline 81 & 299. & 0.54 & $2.82 \mathrm{E}-10$ & 1. & 7.30 & 0.31 & 0.9992 & $1.56 \mathrm{E}-10$ & $2.65 \mathrm{E}-10$ & $9.0 \mathrm{E}-13$ & 0.914 & 0.917 & 0.133 \\
\hline 82 & 299. & 0.54 & $1.89 \mathrm{E}-10$ & 2. & 7.14 & 0.72 & 0.9993 & $1.19 \mathrm{E}-10$ & $2.04 \mathrm{E}-10$ & $8.2 \mathrm{E}-13$ & 0.883 & 0.885 & 0.167 \\
\hline 83 & $\mathrm{Ramp}$ & 1.08 & $1.09 \mathrm{E}-09$ & 0 . & 8.25 & 0.19 & 0.9998 & $6.13 \mathrm{E}-10$ & $1.09 \mathrm{E}-09$ & $1.1 \mathrm{E}-12$ & 1.028 & 1.030 & 0.030 \\
\hline 84 & 425 . & 0.54 & $2.74 \mathrm{E}-10$ & 1. & 7.85 & 0.33 & 0.9999 & $8.58 \mathrm{E}-10$ & $1.60 \mathrm{E}-09$ & 3. $2 \mathrm{E}-12$ & 0.995 & 0.997 & 0.070 \\
\hline 85 & 425. & 2.16 & $2.18 \mathrm{E}-10$ & 2 . & 7.90 & 0.43 & 1.0000 & & & & & & \\
\hline & & & & & & & & & & & & & \\
\hline
\end{tabular}


Experiment \#6

Alv892-1a MORB tholeite glass

$38-53$ um sieve fraction, $110.6 \mathrm{mg}$

Grain size data (um):

100 "mean diameter" measurements grouped by size

Side lengths approximated from inspection as : $x, .75 x, .75 x$

$\begin{array}{llllllllllr}\text { Bin size:18 } & 24 & 30 & 36 & 42 & 48 & 54 & 60 & 66 & 72 \\ \text { \# in bin: } & 8 & 16 & 25 & 13 & 17 & 9 & 6 & 1 & 3\end{array}$

Bulk helium contents: He $=8.87 \mathrm{E}-07$ c CSTP $3 \mathrm{He} / 4 \mathrm{He}=8.31+0.01 \times \mathrm{Ra}$ prior loss corrected: $\mathrm{He}=9.78 \mathrm{E}-07 \mathrm{ccSTP} \quad 3 \mathrm{He} / 4 \mathrm{He}=8.35+0.01$ $9.3 \%$ with $3 \mathrm{He} / 4 \mathrm{He}$ diffusivity ratio of 1.054 .

sequential release data:

\begin{tabular}{|c|c|c|c|c|c|c|c|c|c|c|c|c|c|}
\hline \# & $\begin{array}{l}\text { Temp. } \\
\text { C }\end{array}$ & $\begin{array}{c}\text { Time } \\
\text { hr }\end{array}$ & $\begin{array}{l}4 \mathrm{He} \\
\operatorname{cCSTP}\end{array}$ & $\begin{array}{l}1 \mathrm{~s} \\
0 / 00\end{array}$ & $\underset{\mathrm{Ra}}{3 \mathrm{He} / 4 \mathrm{He}}$ & $\begin{array}{l}1 \mathrm{~s} \\
\mathrm{Ra}\end{array}$ & $\begin{array}{l}F(4 \mathrm{He}) \\
(\text { total })\end{array}$ & $\begin{array}{c}\text { Dsm } \\
\text { Cm } 2 / \mathrm{s}\end{array}$ & $\begin{array}{l}\text { Drm } \\
\operatorname{cm} 2 / s\end{array}$ & $\begin{array}{l}1 \mathrm{~s} \\
\mathrm{~cm} 2 / \mathrm{s}\end{array}$ & $\begin{array}{c}\mathrm{D} 3 / \mathrm{D} 4 \\
\text { s m }\end{array}$ & $\underset{\mathrm{rm}}{\mathrm{D} 3 / \mathrm{D} 4}$ & $\begin{array}{l}1 \mathrm{~s} \\
\mathrm{rm}\end{array}$ \\
\hline 1 & 26. & 3.25 & 5. $22 \mathrm{E}-10$ & 0 . & 8.82 & 0.30 & 0.0935 & Prior loss & & d fractic & nal $r e 1$ & 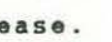 & \\
\hline 2 & 26. & 11.39 & 1. $66 \mathrm{E}-09$ & 1. & 8.65 & 0.11 & 0.0952 & $3.57 \mathrm{E}-15$ & $2.89 \mathrm{E}-15$ & 2. $3 E-17$ & 1.095 & & 0.264 \\
\hline 3 & 26. & 12.47 & $2.12 \mathrm{E}-09$ & 0. & 8.40 & 0.11 & 0.0974 & 4. $25 \mathrm{E}-15$ & $3.44 \mathrm{E}-15$ & 2. $3 E-17$ & 1.064 & 1.066 & 0.207 \\
\hline 4 & 26. & 12.47 & $1.64 E-09$ & 0. & 8.79 & 0.09 & 0.0991 & $3.37 E-15$ & $2.73 E-15$ & 2. $2 \mathrm{E}-17$ & 1.112 & 1.113 & 0.266 \\
\hline 5 & 26 . & 13.57 & $2.10 \mathrm{E}-09$ & 0. & 8.75 & 0.09 & 0.1012 & $4.05 E-15$ & $3.28 E-15$ & 2. $2 E-17$ & 1.107 & 1.109 & 0.209 \\
\hline 6 & 26 & 15.64 & $2.33 E-09$ & 0. & 8.37 & 0.10 & 0.1036 & 3. $98 \mathrm{E}-15$ & 3. $23 \mathrm{E}-15$ & $2.0 \mathrm{E}-17$ & 1.059 & 1.060 & 0.189 \\
\hline 7 & Ramp & 0.54 & $7.79 \mathrm{E}-10$ & 1. & 8.53 & 0.18 & 0.1044 & $3.91 \mathrm{E}-14$ & $3.18 \mathrm{E}-14$ & $6.0 \mathrm{E}-16$ & 1.079 & 1.081 & 0.561 \\
\hline 8 & 50 . & 1.08 & $2.54 \mathrm{E}-09$ & 0. & 8.67 & 0.11 & 0.1070 & $6.49 \mathrm{E}-14$ & 5. $28 \mathrm{E}-14$ & 3. $2 \mathrm{E}-16$ & 1.095 & 1.098 & 0.174 \\
\hline 9 & 50. & 2.17 & 5. $39 \mathrm{E}-09$ & 0. & 8.61 & 0.08 & 0.1125 & $7.17 \mathrm{E}-14$ & $5.83 \mathrm{E}-14$ & 1. $8 \mathrm{E}-16$ & 1.087 & 1.089 & 0.083 \\
\hline 10 & 50. & 2.71 & $5.78 \mathrm{E}-09$ & 1. & 8.68 & 0.08 & 0.1184 & $6.50 \mathrm{E}-14$ & $5.31 \mathrm{E}-14$ & 1. $6 \mathrm{E}-16$ & 1.096 & 1.098 & 0.078 \\
\hline 11 & 50 . & 2.71 & $4.63 \mathrm{E}-09$ & 0 . & 8.56 & 0.08 & 0.1232 & $5.47 E-14$ & $4.47 E-14$ & 1. $7 \mathrm{E}-16$ & 1.080 & 1.083 & 0.097 \\
\hline 12 & 50. & 4.88 & $7.85 \mathrm{E}-09$ & 0. & 8.46 & 0.06 & 0.1312 & $5.46 \mathrm{E}$ & 4.471 & 1. $1 \mathrm{E}-16$ & 1.067 & 1.068 & 0.058 \\
\hline 13 & 50. & 4.88 & $7.54 \mathrm{E}-09$ & 0 . & 8.57 & 0.07 & 0.1389 & $5.60 \mathrm{E}-14$ & $4.60 \mathrm{E}-14$ & 1. $3 E-16$ & 1.079 & 1.081 & 0.061 \\
\hline 14 & Ramp & 0.54 & $1.82 \mathrm{E}-08$ & 0. & 8.75 & 0.05 & 0.1575 & 1. $35 E-12$ & 1. $11 \mathrm{E}-12$ & 1. $5 \mathrm{E}-15$ & 1.102 & 1.104 & 0.027 \\
\hline 15 & 118. & 0.54 & $2.66 \mathrm{E}-08$ & 0. & 8.61 & 0.05 & 0.1847 & $2.34 E-12$ & $1.95 \mathrm{E}-12$ & 2. $2 \mathrm{E}-15$ & 1.084 & 1.087 & 0.020 \\
\hline 16 & 118. & 1.08 & $4.14 \mathrm{E}-08$ & 0 . & 8.53 & 0.05 & 0.2270 & 2. $26 \mathrm{E}-12$ & $1.90 \mathrm{E}-12$ & 1. $8 \mathrm{E}-15$ & 1.072 & 1.076 & 0.014 \\
\hline 17 & 118. & 0.54 & $1.63 \mathrm{E}-08$ & 0 . & 8.56 & 0.05 & 0.2437 & $2.10 \mathrm{E}-12$ & 1. $78 \mathrm{E}-12$ & 4. $2 \mathrm{E}-15$ & 1.074 & 1.077 & 0.033 \\
\hline 18 & 118. & 0.54 & $1.45 \mathrm{E}-08$ & 0 . & 8.51 & 0.05 & 0.2585 & $2.02 \mathrm{E}-12$ & $1.72 \mathrm{E}-12$ & $4.8 \mathrm{E}-15$ & 1.067 & 1.072 & 0.037 \\
\hline 19 & 118. & 1.09 & $2.57 E-08$ & 0 . & 8.43 & 0.05 & 0.2848 & $1.98 \mathrm{E}-12$ & 1. $71 \mathrm{E}-12$ & $3.0 \mathrm{E}-15$ & 1.057 & 1.061 & 0.022 \\
\hline 20 & 118. & 0.54 & 1. $12 \mathrm{E}-08$ & 0 . & 8.53 & 0.06 & 0.2962 & $1.88 \mathrm{E}-12$ & $1.63 \mathrm{E}-12$ & $6.7 \mathrm{E}-15$ & 1.067 & 1.072 & 0.050 \\
\hline 21 & Ramp & 0.54 & $5.19 \mathrm{E}-08$ & 0. & 8.60 & 0.05 & 0.3494 & $1.01 \mathrm{E}-11$ & $8.86 \mathrm{E}-12$ & $9.8 \mathrm{E}-15$ & 1.078 & 1.083 & 0.013 \\
\hline 22 & 150 . & 0.54 & $7.51 \mathrm{E}-08$ & 1. & 8.24 & 0.05 & 0.4262 & $1.90 \mathrm{E}-11$ & $1.71 \mathrm{E}-11$ & $2.0 \mathrm{E}-14$ & 1.029 & 1.034 & 0.010 \\
\hline 23 & 150. & 1.08 & $9.48 \mathrm{E}-08$ & 0. & 8.45 & 0.04 & 0.5232 & $1.65 E-11$ & $1.56 \mathrm{E}-11$ & 1. $3 \mathrm{E}-14$ & 1.053 & 1.059 & 0.008 \\
\hline 24 & 150. & 0.54 & $3.73 \mathrm{E}-08$ & 0 . & 8.45 & 0.05 & 0.5614 & $1.64 \mathrm{E}-11$ & $1.61 \mathrm{E}-11$ & $2.9 \mathrm{E}-14$ & 1.056 & 1.064 & 0.017 \\
\hline 25 & 150. & 1.08 & $5.86 E-08$ & 1. & 8.35 & 0.05 & 0.6213 & $1.54 \mathrm{E}-11$ & $1.56 \mathrm{E}-11$ & 1. $6 \mathrm{E}-14$ & 1.045 & 1.054 & 0.011 \\
\hline 26 & 150. & 0.54 & $2.39 \mathrm{E}-08$ & 0 . & 8.45 & 0.05 & 0.6458 & $1.46 \mathrm{E}-11$ & $1.52 \mathrm{E}-11$ & 3. $6 \mathrm{E}-14$ & 1.059 & 1.069 & 0.024 \\
\hline 27 & 150. & 1.08 & $4.03 E-08$ & 0 . & 8.35 & 0.05 & 0.6870 & $1.40 \mathrm{E}-11$ & $1.49 \mathrm{E}-11$ & $2.0 \mathrm{E}-14$ & 1.050 & 1.061 & 0.014 \\
\hline
\end{tabular}

continued 
Experiment \#6 (continued)

\begin{tabular}{|c|c|c|c|c|c|c|c|c|c|c|c|c|c|}
\hline \# & $\begin{array}{l}\text { Temp. } \\
\text { C }\end{array}$ & $\begin{array}{c}\text { Time } \\
\mathrm{hr}\end{array}$ & $\begin{array}{l}4 \mathrm{He} \\
\text { ccSTP }\end{array}$ & $\begin{array}{l}1 \mathrm{~s} \\
0 / 00\end{array}$ & $\begin{array}{c}3 \mathrm{He} / 4 \mathrm{He} \\
\mathrm{Ra}\end{array}$ & $\begin{array}{l}1 \mathrm{~s} \\
\mathrm{Ra}\end{array}$ & $\begin{array}{l}F(4 \mathrm{He}) \\
(\operatorname{total})\end{array}$ & $\begin{array}{c}\text { Dsm } \\
\mathrm{cm} 2 / \mathrm{s}\end{array}$ & $\begin{array}{l}\text { Drm } \\
\operatorname{cm} 2 / \mathrm{s}\end{array}$ & $\begin{array}{l}1 \mathrm{~s} \\
\mathrm{~cm} 2 / \mathrm{s}\end{array}$ & $\begin{array}{l}\mathrm{D} 3 / \mathrm{D} 4 \\
\text { s a }\end{array}$ & $\begin{array}{c}\mathrm{D} 3 / \mathrm{D} 4 \\
\mathrm{rm}\end{array}$ & $\begin{array}{l}1 \mathrm{~s} \\
\mathrm{rm}\end{array}$ \\
\hline 28 & Ramp & 13.01 & $4.71 \mathrm{E}-08$ & 0. & 8.31 & 0.05 & 0.7351 & $1.63 \mathrm{E}-12$ & $1.80 \mathrm{E}-12$ & 1. $9 \mathrm{E}-15$ & 1.049 & 1.061 & 0.012 \\
\hline 29 & 211. & 0.54 & $4.01 \mathrm{E}-08$ & 0. & 8.33 & 0.05 & 0.7761 & $4.04 \mathrm{E}-11$ & $4.68 \mathrm{E}-11$ & $5.0 E-14$ & 1.057 & 1.071 & 0.012 \\
\hline 30 & 211. & 0.54 & $3.86 E-08$ & 0 . & 8.24 & 0.04 & 0.8157 & $4.76 \mathrm{E}-11$ & $5.77 \mathrm{E}-11$ & $5.6 \mathrm{E}-14$ & 1.053 & 1.068 & 0.012 \\
\hline 31 & 211. & 1.08 & $5.53 \mathrm{E}-08$ & 0 . & 8.10 & 0.04 & 0.8722 & $4.60 \mathrm{E}-11$ & $5.89 \mathrm{E}-11$ & 3. $4 \mathrm{E}-14$ & 1.042 & 1.062 & 0.007 \\
\hline 32 & 211. & 1.08 & $3.60 \mathrm{E}-08$ & 0 . & 7.97 & 0.04 & 0.9090 & $4.27 \mathrm{E}-11$ & $5.94 \mathrm{E}-11$ & 3. $6 \mathrm{E}-14$ & 1.042 & 1.061 & 0.008 \\
\hline 33 & 211. & 0.54 & $1.30 \mathrm{E}-08$ & 0 . & 7.94 & 0.05 & 0.9223 & 3. $96 \mathrm{E}-11$ & $5.78 E-11$ & $7.6 \mathrm{E}-14$ & 1.049 & 1.068 & 0.017 \\
\hline 34 & 211. & 0.54 & $1.07 \mathrm{E}-08$ & 0 . & 7.82 & 0.05 & 0.9332 & $3.81 \mathrm{E}-11$ & $5.70 \mathrm{E}-11$ & 7. $9 \mathrm{E}-14$ & 1.041 & 1.059 & 0.019 \\
\hline 35 & 211. & 1.08 & $1.67 \mathrm{E}-08$ & 0. & 7.71 & 0.04 & 0.9503 & $3.71 \mathrm{E}-11$ & $5.76 \mathrm{E}-11$ & $4.5 \mathrm{E}-14$ & 1.034 & 1.052 & 0.011 \\
\hline 36 & 211. & 1.08 & $1.19 \mathrm{E}-08$ & 0 . & 7.65 & 0.04 & 0.9625 & $3.52 \mathrm{E}-11$ & $5.70 E-11$ & $5.1 \mathrm{E}-14$ & 1.037 & 1.054 & 0.014 \\
\hline 37 & 211. & 1.08 & $8.50 \mathrm{E}-09$ & 0 . & 7.56 & 0.06 & 0.9711 & 3. $31 \mathrm{E}-11$ & $5.54 \mathrm{E}-11$ & $6.2 \mathrm{E}-14$ & 1.035 & 1.051 & 0.018 \\
\hline 38 & 211. & 1.08 & $6.11 \mathrm{E}-09$ & 0. & 7.59 & 0.07 & 0.9774 & $3.07 \mathrm{E}-11$ & $5.29 \mathrm{E}-11$ & 7. $4 \mathrm{E}-14$ & 1.050 & 1.067 & 0.021 \\
\hline 39 & Ramp & 2.71 & $1.79 \mathrm{E}-08$ & 0 . & 7.32 & 0.04 & 0.9957 & $8.36 \mathrm{E}-11$ & 1. $58 \mathrm{E}-10$ & $4.0 \mathrm{E}-14$ & 1.052 & 1.068 & 0.010 \\
\hline 40 & 310. & 0.54 & $2.93 \mathrm{E}-09$ & 0 . & 6.58 & 0.08 & 0.9987 & $3.02 \mathrm{E}-10$ & $6.24 \mathrm{E}-10$ & $4.6 \mathrm{E}-13$ & 0.996 & 1.007 & 0.037 \\
\hline 41 & 310. & 0.54 & $4.47 \mathrm{E}-10$ & 1. & 6.88 & 0.27 & 0.9992 & 1. $10 \mathrm{E}-10$ & 2. $36 \mathrm{E}-10$ & $6.5 \mathrm{E}-13$ & 1.050 & 1.059 & 0.147 \\
\hline 42 & 310. & 1.08 & $2.00 \mathrm{E}-10$ & 1. & 7.46 & 0.47 & 0.9994 & 3. $55 \mathrm{E}-11$ & $7.70 \mathrm{E}-11$ & 4. $1 \mathrm{E}-13$ & 1.180 & 1.189 & 0.304 \\
\hline 43 & 310. & 0.54 & $4.59 \mathrm{E}-11$ & 4. & 7.99 & 1.76 & 0.9994 & $1.97 \mathrm{E}-11$ & $4.28 \mathrm{E}-11$ & $8.7 E-13$ & 1.311 & 1.323 & 1.175 \\
\hline 44 & 310. & 0.54 & $2.91 \mathrm{E}-11$ & 10. & 9.06 & 3.41 & 0.9995 & 1. $33 \mathrm{E}-11$ & $2.90 \mathrm{E}-11$ & $8.8 \mathrm{E}-13$ & 1.523 & 1.538 & 1.697 \\
\hline 45 & 310. & 1.08 & $5.84 \mathrm{E}-11$ & 7. & 10.99 & 1.57 & 0.9995 & $1.45 E-11$ & $3.16 \mathrm{E}-11$ & 4. $3 \mathrm{E}-13$ & 1.991 & 2.015 & 0.969 \\
\hline 46 & 310. & 1.63 & $6.02 \mathrm{E}-11$ & 5. & 10.00 & 1.63 & 0.9996 & $1.13 \mathrm{E}-11$ & $2.48 \mathrm{E}-11$ & 3. $1 \mathrm{E}-13$ & 2.058 & 2.084 & 1.011 \\
\hline 47 & 310. & 1.63 & $4.77 \mathrm{E}-11$ & 6. & 5.95 & 1.73 & 0.9996 & $1.02 \mathrm{E}-11$ & $2.25 \mathrm{E}-11$ & 3. $2 \mathrm{E}-13$ & 1.346 & 1.361 & 1.230 \\
\hline 48 & 310. & 1.63 & $3.37 \mathrm{E}-11$ & 9. & 7.37 & 2.00 & 0.9997 & $8.00 \mathrm{E}-12$ & $1.76 \mathrm{E}-11$ & $3.1 \mathrm{E}-13$ & 1.765 & 1.790 & 1.759 \\
\hline 49 & Ramp & 2.17 & $3.18 \mathrm{E}-10$ & 1. & 4.17 & 0.22 & 1.0000 & $1.85 E-10$ & $4.19 \mathrm{E}-10$ & 3. $4 \mathrm{E}-12$ & 1.363 & 1.378 & 0.685 \\
\hline 50 & 619. & 0.54 & 7. $35 \mathrm{E}-12$ & 54. & 0.002 & 1.97 & 1.0000 & 1. $36 \mathrm{E}-10$ & $3.13 \mathrm{E}-10$ & 1. $3 \mathrm{E}-11$ & 0.000 & 0.000 & 0.000 \\
\hline 51 & 619. & 1.08 & $1.03 \mathrm{E}-11$ & 19. & 2.37 & 5.23 & 1.0000 & & & & & & \\
\hline
\end{tabular}




\section{Experiment \#7}

Charcog8-11T MORB tholeitic glass 8 chips, $15.7 \mathrm{mg}$

Grain size data (um): short side, "visual average", long side.

$\begin{array}{llllllll}914 & 787 & 406 & 711 & 1016 & 965 & 889 & 889\end{array}$

$\begin{array}{llllllll}1321 & 940 & 1067 & 1524 & 1016 & 1143 & 1270 & 1270\end{array}$

$\begin{array}{lllllllll}1651 & 2134 & 1930 & 2667 & 2235 & 1626 & 1727 & 2083\end{array}$

Bulk helium contents: He $=1.52 \mathrm{E}-07 \mathrm{ccSTP} \quad 3 \mathrm{He} / 4 \mathrm{He}=8.77+0.04 \times \mathrm{Ra}$

Sequential release data:

\begin{tabular}{|c|c|c|c|c|c|c|c|c|c|c|c|c|c|}
\hline \# & $\begin{array}{l}\text { Temp. } \\
\text { c }\end{array}$ & $\underset{\mathrm{Tr}}{\mathrm{Time}}$ & $\begin{array}{l}4 \mathrm{He} \\
\operatorname{ccSTP}\end{array}$ & $\begin{array}{l}1 \mathrm{~s} \\
0 / 00\end{array}$ & $\begin{array}{c}3 \mathrm{He} / 4 \mathrm{He} \\
\mathrm{Ra} \\
\end{array}$ & $\begin{array}{l}1 \mathbf{s} \\
\mathrm{Ra}\end{array}$ & $\begin{array}{l}\mathrm{F}(4 \mathrm{He}) \\
(\operatorname{tota} 1)\end{array}$ & $\begin{array}{c}\text { Dsm } \\
\operatorname{cm} 2 / s\end{array}$ & $\begin{array}{l}\text { Drm } \\
\operatorname{cra} 2 / s\end{array}$ & $\begin{array}{l}1 \mathrm{~s} \\
\mathrm{~cm} 2 / \mathrm{s}\end{array}$ & $\begin{array}{l}\mathrm{D} 3 / \mathrm{D} 4 \\
\mathrm{sm}\end{array}$ & $\begin{array}{c}\mathrm{D} 3 / \mathrm{D} 4 \\
\mathrm{r}=\end{array}$ & $\begin{array}{l}1 \mathrm{~s} \\
\mathrm{r} \text { m }\end{array}$ \\
\hline 1 & 106. & 0.53 & $1.62 \mathrm{E}-10$ & 2 . & 7.11 & 0.56 & 0.0011 & $8.70 E-14$ & $1.78 \mathrm{E}-13$ & 9. $3 \mathrm{E}-16$ & 0.657 & 0.657 & 0.104 \\
\hline 2 & 106. & 7.03 & $9.15 \mathrm{E}-10$ & 1. & 8.96 & 0.16 & 0.0071 & $2.84 \mathrm{E}-13$ & $5.82 \mathrm{E}-13$ & $1.1 \mathrm{E}-15$ & 0.987 & 0.987 & 0.038 \\
\hline 3 & 106. & 17.85 & $1.05 E-09$ & 1. & 9.20 & 0.19 & 0.0140 & 3. $33 \mathrm{E}-13$ & $6.86 \mathrm{E}-13$ & 1. $5 E-15$ & 1.059 & 1.059 & 0.042 \\
\hline 4 & 106. & 25.42 & $9.13 \mathrm{E}-10$ & 19. & 9.38 & 4.66 & 0.0200 & 3. $27 \mathrm{E}-13$ & $6.78 \mathrm{E}-13$ & $8.6 E-14$ & 1.099 & 1.100 & 0.715 \\
\hline 5 & $\mathrm{CoO}$ & 21.64 & $1.58 \mathrm{E}-09$ & 1. & 7.02 & 0.10 & 0.0304 & $9.89 \mathrm{E}-13$ & $2.07 \mathrm{E}-12$ & 1. $5 E-13$ & 0.789 & 0.788 & 0.384 \\
\hline 6 & Ramp & 0.54 & $2.08 \mathrm{E}-11$ & 14. & 12.26 & 3.07 & 0.0306 & $6.27 E-13$ & 1. $31 \mathrm{E}-12$ & $3.8 \mathrm{E}-12$ & 1.335 & 1.334 & 13.930 \\
\hline 7 & 151. & 0.54 & $1.57 \mathrm{E}-10$ & 3. & 10.45 & 0.71 & 0.0316 & $4.84 E-12$ & $1.02 \mathrm{E}-11$ & $6.4 \mathrm{E}-12$ & 1.144 & 1.143 & 2.393 \\
\hline 8 & 151. & 18.39 & $4.08 \mathrm{E}-09$ & 0 . & 8.86 & 0.08 & 0.0584 & $5.55 \mathrm{E}-12$ & $1.14 \mathrm{E}-11$ & 3. $4 \mathrm{E}-13$ & 0.986 & 0.986 & 0.163 \\
\hline 9 & 151. & 17.72 & $2.63 \mathrm{E}-09$ & 1. & 9.08 & 0.10 & 0.0757 & $5.64 \mathrm{E}-12$ & $1.16 \mathrm{E}-11$ & $4 \cdot 6 E-13$ & 1.025 & 1.025 & 0.219 \\
\hline 10 & 151. & 28.55 & 3. $26 \mathrm{E}-09$ & 0 . & 8.97 & 0.06 & 0.0972 & $5.68 \mathrm{E}-12$ & $1.17 \mathrm{E}-11$ & 3. $7 E-13$ & 1.022 & 1.022 & 0.173 \\
\hline 11 & 151. & 28.01 & $2.62 \mathrm{E}-09$ & 0 . & 9.04 & 0.06 & 0.1144 & $5.80 \mathrm{E}-12$ & $1.20 \mathrm{E}-11$ & $4.4 \mathrm{E}-13$ & 1.036 & 1.036 & 0.204 \\
\hline 12 & Ramp & 1.08 & $3.99 \mathrm{E}-10$ & 1. & 9.33 & 0.27 & 0.1171 & $2.52 \mathrm{E}-11$ & $5.23 \mathrm{E}-11$ & 1. $2 \mathrm{E}-11$ & 1.072 & 1.073 & 1.181 \\
\hline 13 & 223 . & 6.39 & $6.12 \mathrm{E}-09$ & 0 . & 9.21 & 0.06 & 0.1573 & $7.91 \mathrm{E}-11$ & $1.65 \mathrm{E}-10$ & 2. $7 \mathrm{E}-12$ & 1.067 & 1.067 & 0.091 \\
\hline 14 & 223. & 8.65 & $5.80 \mathrm{E}-09$ & 0 . & 8.83 & 0.07 & 0.1955 & $7.38 \mathrm{E}-11$ & $1.55 \mathrm{E}-10$ & 2. $4 \mathrm{E}-12$ & 1.027 & 1.028 & 0.089 \\
\hline 15 & 223. & 8.65 & $4.55 E-09$ & 0 . & 8.97 & 0.07 & 0.2254 & $7.14 \mathrm{E}-11$ & $1.50 \mathrm{E}-10$ & 2. $8 \mathrm{E}-12$ & 1.043 & 1.043 & 0.106 \\
\hline 16 & 223. & 11.35 & $4.94 \mathrm{E}-09$ & 0 . & 8.94 & 0.07 & 0.2578 & $6.99 \mathrm{E}-11$ & $1.48 \mathrm{E}-10$ & $2.4 \mathrm{E}-12$ & 1.041 & 1.042 & 0.094 \\
\hline 17 & 223. & 11.90 & $4.60 \mathrm{E}-09$ & 0 . & 9.04 & 0.08 & 0.2881 & $7.27 \mathrm{E}-11$ & 1. $55 \mathrm{E}-10$ & $2.6 \mathrm{E}-12$ & 1.054 & 1.055 & 0.096 \\
\hline 18 & Ramp & 1.08 & $1.06 \mathrm{E}-08$ & 1. & 9.30 & 0.06 & 0.3581 & $2.31 \mathrm{E}-09$ & $4.98 \mathrm{E}-09$ & $3 \cdot 5 E-11$ & 1.095 & 1.097 & 0.042 \\
\hline 19 & 413. & 1.08 & $2.54 \mathrm{E}-08$ & 0 . & 9.05 & 0.05 & 0.5250 & $8.85 \mathrm{E}-09$ & $1.95 \mathrm{E}-08$ & $5.0 E-11$ & 1.080 & 1.083 & 0.016 \\
\hline 20 & 413. & 0.54 & $8.02 \mathrm{E}-09$ & 0 . & 8.97 & 0.07 & 0.5778 & $8.19 \mathrm{E}-09$ & $1.84 E-08$ & 1. $1 \mathrm{E}-10$ & 1.080 & 1.085 & 0.039 \\
\hline 21 & 413. & 0.54 & $6.44 \mathrm{E}-09$ & 0 . & 8.86 & 0.07 & 0.6201 & $7.79 \mathrm{E}-09$ & $1.77 \mathrm{E}-08$ & 1. $2 \mathrm{E}-10$ & 1.072 & 1.077 & 0.044 \\
\hline 22 & 413. & 1.62 & $1.45 \mathrm{E}-08$ & 1. & 8.83 & 0.05 & 0.7152 & $7.57 \mathrm{E}-09$ & $1.76 E-08$ & $4.6 \mathrm{E}-11$ & 1.075 & 1.081 & 0.019 \\
\hline 23 & Ramp & 0.54 & $2.47 \mathrm{E}-08$ & 0 . & 8.52 & 0.05 & 0.8776 & $7.38 \mathrm{E}-08$ & 1. $76 E-07$ & 1. $6 E-10$ & 1.053 & 1.064 & 0.008 \\
\hline 24 & 617. & 0.54 & $1.17 \mathrm{E}-08$ & 1. & 7.82 & 0.06 & 0.9544 & $8.64 E-08$ & $2.21 \mathrm{E}-07$ & 1. $6 \mathrm{E}-10$ & 0.987 & 0.991 & 0.008 \\
\hline 25 & 617. & 0.54 & $2.46 \mathrm{E}-09$ & 0 . & 7.09 & 0.07 & 0.9706 & $3.84 \mathrm{E}-08$ & $1.00 \mathrm{E}-07$ & 1. $7 \mathrm{E}-10$ & 0.864 & 0.865 & 0.020 \\
\hline 26 & 617. & 1.08 & $1.15 \mathrm{E}-09$ & 1. & 7.81 & 0.11 & 0.9782 & $1.30 \mathrm{E}-08$ & $3.42 \mathrm{E}-08$ & $8.8 E-11$ & 0.910 & 0.910 & 0.034 \\
\hline 27 & 617. & 1.62 & $7.97 \mathrm{E}-10$ & 1. & 9.25 & 0.15 & 0.9834 & $8.01 \mathrm{E}-09$ & $2.11 \mathrm{E}-08$ & $6.4 \mathrm{E}-11$ & 1.074 & 1.074 & 0.043 \\
\hline 28 & 617. & 6.49 & $1.72 \mathrm{E}-09$ & 1. & 8.72 & 0.10 & 0.9947 & $8.31 \mathrm{E}-09$ & $2.21 \mathrm{E}-08$ & $1.8 \mathrm{E}-11$ & 1.043 & 1.043 & 0.024 \\
\hline 29 & 617. & 9.10 & $8.07 \mathrm{E}-10$ & 1. & 8.12 & 0.21 & 1.0000 & & & & & & \\
\hline
\end{tabular}


Experiment \#8

Aftermath D9 alkali basalt glass

17 chips, $49.1 \mathrm{mg}$

Grain size data (um): short side, "visual average", long side.

$864 \quad 1397838 \quad 1016 \quad 1016 \quad 711 \quad 1219 \quad 1041889965 \quad 1067813 \quad 8386351016965 \quad 1245$

$\begin{array}{lllllllllllllllll}1600 & 1473 & 1651 & 1067 & 1422 & 1194 & 1346 & 1321 & 1524 & 1143 & 1270 & 1067 & 1067 & 17271422 & 1295 & 1372\end{array}$

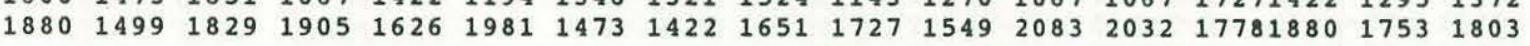

Bulk helium contents: He $=3.99 \mathrm{E}-08 \mathrm{ccSTP} 3 \mathrm{He} / 4 \mathrm{He}=7.23+0.02 \times \mathrm{Ra}$

Sequential release data:

\begin{tabular}{|c|c|c|c|c|c|c|c|c|c|c|c|c|c|}
\hline & $\begin{array}{l}\text { Temp. } \\
\text { c }\end{array}$ & $\begin{array}{c}\mathrm{Time} \\
\mathrm{hr}\end{array}$ & $\begin{array}{l}4 \mathrm{HO} \\
\operatorname{ccSTP}\end{array}$ & $\begin{array}{l}1 \mathrm{~s} \\
0 / 00\end{array}$ & $\begin{array}{c}3 \mathrm{He} / 4 \mathrm{He} \\
\mathrm{Ra} \\
.\end{array}$ & $\begin{array}{l}1 \mathrm{~s} \\
\mathrm{Ra}\end{array}$ & $\begin{array}{l}F(4 \mathrm{He}) \\
(\operatorname{total})\end{array}$ & $\begin{array}{c}\text { Ds m } \\
\text { Cm } 2 / \mathrm{s}\end{array}$ & $\begin{array}{l}\text { Drm } \\
\operatorname{cm} 2 / \mathrm{s}\end{array}$ & $\begin{array}{l}1 \mathrm{~s} \\
\mathrm{c}=2 / \mathrm{s}\end{array}$ & $\begin{array}{l}\mathrm{D} 3 / \mathrm{D} 4 \\
\mathbf{3 m}\end{array}$ & $\underset{r=}{\mathrm{D} 3 / \mathrm{D} 4}$ & $\begin{array}{l}1 \mathrm{~s} \\
\mathrm{rm}\end{array}$ \\
\hline 1 & $R a m p$ & 0.53 & 3. $72 \mathrm{E}-10$ & 1. & 7.09 & 0.28 & 0.0093 & $9.48 \mathrm{E}-12$ & 1. $60 \mathrm{E}-11$ & $3.7 E-14$ & 0.960 & 0.960 & 0.076 \\
\hline 2 & 150. & 2.71 & $7.45 \mathrm{E}-10$ & 1. & 7.32 & 0.25 & 0.0280 & 1. $50 \mathrm{E}-11$ & $2.56 \mathrm{E}-11$ & $4.0 \mathrm{E}-14$ & 1.009 & 1.009 & 0.060 \\
\hline 3 & 150. & 3.79 & $9.18 \mathrm{E}-10$ & 1. & 7.52 & 0.15 & 0.0511 & $2.89 \mathrm{E}-11$ & $4.87 \mathrm{E}-11$ & 7. $3 \mathrm{E}-14$ & 1.054 & 1.054 & 0.050 \\
\hline 4 & 150. & 4.87 & $7.92 \mathrm{E}-10$ & 1. & 7.54 & 0.13 & 0.0709 & $3.04 \mathrm{E}-11$ & $5.14 \mathrm{E}-11$ & $9.5 E-14$ & 1.068 & 1.068 & 0.057 \\
\hline 5 & 150. & 4.87 & $5.90 E-10$ & 1. & 7.80 & 0.13 & 0.0857 & $2.95 \mathrm{E}-11$ & $5.01 \mathrm{E}-11$ & 1. $3 E-13$ & 1.113 & 1.114 & 0.074 \\
\hline 6 & 150. & 4.87 & $5.24 \mathrm{E}-10$ & 1. & 7.32 & 0.15 & 0.0989 & $3.13 \mathrm{E}-11$ & $5.32 \mathrm{E}-11$ & $1.6 \mathrm{E}-13$ & 1.049 & 1.050 & 0.084 \\
\hline 7 & Ramp & 0.54 & $5.52 \mathrm{E}-10$ & 1. & 7.23 & 0.18 & 0.1127 & 3. $44 \mathrm{E}-10$ & $5.86 \mathrm{E}-10$ & 1. $8 \mathrm{E}-12$ & 1.033 & 1.034 & 0.083 \\
\hline 8 & 278 . & 2.17 & $5.16 \mathrm{E}-09$ & 0 . & 7.54 & 0.08 & 0.2420 & $1.45 \mathrm{E}-09$ & $2.51 \mathrm{E}-09$ & 1. $6 \mathrm{E}-12$ & 1.085 & 1.086 & 0.017 \\
\hline 9 & 278 . & 3.25 & $4.96 \mathrm{E}-09$ & 0 . & 7.43 & 0.07 & 0.3665 & $1.81 \mathrm{E}-09$ & $3.23 \mathrm{E}-09$ & 2. $2 \mathrm{E}-12$ & 1.076 & 1.078 & 0.017 \\
\hline 10 & $R a m p$ & 0.54 & $2.80 \mathrm{E}-09$ & 1. & 7.33 & 0.07 & 0.4367 & $9.09 \mathrm{E}-09$ & $1.65 \mathrm{E}-08$ & $1.7 \mathrm{E}-11$ & 1.063 & 1.067 & 0.027 \\
\hline 11 & 414 . & 1.08 & $7.05 \mathrm{E}-09$ & 0 . & 7.35 & 0.05 & 0.6134 & $1.81 \mathrm{E}-08$ & 3. $39 \mathrm{E}-08$ & 1. $6 \mathrm{E}-11$ & 1.072 & 1.076 & 0.013 \\
\hline 12 & 414. & 1.08 & $3.75 \mathrm{E}-09$ & 0 . & 7.00 & 0.07 & 0.7075 & $1.56 \mathrm{E}-08$ & $3.03 E-08$ & 2. $2 \mathrm{E}-11$ & 1.024 & 1.029 & 0.019 \\
\hline 13 & 414. & 2.16 & $3.55 \mathrm{E}-09$ & 0 . & 6.78 & 0.07 & 0.7967 & $1.08 \mathrm{E}-08$ & $2.17 \mathrm{E}-08$ & 1. $6 \mathrm{E}-11$ & 0.984 & 0.987 & 0.019 \\
\hline 14 & 414. & 3.25 & $2.16 \mathrm{E}-09$ & 0 . & 6.77 & 0.09 & 0.8508 & $6.35 E-09$ & 1. $31 \mathrm{E}-08$ & 1. $4 \mathrm{E}-11$ & 0.970 & 0.973 & 0.026 \\
\hline 15 & 414. & 6.50 & $1.75 \mathrm{E}-09$ & 0 . & 6.93 & 0.10 & 0.8947 & 3. $62 \mathrm{E}-09$ & $7.63 \mathrm{E}-09$ & $9.7 \mathrm{E}-12$ & 0.982 & 0.984 & 0.029 \\
\hline 16 & 414. & 4.87 & $6.78 \mathrm{E}-10$ & 1. & 7.11 & 0.25 & 0.9117 & $2.44 \mathrm{E}-09$ & $5.26 \mathrm{E}-09$ & $1.6 \mathrm{E}-11$ & 1.006 & 1.007 & 0.059 \\
\hline 17 & Ramp & 0.54 & $6.68 \mathrm{E}-10$ & 1. & 7.77 & 0.17 & 0.9284 & $2.62 \mathrm{E}-08$ & $5.72 \mathrm{E}-08$ & $1.6 \mathrm{E}-10$ & 1.111 & 1.114 & 0.057 \\
\hline 18 & 618. & 1.63 & 1. $33 \mathrm{E}-09$ & 1. & 7.27 & 0.13 & 0.9617 & $2.59 \mathrm{E}-08$ & $5.79 \mathrm{E}-08$ & $8.1 \mathrm{E}-11$ & 1.074 & 1.078 & 0.028 \\
\hline 19 & 618. & 13.47 & $1.45 \mathrm{E}-09$ & 1. & 6.74 & 0.11 & 0.9980 & $1.49 \mathrm{E}-08$ & $3.56 \mathrm{E}-08$ & $4.3 E-11$ & 1.189 & 1.202 & 0.080 \\
\hline 20 & 618. & 8.12 & $7.83 E-11$ & 4. & 3.76 & 0.63 & 1.0000 & & & & & & \\
\hline
\end{tabular}


Experiment \#9

Aftermath D9 alkali basalt glass 26 chips, $44.5 \mathrm{mg}$

Grain size data (um): short side, "visual average", long side.

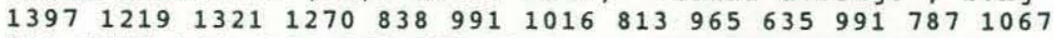

$8891168 \quad 8381143 \quad 965 \quad 787 \quad 1016 \quad 8138899658891143 \quad 889$

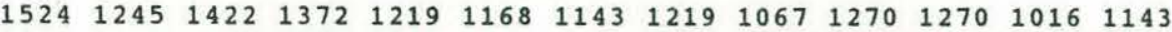
$\begin{array}{llllllllllllll}1143 & 1321 & 1295 & 1270 & 1041 & 1473 & 1143 & 1295 & 1295 & 1397 & 889 & 1397 & 1346\end{array}$

$\begin{array}{llllllllllllll}2159 & 1346 & 1854 & 1397 & 1880 & 1778 & 1346 & 1397 & 1676 & 1600 & 1372 & 1473 & 1372\end{array}$ $\begin{array}{lllllllllllll}1930 & 1778 & 1321 & 1473 & 1346 & 1778 & 1270 & 1753 & 1575 & 1676 & 1295 & 1473 & 1524\end{array}$

Bulk helium contents: He $=7.39 \mathrm{E}-08 \mathrm{ccSTP} \quad 3 \mathrm{He} / 4 \mathrm{He}=7.25+0.07 \times \mathrm{Ra}$

Sequential release data:

\begin{tabular}{|c|c|c|c|c|c|c|c|c|c|c|c|c|c|}
\hline$\#$ & $\begin{array}{l}\text { Temp. } \\
\text { c }\end{array}$ & $\begin{array}{c}\mathrm{Time} \\
\mathrm{hr}\end{array}$ & $\begin{array}{l}4 \mathrm{He} \\
\operatorname{ccSTP}\end{array}$ & $\begin{array}{l}1 \mathrm{~s} \\
0 / 00\end{array}$ & $\begin{array}{c}3 \mathrm{He} / 4 \mathrm{He} \\
\mathrm{Ra}\end{array}$ & $\begin{array}{l}1 \mathrm{~s} \\
\mathrm{Ra}\end{array}$ & $\begin{array}{l}F(4 \mathrm{He}) \\
(\operatorname{tota} 1)\end{array}$ & $\begin{array}{c}\text { Dsm } \\
\operatorname{cm} 2 / s\end{array}$ & $\begin{array}{l}\text { Drm } \\
\operatorname{cm} 2 / s\end{array}$ & $\begin{array}{l}1 \mathrm{~s} \\
\mathrm{~cm} 2 / \mathrm{s}\end{array}$ & $\begin{array}{l}\mathrm{D} 3 / \mathrm{D} 4 \\
\mathrm{sm}\end{array}$ & $\begin{array}{c}\mathrm{D} 3 / \mathrm{D} 4 \\
\mathrm{r}=\mathrm{m}\end{array}$ & $\begin{array}{l}1 \mathrm{~s} \\
\mathrm{rm}\end{array}$ \\
\hline 1 & 53. & 41.24 & $1.25 \mathrm{E}-10$ & 4. & 8.81 & 0.25 & 0.0017 & $5.62 \mathrm{E}-15$ & $6.44 \mathrm{E}-15$ & $4.8 \mathrm{E}-17$ & 1.478 & 1.478 & 0.169 \\
\hline 2 & 53. & 29.28 & $8.72 \mathrm{E}-11$ & 8. & 10.18 & 0.60 & 0.0029 & $1.50 \mathrm{E}-14$ & 1. $72 E-14$ & $2.1 \mathrm{E}-16$ & 1.775 & 1.776 & 0.317 \\
\hline 3 & Ramp & 1.63 & $3.31 \mathrm{E}-11$ & 15. & 8.23 & 2.23 & 0.0033 & 1. $39 \mathrm{E}-13$ & $1.59 E-13$ & $5.2 \mathrm{E}-15$ & 1.456 & 1.457 & 0.851 \\
\hline 4 & 125. & 15.73 & $1.29 \mathrm{E}-09$ & 3. & 7.61 & 0.13 & 0.0208 & $2.18 \mathrm{E}-12$ & $2.53 \mathrm{E}-12$ & 1. $4 \mathrm{E}-14$ & 1.166 & 1.167 & 0.051 \\
\hline 5 & 125. & 23.71 & $1.07 \mathrm{E}-09$ & 7. & 7.20 & 0.16 & 0.0353 & $2.80 \mathrm{E}-12$ & 3. $30 \mathrm{E}-12$ & $3.1 \mathrm{E}-14$ & 1.053 & 1.055 & 0.059 \\
\hline 6 & Ramp & 1.63 & $7.21 \mathrm{E}-11$ & 6. & 4.77 & 1.19 & 0.0363 & $3.49 \mathrm{E}-12$ & $4.15 \mathrm{E}-12$ & $4.6 \mathrm{E}-13$ & 0.686 & 0.687 & 0.708 \\
\hline 7 & 152. & 6.51 & $7.43 \mathrm{E}-10$ & 1. & 7.56 & 0.17 & 0.0463 & $1.07 \mathrm{E}-11$ & 1. $24 \mathrm{E}-11$ & 1. $5 \mathrm{E}-13$ & 1.084 & 1.084 & 0.088 \\
\hline 8 & 151. & 14.10 & $1.32 \mathrm{E}-09$ & 2 . & 7.46 & 0.11 & 0.0641 & $1.19 \mathrm{E}-11$ & $1.38 \mathrm{E}-11$ & $1.1 \mathrm{E}-13$ & 1.069 & 1.069 & 0.062 \\
\hline 9 & 151. & 3.25 & $2.55 \mathrm{E}-10$ & 3. & 6.65 & 0.36 & 0.0676 & $1.20 \mathrm{E}-11$ & 1. $39 \mathrm{E}-11$ & $5.0 \mathrm{E}-13$ & 0.949 & 0.950 & 0.288 \\
\hline 10 & Ramp & 1.63 & $2.19 \mathrm{E}-10$ & 3. & 6.62 & 0.38 & 0.0705 & $2.17 \mathrm{E}-11$ & $2.52 \mathrm{E}-11$ & 1. $1 \mathrm{E}-12$ & 0.940 & 0.941 & 0.341 \\
\hline 11 & 189. & 10.85 & $2.31 \mathrm{E}-09$ & 3. & 7.36 & 0.08 & 0.1018 & $4.35 E-11$ & $5.08 \mathrm{E}-11$ & 3. $0 \mathrm{E}-13$ & 1.041 & 1.041 & 0.048 \\
\hline 12 & 189. & 9.25 & $1.47 \mathrm{E}-09$ & 3. & 7.21 & 0.12 & 0.1217 & $4.30 \mathrm{E}-11$ & $5.05 \mathrm{E}-11$ & 4. $7 \mathrm{E}-13$ & 1.016 & 1.017 & 0.079 \\
\hline 13 & Ramp & 1.63 & $5.08 \mathrm{E}-10$ & 2 . & 7.30 & 0.27 & 0.1286 & $9.57 \mathrm{E}-11$ & 1. $13 \mathrm{E}-10$ & 2. $9 \mathrm{E}-12$ & 1.026 & 1.027 & 0.225 \\
\hline 14 & 240 . & 8.14 & $3.80 \mathrm{E}-09$ & 1. & 7.53 & 0.09 & 0.1799 & $1.81 \mathrm{E}-10$ & $2.15 \mathrm{E}-10$ & 1. $0 \mathrm{E}-12$ & 1.063 & 1.064 & 0.047 \\
\hline 15 & 240 . & 13.02 & $3.79 \mathrm{E}-09$ & 1. & 7.33 & 0.07 & 0.2312 & $1.58 \mathrm{E}-10$ & $1.90 \mathrm{E}-10$ & $1.0 \mathrm{E}-12$ & 1.038 & 1.039 & 0.056 \\
\hline 16 & Ramp & 1.63 & 1. $45 \mathrm{E}-09$ & 1. & 7.43 & 0.14 & 0.2508 & $5.88 \mathrm{E}-10$ & $7.12 \mathrm{E}-10$ & 9. $7 E-12$ & 1.053 & 1.054 & 0.142 \\
\hline 17 & 329. & 3.26 & $4.13 \mathrm{E}-09$ & 2 . & 7.38 & 0.07 & 0.3068 & $1.01 \mathrm{E}-09$ & 1. $23 \mathrm{E}-09$ & 7. $7 \mathrm{E}-12$ & 1.045 & 1.047 & 0.065 \\
\hline 18 & 329. & 4.88 & $3.41 \mathrm{E}-09$ & 2. & 7.29 & 0.06 & 0.3528 & $6.94 \mathrm{E}-10$ & $8.60 E-10$ & 7. $1 \mathrm{E}-12$ & 1.033 & 1.035 & 0.086 \\
\hline 19 & 328. & 13.02 & $3.75 E-09$ & 3 . & 6.70 & 0.06 & 0.4036 & $3.49 \mathrm{E}-10$ & $4.38 \mathrm{E}-10$ & 3. $7 E-12$ & 0.941 & 0.942 & 0.086 \\
\hline 20 & Ramp & 1.63 & $8.75 E-09$ & 2. & 7.63 & 0.05 & 0.5219 & $8.95 E-09$ & $1.15 \mathrm{E}-08$ & 5. $7 E-11$ & 1.076 & 1.078 & 0.054 \\
\hline 21 & 618. & 3.80 & 1. $58 \mathrm{E}-08$ & 3. & 7.56 & 0.26 & 0.7358 & $1.29 \mathrm{E}-08$ & $1.77 \mathrm{E}-08$ & $5.1 \mathrm{E}-11$ & 1.102 & 1.108 & 0.034 \\
\hline 22 & 618. & 0.54 & 1. $50 \mathrm{E}-09$ & 3. & 7.27 & 0.28 & 0.7562 & $1.33 \mathrm{E}-08$ & $1.90 \mathrm{E}-08$ & $4.0 \mathrm{E}-10$ & 1.090 & 1.100 & 0.243 \\
\hline 23 & 618. & 5.97 & $9.98 \mathrm{E}-09$ & 3. & 7.04 & 0.24 & 0.8911 & $1.28 \mathrm{E}-08$ & $1.89 \mathrm{E}-08$ & $4.9 \mathrm{E}-11$ & 1.078 & 1.095 & 0.030 \\
\hline 24 & 618. & 5.97 & $4.45 E-09$ & 2 . & 6.65 & 0.23 & 0.9513 & $1.28 \mathrm{E}-08$ & $2.08 \mathrm{E}-08$ & $6.2 \mathrm{E}-11$ & 1.087 & 1.105 & 0.035 \\
\hline 25 & 618. & 7.05 & $2.15 \mathrm{E}-09$ & 3. & 6.22 & 0.23 & 0.9805 & 1. $23 \mathrm{E}-08$ & $2.15 \mathrm{E}-08$ & 7. $7 E-11$ & 1.101 & 1.123 & 0.033 \\
\hline 26 & 618. & 11.50 & $1.08 \mathrm{E}-09$ & 4. & 5.62 & 0.12 & 0.9951 & $1.14 \mathrm{E}-08$ & $2.19 \mathrm{E}-08$ & 7. $6 \mathrm{E}-11$ & 1.115 & 1.146 & 0.076 \\
\hline 27 & 618. & 13.01 & $3.39 \mathrm{E}-10$ & 6. & 4.83 & 0.35 & 0.9997 & $2.05 \mathrm{E}-08$ & $4.47 E-08$ & $3.5 E-10$ & 1.818 & 1.924 & 1.152 \\
\hline 28 & 618. & 1.63 & $2.14 \mathrm{E}-11$ & 19. & 0.45 & 3.36 & 1.0000 & & & & & & \\
\hline
\end{tabular}


Experiment \#10

Alv1389-1854B alkalic basalt glass

Radiogenic * He

9 chips, $44.0 \mathrm{mg}$

Grain size data (um): short side, "visual average", long side.

$\begin{array}{lllllllll}1626 & 1524 & 1270 & 1194 & 787 & 1270 \quad 737 & 1270 & 813\end{array}$

$\begin{array}{llllllllll}1728 & 1854 & 1956 & 1880 & 1702 & 1549 & 1905 & 1829 & 1575\end{array}$

$289622352032 \quad 348022352362266724132007$

Bulk helium contents: He $=1.03 \mathrm{E}-08 \mathrm{ccSTP} 3 \mathrm{He} / 4 \mathrm{He}=0.08+0.04 \times \mathrm{Ra}$

Sequential release data:

\begin{tabular}{|c|c|c|c|c|c|c|c|c|c|c|}
\hline & $\begin{array}{l}\text { Temp. } \\
\text { c }\end{array}$ & $\begin{array}{c}\mathrm{Time} \\
\mathrm{hr}\end{array}$ & $\begin{array}{l}4 \mathrm{He} \\
\operatorname{ccSTP}\end{array}$ & $\begin{array}{c}1 \mathrm{~s} \\
0 / 00\end{array}$ & $\begin{array}{c}3 \mathrm{He} / 4 \mathrm{He} \\
\mathrm{Ra}\end{array}$ & $\begin{array}{l}1 \mathrm{~s} \\
\mathrm{Ra}\end{array}$ & $\begin{array}{l}F \quad(4 \mathrm{He}) \\
(\text { total) }\end{array}$ & $\begin{array}{c}\text { Ds m } \\
\operatorname{cm} 2 / \mathrm{s}\end{array}$ & $\begin{array}{l}\text { Drm } \\
\operatorname{cm} 2 / \mathrm{s}\end{array}$ & $\begin{array}{l}1 \mathrm{~s} \\
\mathrm{~cm} 2 / \mathrm{s}\end{array}$ \\
\hline 1 & 123. & 16.81 & $9.63 \mathrm{E}-11$ & 3. & 0.01 & 0.57 & 0.0094 & $4.27 \mathrm{E}-13$ & $8.80 \mathrm{E}-13$ & $6.0 \mathrm{E}-15$ \\
\hline 2 & Ramp & 0.54 & $2.71 \mathrm{E}-11$ & 11. & 0.00 & 3.09 & 0.0120 & $8.53 E-12$ & 1. $76 \mathrm{E}-11$ & 3. $2 \mathrm{E}-13$ \\
\hline 3 & 220. & 0.54 & $8.11 \mathrm{E}-11$ & 4. & 0.00 & 0.89 & 0.0199 & $3.80 \mathrm{E}-11$ & $7.91 \mathrm{E}-11$ & $6.7 \mathrm{E}-13$ \\
\hline 4 & 220. & 2.71 & 7. $30 \mathrm{E}-10$ & 1. & 0.42 & 0.12 & 0.0912 & $2.51 \mathrm{E}-10$ & $5.19 \mathrm{E}-10$ & $7.8 \mathrm{E}-13$ \\
\hline 5 & 220. & 2.71 & $4.98 \mathrm{E}-10$ & 1. & 0.80 & 0.21 & 0.1397 & 3. $72 \mathrm{E}-10$ & $7.76 \mathrm{E}-10$ & 1. $5 E-12$ \\
\hline 6 & 220. & 2.71 & $3.78 \mathrm{E}-10$ & 1. & 0.00 & 0.14 & 0.1765 & $4.01 \mathrm{E}-10$ & $8.45 E-10$ & 2. $0 \mathrm{E}-12$ \\
\hline 7 & 220. & 2.71 & $2.97 \mathrm{E}-10$ & 1. & 0.00 & 0.22 & 0.2055 & $3.93 \mathrm{E}-10$ & $8.33 \mathrm{E}-10$ & 2. $5 \mathrm{E}-12$ \\
\hline 8 & R a mp & 0.54 & $1.03 \mathrm{E}-09$ & 0 . & 0.00 & 0.06 & 0.3060 & $9.78 \mathrm{E}-09$ & $2.10 \mathrm{E}-08$ & 2. $3 \mathrm{E}-11$ \\
\hline 9 & 312. & 0.54 & $1.69 \mathrm{E}-09$ & 0 . & 0.02 & 0.04 & 0.4709 & $2.86 E-08$ & $6.32 \mathrm{E}-08$ & 4. $8 \mathrm{E}-11$ \\
\hline 10 & 312. & 1.08 & $2.96 \mathrm{E}-09$ & 0 . & 0.00 & 0.02 & 0.7600 & $5.97 \mathrm{E}-08$ & $1.41 \mathrm{E}-07$ & 7. $2 \mathrm{E}-11$ \\
\hline 11 & 312. & 0.54 & $6.01 \mathrm{E}-10$ & 0 . & 0.00 & 0.12 & 0.8186 & $4.75 E-08$ & $1.21 \mathrm{E}-07$ & 1. $9 \mathrm{E}-10$ \\
\hline 12 & Ramp & 9.76 & $2.00 \mathrm{E}-10$ & 1. & 0.02 & 0.32 & 0.8381 & $1.09 \mathrm{E}-09$ & $2.84 \mathrm{E}-09$ & 1. $2 \mathrm{E}-11$ \\
\hline 13 & 312. & 0.54 & $2.47 \mathrm{E}-10$ & 1. & 0.01 & 0.36 & 0.8622 & $2.81 \mathrm{E}-08$ & $7.40 \mathrm{E}-08$ & 2. $5 E-10$ \\
\hline 14 & 312. & 1.08 & $3.09 \mathrm{E}-10$ & 1. & 0.00 & 0.18 & 0.8923 & $2.16 \mathrm{E}-08$ & $5.84 E-08$ & 1. $5 \mathrm{E}-10$ \\
\hline 15 & 312. & 1.63 & $2.05 \mathrm{E}-10$ & 1. & 0.08 & 0.41 & 0.9123 & $1.19 \mathrm{E}-08$ & $3.31 \mathrm{E}-08$ & 1. $2 \mathrm{E}-10$ \\
\hline 16 & Ramp & 0.54 & $3.91 \mathrm{E}-10$ & 1. & 0.00 & 0.34 & 0.9504 & $9.97 \mathrm{E}-08$ & $2.86 E-07$ & 5. $8 \mathrm{E}-10$ \\
\hline 17 & 416. & 0.54 & 1. $21 \mathrm{E}-10$ & 2 . & 0.04 & 0.57 & 0.9622 & $4.76 E-08$ & $1.40 \mathrm{E}-07$ & 6. $2 \mathrm{E}-10$ \\
\hline 18 & 416. & 1.08 & $9.20 \mathrm{E}-11$ & 3. & 0.00 & 0.88 & 0.9712 & $2.37 \mathrm{E}-08$ & $7.05 E-08$ & 3. $5 \mathrm{E}-10$ \\
\hline 19 & Ramp & 0.54 & $2.95 \mathrm{E}-10$ & 1. & 0.27 & 0.29 & 1.0000 & & & \\
\hline
\end{tabular}


Experiment \#11

ALV1389-1854B Alkali Basalt glass

Radiogenic * $4 \mathrm{He}$

Grain size data (um): short side, "visual average", long side.

$\begin{array}{llllllllll}246 & 216 & 293 & 169 & 185 & 185 & 277 & 231 & 169 & 185\end{array}$

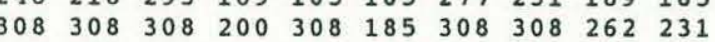

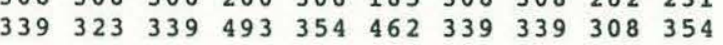

Bulk helium contents: He $=4.10 \mathrm{E}-09 \operatorname{ccSTP} 3 \mathrm{He} / 4 \mathrm{He}=0.81+0.06 \times \mathrm{Ra}$

Sequential release data:

\begin{tabular}{|c|c|c|c|c|c|c|c|c|c|c|}
\hline & $\begin{array}{l}\text { Temp. } \\
\text { c }\end{array}$ & $\begin{array}{c}\text { Time } \\
\text { hr }\end{array}$ & $\begin{array}{l}4 \mathrm{He} \\
\text { CCSTP }\end{array}$ & $\begin{array}{c}1 \mathrm{~s} \\
0 / 00\end{array}$ & $\begin{array}{c}3 \mathrm{He} / 4 \mathrm{He} \\
\mathrm{Ra}\end{array}$ & $\begin{array}{l}1 \mathrm{~s} \\
\mathrm{Ra}\end{array}$ & $\begin{array}{l}F(4 \mathrm{He}) \\
(\operatorname{total})\end{array}$ & $\begin{array}{c}\text { Dsm } \\
\mathrm{cm} 2 / \mathrm{s}\end{array}$ & $\begin{array}{l}\operatorname{Drm} \\
\mathrm{Cm} 2 / \mathrm{s}\end{array}$ & $\begin{array}{l}1 \mathrm{~s} \\
\mathrm{~cm} 2 / \mathrm{s}\end{array}$ \\
\hline 1 & Ramp & 2.20 & $3.13 \mathrm{E}-11$ & 13. & 2.18 & 1.48 & 0.0076 & $8.49 \mathrm{E}-14$ & $1.18 \mathrm{E}-13$ & 3. $5 \mathrm{E}-15$ \\
\hline 2 & 127. & 49.94 & 1. $26 \mathrm{E}-10$ & 4. & 5.41 & 0.27 & 0.0385 & & 1.30 & -15 \\
\hline 3 & Cool & 26.88 & $1.04 \mathrm{E}-11$ & 67. & 16.48 & 0.65 & 0.0410 & $2.48 \mathrm{E}-14$ & $3.47 \mathrm{E}-14$ & $4.0 \mathrm{E}-15$ \\
\hline 4 & $\mathrm{Ramp}$ & 1.63 & below det & tect & ion $\mathrm{l}$ im & it & & & & \\
\hline 5 & 127. & 63.11 & $5.12 \mathrm{E}-11$ & 20. & 7.82 & 0.35 & 0.0535 & $6.22 \mathrm{E}-14$ & $8.47 E-14$ & 3. $2 \mathrm{E}-15$ \\
\hline 6 & Ramp & 1.61 & $1.53 \mathrm{E}-11$ & 20. & 0.96 & 4.67 & 0.0572 & $8.60 \mathrm{E}-13$ & 1. $21 \mathrm{E}-12$ & 1. $4 \mathrm{E}-13$ \\
\hline 7 & 162 . & 36.89 & $2.76 \mathrm{E}-10$ & 7. & 1.97 & 0.10 & 0.1245 & $1.15 \mathrm{E}-12$ & $1.63 \mathrm{E}-12$ & 2. $4 \mathrm{E}-14$ \\
\hline 8 & 162. & 16.81 & $6.62 \mathrm{E}-11$ & 12. & 4.92 & 0.65 & 0.1407 & $9.12 \mathrm{E}-13$ & 1. $30 \mathrm{E}-12$ & 6. $6 \mathrm{E}-14$ \\
\hline 9 & Ramp & 2.17 & $3.53 \mathrm{E}-09$ & 6. & 0.31 & 0.03 & 1.0000 & & & \\
\hline & & & at 600 & & & 10 mea & surable & & & \\
\hline
\end{tabular}


APPENDIX C: Helium emanation data and diffusivity results for olivine and pyroxene at high temperature (experiments \#1-8, chapter 4). 
Experiment \#1

$113987-107$ olivine

500-710um sieve fraction, $127.0 \mathrm{mg}, r=303 .(27$.$) um$

Bulk helium contents: $\mathrm{He}=2.24 \mathrm{E}-08 \mathrm{cCSTP} 3 \mathrm{He} / 4 \mathrm{He}=8.89+0.07 \times \mathrm{Ra}$

Sequential release data:

\begin{tabular}{|c|c|c|c|c|c|c|c|c|c|c|c|c|}
\hline \# & $\begin{array}{c}\text { Temp. } \\
\text { C }\end{array}$ & $\begin{array}{r}1 \mathrm{~s} \\
\mathrm{C}\end{array}$ & $\begin{array}{c}\text { Time } \\
\text { hr }\end{array}$ & $\begin{array}{c}4 \mathrm{He} \\
\operatorname{ccSTP}\end{array}$ & $\begin{array}{c}1 \mathrm{~s} \\
\operatorname{ccsTp}\end{array}$ & $\begin{array}{c}3 \mathrm{He} / 4 \mathrm{He} \\
\mathrm{Ra}\end{array}$ & $\begin{array}{l}1 \mathrm{~s} \\
\mathrm{Ra}\end{array}$ & $\begin{array}{l}F(4 \mathrm{He}) \\
(\operatorname{total})\end{array}$ & $\begin{array}{c}\text { Dsm } \\
\mathrm{cm} 2 / \mathrm{s}\end{array}$ & $\begin{array}{c}1 \mathrm{~s} \\
\mathrm{~cm} 2 / \mathrm{s}\end{array}$ & $\begin{array}{c}\mathrm{D} 3 / \mathrm{D} 4 \\
\mathrm{sm}\end{array}$ & $\begin{array}{l}1 \mathrm{~s} \\
\mathrm{~s} \text { m }\end{array}$ \\
\hline 1 & Load & - & 1.63 & $1.56 \mathrm{E}-10$ & 2. E-12 & 11.40 & 1.03 & 0.0070 & $6.64 \mathrm{E}-13$ & 1. $2 \mathrm{E}-13$ & 1.64 & 0.30 \\
\hline 2 & 965. & 10. & 12.48 & 1. $76 \mathrm{E}-10$ & 2. $E-12$ & 9.38 & 0.96 & 0.0148 & $3.06 \mathrm{E}-13$ & 5. $4 \mathrm{E}-14$ & 1.27 & 0.24 \\
\hline 3 & 965. & 10. & 27.09 & 1. $71 \mathrm{E}-10$ & $6 \cdot E-13$ & 8.69 & 0.54 & 0.0225 & $2.34 \mathrm{E}-13$ & 4. $2 \mathrm{E}-14$ & 1.10 & 0.22 \\
\hline 4 & 965. & 10. & 26.04 & $1.64 \mathrm{E}-10$ & $5 \cdot E-13$ & 8.72 & 0.50 & 0.0298 & 3. $26 \mathrm{E}-13$ & $5.8 E-14$ & 1.06 & 0.22 \\
\hline 5 & Melt & - & 1.63 & $2.17 \mathrm{E}-08$ & 9. E-11 & 8.88 & 0.05 & 0.9984 & & & & \\
\hline 6 & Left & - & 1.63 & $3.68 \mathrm{E}-11$ & 4. $E-13$ & 4.86 & 1.83 & 1.0000 & & & & \\
\hline
\end{tabular}

Experiment \#2

$113987-107$ olivine

500-710um sieve fraction, $93.3 \mathrm{mg}, r=303(27$.)

Bulk helium contents: $\mathrm{He}=2.68 \mathrm{E}-08 \mathrm{ccSTP} 3 \mathrm{He} / 4 \mathrm{He}=8.83+0.08 \times \mathrm{Ra}$ Sequential release data:

\begin{tabular}{|c|c|c|c|c|c|c|c|c|c|c|c|c|}
\hline \# & $\begin{array}{c}\text { Temp. } \\
\text { c }\end{array}$ & $\begin{array}{r}1 \mathrm{~s} \\
\mathrm{C}\end{array}$ & $\begin{array}{c}\mathrm{Time} \\
\mathrm{hr}\end{array}$ & $\begin{array}{c}4 \mathrm{H} \\
\operatorname{ccSTP}\end{array}$ & $\begin{array}{c}1 \mathrm{~s} \\
\operatorname{ccSTP}\end{array}$ & $\begin{array}{c}3 \mathrm{He} / 4 \mathrm{He} \\
\mathrm{Ra}\end{array}$ & $\begin{array}{l}1 \mathrm{~s} \\
\mathrm{Ra}\end{array}$ & $\begin{array}{l}F(4 \mathrm{He}) \\
(\operatorname{tota} 1)\end{array}$ & $\begin{array}{c}\text { Dsm } \\
\operatorname{cm} 2 / s\end{array}$ & $\begin{array}{c}1 \mathrm{~s} \\
\mathrm{~cm} 2 / \mathrm{s}\end{array}$ & $\begin{array}{c}\mathrm{D} 3 / \mathrm{D} 4 \\
\mathrm{~s} \text { m }\end{array}$ & $\begin{array}{l}1 \mathrm{~s} \\
\mathrm{~s} \mathrm{~m}\end{array}$ \\
\hline 1 & Load & - & 0.53 & $2.62 \mathrm{E}-10$ & $4 \cdot E-13$ & 8.78 & 0.39 & 0.0098 & $4.00 \mathrm{E}-12$ & $7.1 \mathrm{E}-13$ & 0.99 & 0.09 \\
\hline 2 & 1084 . & 20. & 20.62 & $1.44 E-09$ & 9.E-13 & 9.21 & 0.13 & 0.0636 & 4. $42 \mathrm{E}-12$ & 7. $9 \mathrm{E}-13$ & 1.08 & 0.04 \\
\hline 3 & 1084 . & 20. & 24.96 & $1.61 \mathrm{E}-09$ & 8. $E-12$ & 9.44 & 0.13 & 0.1239 & $1.09 \mathrm{E}-11$ & 1. $9 \mathrm{E}-12$ & 1.12 & 0.04 \\
\hline 4 & 1084 . & 20. & 26.51 & $1.10 \mathrm{E}-09$ & 7. $E-12$ & 9.36 & 0.12 & 0.1650 & $1.13 \mathrm{E}-11$ & $2.0 \mathrm{E}-12$ & 1.12 & 0.06 \\
\hline 5 & Melt & - & 1.69 & 2. $23 E-08$ & 1. $E-10$ & 8.74 & 0.06 & 0.9980 & & & & \\
\hline 6 & Left & - & 1.63 & $5.48 \mathrm{E}-11$ & 7. $E-13$ & 7.13 & 1.98 & 1.0000 & & & & \\
\hline
\end{tabular}




\section{Experiment \#3}

$113987-107$ olivine

$500-710$ um sieve fraction, $101.5 \mathrm{mg}, \mathrm{r}=303 .(27) \mathrm{um}$

Bulk helium contents: Ho $=3.23 \mathrm{E}-08$.

Sequential release data:

\begin{tabular}{|c|c|c|c|c|c|c|c|c|c|c|c|c|}
\hline$\#$ & $\begin{array}{c}\text { Te mp. } \\
C\end{array}$ & $\begin{array}{r}1 \mathrm{~s} \\
\mathrm{C}\end{array}$ & $\begin{array}{c}\mathrm{Ti} m \theta \\
\mathrm{hr}\end{array}$ & $\begin{array}{c}4 \mathrm{H} \bullet \\
\operatorname{CCSTP}\end{array}$ & $\begin{array}{c}1 \mathrm{~s} \\
\operatorname{ccSTP}\end{array}$ & $\begin{array}{c}3 \mathrm{He} / 4 \mathrm{He} \\
\mathrm{Ra}\end{array}$ & $\begin{array}{l}1 \mathrm{~s} \\
\mathrm{Ra}\end{array}$ & $\begin{array}{l}F(4 \mathrm{He}) \\
(\operatorname{tota} l)\end{array}$ & $\begin{array}{c}\text { Dsm } \\
\operatorname{cm} 2 / s\end{array}$ & $\begin{array}{c}1 \mathrm{~s} \\
\mathrm{~cm} 2 / \mathrm{s}\end{array}$ & $\begin{array}{c}\mathrm{D} 3 / \mathrm{D} 4 \\
\mathrm{sm}\end{array}$ & $\begin{array}{l}1 \mathrm{~s} \\
\mathrm{~s} \mathrm{~m}\end{array}$ \\
\hline 1 & Load & - & 0.53 & $4.76 \mathrm{E}-09$ & 1. $E-11$ & 9.22 & 0.06 & 0.1475 & $9.86 \mathrm{E}-10$ & 1. $8 \mathrm{E}-10$ & 1.07 & 0.02 \\
\hline 2 & 1255. & 20. & 1.63 & $4.78 \mathrm{E}-09$ & 1. $E-11$ & 9.20 & 0.07 & 0.2955 & $1.10 \mathrm{E}-09$ & 2. $0 \mathrm{E}-10$ & 1.07 & 0.02 \\
\hline 3 & 1255. & 20. & 3.26 & $5.87 \mathrm{E}-09$ & 2. $E-11$ & 9.06 & 0.07 & 0.4773 & $1.43 \mathrm{E}-09$ & 2. $5 E-10$ & 1.06 & 0.02 \\
\hline 4 & 1255 . & 20 . & 4.88 & $4.82 \mathrm{E}-09$ & 1.E-11 & 9.01 & 0.09 & 0.6265 & $1.42 \mathrm{E}-09$ & 2. $5 E-10$ & 1.06 & 0.02 \\
\hline 5 & 1255. & 20. & 8.14 & $3.91 \mathrm{E}-09$ & 1. $\mathrm{E}-11$ & 8.85 & 0.07 & 0.7476 & $1.14 \mathrm{E}-09$ & $2.0 \mathrm{E}-10$ & 1.04 & 0.02 \\
\hline 6 & Melt & - & 1.63 & $8.15 \mathrm{E}-09$ & 4. $E-11$ & 8.49 & 0.05 & 1.0000 & & & & \\
\hline
\end{tabular}

\section{Experiment \#4}

$113987-107$ olivine

500-710um sieve fraction, approx.80mg (partial load), $r=303(27)$ um

Gold in crucible

Bulk helium contents: He $=2.51 \mathrm{E}-08$ ccSTP $3 \mathrm{He} / 4 \mathrm{He}=8.81+0.03 \times \mathrm{Ra}$

Sequential release data:

\begin{tabular}{|c|c|c|c|c|c|c|c|c|c|c|c|c|}
\hline$\#$ & $\begin{array}{l}\text { Temp. } \\
\text { c }\end{array}$ & $\begin{array}{r}1 \mathrm{~s} \\
\mathrm{C}\end{array}$ & $\begin{array}{c}\text { Time } \\
\text { hr }\end{array}$ & $\begin{array}{c}\begin{array}{c}4 \mathrm{He} \\
\operatorname{ccSTP}\end{array}\end{array}$ & $\begin{array}{c}1 \mathrm{~s} \\
\operatorname{ccSTP}\end{array}$ & $\begin{array}{c}3 \mathrm{He} / 4 \mathrm{He} \\
\mathrm{Ra}\end{array}$ & $\begin{array}{l}1 \mathrm{~s} \\
\mathrm{Ra}\end{array}$ & $\begin{array}{l}F(4 \mathrm{He}) \\
(\operatorname{total})\end{array}$ & $\begin{array}{c}\text { Dsm } \\
\mathrm{cm} 2 / \mathrm{s}\end{array}$ & $\begin{array}{c}1 \mathrm{~s} \\
\mathrm{~cm} 2 / \mathrm{s}\end{array}$ & $\begin{array}{c}\mathrm{D} 3 / \mathrm{D} 4 \\
\mathrm{~s} \text { m }\end{array}$ & $\begin{array}{l}1 \mathrm{~s} \\
\mathrm{~s} \text { m }\end{array}$ \\
\hline 1 & Load & - & 0.53 & $9.84 \mathrm{E}-11$ & $6 \cdot E-13$ & 6.84 & 0.94 & 0.0039 & $6.41 \mathrm{E}-13$ & $1.1 \mathrm{E}-13$ & 0.60 & 0.17 \\
\hline 2 & 1095. & 10. & 0.54 & $7.80 \mathrm{E}-11$ & 5. $E-13$ & 11.45 & 1.24 & 0.0070 & $1.40 \mathrm{E}-12$ & 2. $5 E-13$ & 1.20 & 0.25 \\
\hline 3 & 1095. & 10. & 6.47 & 7. $32 \mathrm{E}-10$ & 2. $E-12$ & 9.28 & 0.20 & 0.0362 & $4.33 \mathrm{E}-12$ & $7.7 E-13$ & 1.09 & 0.05 \\
\hline 4 & 1095. & 10. & 11.86 & $1.05 \mathrm{E}-09$ & 4. E-12 & 9.24 & 0.22 & 0.0780 & $9.39 \mathrm{E}-12$ & 1. $7 E-12$ & 1.10 & 0.05 \\
\hline 5 & 1095. & 10. & 23.11 & $1.76 \mathrm{E}-09$ & $3 \cdot E-12$ & 9.08 & 0.11 & 0.1480 & $1.68 \mathrm{E}-11$ & 3. $0 \mathrm{E}-12$ & 1.08 & 0.03 \\
\hline 6 & 1095. & 10. & 23.58 & $1.36 \mathrm{E}-09$ & $6 \cdot E-12$ & 9.04 & 0.13 & 0.2023 & $2.09 E-11$ & 3. $7 \mathrm{E}-12$ & 1.07 & 0.04 \\
\hline 7 & 1095. & 10. & 1.08 & $5.63 \mathrm{E}-11$ & $6 \cdot E-13$ & 10.25 & 0.98 & 0.2045 & $2.25 E-11$ & $4.0 \mathrm{E}-12$ & 1.21 & 0.75 \\
\hline 8 & $\mathrm{COO} 1$ & - & 4.28 & $7.14 \mathrm{E}-11$ & $4 \cdot E-13$ & 9.27 & 1.48 & 0.2073 & $7.29 \mathrm{E}-12$ & 1. $3 \mathrm{E}-12$ & 1.10 & 0.61 \\
\hline 9 & Ramp & - & 0.54 & $2.82 \mathrm{E}-09$ & 2. E-12 & 9.09 & 0.09 & 0.3195 & $3.12 \mathrm{E}-09$ & $5.6 \mathrm{E}-10$ & 1.08 & 0.02 \\
\hline 10 & 1385. & 30. & 0.54 & $3.19 \mathrm{E}-09$ & 2. $E-12$ & 8.92 & 0.05 & 0.4467 & $5.91 \mathrm{E}-09$ & $1.1 \mathrm{E}-09$ & 1.06 & 0.02 \\
\hline 11 & 1385. & 30. & 1.08 & $4.04 \mathrm{E}-09$ & 3. $E-12$ & 8.91 & 0.06 & 0.6074 & $6.34 \mathrm{E}-09$ & $1.1 \mathrm{E}-09$ & 1.06 & 0.01 \\
\hline 12 & 1385. & 30. & 2.67 & $4.14 \mathrm{E}-09$ & 3. $E-12$ & 8.67 & 0.07 & 0.7725 & $4.84 E-09$ & 8. $6 E-10$ & 1.04 & 0.01 \\
\hline 13 & Melt & - & 0.54 & $5.71 \mathrm{E}-09$ & 4. $E-12$ & 8.34 & 0.04 & 1.0000 & & & & \\
\hline
\end{tabular}


Experiment \#5

69 Sal219 clinopyroxine

$500-710 u m$ sieve fraction, $29.9 \mathrm{mg}, \mathrm{r}=303 .(27)$

$23 / 83$ grains trapped in crack

Bulk helium contents: He $=2.14 \mathrm{E}-08 \mathrm{ccSTP} \quad 3 \mathrm{He} / 4 \mathrm{He}=8.66+0.08 \mathrm{xa}$

Sequential release data:

\begin{tabular}{|c|c|c|c|c|c|c|c|c|c|c|c|c|}
\hline \# & $\begin{array}{l}\text { Temp. } \\
\text { C }\end{array}$ & $\begin{array}{r}1 \mathrm{~s} \\
\mathrm{C}\end{array}$ & $\begin{array}{c}\text { Time } \\
\text { hr }\end{array}$ & $\begin{array}{c}4 \mathrm{He} \\
\operatorname{ccSTP}\end{array}$ & $\begin{array}{c}1 \mathrm{~s} \\
\operatorname{ccSTP}\end{array}$ & $\begin{array}{c}3 \mathrm{He} / 4 \mathrm{He} \\
\mathrm{Ra}\end{array}$ & $\begin{array}{l}1 \mathrm{~s} \\
\mathrm{Ra}\end{array}$ & $\begin{array}{l}F(4 \mathrm{He}) \\
(\operatorname{total})\end{array}$ & $\begin{array}{c}\text { Dsm } \\
\mathrm{cm} 2 / \mathrm{s}\end{array}$ & $\begin{array}{c}1 \mathrm{~s} \\
\operatorname{cm} 2 / \mathrm{s}\end{array}$ & $\begin{array}{c}\mathrm{D} 3 / \mathrm{D} 4 \\
\mathrm{~s} \mathrm{~m}\end{array}$ & $\begin{array}{l}1 \mathrm{~s} \\
\mathrm{sm}\end{array}$ \\
\hline 1 & Load & - & 0.53 & $1.17 \mathrm{E}-09$ & 4. E-12 & 8.24 & 0.13 & 0.0549 & 1. $30 \mathrm{E}-10$ & $2.3 E-11$ & 0.90 & 0.03 \\
\hline 2 & 965. & 10. & 0.54 & 4. $73 \mathrm{E}-10$ & 2. $E-12$ & 9.04 & 0.36 & 0.0770 & 1. $26 \mathrm{E}-10$ & 2. $2 \mathrm{E}-11$ & 1.01 & 0.07 \\
\hline 3 & 965. & 10. & 5.43 & $2.35 \mathrm{E}-09$ & 8.E-12 & 9.03 & 0.12 & 0.1870 & 1. $34 \mathrm{E}-10$ & 2. $4 \mathrm{E}-11$ & 1.05 & 0.03 \\
\hline 4 & 965. & 10. & 6.51 & $1.52 \mathrm{E}-09$ & $5 \cdot E-12$ & 8.47 & 0.12 & 0.2581 & 1. $32 \mathrm{E}-10$ & 2. $4 \mathrm{E}-11$ & 0.99 & 0.05 \\
\hline 5 & 965. & 10. & 7.60 & $1.15 \mathrm{E}-09$ & 5. E-12 & 8.86 & 0.16 & 0.3119 & $1.17 \mathrm{E}-10$ & $2.1 \mathrm{E}-11$ & 1.03 & 0.07 \\
\hline 6 & Melt & - & 1.63 & $1.47 \mathrm{E}-08$ & $6 . E-11$ & 8.64 & 0.10 & 0.9987 & & & & \\
\hline 7 & Left & - & 0.54 & $2.72 \mathrm{E}-11$ & 4. $E-13$ & 1.44 & 3.17 & 1.0000 & & & & \\
\hline
\end{tabular}

\section{Experiment \#6}

9 Sal219 clinopyroxine

$500-710$ um sieve fraction, $29.2 \mathrm{mg}, \mathrm{r}=303.127$

$7 / 50$ grains trapped in crack

Bulk helium contents: $\mathrm{He}=1.32 \mathrm{E}-08$ ccSTP $3 \mathrm{He} / 4 \mathrm{He}=8.77+0.04 \times \mathrm{Ra}$ Sequential release data:

\begin{tabular}{|c|c|c|c|c|c|c|c|c|c|c|c|c|}
\hline \# & $\begin{array}{l}\text { Temp. } \\
\text { C }\end{array}$ & $\begin{array}{r}1 \mathrm{~s} \\
\mathrm{C}\end{array}$ & $\begin{array}{c}\mathrm{Time} \\
\mathrm{hr}\end{array}$ & $\begin{array}{c}4 \mathrm{He} \\
\operatorname{ccSTP}\end{array}$ & $\begin{array}{c}1 \mathrm{~s} \\
\operatorname{ccSTP}\end{array}$ & $\begin{array}{c}3 \mathrm{He} / 4 \mathrm{He} \\
\mathrm{Ra}\end{array}$ & $\begin{array}{l}1 \mathrm{~s} \\
\mathrm{Ra}\end{array}$ & $\begin{array}{l}F(4 \mathrm{He}) \\
(\text { total })\end{array}$ & $\begin{array}{c}\text { Dsm } \\
\mathrm{cm} 2 / \mathrm{s}\end{array}$ & $\begin{array}{c}1 \mathrm{~s} \\
\operatorname{cm} 2 / \mathrm{s}\end{array}$ & $\begin{array}{c}\mathrm{D} 3 / \mathrm{D} 4 \\
\mathrm{sm}\end{array}$ & $\begin{array}{l}1 \mathrm{~s} \\
\mathrm{~s} \mathrm{man}\end{array}$ \\
\hline 1 & Load & - & 0.53 & $1.46 \mathrm{E}-09$ & 6. $E-12$ & 8.67 & 0.09 & 0.1106 & $5.42 \mathrm{E}-10$ & $9.7 \mathrm{E}-11$ & 0.98 & 0.02 \\
\hline 2 & 1070 . & 20. & 0.54 & $7.44 \mathrm{E}-10$ & 3. $E-12$ & 8.32 & 0.15 & 0.1671 & $7.24 \mathrm{E}-10$ & 1. $3 \mathrm{E}-10$ & 0.93 & 0.03 \\
\hline 3 & 1070 . & 20. & 5.43 & $3.59 \mathrm{E}-09$ & 1. $E-11$ & 9.00 & 0.07 & 0.4399 & $9.29 E-10$ & 1. $7 E-10$ & 1.02 & 0.02 \\
\hline 4 & 1070. & 20. & 8.69 & $2.19 \mathrm{E}-09$ & 9. $E-12$ & 8.81 & 0.10 & 0.6063 & $8.04 \mathrm{E}-10$ & 1. $4 \mathrm{E}-10$ & 1.02 & 0.02 \\
\hline 5 & 1070 . & 20. & 9.77 & 1. $25 E-09$ & 3. $E-12$ & 8.59 & 1) 16 & 0.7016 & $6.52 \mathrm{E}-10$ & 1. $2 \mathrm{E}-10$ & 0.99 & 0.03 \\
\hline 6 & Melt & - & 1.63 & $3.93 \mathrm{E}-09$ & 8. $E-12$ & 8.72 & 0.08 & 1.0000 & & & & \\
\hline
\end{tabular}


Experiment \#7

69 Sal219 clinopyroxine

$500-710 u m$ sieve fraction, $38.8 \mathrm{mg}, \mathrm{r}=303 .(27)$

Bulk helium contents: $\mathrm{He}=2.69 \mathrm{E}-08 \mathrm{ccSTP} \quad 3 \mathrm{He} / 4 \mathrm{He}=8.65+0.04 \times \mathrm{Ra}$

Sequential release data:

\begin{tabular}{|c|c|c|c|c|c|c|c|c|c|c|c|c|}
\hline \# & $\begin{array}{l}\text { Temp. } \\
\text { C }\end{array}$ & $\begin{array}{r}1 \mathrm{~s} \\
\mathrm{C}\end{array}$ & $\begin{array}{c}\mathrm{Time} \\
\mathrm{hr}\end{array}$ & $\begin{array}{c}4 \mathrm{He} \\
\text { CCSTP }\end{array}$ & $\begin{array}{c}1 \mathrm{~s} \\
\operatorname{ccSTP}\end{array}$ & $\begin{array}{c}3 \mathrm{He} / 4 \mathrm{He} \\
\mathrm{Ra}\end{array}$ & $\begin{array}{l}1 \mathrm{~s} \\
\mathrm{Ra}\end{array}$ & $\begin{array}{l}F(4 \mathrm{He}) \\
(\text { total })\end{array}$ & $\begin{array}{c}\text { Dsm } \\
\mathrm{Cm} 2 / \mathrm{s}\end{array}$ & $\begin{array}{c}1 \mathrm{~s} \\
\mathrm{~cm} 2 / \mathrm{s}\end{array}$ & $\begin{array}{c}\mathrm{D} 3 / \mathrm{D} 4 \\
\mathrm{~s} \text { m }\end{array}$ & $\begin{array}{l}1 \mathrm{~s} \\
\mathrm{sm}\end{array}$ \\
\hline 1 & Load & - & 0.53 & $1.64 \mathrm{E}-08$ & $6 \cdot E-11$ & 8.71 & 0.04 & 0.6097 & $2.42 \mathrm{E}-08$ & $4.3 \mathrm{E}-09$ & 1.02 & 0.01 \\
\hline 2 & 1170 . & 30. & 0.54 & $3.06 \mathrm{E}-09$ & 1. $E-11$ & 8.82 & 0.09 & 0.7234 & 1. $47 \mathrm{E}-08$ & $2.6 \mathrm{E}-09$ & 1.04 & 0.02 \\
\hline 3 & 1170. & 30. & 0.54 & $2.06 \mathrm{E}-09$ & 8. E-12 & 8.71 & 0.13 & 0.7999 & $1.48 \mathrm{E}-08$ & $2.6 \mathrm{E}-09$ & 1.04 & 0.03 \\
\hline 4 & 1170. & 30. & 2.17 & $2.90 \mathrm{E}-09$ & 1. E-11 & 8.47 & 0.11 & 0.9076 & $9.19 \mathrm{E}-09$ & 1. $6 \mathrm{E}-09$ & 1.02 & 0.01 \\
\hline 5 & 1170. & 30. & 5.43 & $1.63 \mathrm{E}-09$ & $6 \cdot E-12$ & 8.42 & 0.10 & 0.9681 & $5.06 \mathrm{E}-09$ & $9.0 \mathrm{E}-10$ & 1.04 & 0.02 \\
\hline 6 & 1170. & 30. & 6.64 & $5.60 \mathrm{E}-10$ & 1. E-12 & 7.70 & 0.19 & 0.9889 & $4.12 \mathrm{E}-09$ & 7. $3 E-10$ & 0.96 & 0.03 \\
\hline 7 & Melt & - & 24.00 & $2.98 \mathrm{E}-10$ & 8. E-13 & 8.21 & 0.24 & 1.0000 & & & & \\
\hline
\end{tabular}

Experiment \#8

69 SAL 219 Clinopyroxene

$.5-1 . \mathrm{mm}$ sieve fraction, $72.1 \mathrm{mg}, \mathrm{r}=375(125) \mathrm{um}$

Sample in Ta boat at $770 \mathrm{C}, 100 \mathrm{se}$ at $1110 \mathrm{C}$

Bulk helium contents: He $=4.47 \mathrm{E}-08$ c cSTP $3 \mathrm{He} / 4 \mathrm{He}=8.68+0.03 \times \mathrm{Ra}$ Sequential release data:

\begin{tabular}{|c|c|c|c|c|c|c|c|c|c|c|c|c|}
\hline \# & $\begin{array}{l}\text { Temp. } \\
\text { C }\end{array}$ & $\begin{array}{r}1 \mathrm{~s} \\
\mathrm{C}\end{array}$ & $\begin{array}{c}\text { Time } \\
\text { hr }\end{array}$ & $\begin{array}{c}4 \mathrm{He} \\
\operatorname{ccSTP}\end{array}$ & $\begin{array}{c}1 \mathrm{~s} \\
\operatorname{ccsTP}\end{array}$ & $\begin{array}{c}3 \mathrm{He} / 4 \mathrm{He} \\
\mathrm{Ra}\end{array}$ & $\begin{array}{l}1 \mathrm{~s} \\
\mathrm{Ra}\end{array}$ & $\begin{array}{l}F(4 \mathrm{He}) \\
(\operatorname{tota} l)\end{array}$ & $\begin{array}{c}\text { Ds m } \\
\operatorname{cm} 2 / s\end{array}$ & $\begin{array}{c}1 \mathrm{~s} \\
\mathrm{~cm} 2 / \mathrm{s}\end{array}$ & $\begin{array}{l}\mathrm{D} 3 / \mathrm{D} 4 \\
\mathrm{~s} \text { m }\end{array}$ & $\begin{array}{l}1 \mathrm{~s} \\
\mathrm{sin}\end{array}$ \\
\hline 1 & Load & - & 0.53 & $4.67 \mathrm{E}-10$ & 1. $E-12$ & 6.70 & 0.30 & 0.0104 & $6.97 \mathrm{E}-12$ & $4.6 \mathrm{E}-12$ & 0.60 & 0.05 \\
\hline 2 & 770. & 20. & 2.57 & $2.95 \mathrm{E}-10$. & 1. $E-12$ & 7.78 & 0.45 & 0.0170 & $2.41 \mathrm{E}-12$ & 1. $6 \mathrm{E}-12$ & 0.72 & 0.08 \\
\hline 3 & 770. & 20. & 2.69 & $1.63 \mathrm{E}-10$ & 6. $E-13$ & 8.75 & 0.52 & 0.0207 & 1. $74 \mathrm{E}-12$ & 1. $2 \mathrm{E}-12$ & 0.84 & 0.14 \\
\hline 4 & 770. & 20. & 2.69 & 1. $35 \mathrm{E}-10$ & 5. $E-13$ & 8.77 & 0.63 & 0.0237 & $1.69 \mathrm{E}-12$ & 1. $1 \mathrm{E}-12$ & 0.87 & 0.18 \\
\hline 5 & Ramp & - & 21.57 & $7.06 \mathrm{E}-09$ & $3 \cdot E-11$ & 8.78 & 0.05 & 0.1817 & $5.69 \mathrm{E}-11$ & 3. $8 \mathrm{E}-11$ & 0.99 & 0.01 \\
\hline 6 & 1110. & 20. & 0.53 & $3.12 \mathrm{E}-09$ & 1. E-11 & 8.75 & 0.07 & 0.2514 & $2.36 \mathrm{E}-09$ & $1.6 \mathrm{E}-09$ & 1.00 & 0.02 \\
\hline 7 & 1110. & 20. & 1.62 & $5.22 \mathrm{E}-09$ & 2. $E-11$ & 8.79 & 0.07 & 0.3681 & $2.05 \mathrm{E}-09$ & 1. $4 \mathrm{E}-09$ & 1.01 & 0.02 \\
\hline 8 & 1110. & 20. & 1.08 & $2.47 \mathrm{E}-09$ & $9 . E-12$ & 8.87 & 0.11 & 0.4233 & $2.05 \mathrm{E}-09$ & 1. $4 \mathrm{E}-09$ & 1.03 & 0.03 \\
\hline 9 & 1110. & 20. & 1.08 & $1.89 \mathrm{E}-09$ & 7. $E-12$ & 8.78 & 0.09 & 0.4655 & $1.87 \mathrm{E}-09$ & 1. $2 \mathrm{E}-09$ & 1.02 & 0.04 \\
\hline 10 & 1110. & 20. & 4.85 & $5.81 \mathrm{E}-09$ & 2. $E-11$ & 8.76 & 0.07 & 0.5954 & $1.76 \mathrm{E}-09$ & 1. $2 \mathrm{E}-09$ & 1.02 & 0.02 \\
\hline 11 & 1110. & 20. & 4.85 & $3.34 \mathrm{E}-09$ & 1. $E-11$ & 8.83 & 0.11 & 0.6700 & $1.45 \mathrm{E}-09$ & $9.7 \mathrm{E}-10$ & 1.03 & 0.03 \\
\hline 12 & 1110. & 20. & 5.55 & $2.31 \mathrm{E}-09$ & 3. E-12 & 8.71 & 0.10 & 0.7217 & $1.12 \mathrm{E}-09$ & 7. $5 \mathrm{E}-10$ & 1.02 & 0.03 \\
\hline 13 & Cool & - & 4.22 & $9.92 \mathrm{E}-11$ & 4. $E-13$ & 9.18 & 0.62 & 0.7239 & $7.05 E-11$ & 4. $7 E-11$ & 1.08 & 0.73 \\
\hline 14 & Melt & - & 6.17 & 1. $23 \mathrm{E}-08$ & 7. $E-12$ & 8.52 & 0.06 & 1.0000 & & & & \\
\hline
\end{tabular}


APPENDIX D: Finite difference determination of the fraction of helium remaining in a spherical grain undergoing constant production and volume diffusion loss. 
For a mineral grain undergoing both diffusive loss and constant production by cosmic ray spallation the helium concentration is governed by :

$$
\mathrm{dC} / \mathrm{dt}=\mathrm{D} \nabla^{2} \mathrm{C}+\mathrm{P}
$$

where $P$ is the production rate (per unit volume). The fraction of produced helium which remains at time $t$ can then be written:

$$
\mathrm{F}_{\mathrm{t}}=\mathrm{C} / \mathrm{Pt}=\left\{\mathrm{Pt}-\int_{0}^{\mathrm{t}}(\mathrm{dC} / \mathrm{dt}) \mathrm{dt}\right\} / \mathrm{Pt}
$$

Equation 5.1 was solved numerically using an explicit, spatially centered, finite-difference approach to obtain concentration, C, as a function of time and radial distance within the quartz grains. $\mathrm{F}_{\mathrm{t}}$ was then obtained by integrating these curves over the crystal volume (these routines are provided, in Fortran 77, in the program "Findif". The numerical solution was verified by comparison to an infinite series analytical solution for $F_{t}$ (Tilton, 1960) and has the advantage of easily determining concentration profiles. 


\section{Program Findif}

c Tom Trull Nov. 14, 1988

c Findif uses an explicit, spatially-centered finite difference

c algorithm to obtain the time-dependent solution for a sphere

c undergoing constant production and volume diffusion loss of

c 3 He. It calculates a radial concentration profile as a function

c

of time which is then integrated to obtain the helium content of the crystal. As with diffusion alone, the problem can be scaled with $\mathrm{D} / \mathrm{a} 2$, where $\mathrm{D}$ is the diffusion coefficient and a the radius of the sphere, i.e. the solution is independent of production rate.

Parameter (ngrid $=500$, ngridp $=501$, daysec $=86400$, , yrday $=365 ., p i=3.14159$ ) Dimension cold(ngridp), cnew(ngridp)

Open (uni $t=1$, file $={ }^{\prime}$ FDFp12. dat ${ }^{\prime}$, status $={ }^{\prime}$ new' $)$

Difco $=5 . E-20$

rho $=2.5$

Pgy $=360$.

d $\quad \mathrm{Pgy}=0$

Pvs $=$ Pgy/yrday/daysec/rho $\mathrm{a}=.0250$

c

vspher $=4 \cdot / 3 . * p i * a * * 3$

yrs $=1 . E+06$

diftim=daysec*yrday*yrs nlooks $=4$

$\mathrm{dr}=\mathrm{a} /($ ngrid -1$)$

$\mathrm{dt}=\mathrm{dr} * * 2 / \mathrm{Difco} / 100$.

$\mathrm{dc}=\mathrm{Difco} * \mathrm{dt} / \mathrm{dr} * * 2$

nts tep $=\mathrm{diftim} / \mathrm{dt} /$ nlooks
!Diffusion coefficient in $\mathrm{cm} 2 / \mathrm{s}$. !density in $\mathrm{g} / \mathrm{cc}$

!production rate in atoms/gr/yr

!zero production test option

! production rate in atoms $/ \mathrm{cc} / \mathrm{s}$

!radius in $\mathrm{cm}$

!crystal volume

! time in years

! time in seconds

!\# looks at profile during diftim

!grid spacing

!numeric stability

!flux increment

!\# of time steps between looks

c

C

Write $(5, *)$ diftim, ntstep, dt, dr, dc, Pvs

d

Do $50 \mathrm{nr}=1$, ngrid

! boundary conditions

$\operatorname{cold}(\mathrm{nr})=0$.

50 $\operatorname{cold}(\mathrm{nr})=1$ cnew $(\mathrm{nr})=0$.

!0 prod., diffusive loss option

Continue

$\operatorname{cold}($ ngrid +1$)=0$.

c cnew (ngrid +1$)=0$.

Do 300 nlook=1, nlooks

Do $200 \mathrm{nt}=1$, ntstep

c

Do $100 \mathrm{nr}=2$, ngrid $\operatorname{cnew}(n r)=\operatorname{cold}(n r)+d c / n r$

$\&$

$\&$

Continue

$$
*((n r+1) * \operatorname{cold}(n r+1)-2 * n r * \operatorname{col} d(n r)+(n r-1) * \operatorname{cold}(n r-1))
$$

$+\mathrm{Pvs} * \mathrm{dt}$ 


$$
\operatorname{cnew}(1)=\operatorname{cold}(1)+6 * d c *(\operatorname{cold}(2)-\operatorname{cold}(1))+\mathrm{Pvs} * d t \quad ! \text { center point }
$$

C

150 Continue

Do $150 \mathrm{nr}=1$, ngrid $\quad$ !reset old and new, step again

$\mathrm{C}$

200 Continue

$\mathrm{C}$

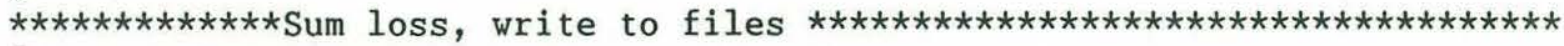

C

time $=($ nlook $) *$ ntstep $*$ dt $/$ daysec/yrday $\quad$ !years

write $(1,1000)$ nlook, time totrem=0.

C

Do $250 \mathrm{nr}=1$, ngrid !use conc. at shell midpoint to integrate $\operatorname{posn}=(\mathrm{nr}-1) * \mathrm{dr}$

fposn=posn $/ \mathrm{a}$

vshell $=4 . / 3 . * \mathrm{pi} *(((\mathrm{nr}-1) * \mathrm{dr}+.5 * \mathrm{dr}) * * 3-((\mathrm{nr}-1) * \mathrm{dr}-.5 * \mathrm{dr}) * * 3)$

IF $(\mathrm{nr}$. eq. 1$) \mathrm{vshel}=4 . / 3 . * \mathrm{pi} *(.5 * \mathrm{dr}) * * 3$

IF (nr.eq.ngrid) vshell $=4 . / 3 . * p i *$

\& $\quad(((n r-1) * d r) * * 3-((n r-1) * d r-.5 * d r) * * 3)$

d cnew $(\mathrm{nr})=1$

amoun $\mathrm{t}=\mathrm{cnew}(\mathrm{nr}) * \mathrm{vshell}$

totrem $=$ to trem+amount

!volume integration test option

C

write $(5, *)(n r-1) * d r$, totrem, amount, vshell, cnew(nr)

write $(1,2000) \mathrm{nr}$, posn, cnew $(\mathrm{nr}) /($ Pvs*nlook*ntstep*dt) write $(1,2000) n r$, Fposn, cnew(nr) $! 0$ prod. option

250 Continue

Totprd=Pvs*(nlook*ntstep*dt)*vspher ! total prod. in sphere

flost $=1 .-($ totrem/totprd $)$

d flost $\quad$ 1.-( totrem/vspher $)$

IF (flost.lt.(0.00001)) THEN

10 prod. option $\mathrm{flos} \mathrm{t}=0$.

ENDIF

write $(5, *)$ time, totprd, vspher, totrem, flost

C

write $(1,3000)$ time, flost

300 Continue

$\mathrm{C}$

1000 Format $\left(1 \mathrm{x},{ }^{\prime}\right.$ findif output', $/, 1 \mathrm{x},{ }^{\prime}$ nlook $={ }^{\prime}, \mathrm{I} 3,2 \mathrm{x},{ }^{\prime}$ years $\left.={ }^{\prime}, \mathrm{F} 10.1\right)$

2000 Format (1x, I4, 1x, F10.5, 1x, F10.5)

3000 Format $\left(1 \mathrm{x},{ }^{\prime}\right.$ years $={ }^{\prime}, \mathrm{F} 10.1,1 \mathrm{x},{ }^{\prime}$ flost $=$ ', F8.4)

Stop

End 